\title{
MWTF Jumper Connector Integral Seal Block Development and Lealk Testing
}

Prepared for the U.S. Department of Energy Office of Environmental Restoration and Waste Management

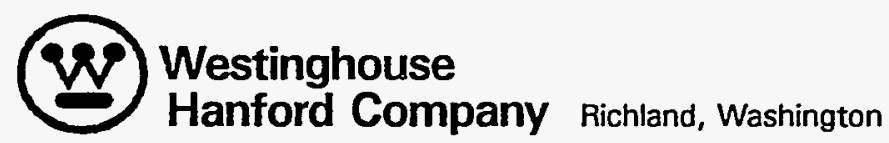

Henford Operations and Engineering Contractor for the

U.S. Department of Energy under Contract DE-AC06-87RL10930

Approved for Public Release 
DISTRIBUTION SHEET

\begin{tabular}{l|l|l}
\hline To & From \\
Distribution & E. S. Ruff & Page 1 of 2 \\
\cline { 2 - 3 } $\begin{array}{l}\text { Project Title/Work Order } \\
\text { WHC-SD-WM-TRP-223 REV O, MWTF Jumper Connector Integral Sea] } \\
\text { Block Development and Leak Testing }\end{array}$ & EDT No. 608777 \\
\cline { 2 - 3 } & ECN No. N/A \\
\hline
\end{tabular}

\begin{tabular}{|c|c|c|c|c|c|}
\hline Name & MSIN & $\begin{array}{c}\text { Text } \\
\text { With All } \\
\text { Attach. }\end{array}$ & Text Only & $\begin{array}{c}\text { Attach./ } \\
\text { Appendix } \\
\text { Only }\end{array}$ & $\begin{array}{c}\text { EDT/ECN } \\
\text { Only }\end{array}$ \\
\hline $\begin{array}{l}\text { M. M. Ahmed } \\
\text { E. G. Allen III (2) } \\
\text { D. L. Becker } \\
\text { A. W. Bjorkedal } \\
\text { L. D. Blackburn } \\
\text { P. J. Brackenbury } \\
\text { L. J. Bray } \\
\text { H. H. Byers } \\
\text { H. C. Carlos } \\
\text { J. R. Collins } \\
\text { J. E. Conner } \\
\text { T. J. Conrads } \\
\text { D. L. Cooley } \\
\text { V. J. Cruz (5) } \\
\text { S. K. Farnworth } \\
\text { P. Felise } \\
\text { J. C. Fordham } \\
\text { R. L. Fritz } \\
\text { J. E. Geary } \\
\text {.E. Hal1 } \\
\text { J. L. Henderson } \\
\text { F. H. Huang } \\
\text { M. T. Husain } \\
\text { D. L. Isakson } \\
\text { M. A. Islam } \\
\text { C. D. Jones } \\
\text { S. R. Jordan (3) } \\
\text { J. L. Julyk } \\
\text { S. K. Kanjilal } \\
\text { D. L. Koreis } \\
\text { D. B. Lichy } \\
\text { J. M. Light } \\
\text { T. L. Moore, Jr. } \\
\text { T. L. Moore, Sr. } \\
\text { J. R. Nichoison } \\
\text { E. C. Ocoma } \\
\text { T. C. Oten } \\
\text { M. B. Palm } \\
\text { R. J. Parazin } \\
\text { R. D. Pierce } \\
\text { R. S. Popielarczyk } \\
\text { M. A. Przyby Tski }\end{array}$ & $\begin{array}{l}\text { B4-40 } \\
G 6-02 \\
H 5-57 \\
B 4-40 \\
H 5-67 \\
G 6-06 \\
T 2-08 \\
T 2-08 \\
H 5-67 \\
E 6-22 \\
T 2-10 \\
H 5-55 \\
X 3-76 \\
B 4-08 \\
H 5-55 \\
G 6-06 \\
\text { N1-32 } \\
B 4-08 \\
S 5-01 \\
E 6-25 \\
E 6-22 \\
H 5-67 \\
E 6-37 \\
T 2-10 \\
H 5-55 \\
S 3-07 \\
L 6-13 \\
H 5-55 \\
H 5-57 \\
H 5-53 \\
S 5-66 \\
B 4-08 \\
R 2-82 \\
H 5-09 \\
E 6-37 \\
H 5-55 \\
E 6-50 \\
H 5-67 \\
H 5-49 \\
H 5-11 \\
R 1-30 \\
B 4-40\end{array}$ & $\begin{array}{l}x \\
x \\
x \\
x \\
x \\
x \\
x \\
x \\
3 \\
x \\
x \\
x \\
x \\
x \\
\\
x \\
x \\
x\end{array}$ & $\begin{array}{l}X \\
X \\
x \\
X \\
X \\
X \\
X \\
x \\
X\end{array}$ & & \\
\hline
\end{tabular}


Page 2 of 2

\begin{tabular}{c|c|c|c|c|c|c}
\hline Name & MSIN & $\begin{array}{c}\text { Text } \\
\text { With All } \\
\text { Attach. }\end{array}$ & Text Only & $\begin{array}{c}\text { Attach./ } \\
\text { Appendix } \\
\text { Only }\end{array}$ & $\begin{array}{c}\text { EDT/ECN } \\
\text { Only }\end{array}$ \\
\hline
\end{tabular}

R. E. Raymond

E. J. Renkey

H. L. Roach

H. E. Ross

E. S. Ruff (6)

R. E. Russe11

T. B. Salzano

J. B. Truitt

E. B. Schwenk

H. P. Shrivastava

J. J. Sisk

D. M. Squier

J. F. Thompson

T. A. Tomaszewski

R. N. Wagner

E. N. Wegener

E. O. Weiner

OSTI (2)

TWRS/DPC (2)

W236A Document Control
R2-54

$\mathrm{H} 5-72$

$\mathrm{H} 5-72$

S5-07

H5-67

H5-70

E6-37

H5-56

H5-52

H5-55

S2-03

L6-13

H5-70

H5-72

L4-96

L6-37

H5-55

L8-07

L8-04

G6-51

E6-50 $x$

$\begin{array}{ll}X & \\ X & X \\ 3 & 3 \\ X & \\ X & \\ X & X \\ & X \\ & X \\ & \\ & \\ & \end{array}$

$X$
$X$

X

$x$

$X$
$X$

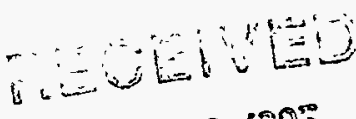

APR 03 13:3

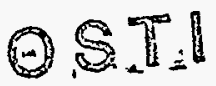

$x$
$X$
$X$

$x$

$x$
$x$
$x$ 


\section{DISCLAIMER}

Portions of this document may be illegible in electronic image products. Images are produced from the best available original document. 


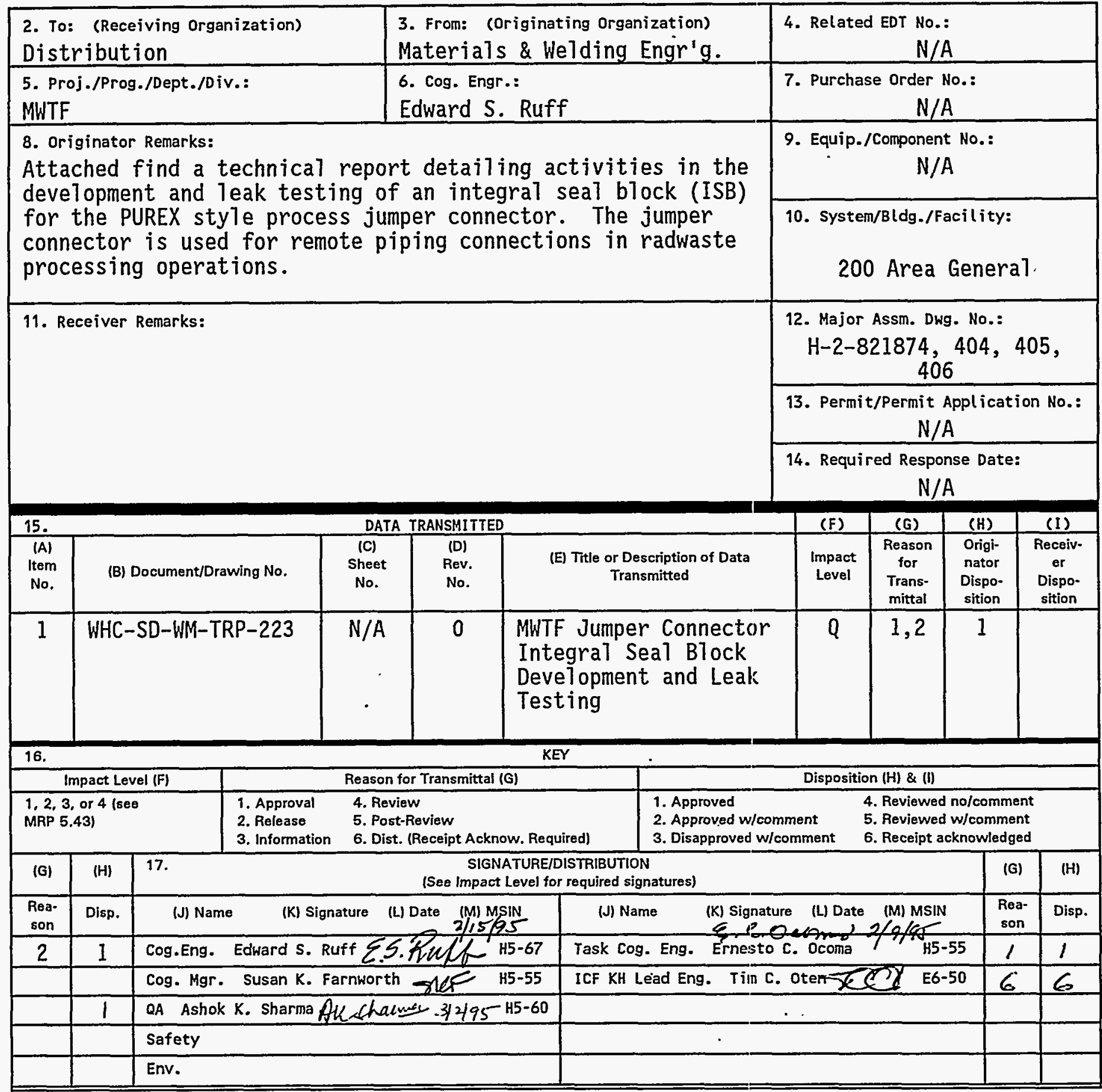

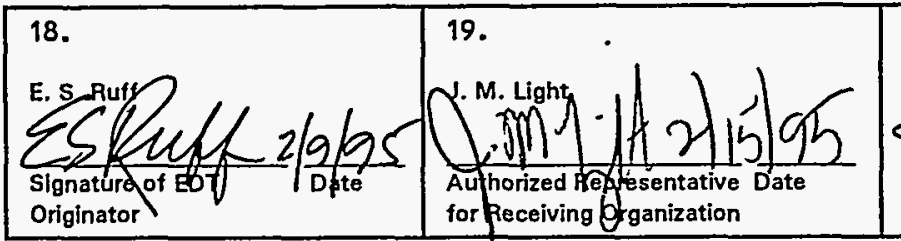

BD-7400-172-2 (07/91) GEF097

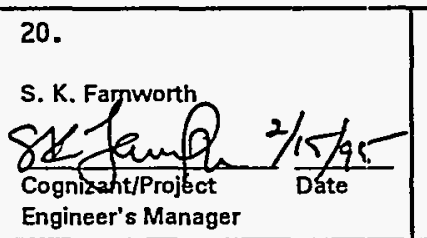

21. DOE APPROVAL (if required)

Ltr. No. N/A

[] Approved

[] Approved w/comments

[] Disapproved w/comments 
2. Title

MWTF Jumper Connector Integral Seal Block Development and Leak Testing

5. Key Words

Jumper, Connector, PUREX Connector, Gasket

Integral Seal BTock, BTock

o-Ring, Seal, Seal Development, 0-Ring Seal

3. Number

HHC-SD-WM-TRP-223

4. Rev No.

0
6. Author

Name: Edward S. Ruff

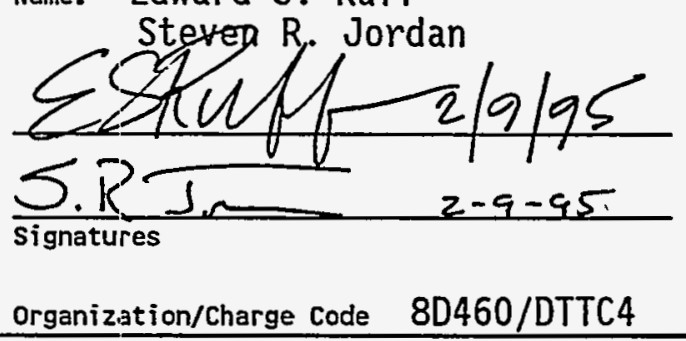

7. Abstract

This technical report summarizes activities and tests for the development of an integral seal block for the PUREX style process jumper connector. The integral seal block has greater resistance to leakage, is easier to manufacture, and has fewer parts than the standard seal.

8. RELEASE STAMP

OFFICIALPE:ESSE

EYWS

DATE

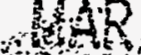


WHC-SD-WM-TRP-223

Rev. 0

\section{MWTF JUMPER CONNECTOR INTEGRAL SEAL BLOCK}

DEVELOPHENT AND LEAK TESTING

January 1995

Edward S. Ruff

\section{ABSTRACT}

In fiscal year 1993, tests of an o-ring/tetraseal retainer designed to replace a gasket-type seal used in PUREX-type process jumper connectors encouraged the design of an improved seal block. This new seal block combines several parts into one unitized component called an integral seal block.

This report summarizes development and leak testing of the new integral seal block. The integral seal block uses a standard o-ring nested in a groove to accomplish leak tightness. This seal block eliminates the need to machine acme threads into the lower skirt casting and seal retainers, eliminates tolerance stack-up, reduces parts inventory, and eliminates an unnecessary leak path in the jumper connector assembly.

This report also includes test data on various types of o-ring materials subjected to heat and pressure. Materials tested included Viton, Kalrez-1, and fluorosilicone, with some incidental data on teflon coated silicone o-rings. Test experience clearly demonstrates the need to test each seal material for temperature and pressure in its intended application. Some materials advertised as being "better" at higher temperatures did not perform up to expectations.

Inspection of the fluorosilicone and Kalrez seals after thermal testing indicates that they are much more susceptible to heat softening than Viton.

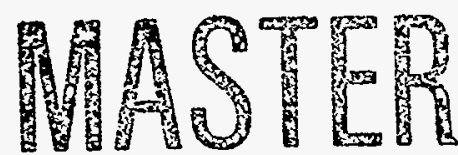

${ }^{1}$ Kalrez is a registered trademark of E.I. du Pont cle Nemours and Company, Wilmington, Delaware. 
WHC-SD-WM-TRP-223

Rev. 0

The specified purpose of this series of tests (per WHC-SD-WM-TP-256 REV 0 , "Jumper Connector Integral Seal Block Test Plan and Procedure") was to:

1) To investigate the effect of clamping force on ISB jumper connector leak integrity.

2) To investigate the effect of externally applied loads and moments on the leak resistance of ISB jumper connector assemblies.

3) To determine the effect of temperature and pressure on the leak resistance of ISB jumper connector seals.

4) To determine the seal compression properties of o-ring seals used in the ISB jumper connector assemblies.

5) To qualify the ISB jumper connector and various o-ring elastomers for plant service.

Objectives 1 thru 4 have been accomplished. Objective 5, qualification by test of the ISB jumper connector and various o-ring elastomers for plant service, encompasses many other areas beyond the scope of this report. These may include: radiation testing, chemical testing, corrosion testing, destructive testing, and seismic testing. This test report pertains only to leak testing under conditions of temperature, pressure, and applied moments. These leak tests were performed at temperatures and pressures that exceed operating conditions stated in the design criteria for the MWTF project.

Radiation and chemical tests of various o-ring polymers were not performed because no funds were available to do so. Hence, chemical resistance to MWTF process fluids, and radiation resistance of seal materials are not specifically addressed in this leak test report. However, the 3 primary oring seal materials tested (Viton, Kalrez, and Fluorosilicone) were selected on an overview basis - that is, general chemical resistance to either water or highly caustic solutions expected to be found in the tank waste. Kalrez was selected for testing primarily for it's advertised combined chemical and radiation resistance to $10^{\underline{8}}$ rads..

Nonetheless, for each specific MWTF process piping application, the piping system designer should refer to vendor data on chemical and radiation resistance of Viton, Fluorosilicone, or Kalrez, and verify seal material selection for compatibility with chemical and radiation service conditions. 
WHC-SD-WM-TRP-223

Rev. 0

Where possible, the piping designer should try to use Viton, Kalrez, or Fluorosilicone as they have been leak tested at temperature and pressure in the laboratory, in the ISB jumper configuration.

Moment loads used during this leak testing may not envelope those loads anticipated for some MWTF jumpers. There are two reasons for this: 1) design moment loads for MWTF jumper connectors were not available prior to the start of leak testing, and, 2) moment loads applied during testing were limited by the capacity of the existing test equipment. To help assure leak-tightness in actual plant operating systems, the piping designer should strive to keep design moments within the limits shown effective by this testing.

\title{
DISCLAIMER
}

This report was prepared as an account of work sponsored by an agency of the United States Government. Neither the United States Government nor any agency thereof, nor any of their employees, makes any warranty, express or implied, or assumes any legal liability or responsibility for the accuracy, completeness, or usefulness of any information, apparatus, product, or process disclosed, or represents that its use would not infringe privately owned rights. Reference herein to any specific commercial product, process, or service by trade name, trademark, manufacturer, or otherwise does not necessarily constitute or imply its endorsement, recommendation, or favoring by the United States Government or any agency thereof. The views and opinions of authors expressed herein do not necessarily state or reflect those of the United States Government or any agency thereof.

\section{DISTRIRUTION OF THIS DOCUMENT IS UNLIMITEOS $\mathrm{i} / \mathrm{V}$}

\author{
Westinghouse Hanford Company \\ P.0. Box 1970 \\ Richland, Washington 9935
}


WHC-SD-WM-TRP-223

Rev. 0

Document Tit7e: WHC-SD-WM-TRP-223

MWTF JUMPER CONNECTOR

INTEGRAL SEAL BLOCK

DEVELOPMENT AND LEAK TESTING

Multi-Function Waste Tank Facility Program (MWTF)

Prepared by:

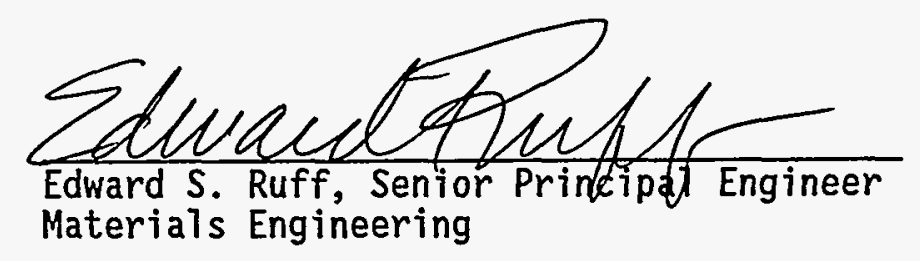

$\frac{\text { Fen. 9,1995 }}{\text { Date }}$

Edward S. Ruff, Senior Printelpal Engineer
Materials Engineering

Assisted by:

$S 2 R \overline{J_{n}}$

Steven R. Jordan, Test Technician

Equipment Testing Laboratory

Reviewed by:

Approved by:
Vuite \& Cur

Victor . Cruz, Rog. Project Engineer MWTF Project Engineering

E.e.Oeoma

Ernesto C. Ocoma, Task Cog. Engineer

Engineering Analysis, Structural Assessments

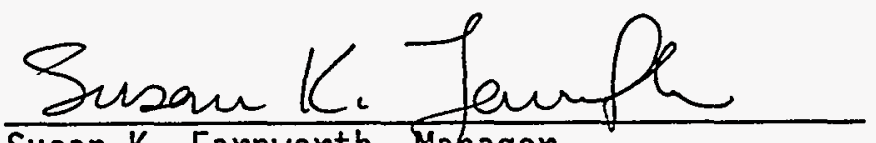

Susan K. Farnworth, Mahager

Engineering Analysis, Structural Assessments

Larry D. Blackburn, Acting Manager

Materials Engineering

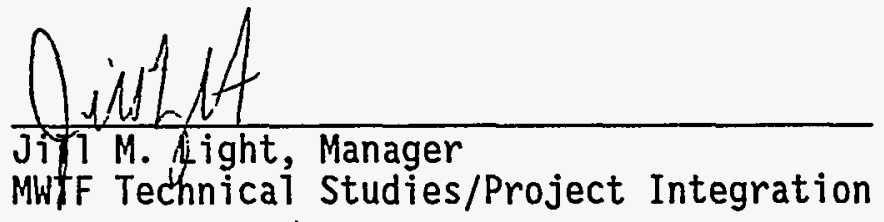

$\frac{2-9-95}{\text { Date }}$

$\frac{2-15-95}{\text { Date }}$

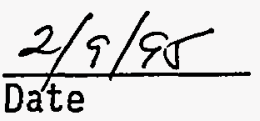

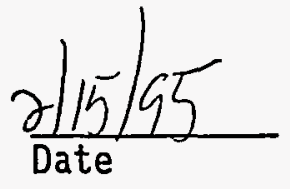


WHC-SD-WM-TRP-223

Rev. 0

\section{ACKNOHLEDGEMENTS}

The contribution of the following individuals in integral seal block development is gratefully acknowledged:

Steven R. Jordan, Test Technician, Equipment Testing Laboratory, who built the test equipment and performed these tests. Steve persevered through many difficulties. He made many key observations and contributions throughout the test program. The graphs in the data appendix indicate the extent of his labor, his dedication, and his achievement.

Robert D. Pierce, Designer, who prepared the design/fabrication blueprints for the integral seal block components and test fixtures. Bob's design talent and patient thoroughness were invaluable. He also did the initial three-dimensional computer modeling of the one-piece-body connector concept.

Larry J. Robinson, Design Services Checker, who performed an exacting and exhaustive review of the component fabrication drawings to ensure accurate fit, function, and compliance to drafting standards.

Harold Byers, Larry Bray, Gordon Cox, and Farrell Coulsion, Developmental Machinists, who developed the computer numerical control (CNC) machining programs and fabricated the prototype integral seal block retainers. They overcame many difficulties to produce the unique machining shapes required.

Victor J. Cruz, Susan K. Farnworth, and Thomas J. Conrads, MWTF Project Engineering and Engineering Services, who organized several key project meetings that enabled acceptance of the ISB concept in the engineering design community.

Robert L. Fritz, MWTF Project Manager, and Jill M. Light, Manager of MWTF Technical Studies, who early-on gave approval to proceed with ISB development and granted resources for fabrication and testing of the prototype. 
WHC-SD-WM-TRP-223

Rev. 0

CONTENTS

GLOSSARY

ix

1.0 MWTF ISB LEAK TESTING: PROJECT ORGANIZATION AND ADMINISTRATION • . 1

2.0 INTEGRAL SEAL BLOCK DEVELOPMENT: BACKGROUND INFORMATION . . . . . 2

2.1 SEAL COSTS ....................... . . . 2

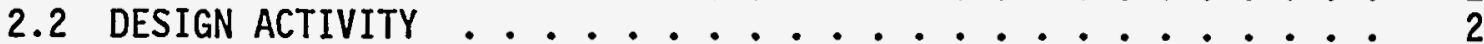

2.2.1 Tasks Carried Over from FY 1993 .......... . 2

2.2.2 Single-Port Connectors ............ 5

2.2.3 Three-way Connectors ............. 7

3.0 DESIGN CONSIDERATIONS, INTEGRAL SEAL BLOCK .......... 9

3.1 INTERCHANGEABILITY ..................... 9

3.2 LEAK PATHS ....................... . . 9

3.3 PRESSURIZATION ................... . . 9

3.4 MINIMAL STRETCH ................... 9

3.5 SEAL GROOVE ................... 9

4.0 DESIGN REQUIREMENTS AND TEST CONDITIONS . . . . . . . . . . 11

5.0 INTEGRAL SEAL BLOCK PROCUREMENT ACTIVITIES . . . . . . . . . 13

5.1 SEAL PROCUREMENT . . . . . . . . . . . . . . . . 13

5.2 CONNECTOR HARDWARE AND MISCELLANEOUS TEST EQUIPMENT $\ldots . \cdots 13$

6.0 STRESS ANALYSIS SUPPORT ACTIVITY . . . . . . . . . . 18

7.0 INTEGRAL SEAL BLOCK MACHINING ................ 19

8.0 EQUIPMENT TESTING LABORATORY - INITIAL SETUP . . . . . . . . 22

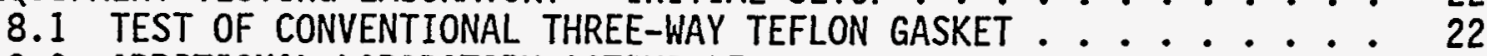

8.2 ADDITIONAL LABORATORY ACTIVITIES ........... 26

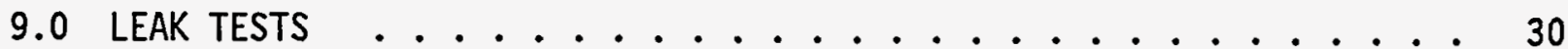

9.1 2-IN. ISB 0-RING SEAL ................ 30

9.23 -IN. ISB O-RING SEAL ................ 34

9.34 -IN. ISB 0 -RING SEAL .............. 37

9.43 -WAY ISB O-RING SEAL .................. 40

10.0 CONCLUSIONS . . . . . . . . . . . . . . . . . 42

10.1 ADVANTAGES OF INTEGRAL SEAL BLOCK DESIGN FOR JUMPER
CONNECTORS $\ldots \ldots . .$.

10.2 ENABLING TECHNOLOGY FOR REMOTE APPLICATIONS . . . . . . . . . 43

10.3 O-RING CROSS SECTION ................. 43

10.4 SEAL DUROMETER ................... 43

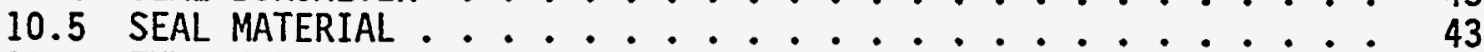

10.6 FUTURE IMPROVED DESIGN - JUMPER CONNECTORS WITH ONEBODIES ...................... 45

10.7 RECOMMENDATIONS FOR FUTURE DEVELOPMENT WORK ON JUMPER CONNECTORS ..................... 49

11.0 REFERENCES .......................... 50 
APPENDIX A: PLANNING CHARTS ...................... A-1 APPENDIX B: DRAWINGS FOR 2-, 3-, and 4-IN. AND THREE-HAY

INTEGRAL SEAL BLOCKS ................... B-1

APPENDIX C: DRAWINGS FOR JUMPER CONNECTOR LEAK-TEST FIXTURE . . . . c-1

APPENDIX D: DRAWINGS FOR JUMPER CONNECTOR BLOCK MOMENT ARM : . . . . D-1

APPENDIX E: GRAPHS OF 2-IN. FLUROSILICONE TESTS ......... E-1

APPENDIX F: GRAPHS OF 2-IN. KALREZ TESTS ........... F-1

APPENDIX G: GRAPHS OF 2-IN. VITON TESTS ............ G-1

APPENDIX H: GRAPHS OF 3 -IN. FLUOROSILICONE TESTS ......... . H-1

APPENDIX I： GRAPHS OF 3 -IN. KALREZ TESTS ........... I-1

APPENDIX J: GRAPHS OF $3-I N$. VITON TESTS ........... . J-1

APPENDIX K: GRAPHS OF 4-IN. FLUOROSILICONE TESTS . . . . . . . . K-1

APPENDIX L: GRAPHS OF 4 -IN. KALREZ TESTS ............. . L-1

APPENDIX M: GRAPHS OF 4 -IN. VITON TESTS ............ . . . . .

APPENDIX N: GRAPHS OF 4-IN. TFE-0-SIL TESTS $\ldots \ldots \ldots$

APPENDIX 0: GRAPHS OF THREE-WAY FLUOROSILICONE TESTS . . . . . . . 0-1

APPENDIX P: GRAPHS OF THREE-WAY KALREZ TESTS . . . . . . . . . P-1

APPENDIX Q: GRAPHS OF THREE-WAY VITON TESTS .......... Q Q

APPENDIX R: GRAPHS OF THREE-WAY SILICONE TESTS .......... R-1

APPENDIX S: GRAPHS OF THREE-WAY EPDM TESTS ........... . S-1

APPENDIX T: GRAPHS OF THREE-WAY CONVENTIONAL TEFLON GASKET TESTS . . . T-1

APPENDIX U: STATEMENT ATTESTING TO TEST PROCEDURES USED AND DATA CHECKS

PERFORMED ....................... U-

APPENDIX V: SOURCE REFERENCES FOR TEST CRITERIA . . . . . . . V-1 
WHC-SD-WM-TRP-223

Rev. 0

\section{LIST OF FIGURES}

Figure 1. Jumper Connector AssembTy. . . . . . . . . . . 6

Figure 2. Project Design Conditions Used to Determine Leak Test

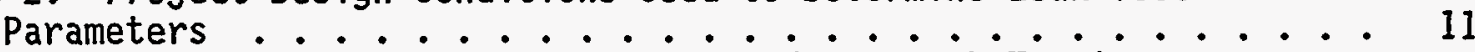

Figure 3. Applied Moments and Pressures Used For Leak Testing

ISB Jumper Connectors ................. 12

Figure 4. Schematic for Process Jumper Connector Test Loop. . . . . 16

Figure 5. Results of Leak Tests on 2-In. Integral Seal BTock . . . . . 31

Figure 6 . Results of Leak Tests on 3-In. Integral Seal Block . . . 35

Figure 7. Results of Leak Tests on 4-In. Integral Seal Block .... 39

Figure 8. Results of Leak Tests on Three-Hay Integral Seal Block . . . 41

Figure 9. Summary Results of Leak Tests on ISB Jumper Connectors

Using 0 -Ring Sears. ................ 44

Figure 10. One-Piece Body, Machined from Sojid Bar Stock - Side View. . 47

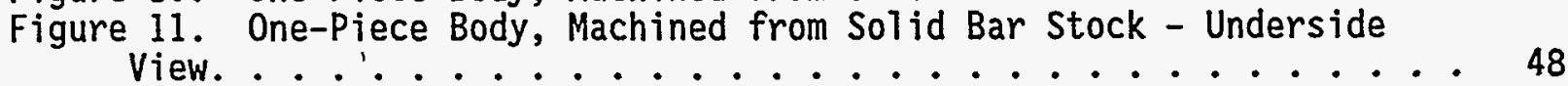

\section{LIST OF PHOTOGRAPHS}

Photograph 1. Seal Retainer Removal Tool - Four Sizes. . . . . . . . 3

Photograph 2. Demonstrating the Function of the Seal Retainer Removal

Tool. .................... 4

Photograph 3. Test Assembiy for Three-Way Nozzle and Baseplate

- Closeup View. ................ 8

Photograph 4. Nozzle Baseplate Showing Wire Bundles for Thermocouples

and Heating-Element Electrical Power. .......... 15

Photograph 5. Test Equipment Setup, High-Pressure Pump and

Instrumentation. .................... 17

Photograph 6. Closeup View of Three-Way Integral Seal Block Installed

in Vertical Skirt, Showing 0-Ring Seals. ....... 20

Photograph 7. Single-Port Integral Seal Blocks (2-, 3-, and 4-In.)

Welded to Block Moment Arms. ............ 21

Photograph 8. Test Stand Arranged for Lateral Moment Testing. $\ldots 24$

Photograph 9. Three-Way Conventional Connector with 100\% Tefion Gasket, $2501 \mathrm{bf} / \mathrm{in}^{2}, 400{ }^{\circ} \mathrm{F}$.

Photograph 10. Using a Wrench to Tighten a Threaded Rod Siowly Appl ies

a Lateral Moment to the ISB Jumper Connector Test Assembly. . . .

Photograph 11. Post-Test Visual Examination of O-Ring Using a Desk

Microscope with 15-Power Magnification. . .......................

Photograph 12. Quality Control Engineer Verifies that Lab Notebook Data

Matches Information in the Computer Database. . . ......

Photograph 13, 2-In. 0-Ring Seal, 70-Durometer Viton, $1,000 \mathrm{lbf} / \mathrm{in}^{2}, 400^{\circ} \mathrm{F}$.

Photograph 14. 2-In. 0-Ring Seal, $7 \dot{0}$-Durometer Fiuorositicone,

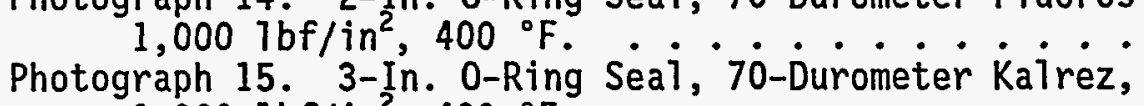
$1,000 \mathrm{lbf} / \mathrm{in}^{2}, 400{ }^{\circ} \mathrm{F}$. . . . . . . . . . . . . . . . .

Photograph 16. 4-In. 0-Ring Seai, TFE-0-SIL (Sijicone core with Tefion Outer coating), 70 -Durometer, $1,000 \mathrm{Ibf} / \mathrm{in}^{2}$ (gauge), $400^{\circ} \mathrm{F}$. 
WHC-SD-WM-TRP-223

Rev. 0

\section{GLOSSARY}

ISB Integral seal block - the part of the jumper connector containing the o-ring seal. It is called "integral" because a previously separate seal retainer has been made integral with the block.

MWTF Multi-Function Waste Tank Facility - a Hanford project to construct new double-she11 underground radioactive-waste storage tanks.

OPB One-piece-body - a jumper connector design that combines a number of separate parts into a unitized component. 
WHC-SD-WM-TRP-223

Rev. 0

\section{PUREX JUMPER CONNECTOR INTEGRAL SEAL BLOCK DEVELOPMENT AND LEAK TESTING}

\subsection{MWTF ISB LEAK TESTING: PROJECT ORGANIZATION AND ADMINISTRATION}

The decision to use an integral seal block (ISB) type of jumper connector on the Multi-Function Waste Tank Facility (MWTF) Project evolved from work on the o-ring/tetraseal type of connector (Ruff 1994a). At a meeting on January 4, 1994, with ICF Kaiser Hanford Company (ICF KH), Westinghouse Hanford Company (WHC) Project Engineering and WHC Materials and Welding Engineering, an overview of o-ring/tetraseal test results was presented. After discussion of the potential advantages of the ISB design, the MWTF Project decided to proceed with testing of the ISB concept.

Following this meeting, cost and schedule estimates were prepared to stipulate requirements for the new task, which includes the following activities:

- Testing integral seal blocks (ISB) instead of tetraseal-type seal retainers,

- Adding lateral moment testing (in addition to up-and-over moments) to the scope of work,

- Developing the design of and testing advanced three-way connector seals (ISB o-ring type).

Some thought was given to the possibility of performing lateral moment cycling tests on the ISBS. D. M. Squier, Jr., of the 305 Equipment Testing Laboratory, was contacted about the possibility of using a computer-controlled hydraulic system to perform lateral moment cycling tests. He stated that the hydraulic system could be adapted to the job and that the adaptation would take from three to five days to set up. He also indicated that the displacement accuracy of the hydraulics could be controlled to within $0.030 \mathrm{in}$.

Further discussion led the stress analysis group to decide not to perform lateral-moment cycling tests on the connector seals. Subsequently, in accordance with these wishes, test technician S. R. Jordan designed and fabricated a simpler fixture to perform lateral puil tests without cycling. 
WHC-SD-WM-TRP-223

Rev. 0

\subsection{INTEGRAL SEAL BLOCK DEVELOPMENT: BACKGROUND INFORMATION}

\subsection{SEAL COSTS}

Six commercial vendors were contacted for price and lead-time information on o-rings. From the outset, plans called for using standard-design, stock-available o-rings for the ISB connectors to minimize development costs. If initial tests with o-rings proved successful, consideration could be given to molding a special seal that would include an enhanced seal groove retainer.

\subsection{DESIGN ACTIVITY}

\subsubsection{Tasks Carried Over from FY 1993}

A1though design work on the new ISB was underway, a few "cleanup activities" remained from the previous year. Following up on FY 1993 testing activities, Design Services completed final check on $\mathrm{H}-2$ drawings of the o-ring/tetraseal seal retainers. Quality Assurance (QA) review and approval were completed. The $\mathrm{H}-2$ drawings of the o-ring/tetraseal seal retainers were released into the archives via an Engineering Data Transmittal. Design Services completed four-color renderings of the o-ring seal assembly to include in the annual test report and also generated a 30-second video animation of the connector. The computer animation was rendered to VHS video tape for convenient viewing.

Another task from the previous year's work was a detailed design for the removal tool for the o-ring/tetraseal seal retainer. The design was based on sketches of the tool submitted by E. G. Allen, a Hanford worker with extensive field experience on jumper connectors. Blueprints of the seal retainer removal tools were issued for seal retainers in 1- through 4-in. sizes. Subsequent fabrication of the seal removal tool prototype was performed by D. L. Powe11, Jr., of the Equipment Testing Laboratory. 
- Photograph 1: Seal Retainer Removal Tool - Four Sizes.

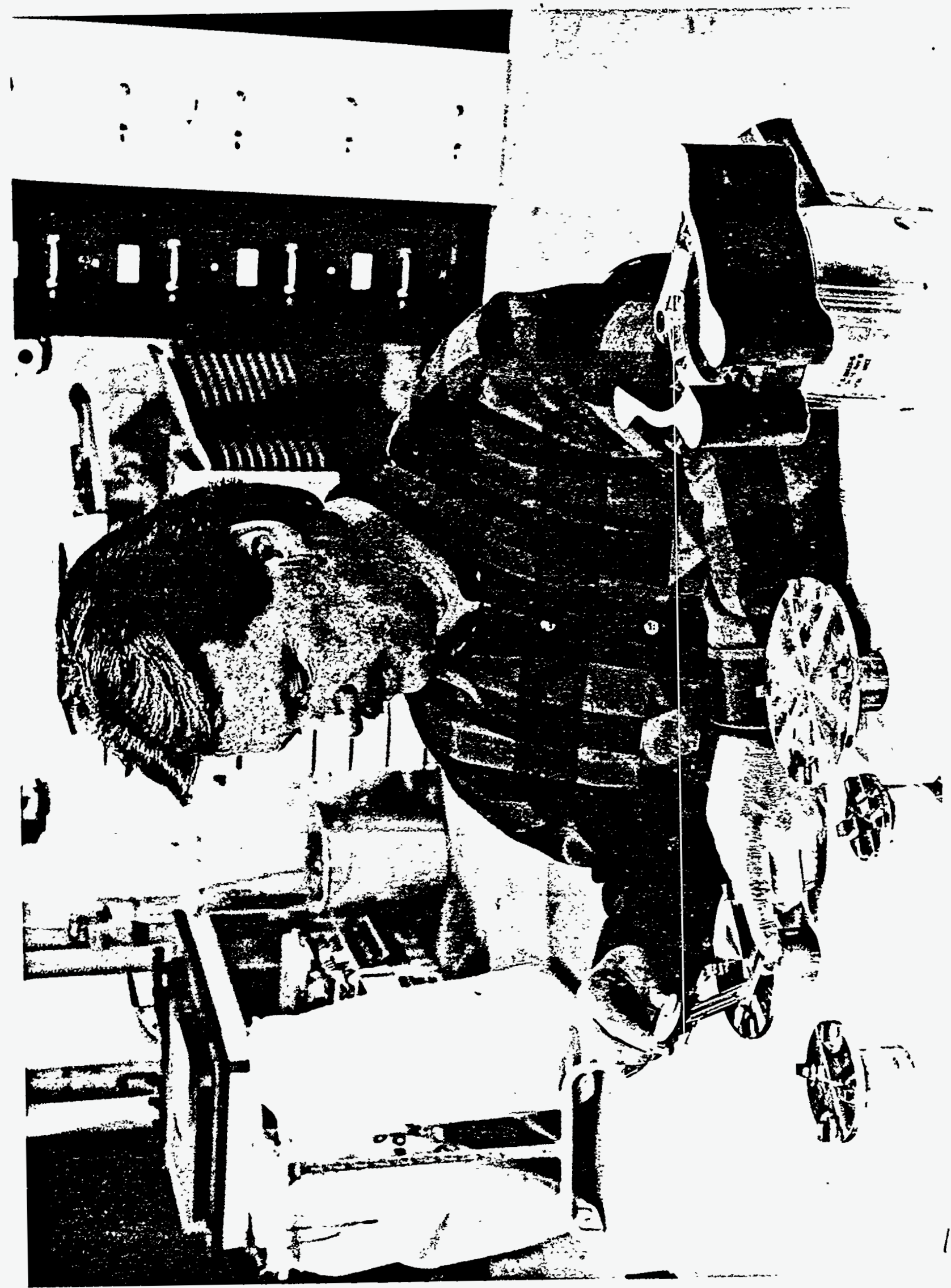


WHC-SD-WM-TRP-223

Photograph 2. Demonstrating the Function of the

Rev. 0

Seal Retainer Removal Tool.

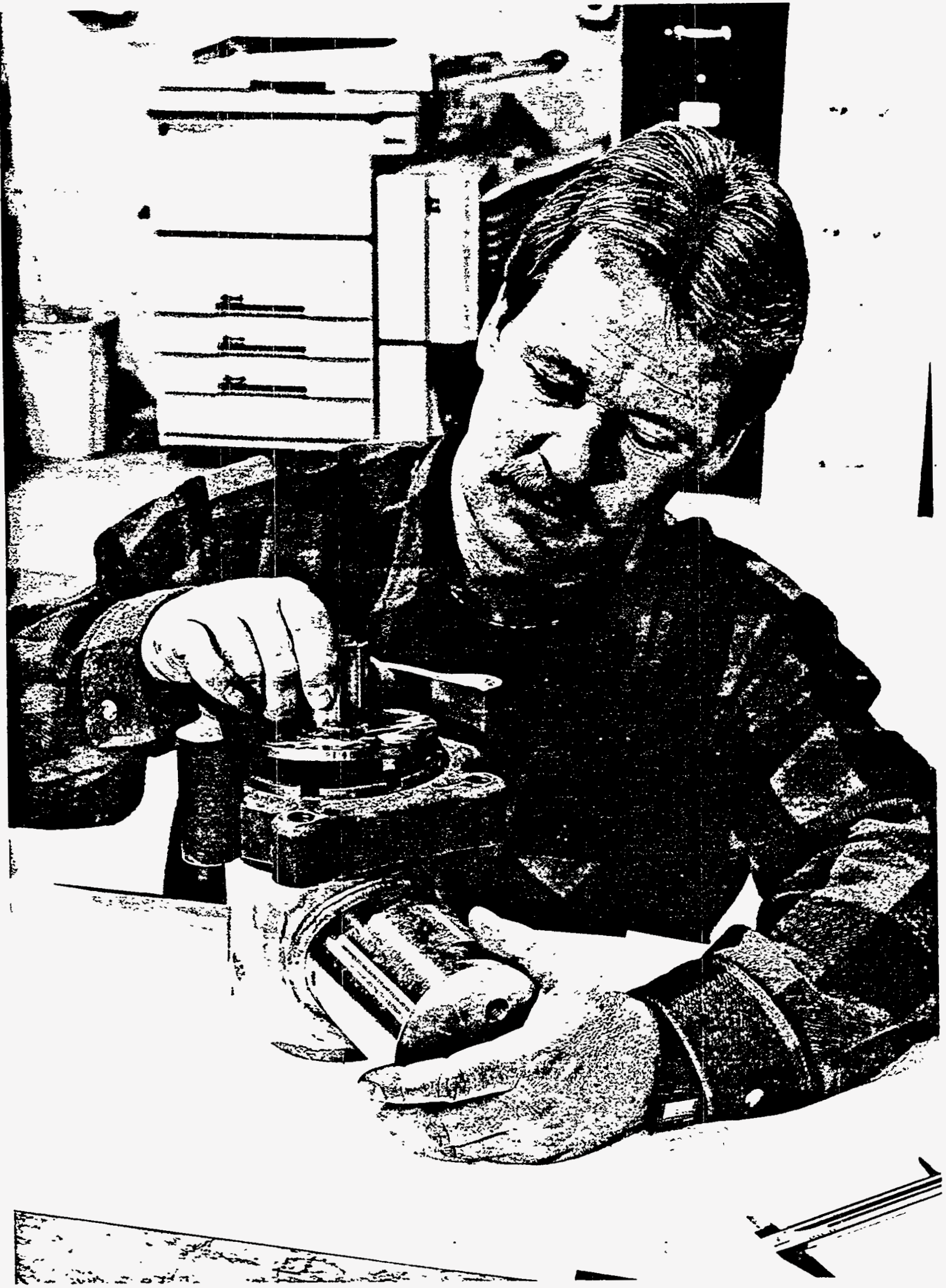




\subsubsection{Single-Port Connectors}

Design began with the smallest jumper connector size; a prototype design for a 2-in. ISB was sent to the jumper connector cognizant engineer, J. E. Conner, and to the fabrication shops for manufacturability review. Review comments were incorporated into the design. After further development, Design Services' R. D. Pierce prepared detailed fabrication drawings for the 2-in. ISB that included both blank blocks and through-flow single-port blocks. Engineering reviewed the drawings, and final comments were incorporated. Once the project was authorized to proceed, the drawings were transmitted to the fabrication shop for cost estimating and machining.

After the ISB had been designed, work began on the drawings for the 2-, 3-, and 4-in. threadless connector skirt and on the final assembly drawings for the ISB jumper connector. ATI design of the ISB jumper connector components, test fixtures, and tools was done on a desktop computer (Intel ${ }^{2} 486 / 586$ processor) with AutoCAD ${ }^{3}$ Release 12 software.

${ }^{2}$ Intel is a registered trademark of the Intel Corporation, Beaverton, Oregon.

${ }^{3}$ AutoCAD is a registered trademark of the Autodesk, Inc., Sausalito, CA. 
WHC-SD-WM-TRP-223

Rev. 0

Figure 1. Jumper Connector Assembly:

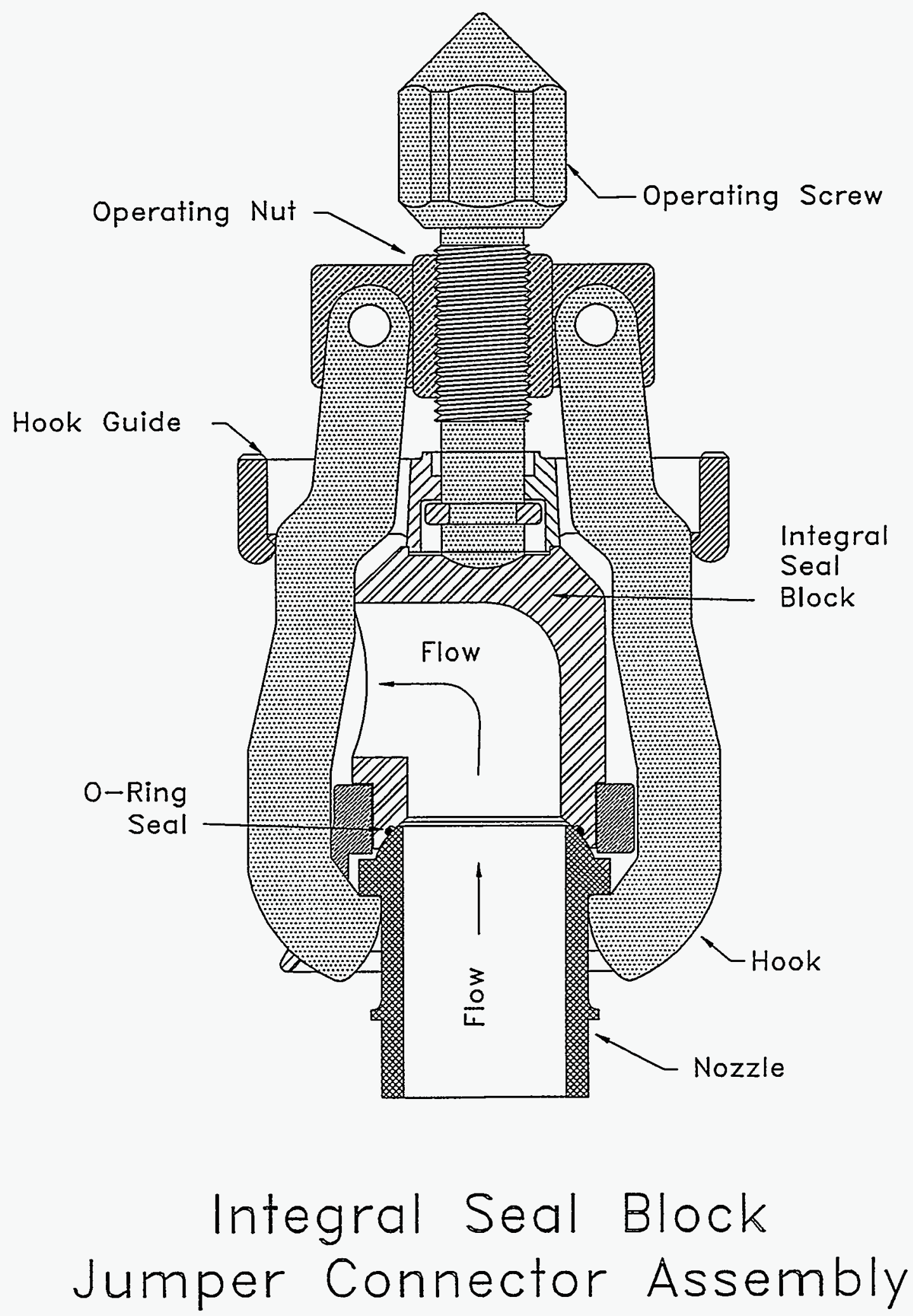

Illustration Showing 0-Ring Seal.

Seal is Compressed Between the Block and Nozzle. 
WHC-SD-WM-TRP-223

Rev. 0

\subsubsection{Three-way Connectors}

Incidental to early-stage design of the ISB was a simplified concept for three-way connectors. Materials and Welding Engineering personnel

J. J. Sisk and J. P. Hauptmann met with Ed Ruff to discuss the feasibility of the design of the simplified three-way connector nozzle. The design sought to eliminate the need for machining the connector-nozzle pipe inserts separately. Accessibility of the attached piping for welding was identified as a problem with the concept. So the concept was set aside and the decision made to continue with a three-way ISB test stand design configured for use with a stock three-way connector nozzle insert.

Detailed engineering design of the test fixture for the three-way connector was very time consuming. Internal flow passages for water, air venting, and the insertion of a heater element required a great deal of attention to ensure proper fit and function. Heating elements were sized and selected. Engineering AutoCAD sketch files were transmitted to Design Services for upgrading into formal engineering drawings. Port configurations and hardware for hydraulic connectors were detailed.

Several minor modifications were made to improve the design of a Teflon ${ }^{4}$ gasket seal for the three-way connector and released via an Engineering Change Notice (ECN). However, prototypes of these gaskets were never produced or tested. To save money, initial leak testing of the threeway connectors used off-the-shelf Teflon gaskets. Because later tests showed the o-ring ISB design to be far more resistant to applied moments than were teflon gaskets, there was no compelling reason to test the "improved" teflon gaskets.

Design work continued on the three-way integral seal block. AutoCAD drawings were forwarded to Engineering for detailed scrutiny of the o-ring groove design. After Engineering review, the o-ring groove design was modified to a $0.103-i n$. cross section. Subsequently, detailed $\mathrm{H}-2$ drawings of the flow-through three-way connector and the three-way blank ISB were completed and forwarded to the machine shop for cost estimates and subsequent fabrication.

"Teflon is a registered trademark of the E.I. du Pont de Nemours Company, Wilmington, Delaware: 
Photograph 3. Test Assembly for Three-Way Nozzle and Baseplate - Closeup View.
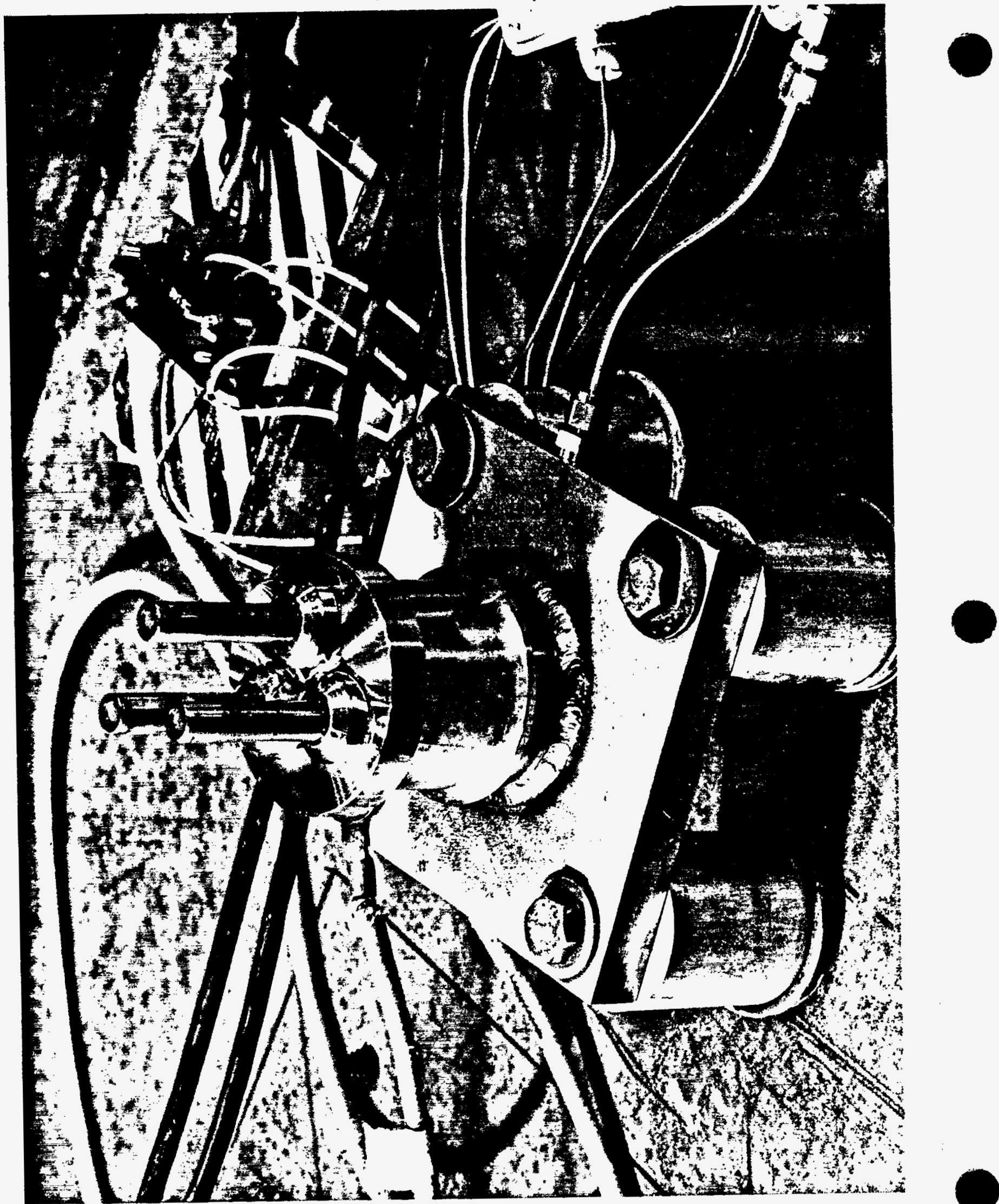
WHC-SD-WM-TRP-223.

Rev. 0

\subsection{DESIGN CONSIDERATIONS, INTEGRAL SEAL BLOCK}

\subsection{INTERCHANGEABILITY}

A fundamental objective of the design process was to produce a design for an integral seal block that could be brought into service with minimal changes to the existing hardware inventory of PUREX-type connectors, so that the new ISB could be used with parts currently stored in the warehouse.

An interchangeable seal design would afford cost savings by eliminating the need to re-design other connector parts, e.g., the nozzle, the block, and the lower skirt casting. It also would simplify the existing parts inventory.

\subsection{LEAK PATHS}

The advantage of the ISB is that there is only one leak path to consider--between the nozzle and the o-ring seal groove--in contrast to the earlier "tetraseal retainer/o-ring" design, which had two leak paths.

\subsection{PRESSURIZATION}

The o-ring seal grooves are designed with enough width so that after compression by clamping, there is space for fluid to enter the groove and pressurize the inside diameter (i.d.) of the seal. Fluid pressure forces the seal material toward the outside, thus seating the o-ring firmly against the outside diameter of the seal groove. This "self-energization" of the seal by the pressurized fluid is key to leak prevention.

\subsection{MINIMAL STRETCH}

The o-ring groove outside diameter (o.d.) was designed initially to match the outside diameter of the o-ring seal to minimize polymer stretch when the o-ring is energized: The design permits the seal to be seated firmly against the outside wall of the groove without excessive polymer stretch and strain that could cause premature failure of the seal.

\subsection{SEAL GROOVE}

The width and depth of the seal groove are critical dimensions for the proper operation of the seal. As a general rule, the depth of the seal groove is designed to allow about 30\% compression in the vertical direction. The groove width must be sufficient (i.e., have enough internal volume) to allow the compressed seal to recess fully into the groove. An additional allowance of $10 \%$ extra volume permits allow fluid pressure to enter the $i . d$. side of the groove and energize the seal.

The o-rings are designed for $0 . d$. contact with the groove at the small end of the o.d. tolerance dimension, at $80 \%$ groove volume, with $30 \%$ diametral squeeze. After compression, approximately $7 \%$ i.d. clearance allows process fluids to energize the o-ring against the $0 . d$. of the groove. 
WHC-SD-WM-TRP-223:

Rev . 0

The technical 1.iterature shows "standard o-ring grooves" as slanted outward from 1 to $5 \mathrm{deg}$. The reason given for this characteristic is "for ease of machining." However, a zero-degree slant theoretically is best for pressure retention in that it minimizes the tendency of the o-ring to extrude from the groove. Also, machinists indicate that they much preferred to machine the groove walls straight (i.e., with zero-degree slant). For these reasons, the decision was to design the grooves with 0 -deg. slant. Standard shop tolerances were acceptable for the perpendicularity of the groove walls. 
WHC-SD-WM-TRP-223.

Rev. 0

\subsection{DESIGN REQUIREMENTS AND TEST CONDITIONS}

Prior to testing, an effort was made to find out what projects on the Hanford site were planning to use jumper connector hardware. Cognizant personnel on these projects were then asked to stipulate what operating conditions would apply to connector installations. The intent was to perform leak tests that would meet or exceed the temperature and pressure requirements for all the projects - not just meet the specific needs of MWTF. This was done in the interest of saving money, and turned out to be a worthwhile excercise.

The table below identifies the temperature and pressure operating conditions for 4 projects that intended to use jumper connectors. As it turned out, HWVP required the highest temperatures $\left(390^{\circ} \mathrm{F}\right)$, and MWTF/C-106 Tank Sluicing required the highest pressures (400 psig).

Figure 2. Project Design Conditions Used to Determine Leak Test Parameters

\begin{tabular}{|c|c|c|c|c|c|c|c|c|}
\hline \multirow[t]{2}{*}{ SIZE } & \multicolumn{2}{|c|}{$\begin{array}{l}W-058 \\
\text { CROSS SITE } \\
\text { TRANSFER }\end{array}$} & \multicolumn{2}{|c|}{$\begin{array}{l}\text { W-028 } \\
\text { HANFORD WASTE } \\
\text { VITRIFICATION } \\
\text { PLANT (HWVP) }\end{array}$} & \multicolumn{2}{|c|}{$\begin{array}{l}\text { W-236A } \\
\text { MULTI-FUNCTION } \\
\text { WASTE TANK } \\
\text { FACILITY } \\
\text { (MWTF) }^{\mathrm{a}}\end{array}$} & \multicolumn{2}{|c|}{$\begin{array}{c}W-320 \\
\text { C-106 } \\
\text { TANK } \\
\text { SLUICING }\end{array}$} \\
\hline & $\begin{array}{c}\text { TEMP. } \\
{ }^{\circ} \mathrm{F} \\
\end{array}$ & $\begin{array}{c}\text { PRESS. } \\
\text { psig } \\
\end{array}$ & $\begin{array}{c}\text { TEMP. } \\
{ }^{\circ} \mathrm{F}\end{array}$ & $\begin{array}{c}\text { PRESS. } \\
\text { psig }\end{array}$ & $\begin{array}{c}\text { TEMP. } \\
{ }^{\circ} \mathrm{F} \\
\end{array}$ & $\begin{array}{r}\text { PRESS. } \\
\text { PS } 1 \mathrm{~g} \\
\end{array}$ & $\begin{array}{c}\text { TEMP. } \\
{ }^{\circ} \mathrm{F} \\
\end{array}$ & $\begin{array}{c}\text { PRESS. } \\
\text { psig } \\
\end{array}$ \\
\hline 2-In. & NS & NS & 390 & 220 & 250 & 400 & 180 & 400 \\
\hline $3-\operatorname{In}$. & 200 & 250 & 390 & 220 & 250 & 400 & 180 & 400 \\
\hline 4-In. & NS & NS & 390 & 220 & 120 & $\begin{array}{c}-10^{\prime \prime} \\
\mathrm{H}_{2} \mathrm{D} \\
\end{array}$ & 180 & 400 \\
\hline $\begin{array}{c}3 \text {-Way } \\
(2-\text { In. })\end{array}$ & NS & NS & 390 & 220 & 125 & 125 & NS & NS \\
\hline
\end{tabular}

NS = Not Specified, presumably because the project did not plan to use this particular size of connector.

aLetter, M. A. Rezvani to V. J. Cruz and J. M. Light, dated Dec. 6, 1993, "Qualification of Mechanical Jumpers and Electrical Connections," comment 2, page 2, MWTF Functional Design Criteria jumper classification.

${ }^{b}$ cc:Mail Message, E. G. Allen III to E. S. Ruff, dated Apri1 14, 1994, "Project W320 Connector Needs."

After considering the above matrix of project needs, it was decided to perform the leak tests on ISB jumper connectors at ambient: temperature and $400^{\circ} \mathrm{F}$, and at pressures incremented at 250, 500, 750, and 1,000 psig. 
WHC-SD-WM-TRP-223.

Rev.-O

Figure 3. Applied Moments and Pressures Used For Leak Testing ISB Jumper Connectors.

\begin{tabular}{|c|c|c|c|c|c|c|c|c|}
\hline \multirow[t]{2}{*}{ Size } & \multicolumn{2}{|c|}{$\begin{array}{c}\text { Tests at } \\
250 \\
\text { psig }\end{array}$} & \multicolumn{2}{|c|}{$\begin{array}{l}\text { Tests at } \\
500 \text { psig }\end{array}$} & \multicolumn{2}{|c|}{$\begin{array}{l}\text { Tests at } \\
750 \mathrm{psig}\end{array}$} & \multicolumn{2}{|c|}{$\begin{array}{c}\text { Tests at } \\
1,000 \text { psig }\end{array}$} \\
\hline & $\begin{array}{l}\text { Lat. } \\
\text { Momt. } \\
\text { ft-1bf }\end{array}$ & $\begin{array}{l}\text { Up \& } \\
\text { Over } \\
\text { Momt. } \\
\text { ft-lbf }\end{array}$ & $\begin{array}{l}\text { Lat. } \\
\text { Momt. } \\
\text { ft-1bf }\end{array}$ & $\begin{array}{l}\text { Up \& } \\
\text { Over } \\
\text { Momt. } \\
\text { ft-lbf }\end{array}$ & $\begin{array}{l}\text { Lat. } \\
\text { Momt. } \\
\text { ft-lbf }\end{array}$ & $\begin{array}{l}\text { Up \& } \\
\text { Over } \\
\text { Momt. } \\
\text { ft-7bf }\end{array}$ & $\begin{array}{l}\text { Lat. } \\
\text { Momt. } \\
\text { ft-1bf }\end{array}$ & $\begin{array}{l}\text { Up \& } \\
\text { Over } \\
\text { Momt. } \\
\text { ft-1bf }\end{array}$ \\
\hline 2-In. & 445 & 1500 & 334 & 1200 & 223 & 900 & 165 & 555 \\
\hline 3-In. & 445 & 1200 . & 445 & 1200 & 445 & 1200 & 445 & 1200 \\
\hline 4-In. & 445 & 1200 & 445 & 1200 & 445 & 1200 & 445 & 1200 \\
\hline 3-Way & 445 & 1500 & 356 & 1200 & 267 & 900 & 165 & 555 \\
\hline
\end{tabular}

Lat. Momt. = Lateral Moment Applied to the Jumper Connector

Up \& Over Momt. = Up and Over Moment Applied to the Jumper Connector 
WHC-SD-WM-TRP-223

Rev. 0

\subsection{INTEGRAL SEAL BLOCK PROCUREMENT ACTIVITIES}

\section{$5 . ' 1$ SEAL PROCUREMENT}

Purchase requisitions were prepared for small quantities of o-rings for leak testing. However, a large batch order was necessary to obtain Aflas ${ }^{5}$ rings. Orders of fewer than a dozen were too small for vendors to consider. To reduce development expense, Aflas was dropped from the roster of test materials. Ultimately, the materials chosen for testing were 70-durometer (durometer is a measure of hardness) Viton, Kalrez, and fluorosilicone. Some silicone and ethylene-propylene-diene-monomer (EPDM) 0-rings were obtained later for testing only with the three-way ISB connector. The o-rings procured for the ISB testing were provided by Erik's West Co. of Seattle, Washington. This vendor proved to be very cooperative and responsive in providing seal materials.

Samples of an unusual type of o-ring, called TFE-0--SIL ${ }^{6}$, were received from Row, Inc., Addison, IL. These o-rings had a silicone elastomer core with a Teflon outer coat. - They were described as having the resilience of an elastomer and the chemical resistance of Teflon. A 4-in. ISB connector using one of these o-rings was leak tested. It performed well at ambient temperature. However, high temperature applications are limited because of the relatively low softening temperature of the Teflon outer coat.

\subsection{CONNECTOR HARDWARE AND MISCELLANEOUS TEST EQUIPMENT}

A Tist of parts was developed for the required connector components, and a store order request for them was submitted to Site Warehousing Services. After the necessary tracking and follow up, Site Warehousing Services processed the request, and the components were shipped to the 305 Equipment Testing Laboratory on February 1, 1994. Examination of the parts for their suitability resulted in the discovery that one component, a vertical single-port skirt, was the wrong item. It was returned to Stores in exchange for a three-way vertical skirt.

The fabrication shops verified that stainless steel bar of the size needed to machine the 2-, 3-, and 4-in. ISB prototypes was in stock. A7so, 1 -in.-thick stainless steel (SS) plate was available for fabrication of nozzle baseplates.

A petty cash voucher was used to purchase hydraulic fittings for the internal water flow passages in the test stand baseplate. The hydraulic fittings were received from the Swagelock Company on February 4, 1994. Another company, the AAR Hardware Company of Cerritos, California, provided complimentary stainless steel port plugs. for the test stand air vents. These port plugs proved to be ideal for the air vents in the ISB test units.

\footnotetext{
${ }^{5}$ Aflas is a registered trademark of the $3 \mathrm{M}$ Company, st. Paul, Minnesota.

${ }^{6}$ TFE-O-SIL is a registered trademark of Row, Inc., Acldison, IL.
} 
WHC-SD-WM-TRP-223

Rev. 0

Three 1,000-W heater elements with internal thermocouples were procured from the Watlow Corporation. They were used as the heat source for the high-temperature test of the three-way jumper connector test assembiy. The heater elements performed very well during testing, withstanding repeated temperature cycles and pressurized hot water to $2,500 \mathrm{lbf} / \mathrm{in}^{2}$ (gauge). 
Photograph 4. Nozzle Baseplate Showing Wire Bundles for Thermocouples and Heating-Element Electrical Power.

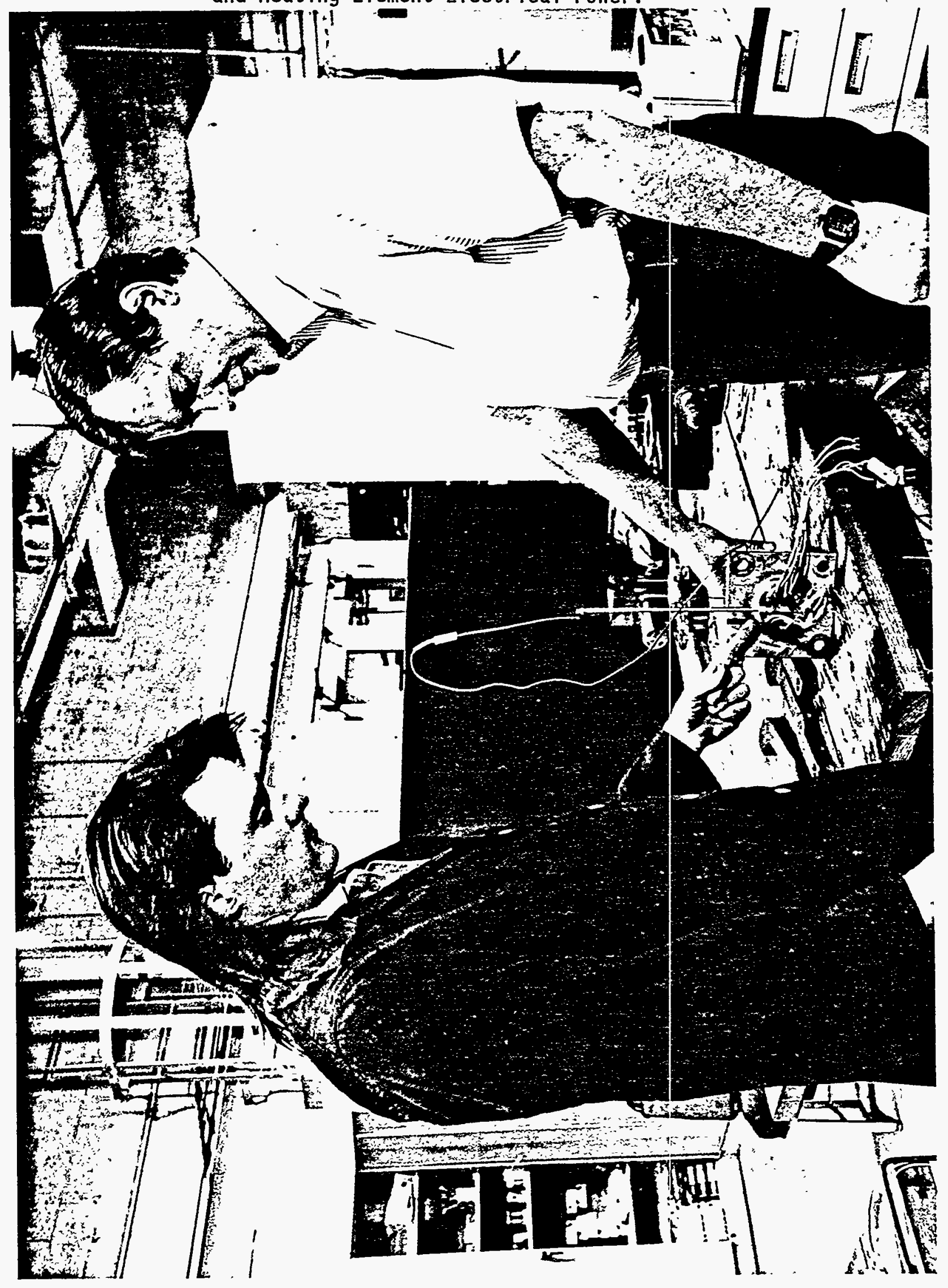




\section{0-RING SEAL TEST LOOP}

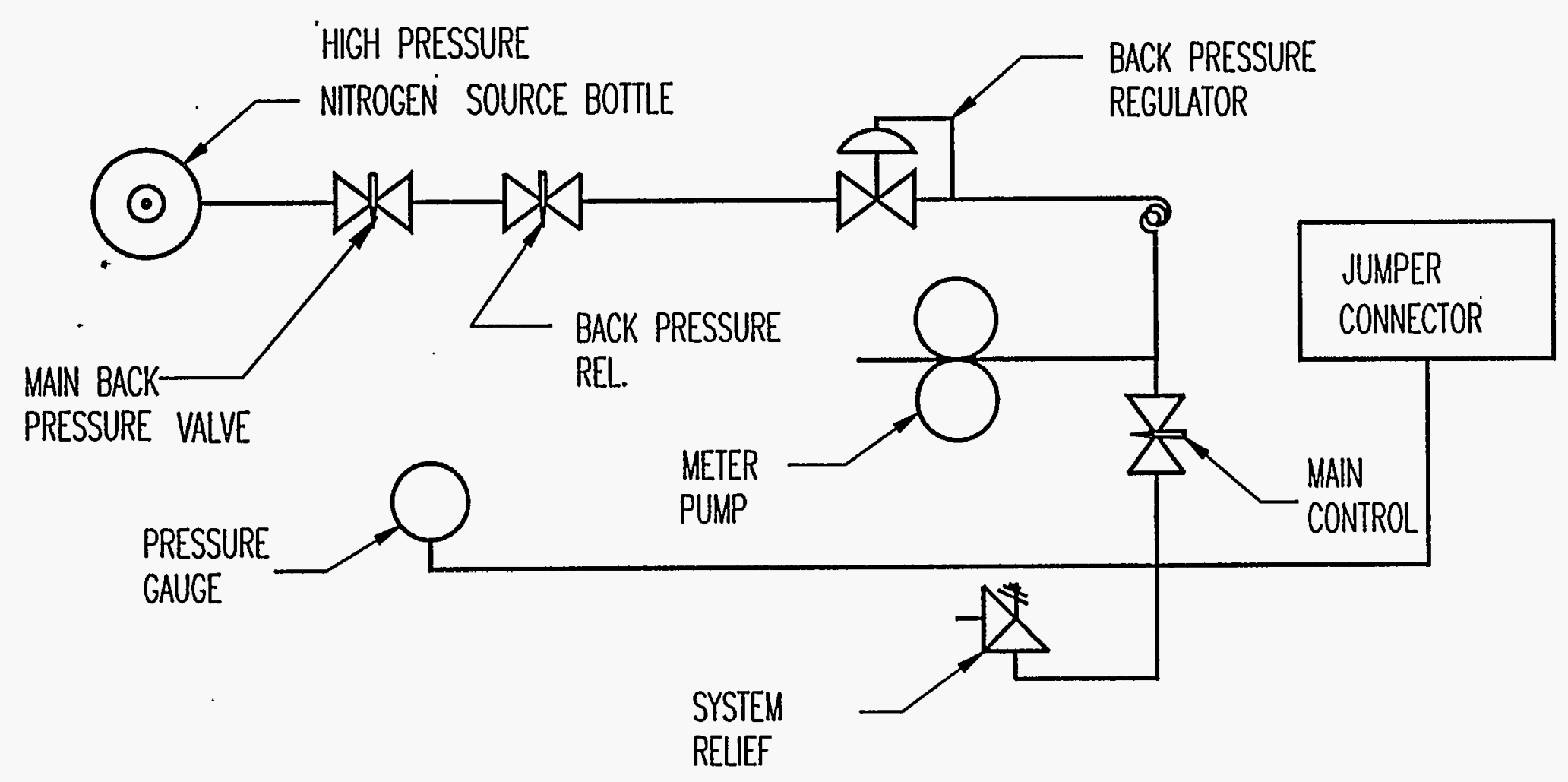


Photograph 5. Test Equipment Setup, High-Pressure Pump and Instrumentation.

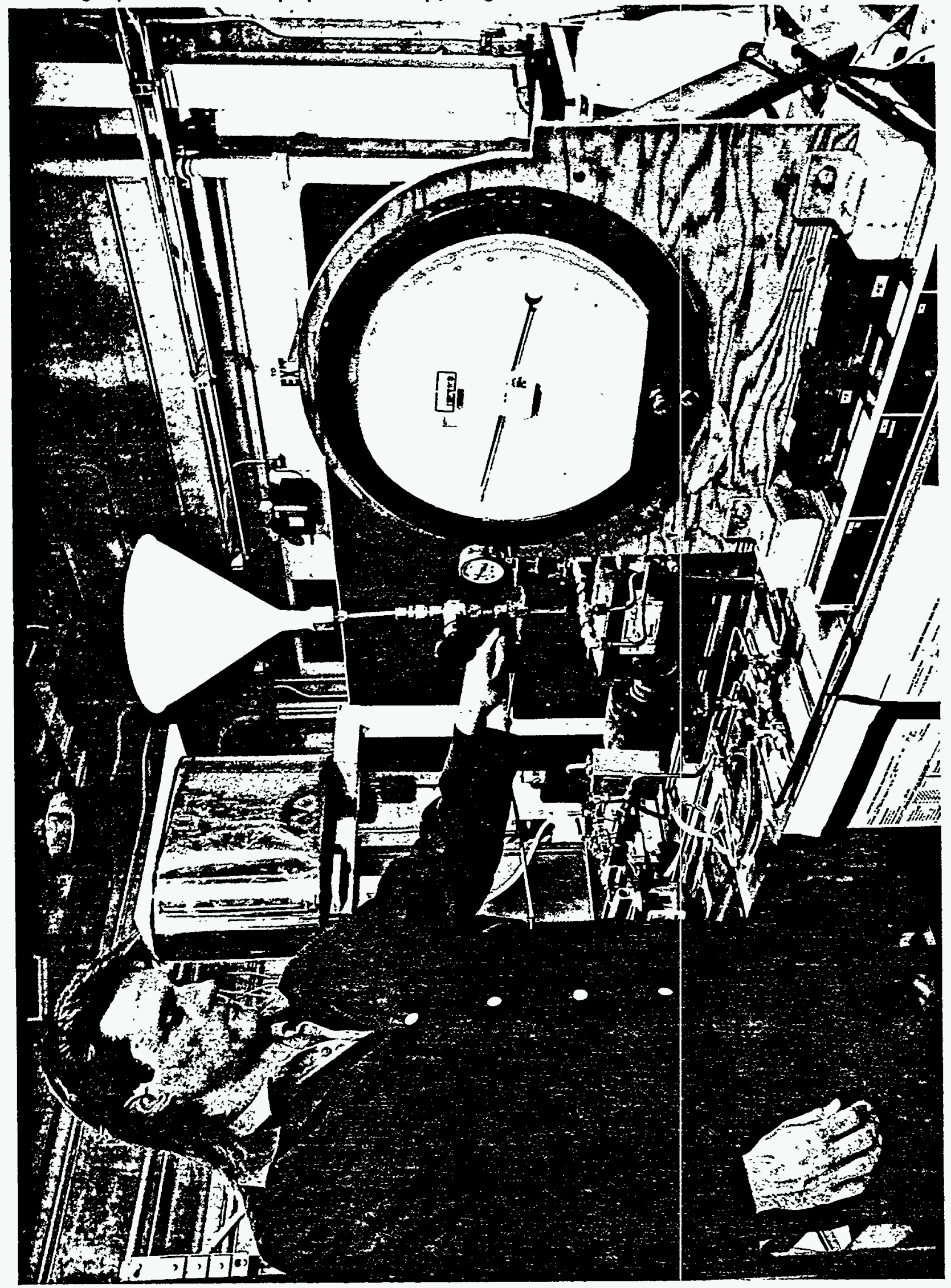


WHC-SD-WM-TRP-223

Rev. 0

\subsection{STRESS ANALYSIS SUPPORT ACTIVITY}

Ed Ruff tried a method for converting AutoCAD drawings to a geometry database suitable for use with the COSMOS ${ }^{7}$ finite-element analysis (FEA) program. Overcoming several minor "glitches" enabled the program to be used successfully. The part modeled for the first conversion experiment was a 3in. jumper connector hook. Use of the converted AutoCAD database reduced the time needed to re-model complex hook geometry with the FEA program. Geometry was exported to both COSMOS and ANSYS ${ }^{8}$ programs. This method aT so saved time in the FEA of other jumper connector components. Models were created for 2-, $3-$, and 4-in. hooks and the 2-in. nozzle. The FEA of the 3- and 4-in. hooks used the COSMOS program and a desktop personal computer (PC).

Tentatively planned was a side experiment on the 2-in. hook model, with the same loading conditions, to compare stress analysis results from the COSMOS PC analysis and the ANSYS workstation version; however, budget and schedule limitations prevented the experiment.

${ }^{7}$ COSMOS is a registered trademark of the Structural Research and Analysis Corporation, Santa Monica, CA.

${ }^{8}$ ANSYS is a registered trademark of Swanson Analysis Systems, Inc., Houston, PA. 


\subsection{INTEGRAL SEAL BLOCK MACHINING}

A J-10 Fabrication Request submitted to the 200 Area. Fabrication Services requested the machining of an updated design three-way gasket retainer. This task was.completed at the 200 West Area machine shop.

The 200 West Area machine shop also fabricated the test fixture for the three-way jumper connector test nozzle and baseplate; the 2-, 3-; and 4-in. ISB's; and the associated thread-less lower skirt castings. On delivery of these parts to the 305 Building Equipment Testing Laboratory, preliminary static leak checks were performed. Then the parts were returned to the machine shop where moment arms were welded onto the 2-, 3-, and 4-in. ISBs.

Generally, machining tolerances on the metal seal retainer can be held to $\pm 0.005 \mathrm{in}$. or better, but molding tolerances on the elastomer 0 -ring are much sloppier - frequentiy as bad as \pm 0.025 in., or worse. Experience shows that, when these two extremes in dimensions are combined in component assembly, there is every chance that the o-ring will not stay in the groove. The solution to this problem is to intentionally machine the nominal seal-groove diameter to match the smallest $0 . d$. in the o-ring tolerance range. This practice tends to "snug up" the o-ring in the seal groove. These provisions for o-ring "snug-up" were incorporated into the design.

After the single-port ISB's were completed, detailed fabrication drawings for the prototype of the three-way ISB were finished and forwarded to the machine shop. The machinist, L. J. Bray, developed ideas for fixtures that could be used to make the component. Basically, an off-centerline chuck setup was used to position the "flow axis" of each of the three ports with the axis of the lathe. This fixture was necessary to machine each of the three o-ring grooves into the concave spherical surface. Just as the three-way setup had been established, work on the three-way prototype was interrupted by a job to machine 1,400 sample valves for underground radioactive waste storage tanks that was of higher priority. Machining of the three-way o-ring connector was delayed for several months.

In anticipation of completion of machining activities, a work order was issued to the 305 Building Equipment Testing Laboratory to begin preparation for leak testing the ISB's. A detailed activities schedule was generated for the tests. The test technician, S. R. Jordan, prepared improvements to the test procedure and ordered additional block heater tubes. Torque wrenches and temperature and pressure instrumentation were calibrated.

Meanwhile, machining of prototypes continued. The machinist, H. H. Byers, continued to refine the CNC program. Perseverance finally resulted in completion of the 2-,3-, and 4-in. stainless steel ISB's. After final de-burring and metrology, the components were delivered to the Equipment Testing Laboratory. Photographs 6 and 7 show the prototype components made by CNC machining. 
Photograph 6. Closeup View of Three-Way Integral Seal Block Installed

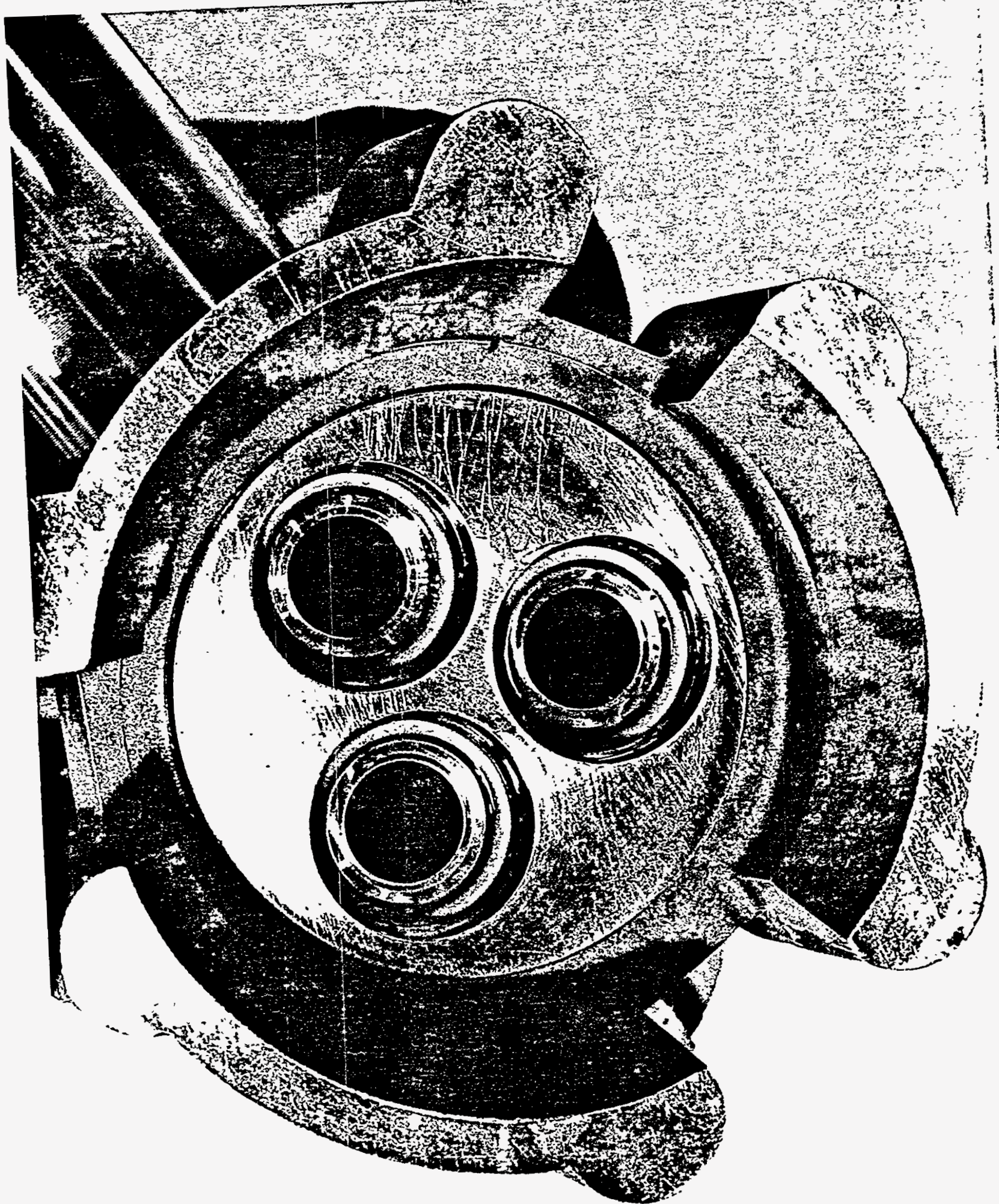


WHC-SD-WM-TRP-223

Rev. 0

Photograph 7. Single-Port Integral seal Blocks (2-, 3-, and 4-In.) Welded to Block Moment Arms.

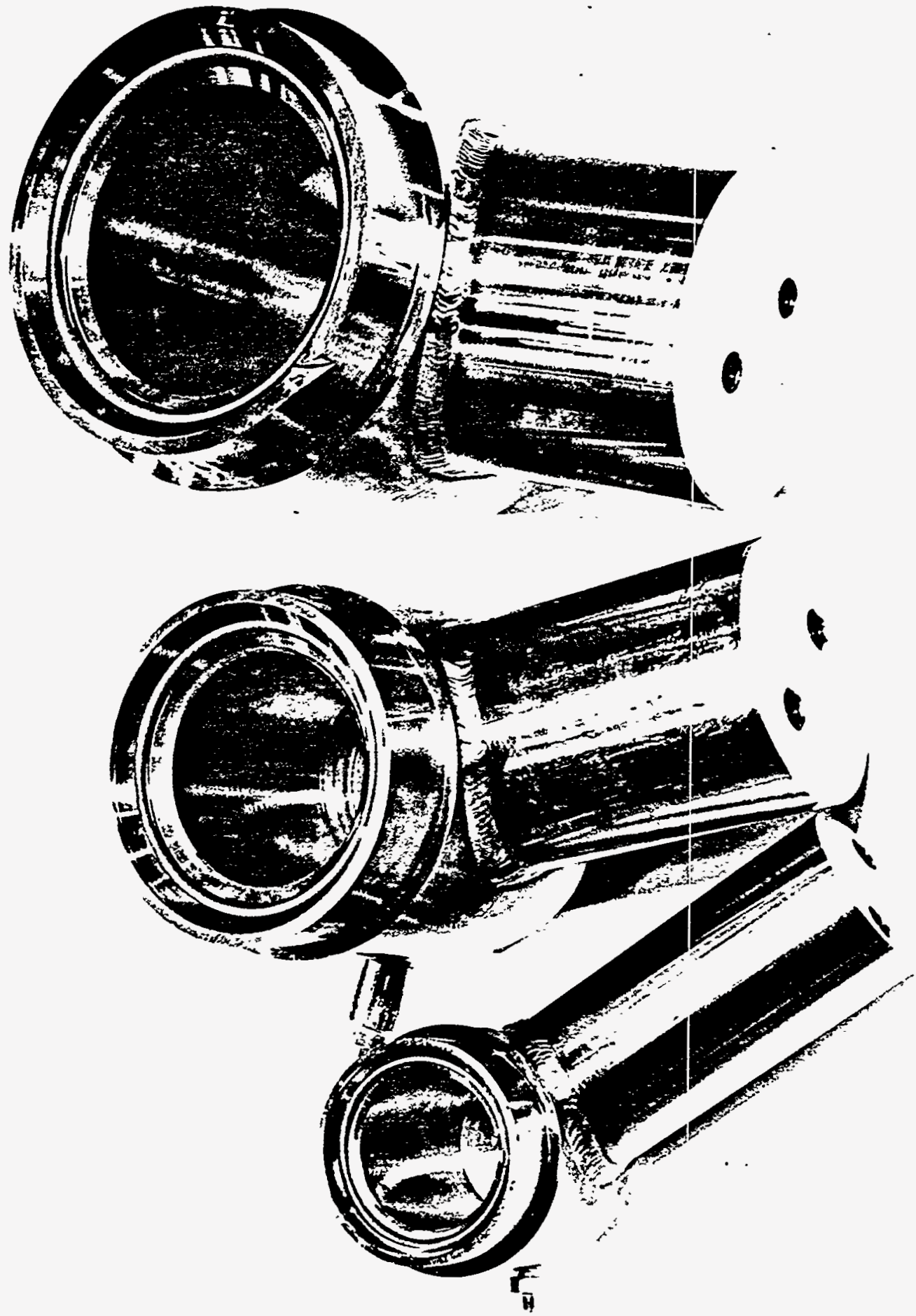


WHC-SD-WM-TRP-223

Rev. 0

\subsection{EQUIPMENT TESTING LABORATORY - INITIAL SETUP}

\subsection{TEST OF CONVENTIONAL THREE-WAY TEFLON GASKET}

The first test fixture to be completed and set up was that for a "conventional Teflon gasket" three-way jumper connector. Once the three-way nozzle assembly arrived from the machine shop, attempts were made to install the newly arrived Watlow heater rods. Minor difficulties with the machined pipe thread on the heater rods led to the return of the heater rods and baseplate to the machine shop for thread adjustment and cleanup.

Meanwhile, high-pressure tubing was installed from the metering pump to the baseplate on the test fixture. The three-way block (with conventional Teflon seals) was assembled to the hook guide and lower skirt, and the size and fit of the air-bleed port plugs were verified.

Heater rod threads were reworked, and Watlow heater rods were installed into the three-way connector test fixture. The use of Grafoil ${ }^{10}$ tape was instrumental in achieving leak-tightness in the heater rod/baseplate pipe thread. Thermocouple connections and power leads were attached to the heater rods.

Once these preparations had been made, leak testing of the conventionaliy gasketed three-way connector began at ambient temperature conditions. The test involved no applied moments, and conventional gaskets of $100 \%$ Teflon were used. The conventional three-way connector performed surprisingly well at ambient temperature when not subjected to moments. Leak-tight operation to $2,300 \mathrm{lbf} / \mathrm{in}^{2}$ was demonstrated at an operating screw clamp torque of $100 \mathrm{ft}-1 \mathrm{~b}$. However, this high pressure leak-tight performance deteriorated significantly when either heat or moments were applied to the Teflon gaskets. This was demonstrated in the next set of leak tests which involved ambient and elevated temperatures (400 ${ }^{\circ} \mathrm{F}$ ) with "up and over" applied moments, using conventional 100\% Teflon gaskets in the connector.

SIGNIFICANT FINDING: The "up and over" moment. tests revealed that the conventional three-way connector is much more susceptible to externally applied moments than the single-port connector is. (That is, the conventional 3-way connector Teaks much sooner.) As shown in Appendix T, Graph 3WAM250M, the teflon-gasketed 3-way connector complete7y failed to hold pressure under the very moderate conditions of ambient temperature, $2501 \mathrm{bf} / \mathrm{in}^{2}$ pressure, and on $7 \mathrm{y} 187 \mathrm{ft}-1 \mathrm{bf}$ of "up and over" moment. This is quite a contrast to the single-port conventional teflon-gasketed connector, which was able to remain leak tight at ambient temperature, $250 \mathrm{ibf} / \mathrm{in}^{2}$ pressure, with $1,250 \mathrm{ft}-1 \mathrm{bf}$ of "up and over" moment applied. (Austin, 1993, Appendix A, page 17). It is thought that the reason for. this difference is that the moment forces in the three-way connector bear on a much smaller area (one of the small three-way nozzle inserts) compared to the single-port connector.

${ }^{9}$ Watlow is a registered trademark of Watlow, Inc., St. Louis, MO.

${ }^{10}$ Grafoil is a registered trademark of the UCAR Carbon Company, Cleveland, $\mathrm{OH}$. 
CONCLUSION: The leak tests of the conventional three-way connector in which external moments were applied revealed a significant weakness in the ability of the connector to take side loads. This finding indicated a need for an improved type of connector block. (Later on in the test program, the new-design three-way ISB using o-rings was to show much greater leak resistance under applied moments.) 
Photograph 8. Test Stand Arranged for Lateral Moment Testing.

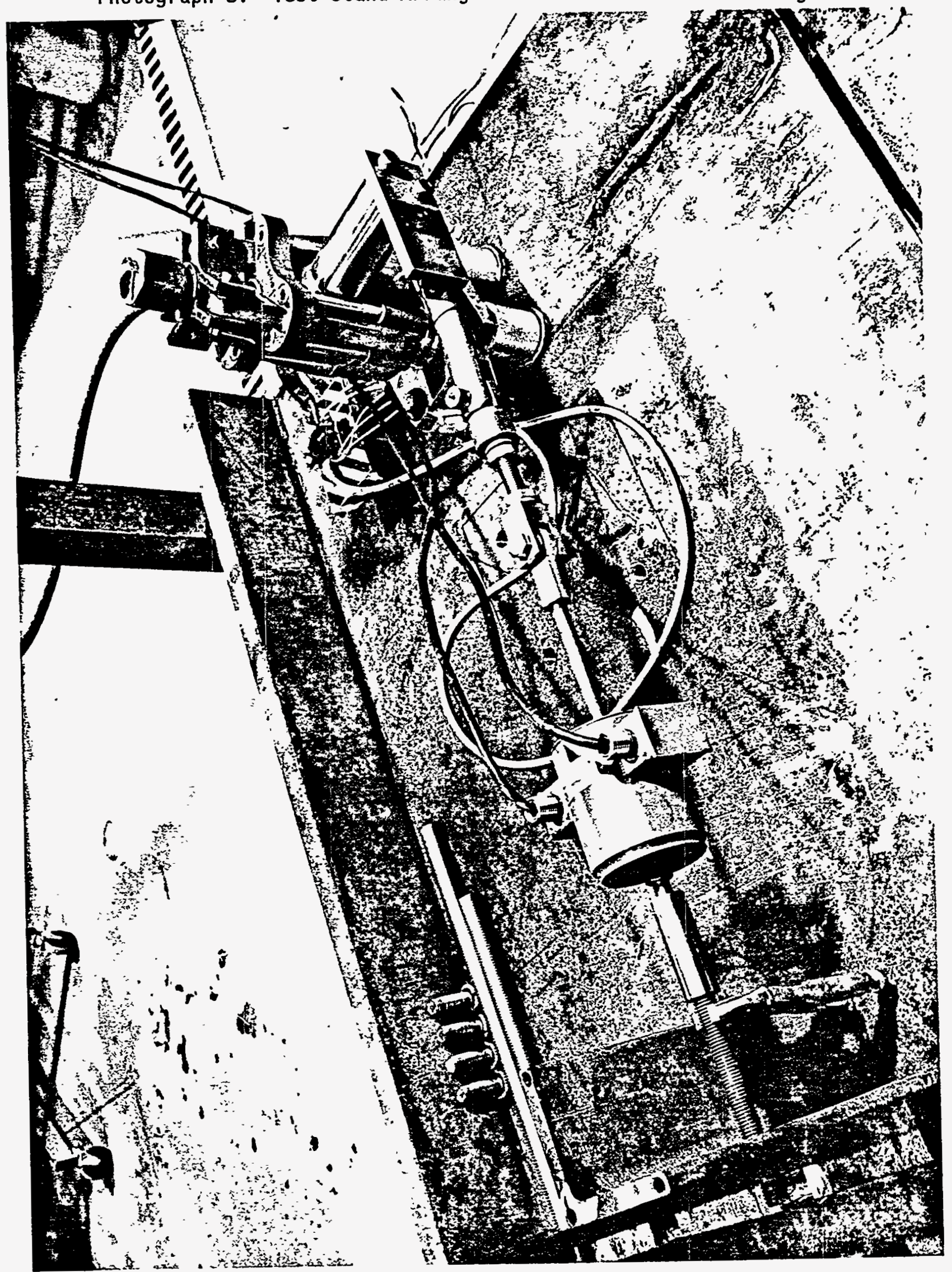



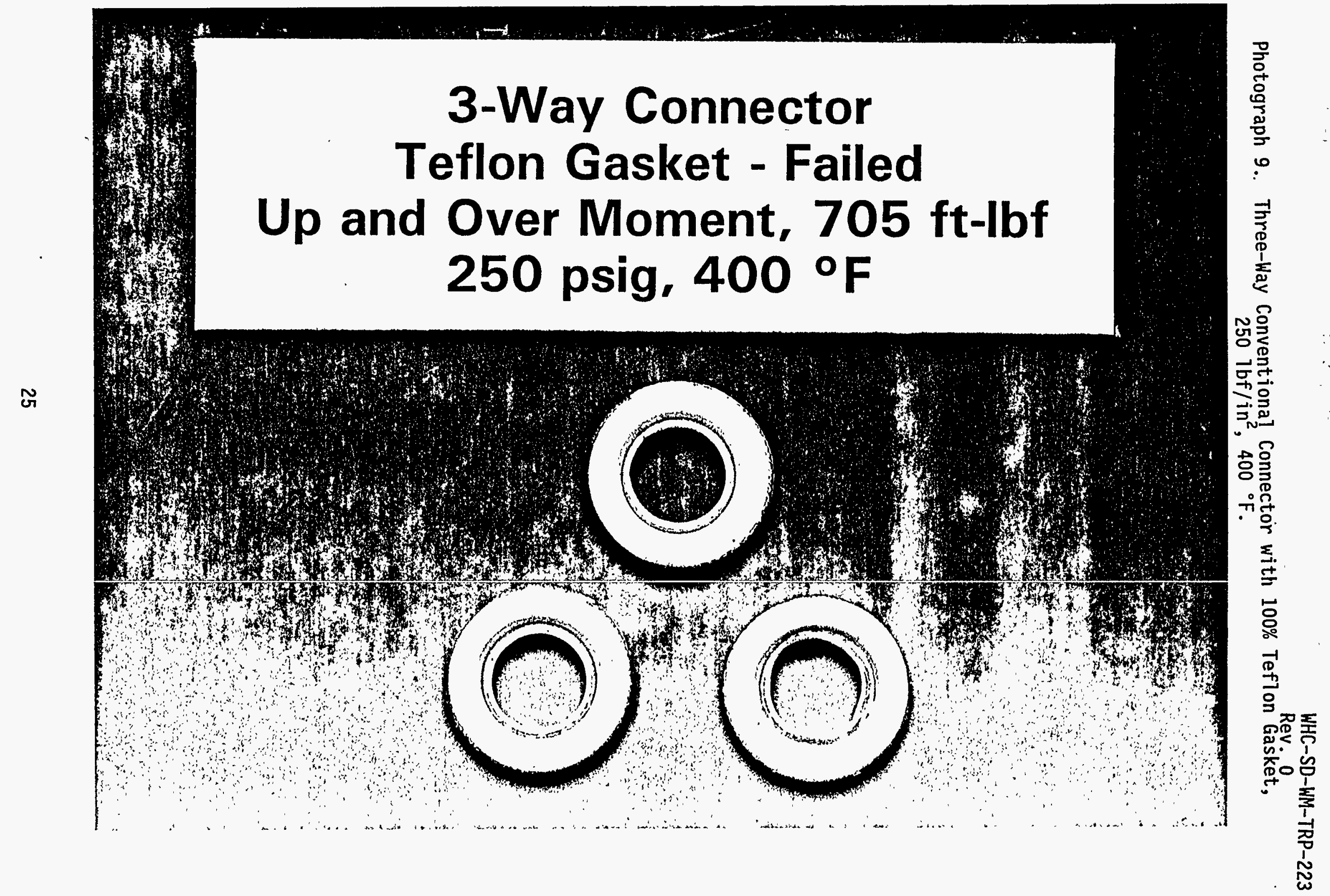
WHC-SD-WM-TRP-223

Rev. 0

\subsection{ADDITIONAL LABORATORY ACTIVITIES}

The jumper connector test loop was modified with a vacuum pump and an isolation valve to enable connectors to be tested under vacuum conditions, should that have been necessary. A digital electronic vacuum transducer was calibrated and installed to measure vacuum levels to $10-i n$. water gauge (w.g.). Several new valves also were installed on the test 10op: an isolation valve on the main pump and a shut-off valve on the water reservoir. Later on in the program, vacuum leak tests were deleted from the scope due to schedule and funding limitations.

The electric heater power supply was rewired to correct a problem with the high-temperature cutout switch. This unit subsequently functioned properiy.

A mechanical linkage and moment-arm adapter plate was made for the lateral-moment test fixture. The structural steel of the lateral-moment fixture (which had been fabricated earlier) was bolted to the laboratory floor. Measurements necessary for fitting the jumper connector to the lateral-moment force transducer were made. Another hinge point was added to the mechanical linkage arm for the laterai-moment test fixture. The addition was to minimize bending moments in the arm during lateral-moment testing.

A new linkage for moment take-up was installed on the test stand. Previous 7 inkages required periodic replacement as the result of thread damage. The new 7 inkage, which had 7 arger threads, eliminated this recurring probiem.

Time and funding limitations led to a decision not to conduct leak tests at pressures from 1,000 to $2,500 \mathrm{ibf} / \mathrm{in}^{2}$ (gauge). Leak tests for elevated temperatures at $1,000 \mathrm{lbf} / \mathrm{in}^{2}$ (gauge) and 300 to $400{ }^{\circ} \mathrm{F}$ were thought to be more directly applicable to plant operations. 
-Photograph 10. Using a Wrench to tighten a threaded Rod STowly Applies a Lateral Moment to the ISB Jumper Connector Test Assembly.

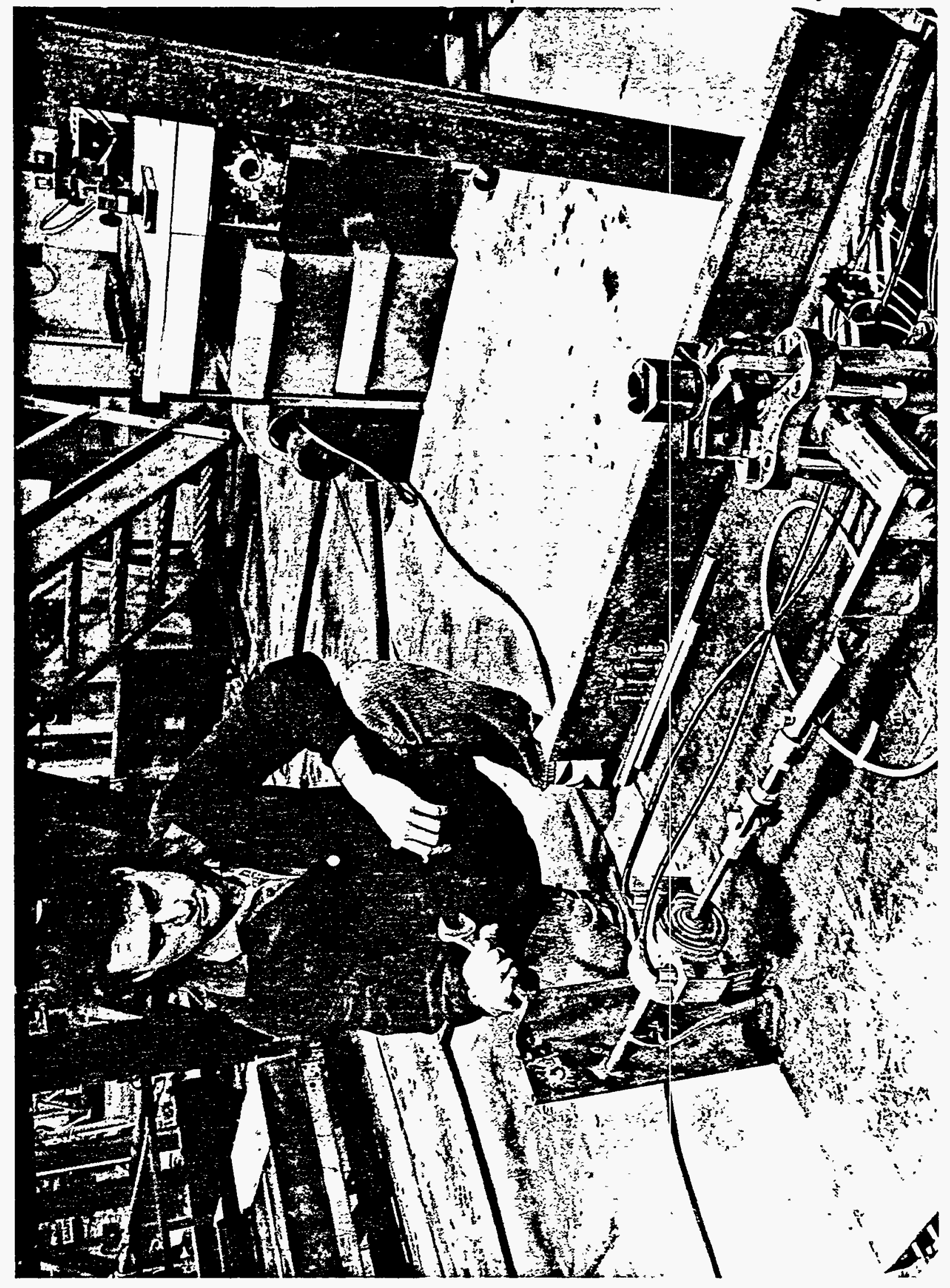


Photograph 11. Post-Test Visual Examination of 0-Ring Using a Desk Microscope with 15-Power Magnification.

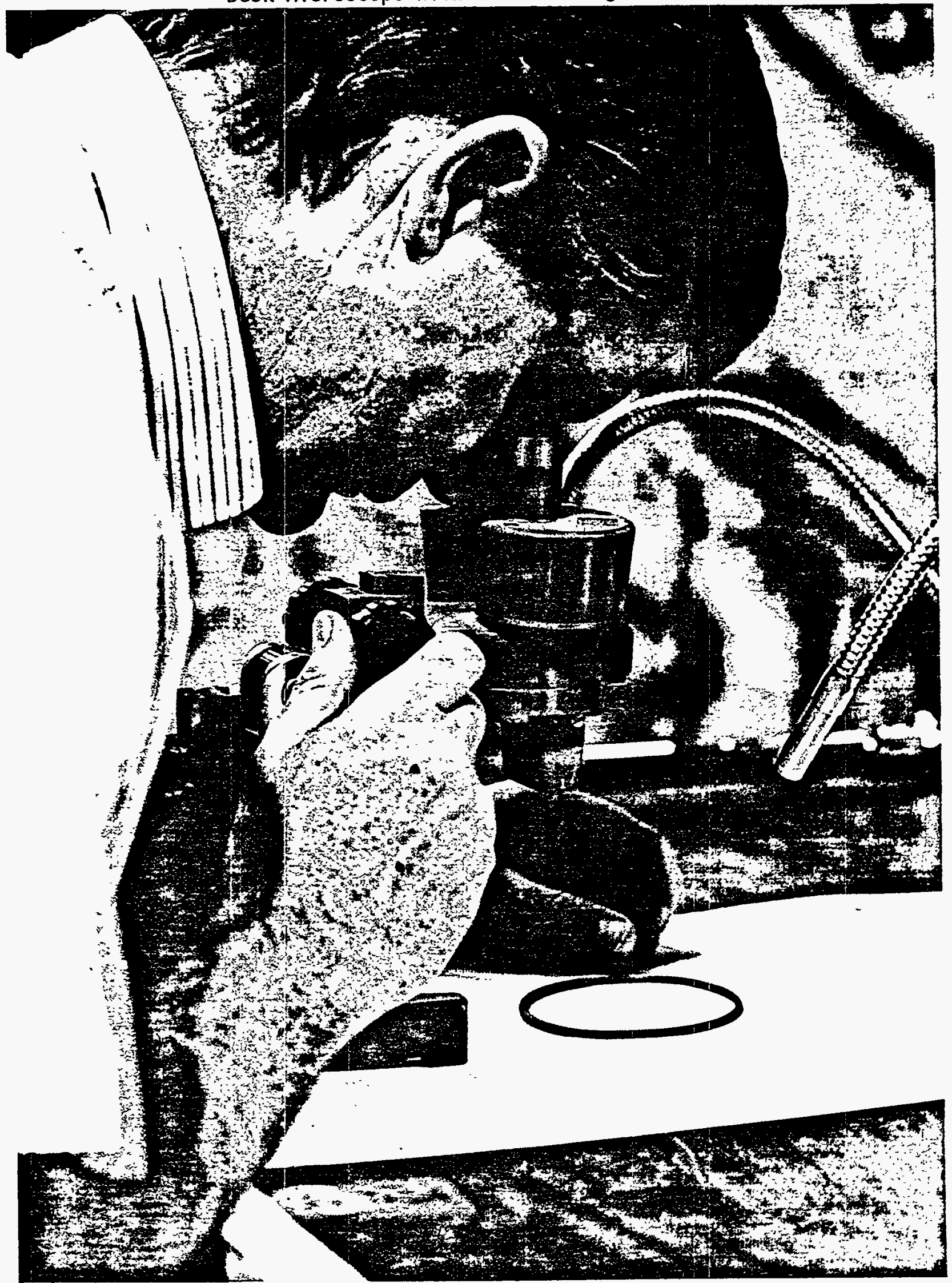


Photograph 12. Quality Control Engineer Verifies that Lab Notebook Data Matches Information in the Computer Database.

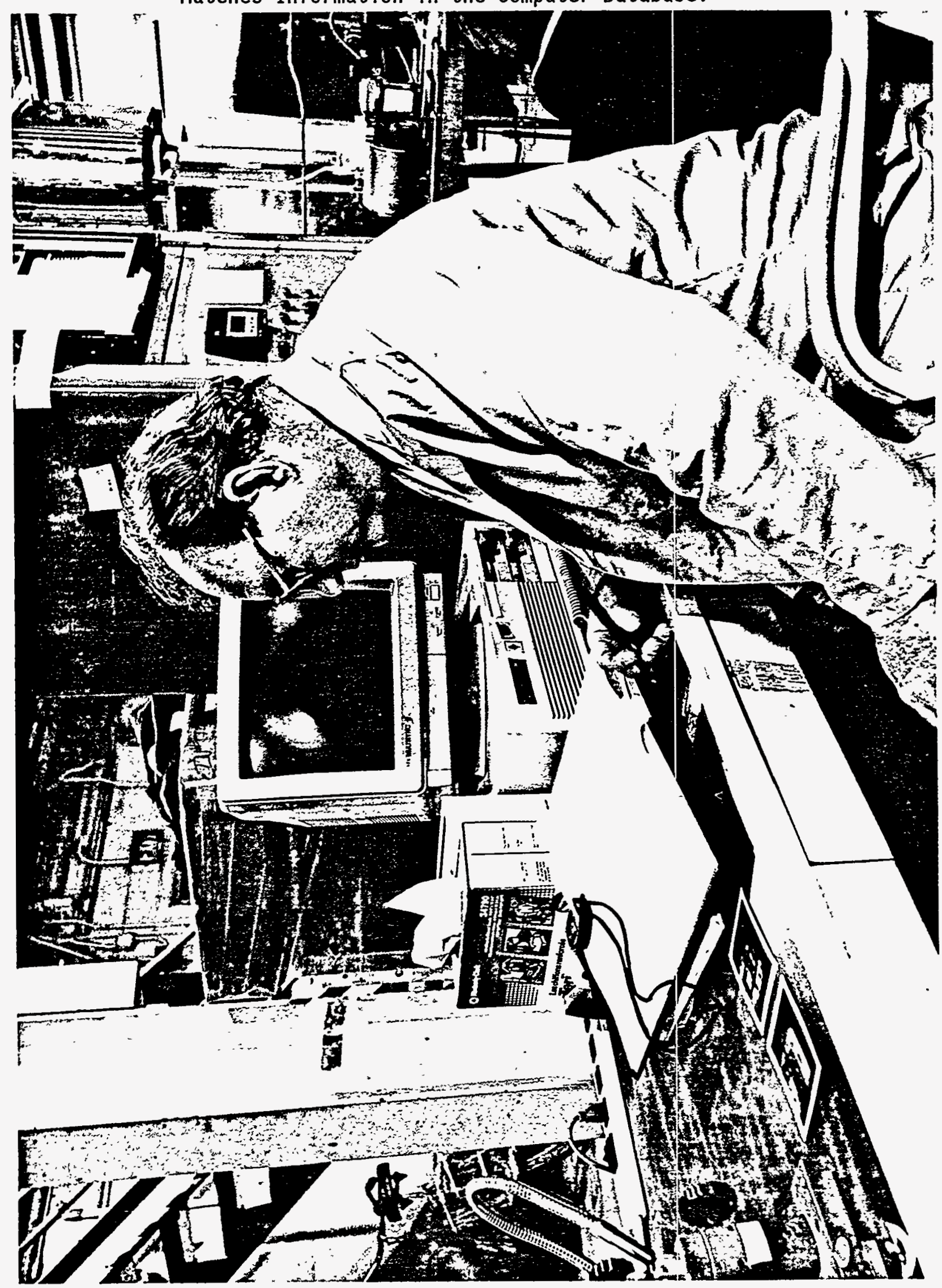


WHC-SD-WM-TRP-223

Rev. 0

\subsection{LEAK TESTS}

\subsection{2-IN. ISB O-RING SEAL}

Once parts arrived, testing of the 2-in. ISB o-ring seals began in earnest. (Refer to Figure 3 for applied moment conditions). The operating screw clamping torque used for leak testing was $50 \mathrm{ft}-1 \mathrm{bf}$ (nominal).

The Equipment Testing Laboratory conducted a series of high-pressure leak tests at ambient and elevated temperatures, with and without external7y applied moments. Results were very good. The 2 -in. 0-ring seal retainer performed successfulty under ambient-temperature test conditions. The hightemperature $\left(300\right.$ and $\left.400{ }^{\circ} \mathrm{F}\right)$ moment test also was successful with no apparent pressure loss at static or moment conditions, depending on the seal material used. Refer to Figure 5. for a summary of 2-In. ISB leak test results

For the elevated temperature tests, total heat-up time for the system was 1.5 hours. The temperature reading on the gasket remained at the elevated test temperature for a total of 2.5 hours (including moment testing). Total test time was approximately 4 hours. Cooling the system with a circulation fan allowed time for two elevated temperature tests per day.

Leak tests that combined high temperatures with externally applied moment constituted more arduous conditions than previous (FY 1994) therma1-cycling tests that did not include applied loads. Hence, thermalcycling tests without an applied moment (as performed on old-style gaskets during the previous year) were judged redundant.

Re-torquing of the connector was not necessary to ensure leak tightness after thermal cycling. Elevated test data indicated good sealing capability for more than four minutes. (Note: Two minutes of hold time was selected as an arbitrary standard for establishing the minimum holding pressure of a seal; therefore, solid sealing for more than four minutes was considered adequate to justify ending the test.)

To visually compare a "good" and "bad" o-ring test result, the reader is encouraged to examine Photographs 13 and 14. In Photograph 13, the Viton o-ring is seen to be intact after testing at $400 \mathrm{\circ}, 1,000 \mathrm{lbf} / \mathrm{in}^{2}$, however, the fluorosilicone o-ring in Photograph 14 has suffered irreparable damage. 


\begin{tabular}{|c|c|c|c|c|c|c|c|c|c|c|c|c|c|c|c|c|}
\hline \multicolumn{17}{|c|}{$\begin{array}{l}\text { PUREX TYPE JUSPER CONNECTOR - INTEGRAL SEAL BLOCK (ISB) LEAK TEST QUALIFICATION } \\
\qquad 2 \text { In. Single Port ISB connector }\end{array}$} \\
\hline & \multicolumn{8}{|c|}{ Ambient Temperature - 70 deg. F. } & \multicolumn{8}{|c|}{ High Temperature ( 400 Deg. F. unless otherwise indicated) } \\
\hline & \multicolumn{4}{|c|}{ Up \& Over Moment } & \multicolumn{4}{|c|}{ Lateral Moment } & \multicolumn{4}{|c|}{ Up \& Over Moment } & \multicolumn{4}{|c|}{ Lateral Moment } \\
\hline $\begin{array}{c}\text { O-Ring } \\
\text { Material }\end{array}$ & $\begin{array}{l}250 \\
\text { psig }\end{array}$ & $\begin{array}{l}500 \\
\text { psig }\end{array}$ & $\begin{array}{l}750 \\
\text { psig }\end{array}$ & $\begin{array}{l}1,000 \\
. \text { psig }\end{array}$ & $\begin{array}{l}250 \\
\text { psig }\end{array}$ & $\begin{array}{l}500 \\
\text { psig }\end{array}$ & $\begin{array}{l}750 \\
\text { psig }\end{array}$ & $\begin{array}{c}1,000 \\
\text { psig }\end{array}$ & $\begin{array}{l}250 \\
\text { psig }\end{array}$ & $\begin{array}{c}500 \\
\text { psig }\end{array}$ & $\begin{array}{l}750 \\
\text { psig }\end{array}$ & $\begin{array}{l}1,000 \\
\text { psig }\end{array}$ & $\begin{array}{l}250 \\
\text { psig }\end{array}$ & $\begin{array}{l}500 \\
\text { psig }\end{array}$ & $\begin{array}{l}750 \\
\text { psig }\end{array}$ & $\begin{array}{l}1,000 \\
\text { psig }\end{array}$ \\
\hline Viton & OK & OK & OK & OK. & OK & OK & OK & OK & $\mathrm{OK}^{\circ}$ & OK & OK & OK & OK & OK & OK & OK \\
\hline Kalrez & OK & OK & OK & OK & OK & OK & OK & OK & $\begin{array}{l}\text { OK } \\
300\end{array}$ & $\begin{array}{l}0 \mathrm{OK} \\
300\end{array}$ & $\begin{array}{l}0 K \\
300\end{array}$ & $\begin{array}{c}\text { OK } \\
300\end{array}$ & $\begin{array}{l}\text { OK } \\
300\end{array}$ & $\begin{array}{c}\text { OK } \\
300\end{array}$ & $\begin{array}{l}\text { OK } \\
300\end{array}$ & $\begin{array}{c}\text { OK } \\
300\end{array}$ \\
\hline Fluorosil. & OK & OK & OK & OK & OK & OK & OK & OK & OK & OK & OK & $\begin{array}{c}\text { FAIL } \\
\text { Q400 } \\
\text { OK } \\
300\end{array}$ & OK & OK & OK & OK \\
\hline
\end{tabular}

FAIL = Seal Failed in Actual Test.

$300=$ Test Performed at 300 Deg. F. 
Photograph 13. 2-In. 0-Ring Sea1, 70-Durometer Viton,

Rev. 0 $1,000 \mathrm{lbf} / \mathrm{in}^{2}, 400^{\circ} \mathrm{F}$.
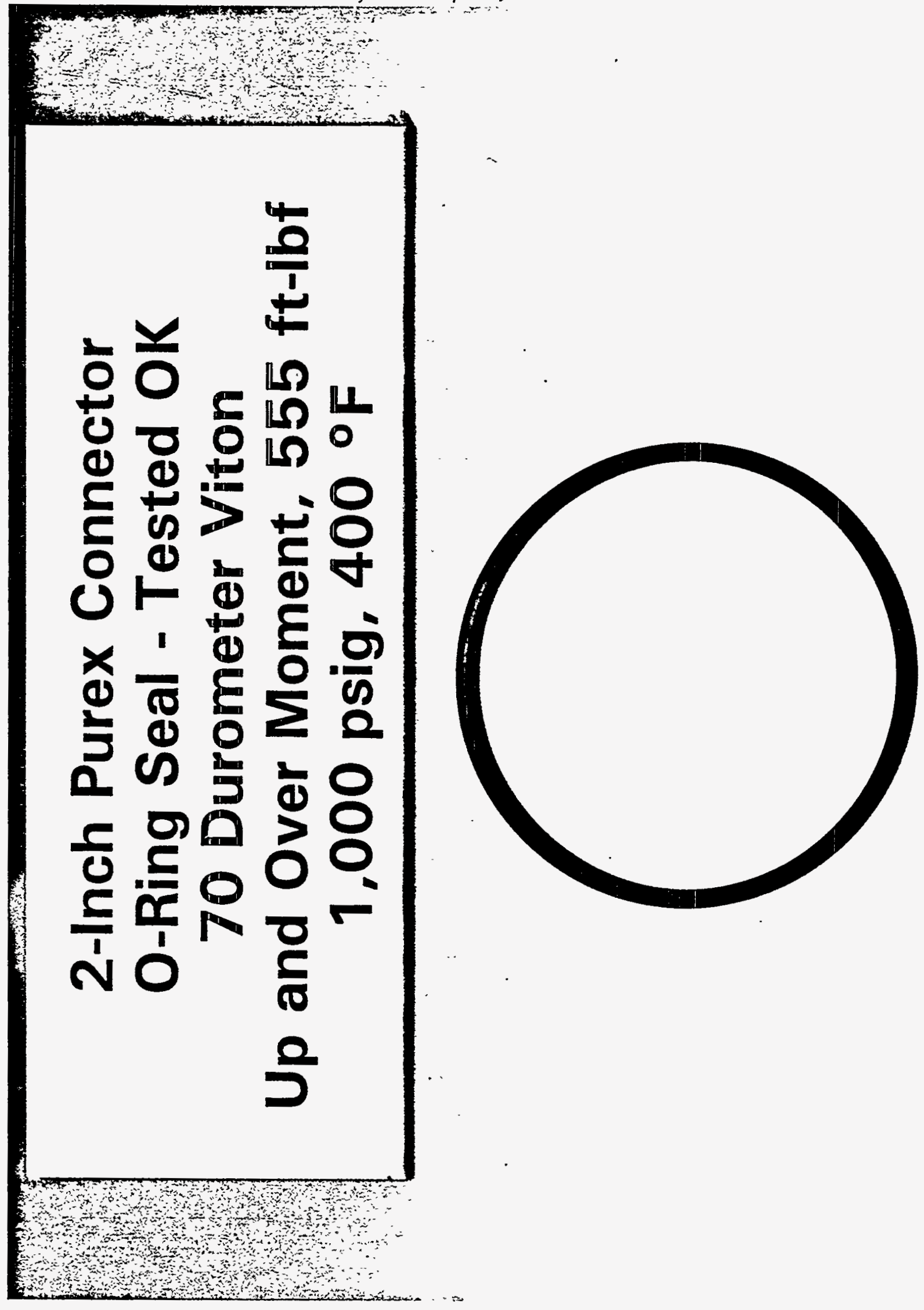


\section{2-Inch Purex Connector O-Ring Seal - Failed 70 Durometer Fluorosilicone Up and Over Moment, $375 \mathrm{ft}$-lbf 1,000 psig, $400^{\circ} \mathrm{F}$}

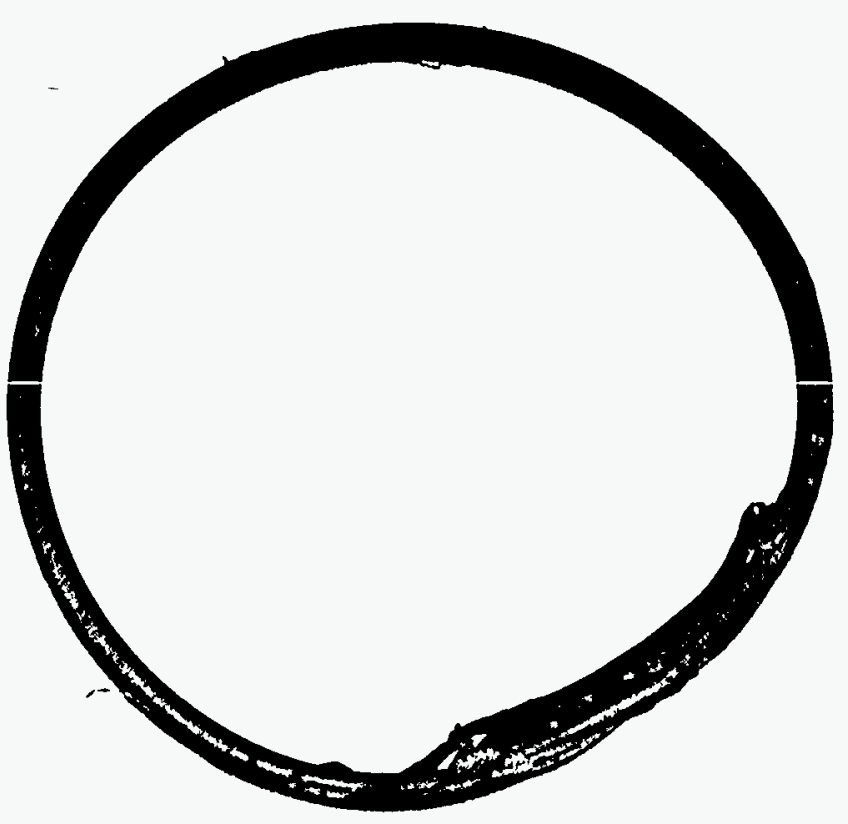


WHC-SD-WM-TRP-223

Rev. 0

\subsection{3-IN. ISB 0-RING SEAL}

Depending on the type of material used, the 3-in. o-ring seals subjected to elevated temperature tests under the following conditions proved adequate: $1,000 \mathrm{lbf} / \mathrm{in}^{2}$ at ambient temperatures and at 300 and $400^{\circ} \mathrm{F}$. (Refer to Figure 3 for applied moments). Refer to Figure 6 for a summary of leak test results on 3-In. ISB connectors.

At the beginning of the test on Kalrez at elevated temperatures $\left(400{ }^{\circ} \mathrm{F}\right)$, a leak was observed underneath the connector block. Removal of the seal retainer exposed o-ring laceration/extrusion, most probably from the high pressure and high temperature in conjunction with the applied moment (see Photograph 15). Kalrez should not be used if temperatures will exceed $300^{\circ} \mathrm{F}$. 


\begin{tabular}{|c|c|c|c|c|c|c|c|c|c|c|c|c|c|c|c|c|}
\hline \multicolumn{17}{|c|}{$\begin{array}{l}\text { PUREX TYPE JUMPER CONNECTOR - INTEGRAL SEAL BLOCK (ISB) LEAK TEST QUALIFICATION } \\
\qquad 3 \mathrm{In} \text {. Single Port ISB Connector }\end{array}$} \\
\hline & \multicolumn{8}{|c|}{ Ambient Temperature -70 deg. $F$. } & \multicolumn{8}{|c|}{ High Temperature ( 400 Deg. F. uniess otherwise indicated) } \\
\hline & \multicolumn{4}{|c|}{ Up \& Over Moment } & \multicolumn{4}{|c|}{ Lateral Moment } & \multicolumn{4}{|c|}{ Up \& Over Moment } & \multicolumn{4}{|c|}{ Lateral Moment } \\
\hline $\begin{array}{c}\text { O-Ring } \\
\text { Material } \\
\end{array}$ & $\begin{array}{l}250 \\
\text { psig }\end{array}$ & $\begin{array}{c}500 \\
\text { psig }\end{array}$ & $\begin{array}{l}750 \\
\text { psig }\end{array}$ & $\begin{array}{l}1,000 \\
\text { psig }\end{array}$ & $\begin{array}{c}250 \\
\text { psig }\end{array}$ & $\begin{array}{c}500 \\
\text { psig }\end{array}$ & $\begin{array}{c}750 \\
\text { psig } \\
\end{array}$ & $\begin{array}{l}1,000 \\
\text { psig }\end{array}$ & $\begin{array}{c}250 \\
\text { psig }\end{array}$ & $\begin{array}{c}500 \\
\text { psig }\end{array}$ & $\begin{array}{c}750 \\
\text { psig }\end{array}$ & $\begin{array}{l}1,000 \\
\text { psig }\end{array}$ & $\begin{array}{c}250 \\
\text { psig }\end{array}$ & $\begin{array}{r}500 \\
\text { psig }\end{array}$ & $\begin{array}{c}750 \\
\text { psig }\end{array}$ & $\begin{array}{l}1,000 \\
\text { psig }\end{array}$ \\
\hline Viton & OK & OK & OK & OK & OK & OK & OK & OK & OK & OK & OK & OK & OK & OK & OK & OK \\
\hline Kalrez & OK & OK & OK & OK & OK, & $\begin{array}{l}\text { OK, } \\
\text { NT }\end{array}$ & $\begin{array}{l}\text { OK, } \\
\text { NT }\end{array}$ & $\begin{array}{l}\text { OK, } \\
\text { NT }\end{array}$ & $\begin{array}{c}\text { OK } \\
300\end{array}$ & $\begin{array}{c}\text { OK } \\
300\end{array}$ & $\begin{array}{c}\text { OK } \\
300\end{array}$ & $\begin{array}{c}\text { FAIL } \\
0400 \\
0 K \\
300 \\
\end{array}$ & $\begin{array}{c}\text { OK } \\
300\end{array}$ & $\begin{array}{c}0 K \\
300\end{array}$ & $\begin{array}{c}\text { OK } \\
300\end{array}$ & $\begin{array}{c}\text { OK } \\
300\end{array}$ \\
\hline Fluorosil. & OK & OK & OK & $\begin{array}{l}\text { OK, } \\
\text { NT }\end{array}$ & OK & OK & OK & OK & $\begin{array}{c}\text { OK } \\
300\end{array}$ & $\begin{array}{c}\text { OK } \\
300\end{array}$ & $\begin{array}{c}\text { OK } \\
300\end{array}$ & $\begin{array}{l}\text { OK } \\
300\end{array}$ & $\begin{array}{c}\text { OK } \\
300\end{array}$ & $\begin{array}{c}\text { OK } \\
300\end{array}$ & $\begin{array}{c}\text { OK } \\
300\end{array}$ & $\begin{array}{c}\text { OK } \\
300\end{array}$ \\
\hline
\end{tabular}

FAIL = Seal Failed in Actual Test.

OK, NT $=$ Not Tested At This Temperature, But OK, Because The Seal Withstood More Rigorous Conditions.

$300=$ Test Performed at 300 Deg. F. 
Photograph 15. 3-In. 0-Ring Sea1, 70-Durometer KaIrez, $1,000 \mathrm{lbf} / \mathrm{in}^{2}, 400^{\circ} \mathrm{F}$.

\section{3-Inch Purex Connector O-Ring Seal - Failed 70 Durometer Kalrez Up and Over Moment 1,000 psig, $400^{\circ} \mathrm{F}$}

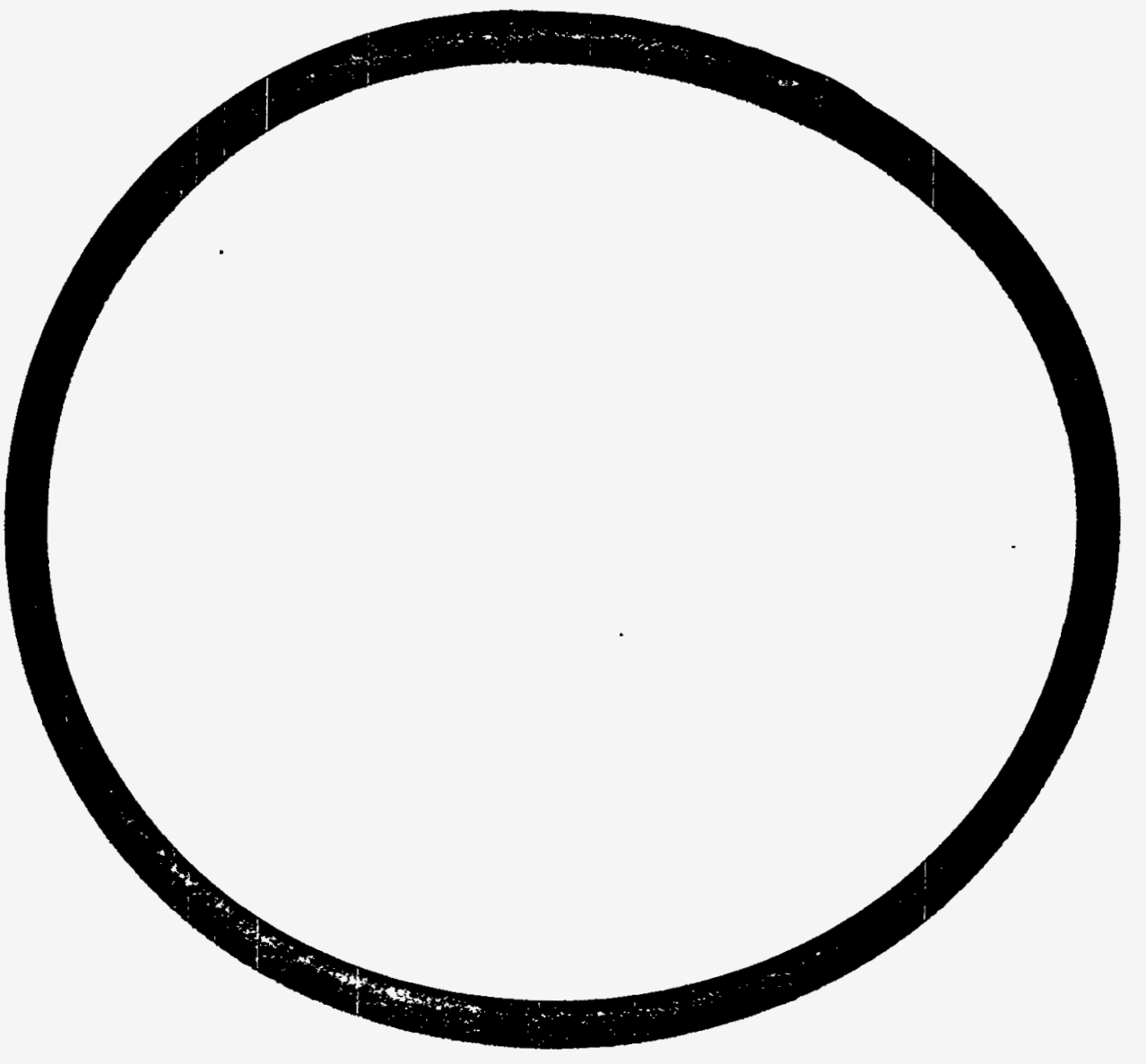


WHC-SD-WM-TRP-223

Rev. 0

\subsection{4-IN. ISB O-RING SEAL}

The initial test protocol involved a standard ambient temperature (no externa7 moments) "pressure ascension": at gauge pressures of 250, then 500 , and finally 1,000 $\mathrm{lbf} / \mathrm{in}^{2}$. The seals performed well. No leaks or pressure drop was noted. The next test in the series involved an elevated temperature of $300{ }^{\circ} \mathrm{F}$ at $1,000 \mathrm{lbf} / \mathrm{in}^{2}$ (gauge), with no external moments applied. Again, the seals performed well.

However, when testing was attempted at $1,000 \mathrm{lbf} / \mathrm{in}^{2}$ and $400^{\circ} \mathrm{F}$, problems developed. When an external moment was applied to the connector, the test technician observed a Teak. After cool down, the connector was disassembled and the seal inspected. The o-ring exhibited severe extrusion damage. Fraying and material separation had occurred. The fluorosilicone o-ring apparently had reached the upper limit of operating temperature.

Moment testing of the 4-in. ISB having Viton o-rings produced very good results. The 4-in. ISB, using Viton o-rings, demonstrated leak tightness both to "up and over" moments and to lateral moments. For an overall summary of 4-in. ISB test results, refer to Figure 7.

There were no leakage problems with the 4-in. ISB, as long as temperature 7 imits of $300^{\circ} \mathrm{F}$ for Kalrez and fluorosilicone o-ring seal material were not exceeded. 
Photograph 16. 4-In. 0-Ring Seal, TFE-0-SIL (Silicone Core with Teflon Outer Coating), 70-Durometer, 1,000 1bf $/ \mathrm{in}^{2}$ (gauge), $400^{\circ} \mathrm{F}$.

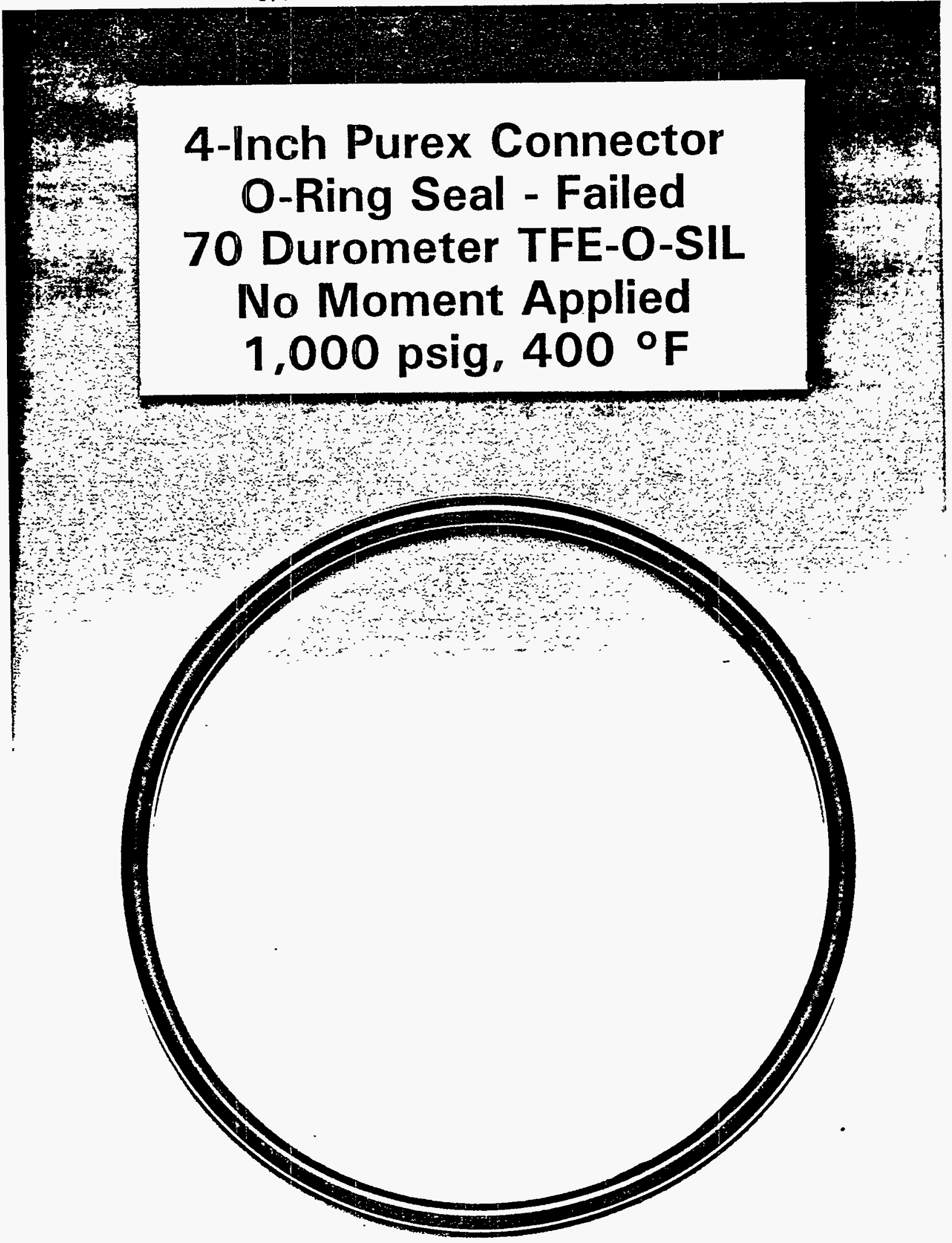




\begin{tabular}{|c|c|c|c|c|c|c|c|c|c|c|c|c|c|c|c|c|}
\hline \multicolumn{17}{|c|}{$\begin{array}{l}\text { PUREX TYPE JUMPER CONNECTOR - INTEGRAL SEAL BLOCK (ISB) LEAK TEST QUALIFICATION } \\
4 \text { In. Single Port ISB Connector }\end{array}$} \\
\hline & \multicolumn{8}{|c|}{ Ambient Temperature $-70 \mathrm{deg} . \mathrm{F}$. } & \multicolumn{8}{|c|}{ High Temperature (400 Deg. F. unless otherwise indicated) } \\
\hline & \multicolumn{4}{|c|}{ Up \& Over Moment } & \multicolumn{4}{|c|}{ Lateral Moment } & \multicolumn{4}{|c|}{ Up \& Over Moment } & \multicolumn{4}{|c|}{ Lateral Moment } \\
\hline $\begin{array}{c}\text { O-Ring } \\
\text { Material }\end{array}$ & $\begin{array}{c}250 \\
\text { psig }\end{array}$ & $\begin{array}{l}500 \\
\text { psig }\end{array}$ & $\begin{array}{c}750 \\
\text { psig }\end{array}$ & $\begin{array}{c}1,000 \\
\text { psig }\end{array}$ & $\begin{array}{c}250 \\
\text { psig }\end{array}$ & $\begin{array}{c}500 \\
\text { psig }\end{array}$ & $\begin{array}{l}750 \\
\text { psig }\end{array}$ & $\begin{array}{c}1,000 \\
\text { psig }\end{array}$ & $\begin{array}{c}250 \\
\text { psig }\end{array}$ & $\begin{array}{c}500 \\
\text { psig }\end{array}$ & $\begin{array}{c}750 \\
\text { psig }\end{array}$ & $\begin{array}{l}1,000 \\
\text { psig }\end{array}$ & $\begin{array}{c}250 \\
\text { psig }\end{array}$ & $\begin{array}{l}500 \\
\text { psig }\end{array}$ & $\begin{array}{l}750 \\
\text { psig }\end{array}$ & $\begin{array}{l}1,000 \\
\text { psig }\end{array}$ \\
\hline Viton & OK & OK & OK & OK & OK. & OK & OK & OK & OK & OK & OK & OK & OK & OK & OK & OK \\
\hline Kalrez & OK & OK & OK & OK & OK & OK & OK & OK & $\begin{array}{c}0 \mathrm{OK} \\
300\end{array}$ & $\begin{array}{l}0 \mathrm{OK} \\
300\end{array}$ & $\begin{array}{c}\text { OK } \\
300\end{array}$ & $\begin{array}{c}\text { OK } \\
300\end{array}$ & $\begin{array}{c}\text { OK } \\
300\end{array}$ & $\begin{array}{c}\text { OK } \\
300\end{array}$ & $\begin{array}{c}\text { OK } \\
300\end{array}$ & $\begin{array}{c}\text { OK } \\
300\end{array}$ \\
\hline Fiuorosit. & OK & OK & OK & OK & OK & OK & OK & OK & $\begin{array}{c}\text { FAIL } \\
\text { O400 } \\
\text { OK } \\
300\end{array}$ & $\begin{array}{l}\text { OK } \\
300\end{array}$ & $\begin{array}{c}\text { OK } \\
300\end{array}$ & $\begin{array}{c}\text { OK } \\
300\end{array}$ & OK & OK & OK & OK \\
\hline
\end{tabular}

FAIL = Seal Failed in Actual Test.

$300=$ Test Performed at 300 Deg. F. 
Rev. 0

\subsection{3-WAY ISB O-RING SEAL}

The 3-way ISB's performed very wel1. In particular, the ISB showed a marked improvement in its capability to withstand appilied moments (compared to that of the conventional three-way teflon gaskets). Again, there were no leakage problems, as long as temperature limits of the o-ring seal material were not exceeded. For an overal1 summary of three-way ISB test results, refer to Figure 8 . For the applied moment conditions used during the test, refer to Figure 3. 


\begin{tabular}{|c|c|c|c|c|c|c|c|c|c|c|c|c|c|c|c|c|}
\hline \multicolumn{17}{|c|}{$\begin{array}{c}\text { PUREX TYPE JUMPER CONNECTOR - INTEGRAL SEAL BLOCK (ISB) LEAK TEST QUALIFICATION } \\
2 \text { In. 3-Way Connector }\end{array}$} \\
\hline & \multicolumn{8}{|c|}{ Ambient Temperature -70 deg. F. } & \multicolumn{8}{|c|}{ High Temperature (400 Deg. F. unless otherwise indicated) } \\
\hline & \multicolumn{4}{|c|}{ Up \& Over Moment } & \multicolumn{4}{|c|}{ Lateral Moment } & \multicolumn{4}{|c|}{ Up \& Over Moment } & \multicolumn{4}{|c|}{ Lateral Moment } \\
\hline $\begin{array}{c}\text { 0-Ring } \\
\text { Material }\end{array}$ & $\begin{array}{l}250 \\
\text { psig }\end{array}$ & $\begin{array}{l}500 \\
\text { psig }\end{array}$ & $\begin{array}{c}750 \\
\text { psig }\end{array}$ & $\begin{array}{l}1,000 \\
\text { psig }\end{array}$ & $\begin{array}{c}250 \\
\text { psig }\end{array}$ & $\begin{array}{c}500 \\
\text { psig }\end{array}$ & $\begin{array}{l}750 \\
\text { psig }\end{array}$ & $\begin{array}{c}1,000 \\
\text { psig }\end{array}$ & $\begin{array}{l}250 \\
\text { psig }\end{array}$ & $\begin{array}{l}500 \\
\text { psig }\end{array}$ & $\begin{array}{c}750 \\
\text { psig }\end{array}$ & $\begin{array}{l}1,000 \\
\text { psig }\end{array}$ & $\begin{array}{c}250 \\
\text { psig }\end{array}$ & $\begin{array}{c}500 \\
\text { psig }\end{array}$ & $\begin{array}{c}750 \\
\text { psig }\end{array}$ & $\begin{array}{l}1,000 \\
\text { psig }\end{array}$ \\
\hline Viton & OK & OK & OK & OK & OK & OK & OK & OK & OK & OK & OK & OK & OK & OK & OK & OK \\
\hline Kairez & OK & OK & OK & OK & OK & OK & OK & OK & $\begin{array}{r}0 K \\
300 \\
\end{array}$ & $\begin{array}{r}0 K \\
300 \\
\end{array}$ & $\begin{array}{r}0 K \\
300 \\
\end{array}$ & $\begin{array}{r}0 K \\
300 \\
\end{array}$ & $\begin{array}{r}0 K \\
300 \\
\end{array}$ & $\begin{array}{r}0 K \\
300 \\
\end{array}$ & $\begin{array}{r}0 K \\
300 \\
\end{array}$ & $\begin{array}{r}0 K \\
300 \\
\end{array}$ \\
\hline Fluorosil. & OK & OK & OK & OK & OK & OK & OK & OK & $\begin{array}{l}0 \mathrm{OK} \\
300\end{array}$ & $\begin{array}{c}\text { OK } \\
300\end{array}$ & $\begin{array}{l}\text { OK } \\
300\end{array}$ & $\begin{array}{l}\text { OK } \\
300\end{array}$ & $\begin{array}{l}\text { OK } \\
300\end{array}$ & $\begin{array}{c}0 K \\
300\end{array}$ & $\begin{array}{l}\text { OK } \\
300\end{array}$ & $\begin{array}{l}\text { OK } \\
300\end{array}$ \\
\hline Silicone & OK & OK & OK & OK & OK & OK & OK & OK & $\begin{array}{c}0 K \\
300 \\
\end{array}$ & $\begin{array}{r}\text { OK } \\
300 \\
\end{array}$ & $\begin{array}{r}0 K \\
300 \\
\end{array}$ & $\begin{array}{l}\text { OK } \\
30\end{array}$ & $\begin{array}{r}0 K \\
300 \\
\end{array}$ & $\begin{array}{r}0 K \\
300 \\
\end{array}$ & $\begin{array}{r}0 K \\
300 \\
\end{array}$ & $\begin{array}{r}0 K \\
300 \\
\& 500 \\
\end{array}$ \\
\hline EPDM & OK & OK & OK & OK & OK & OK & OK & OK & OK & OK & OK & OK & OK & OK & OK & OK \\
\hline $\begin{array}{l}\text { Teflon } \\
\text { Gasket }\end{array}$ & FAIL & (F) & (F) & (F) & OK & NT & NT & NT & $\begin{array}{l}\text { FAIL } \\
212 \\
8400\end{array}$ & (F) & (F) & (F) & NT & NT & NT & NT \\
\hline
\end{tabular}

FAIL = Seal Failed in Actual Test.

$(F)=$ Not Tested, But Failed Under Less Rigorous Conditions, hence Failure Is Probable.

NT $=$ Not Tested.

$300=$ Test Performed at 300 Deg. F.

212 = Test Performed at 212 Deg. F. 
WHC-SD-WM-TRP-223

Rev. 0

\subsection{CONCLUSIONS}

\subsection{ADVANTAGES OF INTEGRAL SEAL BLOCK DESIGN FOR JUMPER CONNECTORS}

The FY 1993 design for seal retainers solved a number of problems associated with the gasket-type seal (Ruff 1994). However, the need for further improvement was obvious. The key advantage of the integral seal block design is that it eliminates a potential leak path.

The additional advantages of the ISB compared to the conventional gasket-type configuration are reiterated here from previous work (Ruff 1994a).

- At clamp loads only $1 / 6$ to $1 / 10$ required by Teflon gaskets, the ISB holds four to ten times higher pressure. Thus, lower wrench torques (50 ft-1bf) can be applied to the connector. These lower torques mean that smaller pneumatic torque wrenches can be used in canyon buildings; the result is easier access to connectors in small, crowded process cells. Also, $50 \mathrm{ft}-1 \mathrm{bf}$ of torque can be applied easily with manual tools. Powered wrenches no longer are an absolute necessity for field applications.

- Lower clamping loads greatly reduce stresses in the nozzle flange, connector hooks, and hook pins (Kanjilal 1992). Lower stresses increase the margin of safety for operation of the connector, i.e., the connector can absorb greater applied moments generated by the piping.

- Lower stresses in the mechanical components enable greater 7 atitude in the selection of materials for hooks and blocks. Alloys may be chosen for corrosion resistance rather than for the high tensile strength required for process service.

- The ISB eliminates.problems associated with deformation and flow of conventional Teflon gasket seal deformation and flow (Squier 1992).

- The o-ring nestles protectively within a circular groove. This configuration permits a greater range of block (connector piping) motion without shear damage to the seal. (Applied moments are less likely to "wipe" the seal.)

- The o-ring provides vastly improved leak tightness during thermat cycling and operation at elevated temperatures. Teflon gasket-connector seals leak after thermal cycling. In fact, with Teflon seals, the operating screw can become so loose after operation at elevated temperatures that it can be turned by hand.

- Only $50 \mathrm{ft}-1 \mathrm{bf}$ of torque is required to seal all three sizes of connectors $(2,3,4 \mathrm{in.})$. Lower torque greatly simplifies procedures for operating remote cells. No special administrative procedures are necessary to ensure that smaller connectors are not over-torqued. Because only one torque wrench is required (instead of two), gantry space on the overhead crane is freed up and the amount of control room wiring is reduced. 
Rev. 0

\subsection{ENABLING TECHNOLOGY FOR REMOTE APPLICATIONS}

Integral seal blocks make it easier to use high-pressure equipment in remote cells, e.g., high-pressure chemical reaction vessels, high-pressure slurry pumps for handiing very viscous mixtures.

\subsection{O-RING CROSS SECTION}

Within given space limits, it is generally better to use as thick an o-ring as possible (in a properly sized groove). A thicker o-ring (one having a 7 arger cross-sectional diameter) has a greater area moment of inertia and is therefore less "flimsy." A thicker o-ring also seats much more firmly into the o-ring groove than does a thinner o-ring. For example, during testing, the o-ring groove on the 4-in. seal-retainer was redesigned to accommodate an o-ring with a 0.210-in. cross-section, as opposed to the previous 0.139-in. cross-section.

\subsection{SEAL DUROMETER}

The 70-durometer material is an industry standard; it is often difficult to get softer (or harder) materials without paying premium batch charges for special polymer formulation.

Testing proceeded with conventional 70 -durometer o-rings. This material worked very wel1 at ambient temperature and pressures of $1,000 \mathrm{lbf} / \mathrm{in}^{2}$ (gauge) and below. This result makes procurement of seals much easier and less expensive.

However, the chemical and radiation resistance required for many process applications may vary. Elastomers must be selected on the basis of expected service conditions (chemical and radiation), and then tested at the temperature and pressure of the intended application.

\subsection{SEAL MATERIAL}

These proof-of-concept tests used 70-durometer Viton, fluorosilicone, and Kalrez elastomer material for the o-rings, as these o-rings are readily available, even in sma17 lot quantities. In actual plant.applications, different o-ring materials would often be needed. Actual material selection must be tailored to meet the service conditions, e.g., $\mathrm{pH}$, temperature, chemical composition of the process stream, and radiation exposure.

These tests led to the following conclusions.

- The ISB o-ring seal design performed as expected. It was leak tight to $1,000 \mathrm{Tbf} / \mathrm{in}^{2}$ (gauge), when kept within the appropriate temperature limits of the o-ring elastomer.

- The most important factor 7 imiting ISB connector performance is temperature resistance of the o-ring material. All o-rings performed well at ambient temperature. However, tests at elevated temperatures quickly revealed weaknesses in fluorosilicone and Kalrez. 
- Viton o-rings did not leak under any of the tested conditions, up to and including $1,000 \mathrm{lbf} / \mathrm{in}^{2}$ (gauge) and $400^{\circ} \mathrm{F}$, even when external moments were applied. Viton was the stellar performer when it came to resisting leaks under al1 conditions.

- Viton seals are very cost effective - reasonably priced and perform very well. Kalrez and fluorosilicone o-rings are more expensive and do not resist elevated temperatures a well as Viton. Viton seals should be used wherever possible for the best, most cost-effective leak resistance in jumper connector ISBs.

- Seal failures occurred with fluorosilicone and KaTrez o-rings, when "up and over" moments were applied at $400^{\circ} \mathrm{F}$ (refer to Figure 3 for applied moments). Fluorosilicone and Kalrez o-rings subsequently were tested at $300^{\circ} \mathrm{F}$ and maintained leak tightness at this temperature.

- Both fluorosilicone and Kalrez o-rings withstood lateral moments at $400{ }^{\circ} \mathrm{F}$ (refer to Figure 3 for applied moments). This information, coupled with the fact that failure occurred when up and over" moments were applied at the same temperature, indicate that the "up and over" moment is a more severe test of leak tightness for an ISB o-ring. However, the performance of these materials indicates that Kalrez and fluorosilicone should NOT be used in applications where temperatures exceed $300^{\circ} \mathrm{F}$. Preferably, long-term temperature exposure of these two materials should be held to temperatures less than $300^{\circ} \mathrm{F}$.

- Kalrez or fluorosilicone seals should be used only at low temperatures and only when necessary for chemical resistance to specific process fluids. This restriction should be observed especially with Kalrez, because each Kalrez o-ring costs hundreds of dollars.

Figure 9. Summary Results of Leak Tests on ISB Jumper Connectors Using 0-Ring Seals.

\begin{tabular}{|c|c|}
\hline \multicolumn{2}{|c|}{$\begin{array}{l}\text { Test Temperatures: } 70,300 \text {, and } 400{ }^{\circ} \mathrm{F} \\
\text { Test Pressures: } 250,500,750 \text {, and } 1,000 \mathrm{lbf} / \mathrm{in}^{2} \\
\text { Test Moments: Refer to Figure } 3 \text {. }\end{array}$} \\
\hline Seal Material & Result \\
\hline Fluorosilicone & OK only to $300^{\circ} \mathrm{F}$ \\
\hline Kalrez & OK only to $300^{\circ} \mathrm{F}$ \\
\hline Viton & OK to $400^{\circ} \mathrm{F}$ \\
\hline EPDM (tested on 3-Way on Ty) & OK to $400^{\circ} \mathrm{F}$ \\
\hline Silicone (tested on 3-Way only) & $\begin{array}{l}\text { OK to } 300^{\circ} \mathrm{F} \\
\text { (see Appendix } R \text { for lateral moment leak test } \\
\text { conducted at } 1,250^{\mathrm{bf} / \text { in }}{ }^{2} \text { and } 500^{\circ} \mathrm{F} \text { ) } \\
\end{array}$ \\
\hline
\end{tabular}




\subsection{FUTURE IMPROVED DESIGN - JUMPER CONNECTORS WITH ONE-PIECE BODIES}

A jumper connector recentiy has been designed to el iminate the need for many separate parts. Dubbed the "one-piece-body" or "OPB" connector (see Figures 7 and 8 ), it exists only as a preliminary three-dimensional computer model.

The one-piece-body (OPB) design has the following significant advantages:

- It improves the "assembled integrity" of the jumper connector, i.e., there are no extraneous pieces to come loose.

- It reduces peak bending stresses in the jumper connector hook by $50 \%$, because it eliminates a protrusion in the lower skirt; thus the hook is straight rather than curved.

- It does not require tie bolts; the elimination of tie bolts eliminates concerns about the possibility of counterfeit fasteners.

- It e7iminates the need for fabrication, inspection, and inventory of long-lead-time castings. The results are cost savings in manufacturing, storage, and overhead.

- It simplifies the manual assembly of the jumper connector. El imination of the need to use tie rods to connect the upper hook guide and the lower skirt casting reduces the chances of failure of the end product.

- As a unified, rigid component machined from a single section of steel, an $\mathrm{OPB}$ enhances the operating integrity of the part. It eliminates backlash and movement associated with assembiy.

- Because there are no tie bolts to interfere with the attached piping, the OPB connector allows greater freedom to the piping designer in the direction of flow discharge from a horizontal connector. Conventional jumper connectors require that a tie bolt be removed and that the lower skirt be partialiy ground away to allow lateral piping attachment.

On the other hand, the one-piece-body (OPB) design has the following disadvantages:

- The greatest challenge it poses is the development of machining methods needed to produce the complex curvatures used in the component. (This, however, could be useful as a "technology driver" which could bring a new level of sophistication to current machining capability.

- Being straight, and not curved, hooks for the OPB could not be used interchangeably with the existing multi-piece connectors.

- The operating screw retaining mechanism would also have to be different than currently existing hardware. This involves designing a relatively small part that would act to keep the operating screw permanently attached to the connector body. Hence, OPB operating screws would not be interchangeable with existing hardware. 
WHC-SD-WM-TRP-223

Rev. 0

- The OPB design would not be applicable for thermocouple-type jumper connectors and leak detector-type jumper connectors. The separate-block jumper connector assembiy is more useful in these 2 specific applications because the block must be removeable to enable assembly of the spring-loaded thermocouple or leak detector element.

However, after considering both the advantages and disadvantages, for fluid flow applications, the $O P B$ design is considered to have relatively more advantages with very low technical risk in performance. A prototype of the one-piece-body (OPB) component for fluid flow applications should be developed and tested to qualify it for operating service. 
Figure 10. One-Piece Body, Machined from Solid Bar Stock - Side View.

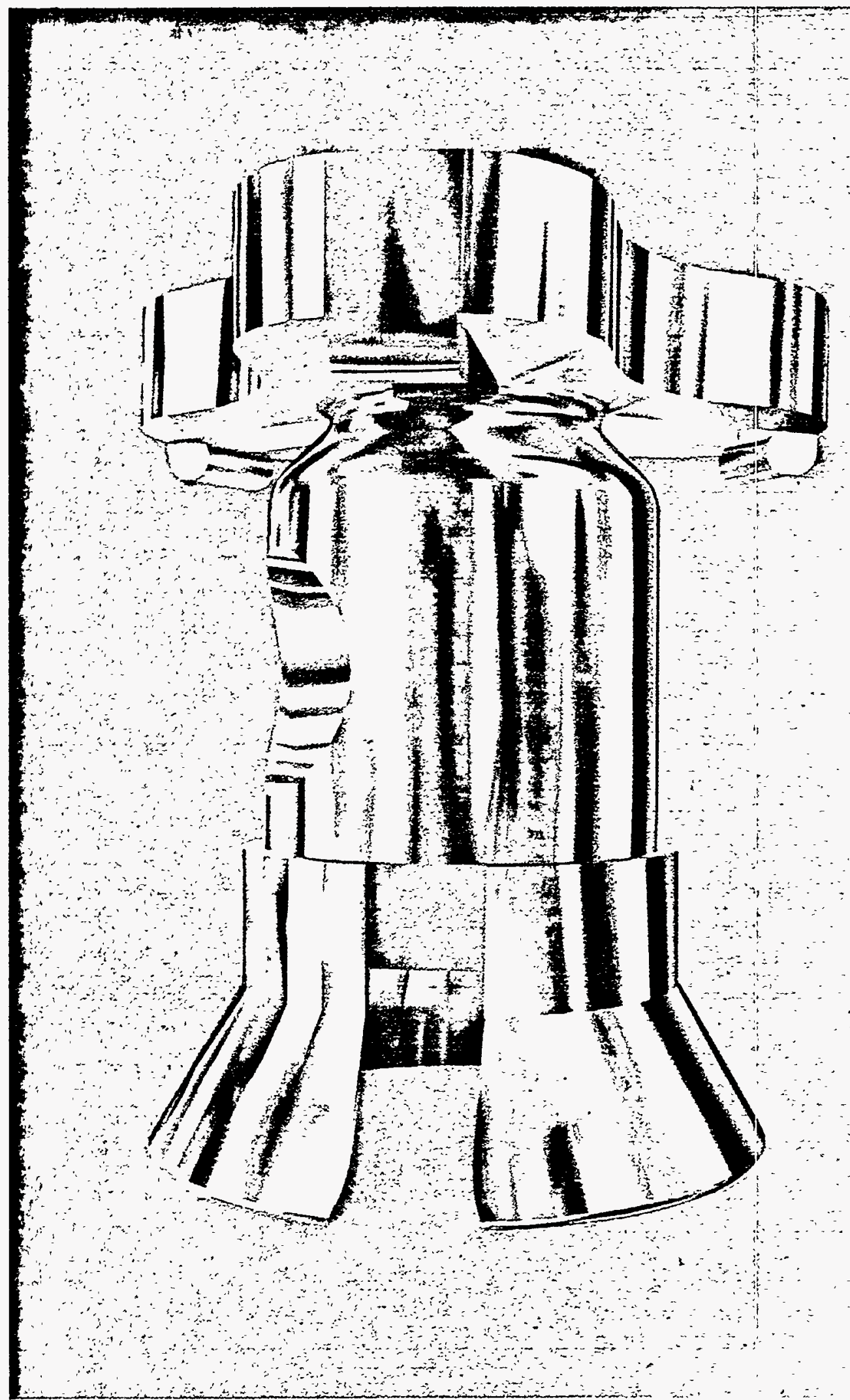


WHC-SD-WM-TRP-223

Rev. 0

Figure 11. One-Piece Body, Machined from Solid Bar Stock - Underside View.

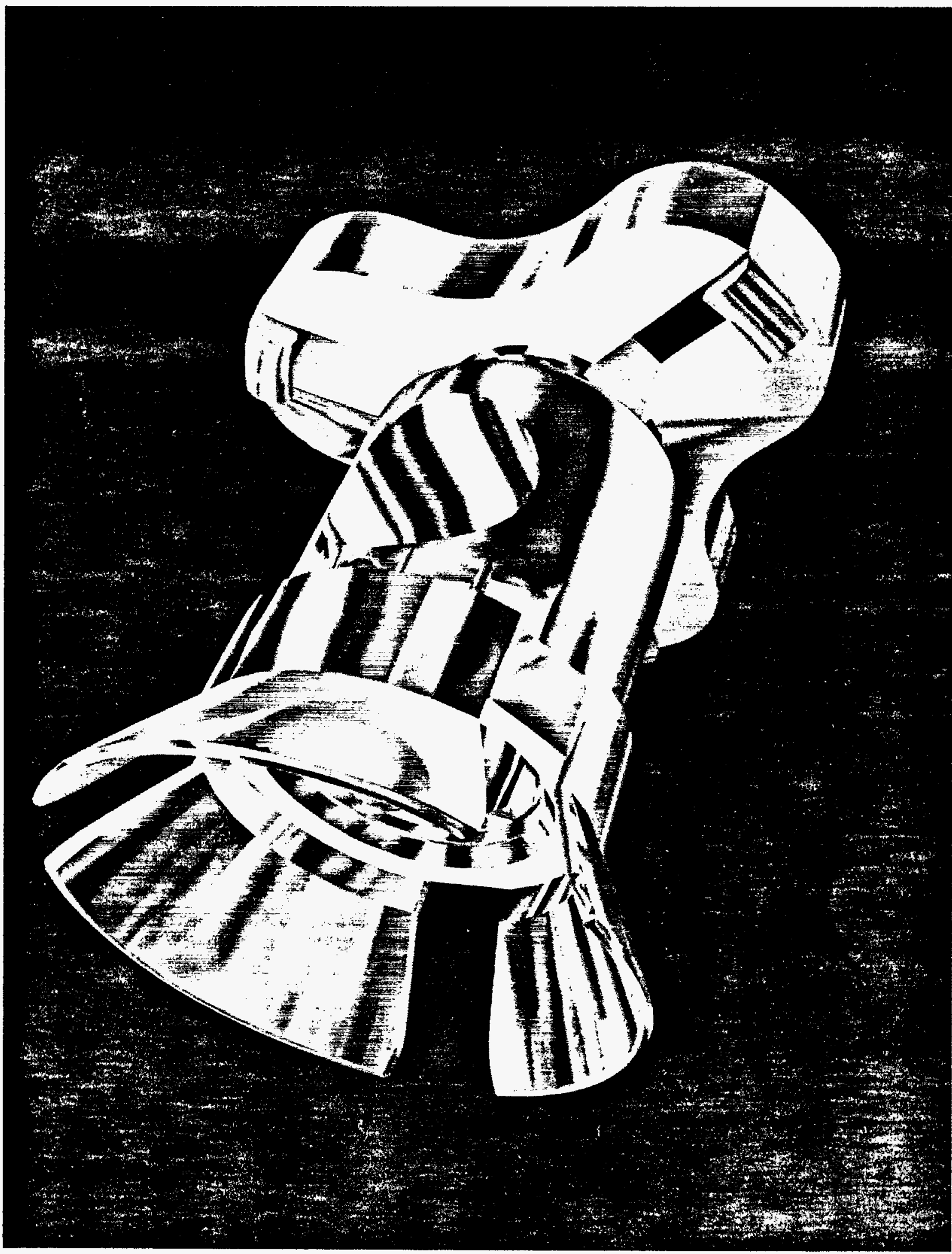


Rev. 0

\subsection{RECOMMENDATIONS FOR FUTURE DEVELOPMENT WORK ON JUMPER CONNECTORS}

While the integral seal block using o-rings has improved jumper connector leak tightness, a number of additional development tasks related to jumper connectors remain. Further investigations, if performed, would expand the range of applications of jumper connectors, and resolve unknowns particularly in the area of load path, ultimate load capacity, rigidity and fit-up.

These tasks include:

- Increase the load capacity of the jumper connector test stand to $10,000 \mathrm{~Tb}$.

- Perform "stiffness testing" of the jumper connector assemblies to enable stress analysts to refine assumptions about connector rigidity during thermal expansion and seismic events.

- Perform strain-gauge testing of jumper connector assemblies that are stressed by externally applied moments to determine the load path in the connector hooks.

- Perform destructive pull testing of machined connector hooks and hook pins on the load frame to determine stress-concentration factors and ultimate breaking limits on these critical components.

- Proof test jumper connector seals that have been specially molded to enhance mechanical retention of the seal during jumper connect and disconnect operations in the field.

- Develop and test horizonta7 jumper connectors with improved hook/cam tolerances that will ensure full hook engagement to the nozzle flange (applies to the bottom hook).

- Develop and test OPB connectors to elimiriate the need for tie-bolts and the long lead-time castings for the jumper assembly.

- Develop (and verify operation of) horizontal jumper connector lower skirts that are designed specifically to allow "side discharge" flow paths to the left and right. Current practice uses a rather crude method of removing one tie bolt, and grinding the lower skirt casting to fit the piping.

- Develop and test o-ring seal specialty connectors such as thermo-wel1, thermocouple, and level-detection instrumentation. 


\subsection{REFERENCES}

Austin, David A., 1993, PUREX Style Jumper Connector Type III Gasket Test Report, WHC-SD-HWV-TRP-006, Rev. 0, Westinghouse Hanford Company, Richland, Washington.

Jordan, Steven R., 1994a, Laboratory Notebook \#WHC-N-817 (Hard Bound and Paginated), pp. 44-142, Westinghouse Hanford Company, Richland, Washington.

Jordan, Steven R., 1994b, Laboratory Notebook \#WHC-N-1088 (Hard Bound and Paginated), "3-Way Integral Seal Block Jumper Connector," Westinghouse Hanford Company, Richland, Washington.

Jordan, Steven R., 1994c, PUREX Jumper Connector Integral Seal Block Leak Test Data, WHC-SD-WM-DP-118, Rev. 0, Westinghouse Hanford Company, Richland, Washington.

Kanjilal, Sunil K., 1992, 2-Inch by 2-Inch PUREX-Type Jumper Connector Analysis, WHC-SD-W028-DA-001, Rev. 0, Westinghouse Hanford Company, Richland, Washington.

Ruff, Edward S., 1994a, PUREX Jumper Connector O-Ring Seal Development, WHC-SD-HWV-TRP-007, Rev. 0, Westinghouse Hanford Company, Richland, Washington.

Ruff, Edward S., 1994b, Jumper Connector Integral Seal Block Test P7an and Procedure, WHC-SD-WM-TP-256, Rev. 0, Westinghouse Hanford Company, Richland, Washington.

Squier, Donald M., 1992, Jumper Connector Test Report, WHC-SD-HWV-TRP-004, Rev. 0, Westinghouse Hanford Company, Richland, Washington.

Squier, Donald M., 1993, Experimental Graphite Gasket for the PUREX Process Pipe Connector, Internal Memo Report \#22360-93-002, dated March 29, Westinghouse Hanfọd Company, Richland, Washington. 
WHC-SD-WM-TRP-223

Rev. 0

\section{Additional References For Further Reading}

Brackenbury, Phil J., et a1., 1992, PUREX Connector Workshop Meeting Minutes, October 2, 1992, HWVP Systems Engineering Internal Correspondence \#7F133-MM92-001, Westinghouse Hanford Company, Richland, Washington.

Cheh1, Sahib S., et a1., 1992, Develop Design Load Ratings For Pipe Jumper Connectors Used at the Hanford Site, Contract No. MLR-SWV-256826, Department of Mechanical Engineering, Southern University, Louisiana.

Kanjila1, Sunil K., 1992, 2-Inch by 2-Inch PUREX-Type Jumper Connector Analysis For Hanford Waste Vitrification Plant, WHC-SD-HWV-DCR-001, Rev. 0, Westinghouse Hanford Company, Richland, Washington.

Ruff, Edward S., 1992a, Jumper Connector Test P7an, WHC-SD-WM-TP-133, Rev. 0, Westinghouse Hanford Company, Richland, Washington.

Ruff, Edward S., and H. H. Byers, 1992b, Machining of Jumper Connector Nozz7es, WHC-SD-HWV-TRP-003, Rev.0, Westinghouse Hanford Company, Richl and, Washington. 
WHC-SD-WM-TRP-223

Rev. 0

APPENDIX A: PLANNING CHARTS

A-1 


\begin{tabular}{|c|c|c|c|c|c|}
\hline \multicolumn{6}{|c|}{ MWTF Integral Seal Block Jumper Connector Development and Leak Testing } \\
\hline ID & Name & \begin{tabular}{l|l} 
Duration \\
\end{tabular} & \% Complate & Scheduled Start & Scheduled Finish \\
\hline 1 & MWTF CONNECTOR SEALS & $186 d$ & $90 \%$ & $1 / 1 / 94$ & $9 / 19 / 94$ \\
\hline 2 & Test Preparation & $111.9 d$ & $100 \%$ & $1 / 1 / 94$ & $6 / 7 / 94$ \\
\hline$\overline{3}$ & Task Planning & $29 d$ & $100 \%$ & $1 / 1 / 94$ & $2 / 10 / 94$ \\
\hline 4 & Initial Cost and Schedule Estimate & $6 d$ & $100 \%$ & $1 / 1 / 94$ & $1 / 10 / 94$ \\
\hline 5 & Updated Cost and Schedule Estimate & 1d & $100 \%$ & $2 / 10 / 94$ & $2 / 10 / 94$ \\
\hline 6 & & & & & \\
\hline$\overline{7}$ & Task Research & $13 d$ & $100 \%$ & $1 / 1 / 94$ & $1 / 19 / 94$ \\
\hline 8 & Request Process Solutions Info & $1 d$ & $100 \%$ & $1 / 1 / 94$ & $1 / 3 / 94$ \\
\hline 9 & Cog Organization Responds & 1d & $100 \%$ & $1 / 4 / 94$ & $1 / 4 / 94$ \\
\hline 10 & Develope List of Process Solutions & 2d & $100 \%$ & $1 / 5 / 94$ & $1 / 6 / 94$ \\
\hline 11 & Review Technical Literature & 1d & $100 \%$ & $1 / 7 / 94$ & $1 / 7 / 94$ \\
\hline 12 & Vendor Information & 1d & $100 \%$ & $1 / 10 / 94$ & $1 / 10 / 94$ \\
\hline 13 & Bibliographic Search & 1d & $100 \%$ & 1/11/94 & $1 / 11 / 94$ \\
\hline 14 & Compare Polymer Performance & $3 d$ & $100 \%$ & $1 / 12 / 94$ & $1 / 14 / 94$ \\
\hline 15 & Identify Data Insufficiencies & 1d & $100 \%$ & $1 / 17 / 94$ & $1 / 17 / 94$ \\
\hline 16 & Select Polymers to $\mathrm{Be}$ Tested & 1d & $100 \%$ & $1 / 18 / 94$ & $1 / 18 / 94$ \\
\hline 17 & Establish Test Parameters & 1d & $100 \%$ & 1/19/94 & 1/19/94 \\
\hline 18 & & & & & \\
\hline 19 & Design Drawing Package & $74 d$ & $100 \%$ & $1 / 17 / 94$ & $4 / 28 / 94$ \\
\hline 20 & 2 Inch 3 Way Connector Fixture & $32 d$ & $100 \%$ & $1 / 17 / 94$ & $3 / 1 / 94$ \\
\hline 21 & Nozzle and Plate & $2 d$ & $100 \%$ & $1 / 17 / 94$ & 1/18/94 \\
\hline 22 & Heater Block & 4d & $100 \%$ & 1/19/94 & $1 / 24 / 94$ \\
\hline 23 & 3 Way Seal Retainer & 1d & $100 \%$ & $1 / 25 / 94$ & $1 / 25 / 94$ \\
\hline 24 & Pressure Fittings/Components & 4d & $100 \%$ & $1 / 26 / 94$ & $1 / 31 / 94$ \\
\hline 25 & Assembly & $3 \mathrm{~d}$ & $100 \%$ & $2 / 1 / 94$ & $2 / 3 / 94$ \\
\hline 26 & Check & 3d & $100 \%$ & $2 / 21 / 94$ & $2 / 23 / 94$ \\
\hline 27 & Approve & 1d & $100 \%$ & $2 / 24 / 94$ & $2 / 24 / 94$ \\
\hline 28 & Cog. Engineer Review & $7 d$ & $100 \%$ & $2 / 21 / 94$ & $3 / 1 / 94$ \\
\hline 29 & & & & & \\
\hline 30 & Prepare Assembly Color Rendering & $2 d$ & $100 \%$ & $2 / 3 / 94$ & $2 / 5 / 94$ \\
\hline 31 & Prepare Engineering Data Transmittal & $1 \mathrm{~d}$ & $100 \%$ & $2 / 7 / 94$ & $2 / 7 / 94$ \\
\hline 32 & Reproduce and Microfilm Drawing Set & $\overline{1 d}$ & $100 \%$ & $2 / 8 / 94$ & $2 / 8 / 94$ \\
\hline 33 & Prepare Mailing Tubes & 1d & $100 \%$ & $2 / 9 / 94$ & $2 / 9 / 94$ \\
\hline 34 & Mail Transmittals & 1d & $100 \%$ & $2 / 10 / 94$ & $2 / 10 / 94$ \\
\hline 35 & & & & & \\
\hline 36 & Design Integral Seal/Block & $22 d$ & $100 \%$ & $2 / 1 / 94$ & $3 / 2 / 94$ \\
\hline 37 & $2^{n}$ Seal Block & $3 d$ & $100 \%$ & $2 / 1 / 94$ & $2 / 3 / 94$ \\
\hline 38 & $3^{n}$ Seal Block & $\overline{3 d}$ & $100 \%$ & $2 / 4 / 94$ & $2 / 8 / 94$ \\
\hline 39 & 4" Seal Block & $3 d$ & $100 \%$ & $2 / 9 / 94$ & $2 / 11 / 94$ \\
\hline 40 & $2^{n}$ Seal Block Moment Arm Assembly & Td & $100 \%$ & $2 / 10 / 94$ & $2 / 14 / 94$ \\
\hline
\end{tabular}




\begin{tabular}{|c|c|c|c|c|c|}
\hline \multicolumn{6}{|c|}{ MWTF Integral Seal Block Jumper Connector Devalopment and Laak Testing } \\
\hline ID & Name & Duration & \% Complete & Schaduled Start & Scheduled Finish \\
\hline 41 & $3^{n}$ Seal Block Moment Arm Assembly & $1 \mathrm{~d}$ & $100 \%$ & $2 / 11 / 94$ & $2 / 14 / 94$ \\
\hline 42 & $4^{n}$ Seal Block Moment Arm Assembly & 1d & $100 \%$ & $2 / 14 / 94$ & $2 / 74 / 94$ \\
\hline 43 & 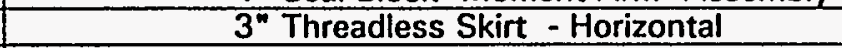 & 6d & $100 \%$ & $2 / 15 / 94$ & $2 / 22 / 94$ \\
\hline 44 & $3^{n \text { Threadless Skirt - Vertical }}$ & 3d & $100 \%$ & $2 / 15 / 94$ & $2 / 23 / 94$ \\
\hline 45 & 4" Threadless Skirt- Horizontal & 5d & $100 \%$ & $2 / 23 / 94$ & $3 / 1 / 94$ \\
\hline 46 & 4" Threadless Skirt-Vertical & $3 d$ & $100 \%$ & $2 / 28 / 94$ & $3 / 2 / 94$ \\
\hline 47 & & & & & \\
\hline 48 & Seal Block Jumper Assembly Dwgs & 10d & $100 \%$ & $4 / 15 / 94$ & $4 / 28 / 94$ \\
\hline 49 & $2^{n}$ Jumper Assembly Drawing & $3 d$ & $100 \%$ & $4 / 15 / 94$ & $4 / 19 / 94$ \\
\hline 50 & 3" Jumper Assembly Drawing & 4d & $100 \%$ & $4 / 20 / 94$ & $4 / 25 / 94$ \\
\hline 51 & 4" Jumper Assembly Drawing & 3d & $100 \%$ & $4 / 26 / 94$ & $4 / 28 / 94$ \\
\hline 52 & & & & & \\
\hline 53 & & & & & \\
\hline 54 & Design Study 3-Way Tetraseal Retainer & 82d & $100 \%$ & $3 / 10 / 94$ & $7 / 1 / 94$ \\
\hline 55 & Project Go-Ahead Decision & $10 \mathrm{~d}$ & $100 \%$ & $3 / 10 / 94$ & $3 / 23 / 94$ \\
\hline 56 & Seal Alternatives & $10 d$ & $100 \%$ & $5 / 2 / 94$ & $5 / 13 / 94$ \\
\hline 57 & Investigate Machinability & $10 \mathrm{~d}$ & $100 \%$ & $5 / 16 / 94$ & $5 / 27 / 94$ \\
\hline 58 & Decide on Feasibility & $5 d$ & $100 \%$ & $5 / 30 / 94$ & $6 / 3 / 94$ \\
\hline 59 & Preliminary AutoCAD Designs & $10 \mathrm{~d}$ & $100 \%$ & $6 / 6 / 94$ & $6 / 17 / 94$ \\
\hline 60 & Formal AutoCAD Design & $10 \mathrm{~d}$ & $100 \%$ & $6 / 20 / 94$ & $7 / 1 / 94$ \\
\hline 61 & & & & & \\
\hline 62 & & & & & \\
\hline 63 & Seal Retainer Removal Tool & $44.4 d$ & $100 \%$ & $5 / 26 / 94$ & $7 / 29 / 94$ \\
\hline 64 & Seal Retainer Removal Tool Dwgs & $27.3 d$ & $100 \%$ & $5 / 26 / 94$ & $7 / 6 / 94$ \\
\hline 65 & $2^{n}$ Removal Tool & $2 \mathrm{~d}$ & $100 \%$ & $5 / 26 / 94$ & $7 / 6 / 94$ \\
\hline 66 & $3^{\prime \prime}$ Removal Tool & $2 \mathrm{2d}$ & $100 \%$ & $5 / 30 / 94$ & $7 / 6 / 94$ \\
\hline 67 & $4^{\prime \prime}$ Removal Tool & $2 d$ & $100 \%$ & $6 / 1 / 94$ & $7 / 6 / 94$ \\
\hline 68 & Fab Removal Tool & 12d & $100 \%$ & $7 / 13 / 94$ & $7 / 29 / 94$ \\
\hline 69 & Submit Request for Cost Estimate & $1 d$ & $100 \%$ & $7 / 13 / 94$ & $7 / 14 / 94$ \\
\hline 70 & Obtain Cost Estimate & 1d & $100 \%$ & $7 / 14 / 94$ & $7 / 15 / 94$ \\
\hline 71 & Prepare J-10 Fab Request & Td & $100 \%$ & $7 / 15 / 94$ & $7 / 16 / 94$ \\
\hline 72 & Approve and Submit J-10 & Td & $100 \%$ & 7/16/94 & $7 / 18 / 94$ \\
\hline 73 & Machine 2" Removal Tool & $4 d$ & $100 \%$ & $7 / 18 / 94$ & $7 / 22 / 94$ \\
\hline 74 & Machine 3" Removal Tool & 3d & $100 \%$ & $7 / 22 / 94$ & $7 / 27 / 94$ \\
\hline 75 & Machine 4" Removal Tool & $4 \mathrm{~d}$ & $100 \%$ & $7 / 25 / 94$ & $7 / 29 / 94$ \\
\hline 76 & & & & & \\
\hline 77 & & & & & \\
\hline 78 & Procure Material & $\overline{77 d}$ & $100 \%$ & $1 / 17 / 94$ & $5 / 3 / 94$ \\
\hline 79 & Write Purchase Requisitions & $20 d$ & $100 \%$ & $1 / 17 / 94$ & $2 / 11 / 94$ \\
\hline 80 & O-Rings & $1 d$ & $100 \%$ & $1 / 19 / 94$ & $1 / 19 / 94$ \\
\hline
\end{tabular}




\begin{tabular}{|c|c|c|c|c|c|}
\hline \multicolumn{6}{|c|}{ MWTF Integral Soel Block Jumper Connector Davelopment and Leak Testing } \\
\hline ID & Namo & Duration & \% Completo & Schoduled Start & Schoduled Finish \\
\hline 81 & $2^{n}$ 3WayConnector Parts - 1 set & $10 \mathrm{~d}$ & $100 \%$ & $1 / 20 / 94$ & $2 / 2 / 94$ \\
\hline 82 & Issue Purchase Requisitions & 5d & $100 \%$ & $1 / 17 / 94$ & $1 / 21 / 94$ \\
\hline 83 & Receive Bids & $15 d$ & $100 \%$ & $1 / 24 / 94$ & $2 / 11 / 94$ \\
\hline 84 & Order Material & 1d & $100 \%$ & $4 / 7 / 94$ & $4 / 7 / 94$ \\
\hline 85 & 0 -Rings, 18 ea. -142 & 1d & $100 \%$ & $4 / 7 / 94$ & $4 / 7 / 94$ \\
\hline 86 & $0-$ Rings, 18 ea. -239 & $1 \mathrm{~d}$ & $100 \%$ & $4 / 7 / 94$ & $4 / 7 / 94$ \\
\hline 87 & O-Rings, 18 ea. -350 & Td & $100 \%$ & $4 / 7 / 94$ & $4 / 7 / 94$ \\
\hline 88 & & & & & \\
\hline 89 & Receive Material & 18d & $100 \%$ & $4 / 8 / 94$ & $5 / 3 / 94$ \\
\hline 90 & Vendor Fulfills Order & $15 d$ & $100 \%$ & $4 / 8 / 94$ & $4 / 28 / 94$ \\
\hline 91 & Inspect Material & $2 d$ & $100 \%$ & $4 / 29 / 94$ & $\frac{7+20 / 2194}{5 / 2 / 94}$ \\
\hline 92 & Deliver to Shop & $\overline{1 \mathrm{~d}}$ & $100 \%$ & $5 / 3 / 94$ & $5 / 3 / 94$ \\
\hline 93 & & & & & \\
\hline 94 & Procure Kalrez Material & $61.9 d$ & $100 \%$ & $3 / 14 / 94$ & $6 / 7 / 94$ \\
\hline 95 & Purchase Requisitions & $40 d$ & $100 \%$ & $3 / 14 / 94$ & $5 / 6 / 94$ \\
\hline 96 & O-Aings & $1 \mathrm{~d}$ & $100 \%$ & $3 / 14 / 94$ & $3 / 14 / 94$ \\
\hline 97 & Issue Purchase Requisitions & $5 d$ & $100 \%$ & $4 / 11 / 94$ & $4 / 15 / 94$ \\
\hline 98 & Receive Bids & $15 d$ & $100 \%$ & $4 / 18 / 94$ & $5 / 6 / 94$ \\
\hline 99 & Order Material & $19.1 d$ & $100 \%$ & $4 / 13 / 94$ & $5 / 10 / 94$ \\
\hline 100 & O-Rings, 3 ea. for 3-way & $1 \mathrm{~d}$ & $100 \%$ & $5 / 9 / 94$ & $5 / 9 / 94$ \\
\hline 101 & O-Rings, 3 ea. -142 & 1d & $100 \%$ & $4 / 13 / 94$ & $5 / 10 / 94$ \\
\hline 102 & O-Rings, 3 ea. -239 & 1d & $100 \%$ & $4 / 13 / 94$ & $5 / 9 / 94$ \\
\hline 103 & O-Rings, 3 ea. -350 & $1 \mathrm{~d}$ & $100 \%$ & $4 / 13 / 94$ & $5 / 9 / 94$ \\
\hline 104 & Receive Material & $38.9 d$ & $100 \%$ & $4 / 14 / 94$ & $6 / 7 / 94$ \\
\hline 105 & Vendor Fulfills Order & $25 d$ & $100 \%$ & $4 / 14 / 94$ & $6 / 7 / 94$ \\
\hline 106 & Inspect Material & $2 d$ & $100 \%$ & $5 / 19 / 94$ & $6 / 7 / 94$ \\
\hline 107 & Deliver to Shop & $\overline{1 d}$ & $100 \%$ & $5 / 23 / 94$ & $6 / 7 / 94$ \\
\hline 108 & & & & & \\
\hline 109 & & & & & \\
\hline 110 & Fab 2" 3Way Connector Test Fixture & $32.5 d$ & $100 \%$ & $2 / 14 / 94$ & $3 / 30 / 94$ \\
\hline 111 & Prepare Work Package & $1 \mathrm{~d}$ & $100 \%$ & $3 / 2 / 94$ & $3 / 2 / 94$ \\
\hline 112 & Obtain Final Cost Estimate & 1d & $100 \%$ & $3 / 3 / 94$ & $3 / 3 / 94$ \\
\hline 113 & Issue Work Order & 1d & $100 \%$ & $3 / 8 / 94$ & $3 / 8 / 94$ \\
\hline 114 & Carbide Inserts - Material Allowance & 1d & $100 \%$ & $3 / 9 / 94$ & $3 / 9 / 94$ \\
\hline 115 & Setup Machine & $1 d$ & $100 \%$ & $3 / 10 / 94$ & $3 / 10 / 94$ \\
\hline 116 & Install Chuck & $0.5 d$ & $100 \%$ & $3 / 11 / 94$ & $3 / 11 / 94$ \\
\hline 117 & Obtain Tool Holders & $0.5 d$ & $100 \%$ & $3 / 11 / 94$ & $3 / 11 / 94$ \\
\hline 118 & Install Inserts & $0.5 d$ & $100 \%$ & $3 / 14 / 94$ & $3 / 14 / 94$ \\
\hline 119 & Install Tool Ensemble & $0.5 d$ & $100 \%$ & $3 / 14 / 94$ & $3 / 14 / 94$ \\
\hline 120 & Section Bar Stock & $0.5 d$ & $100 \%$ & $3 / 15 / 94$ & $3 / 15 / 94$ \\
\hline
\end{tabular}




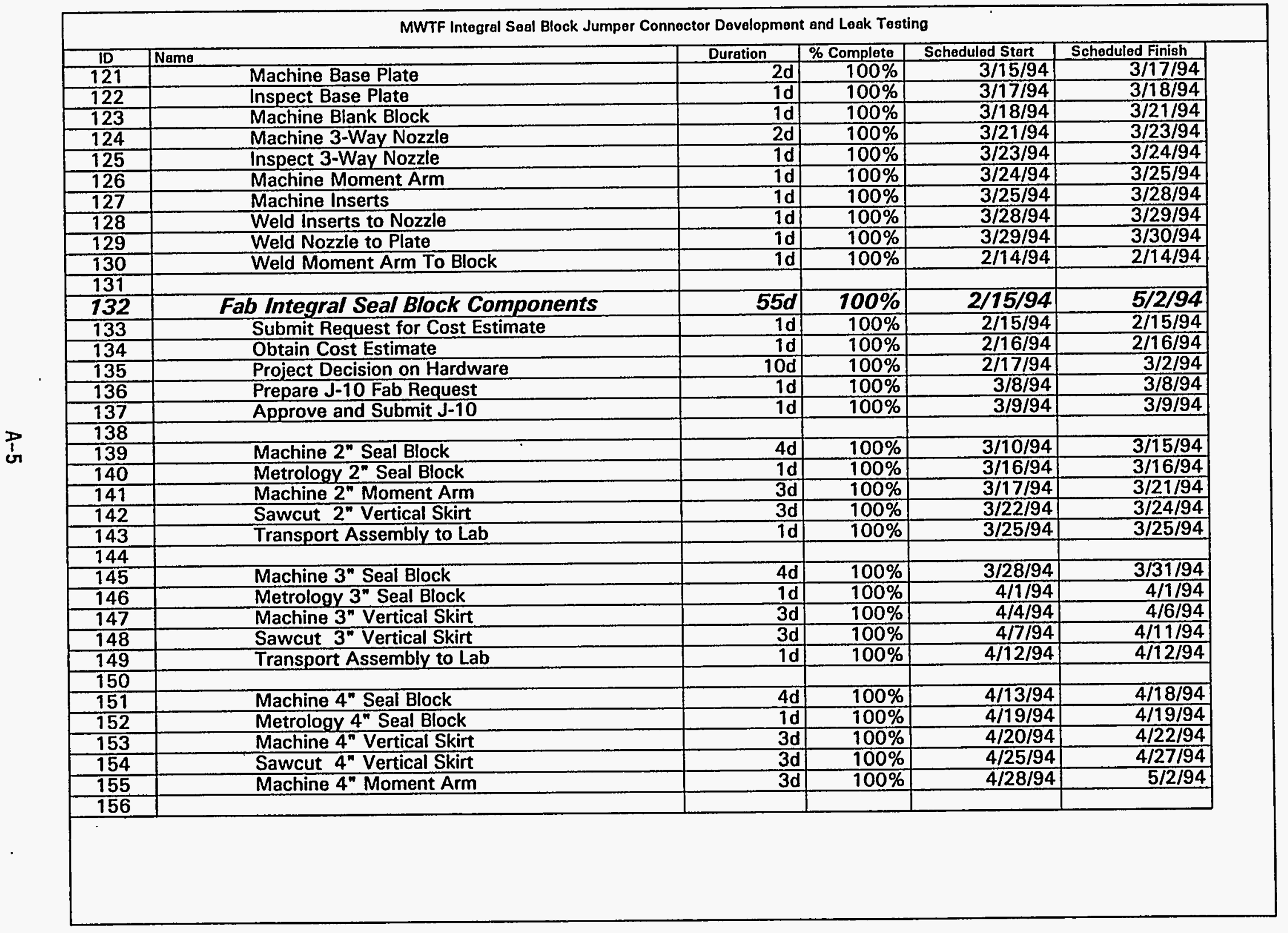




\begin{tabular}{|c|c|c|c|c|c|}
\hline \multicolumn{6}{|c|}{ MWTF Integral Seal Block Jumper Connoctor Development and Loak Testing } \\
\hline ID & ench & Duration & \% Complete & Scheduled Start & Scheduled Finish \\
\hline 157 & Lateral Moments Preparation & $20 d$ & $100 \%$ & $3 / 2 / 94$ & $3 / 29 / 94$ \\
\hline 158 & Establish Lateral Test Criteria & 1d & $100 \%$ & $3 / 2 / 94$ & $3 / 2 / 94$ \\
\hline 159 & Cost Estimate & $\overline{1 d}$ & $100 \%$ & $3 / 3 / 94$ & $3 / 3 / 94$ \\
\hline 160 & Design Lateral Test Fixture & 6d & $100 \%$ & $3 / 10 / 94$ & $3 / 17 / 94$ \\
\hline 161 & Take Floor Measurements & 1d & $100 \%$ & $3 / 10 / 94$ & $3 / 10 / 94$ \\
\hline 162 & Take Load Cell Measurements & 1d & $100 \%$ & $3 / 11 / 94$ & $3 / 11 / 94$ \\
\hline 163 & End Hinge & Td & $100 \%$ & $3 / 14 / 94$ & $3 / 14 / 94$ \\
\hline 164 & Threaded Linkage & 1d & $100 \%$ & $3 / 15 / 94$ & $3 / 15 / 94$ \\
\hline 165 & Reaction Base Plate & 1d & $100 \%$ & $3 / 16 / 94$ & $3 / 16 / 94$ \\
\hline 166 & Reaction Brace & $1 \mathrm{~d}$ & $100 \%$ & $3 / 17 / 94$ & $3 / 17 / 94$ \\
\hline 167 & Fabricate Lateral Test Fixture & $5 d$ & $100 \%$ & $3 / 18 / 94$ & $3 / 24 / 94$ \\
\hline 168 & End Hinge & $2 \mathrm{~d}$ & $100 \%$ & $3 / 18 / 94$ & $3 / 21 / 94$ \\
\hline 169 & Threaded Linkage & 1d & $100 \%$ & $3 / 22 / 94$ & $3 / 22 / 94$ \\
\hline 170 & Reaction Base Plate & 1d & $100 \%$ & $3 / 23 / 94$ & $3 / 23 / 94$ \\
\hline 171 & Reaction Brace & Td & $100 \%$ & $3 / 24 / 94$ & $3 / 24 / 94$ \\
\hline 172 & Install Lateral Test Fixture & $3 d$ & $100 \%$ & $3 / 25 / 94$ & $3 / 29 / 94$ \\
\hline 173 & Drill Concrete Anchor Holes & 1d & $100 \%$ & $3 / 25 / 94$ & $3 / 25 / 94$ \\
\hline 174 & Install Base Plate Anchors & $1 \mathrm{~d}$ & $100 \%$ & $3 / 28 / 94$ & $3 / 28 / 94$ \\
\hline 175 & Install Load Cell & Td & $100 \%$ & $3 / 29 / 94$ & $3 / 29 / 94$ \\
\hline 176 & & & & & \\
\hline 177 & & & & & \\
\hline 178 & Perform Leak Tests & $93 d$ & $100 \%$ & $3 / 8 / 94$ & $7 / 18 / 94$ \\
\hline 179 & Review Test Scope & $1 d$ & $100 \%$ & $3 / 8 / 94$ & $3 / 8 / 94$ \\
\hline 180 & Issue Work Order & $1 d$ & $100 \%$ & $3 / 9 / 94$ & $3 / 9 / 94$ \\
\hline 181 & Setup Equipment & $8.25 d$ & $100 \%$ & $3 / 10 / 94$ & $3 / 22 / 94$ \\
\hline 182 & Check Calibration & $4.13 d$ & $100 \%$ & $3 / 10 / 94$ & $3 / 16 / 94$ \\
\hline 183 & Torque Wrench & $1 d$ & $100 \%$ & $3 / 16 / 94$ & $3 / 17 / 94$ \\
\hline 784 & Pressure Gage & 1d & $100 \%$ & $3 / 17 / 94$ & $3 / 18 / 94$ \\
\hline 185 & Signal Conditioners & $1 \mathrm{~d}$ & $100 \%$ & $3 / 18 / 94$ & $3 / 21 / 94$ \\
\hline 186 & Load Cell & 1d & $100 \%$ & $3 / 21 / 94$ & $3 / 22 / 94$ \\
\hline 187 & Verify Heater Unit & $1 \mathrm{~h}$ & $100 \%$ & $3 / 22 / 94$ & $\frac{3 / 2<1 / 4}{3 / 22 / 94}$ \\
\hline 188 & & & & & \\
\hline 189 & Setup $3^{n}$ Connector - Viton & 1d & $100 \%$ & $4 / 13 / 94$ & $4 / 13 / 94$ \\
\hline 190 & Install Heater Rods & 1d & $100 \%$ & $4 / 14 / 94$ & $4 / 14 / 94$ \\
\hline 191 & $3 \ln$. Ambient & 1d & $100 \%$ & $4 / 15 / 94$ & $4 / 15 / 94$ \\
\hline 192 & Weld 3" Moment Arm & $2 d$ & $100 \%$ & $4 / 18 / 94$ & $4 / 19 / 94$ \\
\hline 193 & 3 In. Thermal Cycle & Td & $100 \%$ & $4 / 20 / 94$ & $4 / 20 / 94$ \\
\hline 194 & 3 In. Thermal Cycle /Lateral & 1d & $100 \%$ & $4 / 21 / 94$ & $4 / 21 / 94$ \\
\hline 195 & Remove $3^{\prime \prime}$ Connector & Td & $100 \%$ & $4 / 22 / 94$ & $4 / 22 / 94$ \\
\hline 196 & & & & & \\
\hline
\end{tabular}




\begin{tabular}{|c|c|c|c|c|c|}
\hline \multicolumn{6}{|c|}{ MWTF Integral Seal Block Jumper Connector Development and Leak Testing } \\
\hline$\frac{1 D}{197}$ & & Duration & \% Complete & Schoduled Start & Scheduled Finish \\
\hline 198 & $\begin{array}{l}\text { Setup } 3^{n} \text { Connector - Aflas } \\
3 \ln \text {. Thermal Cycle } \\
\end{array}$ & $1 d$ & $100 \%$ & $4 / 25 / 94$ & $4 / 25 / 94$ \\
\hline 199 & & 10 & $100 \%$ & $4 / 26 / 94$ & $4 / 26 / 94$ \\
\hline 200 & $\begin{array}{l}3 \text { In. Thermal Cycle /Lateral } \\
\text { Remove } 3^{\prime \prime} \text { Connector }\end{array}$ & 1d & $100 \%$ & $4 / 27 / 94$ & $4 / 27 / 94$ \\
\hline 201 & & $1 d$ & $100 \%$ & $4 / 28 / 94$ & $4 / 28 / 94$ \\
\hline 202 & Setup 3" Connector - Fluorosilicone & & & & \\
\hline 203 & 3 In. Thermal Cycle & $\frac{1 d}{1 d}$ & $\frac{100 \%}{100 \%}$ & $4 / 29 / 94$ & $\frac{4 / 29 / 94}{5 / 2 / 94}$ \\
\hline 204 & 3 In. Thermal Cycle /Lateral & $\frac{1 d}{1 d}$ & $\frac{100 \%}{100 \%}$ & $\begin{array}{l}5 / 2 / 94 \\
5 / 3 / 94 \\
\end{array}$ & $\begin{array}{r}5 / 2 / 94 \\
5 / 3 / 94\end{array}$ \\
\hline$-\frac{205}{206}$ & Remove 3" Connector & $\frac{1 d}{1 d}$ & $100 \%$ & $\frac{5 / 3 / 94}{5 / 4 / 94}$ & $-5 / 3 / 94$ \\
\hline$\frac{206}{207}$ & Setup $3^{m}$ Connector - Kalrez & & 10070 & & \\
\hline 208 & $\begin{array}{l}3 \text { in. Thermal Cycle } \\
\end{array}$ & $\frac{1 d}{1 d}$ & $100 \%$ & $5 / 5 / 94$ & $5 / 5 / 94$ \\
\hline 209 & 3 In. Thermal Cycle /Lateral & $\frac{1 d}{1 d}$ & $100 \%$ & $5 / 6 / 94$ & $5 / 6 / 94$ \\
\hline 210 & Remove $3^{n}$ Connector & $\frac{1 d}{1 d}$ & $100 \%$ & $5 / 9 / 94$ & $5 / 9 / 94$ \\
\hline 211 & & 1d & $100 \%$ & $5 / 10 / 94$ & $5 / 10 / 94$ \\
\hline$\frac{212}{213}$ & Setup 2" Connector -Viton & 1d & & & \\
\hline$\frac{213}{214}$ & Install Heater Rods & $1 \mathrm{~d}$ & $100 \%$ & $\begin{array}{l}5 / 11 / 94 \\
5 / 12 / 94 \\
\end{array}$ & \\
\hline$\frac{214}{215}$ & $\frac{2 \ln . \text { Ambient }}{\text { Weld 2" Moment Arm }}$ & $\frac{1 d}{2 d}$ & $100 \%$ & $\frac{5 / 12 / 94}{5 / 13 / 94}$ & $\frac{5 / 12 / 94}{5 / 13 / 94}$ \\
\hline 216 & & $\frac{2 d}{1 d}$ & $100 \%$ & $\frac{5 / / 3 / 94}{5 / 16 / 94}$ & $\frac{5 / 13 / 94}{5 / 17 / 94}$ \\
\hline 217 & $\begin{array}{l}2 \text { In. Thermal Cycle } \\
2 \text { In. Thermal Cycle /Lateral }\end{array}$ & $\frac{1 d}{1 d}$ & $100 \%$ & $5 / 18 / 94$ & $5 / 17 / 94$ \\
\hline 218 & Remove $2^{\prime \prime}$ Connector & $\frac{1 d}{1 d}$ & $100 \%$ & $5 / 19 / 94$ & $\begin{array}{l}5 / 18 / 94 \\
5 / 19 / 94 \\
\end{array}$ \\
\hline$\frac{219}{220}$ & & & $100 \%$ & $5 / 20 / 94$ & $\begin{array}{l}5 / 19 / 94 \\
5 / 20 / 94 \\
\end{array}$ \\
\hline$\frac{220}{221}$ & $\begin{array}{l}\text { Setup } 2^{\text {" Connector - Aflas }} \\
2 \ln \text {. Thermal Cycle }\end{array}$ & $\frac{1 d}{1 d}$ & & & \\
\hline 222 & $\begin{array}{l}2 \text { In. Thermal Cycle } \\
2 \ln \text {. Thermal Cycle /Lateral }\end{array}$ & $\frac{1 d}{1 d}$ & $\begin{array}{l}100 \% \\
100 \% \\
\end{array}$ & $5 / 23 / 94$ & $5 / 23 / 94$ \\
\hline 223 & Remove $2^{\prime \prime}$ Connector & $\frac{1 d}{1 d}$ & $100 \%$ & $\begin{array}{l}5 / 24 / 94 \\
5 / 25 / 94 \\
\end{array}$ & $5 / 24 / 94$ \\
\hline 224 & & 1d & $100 \%$ & $\begin{array}{l}5 / 25 / 94 \\
5 / 26 / 94 \\
\end{array}$ & $\frac{5 / 25 / 94}{5 / 26 / 94}$ \\
\hline$\frac{225}{236}$ & Setup 2" Connector - Fluorosilicone & 10 & & $5 / 26 / 94$ & $5 / 26 / 94$ \\
\hline$\frac{226}{227}$ & 2 In. Thermal Cycle & $\frac{1 \mathrm{~d}}{1 \mathrm{~d}}$ & $100 \%$ & $5 / 27 / 94$ & \\
\hline$\frac{227}{228}$ & $\begin{array}{l}2 \text { In. Thermal Cycle /Lateral } \\
\text { Remove } 2^{\prime \prime} \text { Connector }\end{array}$ & $\frac{1 d}{1 d}$ & $100 \%$ & $5 / 30 / 94$ & $5 / 27 / 94$ \\
\hline 229 & & 1d & $100 \%$ & $5 / 31 / 94$ & $5 / 30 / 94$ \\
\hline 230 & Setup $2^{n}$ Connector - Kalrez & & & $6 / 1 / 94$ & $\begin{array}{r}5 / 31 / 94 \\
6 / 1 / 94 \\
\end{array}$ \\
\hline 231 & 2 In. Thermal Cycle & & $100 \%$ & & \\
\hline 232 & 2 In. Thermal Cycle /Lateral & & $\frac{100 \%}{100 \%}$ & $\begin{array}{l}6 / 2 / 94 \\
6 / 3 / 94 \\
\end{array}$ & $6 / 2 / 94$ \\
\hline 233 & Remove $2^{n}$ Connector & & & & \\
\hline 234 & & & & & \\
\hline 235 & & & & & \\
\hline 236 & Setup 4" Connector - Viton & & & & \\
\hline
\end{tabular}




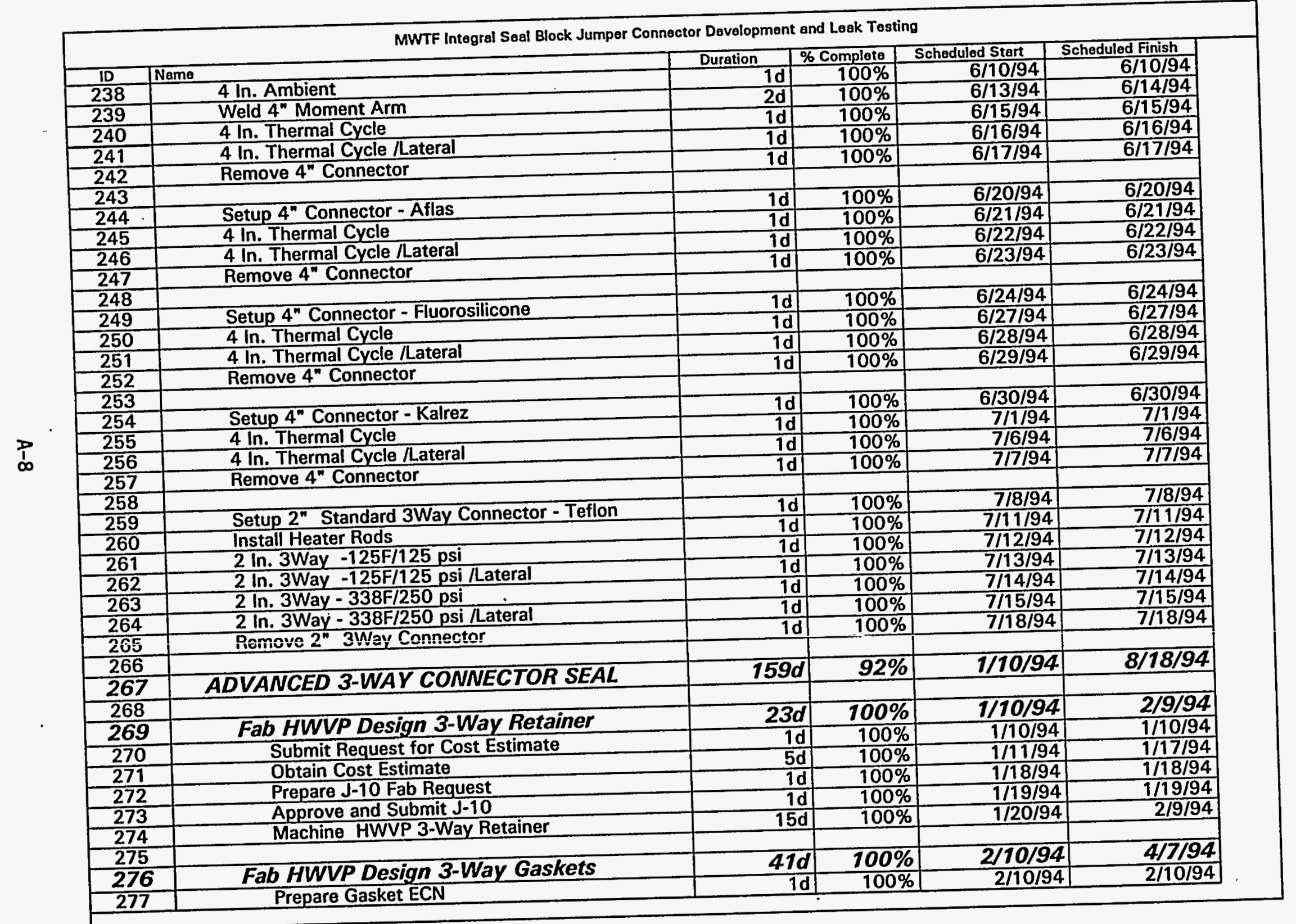




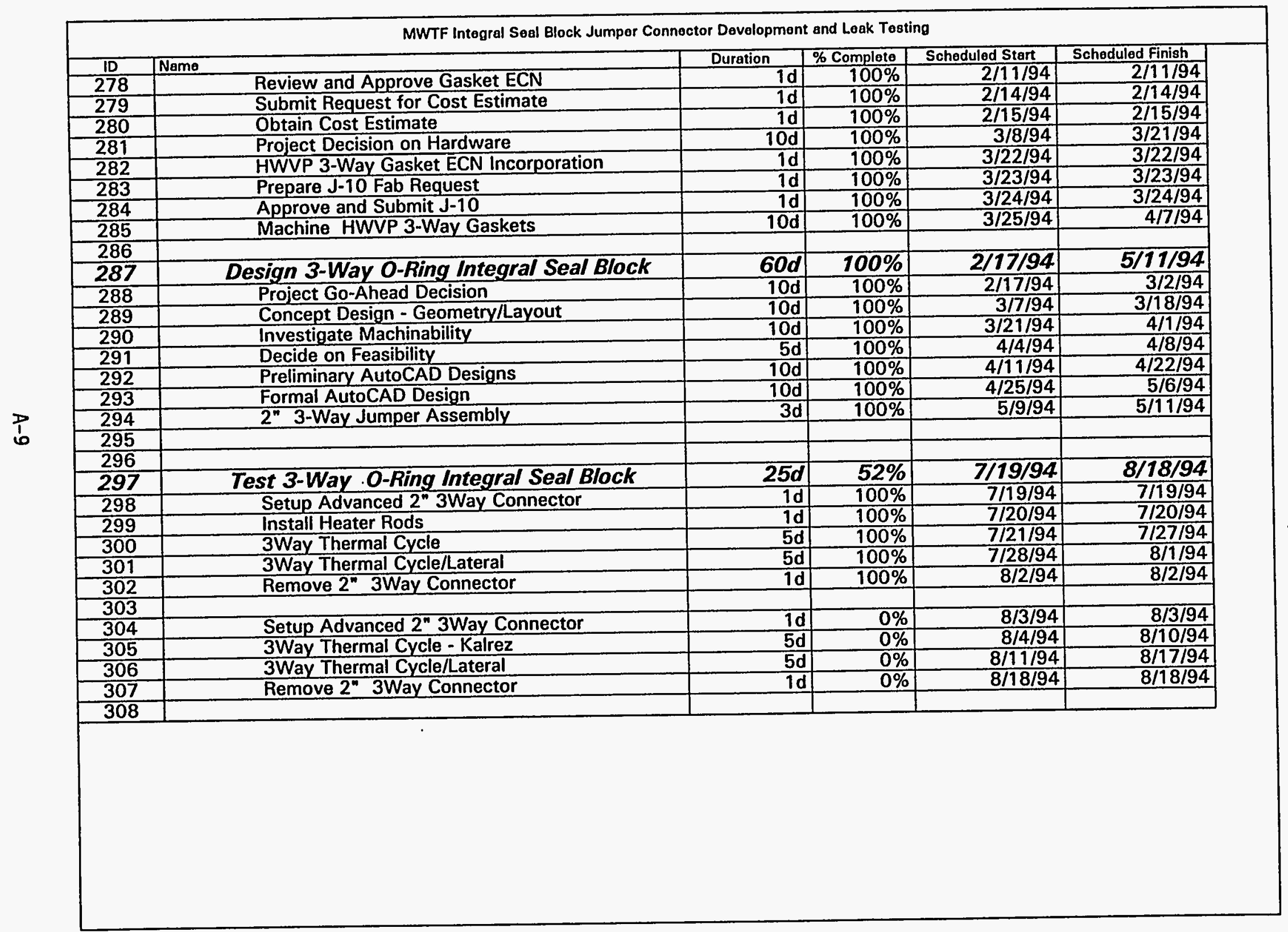




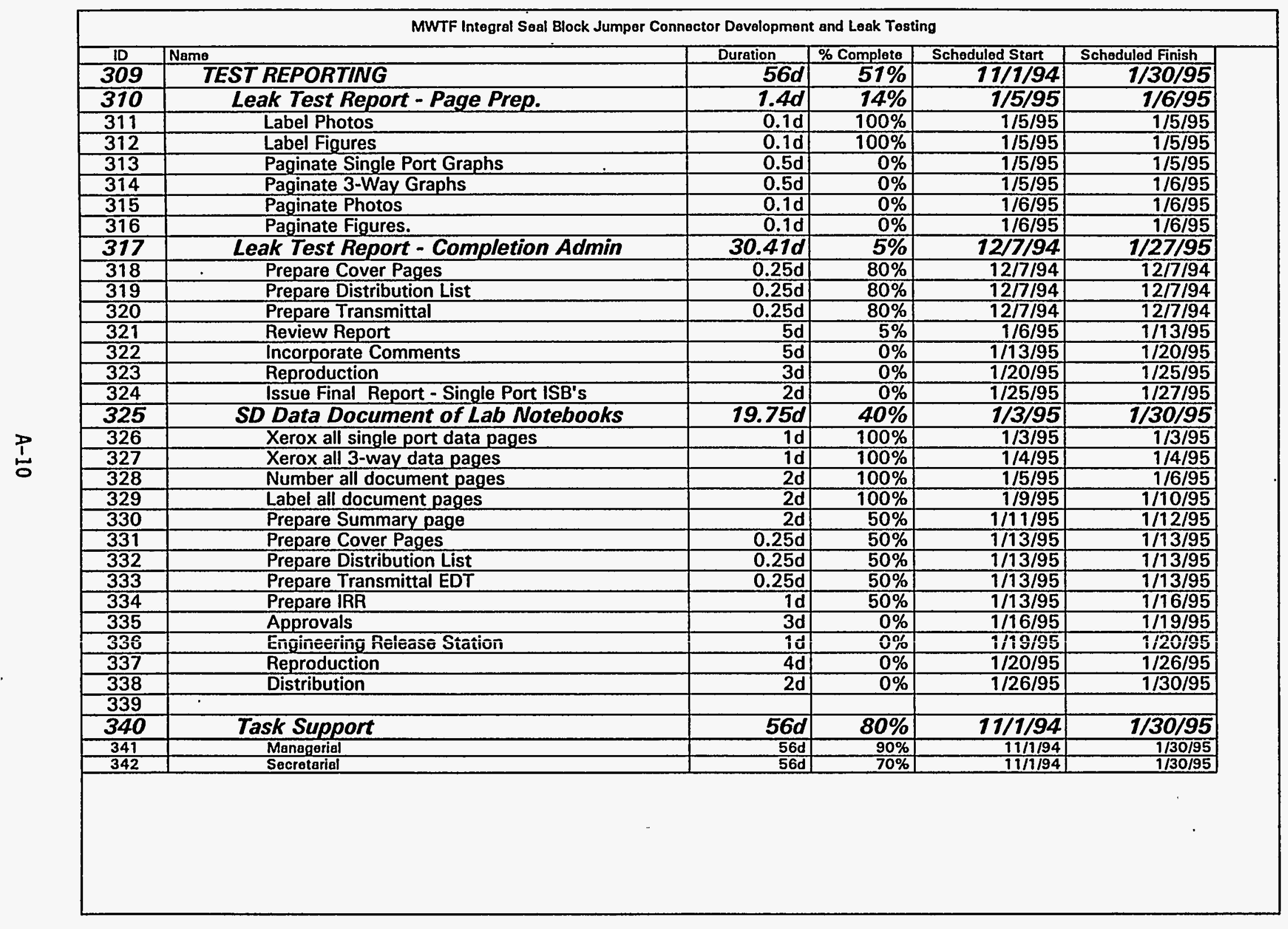


TITLE: Qualification of Mechanical Jumpers with Integral Seal O-Ring Block, Electrical Connections and Remote Flanges

ENGR. GP/

ORG. CODE: Structural Assessment/ 8D440 4 CUSTOMER/ORG CODE: MWTF/7F220 DATE: $\frac{9 / 28 / 93}{2-\varepsilon, a+4} \operatorname{ReV} \cdot \frac{q^{\prime}}{3}$ PERFORMER/SIGNATURE: EC OCOMa/ES Ruff / COG. ENGR./SIGNATURE:VJ Cruz/ ye Cey MANAGER/SIGNATURE TJ ConradS/ IMPACT LEVEL/WORK PLAN NO:

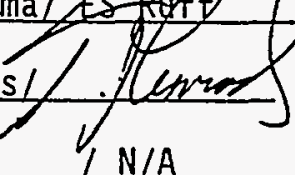
ro/lat 4 MANAGER/SIGNATURE: JM Light

WORK ORDER/TASK PKG:

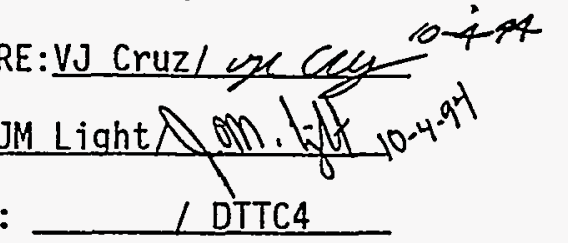

ESR NO:/WORK PLAN NO.: MAR-93-022

SCOPE OF HORK: To qualify the mechanical jumpers with Integral Seal Block (ISB), the electrical connectors, and the remote flanges under operational and seismic conditions.

(A) Qualification of jumper connectors with o-ring integrated seal block; this includes the 2-in. 3-way multi-port, 2-, 3-, and 4-in. single port jumper connector heads. The analysis and testing are defined below.

Task A-I:

Perform structural analysis of jumper connectors with o-ring integrated seal block to verify design adequacy for the following loads: torque load, seismic load, and operational load. Determine the allowable enveloping nozzle loads (see comment 1). The analysis will accept possible spray leak under seismic event at the o-ring seal interface. Therefore, the seismic analysis will only include the piping load transferred from the pipe to the connector heads from structural analysis point of view, i.e, no leak tight consideration is given.

Task A-II:

Perform a series of leak tests for the above listed connector heads. The tests will include temperature, force/moment applied in two directions, and pressure. Tests will be repeated for six thermal cycles. To qualify seal materials, each set of tests will be repeated for different 0 -ring materials. Materials selected for testing are Kalrez, Viton, and Flurositicon (was Aflas, Sylon, and Fluorel).

(B) Qualify the electrical head connectors

\section{Task B-I:}

The electrical connectors are very heavy and strong. The only loads to be considered are the torque load and the load from the strong back attached to the electrical head (dead weight plus seismic load). The main concern in the functionality of the connectors are the springs that transmit the signal. This spring is within the housing of the electrical connector and will not be affected by the load on the connector. The analyses of the strong backs (no configurations as of yet and how many) will evaluate the structural integrity of the connector and the strong back. 
During and after a seismic event, it is essential that the signals would continuousiy transmit and the connector be functioning. This should be tested experimentally and is not addressed within the scope of this work. See comment (2) for details.

(C) Qualify the remote flanges. (deleted by $V$. Cruz at the 9/28/94 meeting in ETC-2 since ICF-KH is already doing it)

\section{Task C-I:}

As of now, there are three different remote $f 1$ anges specified (based on mtg. with Don $0^{\prime}$ Connor and Gary Cleveland from KEH, 3/2/94). These are 6- 8-, and 10-in. remote flanges. The $f 7$ anges have been used in the past and operated satisfactory. The loading would be 60 in. of water pressure at $212^{\circ} \mathrm{F}$ temperature. The ASME Section VIII (or Section III, Appendices) will be used for evaluation.

\section{Task C-II:}

The leakage of the remote $f l$ anges is acceptable after a DBE as a result of the PSAR analysis. The question of possible leakage during operation was raised to see if it is worth performing experiments to verify the leak tightness or leakage rate. It was recommended that a leak test is not required because of the following reasons. (a) The PSAR analysis allows leakage in the pit. There are detectors that would detect leakage beyond the allowable limits. (b) The past experience with PUREX and other facilities that have used the flanges show good results if the gaskets are used and installed properly (phone conversation on $3 / 2 / 94$ with Ed allen, Bob Campbell, and Mike Harty).

(D) Seal retainer removal tool

Task D-I: (completed)

Design and fabricate three tools to remove seal retainers from 2-, 3-, and 4-in. jumper connector lower skirt.

(E) Design study for 3-Way tetraseal type retainer.

\section{Task E-I: (completed)}

Perform a study on the feasibility of a 2-in. 3-way seal retainer that would incorporate an $0-$ ring/tetraseal. The purpose of this retainer would be to allow retrofit of existing 3way teflon type seals.

\section{SCHEDULE/MILESTONES/DUE DATE:}

[Cost tabulation included in Rev. 1 is removed]

COST ESTIMATE: $\$ 489 k$

REFERENCE INFORMATION:

KEY HORDS: MuTti-Function Waste Tank Facility, jumpers, piping
DELIVERABLES: Reports issued as Supporting Documents (SDs):

Test Report

Analysis Report

2, 3, \& 4-in. single port oct. 28, 194 Jan 31, 1995 Connetop-stiffnesses Dee-30, 1994

2-in. 3-way port

JaA. 30 1995 Jan 31, 1995

E7eft. Conn.lstrong backic May 29, 1995

custerner Agreemant by Telecen wit2. V.J.C.c1z 2/5/95 SK farcumb
2/3/95 EShupp

$2 / 8 / 4$ \&e Oerma

Hrkplan7.eco

A-12 
Rev. 1 comments

WHC-SD-WM-TRP-223

Rev. 0

(i) The operational loads (transferred from piping to the head) are not known at this time. Per Tom Salzano's recommendation (cc:mail 2/7/94) the maximum allowable force/moment should be specified at the connector/pipe connection. Since any combination of forces and moments in any direction is possible, the anaiysis will provide unit loads in each direction and resulting stresses that could be scaled to the actual design load.

(2) Discussions have been initiated with the Project and Safety staff to determine the need for connector function during a seismic event.

(3) This work $p 1$ an is a revision to the work $p l$ an dated $9 / 9 / 93$, Rev. 0 . The revision of Rev. 0 work plan is due to a new configuration (o-ring or ISB vs teflon seal) and added work scope. Total cost of rev. 0 is $278.9 \mathrm{~K}$ and the cost of rev. 1 with added analysis and testing is $312.5 \mathrm{~K}$.

ReV 2 comments: REQUEST FOR ADDITIONAL FUNDING AND SCHEDULE EXTENSION

ADDITIONAL FUNDING requested: \$55k testing, \$43k additional for planned-testing $\$ 12 k$ for new stiffness testing

$\$ 122 k$ analysis, additional for planned analysis

$\$ 177 k$ total increase (from $\$ 312.5 \mathrm{~K}$ of Rev. 1)

SCHEDULE EXTENSION (see attached revised scheduTe)

New test work: Stiffness testing deliverables

Drawings of the test fixture and loading mechanism.

Sketches showing the type and direction of loading.

Perform and record loads and deflections.

Provide raw data and tabulated data to the analysts.

Planned testing \& analysis budget increase:

1. Fabrication, test and analysis required more time and more work than was originally planned.

2. The test stand in the 305 Building was forced to relocate two times during the 7 ast two months. The relocation was caused by K-Basin projects moving equipment into our lab space. A7so, the test stand high pressure metering pump had to be replaced.

3. \$10k for $f a b$. and development portion of the 3-way ISB prototype testing was inadvertently omitted from the rev. 0 . This $\$ 10 \mathrm{~K}$ is now included in rev. 2 .

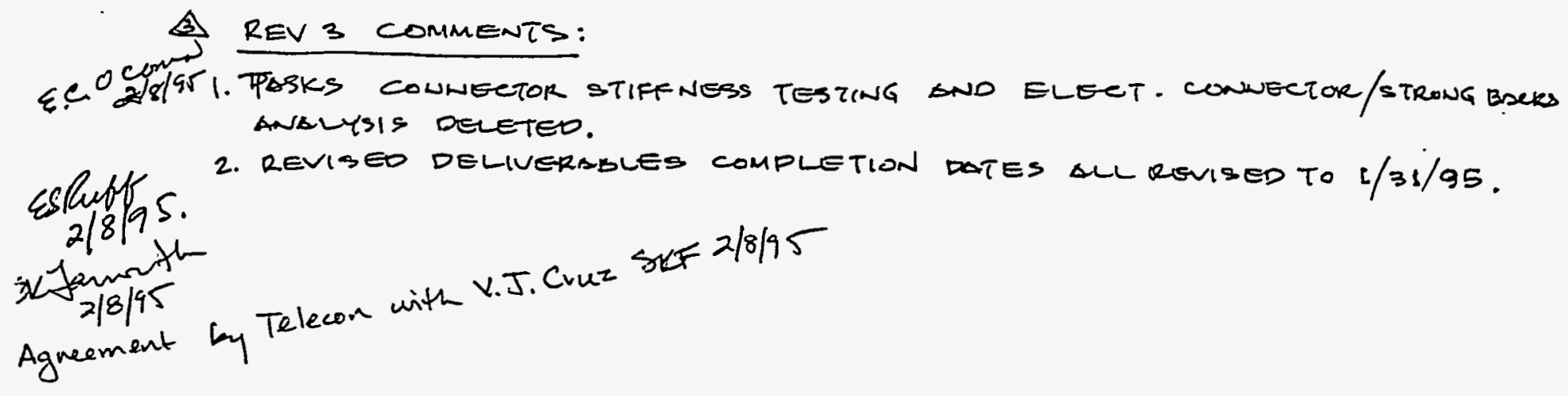

wrkplan7.eco 
STRUCTURAL QUALIFICATION OF MECHANICAL JUMPERS WITH INTEGRAL SEAL BLOCKS (ISB), ELECTRICAL CONNECTORS, AND REMOTE FLANGES

FY94

FY95

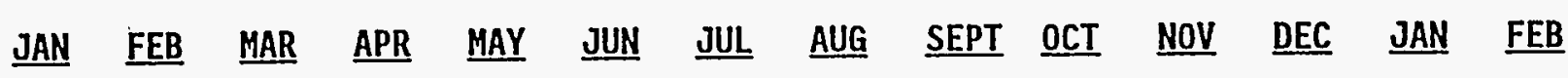

MECHANICAL JUMPERS

Test Preparation

Design/Fabrication

2-in. ISB Single

Port Connector

Analysis

Testing

3-in. ISB Single

Port Connector

Analys is

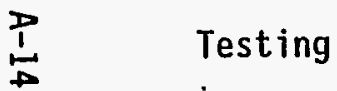

4-in. ISB single

Port Connector

Analys is

Testing

2-in. 3-Way

Port Connector

Analysis*

Testing

Test Report/Document
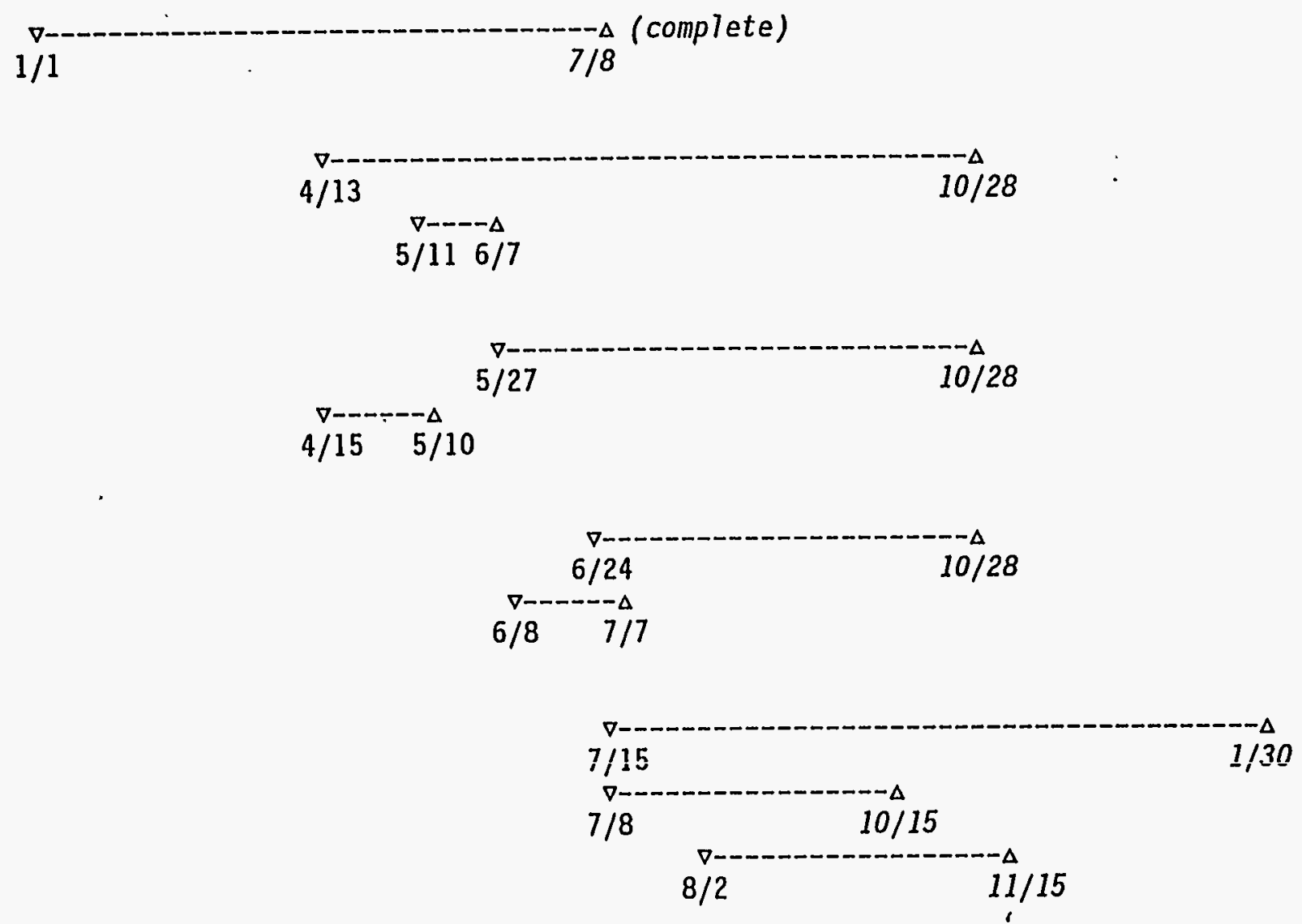

* Each individual loading from the piping configuration needs to be analyzed case by case to find stresses and allowable torque for rigid connection. 
FY95

\section{ELECTRICAL JUMPERS}

\section{$2,3, \& 4-i n$}

Analysis (conn. \& conduit strong back)

Testing (operability after $D B E=T B D$ )

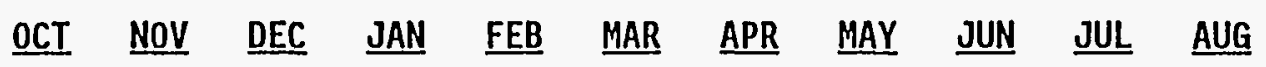

REMOTE FLANGES (deleted 9/28/94 per project's request)

$6-, 8-$, and $10-$ in. flanges

Analys is

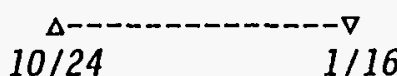

SEAL RETAINER REMOVAL TOOL (TEFLON SEAL)

Analys is (Not Applicable)

Testing

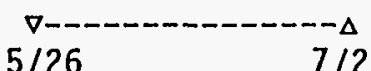

$5 / 26$

DESIGN STUDY OF 3-WAY TETRASEAL RETAINER

Analys is (nothing planned or scheduled)

Testing

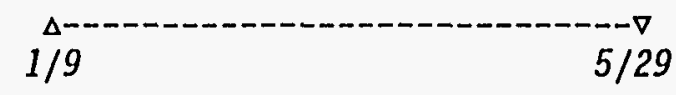

点

STIFFNESS TESTING

2, 3, \& 4-in. single port \& 2-in. 3-way port

Test Preparation

Test Stand Drawings/Sketches

Perform Stiffness Tests

Tabulate Data

Analyze Data/Issue Stiffness Report

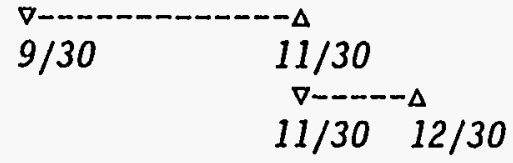


APPENDIX B: DRAWINGS FOR 2-, 3-, and 4-IN. AND THREE-WAY INTEGRAL SEAL BLOĊKS 


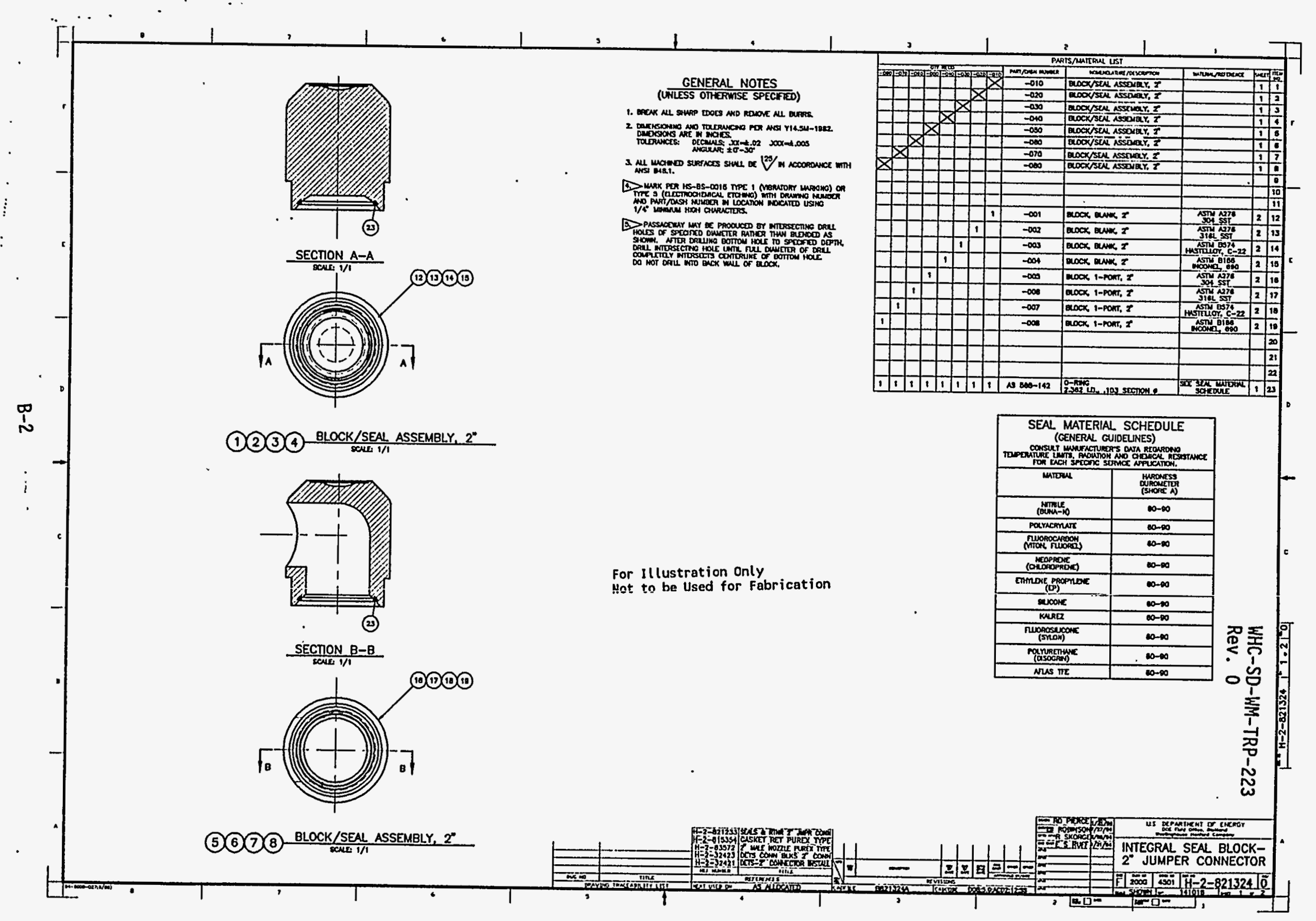




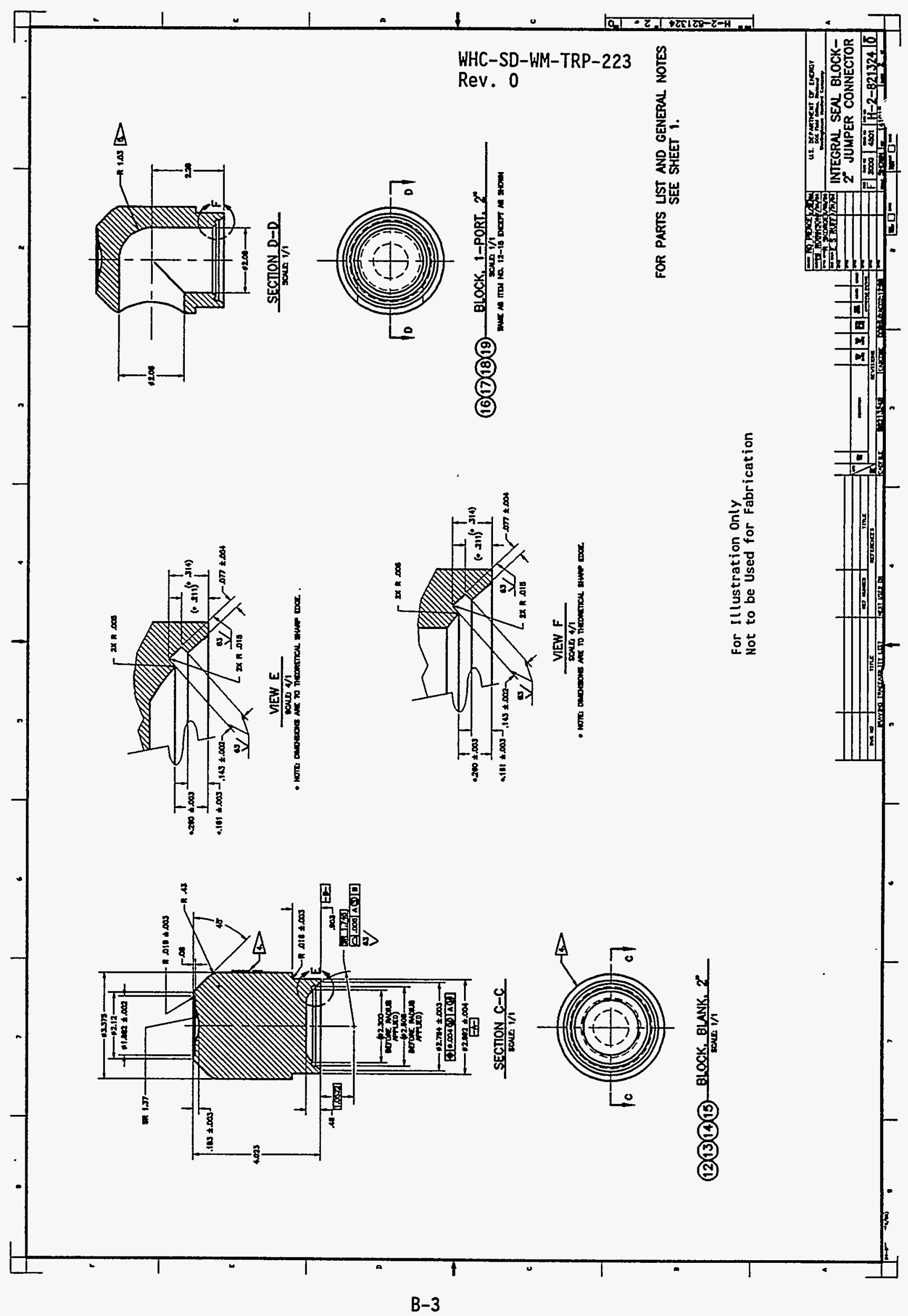




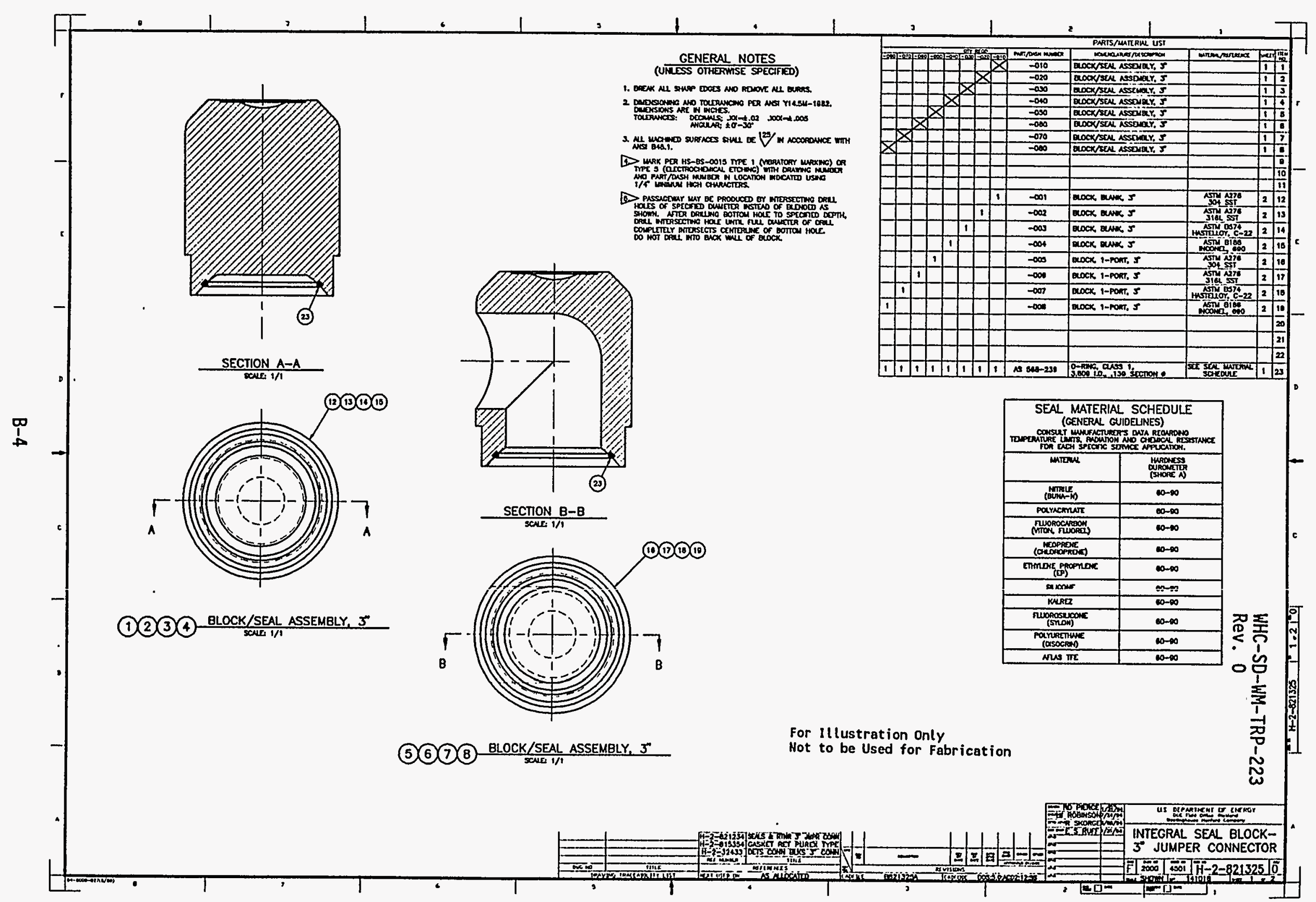




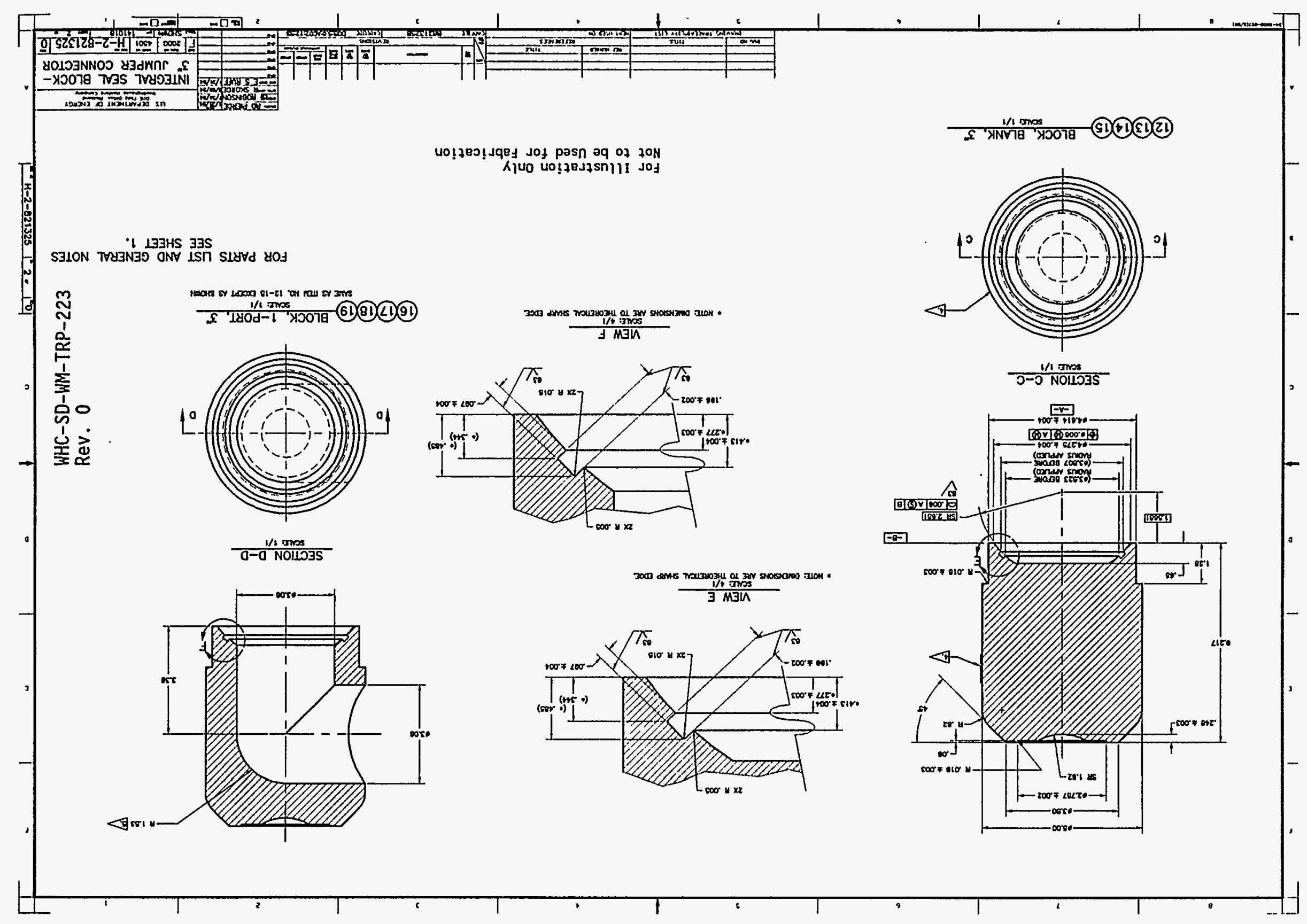




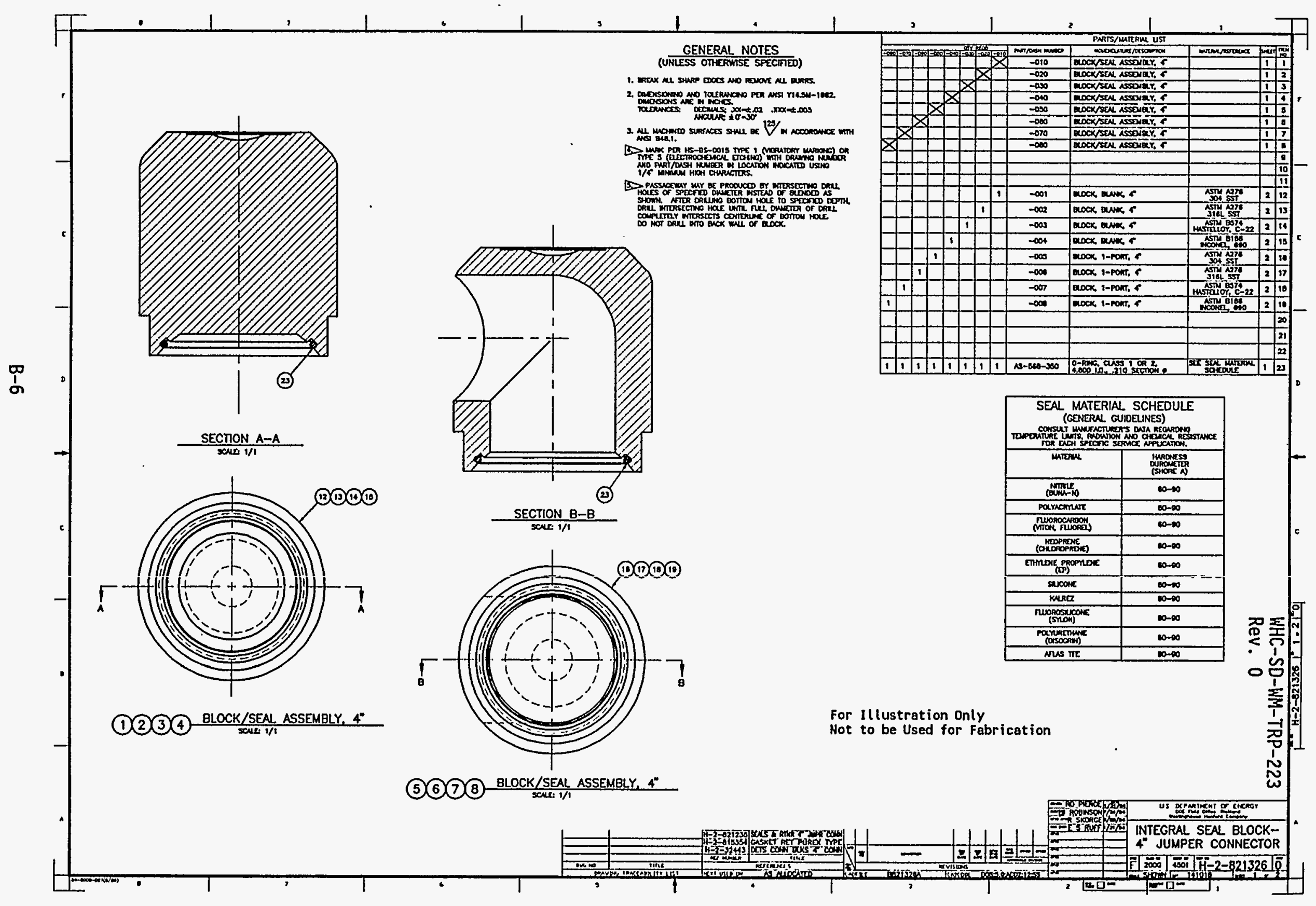




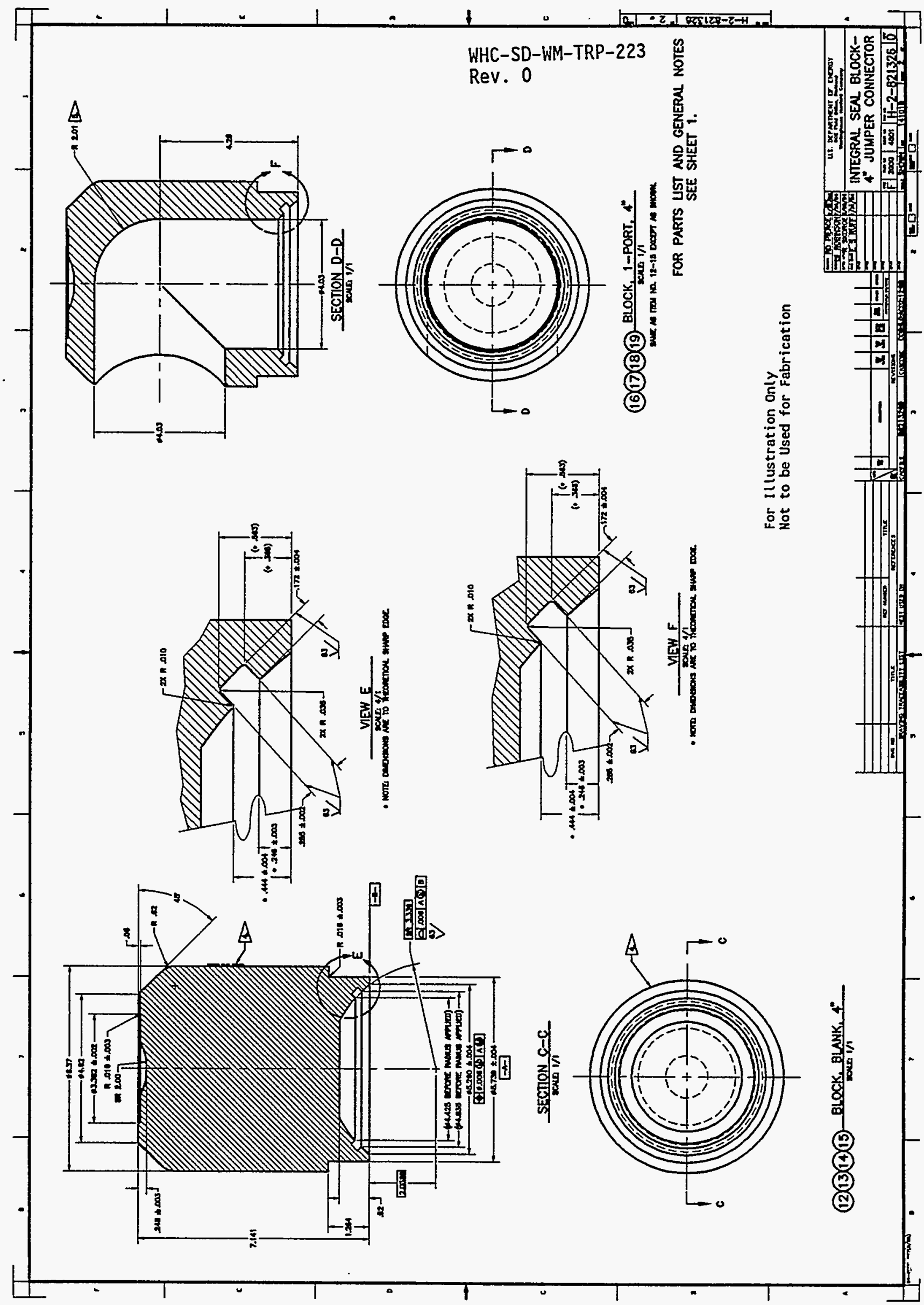




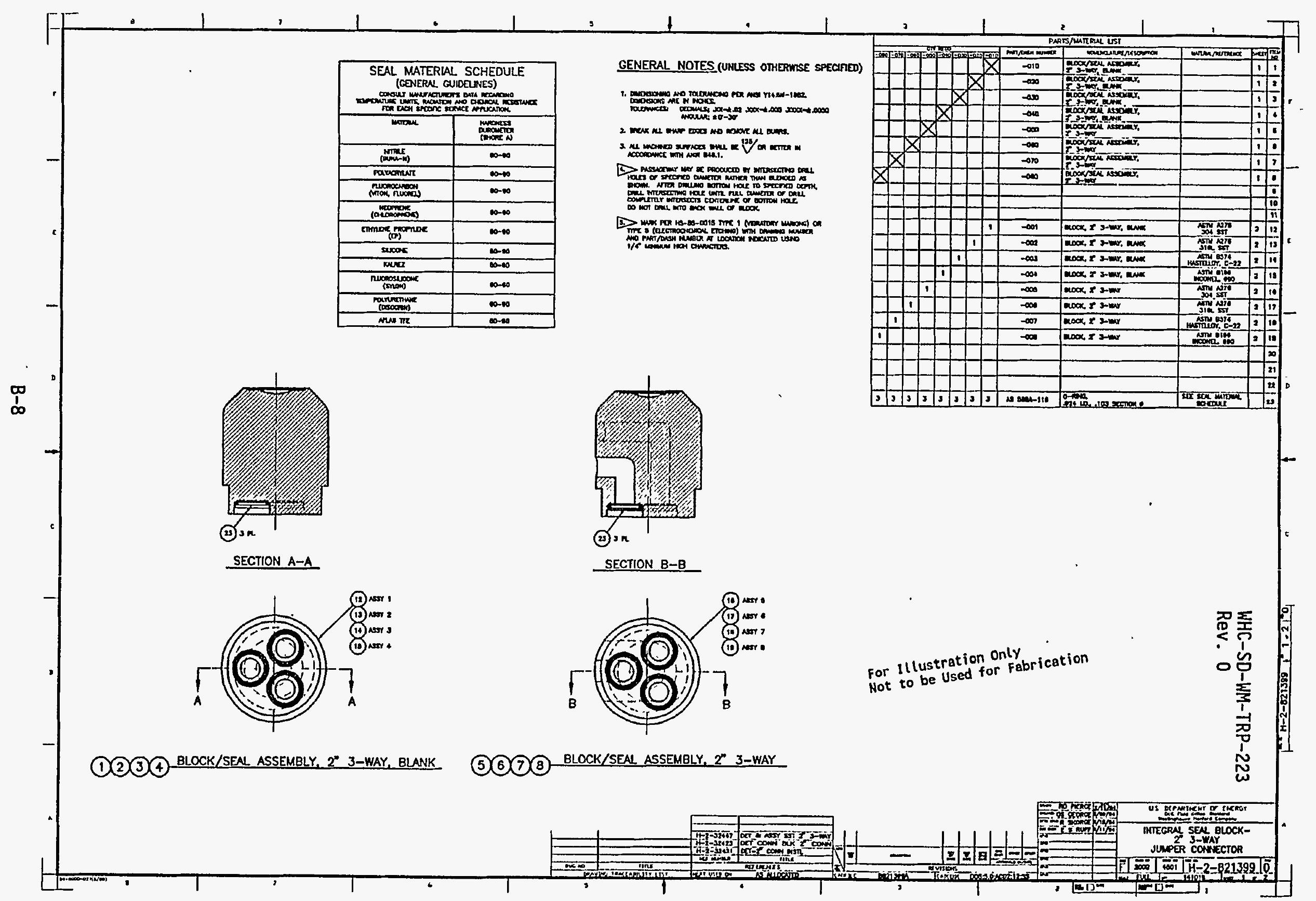




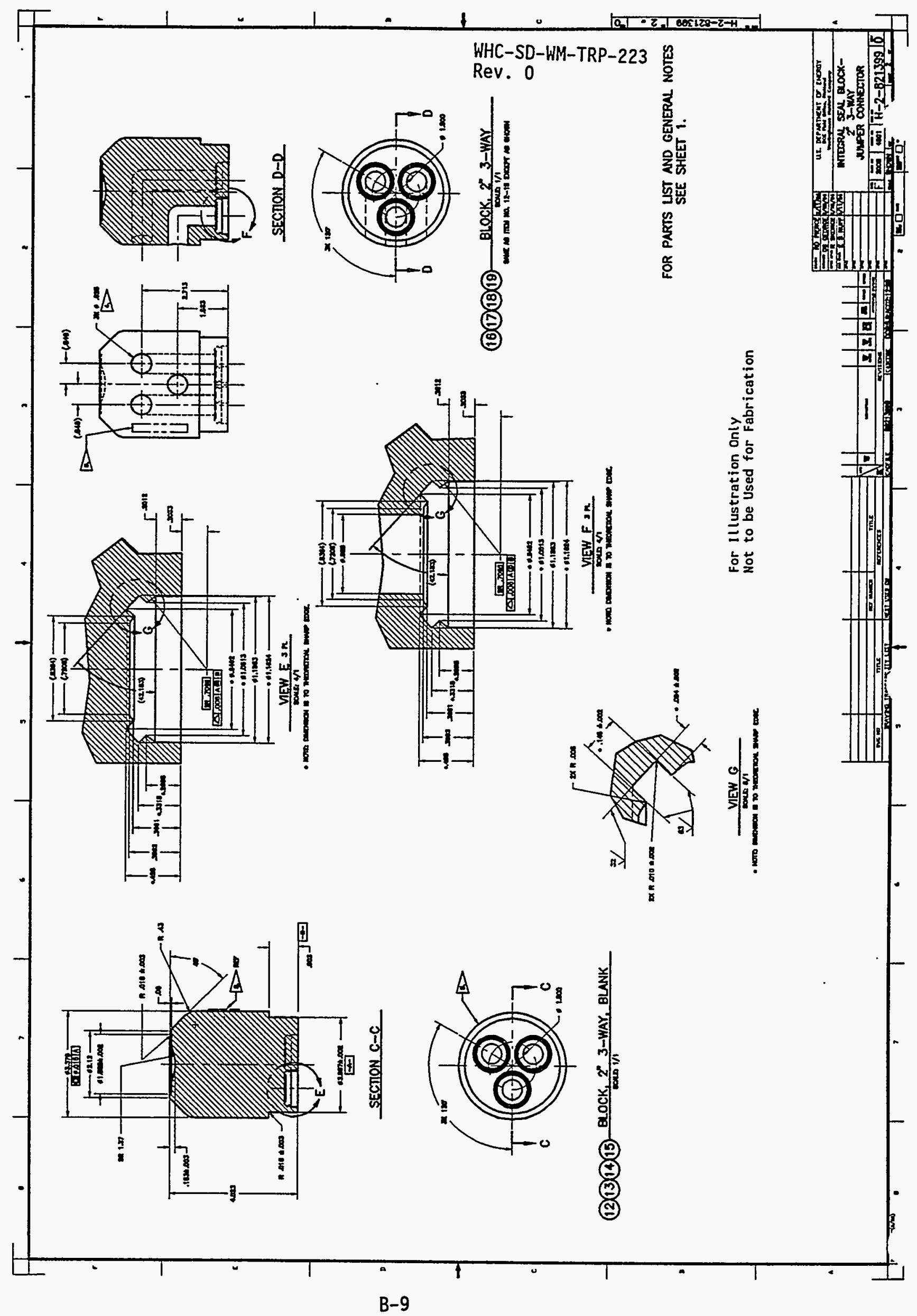


WHC-SD-WM-TRP-223

Rev. 0

$\pi$

APPENDIX C: DRAWINGS FOR JUMPER CONNECTOR LEAK-TEST FIXTURE

)

APPENDIX C: DRAWI

C-1 


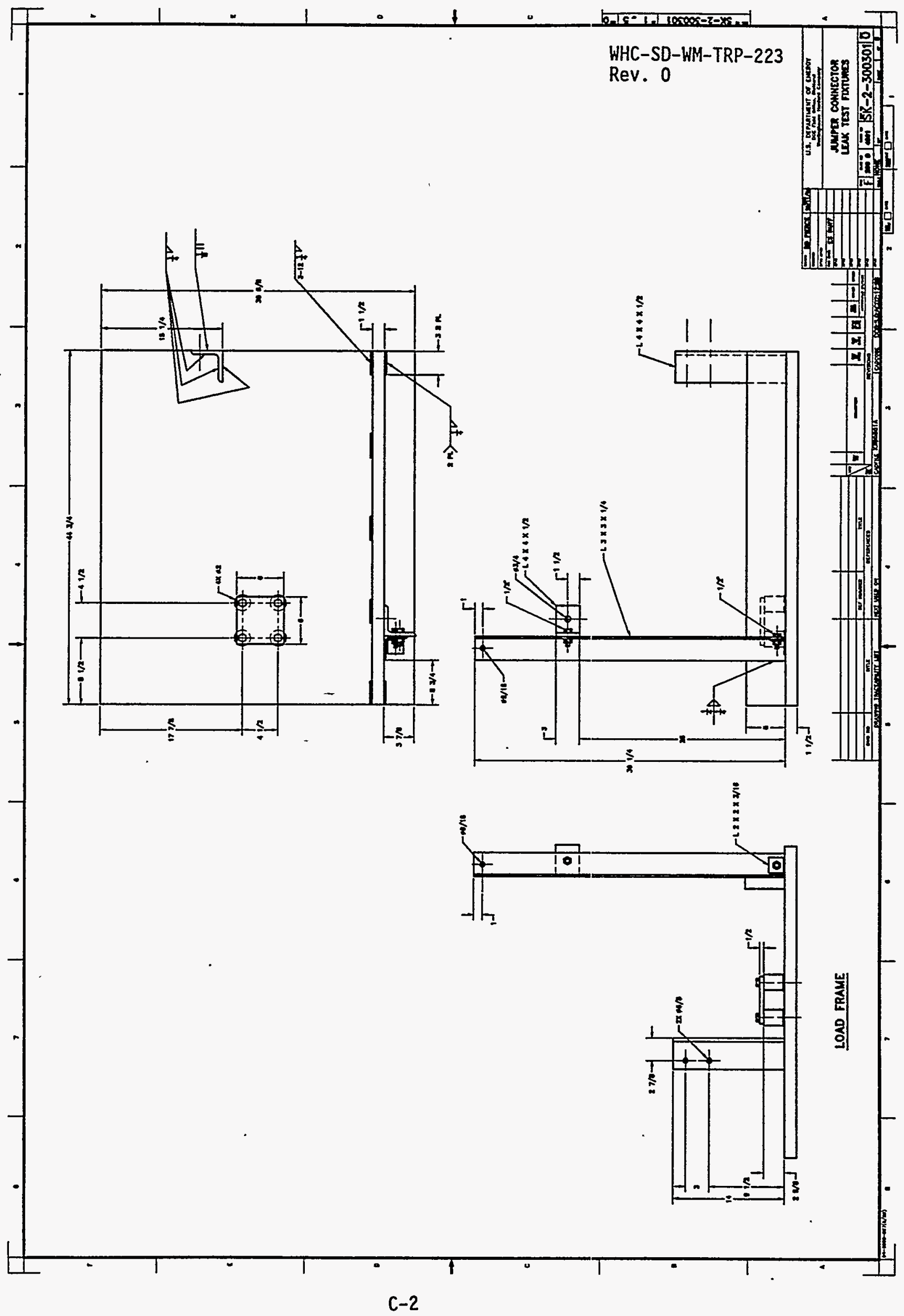




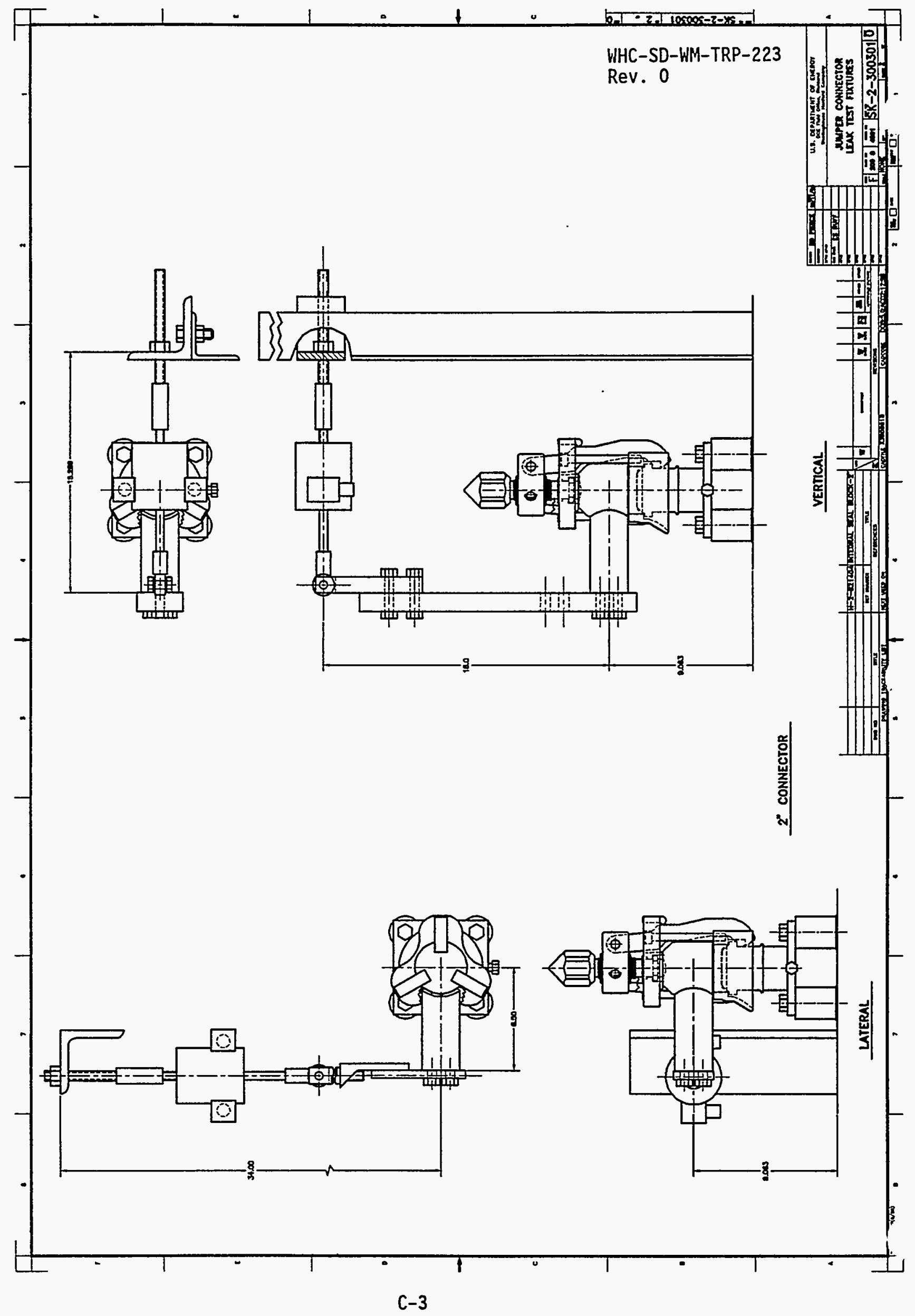




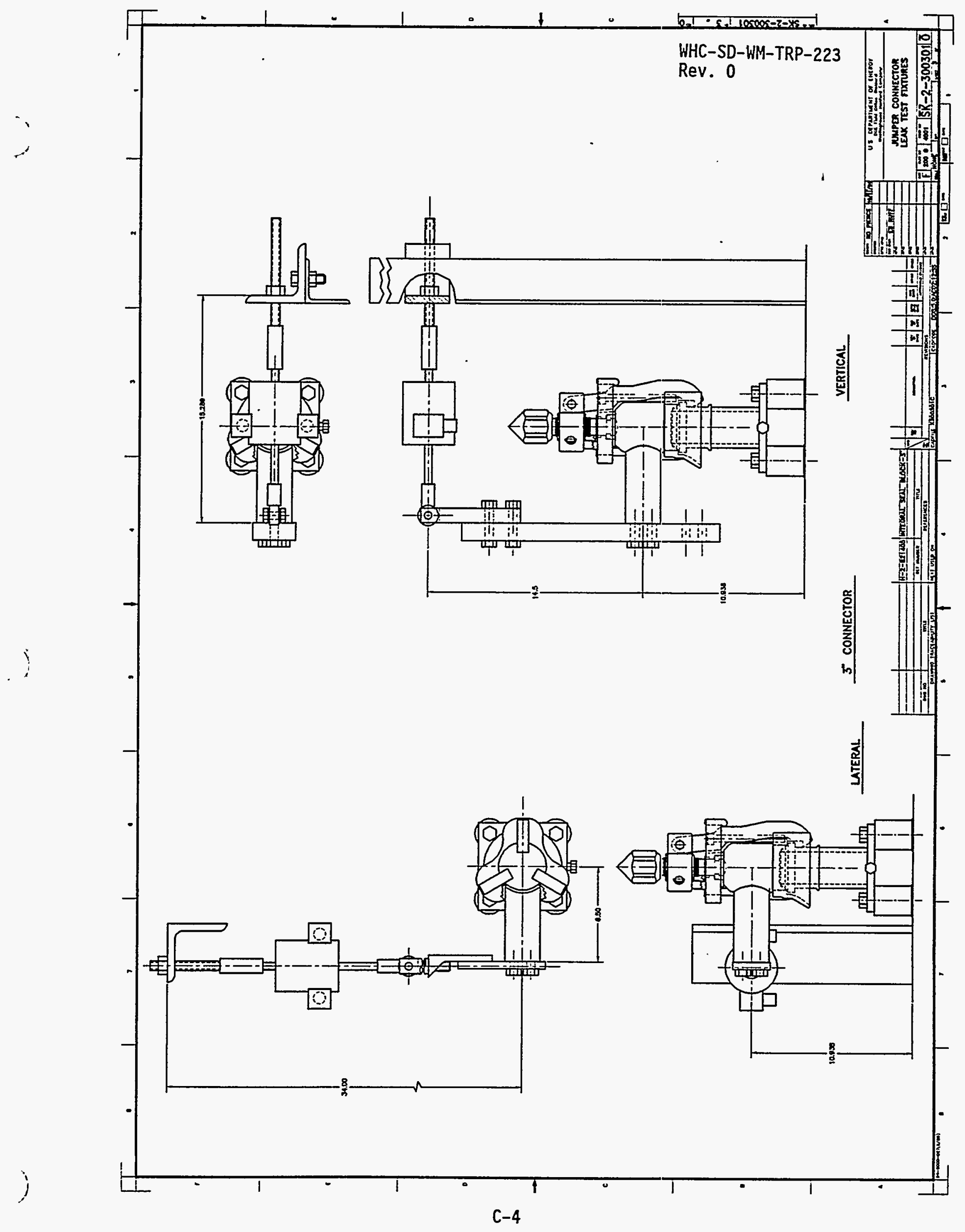




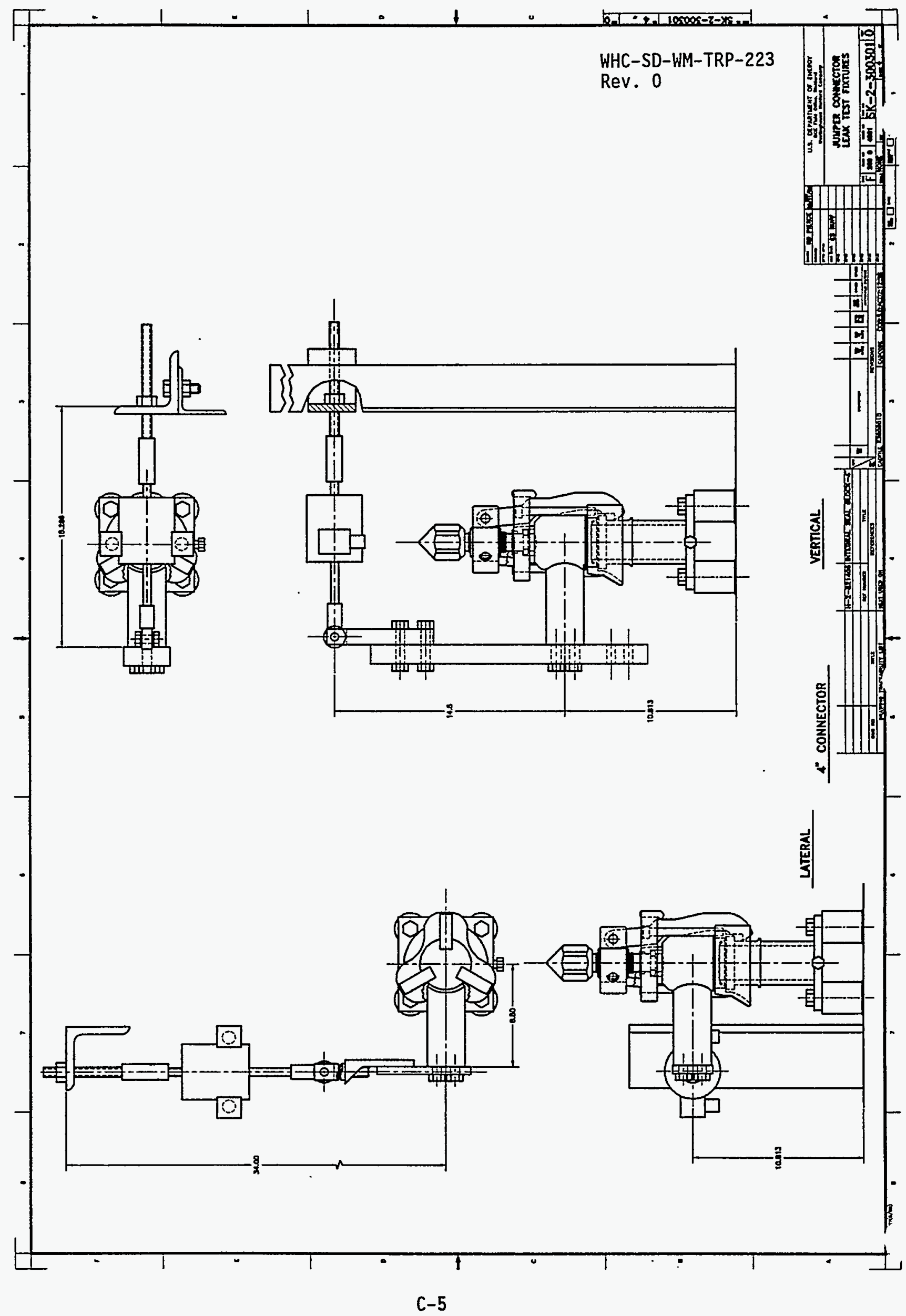




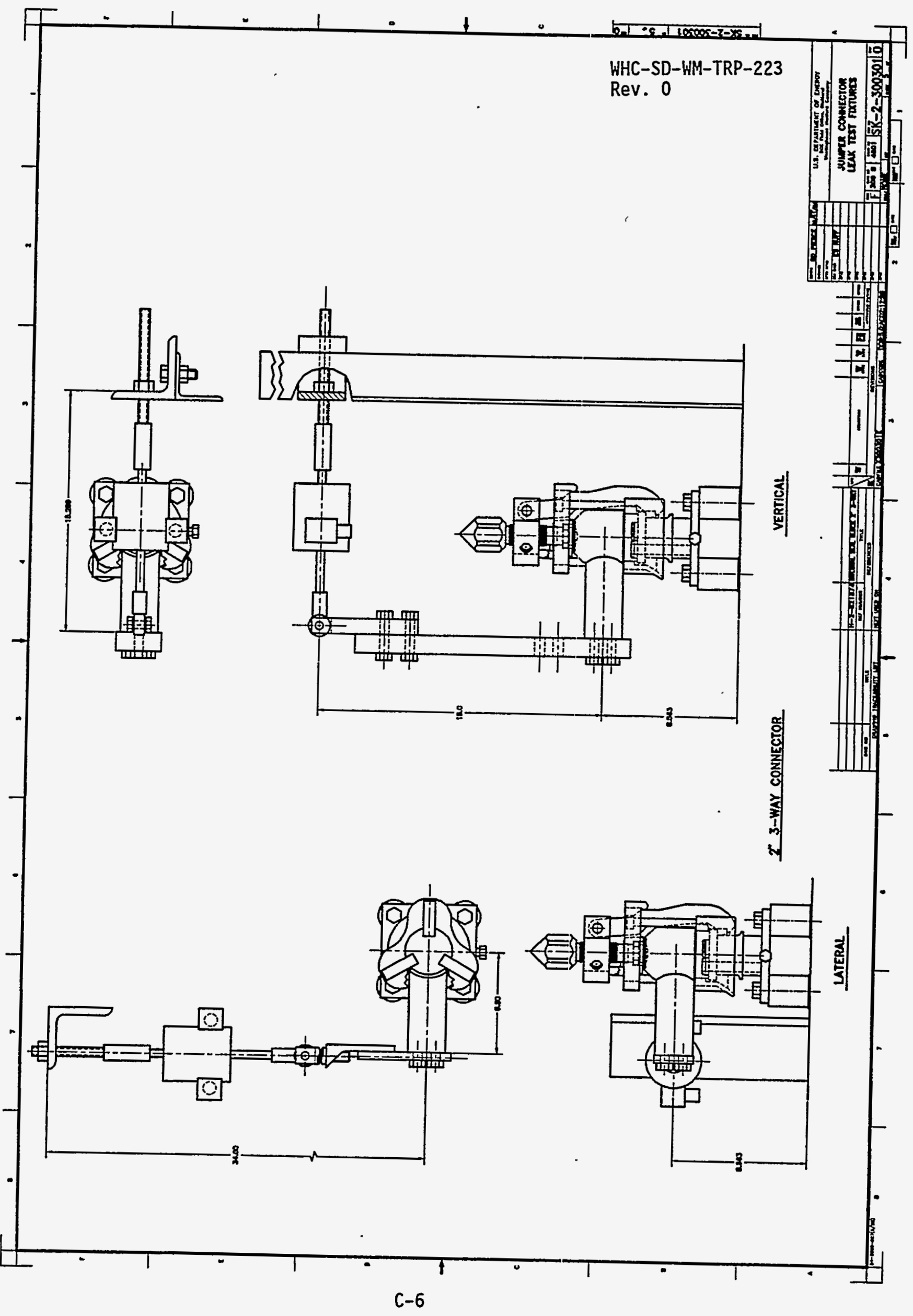


WHC-SD-WM-TRP-223

Rev. 0

APPENDIX D: DRAWINGS FOR JUMPER CONNECTOR BLOCK MOMENT ARM

D-1 


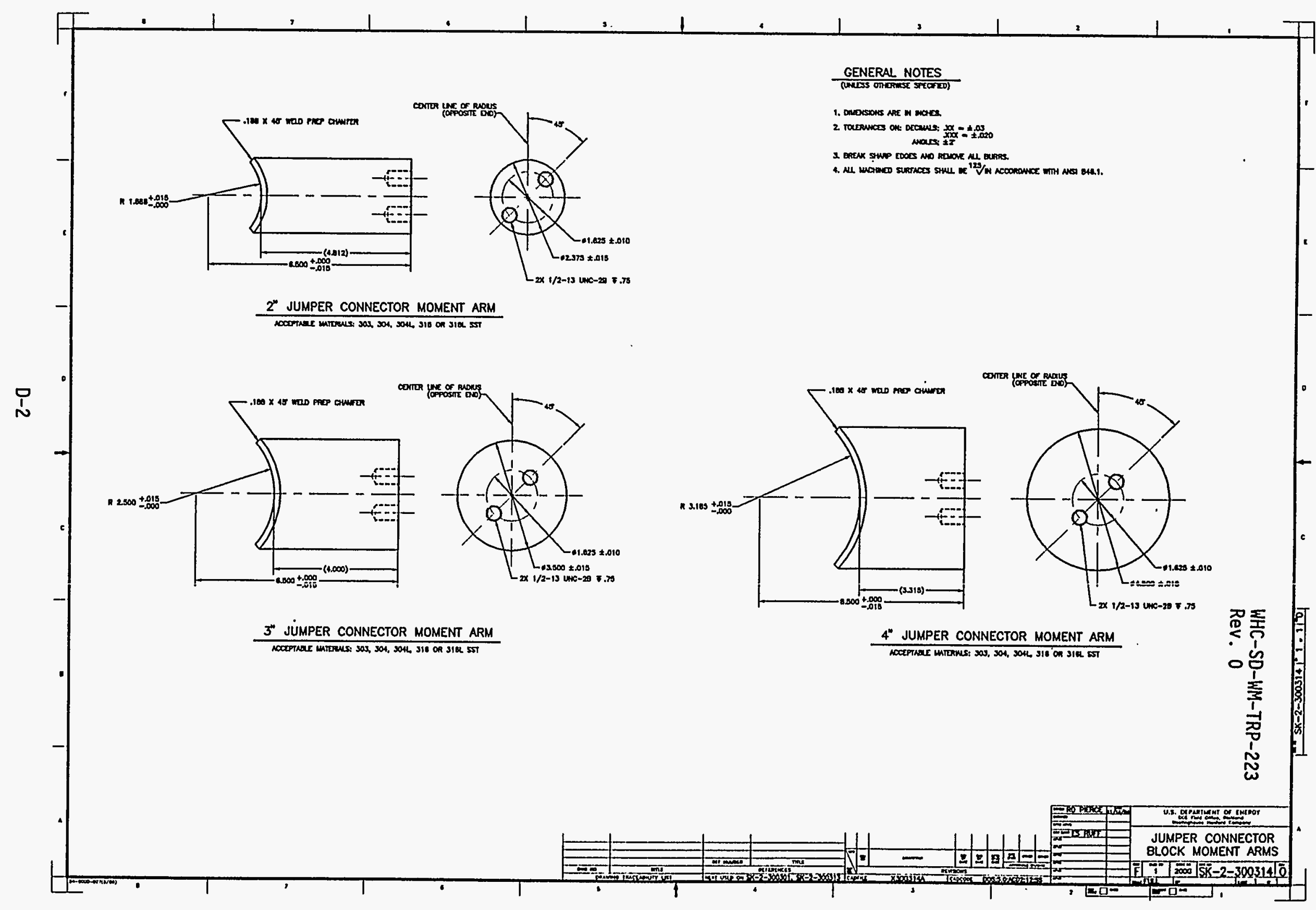


WHC-SD-WM-TRP-223

Rev. 0

APPENDIX E: GRAPHS OF 2-IN. FLUROSILICONE TESTS

E-1 


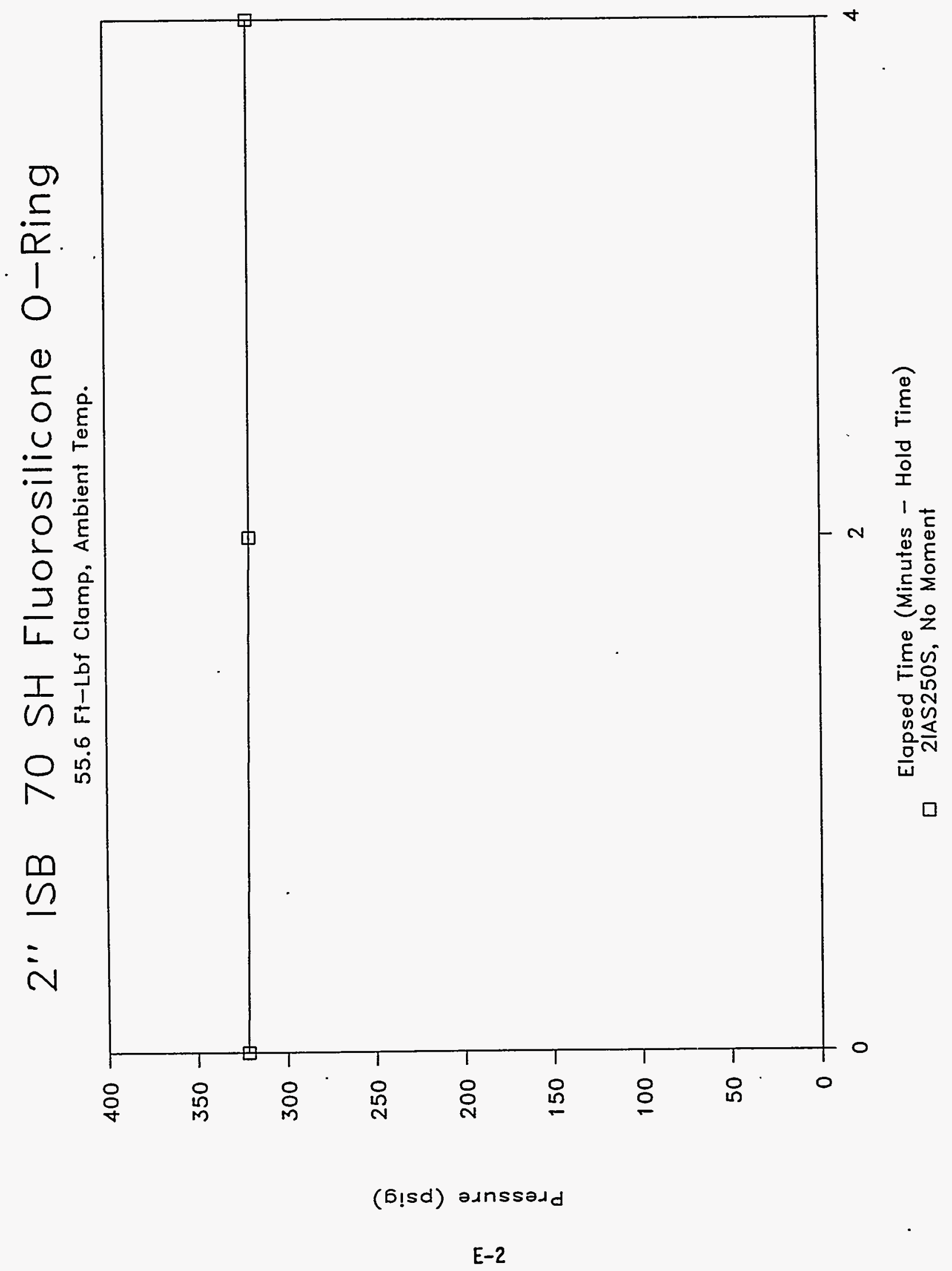


WHC-SD-WM-TRP-223

Rev. 0

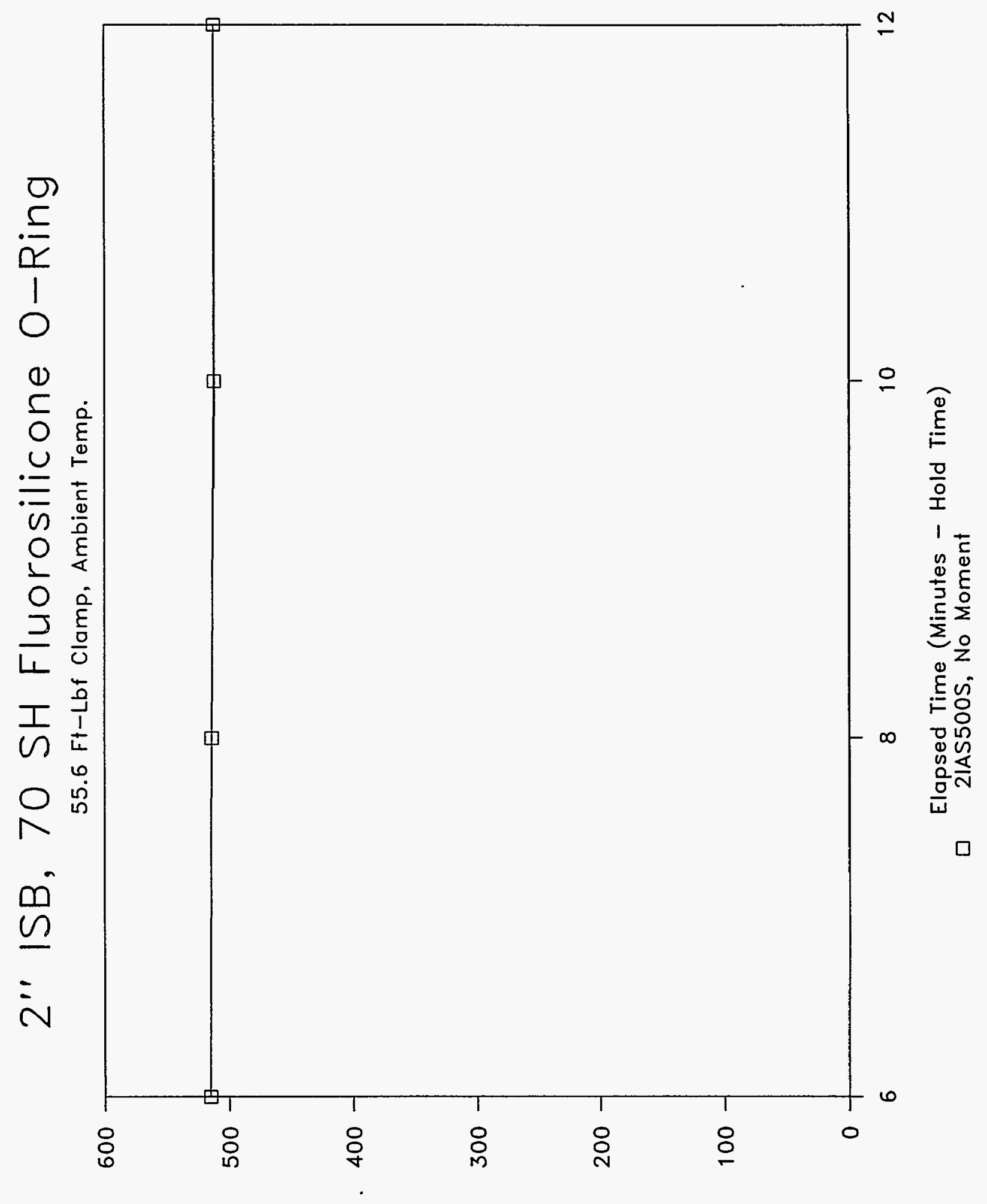

(b!sd) aunssadd

$E-3$ 


\section{2" ISB, 70 SH Fluorosilicone O-Ring}

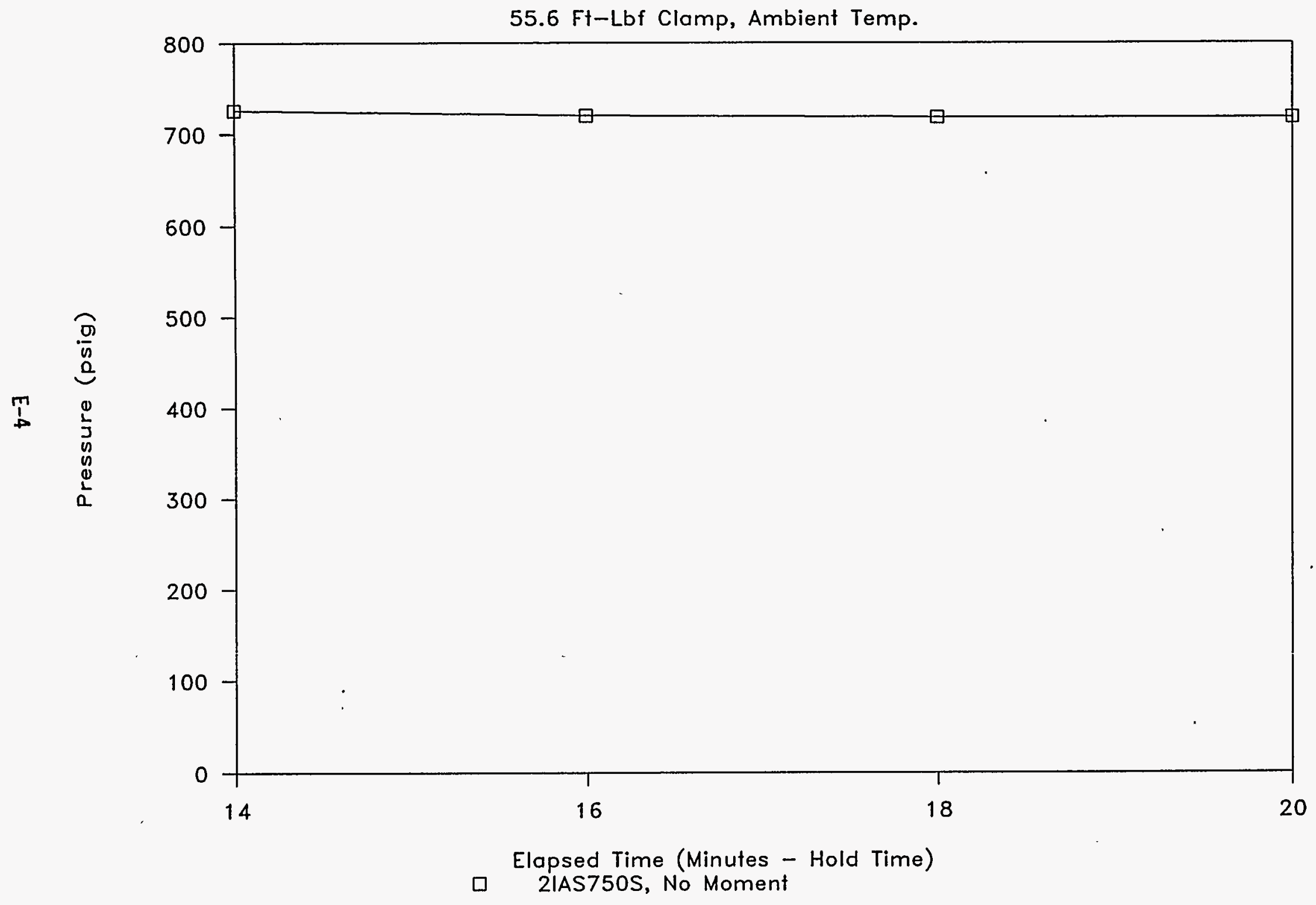


WHC-SD-WM-TRP-223

Rev. 0

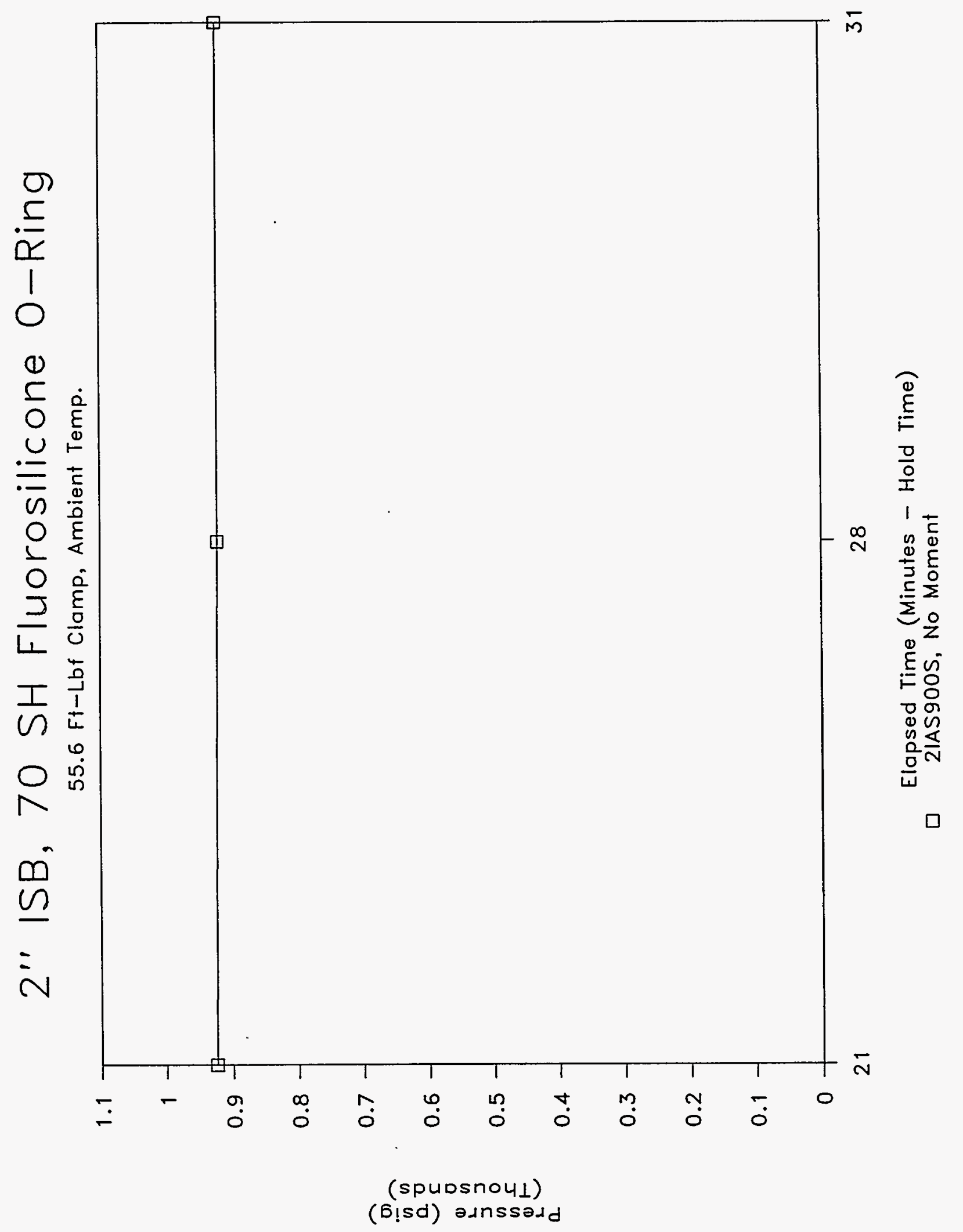


JUNE 23, 1994

2" ISB CONNECTOR, FLUOROSILICONE O-RING, 70 DUROMETER, AMBIENT TEMP. PRESSURE ASCENSION LEAK TEST - NO MOMENT APPIIED

CLAMPING TORQUE $=55.6 \mathrm{FT}-\mathrm{LBF}$

GRAPH NAME $=2$ IAS250S

CHARGE PRESSURE $=250$ PSIG

$\begin{array}{lllll}\text { PRESSURE } & \text { ELAPSED } & \text { ELAPSED } & \text { ELAPSED } & \text { APPLIED } \\ \text { PSIG } & \text { MINUTES } & \text { SECONDS } & \text { DECIMAL } & \text { FORCE } \\ & & & \text { MINUTES } & \text { LBS } \\ \text { INPUT } & \text { INPUT } & \text { INPUT } & \text { COMPUTE } & \text { INPUT }\end{array}$

$\begin{array}{rrrrr}322 & 0 & 0 & 0.00 & 0 \\ 320 & 2 & 10 & 2.17 & 0 \\ 320 & 4 & 30 & 4.50 & 0\end{array}$

INCREASED PRESSURE TO 500 PSIG.

GRAPH NAME $=2$ IAS500S

$\begin{array}{rrrrr}515 & 6 & 35 & 6.58 & 0 \\ 514 & 8 & 18 & 8.30 & 0 \\ 512 & 10 & 28 & 10.47 & 0 \\ 512 & 12 & 30 & 12.50 & 0\end{array}$

INCREASED PRESSURE TO 750 PSIG.

$\begin{array}{lllll}726 & 14 & 25 & 14.42 & 0 \\ 720 & 16 & 27 & 16.45 & 0 \\ 718 & 18 & 25 & 18.42 & 0 \\ 718 & 20 & 26 & 20.43 & 0\end{array}$

INCREASED PRESSURE TO 900 PSIG.

GRAPH NAME $=2$ IAS900S

$\begin{array}{lllll}925 & 21 & 56 & 21.93 & 0 \\ 922 & 28 & 50 & 28.83 & 0 \\ 922 & 31 & 37 & 31.62 & 0\end{array}$


2" ISB, 70 SH Fluorosilicone O-Ring $51.0 \mathrm{Ft}$-Lbf Clamp, Ambient Temp.

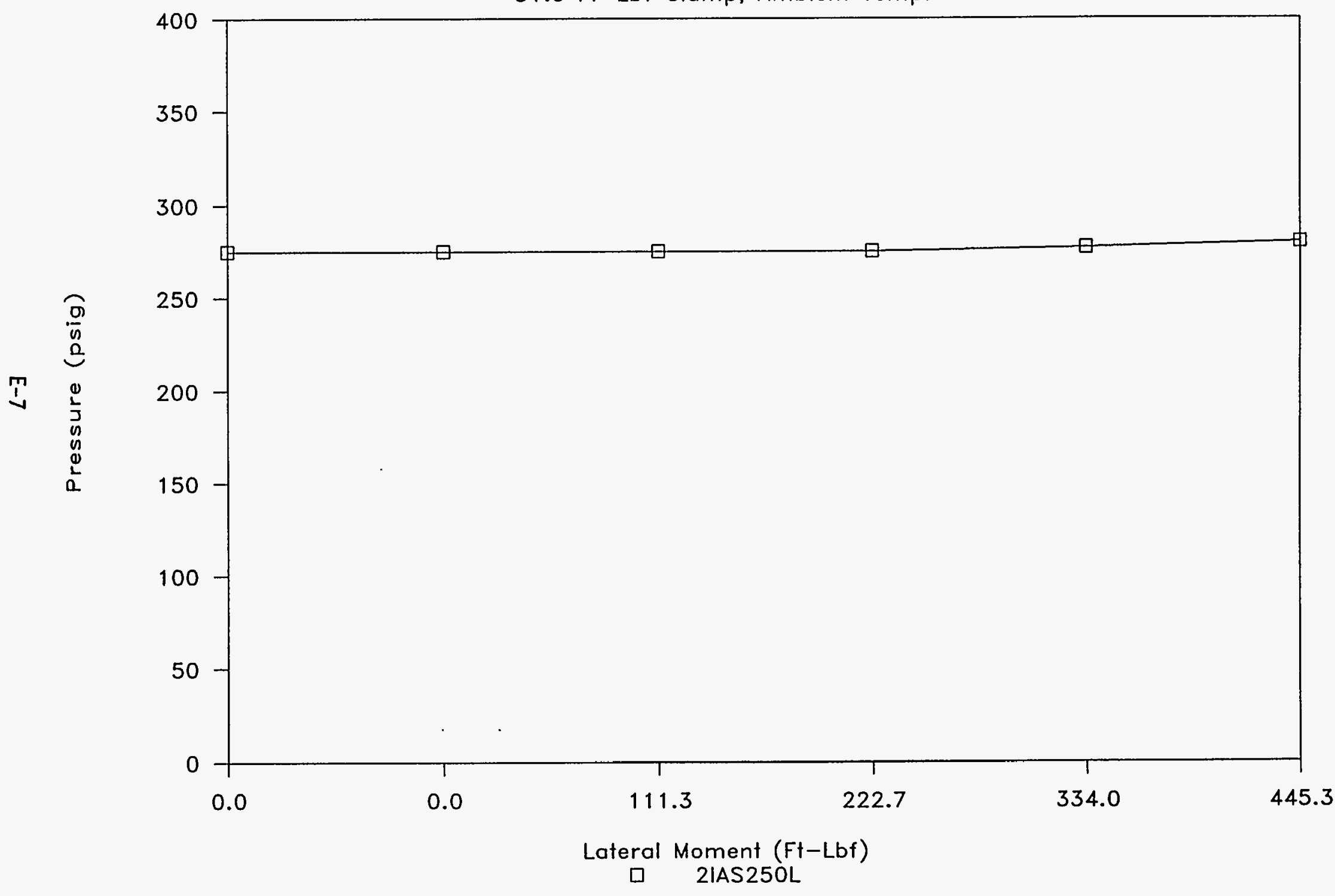




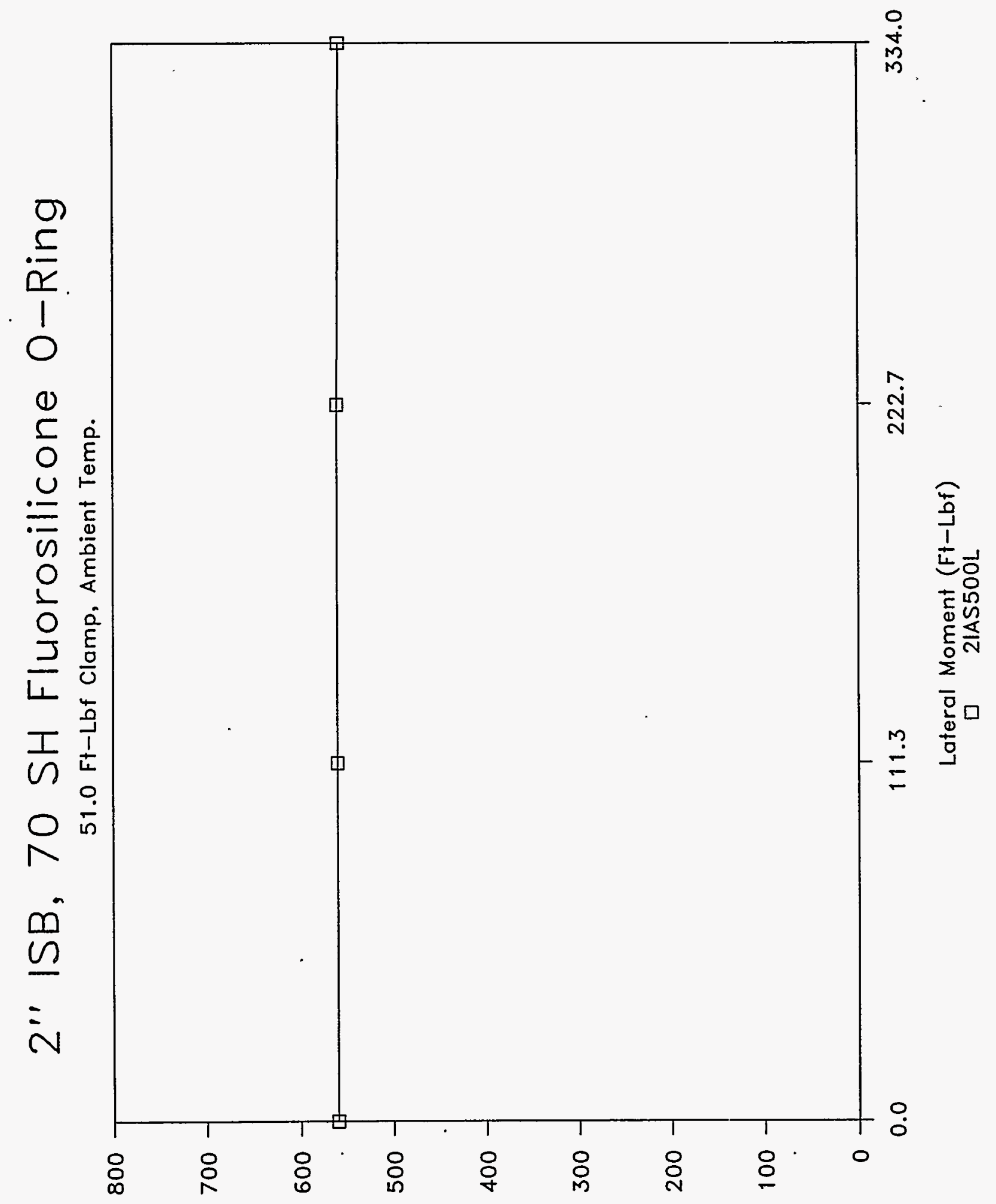

(b!sd) adnssadd

E-8 


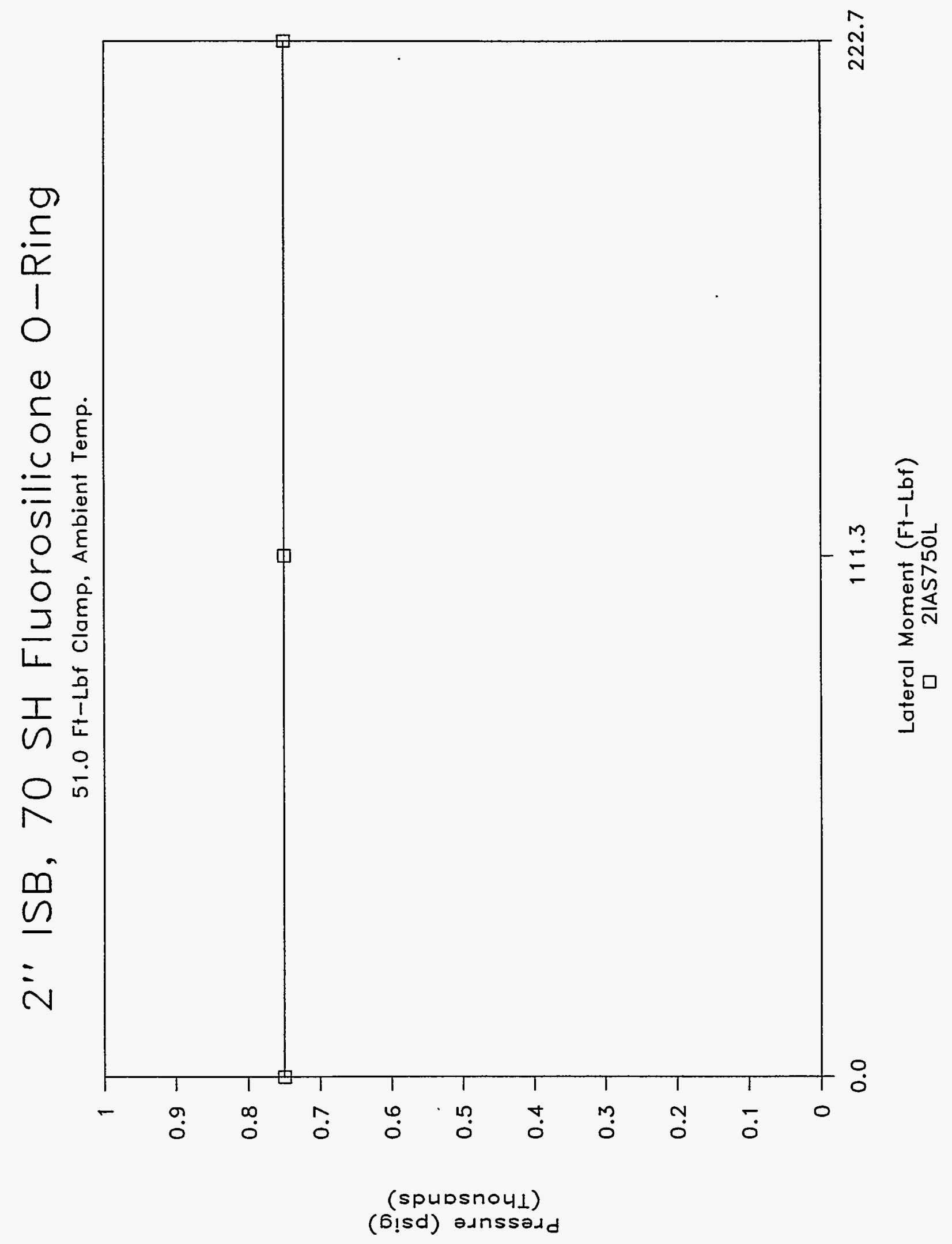

E-9 
2" ISB, 70 SH Fluorosilicone O-Ring

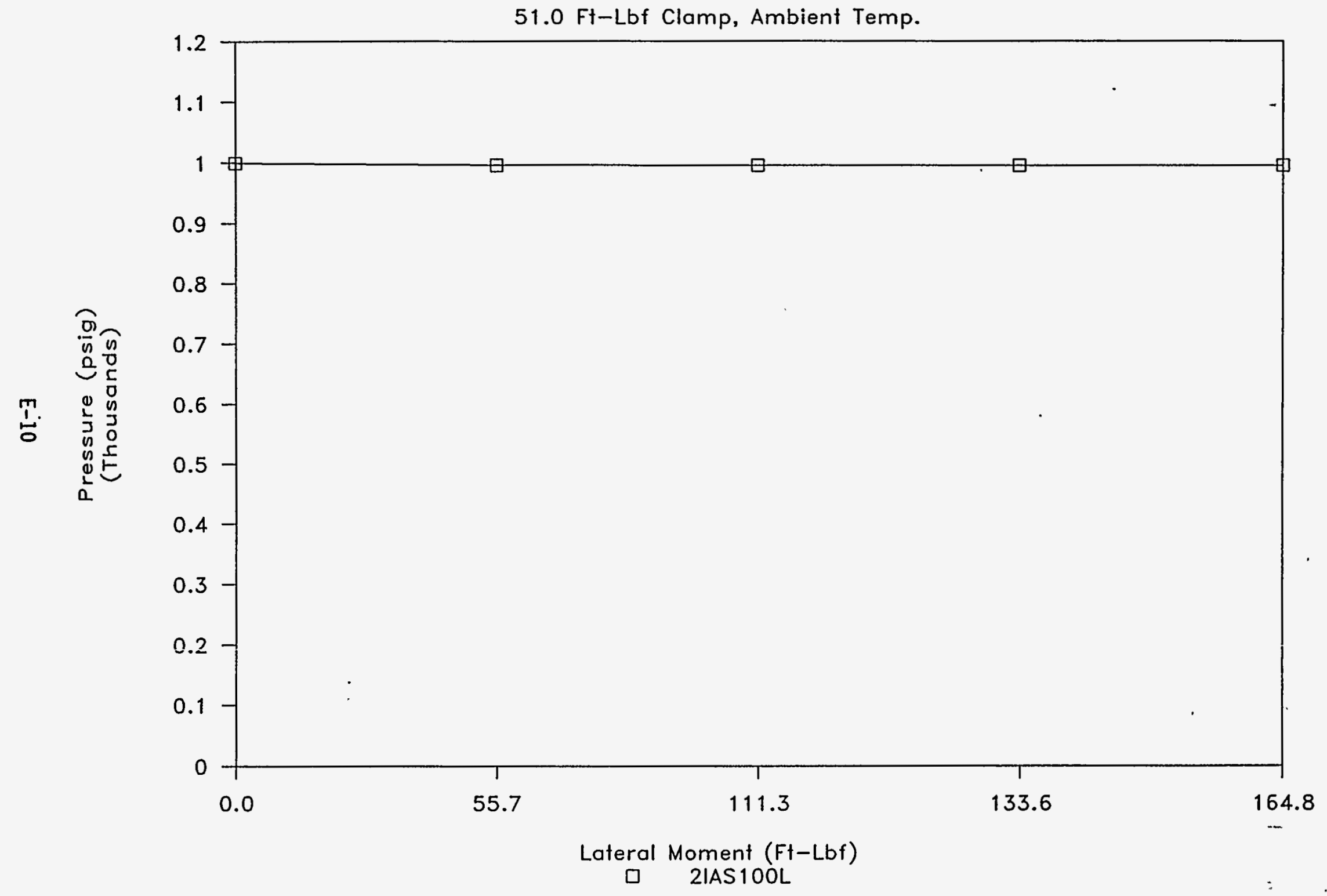


AUGUST 02, 1994

2" ISB CONNECTOR, 70 SH FLUOROSILICONE O-RING, AMBIENT TEMP. PRESSURE ASCENSION LEAK TEST - LATERAL MOMENT (SIDEWAYS ROTATION) CLAMPING TORQUE $=51.0$ FT-LBF CHARGE PRESSURE $=250$ PSTG

GRAPH NAME $=2$ IAS250L

\begin{tabular}{|c|c|c|c|}
\hline PRESSURE & ELAPSED & ELAPSED & ELAPSED \\
\hline & MINUTES & SECONDS & $\begin{array}{l}\text { DECIMAL } \\
\text { MINUTES }\end{array}$ \\
\hline INPUT & INPUT & INPUT & COMPUTE \\
\hline
\end{tabular}

$\begin{array}{rrrr}275 & 0 & 0 & 0.00 \\ 275 & 2 & 2 & 2.03 \\ 275 & 4 & 3 & 4.05 \\ 275 & 6 & 3 & 6.05 \\ 277 & 8 & 2 & 8.03 \\ 280 & 10 & 10 & 10.17\end{array}$

INCREASED PRESSURE TO 500 PSIG

$\begin{array}{llll}560 & 12 & 3 & 12.05 \\ 560 & 14 & 2 & 14.03 \\ 560 & 16 & 2 & 16.03 \\ 558 & 18 & 1 & 18.02\end{array}$

INCREASED PRESSURE TO 750 PSIG

$\begin{array}{rrrrrrr}750 & 20 & 2 & 20.03 & 0 & 0.4453 & 0.0 \\ 750 & 22 & 2 & 22.03 & 250 & 0.4453 & 111.3 \\ 750 & 24 & 2 & 24.03 & 500 & 0.4453 & 222.7\end{array}$

INCREASED PRESSURE TO 1000 PSIG

$\begin{array}{rrrrrrr}1000 & 26 & 3 & 26.05 & 0 & 0.4453 & 0.0 \\ 996 & 28 & 7 & 28.12 & 125 & 0.4453 & 55.7 \\ 995 & 30 & 1 & 30.02 & 250 & 0.4453 & 111.3 \\ 995 & 32 & 2 & 32.03 & 300 & 0.4453 & 133.6 \\ 995 & 34 & 3 & 34.05 & 370 & 0.4453 & 164.8\end{array}$


WHC-SD-WM-TRP-223

Rev. 0

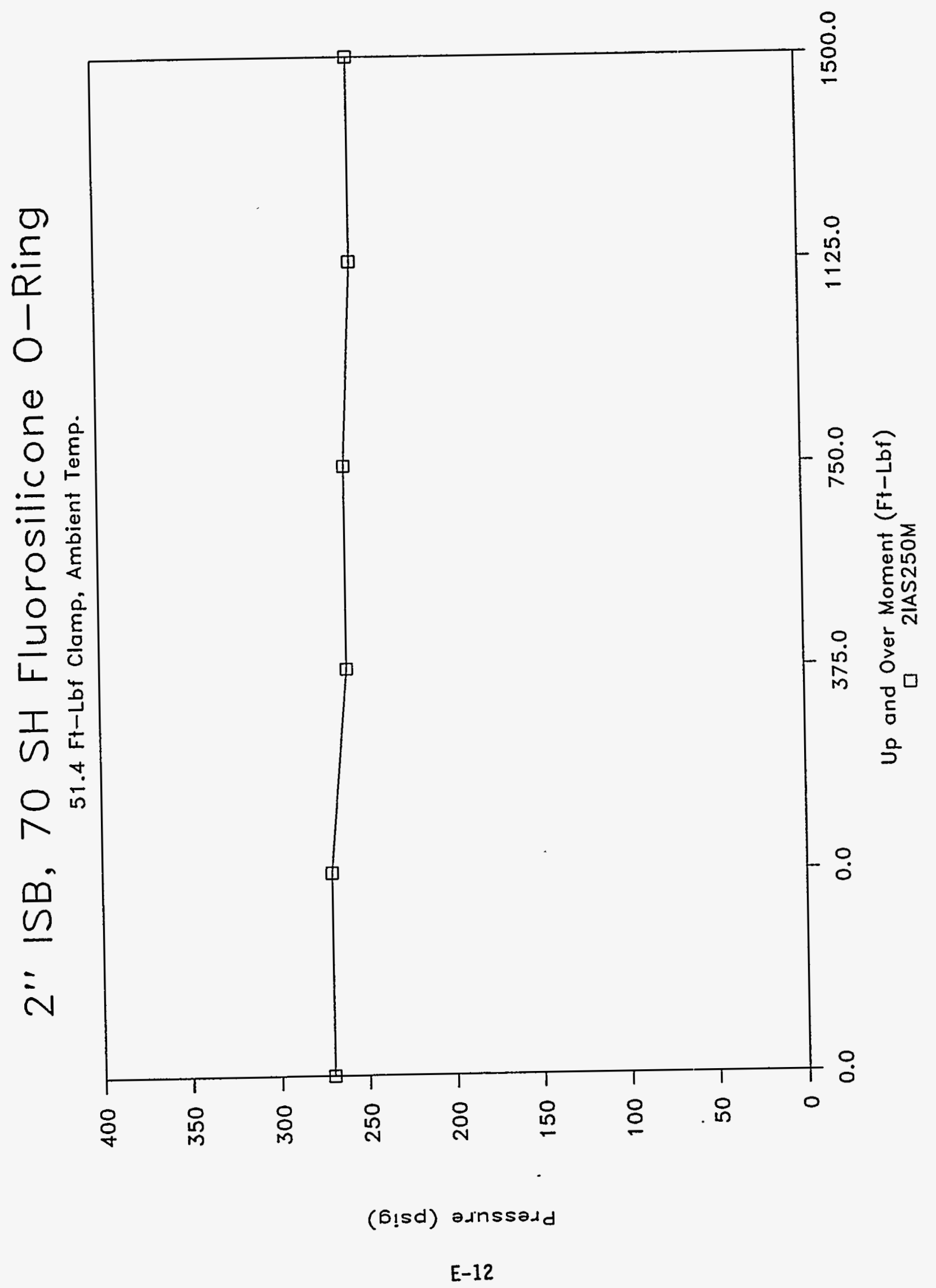


WHC-SD-WM-TRP-223

Rev. 0

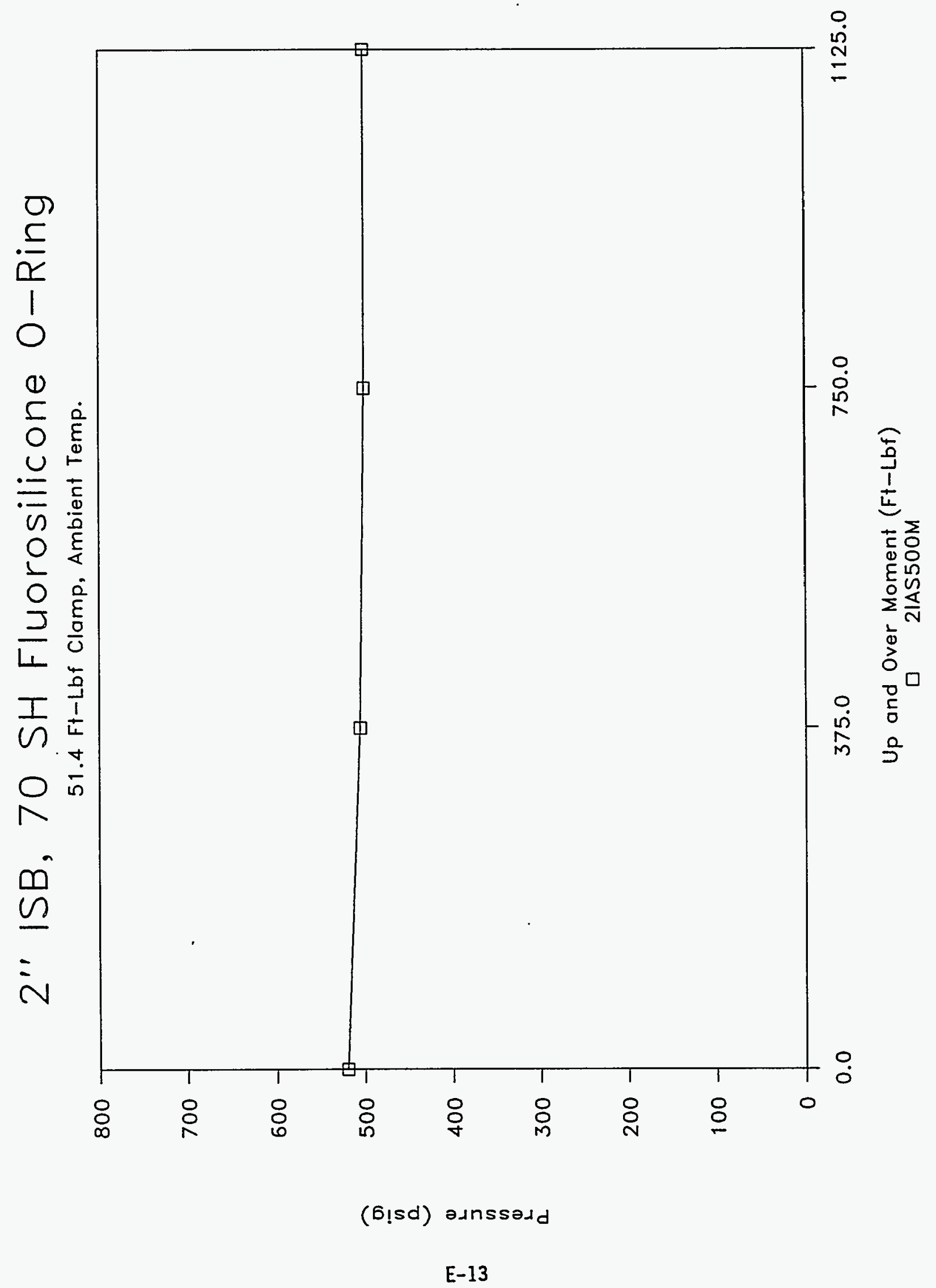




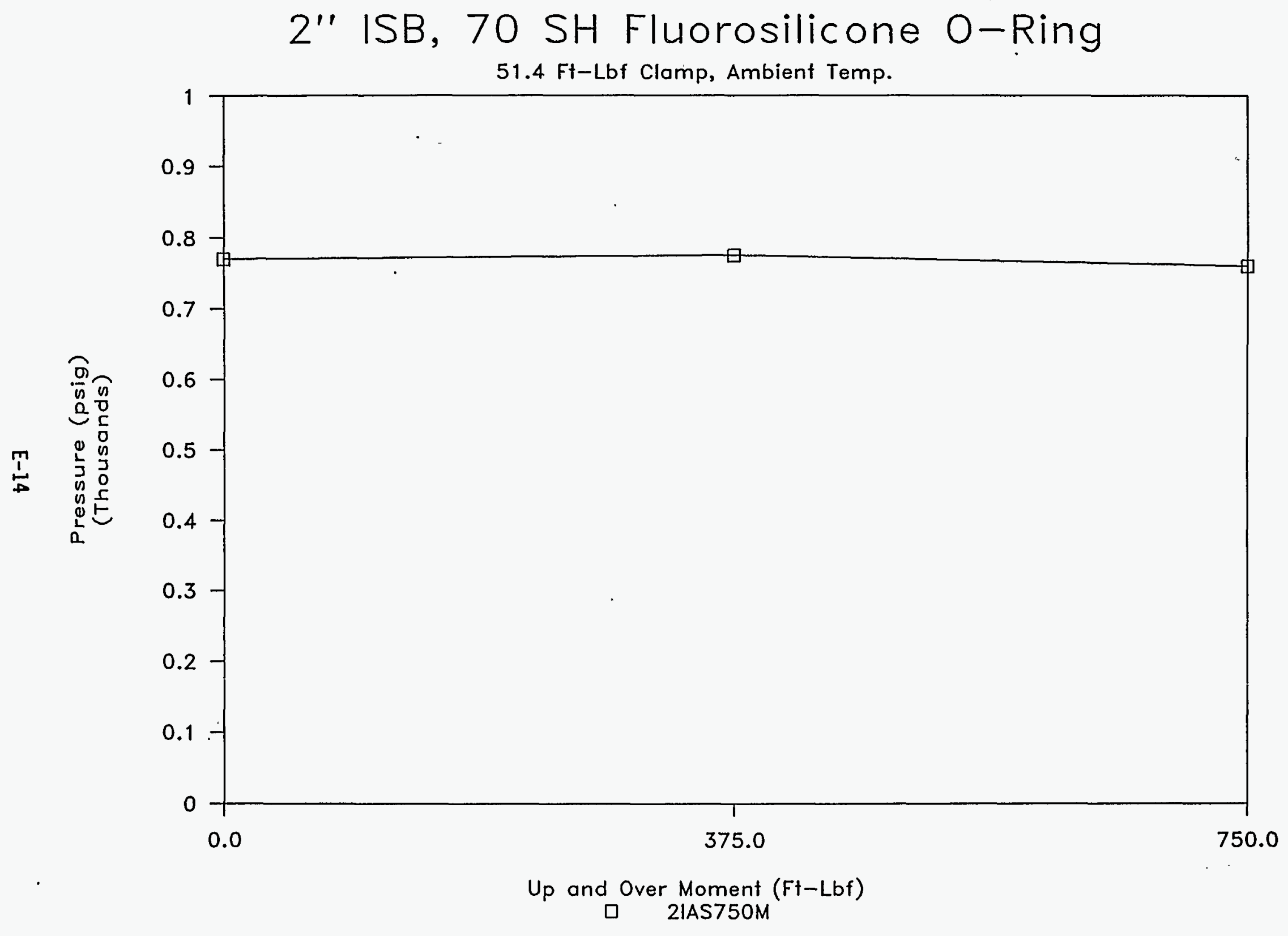


WHC-SD-WM-TRP-223

Rev. 0

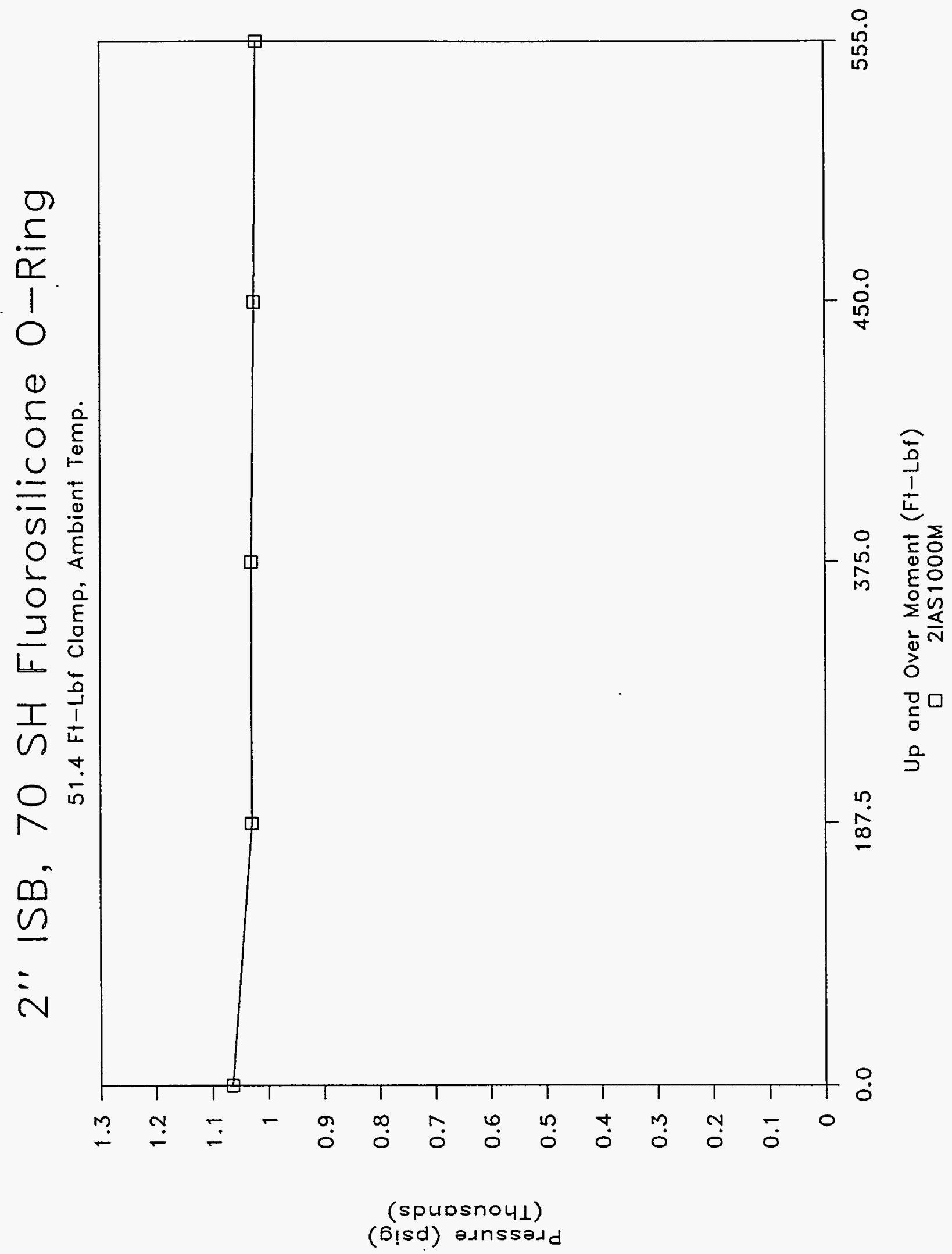

E-15 
AUGUST $03, \quad 1994$

$2 "$ ISB CONNECTOR, 70 SH FLUOROSILICONE O-RING, AMBIENT TEMP. PRESSURE ASCENSION IEAK TEST - UP AND OVER MOMENT (UPWARD ROTATION) CLAMPING TORQUE $=51.4 \mathrm{FT}-\mathrm{LBF} \quad$ GRAPH NAME $=2$ IAS25OM CHARGE PRESSURE $=250$ PSIG

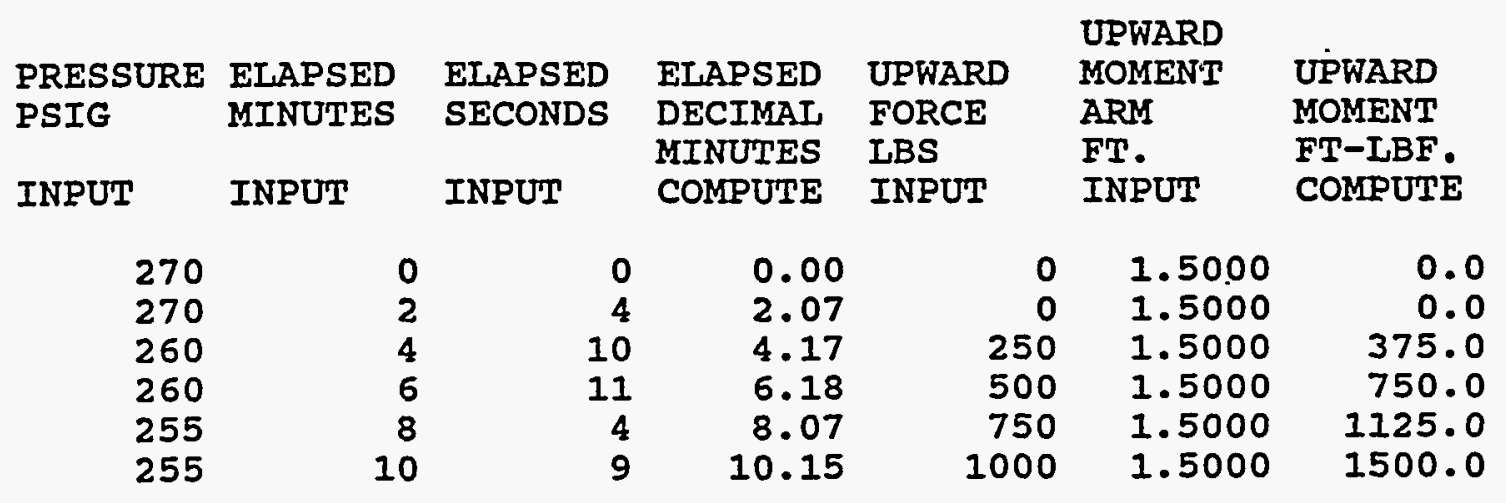

INCREASED PRESSURE TO 500 PSIG

GRAPH NAME $=2$ IAS50OM

$\begin{array}{rrrrrrr}520 & 12 & 6 & 12.10 & 0 & 1.5000 & 0.0 \\ 505 & 14 & 9 & 14.15 & 250 & 1.5000 & 375.0 \\ 500 & 16 & 2 & 16.03 & 500 & 1.5000 & 750.0 \\ 500 & 18 & 6 & 18.10 & 750 & 1.5000 & 1125.0\end{array}$

INCREASED PRESSURE TO 750 PSIG

GRAPH NAME = 2 IAS750M

$\begin{array}{rrrrrrr}770 & 20 & 2 & 20.03 & 0 & 1.5000 & 0.0 \\ 775 & 22 & 13 & 22.22 & 250 & 1.5000 & 375.0 \\ 760 & 24 & 6 & 24.10 & 500 & 1.5000 & 750.0\end{array}$

INCREASED PRESSURE TO 1000 PSIG GRAPH NAME = 2IASI000M

$\begin{array}{rrrrrrr}1065 & 26 & 3 & 26.05 & 0 & 1.5000 & 0.0 \\ 1030 & 28 & 1 & 28.02 & 125 & 1.5000 & 187.5 \\ 1030 & 30 & 5 & 30.08 & 250 & 1.5000 & 375.0 \\ 1025 & 32 & 3 & 32.05 & 300 & 1.5000 & 450.0 \\ 1020 & 34 & 5 & 34.08 & 370 & 1.5000 & 555.0\end{array}$


WHC-SD-WM-TRP-223

Rev. 0

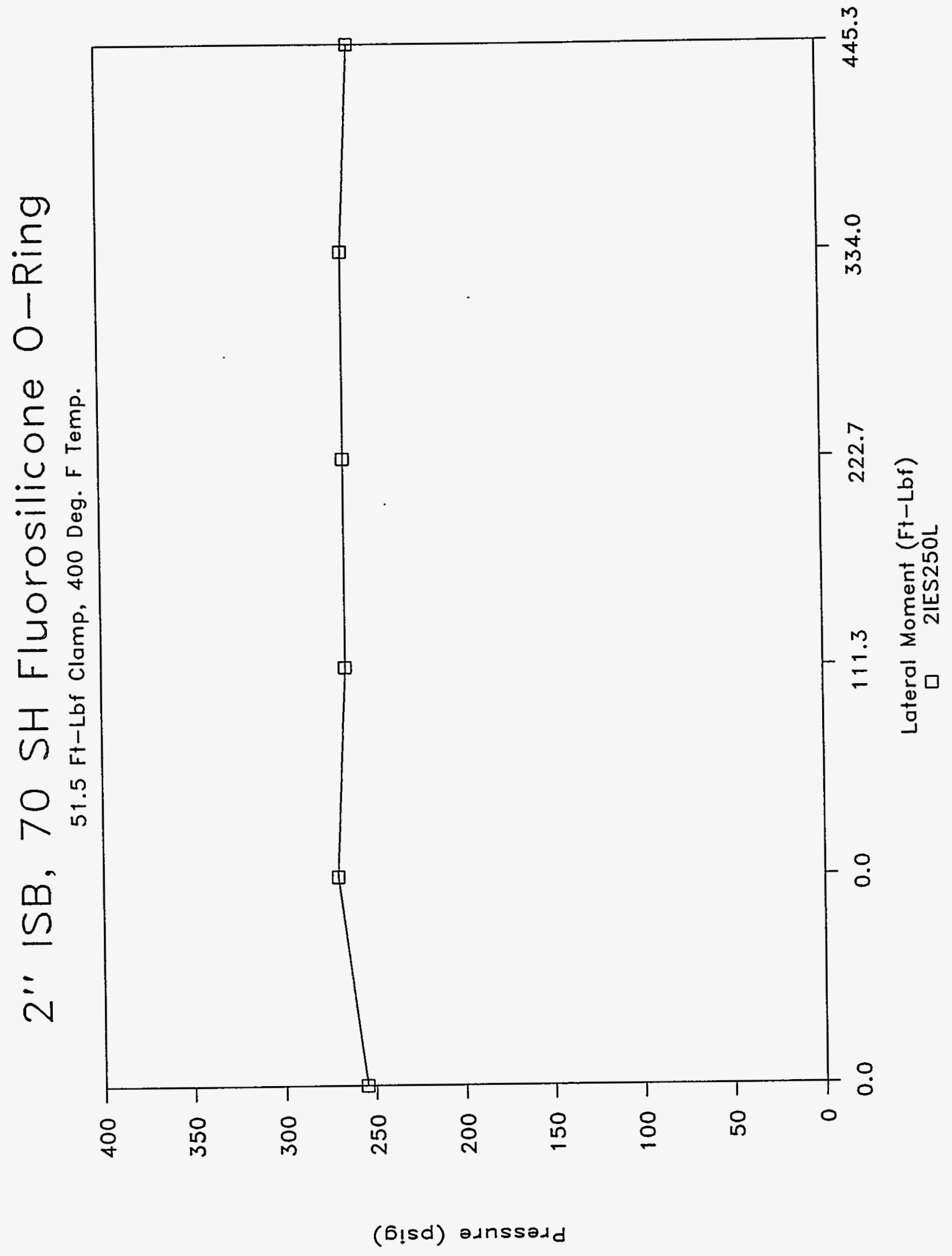




\section{2" ISB, 70 SH Fluorosilicone O-Ring}

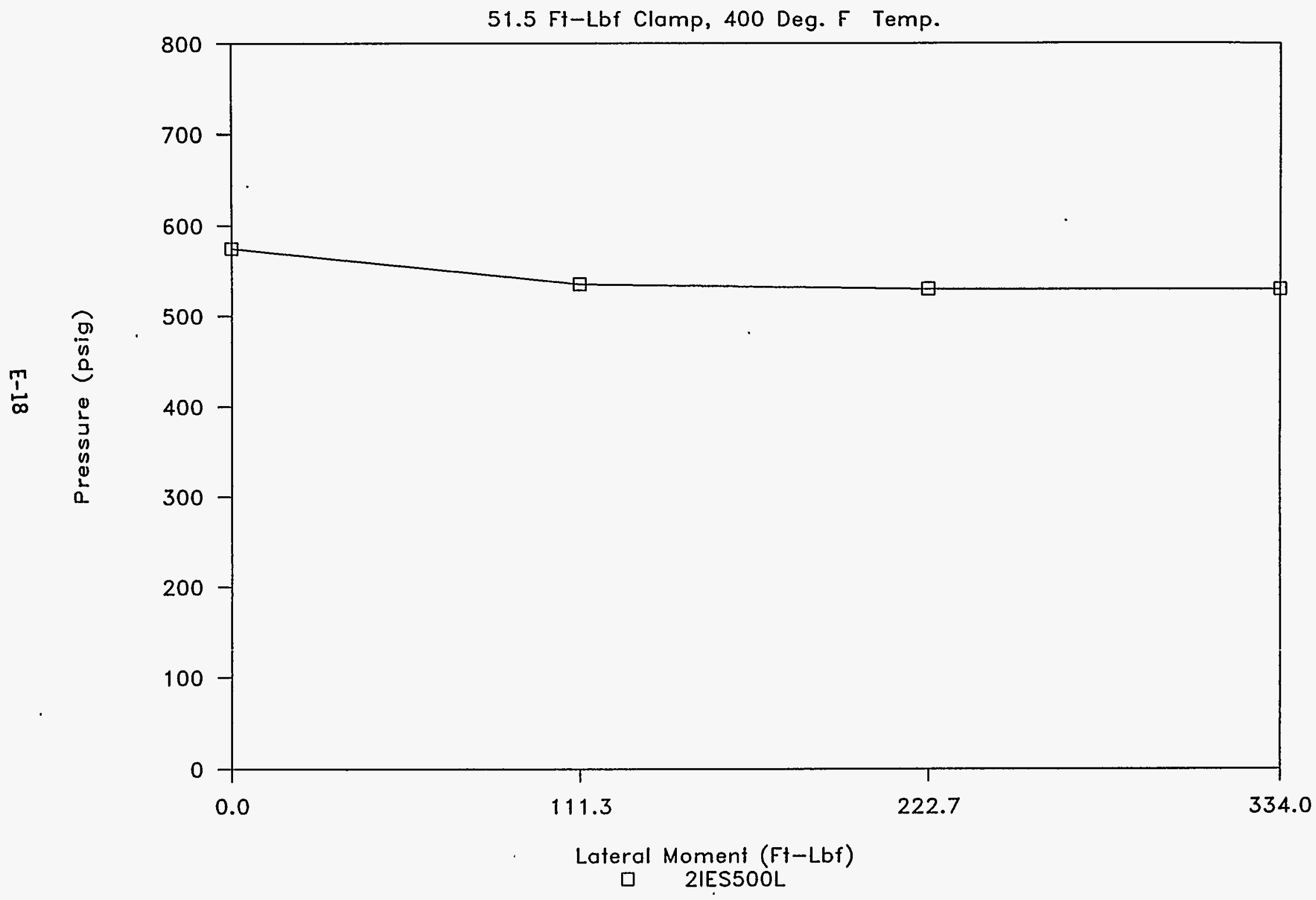


WHC-SD-WM-TRP-223

Rev. 0

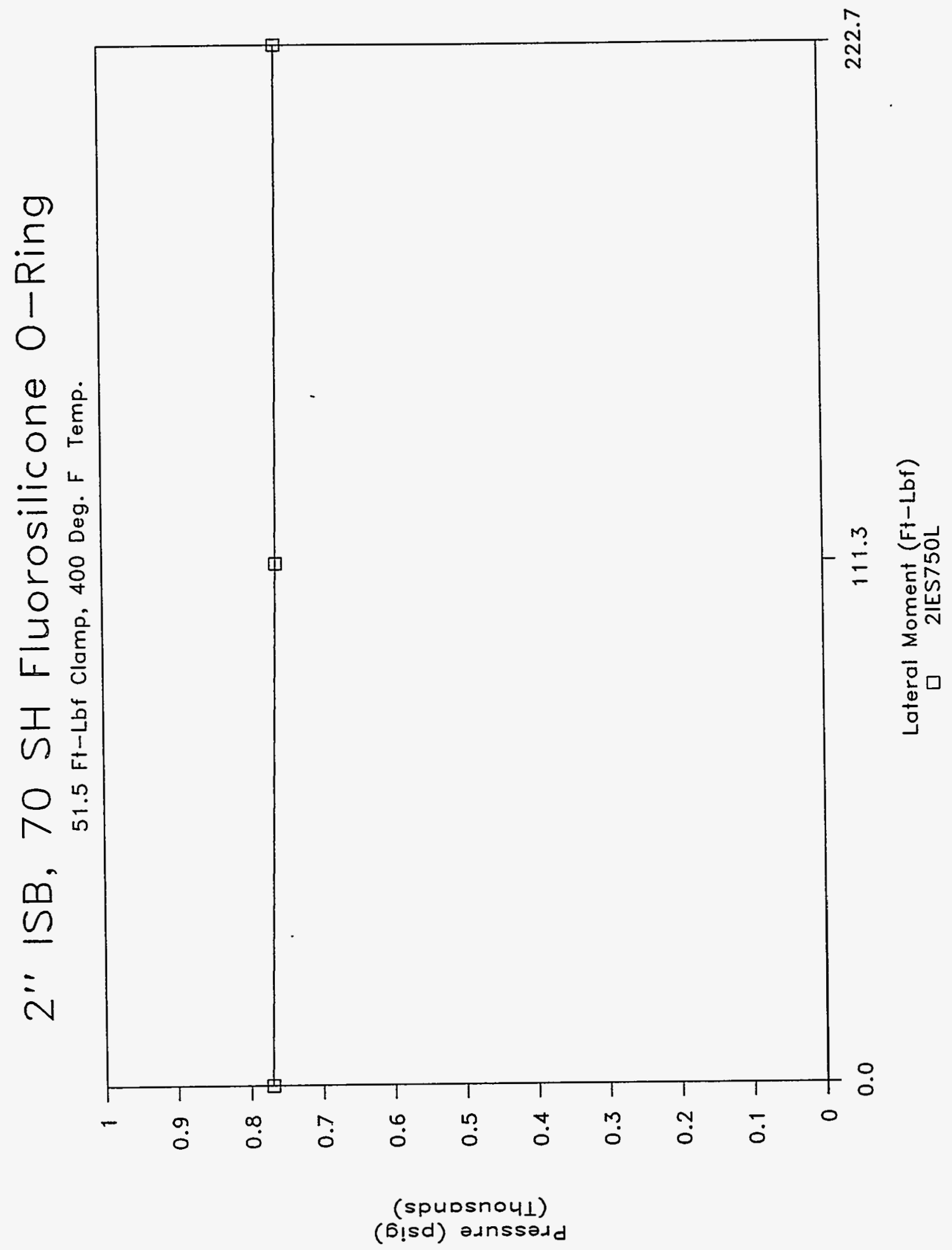

$E-19$ 
2" ISB, 70 SH Fluorosilicone O-Ring $51.5 \mathrm{Ft}$-Lbf Clamp, Elevated Temp.

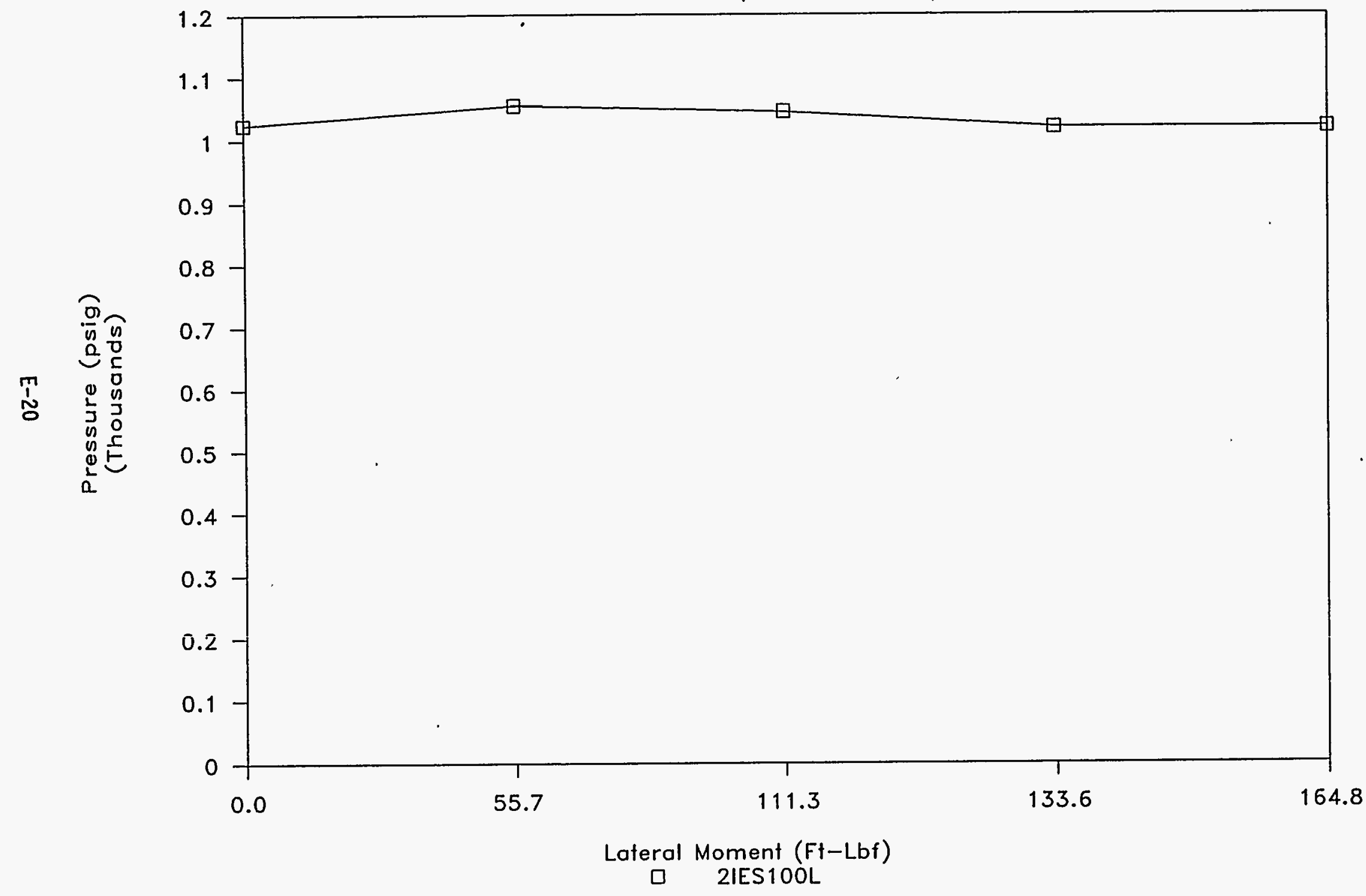


AUGUST 03, 1994

2" ISB CONNECTOR, $70 \mathrm{SH}$ FLUOROSILICONE O-RING, ELEVATED TEMP. PRESSURE ASCENSION LEAK TEST - ILATERAI MOMENT (SIDEWAYS ROTATION) CLAMPING TORQUE $=51.5$ FT-LBF GRAPH NAME $=2$ IES250L

CHARGE PRESSURE $=250$ PSIG

TEMPERATURE $=400$ DEG. $F$

$\begin{array}{lllllll} & & & & & \text { LATERAI } & \\ \text { PRESSURE } & \text { ELAPSED } & \text { ELAPSED } & \text { ELAPSED } & \text { LATERAL } & \text { MOMENT } & \text { LATERAL } \\ \text { PSIG } & \text { MINUTES } & \text { SECONDS } & \text { DECIMAL } & \text { FORCE } & \text { ARM } & \text { MOMENT } \\ & & & \text { MINUTES } & \text { LBS } & \text { FT. } & \text { FT-LBF. } \\ \text { INPUT } & \text { INPUT } & \text { INPUT } & \text { COMPUTE } & \text { INPUT } & \text { INPUT } & \text { COMPUTE }\end{array}$

$\begin{array}{rrrrrrr}255 & 0 & 0 & 0.00 & 0 & 0.4453 & 0.0 \\ 270 & 2 & 17 & 2.28 & 0 & 0.4453 & 0.0 \\ 265 & 4 & 4 & 4.07 & 250 & 0.4453 & 111.3 \\ 265 & 6 & 4 & 0.00 & 500 & 0.4453 & 222.7 \\ 265 & 8 & 5 & 8.08 & 750 & 0.4453 & 334.0 \\ 260 & 10 & 10 & 10.17 & 1000 & 0.4453 & 445.3\end{array}$

INCREASED PRESSURE TO 500 PSIG

GRAPH NAME $=2$ IES50OL

$\begin{array}{rrrrrrr}575 & 12 & 3 & 12.05 & 0 & 0.4453 & 0.0 \\ 535 & 14 & 3 & 14.05 & 250 & 0.4453 & 111.3 \\ 530 & 16 & 1 & 16.02 & 500 & 0.4453 & 222.7 \\ 530 & 18 & 16 & 18.27 & 750 & 0.4453 & 334.0\end{array}$

INCREASED PRESSURE TO 750 PSIG

GRAPH NAME $=2$ IES750L

$\begin{array}{rrrrrrr}770 & 20 & 2 & 20.03 & 0 & 0.4453 & 0.0 \\ 760 & 22 & 3 & 22.05 & 250 & 0.4453 & 111.3 \\ 755 & 24 & 5 & 24.08 & 500 & 0.4453 & 222.7\end{array}$

INCREASED PRESSURE TO 1000 PSIG GRAPH NAME = 2IES100L

$\begin{array}{rrrrrrr}1025 & 26 & 3 & 26.05 & 0 & 0.4453 & 0.0 \\ 1055 & 28 & 3 & 28.05 & 125 & 0.4453 & 55.7 \\ 1045 & 30 & 2 & 30.03 & 250 & 0.4453 & 111.3 \\ 1020 & 32 & 3 & 32.05 & 300 & 0.4453 & 133.6 \\ 1020 & 34 & 5 & 34.08 & 370 & 0.4453 & 164.8\end{array}$




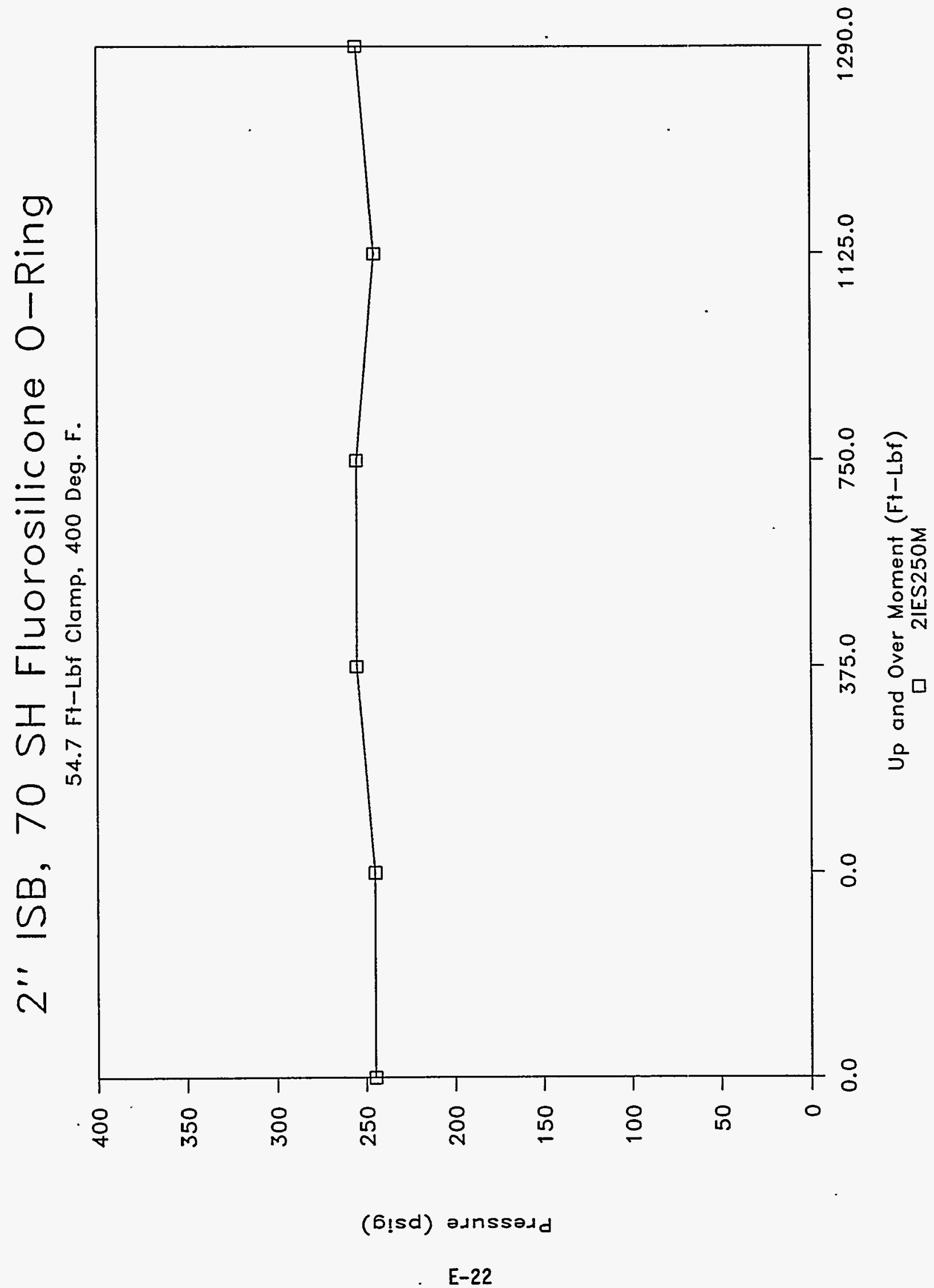


WHC-SD-WM-TRP-223

Rev. 0

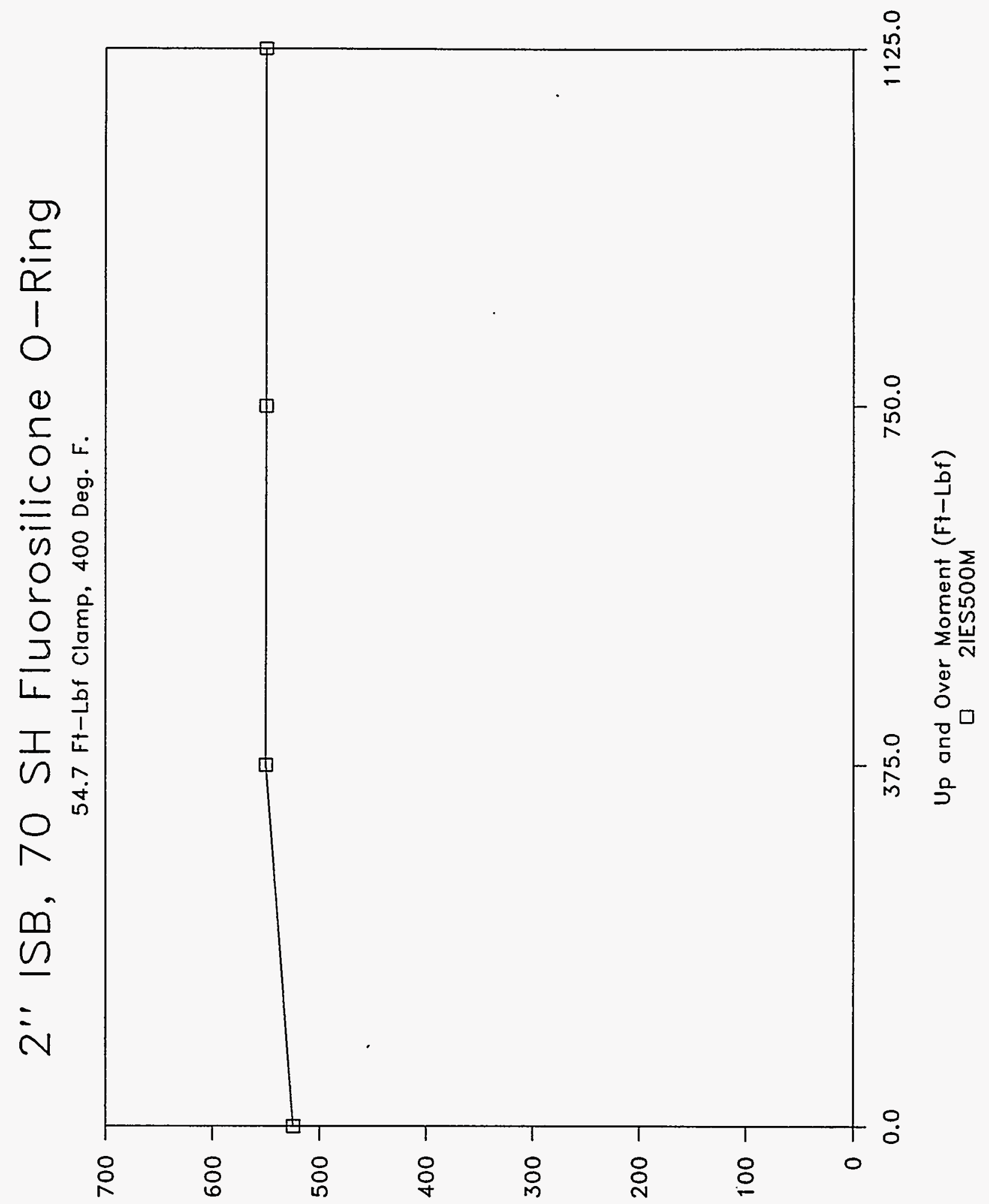

(bisd) atnssadd 


\section{2" ISB, 70 SH Fluorosilicone O-Ring}

54.7 Ft-Lbf Clamp, 400 Deg. F.

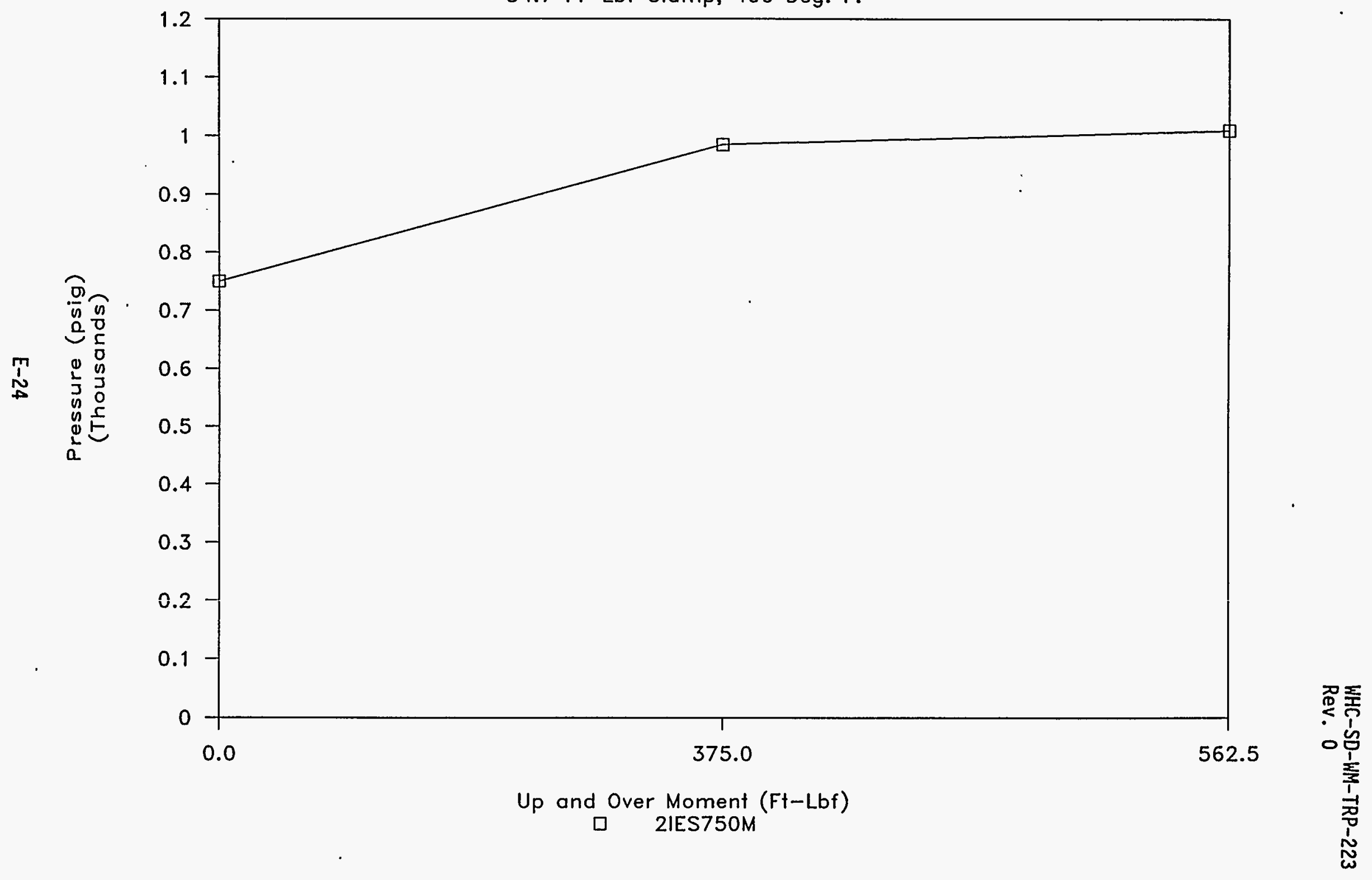


2" ISB, 70 SH Fluorosilicone O-Ring

187.5 Ft-Lbf Upward Moment, 400 Deg. F.

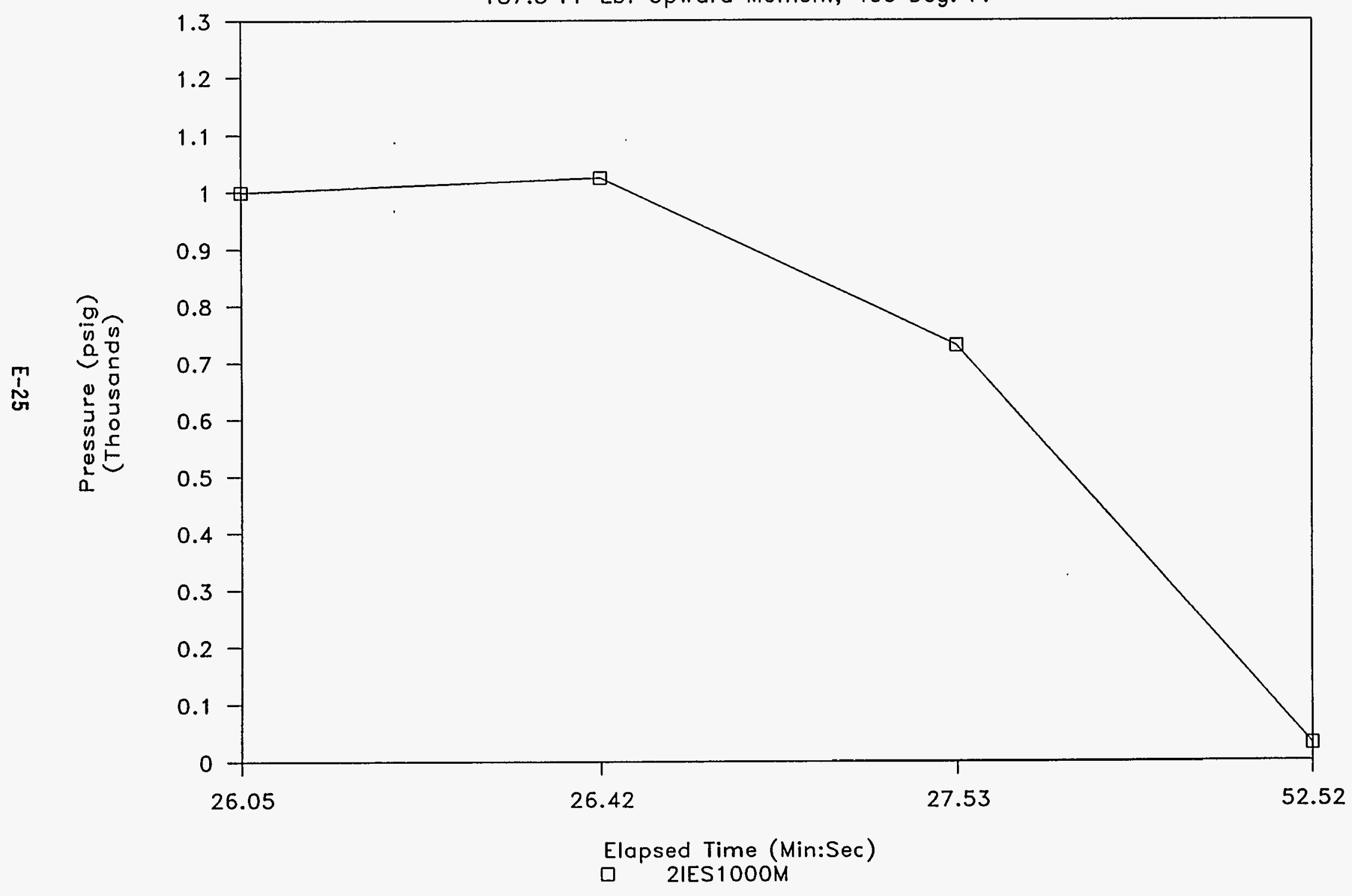


AUGUST 08, 1994

2" ISB CONNECTOR, $70 \mathrm{SH}$ FLUOROSILICONE O-RING, ELEVATED TEMP. PRESSURE ASCENSION LEAK TEST - UP AND OVER MOMENT (UPWARD ROTATION)

CLAMPING TORQUE $=54.7$ FT-IBF GRAPH NAME $=2$ IES250M

CHARGE PRESSURE $=250$ PSIG

TEMPERATURE $=400$ DEG. F

$\begin{array}{lllllll} & & & & & \text { UPWARD } \\ \text { PRESSURE } & \text { ELAPSED } & \text { ELAPSED } & \text { ELAPSED } & \text { UPWARD } & \text { MOMENT } & \text { UPWARD } \\ \text { PSIG } & \text { MINUTES } & \text { SECONDS } & \text { DECIMAL } & \text { FORCE } & \text { ARM } & \text { MOMENT } \\ & & & \text { MINUTES } & \text { LBS } & \text { FT. } & \text { FT-IBF. } \\ \text { INPUT } & \text { INPUT } & \text { INPUT } & \text { COMPUTE } & \text { INPUT } & \text { INPUT } & \text { COMPUTE }\end{array}$

$\begin{array}{rrrrrrr}245 & 0 & 0 & 0.00 & 0 & 1.5000 & 0.0 \\ 245 & 2 & 1 & 2.02 & 0 & 1.5000 & 0.0 \\ 255 & 4 & 2 & 4.03 & 250 & 1.5000 & 375.0 \\ 255 & 6 & 2 & 6.03 & 500 & 1.5000 & 750.0 \\ 245 & 8 & 2 & 8.03 & 750 & 1.5000 & 1125.0 \\ 255 & 10 & 22 & 10.37 & 860 & 1.5000 & 1290.0\end{array}$

INCREASED PRESSURE TO 500 PSIG

GRAPH NAME $=2$ IES50OM

$\begin{array}{rrrrrrr}525 & 12 & 6 & 12.10 & 0 & 1.5000 & 0.0 \\ 550 & 14 & 4 & 14.07 & 250 & 1.5000 & 375.0 \\ 550 & 16 & 1 & 16.02 & 500 & 1.5000 & 750.0 \\ 550 & 18 & 2 & 18.03 & 750 & 1.5000 & 1125.0\end{array}$

INCREASED PRESSURE TO 750 PSIG

GRAPH NAME $=2$ IES750M

$\begin{array}{rrrrrrr}750 & 20 & 1 & 20.02 & 0 & 1.5000 & 0.0 \\ 985 & 22 & 2 & 22.03 & 250 & 1.5000 & 375.0 \\ 1010 & 24 & 3 & 24.05 & 375 & 1.5000 & 562.5\end{array}$

INCREASED PRESSURE TO 1000 PSIG GRAPH NAME = 2IES1000M

$\begin{array}{rrrrrrr}1000 & 26 & 6 & 26.05 & 0 & 1.5000 & 0.0 \\ 1025 & 26 & 25 & 26.42 & 125 & 1.5000 & 187.5 * \text { O-RING } \\ 730 & 27 & 32 & 27.53 & 125 & 1.5000 & 187.5 \text { LEAK } \\ 30 & 52 & 31 & 52.52 & 125 & 1.5000 & 187.5\end{array}$


HHC-SD-WM-TRP-223

Rev. 0

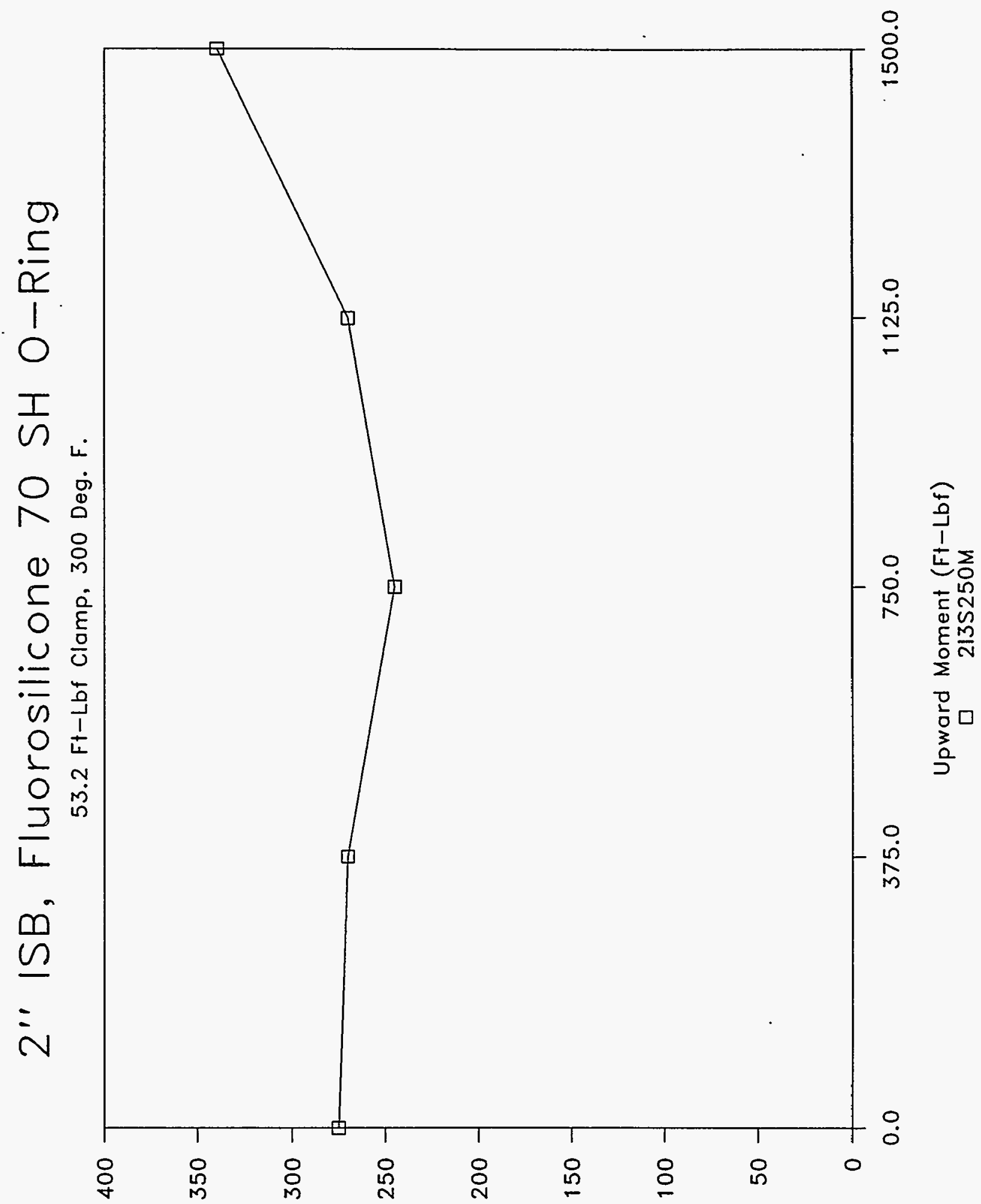

(b!sd) adnssadd 


\section{2" ISB, Fluorosilicone $70 \mathrm{SH} \mathrm{O-Ring}$}

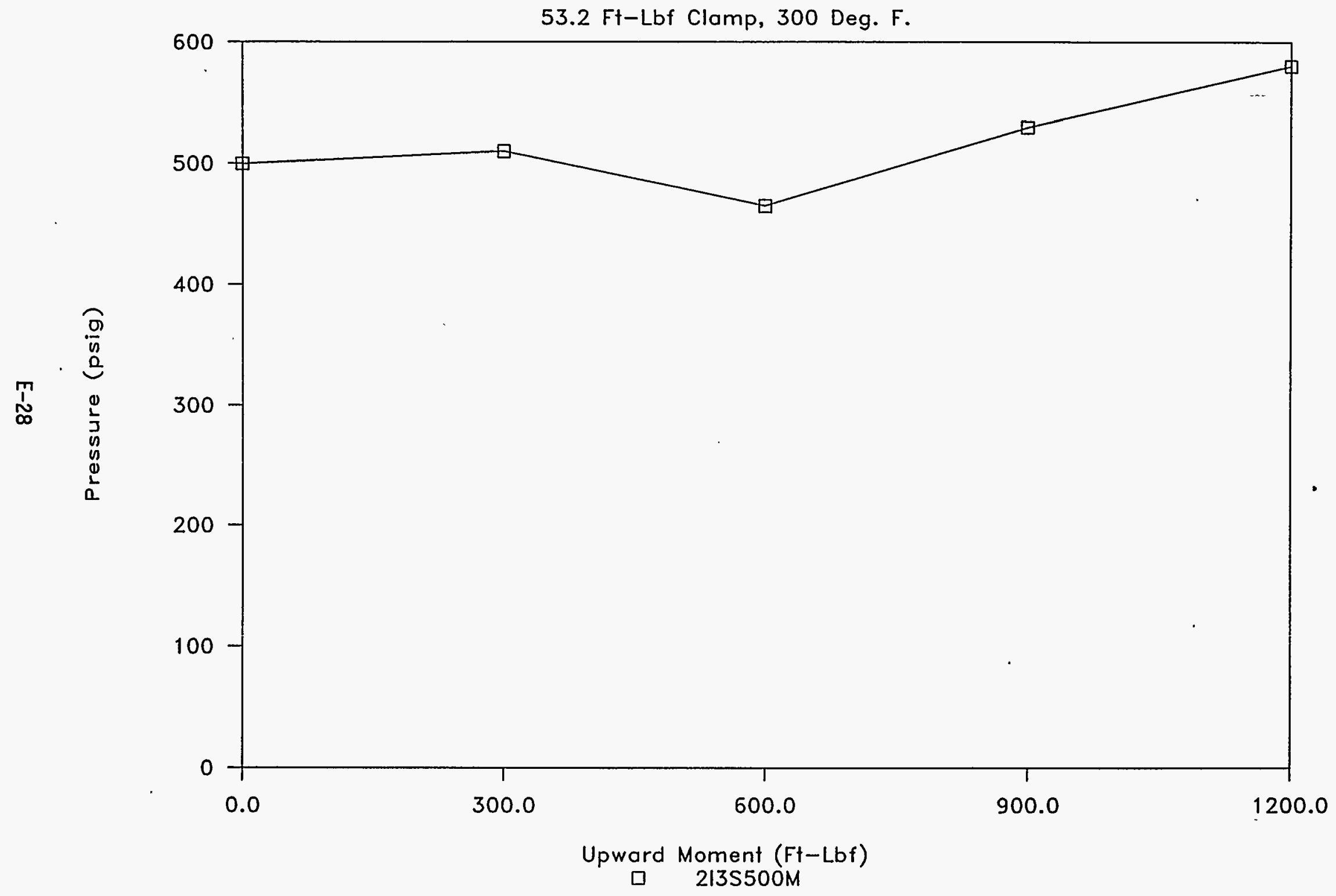


WHC-SD-WM-TRP-223

Rev. 0

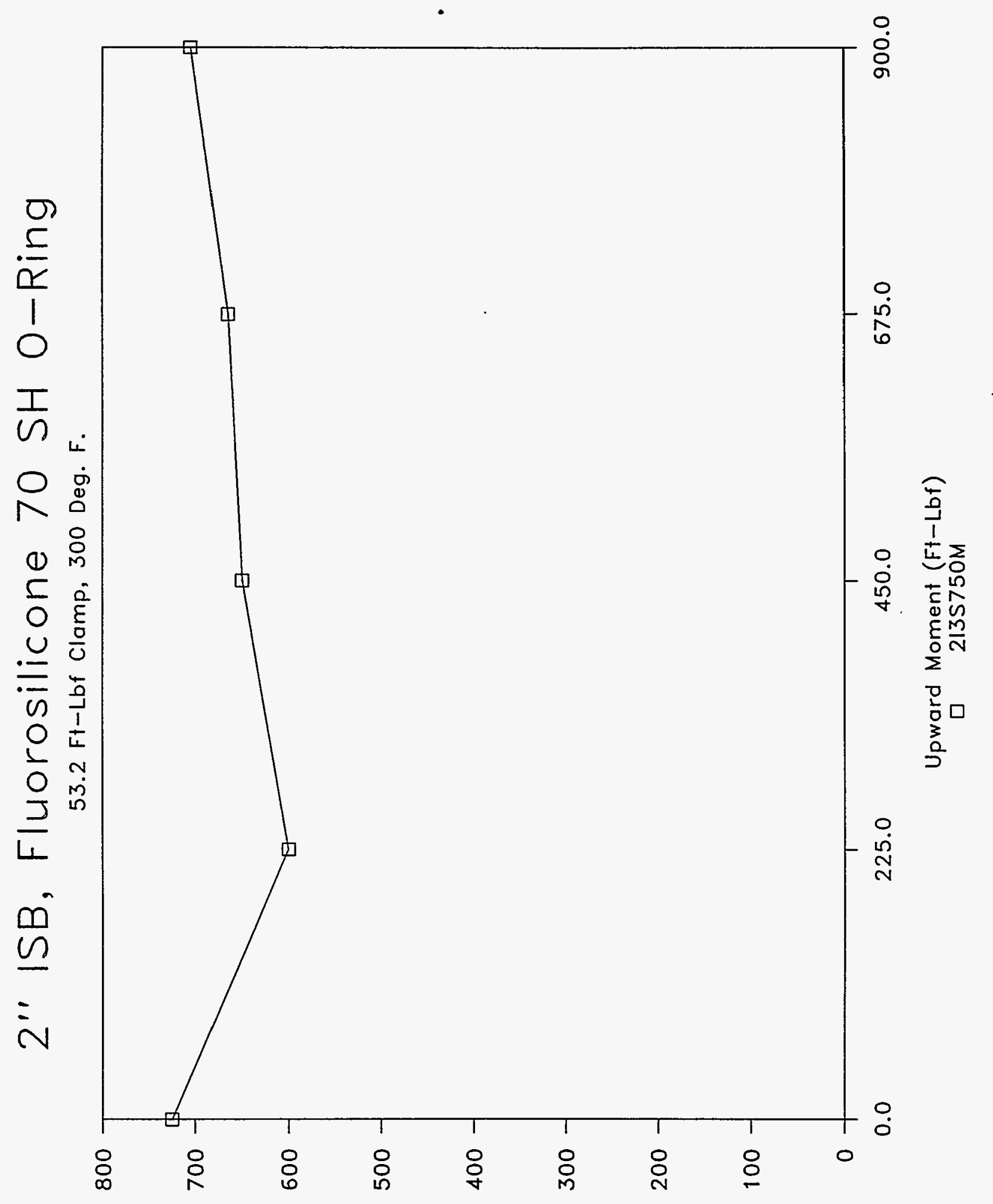

(bisd) adnssadd 


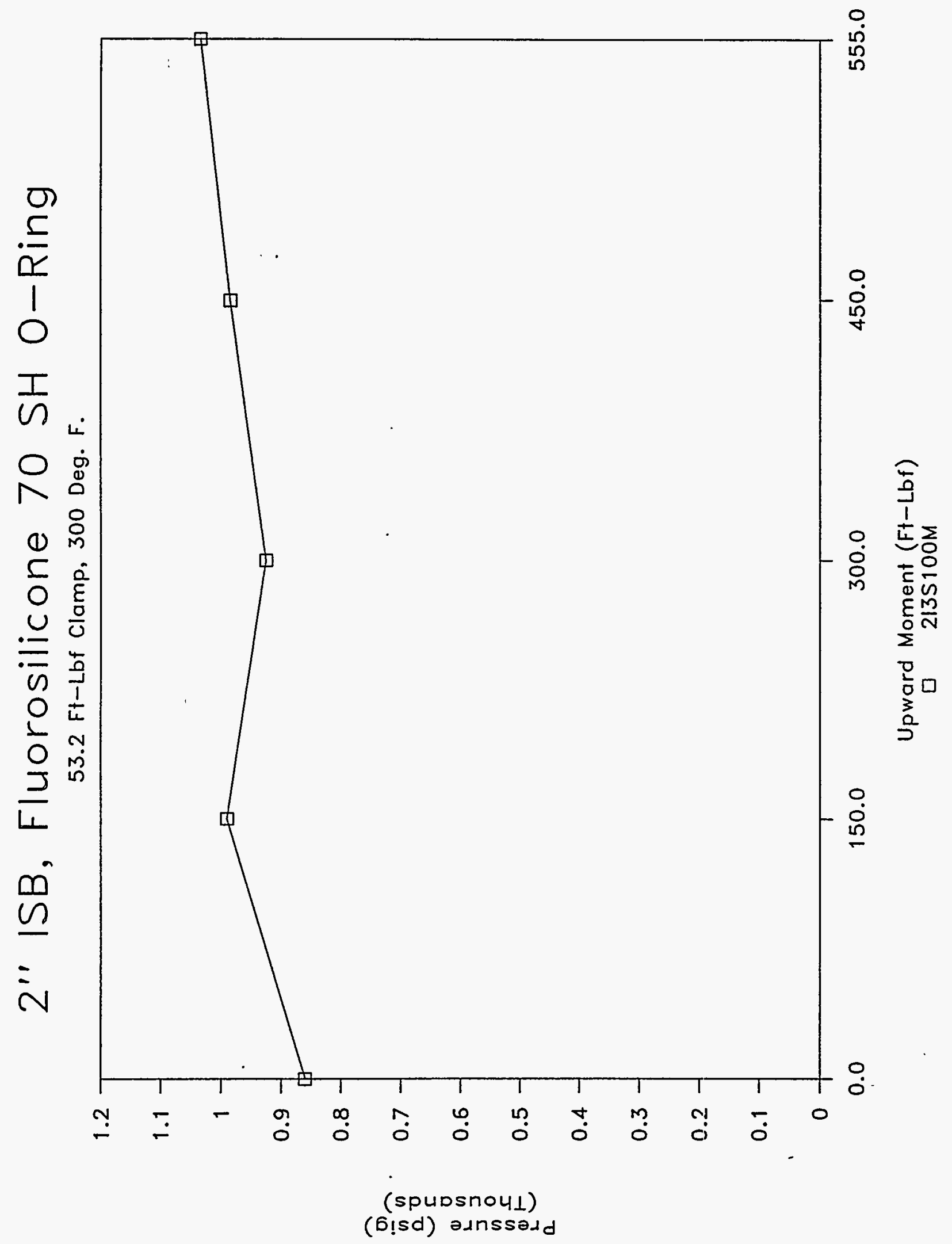


NOVEMBER 14,1994

2" ISB CONNECTOR, FLUOROSILICONE O-RING, 70 DUROMETER, ELEVATED TEMP. PART \# AS568-142, CONQUEST SEAL CO., BATCH PMAF3, CURE DATE 4.93 IEAK TEST - UP AND OVER MOMENT (UPWARD ROTATION)

CLAMPING TORQUE $=53.2$ FT-LBF

CHARGE PRESSURE $=250$ PSIG
GRAPH NAME $=2$ I3S250M

TEMPERATURE: 300 DEG. F.

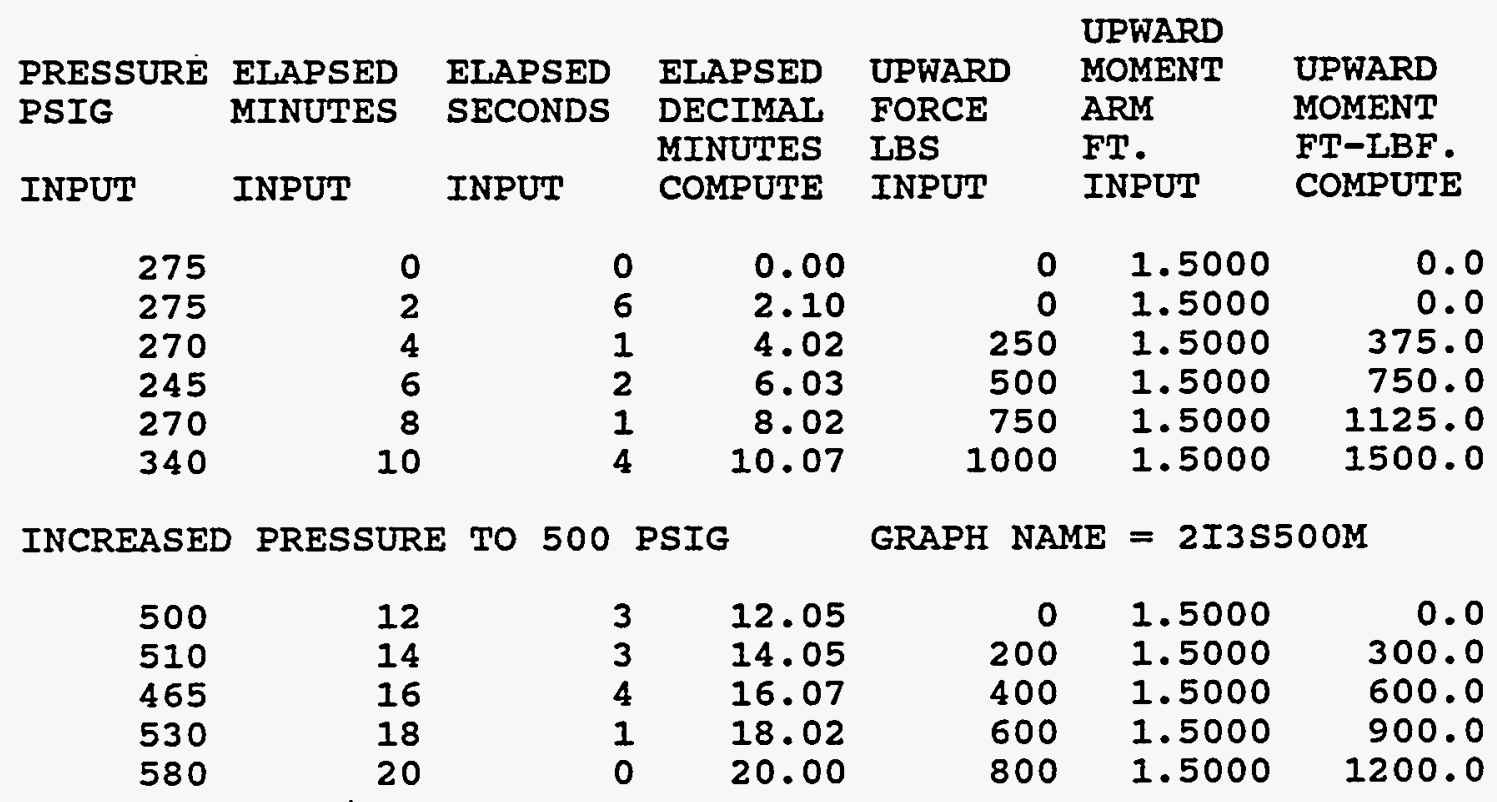

INCREASED PRESSURE TO "750" PSIG

$\begin{array}{ll}725 & 22 \\ 600 & 24 \\ 650 & 26 \\ 665 & 28 \\ 705 & 30\end{array}$

$\begin{array}{ll}6 & 22.10 \\ 2 & 24.03 \\ 1 & 26.02 \\ 1 & 28.02 \\ 3 & 30.05\end{array}$

INCREASED PRESSURE TO 1000 PSIG

$\begin{array}{rr}860 & 32 \\ 990 & 34 \\ 925 & 36 \\ 985 & 38 \\ 1035 & 40\end{array}$

$1 \quad 32.02$

$2 \quad 34.03$

$1 \quad 36.02$

$4 \quad 38.07$

$2 \quad 40.03$
GRAPH NAME $=2$ I3S750M

$\begin{array}{rrr}0 & 1.5000 & 0.0 \\ 150 & 1.5000 & 225.0 \\ 300 & 1.5000 & 450.0 \\ 450 & 1.5000 & 675.0 \\ 600 & 1.5000 & 900.0\end{array}$

GRAPH NAME $=2$ I3S10OM

$\begin{array}{rrr}0 & 1.5000 & 0.0 \\ 100 & 1.5000 & 150.0 \\ 200 & 1.5000 & 300.0 \\ 300 & 1.5000 & 450.0 \\ 370 & 1.5000 & 555.0\end{array}$




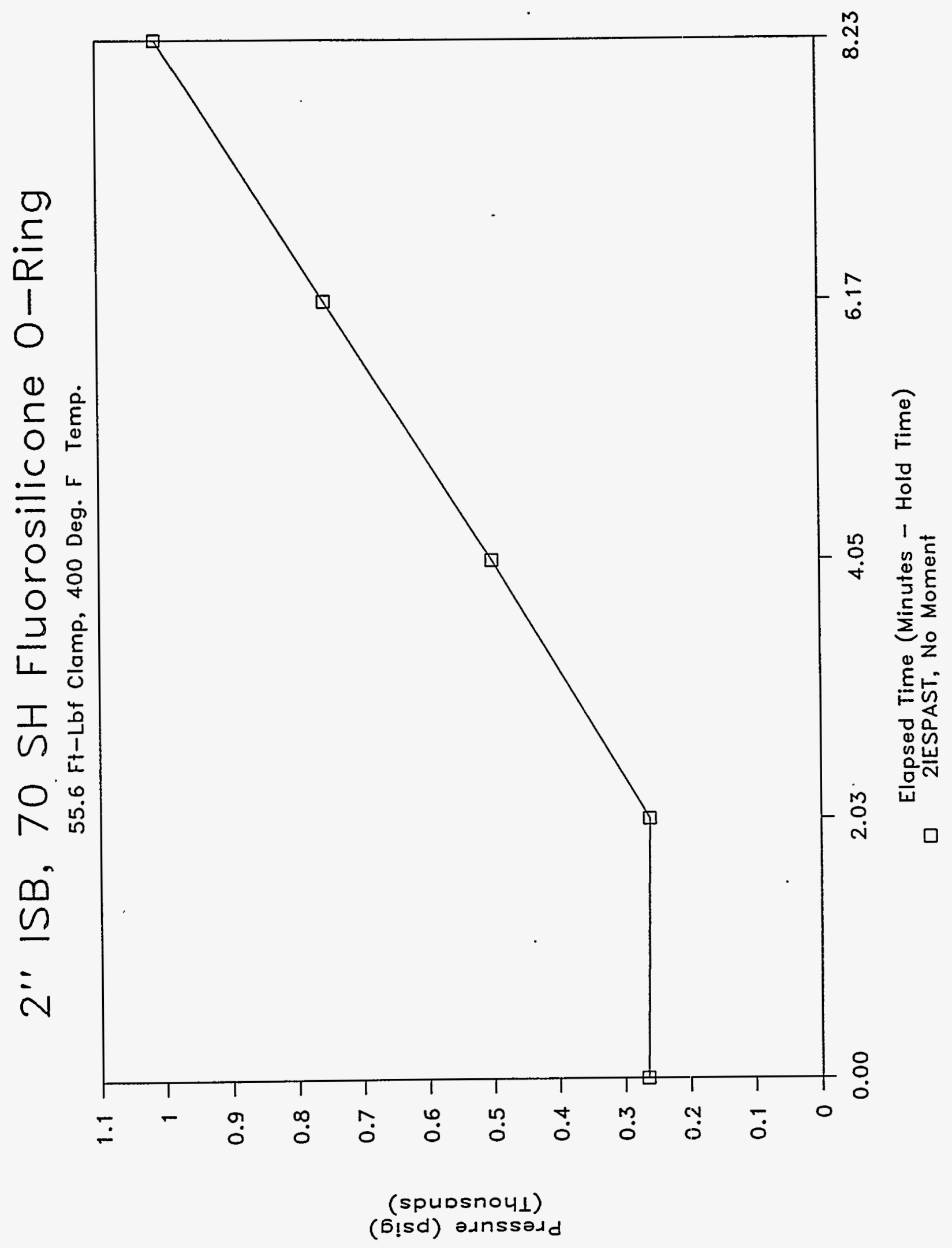


WHC-SD-WM-TRP-223 ReV 0

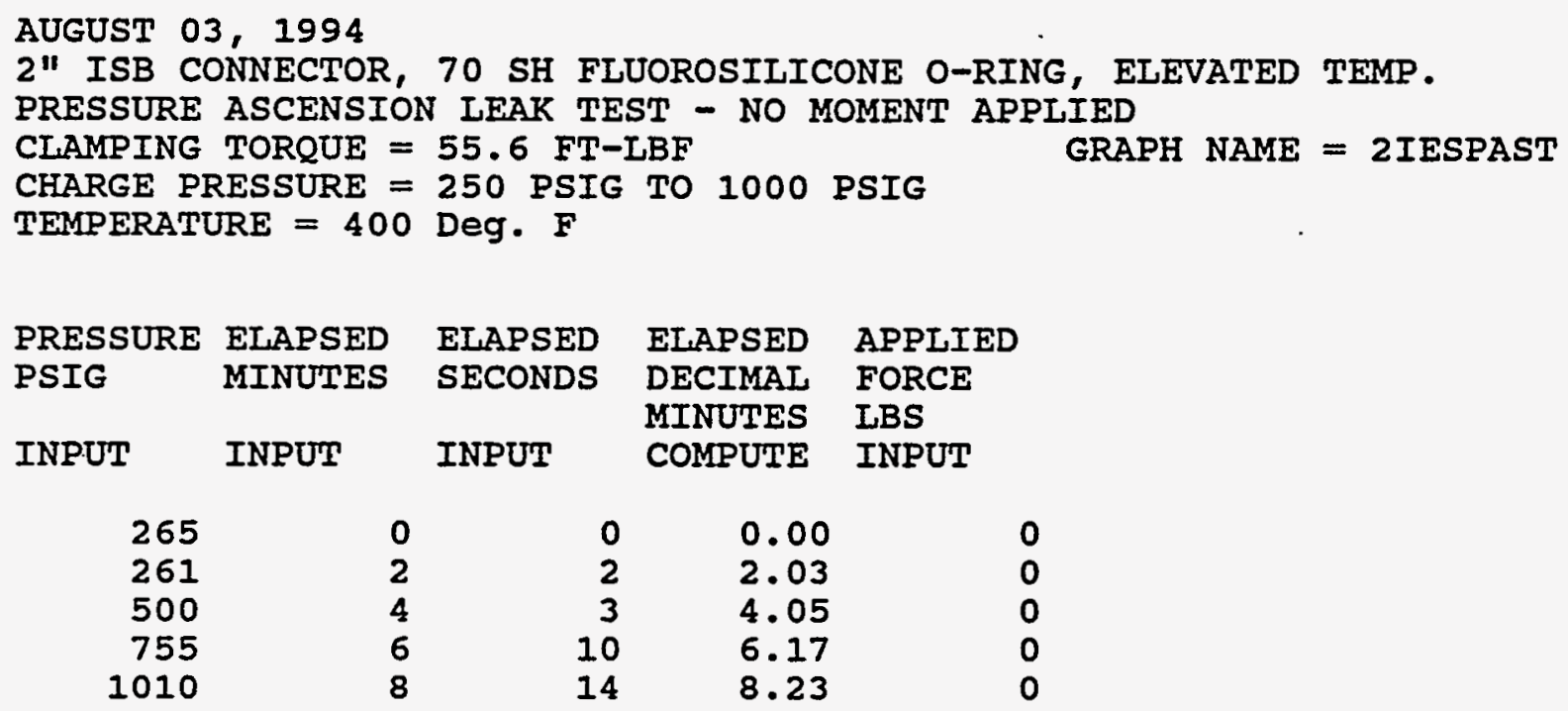


WHC-SD-WM-TRP-223

Rev. 0

APPENDIX F: GRAPHS OF 2-IN. KALREZ TESTS 


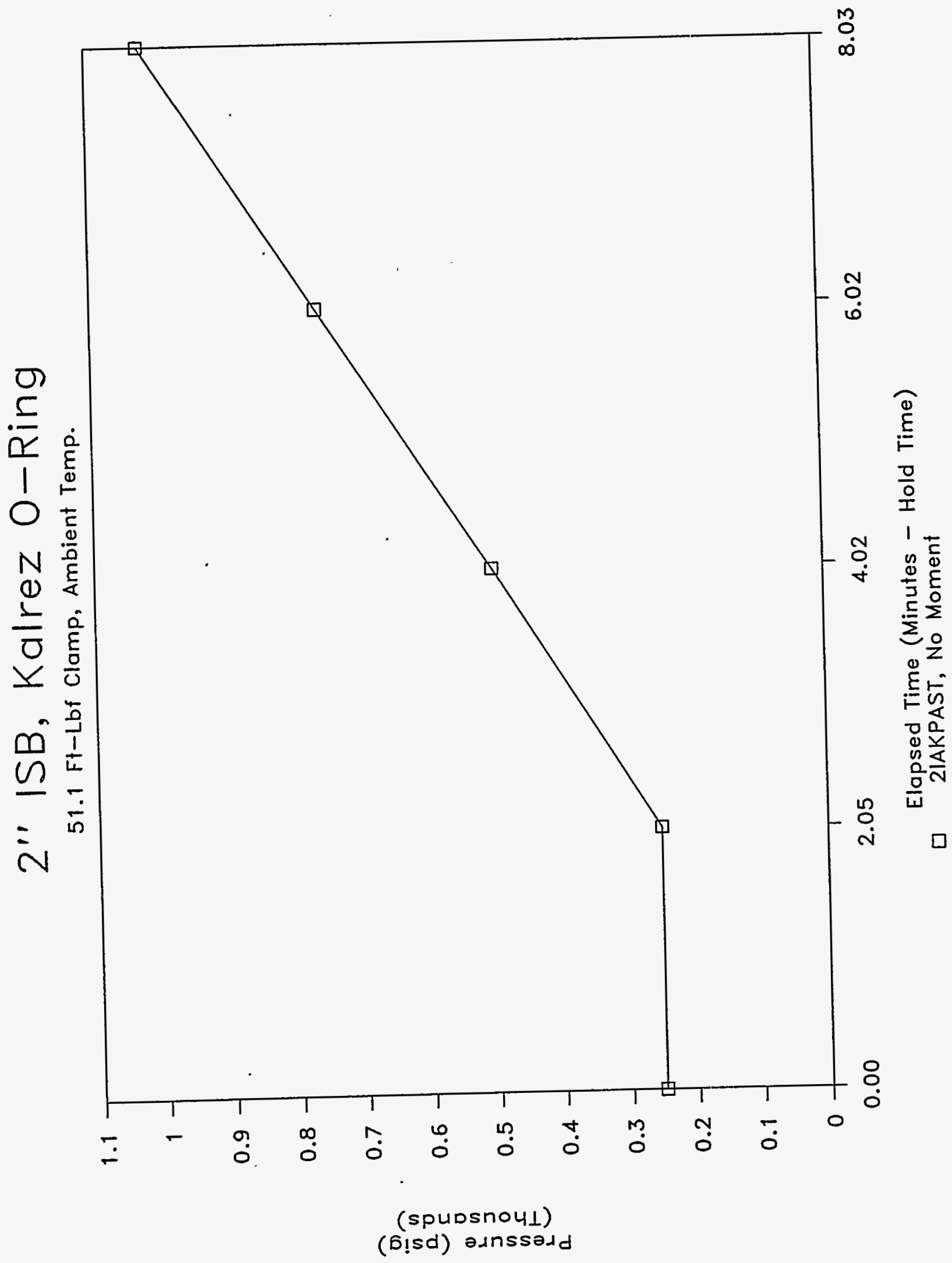


WHC-SD-WM-TRP-223 Rev 0

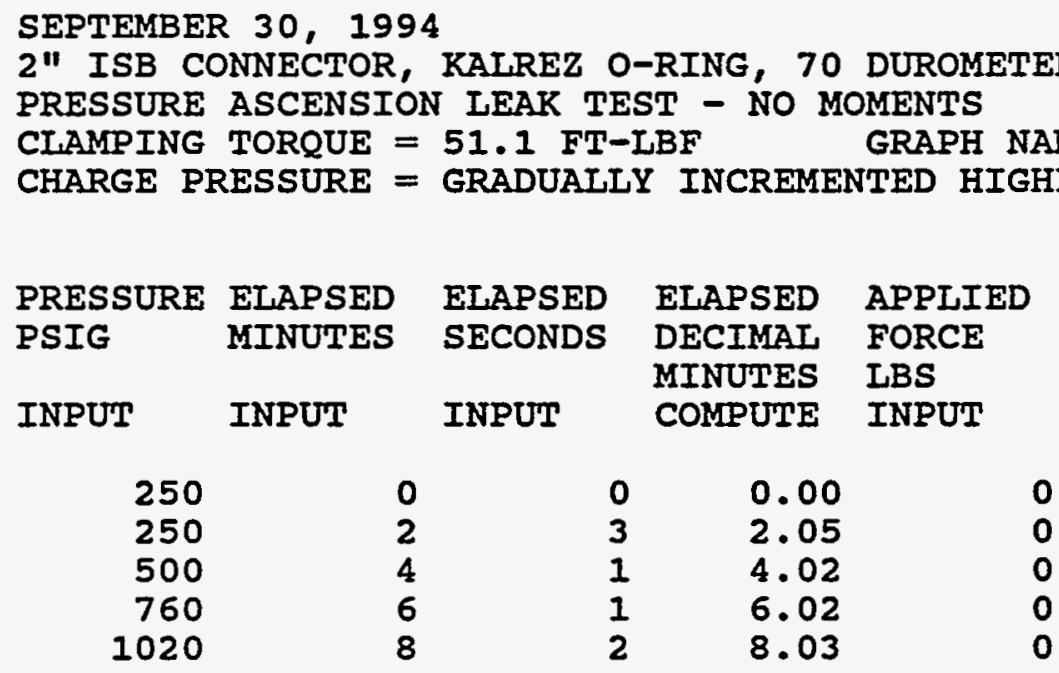


WHC-SD-WM-TRP-223

Rev. 0

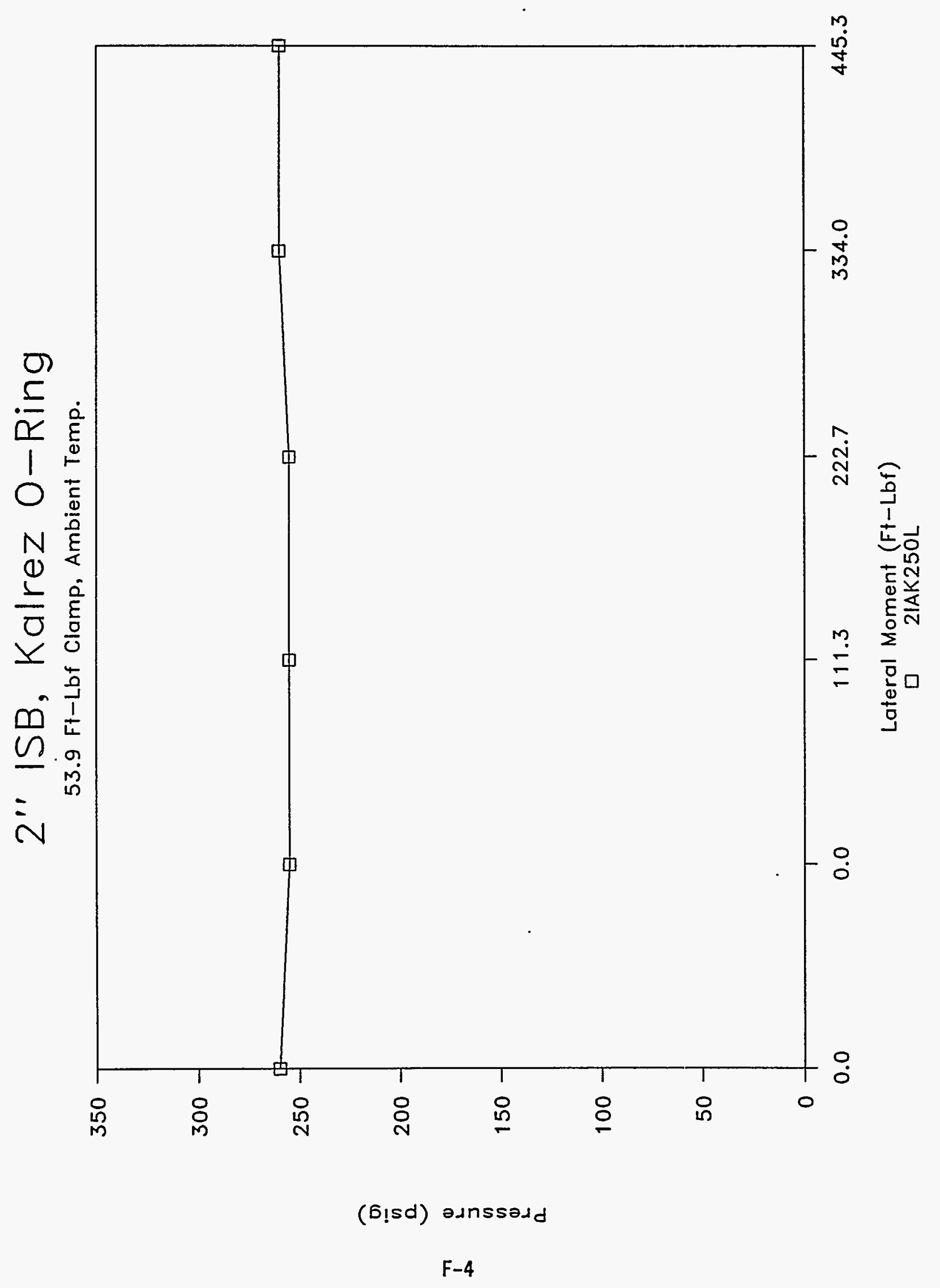




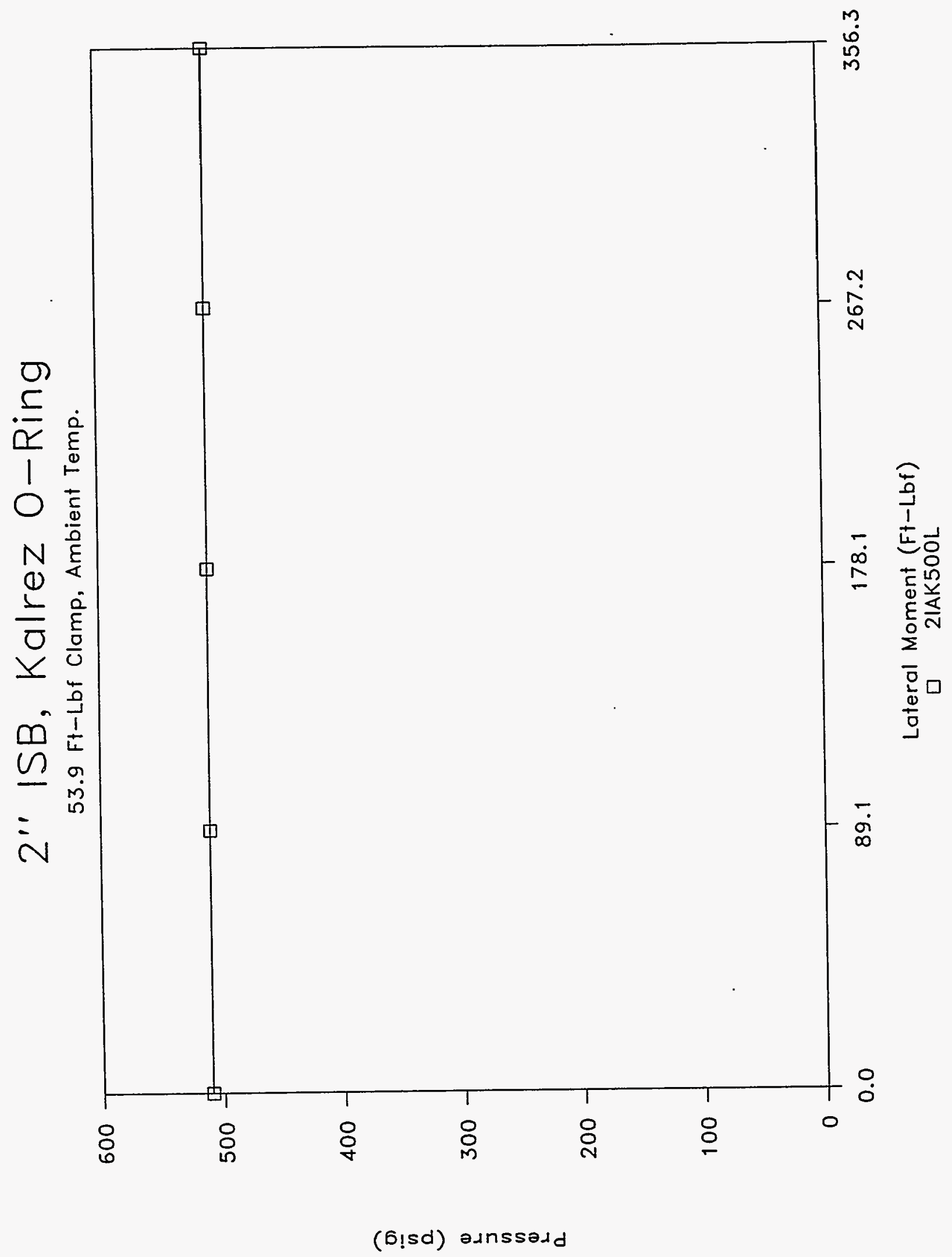

F-5 


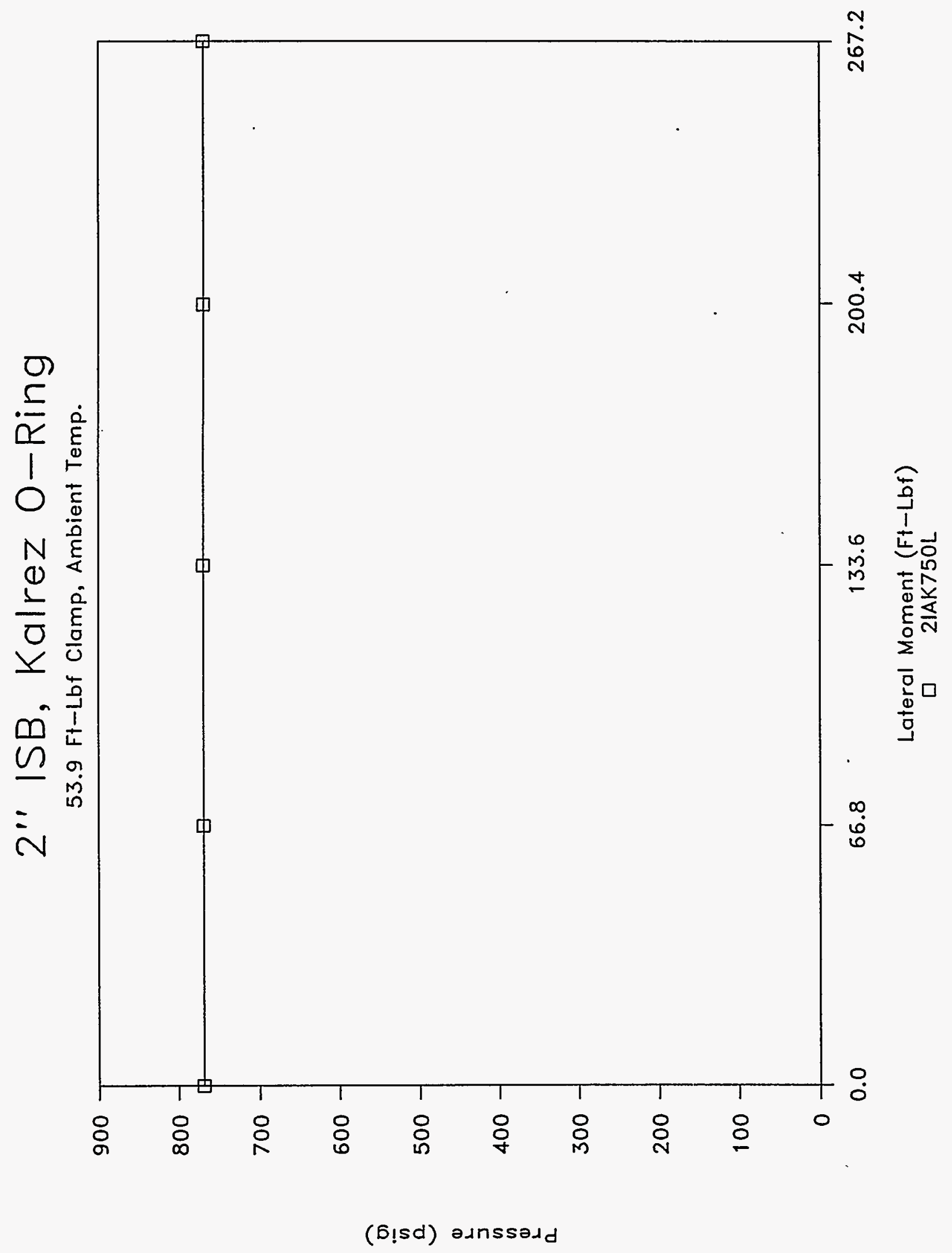


WHC-SD-WM-TRP-223

Rev. 0

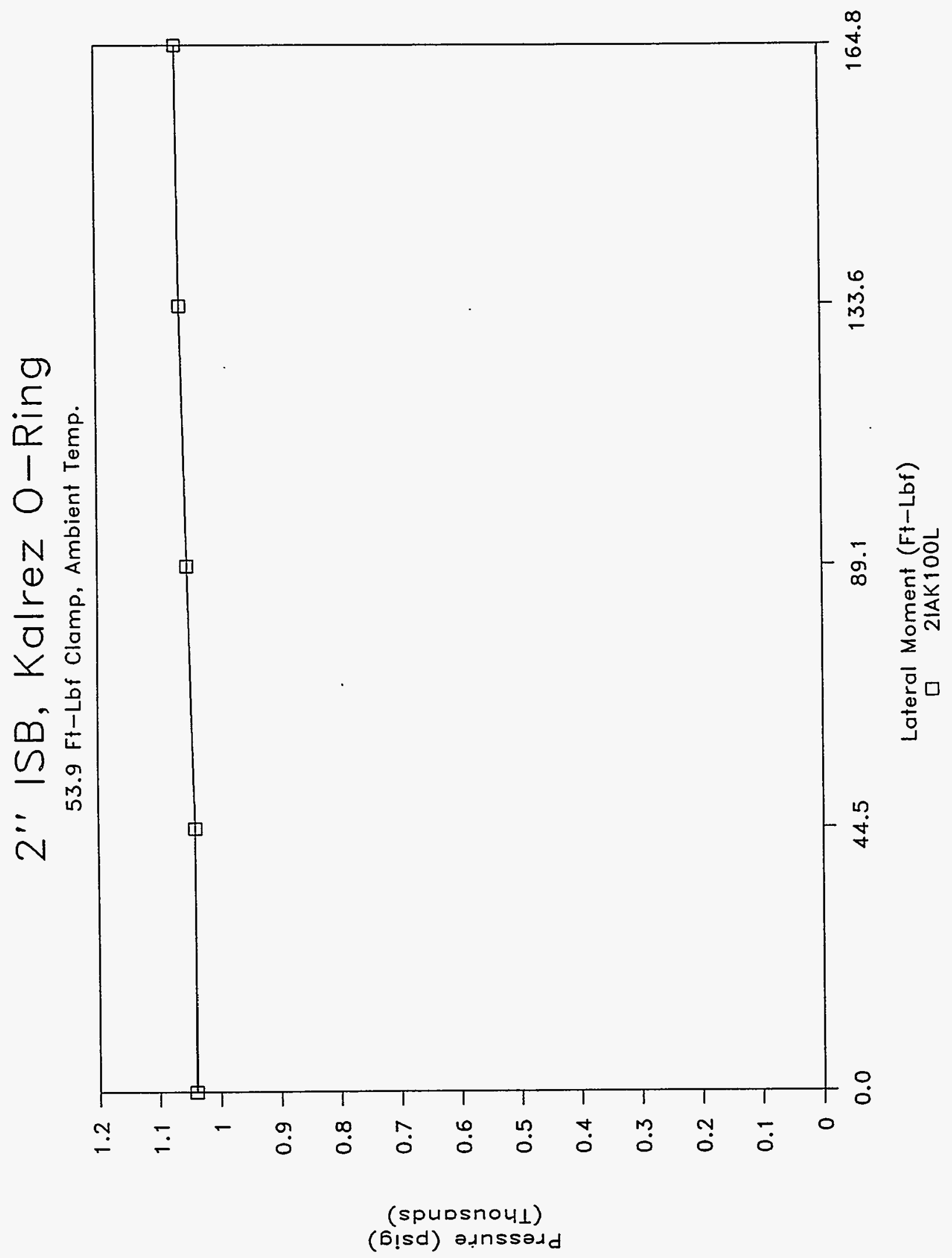

F-7 
OCTOBER 03, 1994

2" ISB CONNECTOR, KALREZ O-RING, 70 DUROMETER, AMBIENT TEMP. PRESSURE ASCENSION IEAK TEST - IATERAL MOMENT (SIDEWAYS ROTATION) CLAMPING TORQUE $=53.9$ FT-LBF CHARGE PRESSURE $=250$ PSIG

$\begin{array}{lllllll} & & & & & \text { LATEIRA } \\ \text { PRESSURE } & \text { ELAPSED } & \text { ELAPSED } & \text { ELAPSED } & \text { LATERAL } & \text { MOMENT } & \text { LATERAI } \\ \text { PSIG } & \text { MINUTES } & \text { SECONDS } & \text { DECIMAL } & \text { FORCE } & \text { ARM } & \text { MOMENT } \\ & & & \text { MINUTES } & \text { LBS } & \text { FT. } & \text { FT-LBF. } \\ \text { INPUT } & \text { INPUT } & \text { INPUT } & \text { COMPUTE } & \text { INPUT } & \text { INPUT } & \text { COMPUTE }\end{array}$

$\begin{array}{rrrrrrr}260 & 0 & 0 & 0.00 & 0 & 0.4453 & 0.0 \\ 255 & 2 & 1 & 2.02 & 0 & 0.4453 & 0.0 \\ 255 & 4 & 2 & 4.03 & 250 & 0.4453 & 111.3 \\ 255 & 6 & 5 & 6.08 & 500 & 0.4453 & 222.7 \\ 260 & 8 & 2 & 8.03 & 750 & 0.4453 & 334.0 \\ 260 & 10 & 7 & 10.12 & 1000 & 0.4453 & 445.3\end{array}$

INCREASED PRESSURE TO 500 PSIG

GRAPH NAME $=2$ IAK50OL

$\begin{array}{rrrrrrr}510 & 12 & 1 & 12.02 & 0 & 0.4453 & 0.0 \\ 510 & 14 & 2 & 14.03 & 200 & 0.4453 & 89.1 \\ 510 & 16 & 1 & 16.02 & 400 & 0.4453 & 178.1 \\ 510 & 18 & 3 & 18.05 & 600 & 0.4453 & 267.2 \\ 510 & 20 & 1 & 20.02 & 800 & 0.4453 & 356.3\end{array}$

INCREASED PRESSURE TO 750 PSIG

$\begin{array}{llll}770 & 22 & 1 & 22.02 \\ 770 & 24 & 1 & 24.02 \\ 770 & 26 & 1 & 26.02 \\ 770 & 28 & 0 & 28.00 \\ 770 & 30 & 1 & 30.02\end{array}$

INCREASED PRESSURE TO 1000 PSIG

$\begin{array}{ll}1040 & 32 \\ 1040 & 34 \\ 1052 & 36 \\ 1061 & 38 \\ 1066 & 40\end{array}$

GRAPH NAME = :IIAK750I

$\begin{array}{rrr}0 & 0.4453 & 0.0 \\ 150 & 0.4453 & 66.8 \\ 300 & 0.4453 & 133.6 \\ 450 & 0.4453 & 200.4 \\ 600 & 0.4453 & 267.2\end{array}$

GRAPH NAME $=2$ IAKI0OL

$\begin{array}{rrr}0 & 0.4453 & 0.0 \\ 100 & 0.4453 & 44.5 \\ 200 & 0.4453 & 89.1 \\ 300 & 0.4453 & 133.6 \\ 370 & 0.4453 & 164.8\end{array}$


WHC-SD-WM-TRP-223

Rev. 0

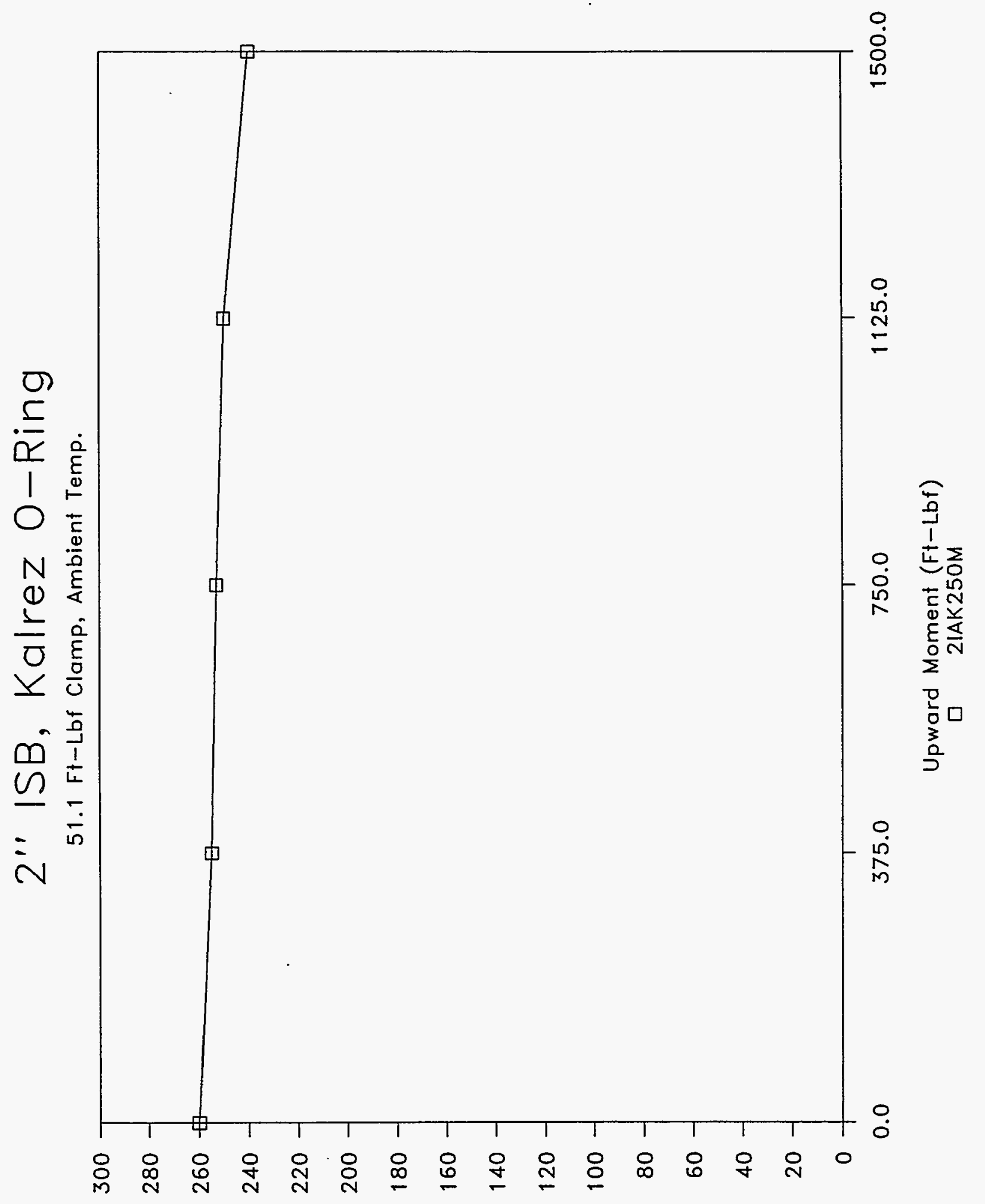

(6!sd) oגnssedd 
WHC-SD-WM-TRP-223

Rev. 0

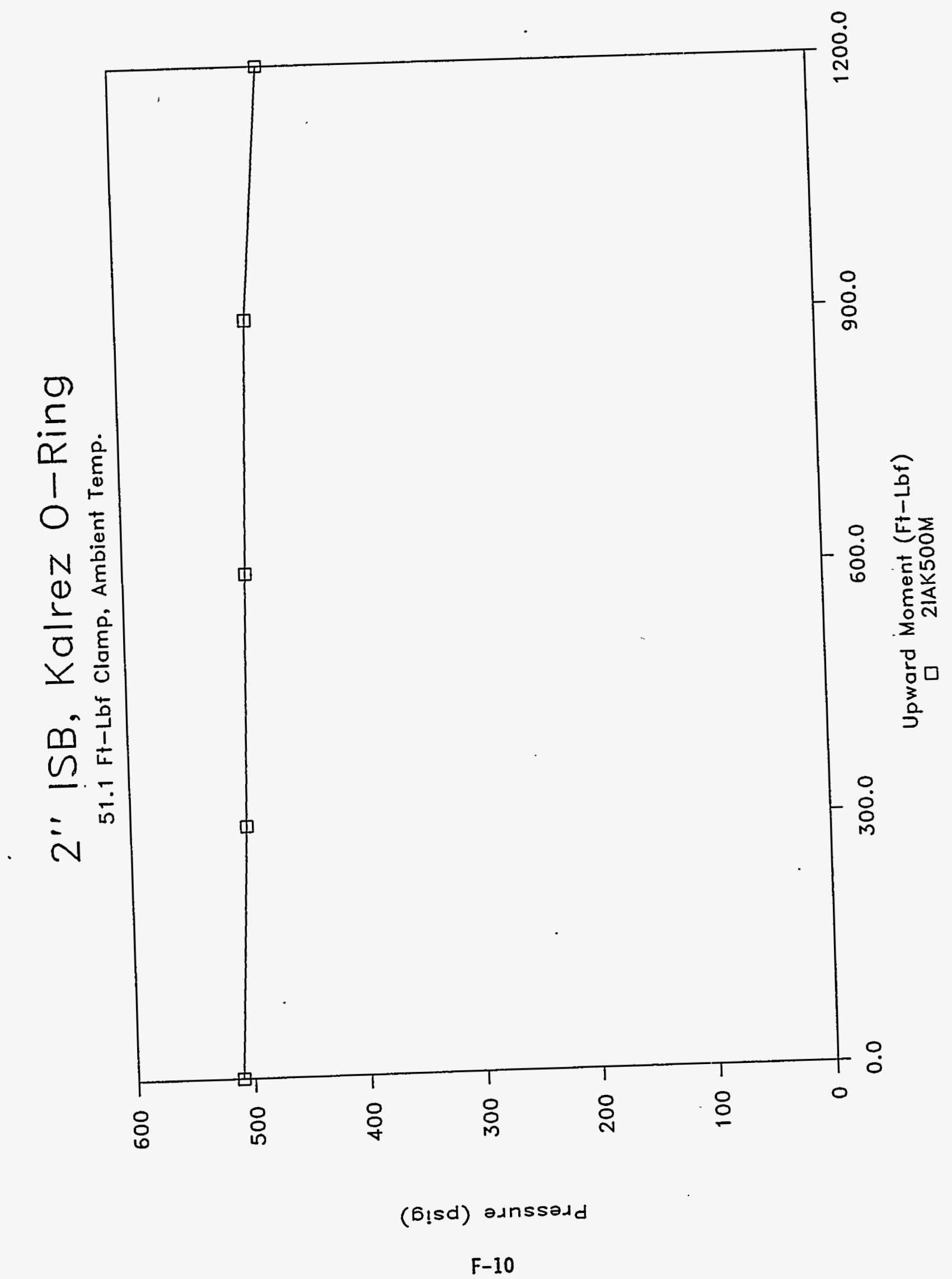




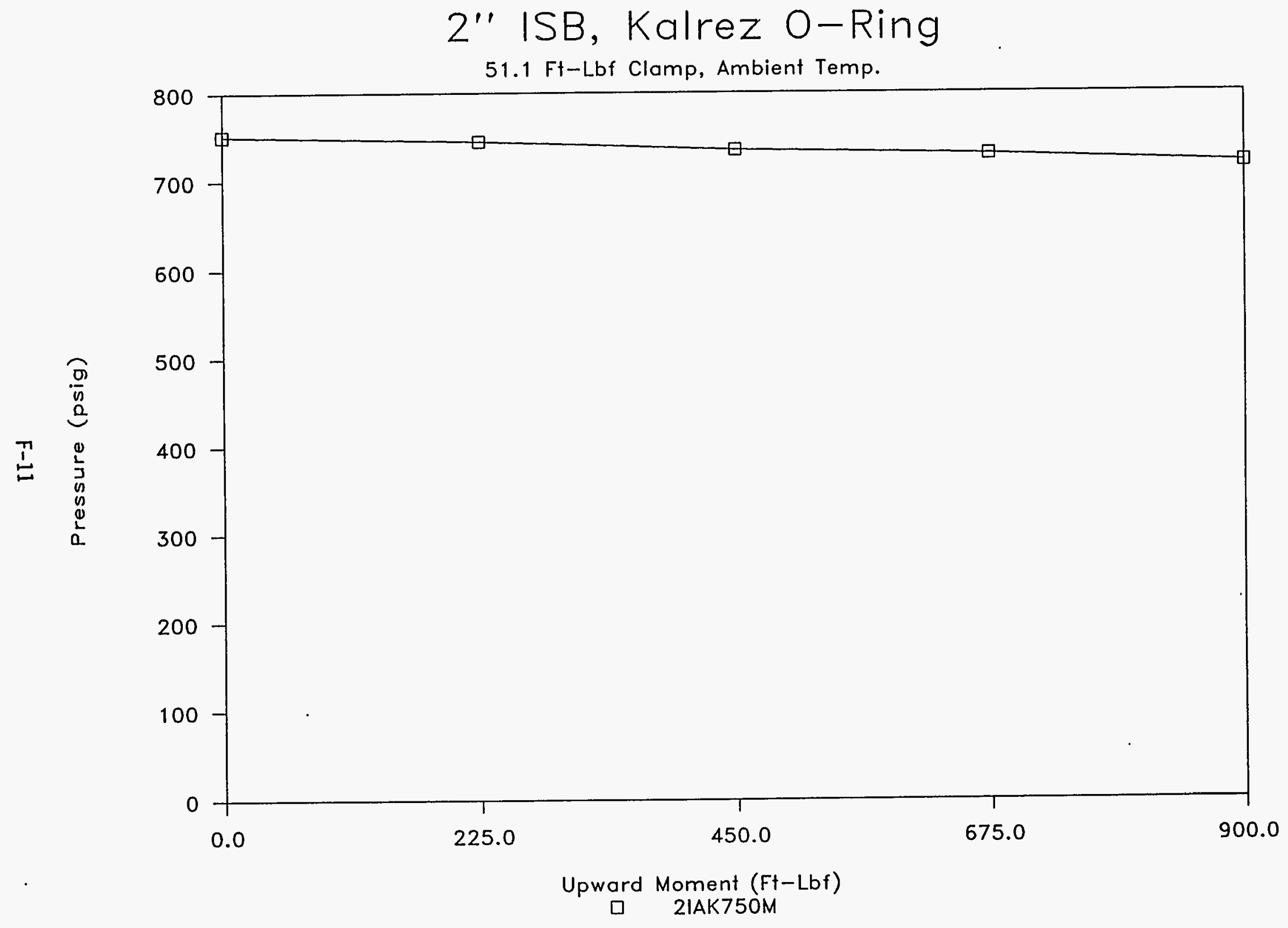


Rev. 0

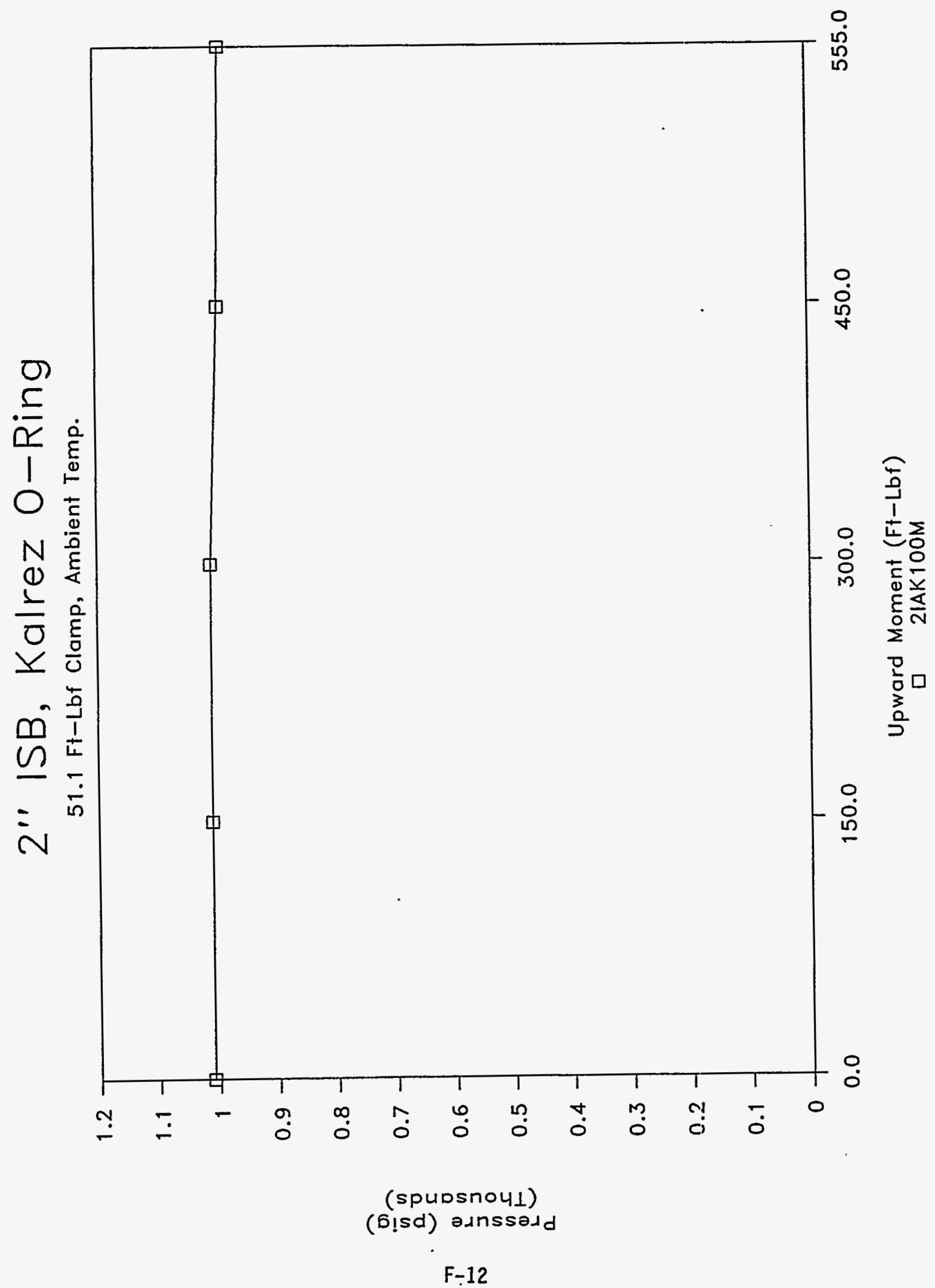


OCTOBER 03, 1994

2" ISB CONNECTOR, KALREZ O-RING, 70 DUROMETER, AMBIENT TEMP. LEAK TEST - UP AND OVER MOMENT (UPWARD ROTATION)

CLAMPING TORQUE $=52.7 \mathrm{FT}-\mathrm{IBF} \quad$ GRAPH NAME $=2$ IAK250M

CHARGE PRESSURE $=250$ PSIG

\begin{tabular}{|c|c|c|c|c|c|c|}
\hline $\begin{array}{l}\text { PRESSURE } \\
\text { PSIG }\end{array}$ & $\begin{array}{l}\text { ELAPSED } \\
\text { MINUTES }\end{array}$ & $\begin{array}{l}\text { ELAPSED } \\
\text { SECONDS }\end{array}$ & $\begin{array}{l}\text { ELAPSED } \\
\text { DECIMAI } \\
\text { MINUTES } \\
\text { COMPUTE }\end{array}$ & $\begin{array}{l}\text { UPWARD } \\
\text { FORCE } \\
\text { LBS } \\
\text { INPUT }\end{array}$ & $\begin{array}{l}\text { UPWARD } \\
\text { MOMENT } \\
\text { ARM } \\
\text { FT. } \\
\text { INPUT }\end{array}$ & $\begin{array}{l}\text { UPWARD } \\
\text { MOMENT } \\
\text { FT-LBF. } \\
\text { COMPUTE }\end{array}$ \\
\hline $\begin{array}{l}260 \\
260 \\
255 \\
253 \\
250 \\
240\end{array}$ & $\begin{array}{r}0 \\
2 \\
4 \\
6 \\
8 \\
10\end{array}$ & $\begin{array}{l}0 \\
0\end{array}$ & $\begin{array}{r}0.00 \\
2.00 \\
4.05 \\
6.03 \\
8.02 \\
10.02\end{array}$ & $\begin{array}{r}0 \\
0 \\
250 \\
500 \\
750 \\
1000\end{array}$ & $\begin{array}{l}1.5000 \\
1.5000 \\
1.5000 \\
1.5000 \\
1.5000 \\
1.5000\end{array}$ & $\begin{array}{r}0.0 \\
0.0 \\
375.0 \\
750.0 \\
1125.0 \\
1500.0\end{array}$ \\
\hline
\end{tabular}

INCREASED PRESSURE TO 500 PSIG

GRAPH NAME $=2$ IAK50OM

$\begin{array}{rrrrrrr}510 & 12 & 1 & 12.02 & 0 & 1.5000 & 0.0 \\ 501 & 14 & 2 & 14.03 & 200 & 1.5000 & 300.0 \\ 495 & 16 & 2 & 16.03 & 400 & 1.5000 & 600.0 \\ 489 & 18 & 1 & 18.02 & 600 & 1.5000 & 900.0 \\ 473 & 20 & 1 & 20.02 & 800 & 1.5000 & 1200.0\end{array}$

INCREASED PRESSURE TO 750 PSIG

GRAPH NAME $=2$ IAK750M

$\begin{array}{llrl}751 & 22 & 17 & 22.28 \\ 745 & 24 & 1 & 24.02 \\ 735 & 26 & 2 & 26.03 \\ 730 & 28 & 2 & 28.03 \\ 720 & 30 & 2 & 30.03\end{array}$

$\begin{array}{rrr}0 & 1.5000 & 0.0 \\ 150 & 1.5000 & 225.0 \\ 300 & 1.5000 & 450.0 \\ 450 & 1.5000 & 675.0 \\ 600 & 1.5000 & 900.0\end{array}$

INCREASED PRESSURE TO 1000 PSIG

GRAPH NAME $=2$ IAK $100 \mathrm{M}$

$\begin{array}{rrrrrrr}1010 & 32 & 2 & 32.03 & 0 & 1.5000 & 0.0 \\ 1010 & 34 & 1 & 34.02 & 100 & 1.5000 & 150.0 \\ 1010 & 36 & 3 & 36.05 & 200 & 1.5000 & 300.0 \\ 996 & 38 & 1 & 38.02 & 300 & 1.5000 & 450.0 \\ 990 & 40 & 3 & 40.05 & 370 & 1.5000 & 555.0\end{array}$


Rev. 0

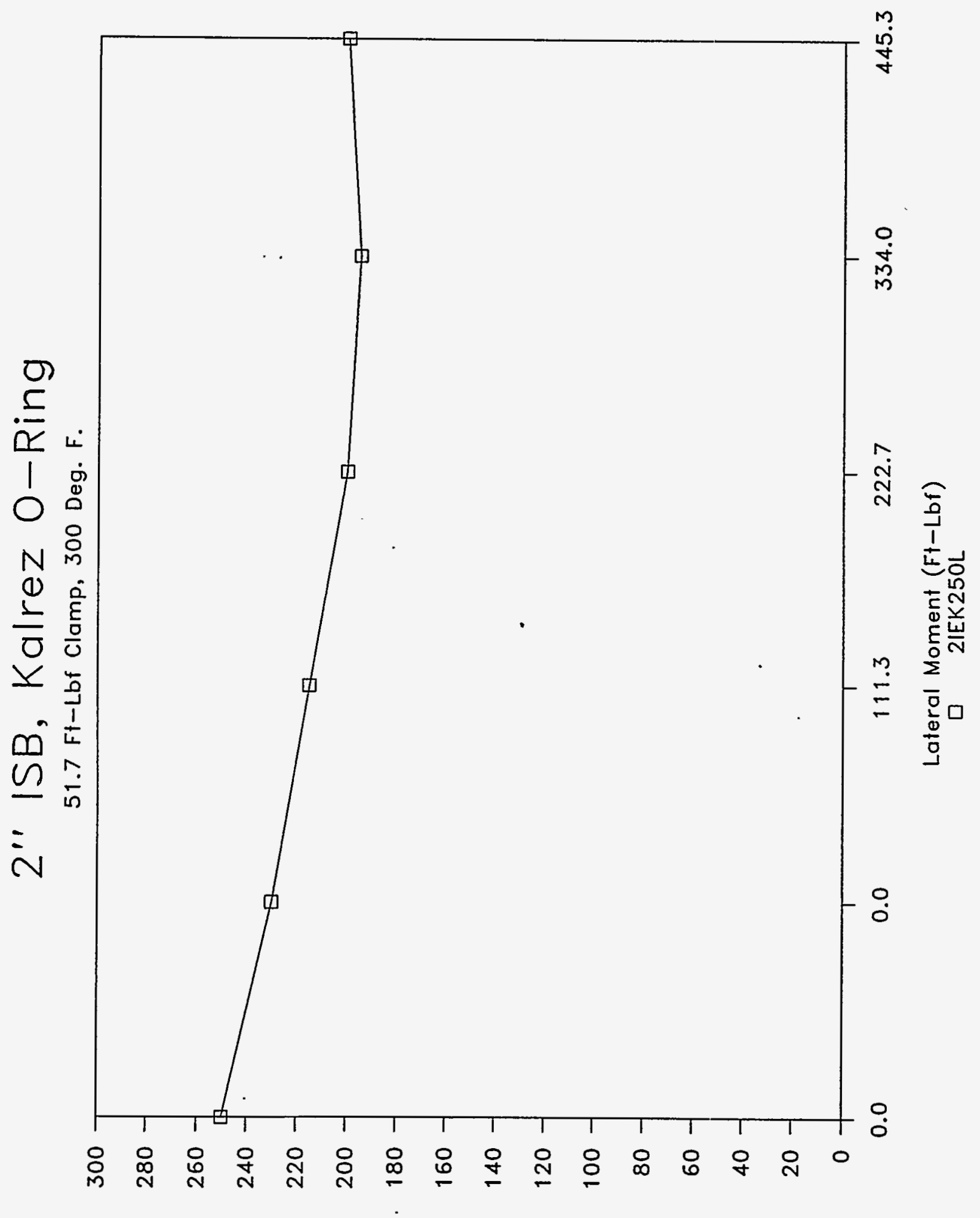

(6!sd) asnssadd 
Rev. 0

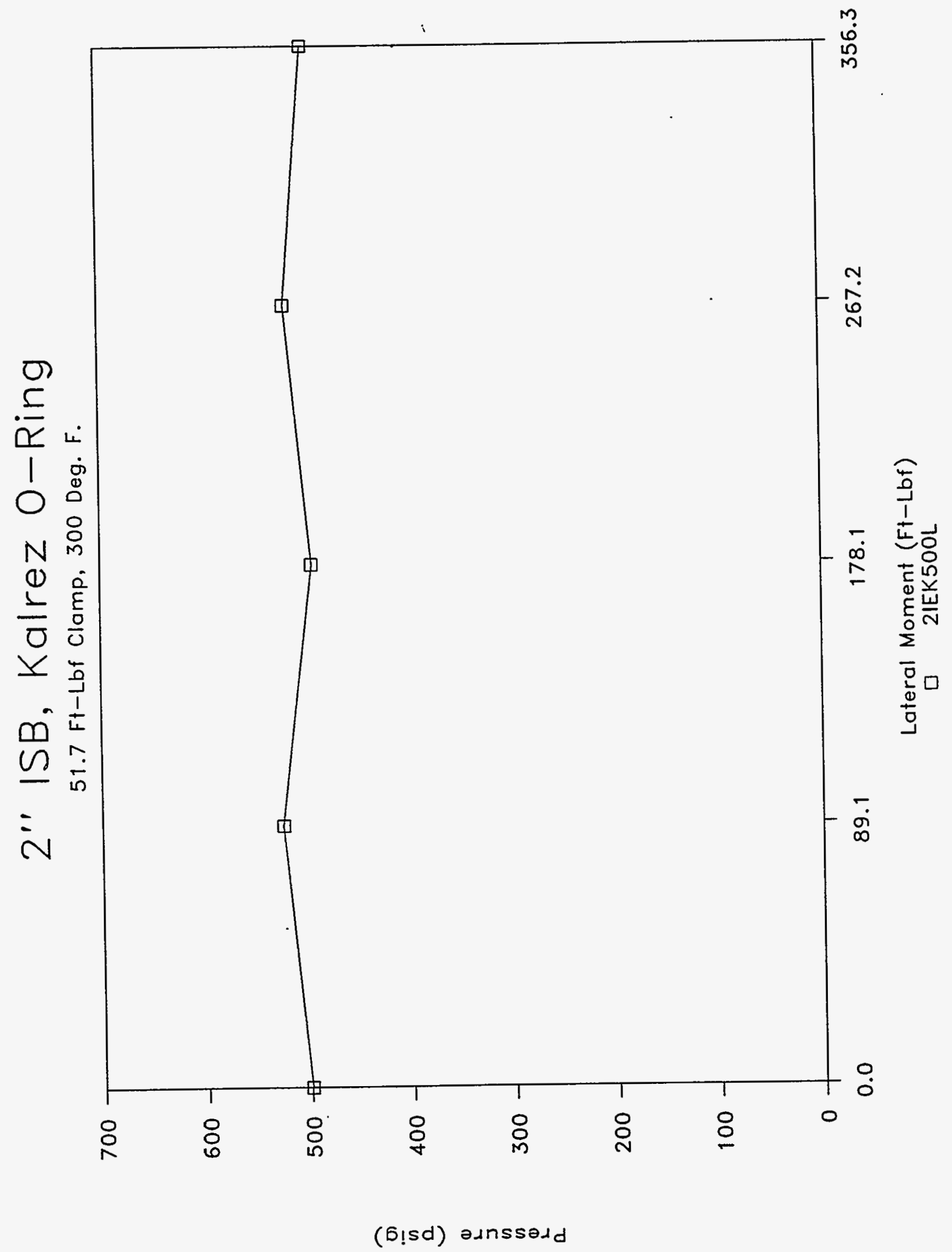

F-15 
WHC-SD-WM-TRP-223

Rev. 0

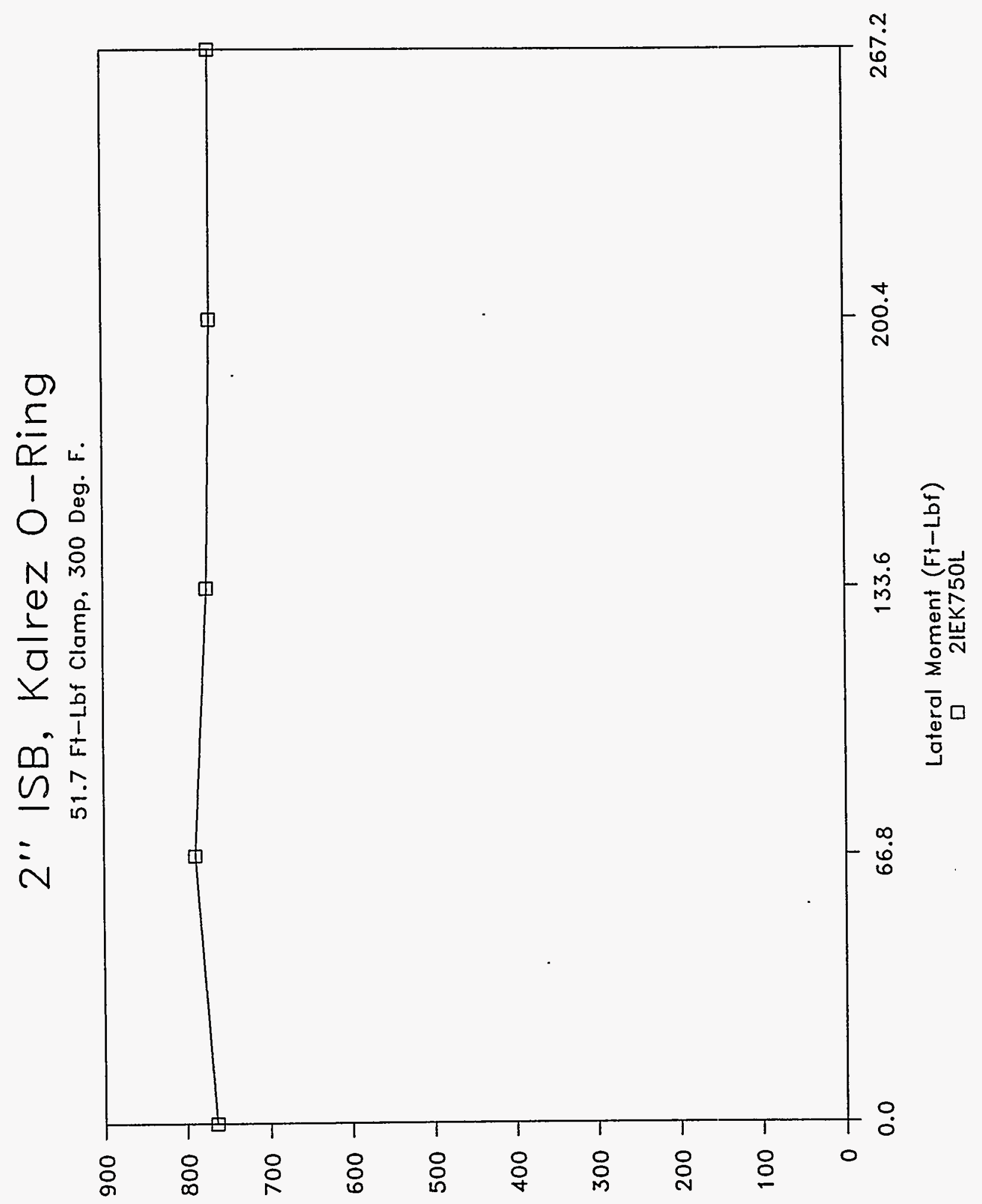

(b!sd) adnssadd

F-16 
WHC-SD-WM-TRP-223

Rev. 0

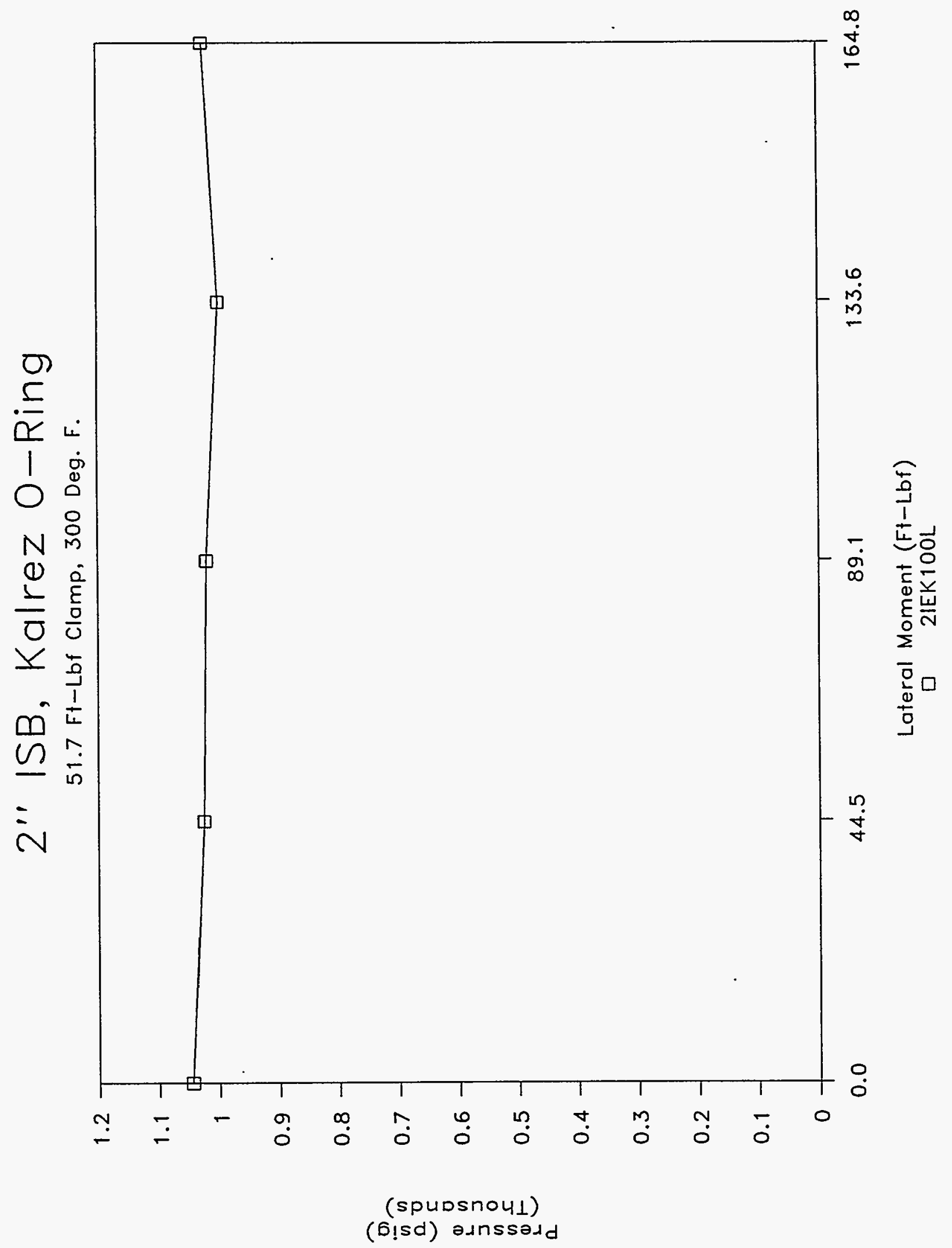

$\mathrm{F}-17$ 
OCTOBER 05, 1994

2" ISB CONNECTOR, KALREZ O-RING, 70 DUROMETER, ELEVATED TEMP. LEAK TEST - LATERAL MOMENT (SIDEWAYS ROTATION)

CLAMPING TORQUE $=51.7$ FT-LBF

CHARGE PRESSURE $=250$ PSIG

GRAPH NAME $=2$ IEK250I

TEMPERATURE: 300 DEG. F.

NOTE: THIS TEST WAS PERFORMED AT 300 DEG. F. INSTEAD OF 400 DEG. F. PREVIOUS TESTS WITH KALREZ SHOWED THAT THE O-RING SUSTAINED

DEFORMATION DAMAGE AND LEAKED AT 400 DEG. F. and 1,000 PSIG.

$\begin{array}{lllllll} & & & & & \text { LATERAL } \\ \text { PRESSURE } & \text { ELAPSED } & \text { ELAPSED } & \text { ELAPSED } & \text { LATERAI } & \text { MOMENT } & \text { LATERAL } \\ \text { PSIG } & \text { MINUTES } & \text { SECONDS } & \text { DECIMAL } & \text { FORCE } & \text { ARM } & \text { MOMENT } \\ & & & \text { MINUTES } & \text { IBS } & \text { FT. } & \text { FT-IBF. } \\ \text { INPUT } & \text { INPUT } & \text { INPUT } & \text { COMPUTE } & \text { INPUT } & \text { INPUT } & \text { COMPUTE }\end{array}$

$\begin{array}{rrrrrrr}250 & 0 & 0 & 0.00 & 0 & 0.4453 & 0.0 \\ 230 & 2 & 2 & 2.03 & 0 & 0.4453 & 0.0 \\ 215 & 4 & 1 & 4.02 & 250 & 0.4453 & 111.3 \\ 200 & 6 & 5 & 6.08 & 500 & 0.4453 & 222.7 \\ 195 & 8 & 1 & 8.02 & 750 & 0.4453 & 334.0 \\ 200 & 10 & 2 & 10.03 & 1000 & 0.4453 & 445.3\end{array}$

INCREASED PRESSURE TO 500 PSIG

GRAPH NAME $=2$ IEK500L

$\begin{array}{rrrrrrr}500 & 12 & 1 & 12.02 & 0 & 0.44453 & 0.0 \\ 525 & 14 & 1 & 14.02 & 200 & 0.44453 & 89.1 \\ 495 & 16 & 1 & 16.02 & 400 & 0.4453 & 178.1 \\ 520 & 18 & 2 & 18.03 & 600 & 0.44453 & 267.2 \\ 500 & 20 & 2 & 20.03 & 800 & 0.44453 & 356.3\end{array}$

INCREASED PRESSURE TO 750 PSIG

GRAPH NAME = 2IEK750L

$\begin{array}{rrrrrrr}765 & 22 & 2 & 22.03 & 0 & 0.4453 & 0.0 \\ 790 & 24 & 1 & 24.02 & 150 & 0.4453 & 66.8 \\ 775 & 26 & 3 & 26.05 & 300 & 0.4453 & 133.6 \\ 770 & 28 & 1 & 28.02 & 450 & 0.4453 & 200.4 \\ 770 & 30 & 2 & 30.03 & 600 & 0.4453 & 267.2\end{array}$

INCREASED PRESSURE TO 1000 PSIG

GRAPH NAME $=2$ IEKI0OL

$\begin{array}{rrrrrrr}1045 & 32 & 2 & 32.03 & 0 & 0.4453 & 0.0 \\ 1025 & 34 & 1 & 34.02 & 100 & 0.4453 & 44.5 \\ 1020 & 36 & 3 & 36.05 & 200 & 0.4453 & 89.1 \\ 1000 & 38 & 2 & 38.03 & 300 & 0.4453 & 133.6 \\ 1025 & 40 & 4 & 40.07 & 370 & 0.4453 & 164.8\end{array}$




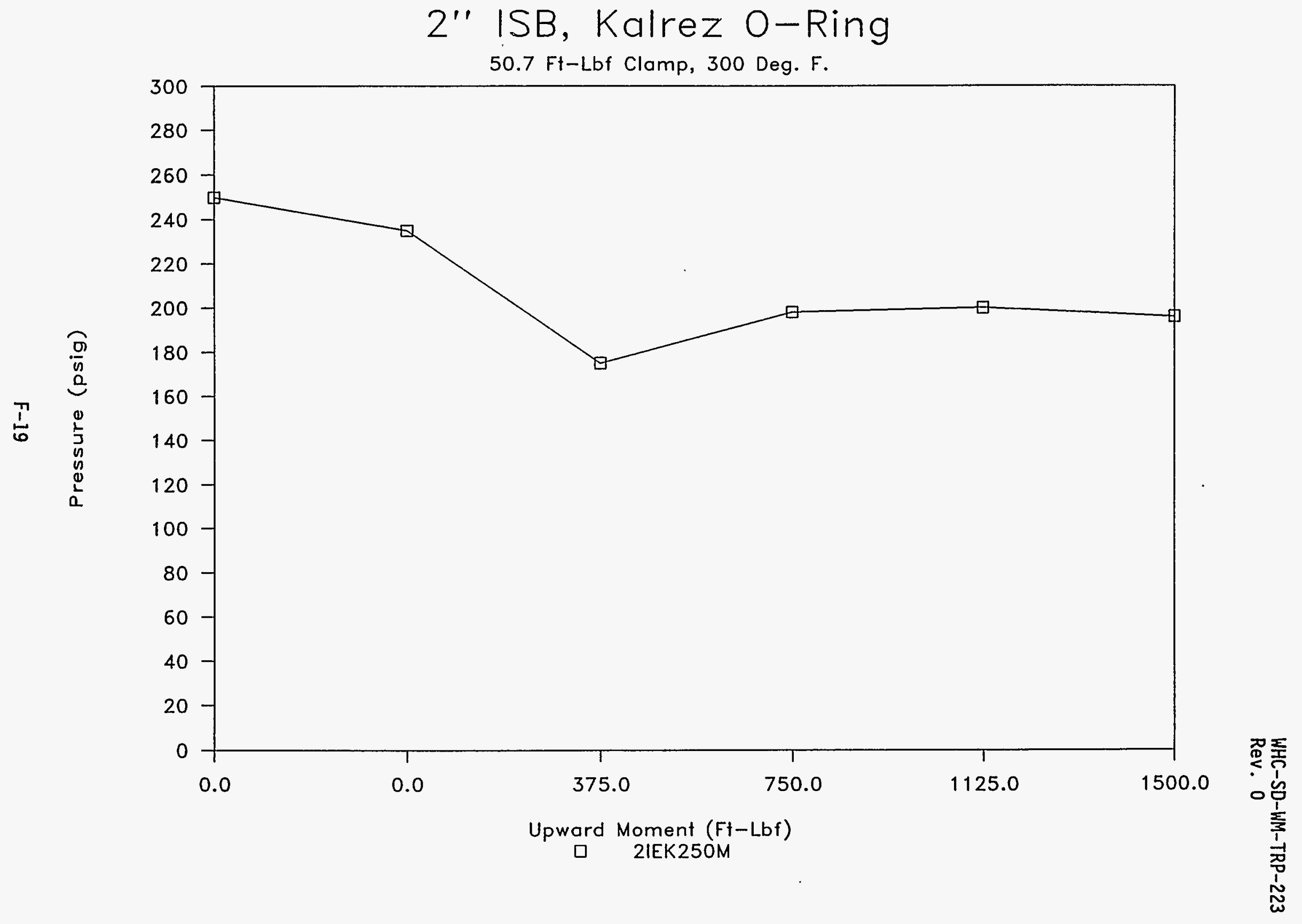


WHC-SD-WM-TRP-223

Rev. 0

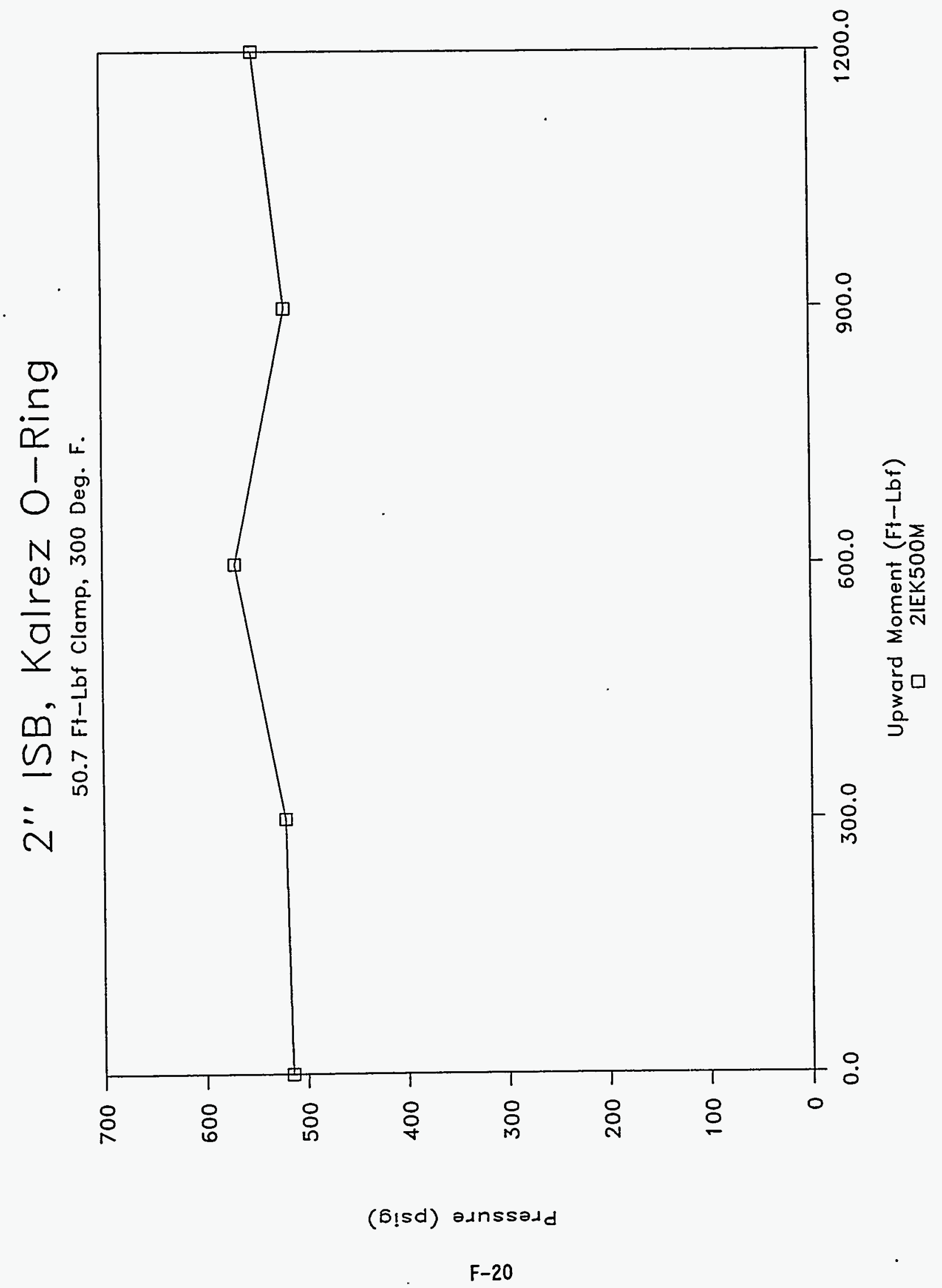


2" ISB, Kalrez O-Ring

$50.7 \mathrm{Ft}$-Lbf Clamp, 300 Deg. F.

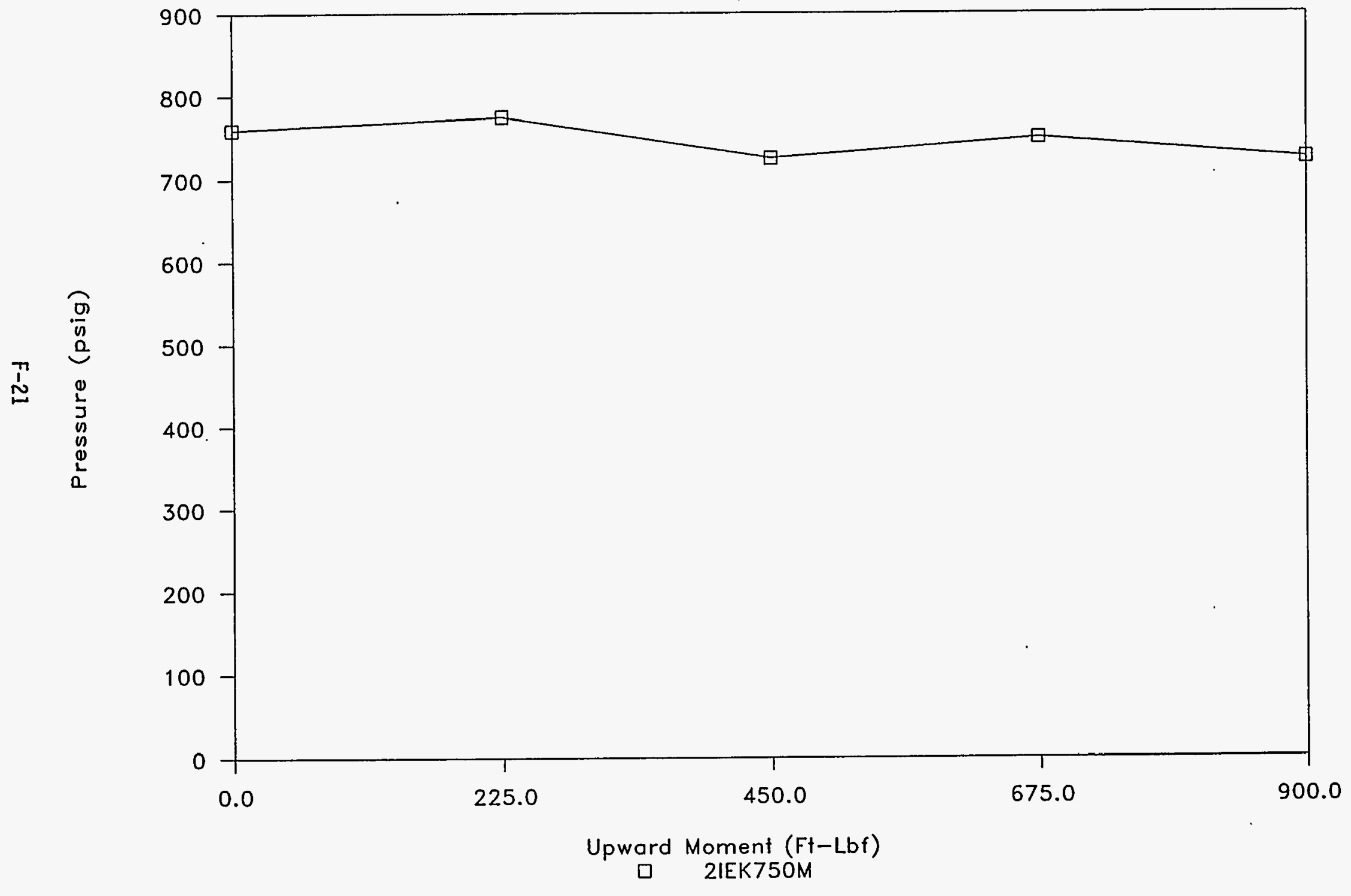


WHC-SD-WM-TRP-223

Rev. 0

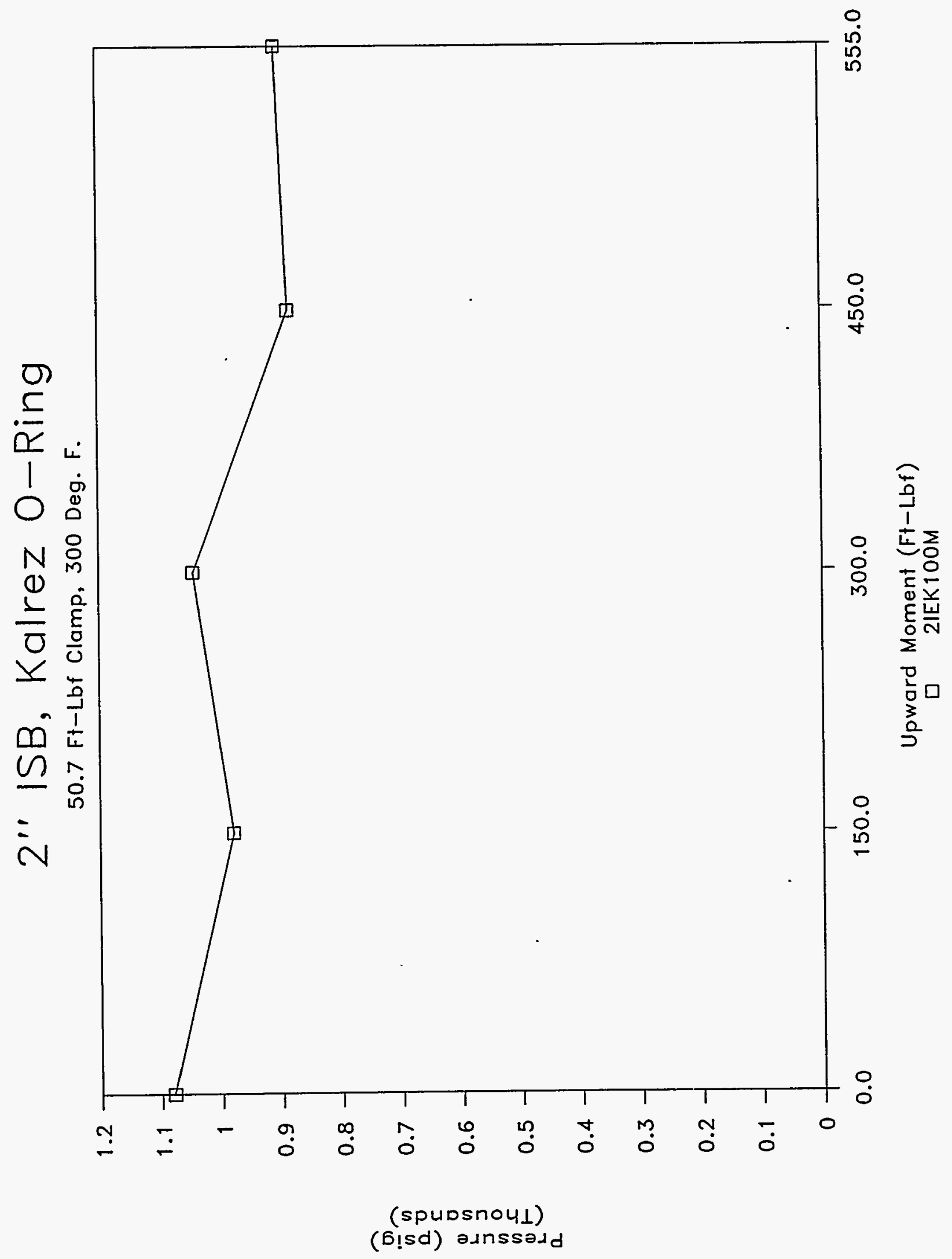


OCTOBER 04, 1994

2" ISB CONNECTOR, KALREZ O-RING, 70 DUROMETER, ELEVATED TEMP. IEAK TEST - UP AND OVER MOMENT (UPWARD ROTATION)

CLAMPING TORQUE $=50.7$ FT-LBF

GRAPH NAME $=2$ IEK250M

CHARGE PRESSURE $=250$ PSIG

TEMPERATURE: 300 DEG. F.

NOTE: THIS TEST WAS PERFORMED AT 300 DEG. F. INSTEAD OF 400 DEG. F. PREVIOUS TESTS WITH KALREZ SHOWED THAT THE O-RING SUSTAINED DEFORMATION DAMAGE AND LEAKED AT 400 DEG. F. and 1,000 PSIG.

\begin{tabular}{|c|c|c|c|c|c|c|}
\hline \multirow{3}{*}{$\begin{array}{l}\text { PRESSURE } \\
\text { PSIG }\end{array}$} & \multirow[b]{2}{*}{ ELAPSED } & \multirow[b]{2}{*}{ ELAPSED } & \multirow[b]{2}{*}{ ELAPSED } & \multicolumn{2}{|r|}{ UPWARD } & \multirow[b]{2}{*}{ UPWARD } \\
\hline & & & & UPWARD & MOMENT & \\
\hline & MINUTES & SECONDS & DECIMAL & $\begin{array}{l}\text { FORCE } \\
\text { RS }\end{array}$ & $\begin{array}{l}\text { ARM } \\
\text { FT. }\end{array}$ & $\begin{array}{l}\text { MOMENT } \\
\text { FT-LBF. }\end{array}$ \\
\hline INPUT & INPUT & INPUT & COMPUTE & INPUT & INPUT & COMPUTE \\
\hline $\begin{array}{l}250 \\
235\end{array}$ & $\begin{array}{l}0 \\
2\end{array}$ & & $\begin{array}{l}0.00 \\
2.02\end{array}$ & $\begin{array}{l}0 \\
0\end{array}$ & $\begin{array}{l}1.5000 \\
1.5000\end{array}$ & $\begin{array}{l}0.0 \\
0.0\end{array}$ \\
\hline 175 & 4 & & 4.02 & 250 & 1.5000 & 375.0 \\
\hline 198 & 6 & & 6.03 & 500 & 1.5000 & 750.0 \\
\hline 200 & 8 & & 8.02 & 750 & 1.5 & 1125.0 \\
\hline 196 & 10 & & 10.02 & 1000 & 1.5000 & 1500.0 \\
\hline
\end{tabular}

INCREASED PRESSURE TO 500 PSIG

GRAPH NAME $=2$ IEK50OM

$\begin{array}{rrrrrrr}515 & 12 & 3 & 12.05 & 0 & 1.5000 & 0.0 \\ 521 & 14 & 1 & 14.02 & 200 & 1.5000 & 300.0 \\ 570 & 16 & 2 & 16.03 & 400 & 1.5000 & 600.0 \\ 520 & 18 & 1 & 18.02 & 600 & 1.5000 & 900.0 \\ 550 & 20 & 1 & 20.02 & 800 & 1.5000 & 1200.0\end{array}$

INCREASED PRESSURE TO 750 PSIG

$\begin{array}{ll}760 & 22 \\ 775 & 24 \\ 725 & 26 \\ 750 & 28 \\ 725 & 30\end{array}$

INCREASED PRESSURE TO 1000 PSIG

$\begin{array}{rr}1080 & 32 \\ 980 & 34 \\ 1045 & 36 \\ 885 & 38 \\ 905 & 40\end{array}$

$\begin{array}{ll}1 & 22.02 \\ 2 & 24.03 \\ 2 & 26.03 \\ 2 & 28.03 \\ 1 & 30.02\end{array}$

GRAPH NAME = 2IEK750M

$\begin{array}{rrr}0 & 1.5000 & 0.0 \\ 150 & 1.5000 & 225.0 \\ 300 & 1.5000 & 450.0 \\ 450 & 1.5000 & 675.0 \\ 600 & 1.5000 & 900.0\end{array}$

GRAPH NAME $=2$ IEK10OM

$\begin{array}{rrr}0 & 1.5000 & 0.0 \\ 100 & 1.5000 & 150.0 \\ 200 & 1.5000 & 300.0 \\ 300 & 1.5000 & 450.0 \\ 370 & 1.5000 & 555.0\end{array}$


WHC-SD-WM-TRP-223

Rev. 0

APPENDIX G: GRAPHS OF 2-IN. VITON TESTS

G-1 


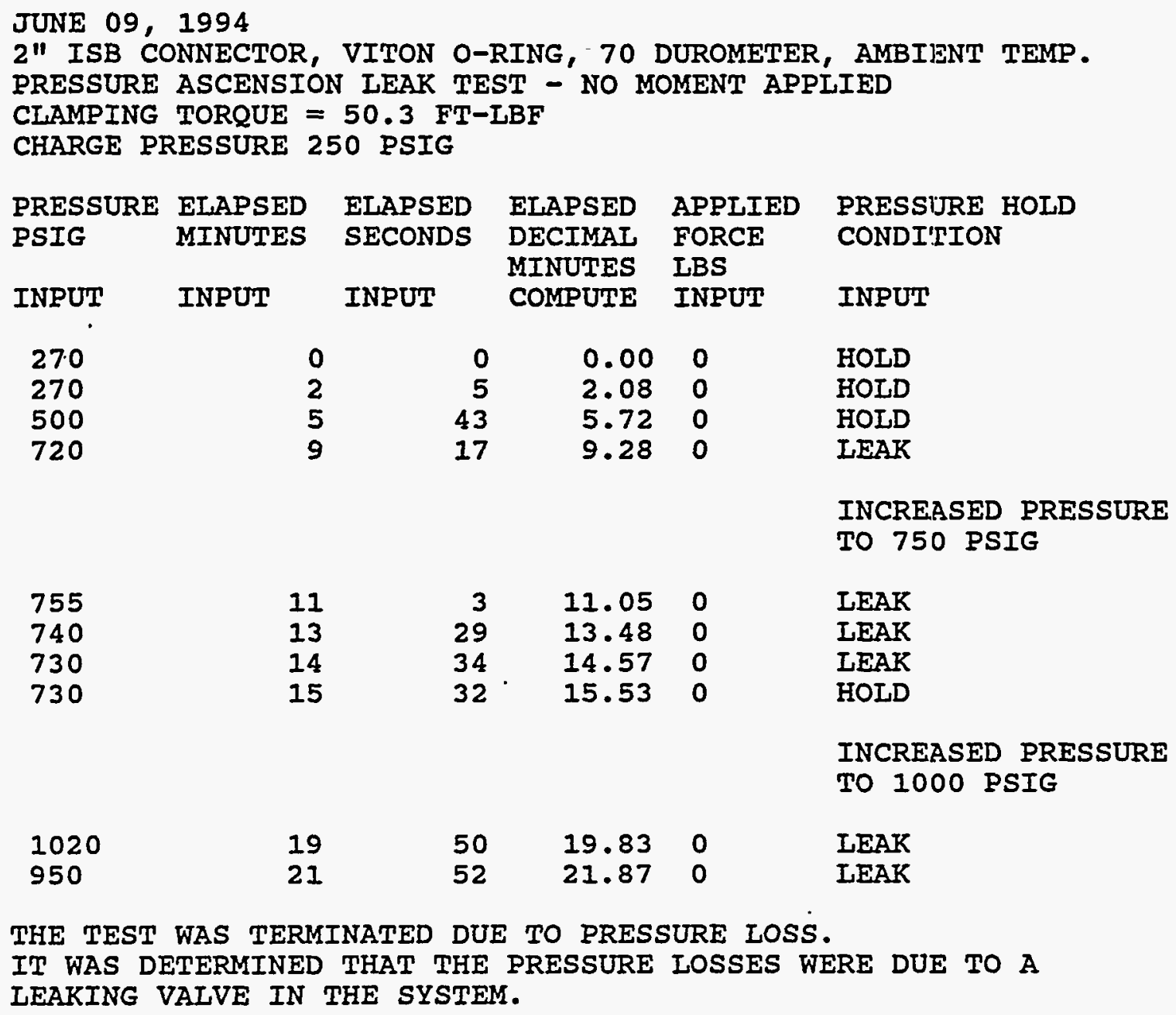

730

730

11

13

14

15

3

11.050

LEAK

IEAK

$\begin{array}{llll}34 & 14.57 & 0 & \text { LEAK } \\ 32 & 15.53 & 0 & \text { HOLD }\end{array}$

INCREASED PRESSURE

TO 1000 PSIG

$\begin{array}{llllll}1020 & 19 & 50 & 19.83 & 0 & \text { LEAK } \\ 950 & 21 & 52 & 21.87 & 0 & \text { LEAK }\end{array}$

THE TEST WAS TERMINATED DUE TO PRESSURE LOSS. IT WAS DETERMINED THAT THE PRESSURE LOSSES WERE DUE TO A LEAKING VALVE IN THE SYSTEM. 
WHC-SD-HM-TRP-223

Rev. 0

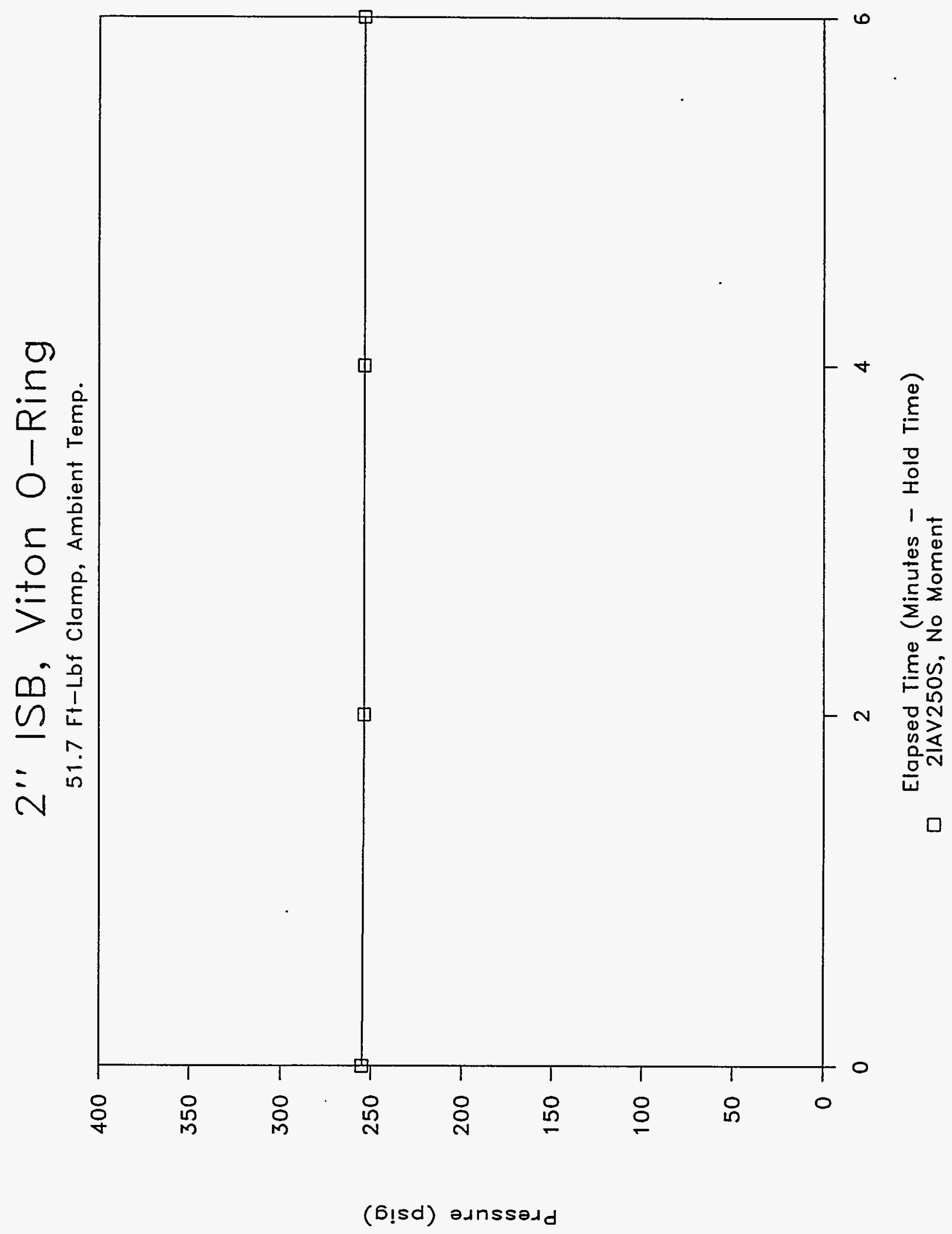

G-3 
WHC-SD-WM-TRP-223

Rev. 0

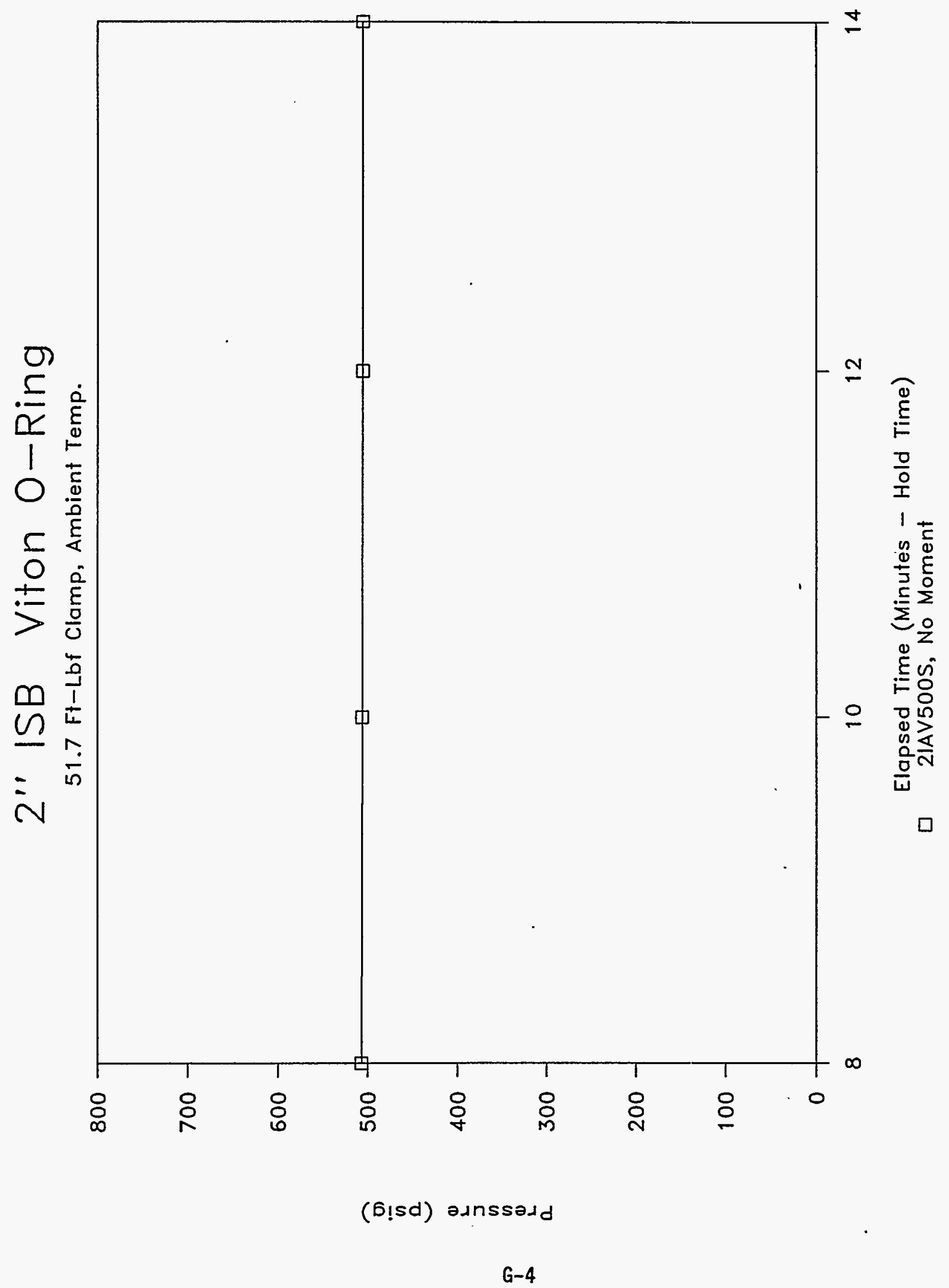




\section{2" ISB Viton O-Ring}

$51.7 \mathrm{Ft}$-Lbf Clamp, Ambient Temp.

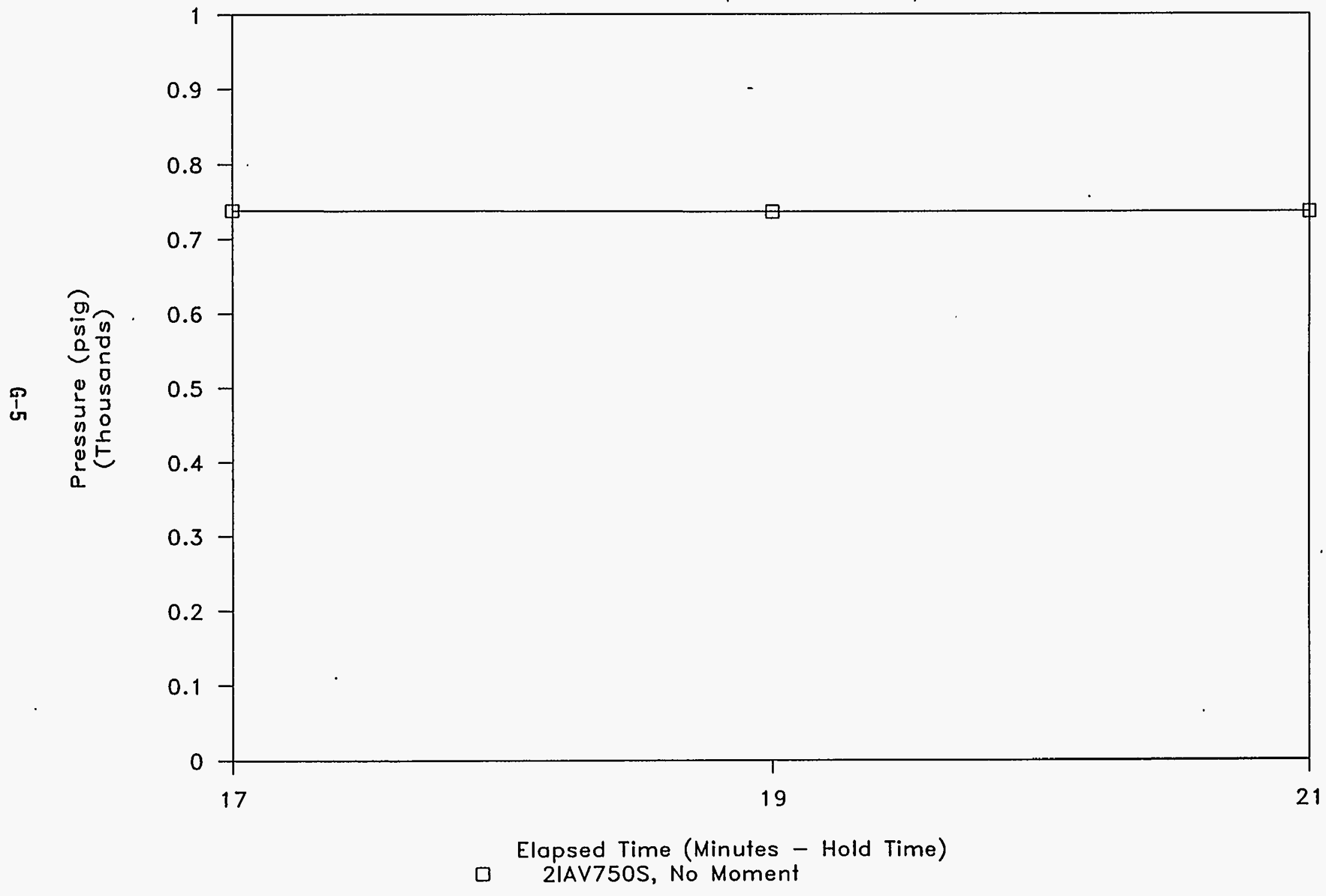




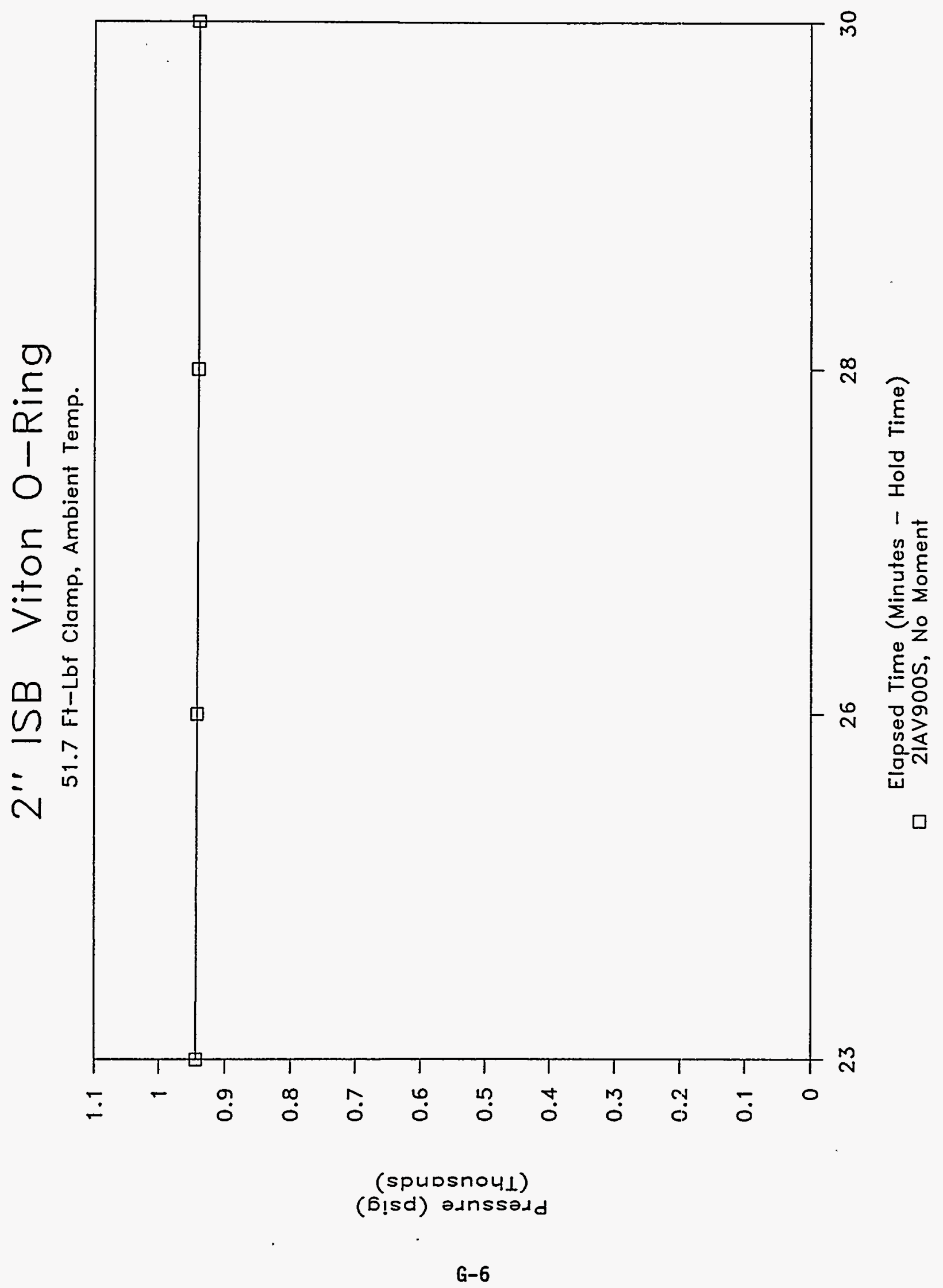


JUNE 23, 1994

2 " ISB CONNECTOR, VITON O-RING, 70 DUROMETER, AMBIENT TEMP. PRESSURE ASCENSION LEAK TEST - NO MOMENT APPIIED

CLAMPING TORQUE $=51.7 \mathrm{FT}-\mathrm{LBF}$

CHARGE PRESSURE $=250$ PSIG

$\begin{array}{llllll}\begin{array}{l}\text { PRESSURE } \\ \text { PSIG }\end{array} & \begin{array}{l}\text { ELAPSED } \\ \text { MINUTES }\end{array} & \begin{array}{l}\text { ELAPSED } \\ \text { SECONDS }\end{array} & \begin{array}{l}\text { ELAPSED } \\ \text { DECIMAL }\end{array} & \begin{array}{l}\text { APPLIED } \\ \text { FORCE }\end{array} \\ \text { INPUT } & \text { INPUT } & \text { INPUT } & \begin{array}{l}\text { MINUTES } \\ \text { COMPUTE }\end{array} & \begin{array}{l}\text { LBS } \\ \text { INPUT }\end{array} \\ & & & & & \\ 255 & 0 & 0 & 0.00 & 0 \\ 254 & 2 & 8 & 2.13 & 0 \\ 254 & 4 & 18 & 4.30 & 0 \\ 254 & 6 & 18 & 6.30 & 0\end{array}$

INCREASED PRESSURE TO 500 PSIG.

GRAPH NAME $=2$ IAV250S

$\begin{array}{rrrr}507 & 8 & 23 & 8.38 \\ 506 & 10 & 24 & 10.40 \\ 505 & 12 & 27 & 12.45 \\ 505 & 14 & 28 & 14.47\end{array}$

INCREASED PRESSURE TO 750 PSIG.

GRAPH NAME $=2$ IAV750S

$\begin{array}{lllll}738 & 17 & 55 & 17.92 & 0 \\ 736 & 19 & 47 & 19.78 & 0 \\ 736 & 21 & 51 & 21.85 & 0\end{array}$

INCREASED PRESSURE TO 900 PSIG.

\author{
GRAPH NAME $=2$ IAV900S
}

$\begin{array}{llrll}945 & 23 & 51 & 23.85 & 0 \\ 943 & 26 & 2 & 26.03 & 0 \\ 941 & 28 & 10 & 28.17 & 0 \\ 941 & 30 & 10 & 30.17 & 0\end{array}$


Rev. 0

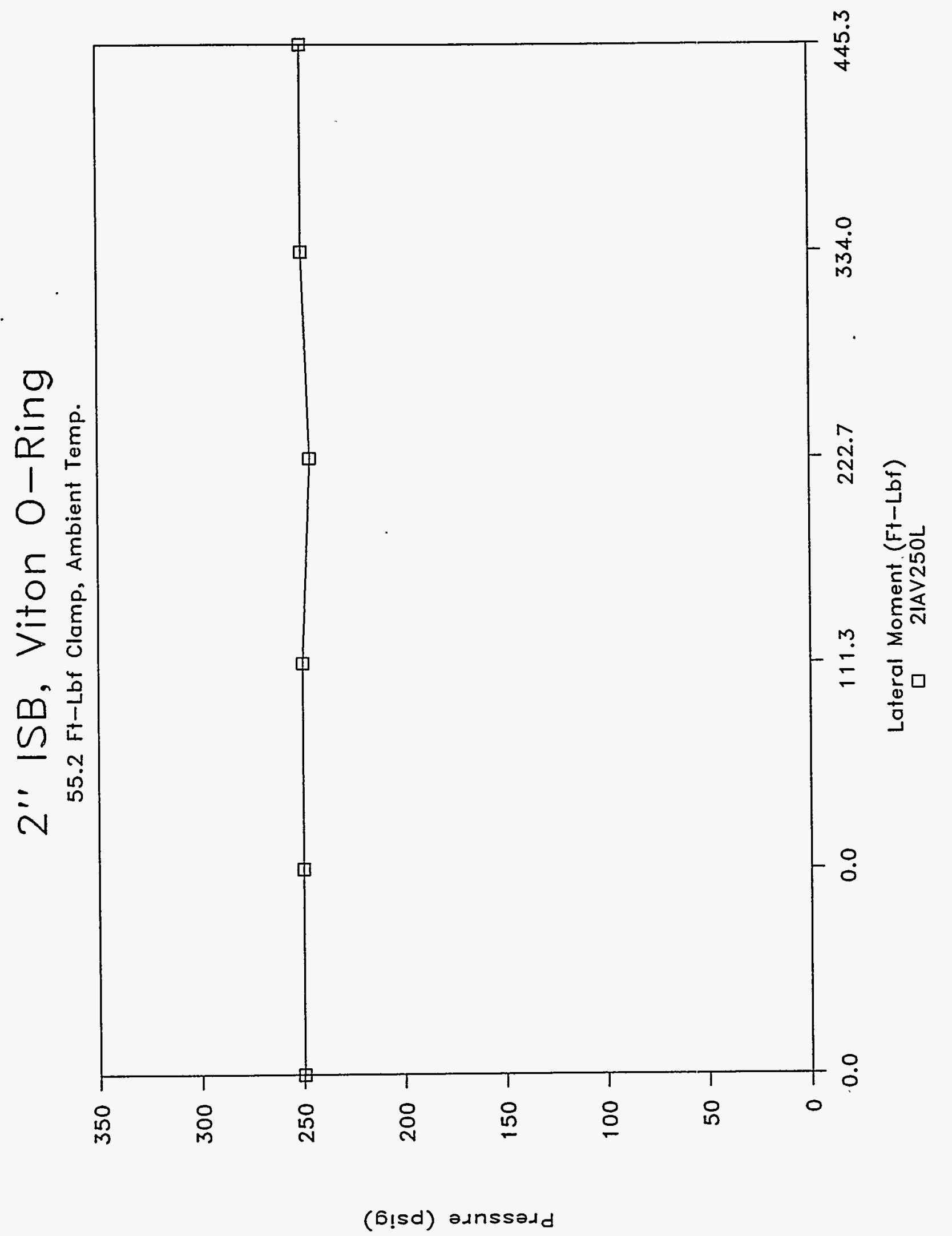

G-8 


\section{2" ISB, Viton O-Ring}

55.2 Ft-Lbf Clamp, Ambient Temp.

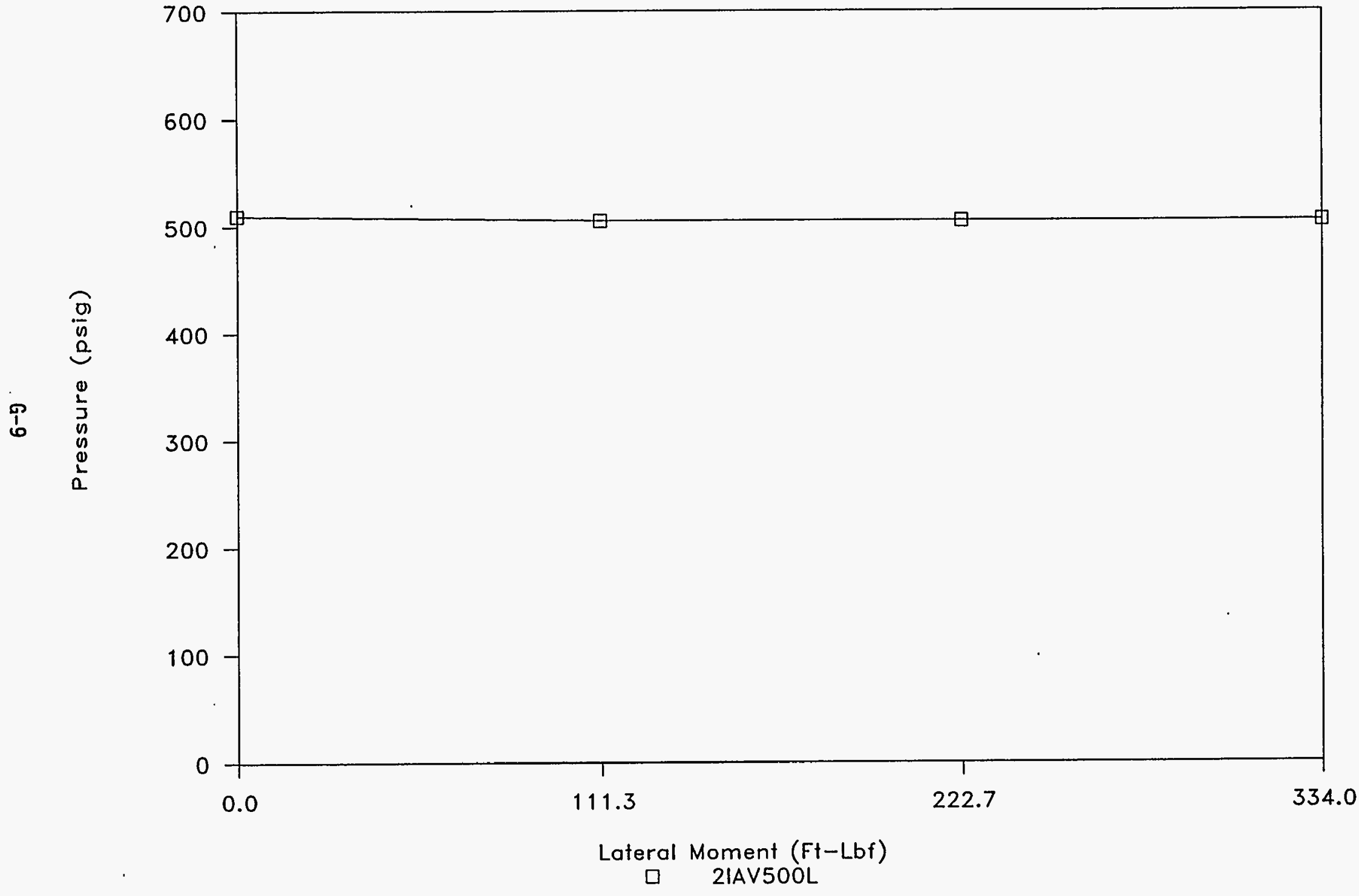


WHC-SD-WM-TRP-223

Rev. 0

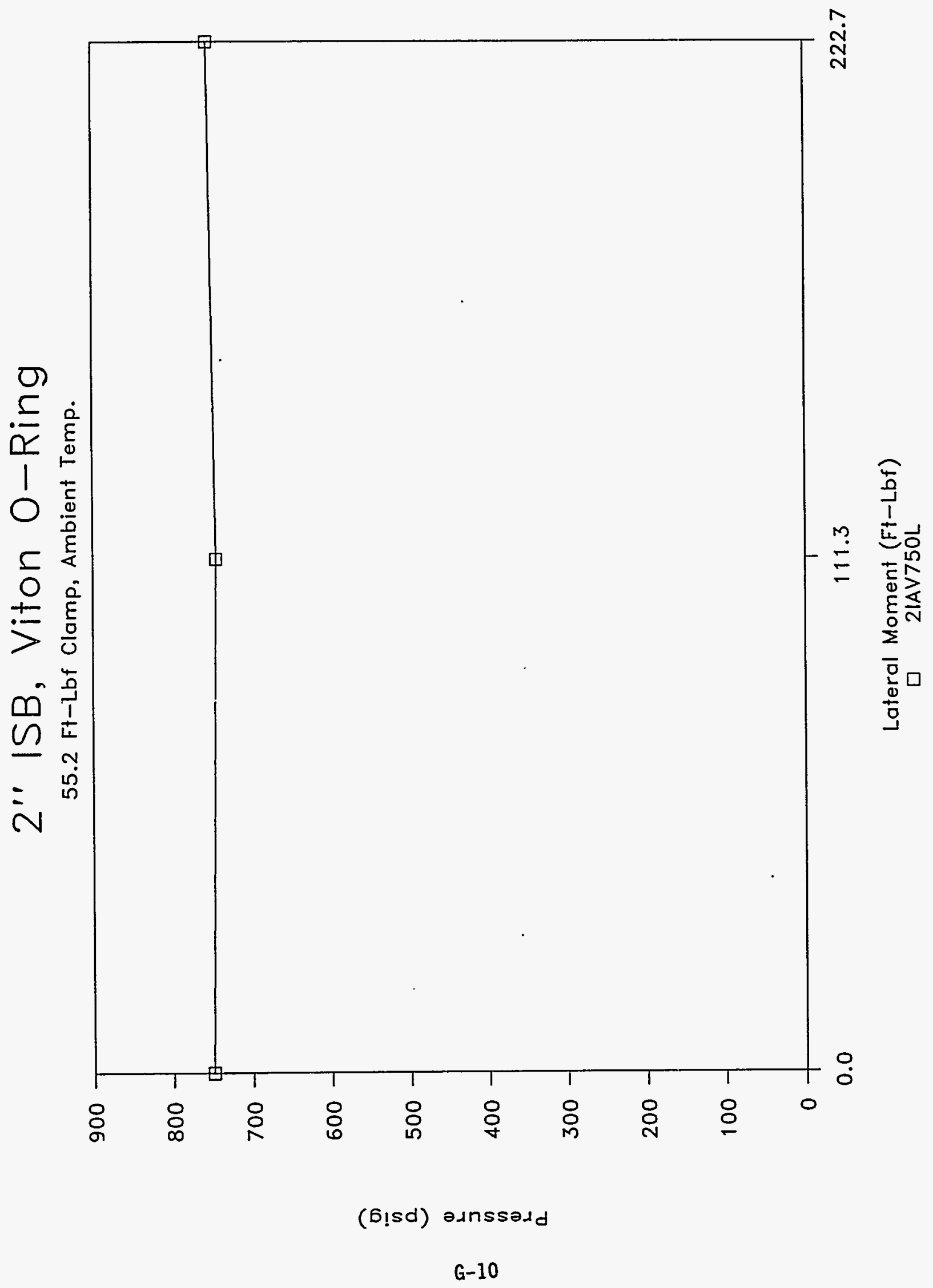


WHC-SD-WM-TRP-223

Rev. 0

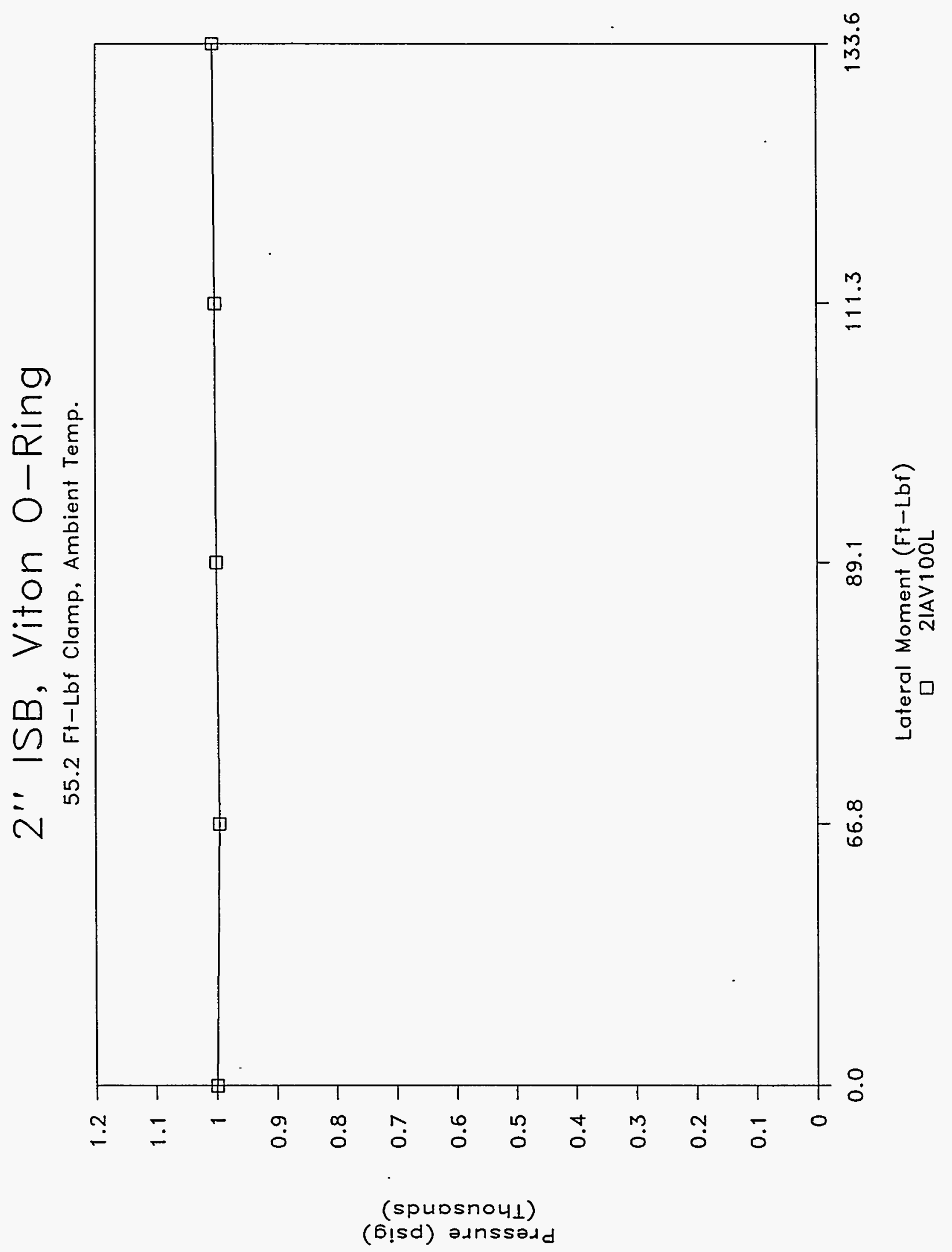


JULY 26,1994

2" ISB CONNECTOR, VITON O-RING, 70 DUROMETER, AMBIENT TEMP. PRESSURE ASCENSION LEAK TEST - LATERAL MOMENT (SILEWAYS ROTATION) CLAMPING TORQUE $=55.2$ FT-LBF GRAPH NAME = ZIAV250L CHARGE PRESSURE $=250$ PSIG

$\begin{array}{lllllll} & & & & \text { LATEFAL } & \\ \text { PRESSURE } & \text { ELAPSED } & \text { ELAPSED } & \text { ELAPSED } & \text { IATERAI } & \text { MOMENT } & \text { LATERAL } \\ \text { PSIG } & \text { MINUTES } & \text { SECONDS } & \text { DECIMAL } & \text { FORCE } & \text { ARM } & \text { MOMENT } \\ & & & \text { MINUTES } & \text { LBS } & \text { FT. } & \text { FT-LBF. } \\ \text { INPUT } & \text { INPUT } & \text { INPUT } & \text { COMPUTE } & \text { INPUT } & \text { INPU!' } & \text { COMPUTE }\end{array}$

$\begin{array}{rrrrrrr}250 & 0 & 0 & 0.00 & 0 & 0.4453 & 0.0 \\ 250 & 2 & 2 & 2.03 & 0 & 0.4453 & 0.0 \\ 250 & 4 & 17 & 4.28 & 250 & 0.4453 & 111.3 \\ 246 & 6 & 5 & 6.08 & 500 & 0.4453 & 222.7 \\ 250 & 8 & 7 & 8.12 & 750 & 0.4453 & 334.0 \\ 250 & 10 & 2 & 10.03 & 1000 & 0.4453 & 445.3\end{array}$

INCREASED PRESSURE TO 500 PSIG

$\begin{array}{rrrr}510 & 12 & 4 & 12.07 \\ 505 & 14 & 3 & 14.05 \\ 505 & 16 & 10 & 16.17 \\ 505 & 18 & 3 & 18.05\end{array}$

INCREASED PRESSURE TO 750 PSIG

$\begin{array}{rrrrrrr}750 & 20 & 2 & 20.03 & 0 & 0.4453 & 0.0 \\ 745 & 22 & 8 & 22.13 & 250 & 0.4453 & 111.3 \\ 755 & 24 & 4 & 24.07 & 500 & 0.4453 & 222.7\end{array}$

INCREASED PRESSURE TO 1000 PSIG

$\begin{array}{rrrrrrr}1000 & 26 & 11 & 26.18 & 0 & 0.4453 & 0.0 \\ 995 & 28 & 3 & 28.05 & 150 & 0.4453 & 66.8 \\ 1000 & 30 & 11 & 30.18 & 200 & 0.4453 & 89.1 \\ 1002 & 32 & 8 & 32.13 & 250 & 0.4453 & 111.3 \\ 1006 & 34 & 15 & 34.25 & 300 & 0.4453 & 133.6\end{array}$

GRAPH NAME = 2IAV50OL

$\begin{array}{rrr}0 & 0.4453 & 0.0 \\ 250 & 0.4453 & 111.3 \\ 500 & 0.4453 & 222.7 \\ 750 & 0.4453 & 334.0\end{array}$

GRAPH NAME = 2IAV750L

GRAPH NAME = 2IAVI00OL 


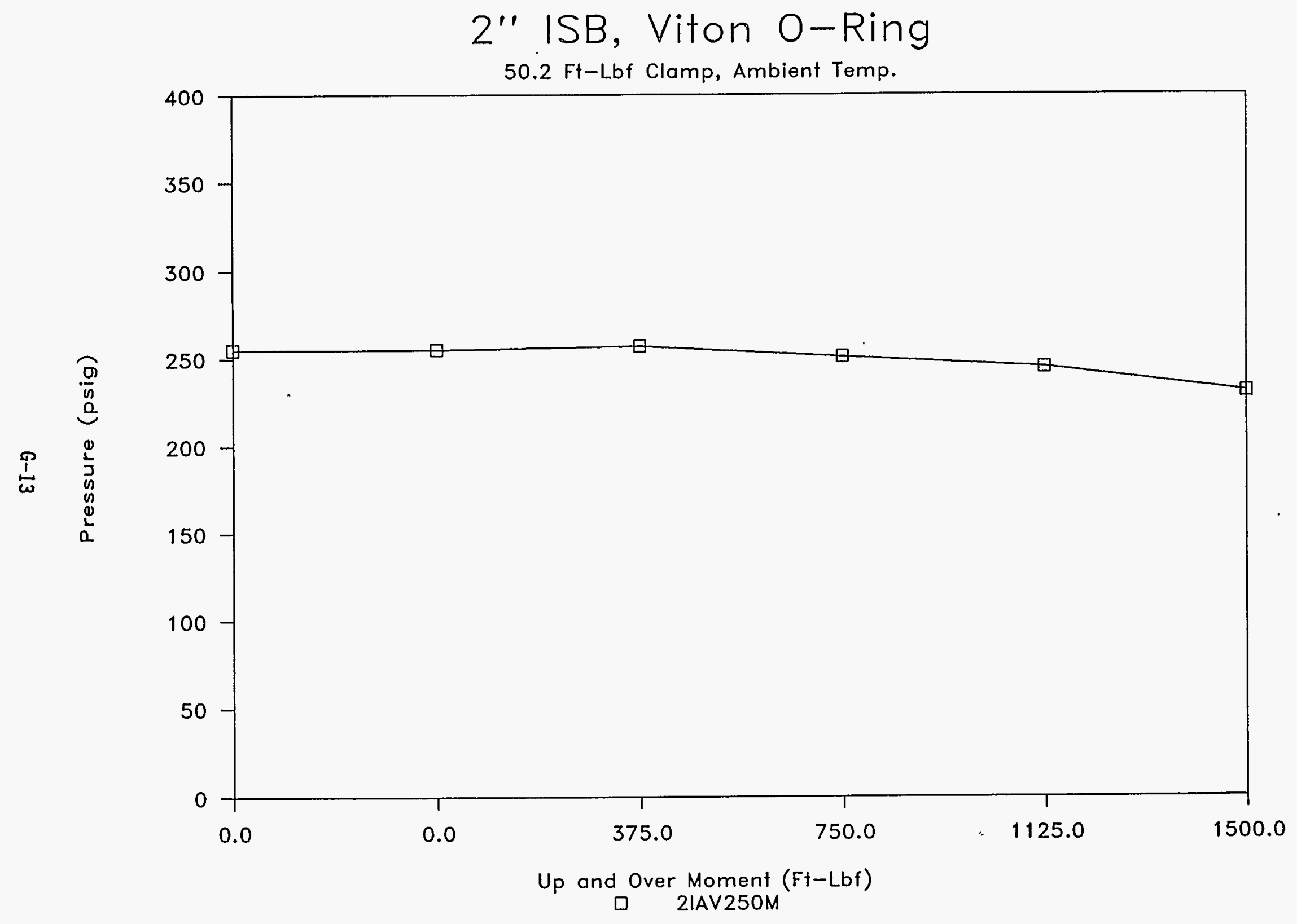

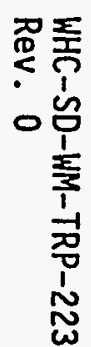


WHC-SD-WM-TRP-223

Rev. 0

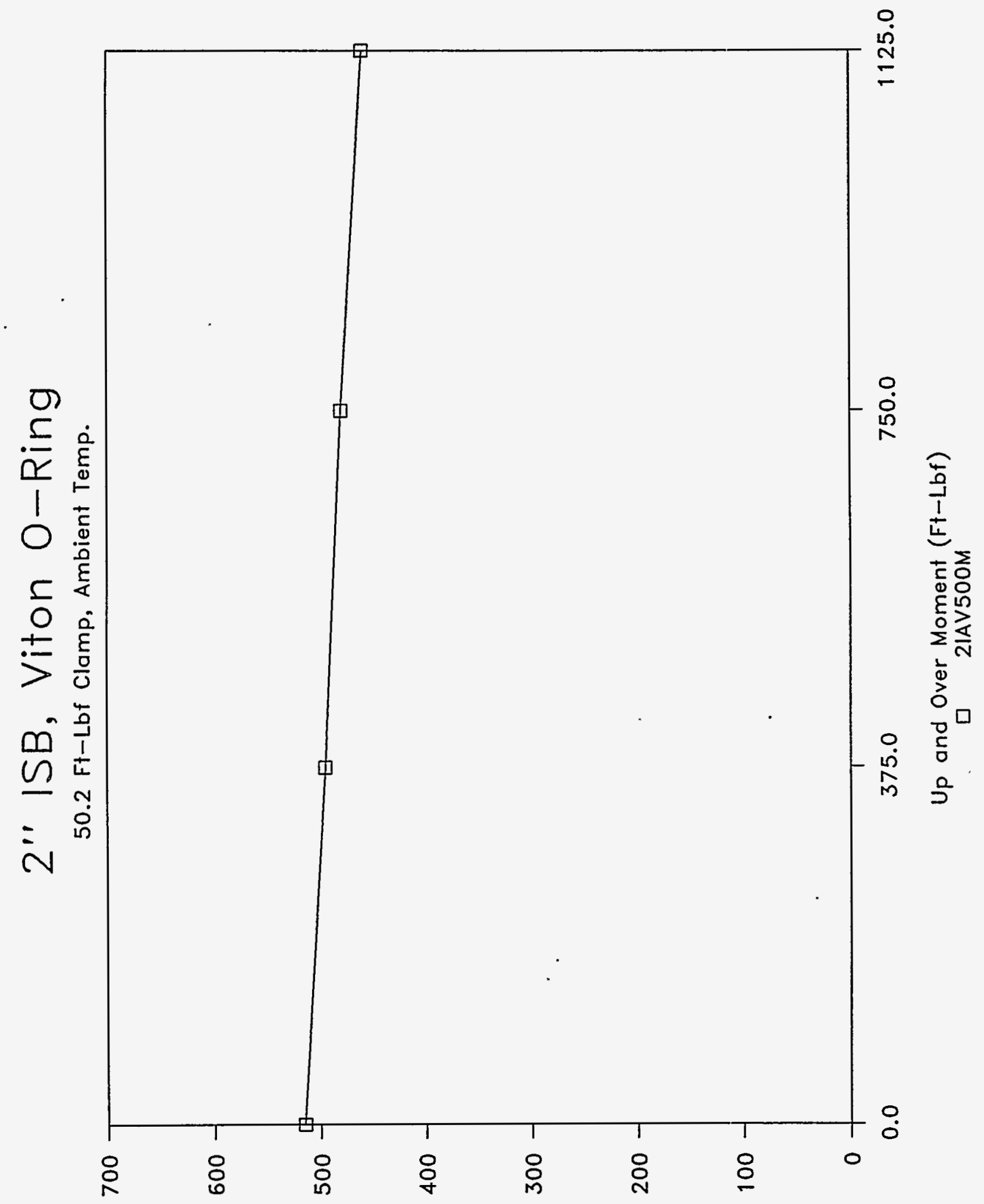

(6!sd) əגnssodd 


\section{2" ISB, Viton O-Ring}

$50.2 \mathrm{Ft}$-Lbf Clamp, Ambient Temp.

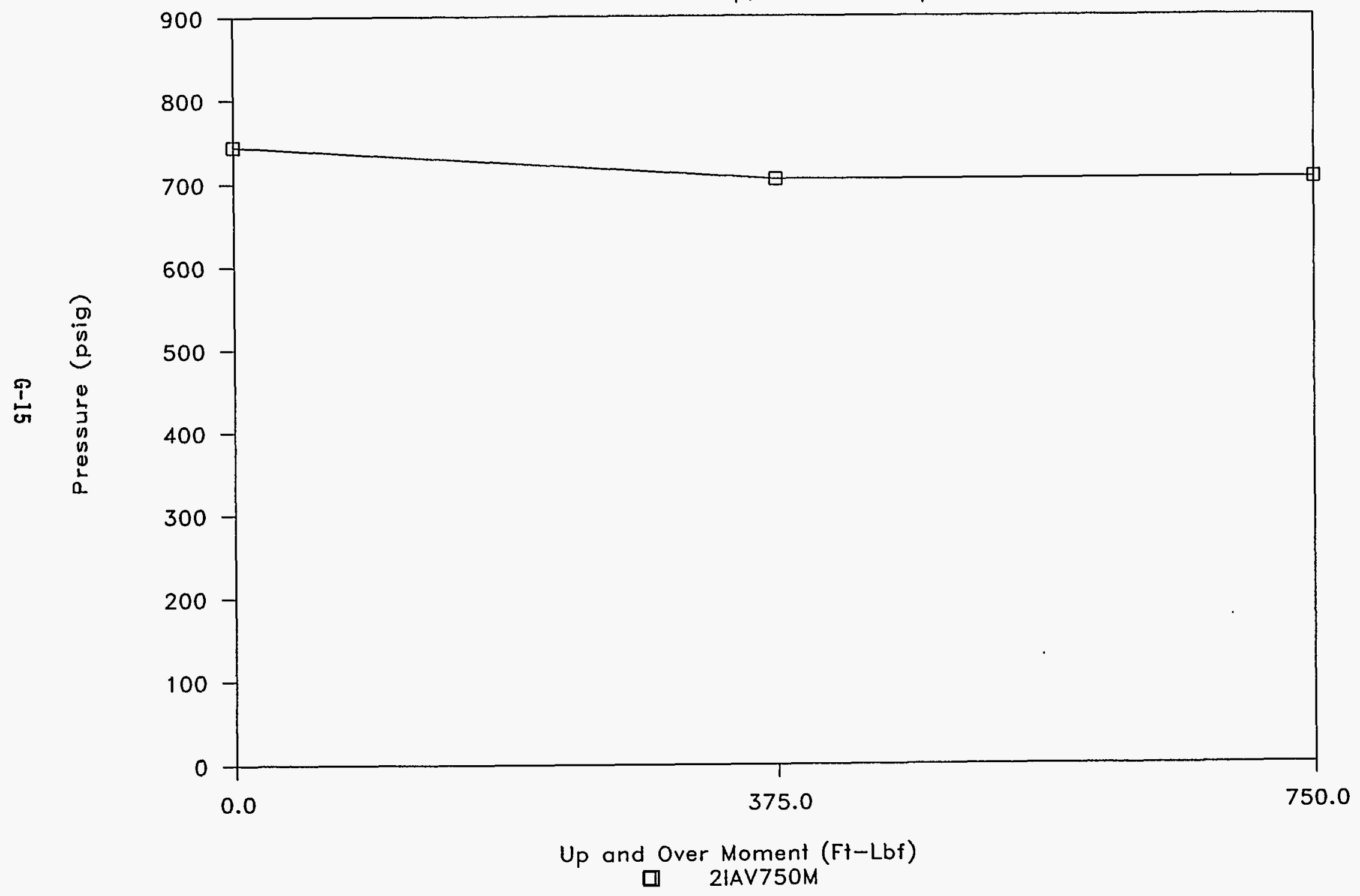


WHC-SD-WM-TRP-223

Rev. 0

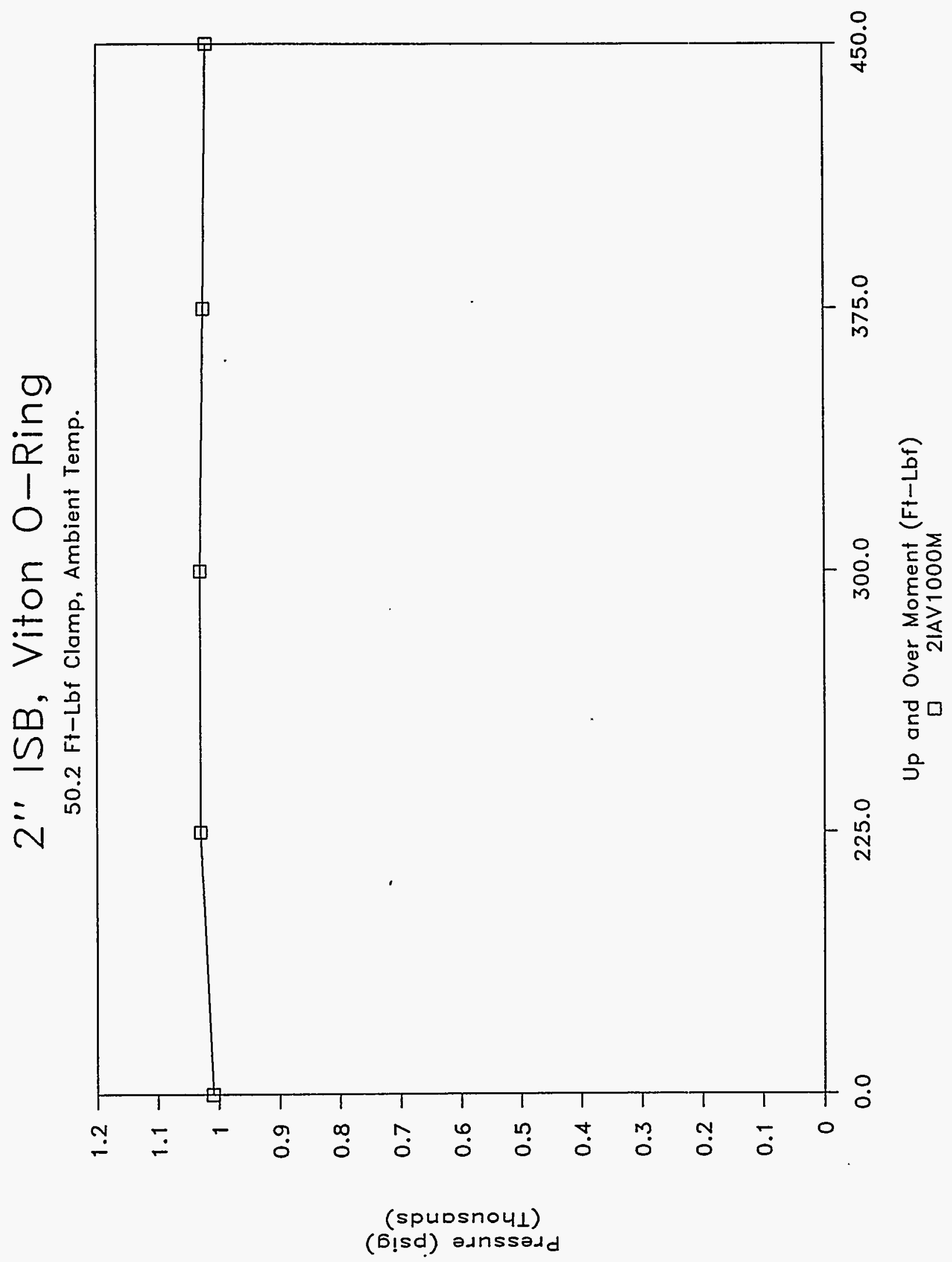

G-16 
JULY 25, 1994

2" ISB CONNECTOR, VITON O-RING, 70 DUROMETER, AMBIENT TEMP. PRESSURE ASCENSION IEAK TEST - UP AND OVER MOMENT (UPWARD ROTATION) CLAMPING TORQUE $=50.2 \mathrm{FT}-\mathrm{IBF}$ GRAPH NAME $=2$ IAV25OM

CHARGE PRESSURE $=250$ PSIG

\begin{tabular}{|c|c|c|c|c|c|c|}
\hline $\begin{array}{l}\text { PESSURE } \\
\text { PIG }\end{array}$ & $\begin{array}{l}\text { ELAPSED } \\
\text { MINUTES }\end{array}$ & $\begin{array}{l}\text { ELAAPSED } \\
\text { SECONDS }\end{array}$ & $\begin{array}{l}\text { ELAPSED } \\
\text { DECIMAI } \\
\text { MINUTES } \\
\text { COMPUTE }\end{array}$ & $\begin{array}{l}\text { UPWARD } \\
\text { FORCE } \\
\text { IBS } \\
\text { INPUT }\end{array}$ & $\begin{array}{l}\text { UPWARD } \\
\text { MOMENT } \\
\text { ARM } \\
\text { FT. } \\
\text { INPUT }\end{array}$ & $\begin{array}{l}\text { UPWARD } \\
\text { MOMENT } \\
\text { FT-IBF. } \\
\text { COMPUTE }\end{array}$ \\
\hline NPUT & INPUT & INPUT & COMPUTE & INPU'T & INPUT & COMPUIE \\
\hline $\begin{array}{l}255 \\
255 \\
257 \\
251 \\
245 \\
231\end{array}$ & $\begin{array}{r}0 \\
2 \\
4 \\
6 \\
8 \\
10\end{array}$ & & $\begin{array}{r}0.00 \\
2.03 \\
4.05 \\
6.08 \\
8.02 \\
10.08\end{array}$ & $\begin{array}{r}0 \\
0 \\
250 \\
500 \\
750 \\
1000\end{array}$ & $\begin{array}{l}1.5000 \\
1.5000 \\
1.5000 \\
1.5000 \\
1.5000 \\
1.5000\end{array}$ & $\begin{array}{r}0.0 \\
0.0 \\
375.0 \\
750.0 \\
1125.0 \\
1500.0\end{array}$ \\
\hline
\end{tabular}

INCREASED PRESSURE TO 500 PSIG

$\begin{array}{llll}515 & 12 & 2 & 12.03 \\ 495 & 14 & 9 & 14.15 \\ 480 & 16 & 2 & 16.03 \\ 460 & 18 & 3 & 18.05\end{array}$

INCREASED PRESSURE TO 750 PSIG

$\begin{array}{llll}745 & 20 & 4 & 20.07 \\ 705 & 22 & 4 & 22.07 \\ 705 & 24 & 6 & 24.10\end{array}$

INCREASED PRESSURE TO 1000 PSIG

$\begin{array}{lrl}26 & 10 & 26.17 \\ 28 & 4 & 28.07 \\ 30 & 4 & 30.07 \\ 32 & 16 & 32.27 \\ 34 & 6 & 34.10\end{array}$

GRAPH NAME = 2IAV50OM

$\begin{array}{rrr}0 & 1.5000 & 0.0 \\ 250 & 1.5000 & 375.0 \\ 500 & 1.5000 & 750.0 \\ 750 & 1.5000 & 1125.0\end{array}$

GRAPH NAME $=2$ IAV75OM

$\begin{array}{rrr}0 & 1.5000 & 0.0 \\ 250 & 1.5000 & 375.0 \\ 500 & 1.5000 & 750.0\end{array}$

GRAPH NAME $=2$ IAV1000M

$\begin{array}{rrr}0 & 1.5000 & 0.0 \\ 150 & 1.5000 & 225.0 \\ 200 & 1.5000 & 300.0 \\ 250 & 1.5000 & 375.0 \\ 300 & 1.5000 & 450.0\end{array}$


WHC-SD-WM-TRP-223

Rev. 0

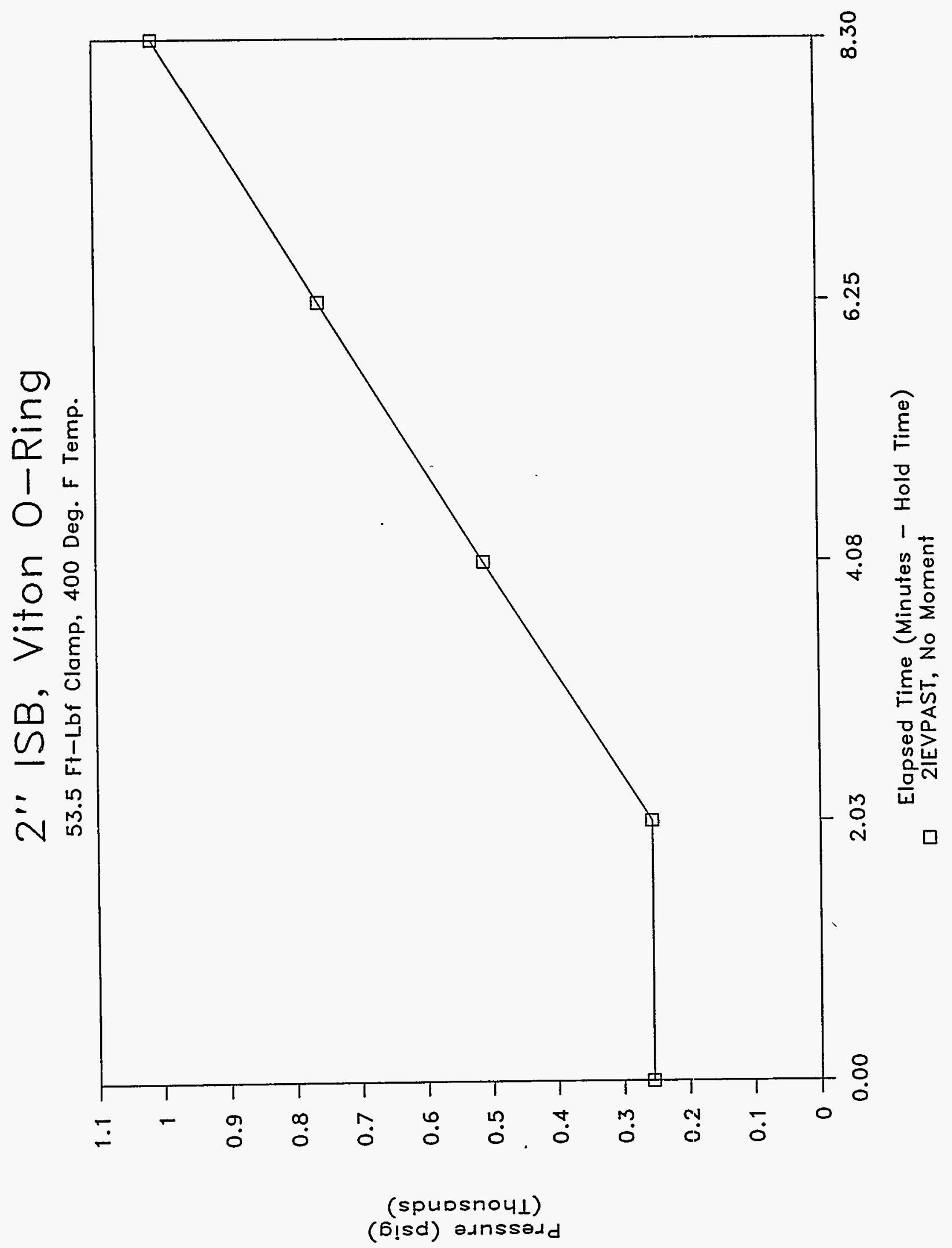

G-18 
JULY 27, 1994

2" ISB CONNECTOR, VITON O-RING, 70 DUROMETER, ELEVATED TEMP. PRESSURE ASCENSION LEAK TEST - NO MOMENT APPLIED

CLAMPING TORQUE $=53.5$ FT-LBF

CHARGE PRESSURE $=250$ PSIG TO 1000 PSIG

GRAPH NAME = 2IEVPAST

TEMPERATURE $=400 \mathrm{Deg} . \mathrm{F}$

\begin{tabular}{|c|c|c|c|c|}
\hline $\begin{array}{l}\text { PRESSURE } \\
\text { PSIG }\end{array}$ & $\begin{array}{l}\text { ELAAPSED } \\
\text { MINUTES }\end{array}$ & $\begin{array}{l}\text { ELAPSED } \\
\text { SECONDS }\end{array}$ & $\begin{array}{l}\text { ELAPSED } \\
\text { DECIMAL } \\
\text { MINUTES }\end{array}$ & $\begin{array}{l}\text { APPLIED } \\
\text { FORCE } \\
\text { LBS }\end{array}$ \\
\hline INPUT & INPUT & INPUT & COMPUTE & INPUT \\
\hline $\begin{array}{r}255 \\
255 \\
510 \\
760 \\
1010\end{array}$ & $\begin{array}{l}0 \\
2 \\
4 \\
6 \\
8\end{array}$ & $\begin{array}{r}0 \\
2 \\
5 \\
15 \\
18\end{array}$ & $\begin{array}{l}0.00 \\
2.03 \\
4.08 \\
6.25 \\
8.30\end{array}$ & $\begin{array}{l}0 \\
0 \\
0 \\
0 \\
0\end{array}$ \\
\hline
\end{tabular}




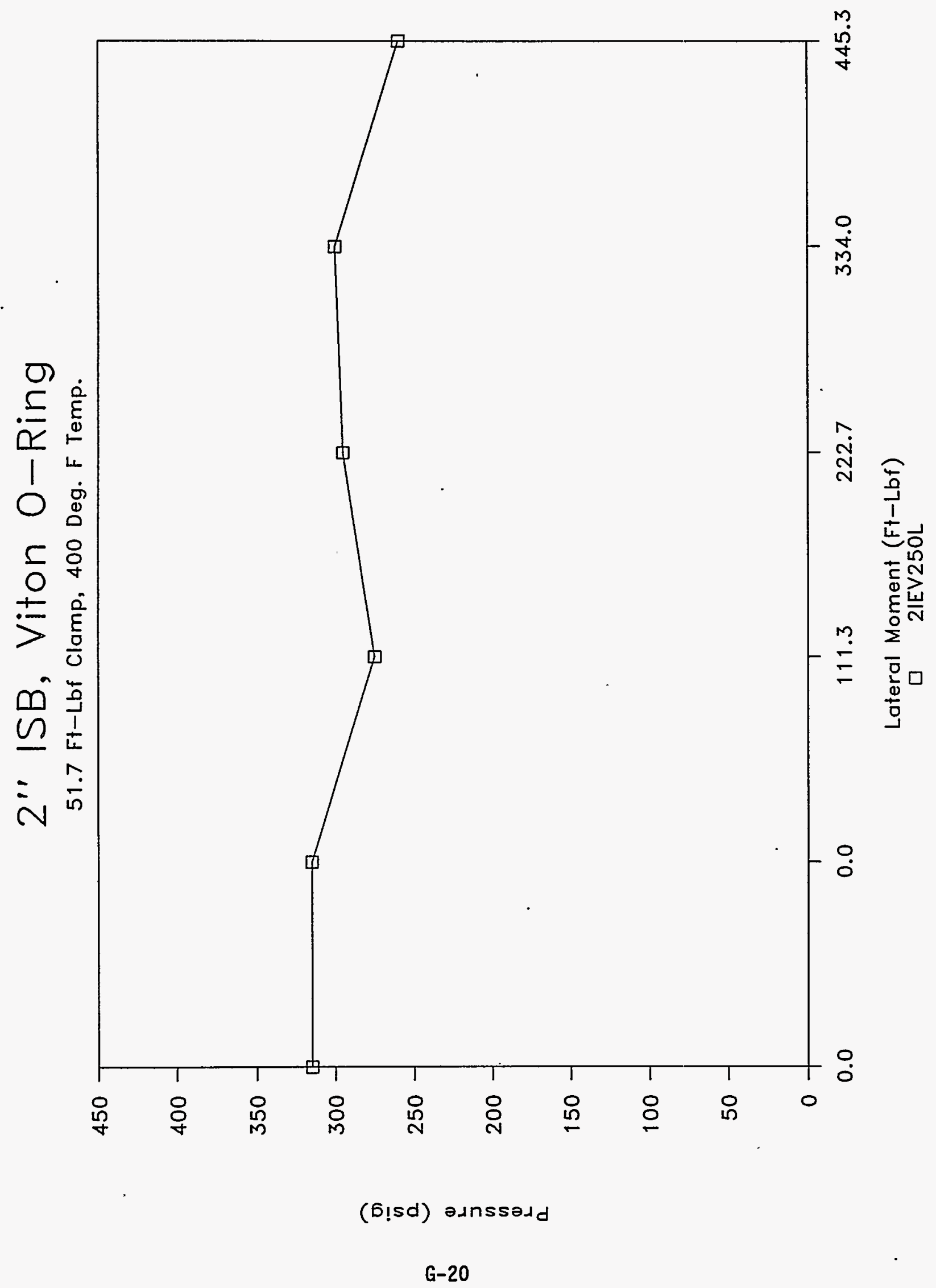


2" ISB, Viton O-Ring

51.7 Ft-Lbf Clamp, 400 Deg. F Temp.

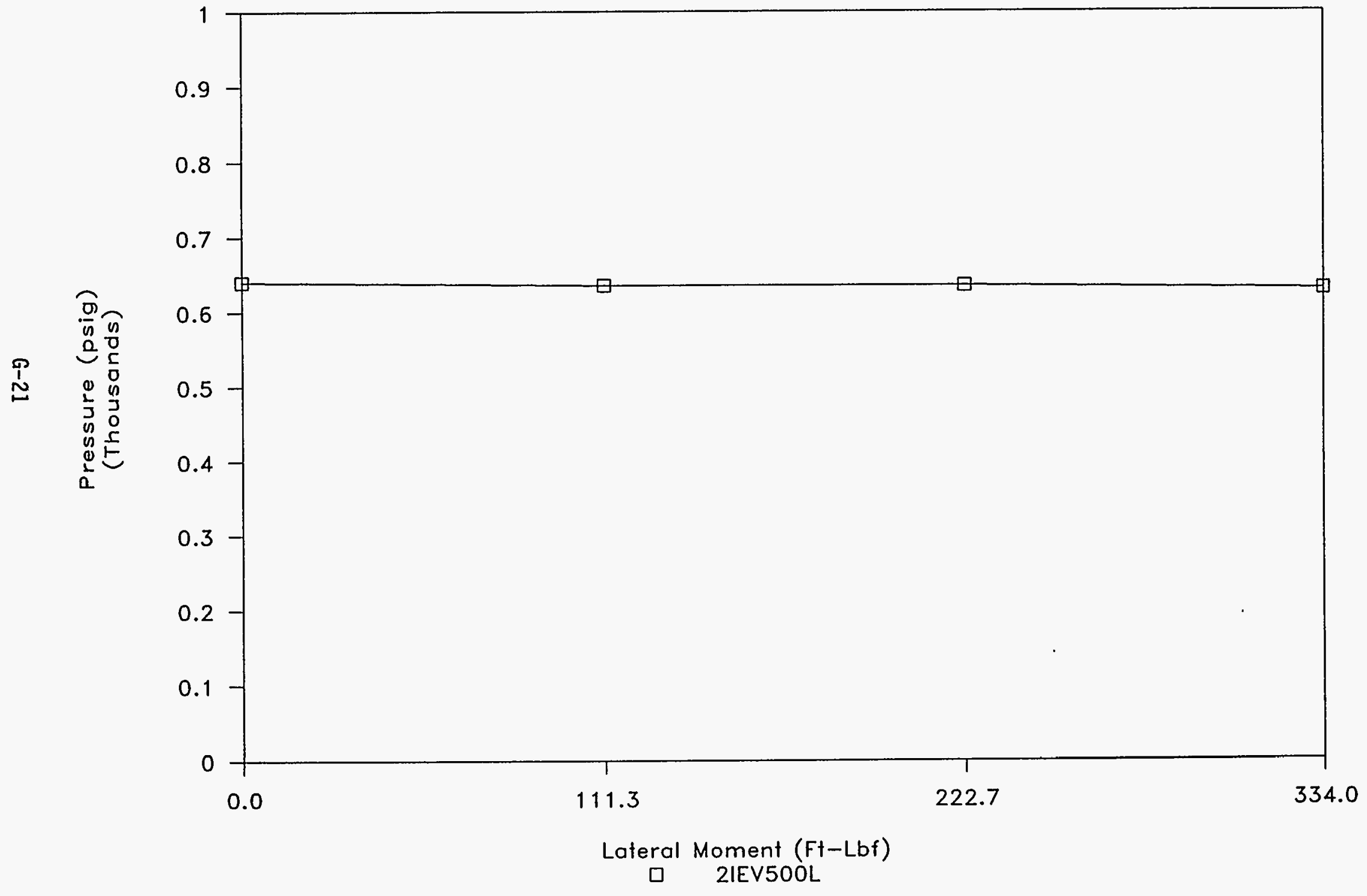




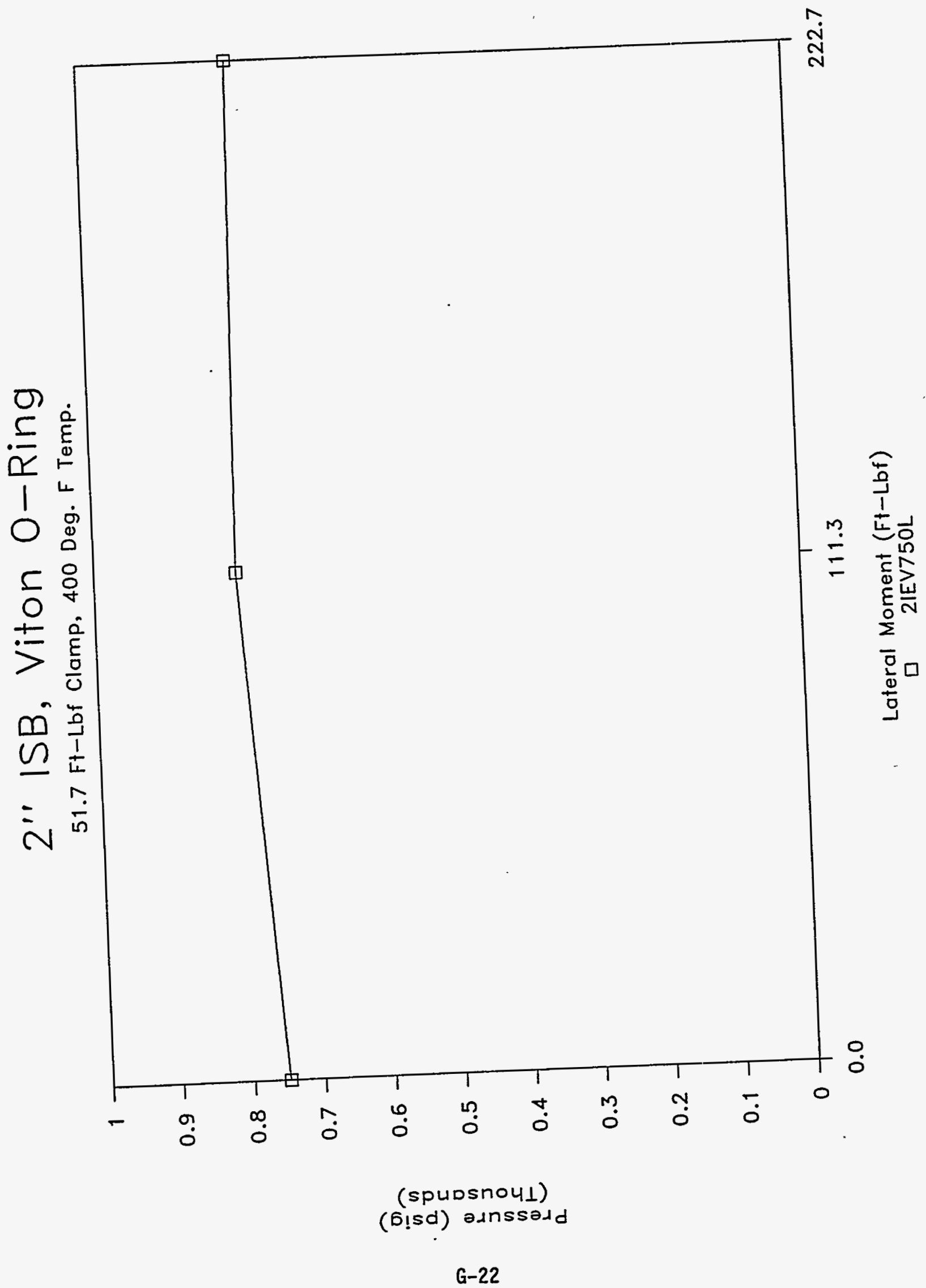




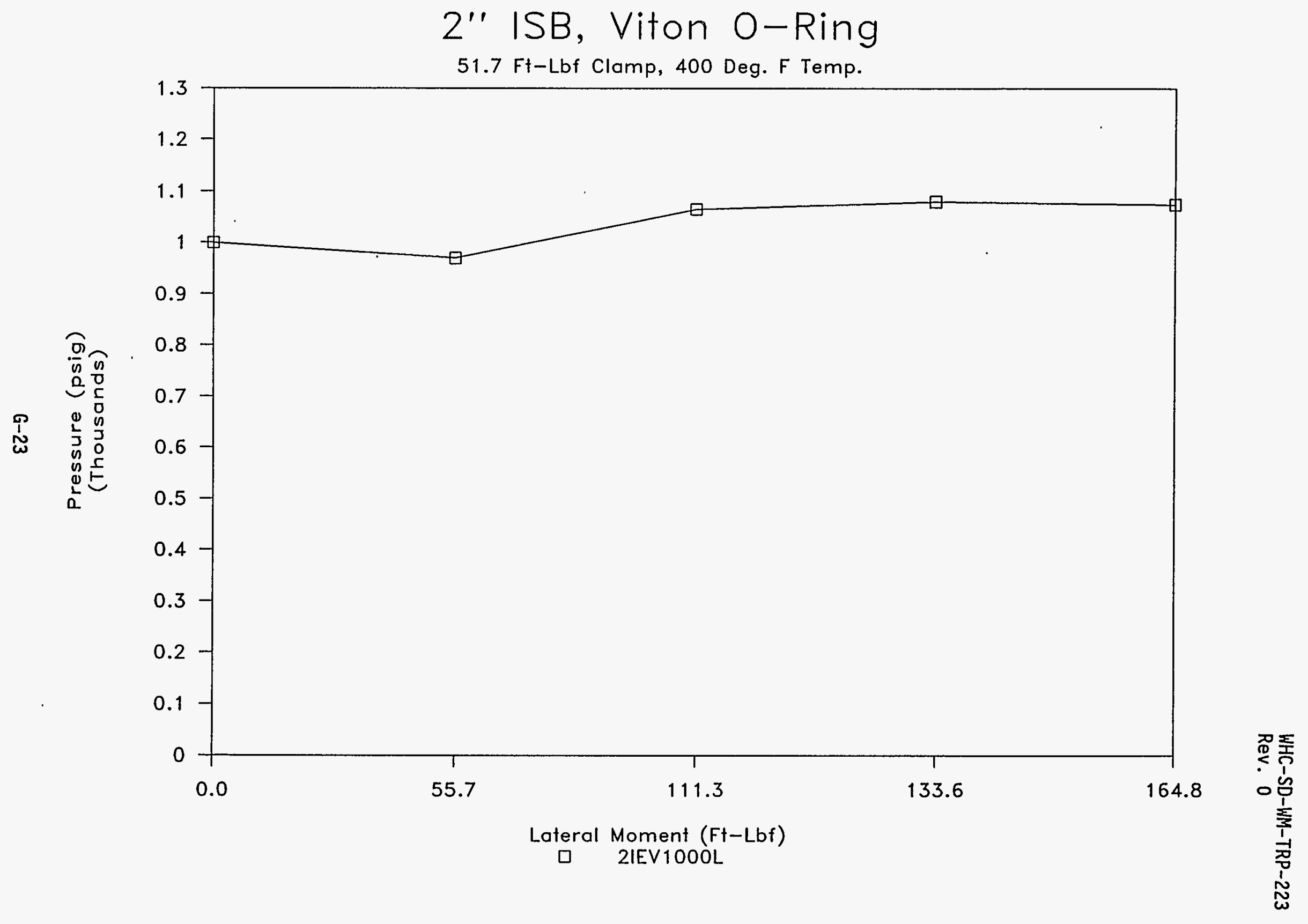


AUGUST 01, 1994

2" ISB CONNECTOR, VITON O-RING, 70 DUROMETER, ELEVATED TEMP. PRESSURE ASCENSION LEAK TEST - IATERAI MOMENT (SIDEWAYS ROTATION) CLAMPING TORQUE $=51.7$ FT-IBF CHARGE PRESSURE $=250$ PSIG TEMPERATURE $=400$ DEG. F

GRAPH NAME $=2$ IEV250L

$\begin{array}{lllllll} & & & & & \text { I.ATERAI } \\ \text { PRESSURE } & \text { ELAPSED } & \text { ELAPSED } & \text { ELAPSED } & \text { LATERAL } & \text { MOMENT } & \text { ILATERAI } \\ \text { PSIG } & \text { MINUTES } & \text { SECONDS } & \text { DECIMAI } & \text { FORCE } & \text { ARM } & \text { MOMENT } \\ & & & \text { MINUTES } & \text { LBS } & \text { FT. } & \text { FT-IBF. } \\ \text { INPUT } & \text { INPUT } & \text { INPUT } & \text { COMPUTE } & \text { INPUT } & \text { INPUT } & \text { COMPUTE }\end{array}$

$\begin{array}{rrrrrrr}315 & 0 & 0 & 0.00 & 0 & 0.4453 & 0.0 \\ 315 & 2 & 4 & 2.07 & 0 & 0.4453 & 0.0 \\ 275 & 4 & 1 & 4.02 & 250 & 0.4453 & 111.3 \\ 295 & 6 & 2 & 6.03 & 500 & 0.4453 & 222.7 \\ 300 & 8 & 2 & 8.03 & 750 & 0.4453 & 334.0 \\ 260 & 10 & 5 & 10.08 & 1000 & 0.4453 & 445.3\end{array}$

INCREASED PRESSURE TO 500 PSIG

$\begin{array}{llll}640 & 12 & 2 & 12.03 \\ 635 & 14 & 4 & 14.07 \\ 635 & 16 & 1 & 16.02 \\ 630 & 18 & 2 & 18.03\end{array}$

INCREASED PRESSURE TO 750 PSIG

$\begin{array}{llll}750 & 20 & 4 & 20.07 \\ 800 & 22 & 1 & 22.02 \\ 790 & 24 & 2 & 24.03\end{array}$

INCREASED PRESSURE TO 1000 PSIG

$\begin{array}{rrrrrrr}1000 & 26 & 2 & 26.03 & 0 & 0.4453 & 0.0 \\ 970 & 28 & 3 & 28.05 & 125 & 0.4453 & 55.7 \\ 1065 & 30 & 2 & 30.03 & 250 & 0.4453 & 111.3 \\ 1080 & 32 & 3 & 32.05 & 300 & 0.4453 & 133.6 \\ 1075 & 34 & 6 & 34.10 & 370 & 0.4453 & 164.8\end{array}$

GRAPH NAME $=2$ IEV5OOL

$\begin{array}{rrr}0 & 0.4453 & 0.0 \\ 250 & 0.4453 & 111.3 \\ 500 & 0.4453 & 222.7 \\ 750 & 0.4453 & 334.0\end{array}$

GRAPH NAME $=2$ IEV750L

$\begin{array}{rrr}0 & 0.4453 & 0.0 \\ 250 & 0.4453 & 111.3 \\ 500 & 0.4453 & 222.7\end{array}$

GRAPH NAME $=2$ IEV1000I 


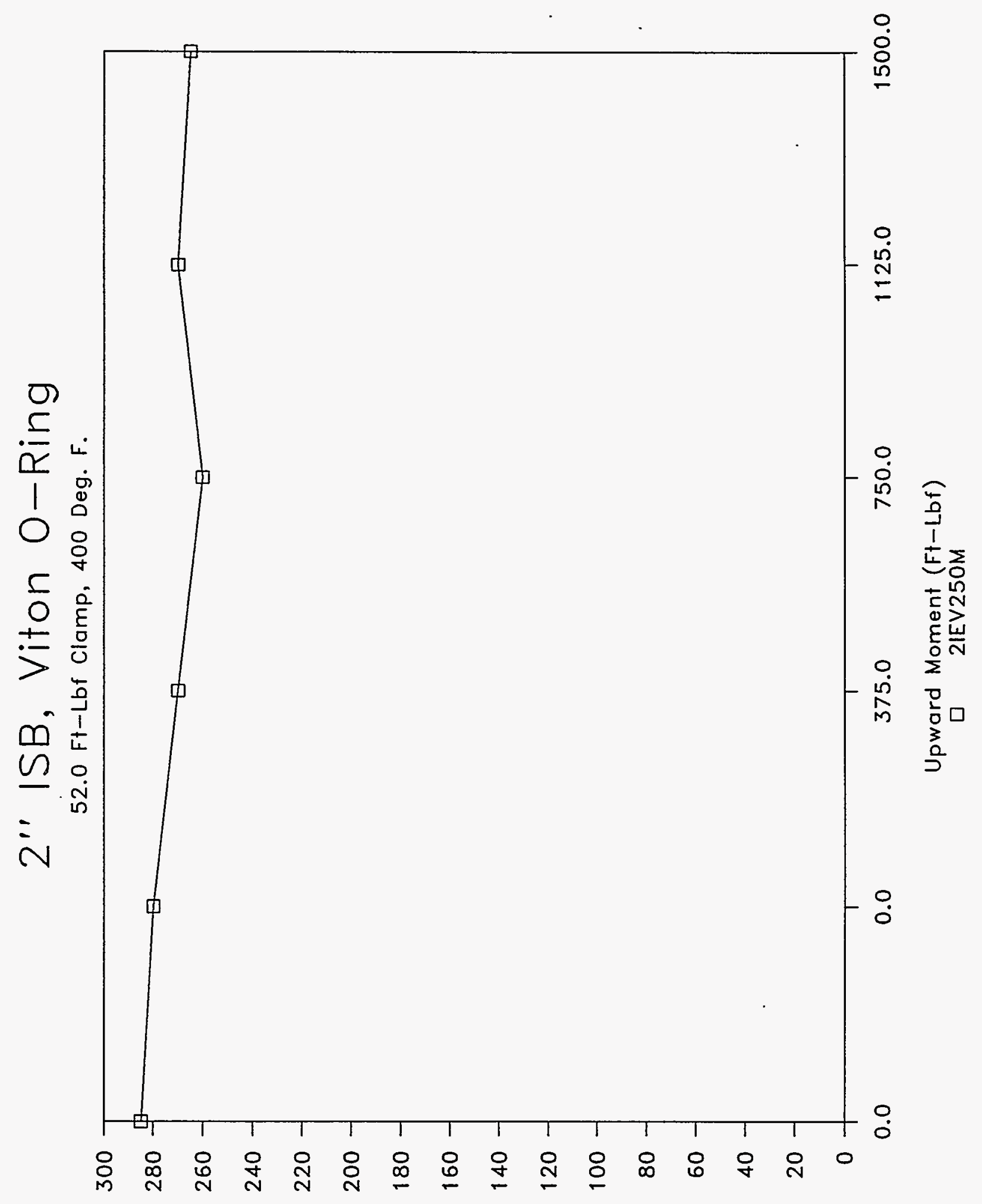

(bisd) adnssadd 
WHC-SD-WM-TRP-223

Rev. 0

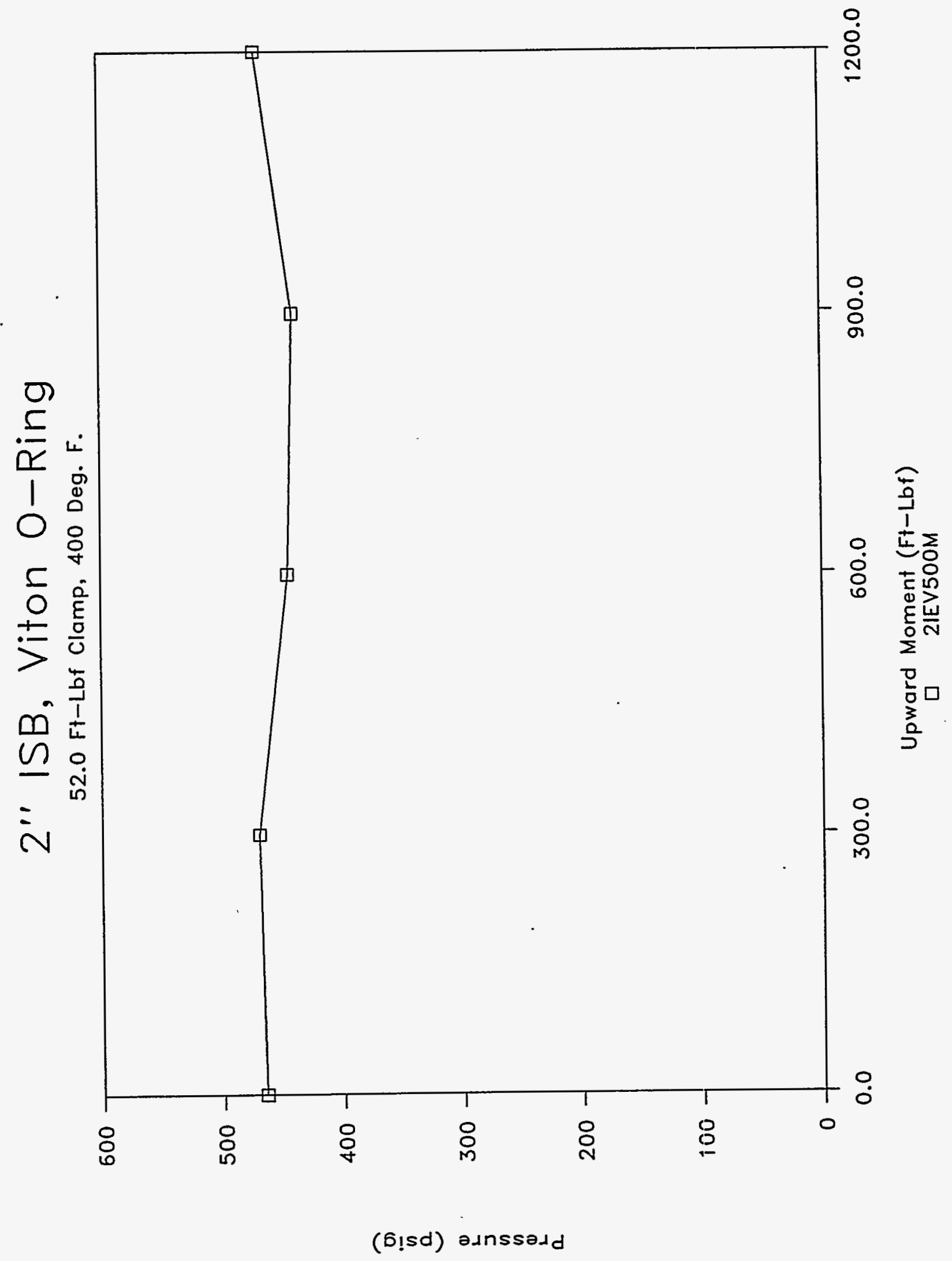




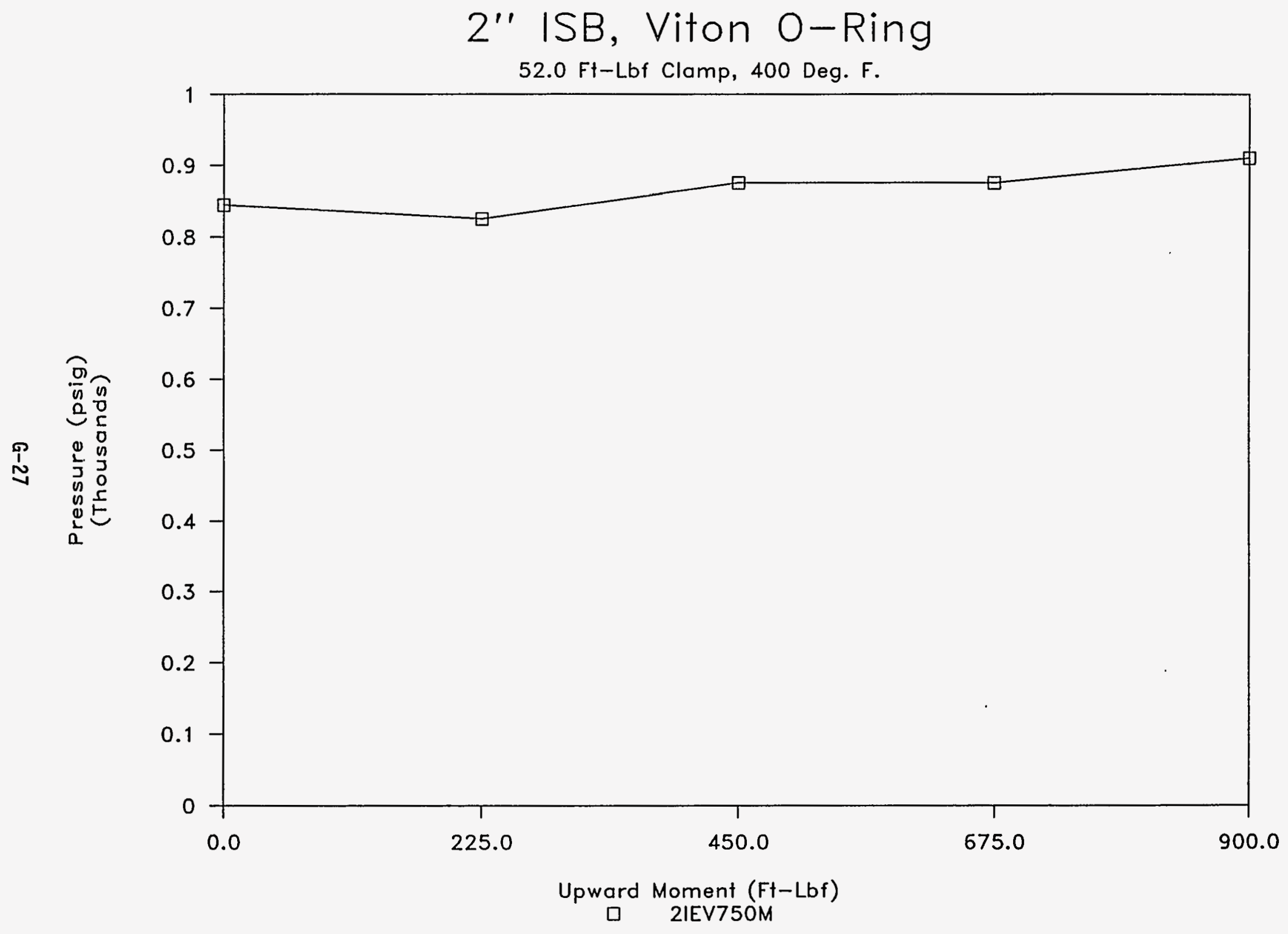


WHC-SD-WM-TRP-223

Rev. 0

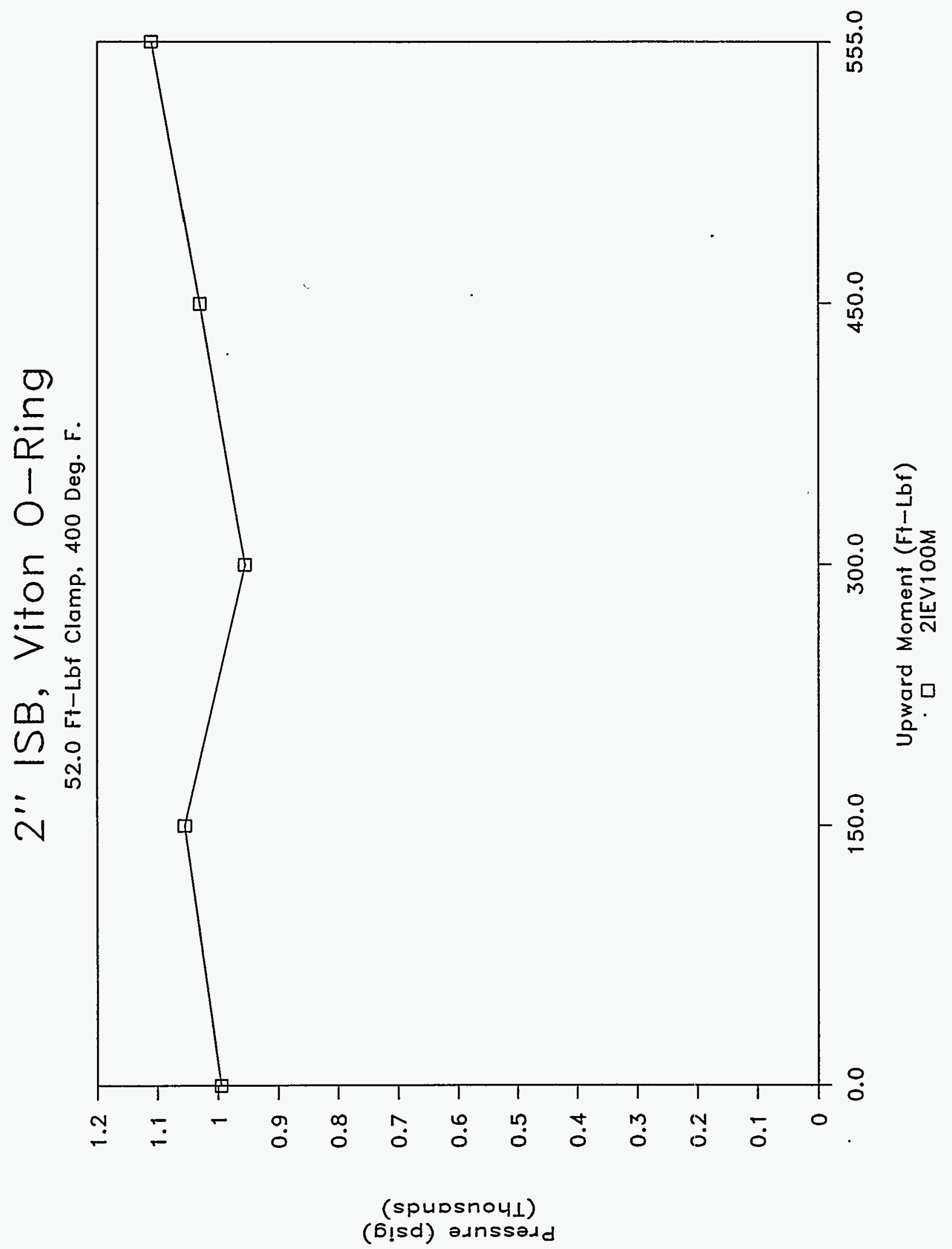


NOVEMBER 14,1994

$2 "$ ISB CONNECTOR, VITON O-RING, 70 DUROMETER, ELEVATED TEMP.

PART \# 2-142 V884-75, PARKER SEAL CO., BATCH 311986, CURE DATE 3Q93

IEAK TEST - UP AND OVER MOMENT (UPWARD ROTATION)

CLAMPING TORQUE $=52.0$ FT-LBF

CHARGE PRESSURE $=250$ PSIG
GRAPH NAME $=2$ IEV250M

TEMPERATURE: 400 DEG. F.

\begin{tabular}{|c|c|c|c|c|c|c|}
\hline \multirow{3}{*}{$\begin{array}{l}\text { PRESSURE } \\
\text { PSIG }\end{array}$} & \multirow{2}{*}{$\begin{array}{l}\text { ELAPSED } \\
\text { MINUTES }\end{array}$} & \multirow[b]{2}{*}{ ELAPSED } & \multirow[b]{2}{*}{ ELAPSED } & \multicolumn{3}{|c|}{ UPWARD } \\
\hline & & & & UPWARD & MOMENT & UPWARD \\
\hline & MINUTES & SECONDS & $\begin{array}{l}\text { DECIMAL } \\
\text { MINUTES }\end{array}$ & $\begin{array}{l}\text { FORCE } \\
\text { LBS }\end{array}$ & $\begin{array}{l}\text { ARM } \\
\text { FT. }\end{array}$ & $\begin{array}{l}\text { MOMENT } \\
\text { FT-LBF. }\end{array}$ \\
\hline NPUT & INPUT & INPUT & COMPUTE & INPUT & INPUT & COMPUTE \\
\hline 285 & 0 & S & 0.00 & 0 & 1.5000 & 0.0 \\
\hline 280 & 2 & 1 & 2.02 & 0 & 1. & 0.0 \\
\hline 270 & 4 & $\mathrm{~J}$ & 4.02 & 250 & 1.5 & 375.0 \\
\hline 260 & 6 & 2 & 6.03 & 500 & 1.5000 & 750.0 \\
\hline 270 & 8 & c & 8.00 & 750 & 1.5000 & 1125.0 \\
\hline 265 & 10 & 1 & 10.02 & 1000 & 1.5000 & 1500.0 \\
\hline
\end{tabular}

INCREASED PRESSURE TO 500 PSIG

GRAPH NAME $=2$ IEV5OOM

$\begin{array}{rrrrrrr}465 & 12 & 2 & 12.03 & 0 & 1.5000 & 0.0 \\ 470 & 14 & 1 & 14.02 & 200 & 1.5000 & 300.0 \\ 445 & 16 & 2 & 16.03 & 400 & 1.5000 & 600.0 \\ 440 & 18 & 3 & 18.05 & 600 & 1.5000 & 900.0 \\ 470 & 20 & 3 & 20.05 & 800 & 1.5000 & 1200.0\end{array}$

INCREASED PRESSURE TO "750" PSIG GRAPH NAME = 2IEV750M

$\begin{array}{rrrrrrr}845 & 22 & 1 & 22.02 & 0 & 1.5000 & 0.0 \\ 825 & 24 & 2 & 24.03 & 150 & 1.5000 & 225.0 \\ 875 & 26 & 2 & 26.03 & 300 & 1.5000 & 450.0 \\ 875 & 28 & 1 & 28.02 & 450 & 1.5000 & 675.0 \\ 910 & 30 & 2 & 30.03 & 600 & 1.5000 & 900.0\end{array}$

INCREASED PRESSURE TO 1000 PSIG

$\begin{array}{rrrrrrr}995 & 32 & 3 & 32.05 & 0 & 1.5000 & 0.0 \\ 1055 & 34 & 1 & 34.02 & 100 & 1.5000 & 150.0 \\ 955 & 36 & 2 & 36.03 & 200 & 1.5000 & 300.0 \\ 1030 & 38 & 1 & 38.02 & 300 & 1.5000 & 450.0 \\ 1110 & 40 & 3 & 40.05 & 370 & 1.5000 & 555.0\end{array}$


WHC-SD-WM-TRP-223

Rev. 0

APPENDIX H: GRAPHS OF 3-IN. FLUOROSILICONE: TESTS

$\mathrm{H}-1$ 


\section{3" ISB, $70 \mathrm{SH}$ Fluorosilicone O-Ring}

50.6 Ft-Lbf Clamp, Ambient Temp.

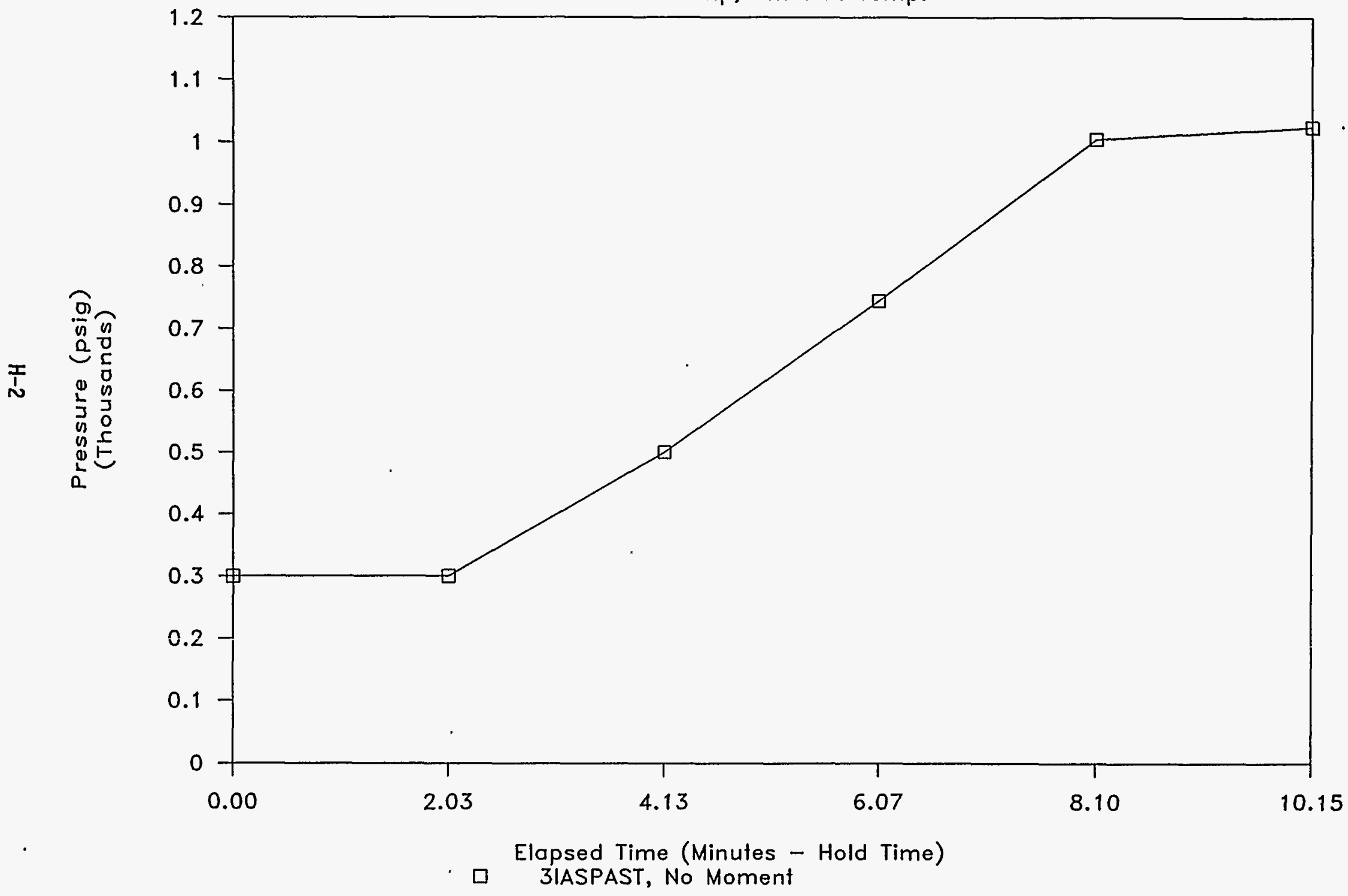


WHC-SD-WM-TRP-223 Rev 0

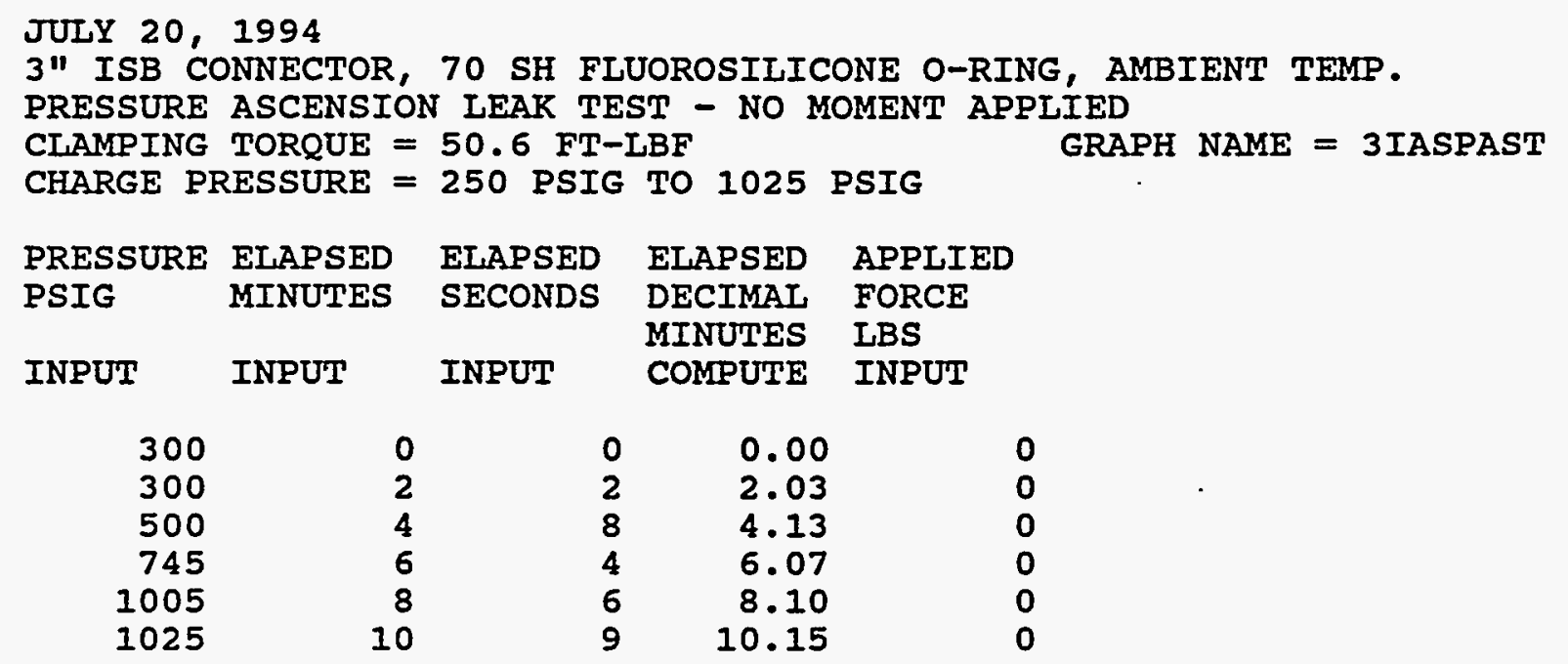


WHC-SD-WM-TRP-223

Rev. 0

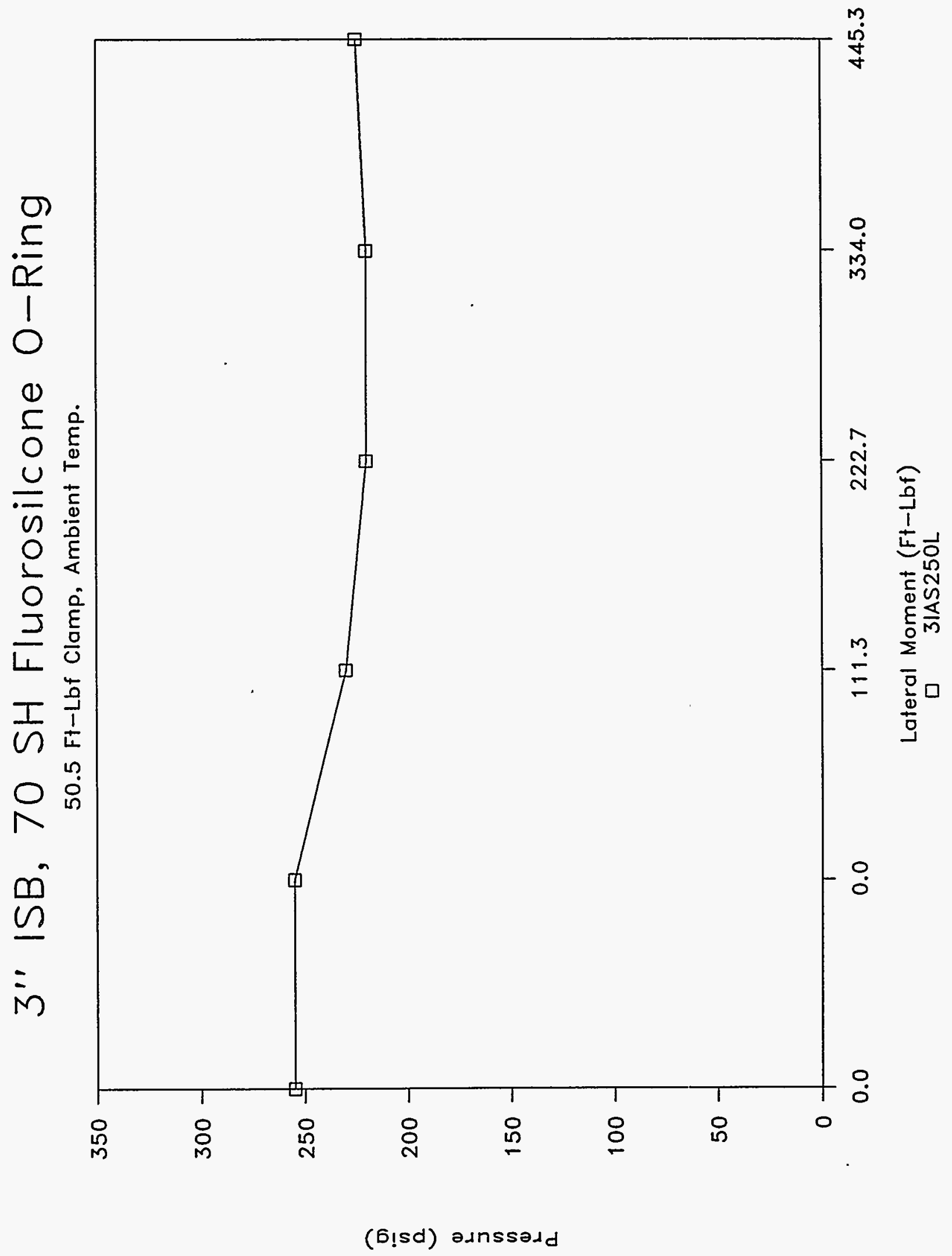

H-4 
WHC-SD-HM-TRP-223

Rev. 0

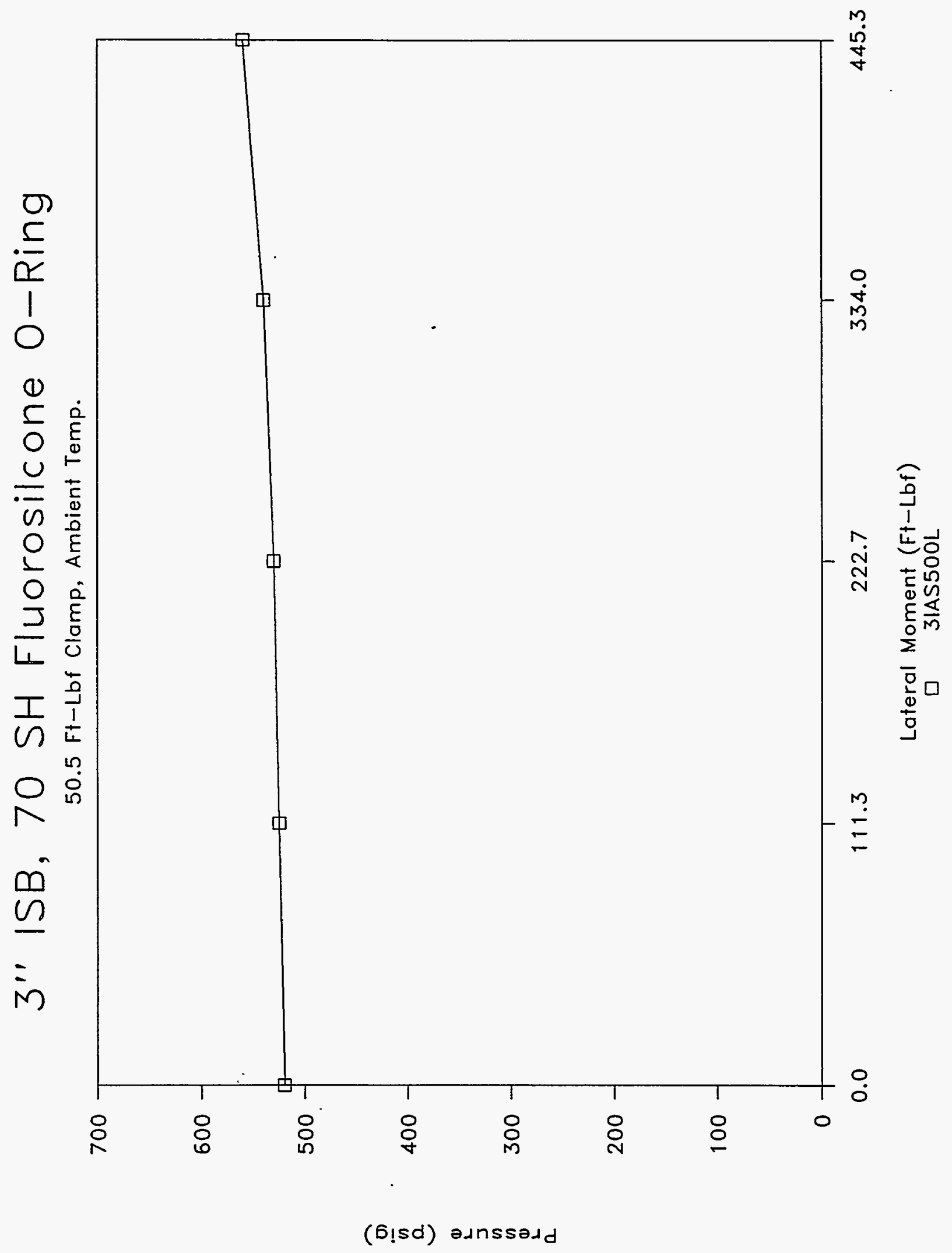

H-5 


\section{3" ISB, 70 SH Fluorosilcone O-Ring}

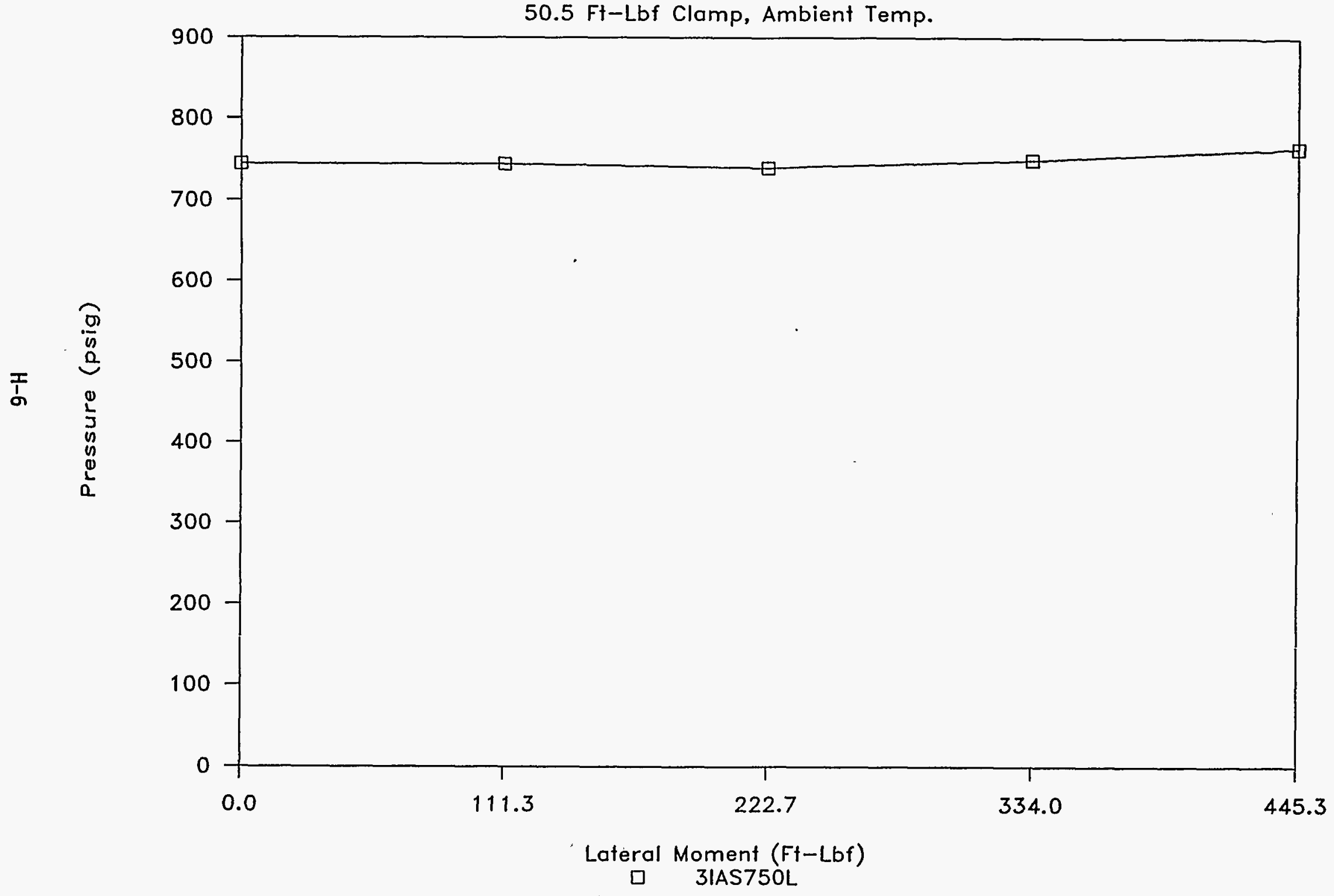


WHC-SD-WM-TRP-223

Rev. 0

$m$
in

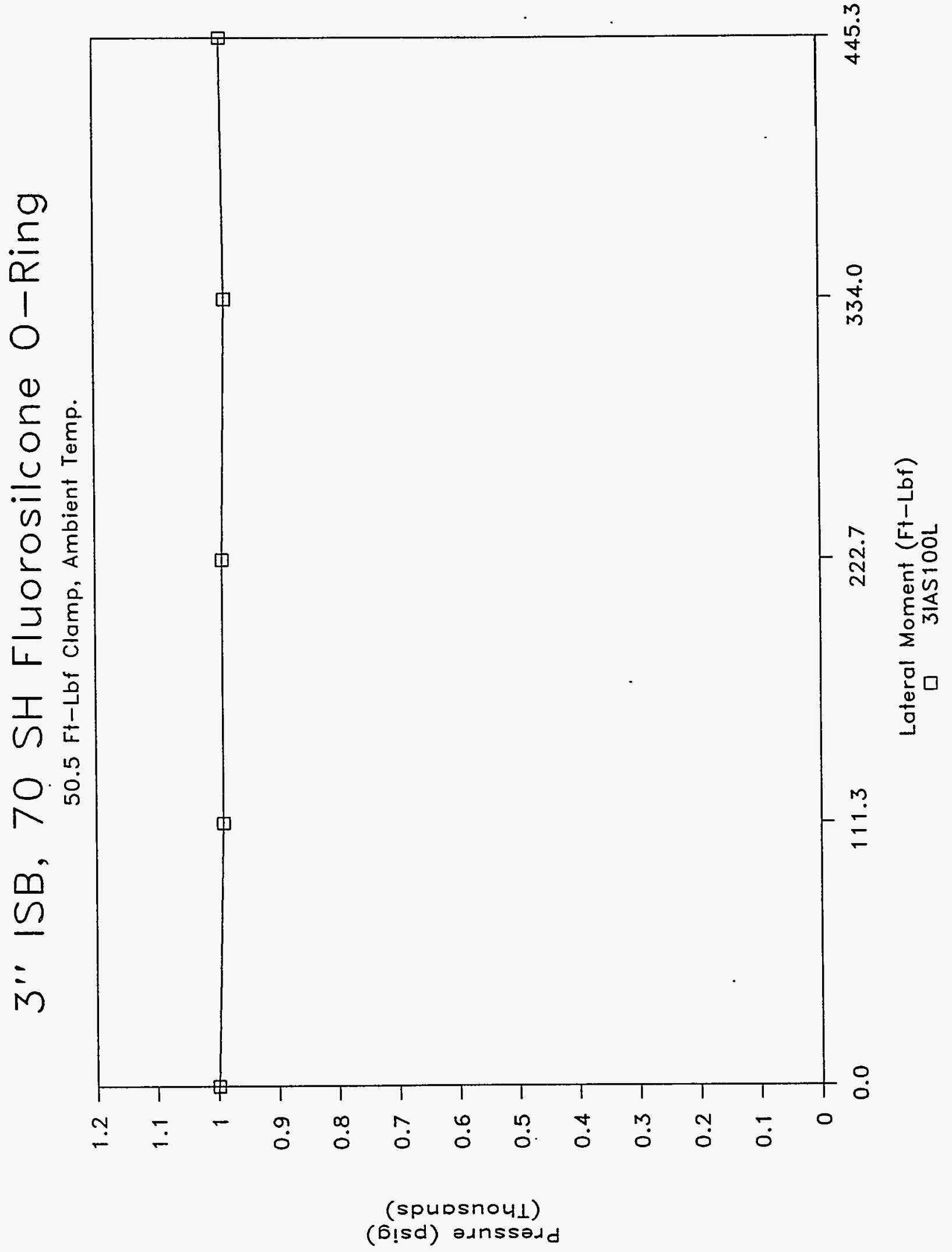


JULY 20, 1994

3" ISB CONNECTOR, 70 SH FLUOROSIIICONE O-RING, AMEIENT TEMP. PRESSURE ASCENSION LEAK TEST - LATERAL MOMENT (SIIEWAYS ROTATION) CLAMPING TORQUE $=52.8$ FT-LBF CHARGE PRESSURE $=250$ PSIG

$\begin{array}{lllllll} & & & & & \text { LATEFAL } \\ \text { PRESSURE } & \text { ELAPSED } & \text { ELAPSED } & \text { ELAPSED } & \text { LATERAI } & \text { MOMENT } & \text { LATERAI } \\ \text { PSIG } & \text { MINUTES } & \text { SECONDS } & \text { DECIMAL } & \text { FORCE } & \text { ARM } & \text { MOMENT } \\ & & & \text { MINUTES } & \text { LBS } & \text { FT. } & \text { FT-IBF, } \\ \text { INPUT } & \text { INPUT } & \text { INPUT } & \text { COMPUTE } & \text { INPUT } & \text { INPUI? } & \text { COMPUTE }\end{array}$

$\begin{array}{rrrrrrr}255 & 0 & 0 & 0.00 & 0 & 0.4453 & 0.0 \\ 255 & 2 & 2 & 2.03 & 0 & 0.4453 & 0.0 \\ 230 & 4 & 3 & 4.05 & 250 & 0.4453 & 111.3 \\ 220 & 6 & 4 & 6.07 & 500 & 0.4453 & 222.7 \\ 220 & 8 & 10 & 8.17 & 750 & 0.4453 & 334.0 \\ 225 & 10 & 8 & 10.13 & 1000 & 0.4453 & 445.3\end{array}$

INCREASED PRESSURE TO 500 PSIG

$\begin{array}{rrrr}520 & 12 & 2 & 12.03 \\ 525 & 14 & 9 & 14.15 \\ 530 & 16 & 13 & 16.22 \\ 540 & 18 & 5 & 18.08 \\ 560 & 20 & 3 & 20.05\end{array}$

INCREASED PRESSURE TO 750 PSIG

$\begin{array}{rrrr}745 & 22 & 3 & 22.05 \\ 745 & 24 & 5 & 24.08 \\ 740 & 26 & 9 & 26.15 \\ 750 & 28 & 10 & 28.17 \\ 765 & 30 & 8 & 30.13\end{array}$

INCREASED PRESSURE TO 1000 PSIG
GRAPH NAME = 3IAS500L

$\begin{array}{rrr}0 & 0.4453 & 0.0 \\ 250 & 0.4453 & 111.3 \\ 500 & 0.4453 & 222.7 \\ 750 & 0.4453 & 334.0 \\ 1000 & 0.4453 & 445.3\end{array}$

GRAPH NAME $=3$ IAS750L

$\begin{array}{rrr}0 & 0.4453 & 0.0 \\ 250 & 0.4453 & 111.3 \\ 500 & 0.4453 & 222.7 \\ 750 & 0.4453 & 334.0 \\ 1000 & 0.4453 & 445.3\end{array}$

GRAPH NAME $=3$ IAS10OL

$\begin{array}{rrrrr}11 & 32.18 & 0 & 0.4453 & 0.0 \\ 8 & 34.13 & 250 & 0.4453 & 111.3 \\ 3 & 36.05 & 500 & 0.4453 & 222.7 \\ 6 & 38.10 & 750 & 0.4453 & 334.0 \\ 10 & 40.17 & 1000 & 0.4453 & 445.3\end{array}$




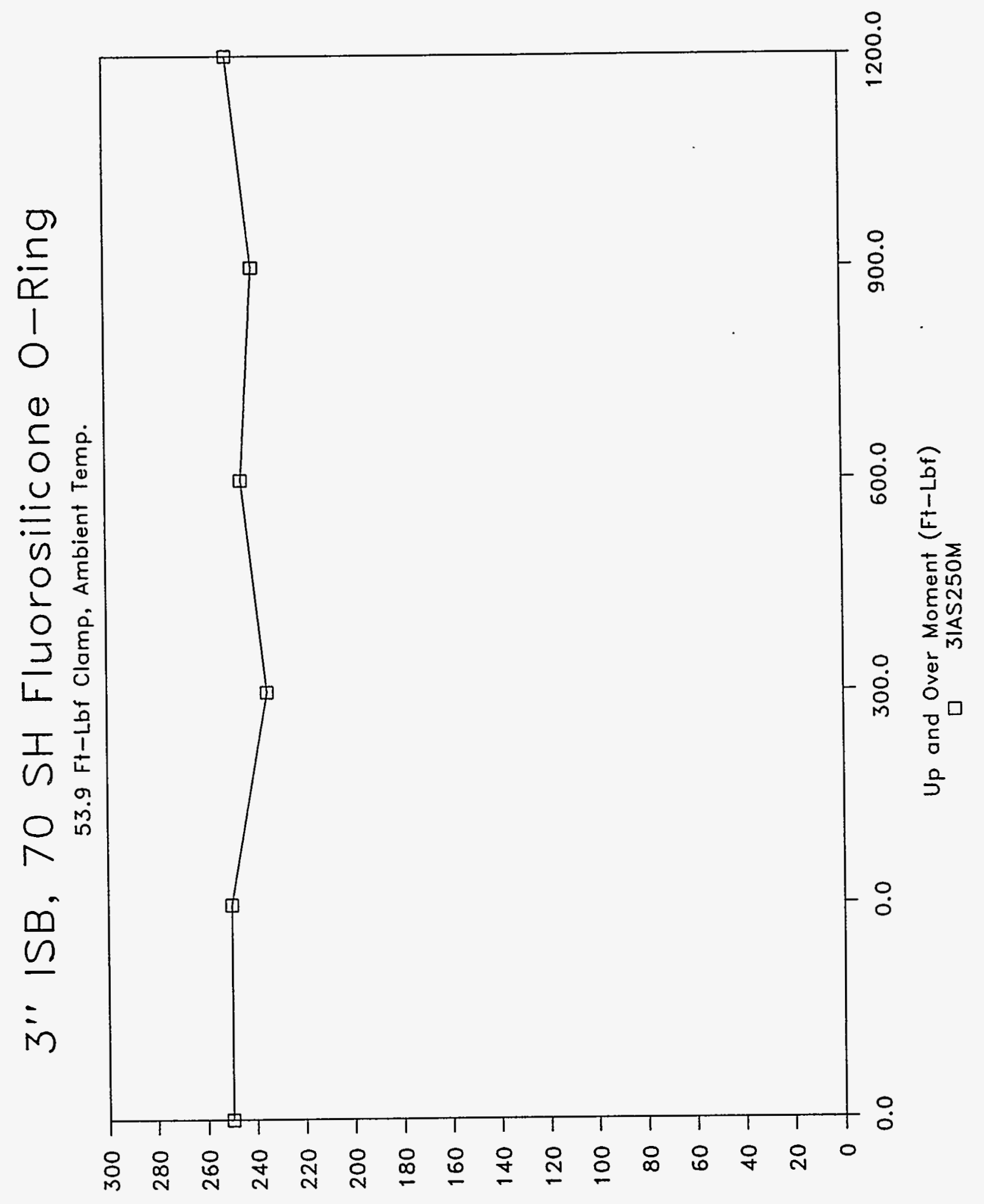

(G!sd) oגnssadd 
HHC-SD-WM-TRP-223

Rev. 0

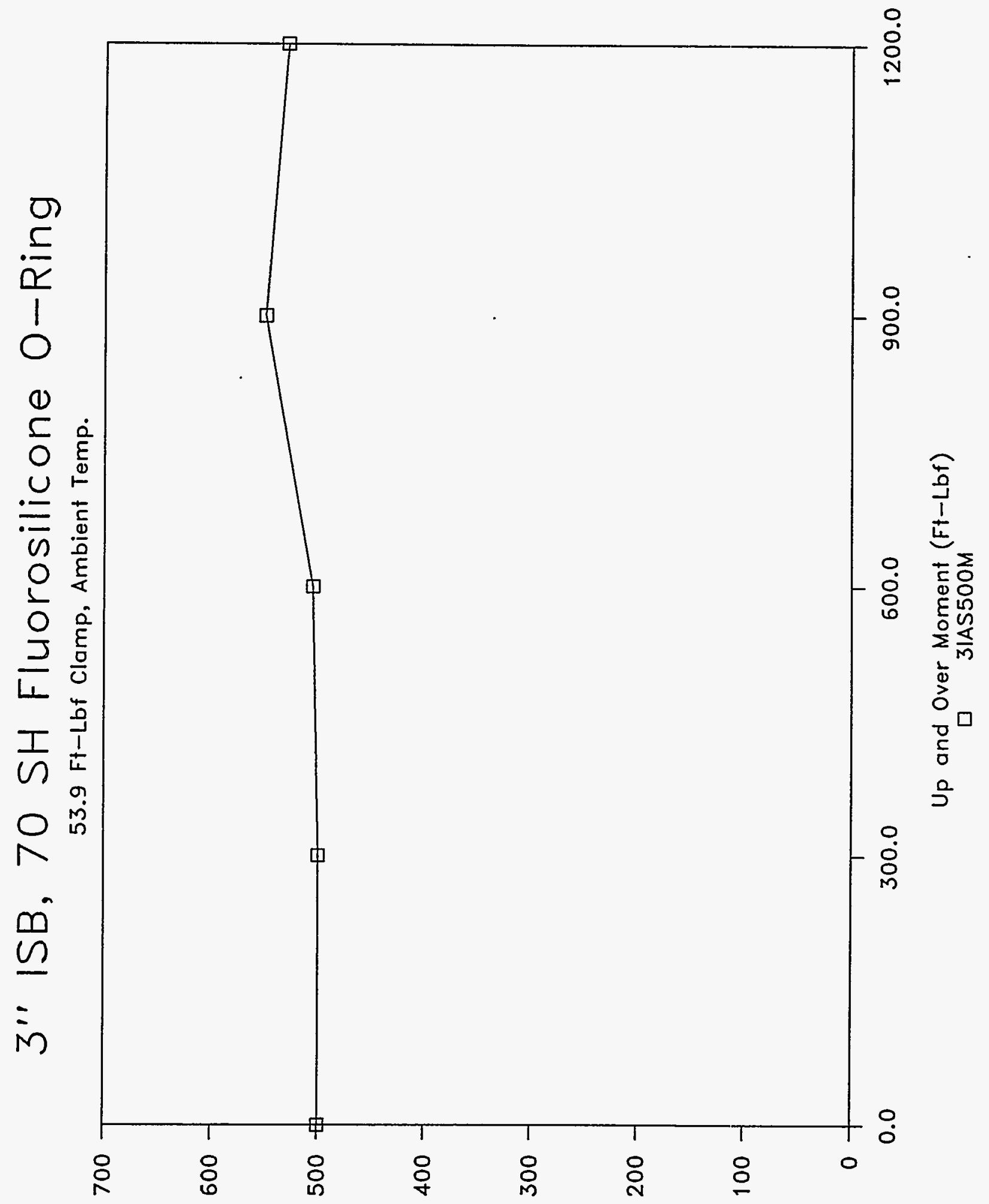

(bisd) ounssadd 
WHC-SD-WM-TRP-223

Rev. 0

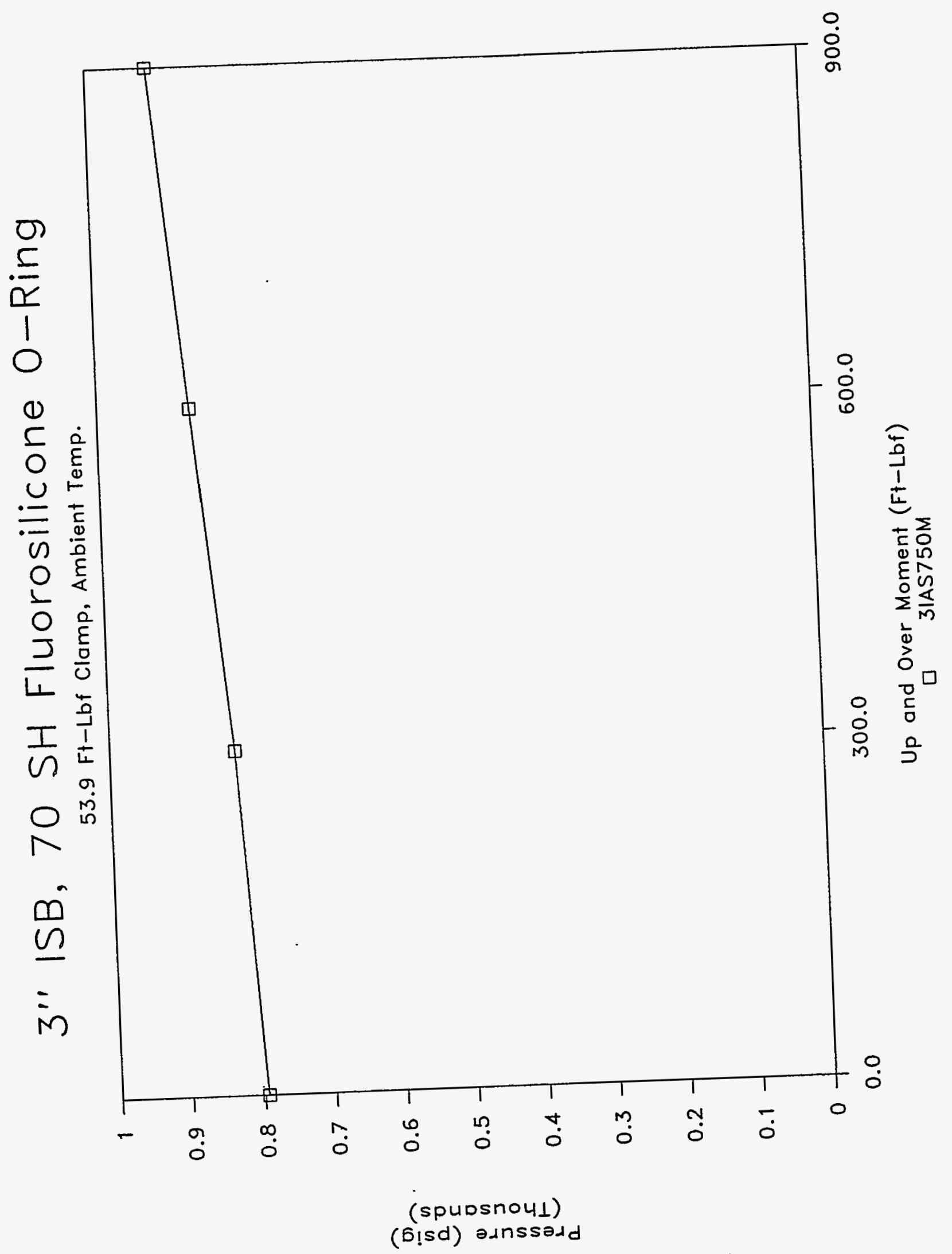

$H-1 I$ 
JUNE 29, 1994

3" ISB CONNECTOR, 70 SH FLUOROSILICONE O-RING, AMBIENT TEMP. PRESSURE ASCENSION LEAK TEST - UP AND OVER MOMENT (UPWARD ROTATION) CLAMPING TORQUE $=53.9$ FT-LBF GRAPH NAME $=3$ IAS250M

CHARGE PRESSURE $=250$ PSIG

$\begin{array}{lllllll} & & & & & \text { UPWARD } & \\ \text { PRESSURE } & \text { ELAPSED } & \text { ELAPSED } & \text { ELAPSED } & \text { UPWARD } & \text { MOMENT } & \text { UPWARD } \\ \text { PSIG } & \text { MINUTES } & \text { SECONDS } & \text { DECIMAL } & \text { FORCE } & \text { ARM } & \text { MOMENT } \\ & & & \text { MINUTES } & \text { LBS } & \text { FT. } & \text { FT-LBF. } \\ \text { INPUT } & \text { INPUT } & \text { INPUT } & \text { COMPUTE } & \text { INPUT } & \text { INPUT } & \text { COMPUTE }\end{array}$

$\begin{array}{rrrrrrr}250 & 0 & 0 & 0.00 & 0 & 1.2000 & 0.0 \\ 250 & 2 & 8 & 2.13 & 0 & 1.2000 & 0.0 \\ 235 & 5 & 3 & 5.05 & 250 & 1.2000 & 300.0 \\ 245 & 8 & 10 & 8.17 & 500 & 1.2000 & 600.0 \\ 240 & 11 & 12 & 11.20 & 750 & 1.2000 & 900.0 \\ 250 & 14 & 6 & 14.10 & 1000 & 1.2000 & 1200.0\end{array}$

INCREASED PRESSURE TO 500 PSIG

GRAPH NAME $=3$ IAS500M

$\begin{array}{llll}500 & 17 & 8 & 17.13 \\ 500 & 20 & 10 & 20.17 \\ 505 & 22 & 12 & 22.20 \\ 550 & 24 & 35 & 24.58 \\ 530 & 26 & 37 & 26.62\end{array}$

INCREASED PRESSURE TO 750 PSIG

$\begin{array}{rrr}0 & 1.2000 & 0.0 \\ 250 & 1.2000 & 300.0 \\ 500 & 1.2000 & 600.0 \\ 750 & 1.2000 & 900.0 \\ 1000 & 1.2000 & 1200.0\end{array}$

GRAPH NAME $=3$ IAS750M

$\begin{array}{rrrrrrr}795 & 28 & 32 & 28.53 & 0 & 1.2000 & 0.0 \\ 825 & 30 & 47 & 30.78 & 250 & 1.2000 & 300.0 \\ 870 & 33 & 13 & 33.22 & 500 & 1.2000 & 600.0 \\ 915 & 35 & 39 & 35.65 & 750 & 1.2000 & 900.0\end{array}$

** ISB LEAKING AT THERMOCOUPLE PORT. TEST TERMINATED. ** 
3" ISB, 70 SH Fluorosilic one O-Ring 52.4 Ft-Lbf Clamp, 300 Deg. F.

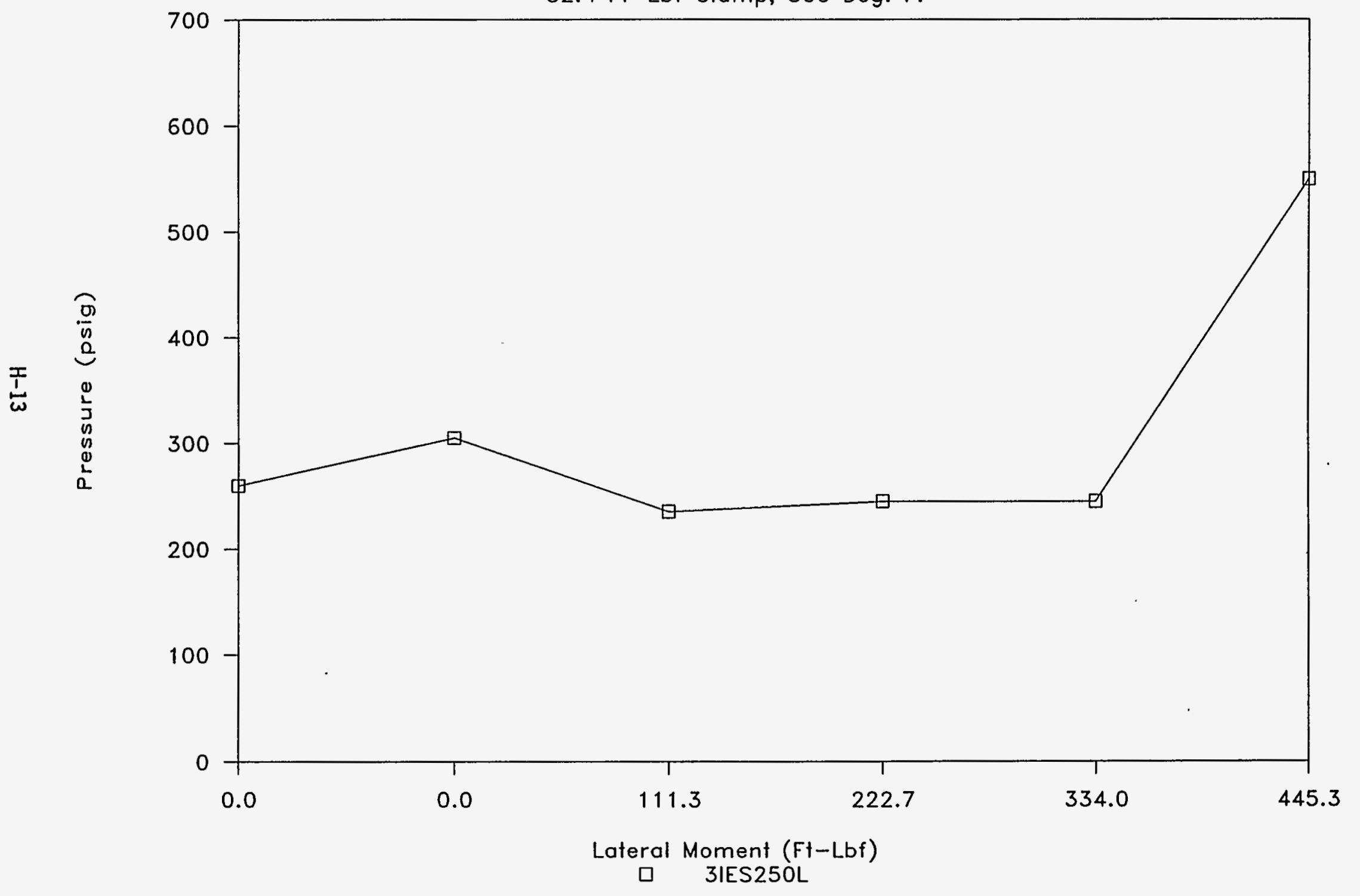




\section{3" ISB, 70 SH Fluorosilicone O-Ring}

52.4 Ft-Lbf Clamp, 300 Deg. F.

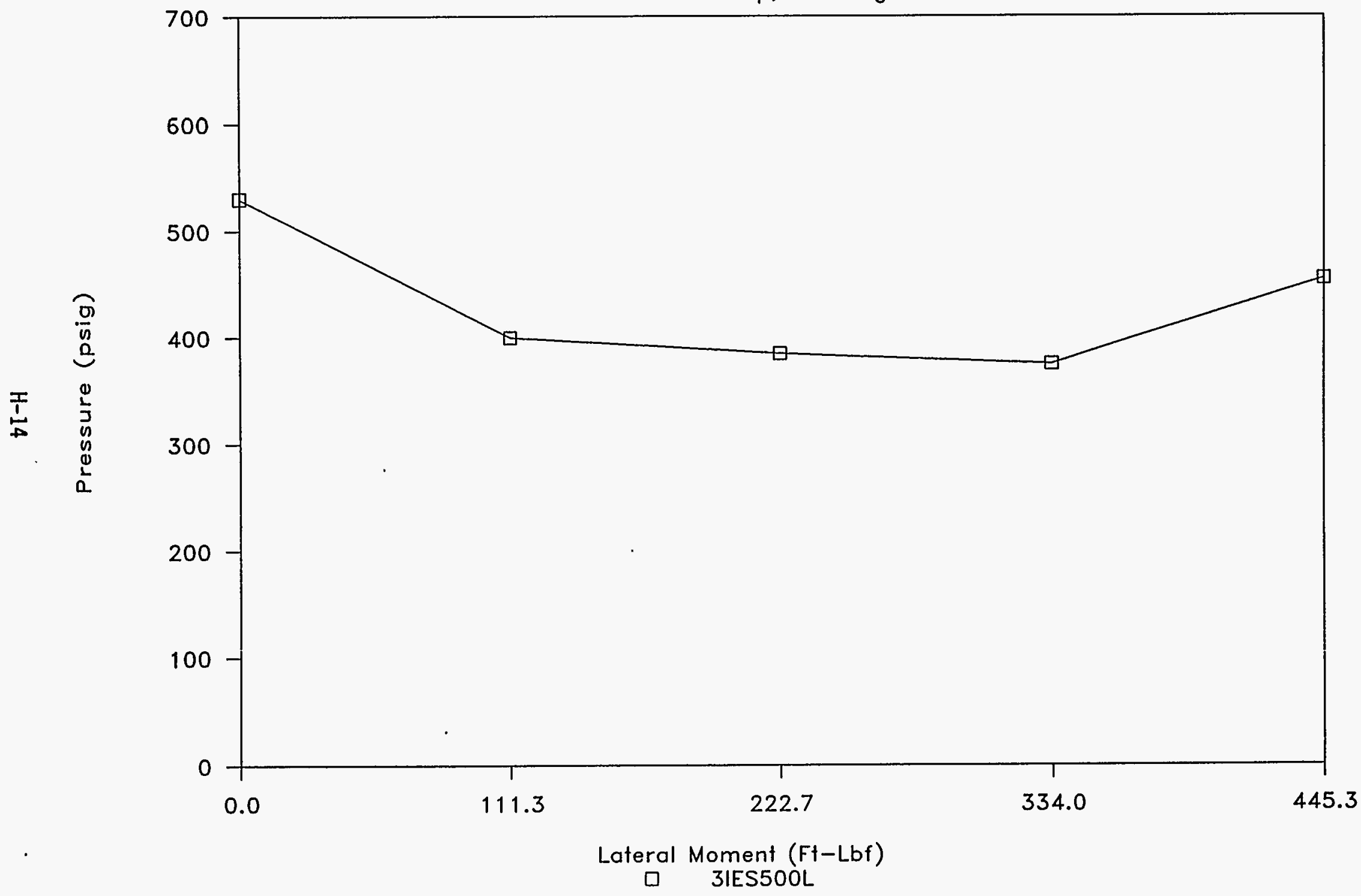


3" ISB, 70 SH Fluorosilicone O-Ring $52.4 \mathrm{Ft}$-Lbf Clamp, 300 Deg. F.

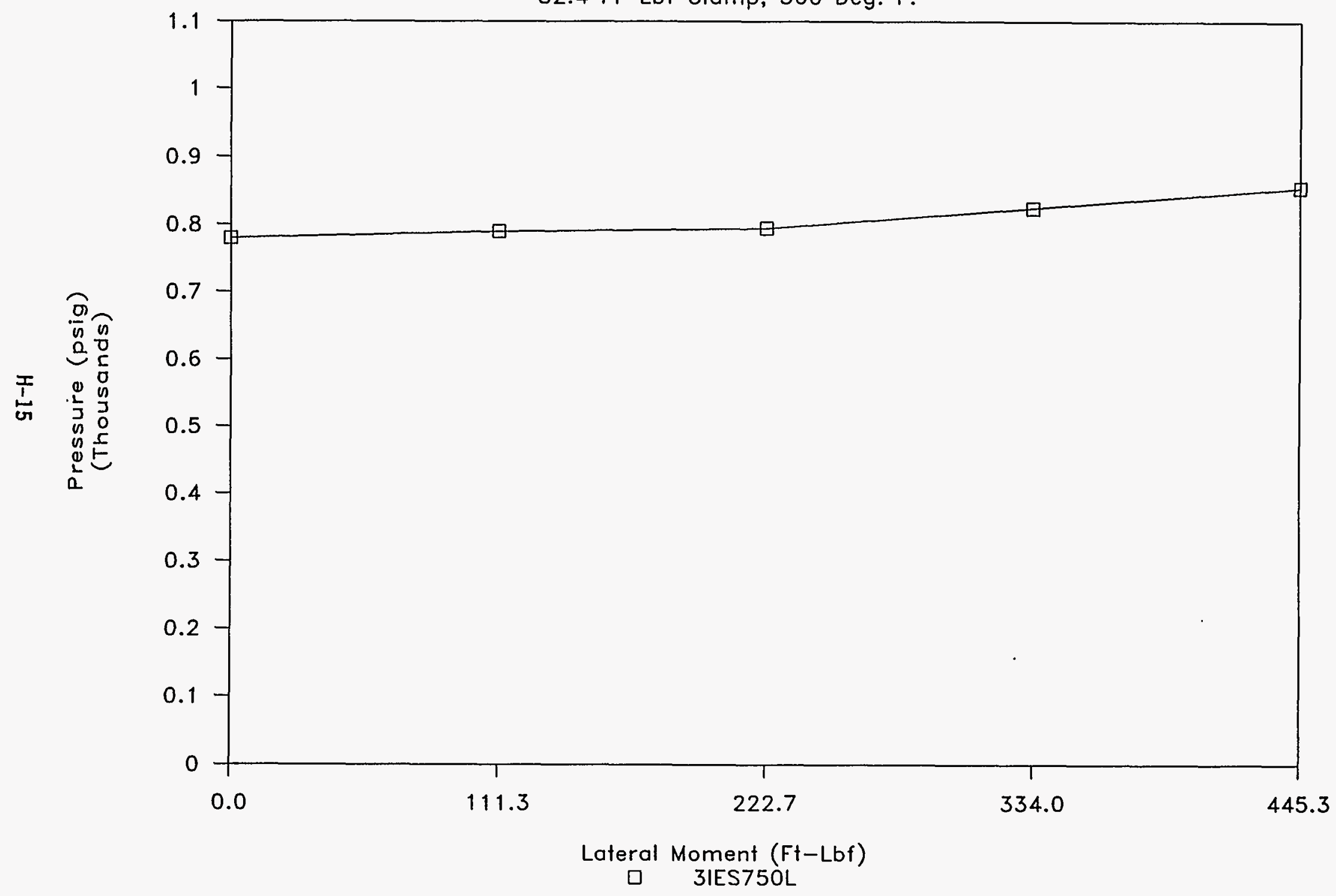




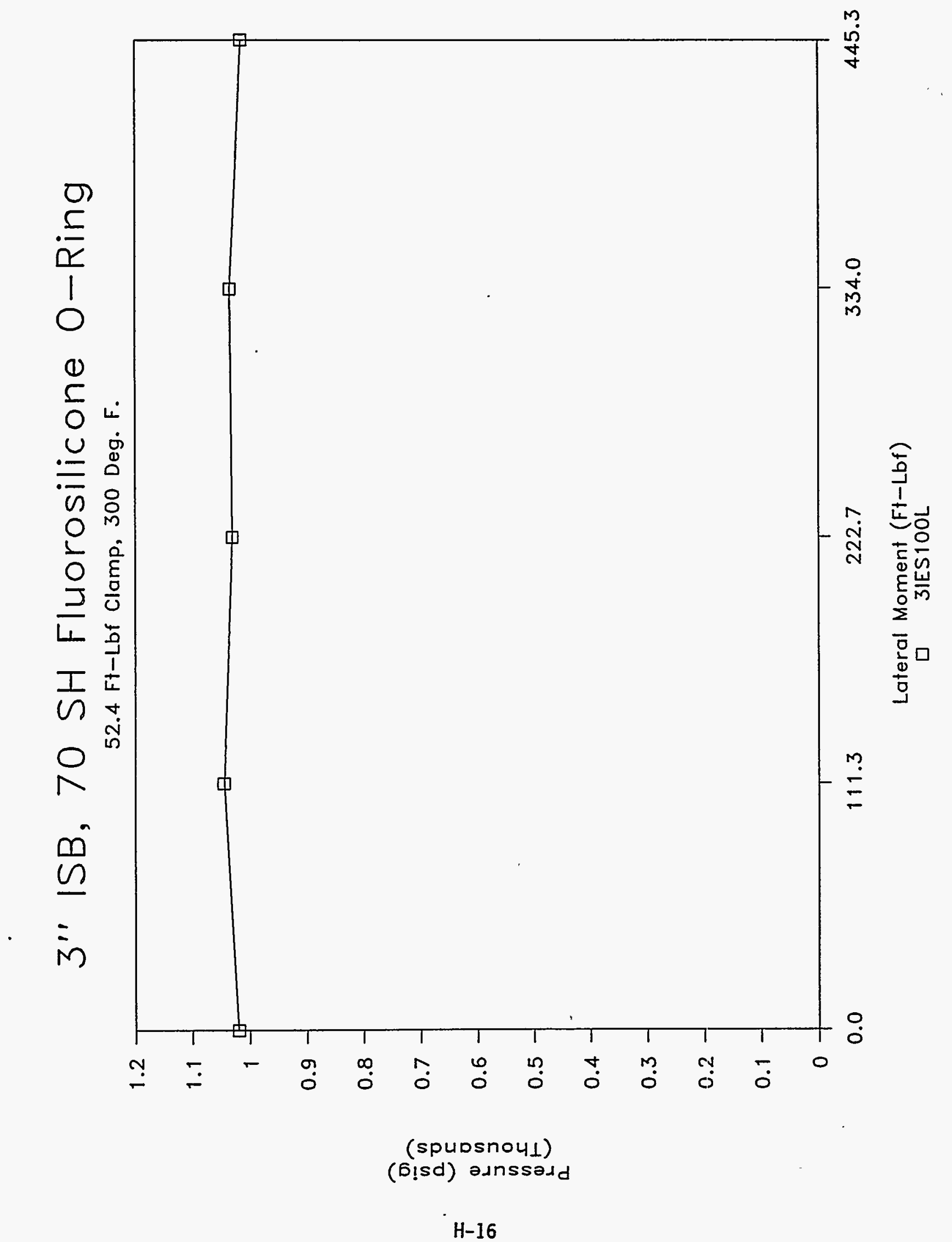


WHC-SD-WM-TRP-223 ReV 0

OCTOBER 31, 1994

3" ISB CONNECTOR, 70 SH FLUOROSILICONE O-RING, ELEVATED TEMP. (300 DEG. F LEAK TEST - LATERAL MOMENT (SIDEWAYS)

CLAMPING TORQUE $=52.4$ FT-IBF GRAPH NAME $=3$ IES25OL

CHARGE PRESSURE $=250$ PSIG

$\begin{array}{lllllll} & & & & \text { IATERAL } \\ \text { PRESSURE } & \text { ELAPSED } & \text { ELAPSED } & \text { ELAPSED } & \text { LATERAI } & \text { MOMENT } & \text { LATERAL } \\ \text { PSIG } & \text { MINUTES } & \text { SECONDS } & \text { DECIMAL } & \text { FORCE } & \text { ARM } & \text { MOMENT } \\ & & & \text { MINUTES } & \text { LBS } & \text { FT. } & \text { FT-LBF. } \\ \text { INPUT } & \text { INPUT } & \text { INPUT } & \text { COMPUTE } & \text { INPUT } & \text { INPUT } & \text { COMPUTE }\end{array}$

$\begin{array}{rrrrrrr}260 & 0 & 0 & 0.00 & 0 & 0.4453 & 0.0 \\ 305 & 2 & 4 & 2.07 & 0 & 0.4453 & 0.0 \\ 235 & 4 & 1 & 4.02 & 250 & 0.4453 & 111.3 \\ 245 & 6 & 3 & 6.05 & 500 & 0.4453 & 222.7 \\ 245 & 8 & 2 & 8.03 & 750 & 0.4453 & 334.0 \\ 550 & 10 & 1 & 10.02 & 1000 & 0.4453 & 445.3\end{array}$

INCREASED PRESSURE TO 500 PSIG

GRAPH NAME $=3$ IES500I

$\begin{array}{rrrrrrr}530 & 12 & 3 & 12.05 & 0 & 0.4453 & 0.0 \\ 400 & 14 & 2 & 14.03 & 250 & 0.4453 & 111.3 \\ 385 & 16 & 4 & 16.07 & 500 & 0.4453 & 222.7 \\ 375 & 18 & 3 & 18.05 & 750 & 0.4453 & 334.0 \\ 455 & 20 & 2 & 20.03 & 1000 & 0.4453 & 445.3\end{array}$

INCREASED PRESSURE TO 750 PSIG

GRAPH NAME $=3$ IES750L

$\begin{array}{rrrrrrr}780 & 22 & 1 & 22.02 & 0 & 0.4453 & 0.0 \\ 790 & 24 & 1 & 24.02 & 250 & 0.4453 & 111.3 \\ 795 & 26 & 2 & 26.03 & 500 & 0.4453 & 222.7 \\ 825 & 28 & 3 & 28.05 & 750 & 0.4453 & 334.0 \\ 855 & 30 & 1 & 30.02 & 1000 & 0.4453 & 445.3\end{array}$

INCREASED PRESSURE TO 1000 PSIG

GRAPH NAME $=3$ IES100L

$\begin{array}{rrrrrrr}1020 & 32 & 3 & 32.05 & 0 & 0.4453 & 0.0 \\ 1045 & 34 & 2 & 34.03 & 250 & 0.4453 & 111.3 \\ 1030 & 36 & 1 & 36.02 & 500 & 0.4453 & 222.7 \\ 1035 & 38 & 2 & 38.03 & 750 & 0.4453 & 334.0 \\ 1015 & 40 & 2 & 40.03 & 1000 & 0.4453 & 445.3\end{array}$


WHC-SD-WM-TRP-223

Rev. 0

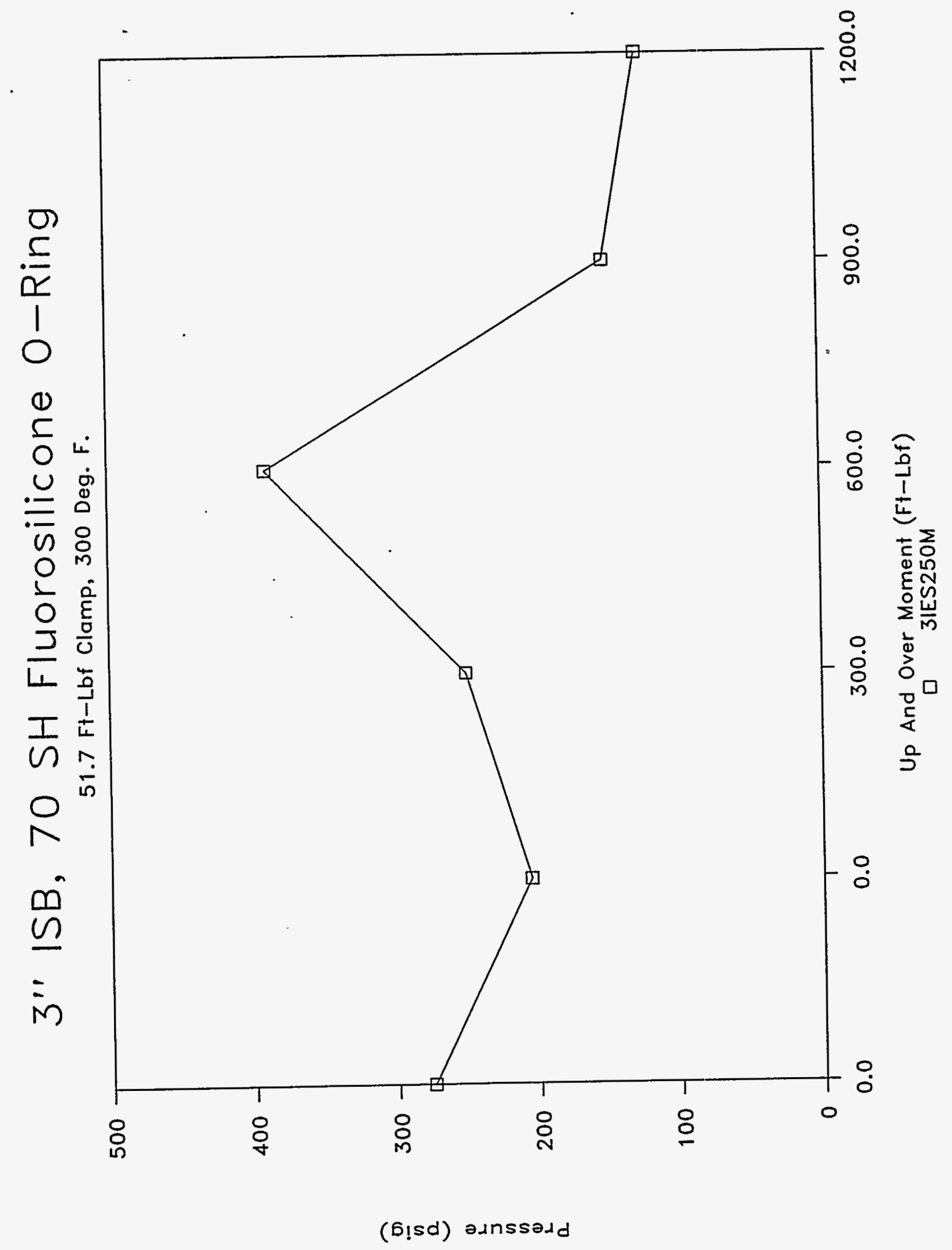

$H-18$ 
WHC-SD-WM-TRP-223

Rev. 0

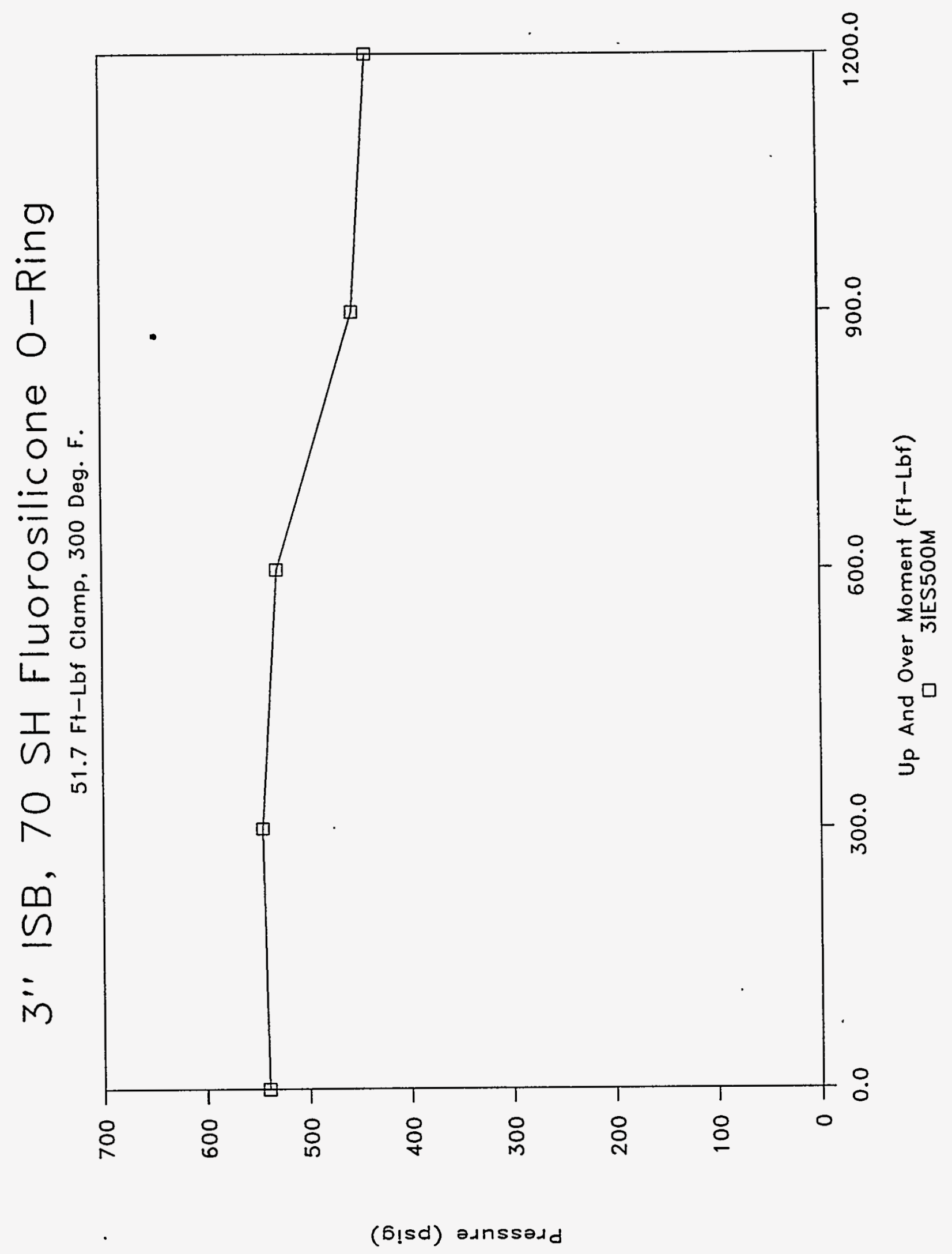




\section{3" ISB, 70 SH Fluorosilicone O-Ring}

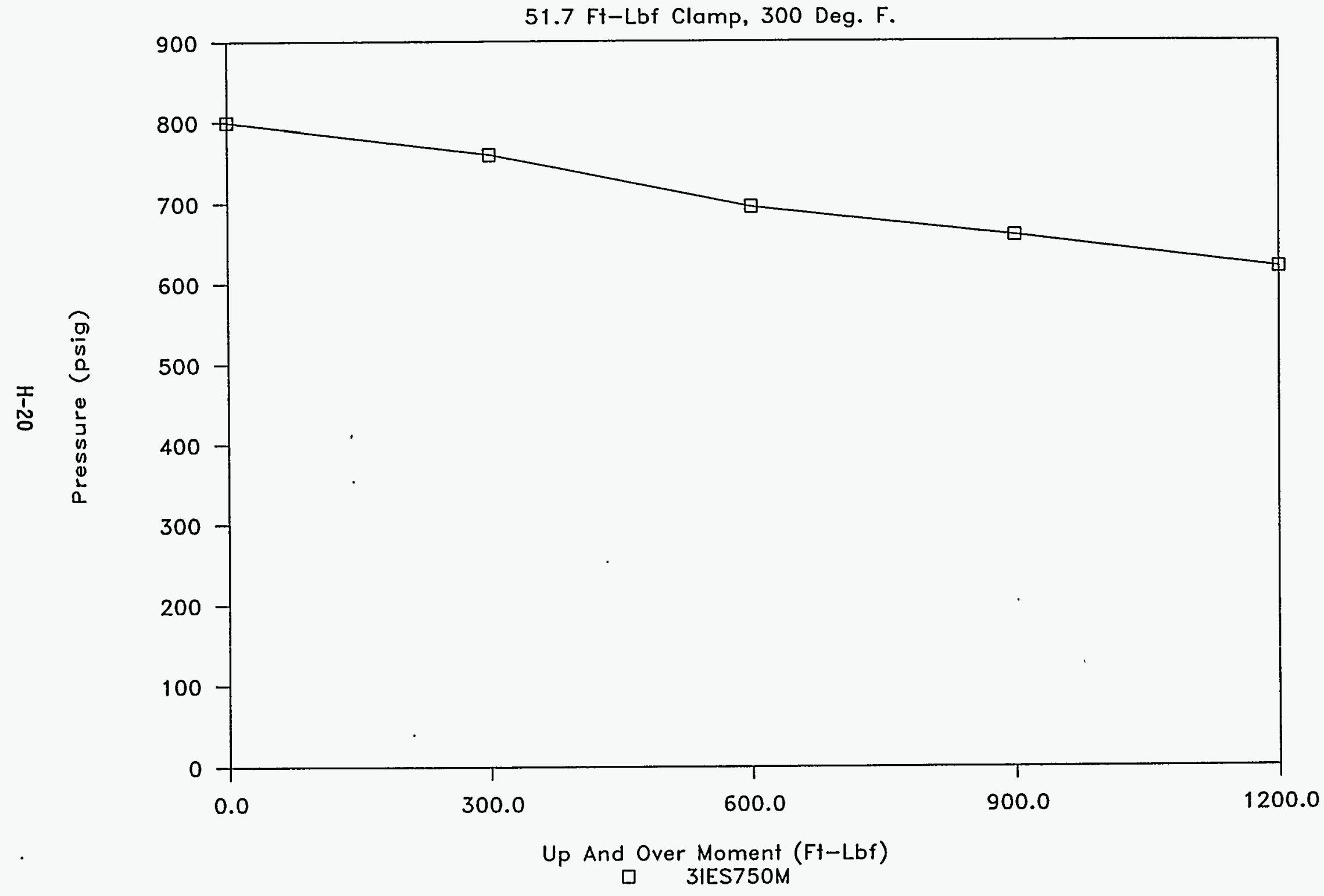




\section{3" ISB, $70 \mathrm{SH}$ Fluorosilicone O-Ring}

$51.7 \mathrm{Ft}$-Lbf Clamp, 300 Deg. F.

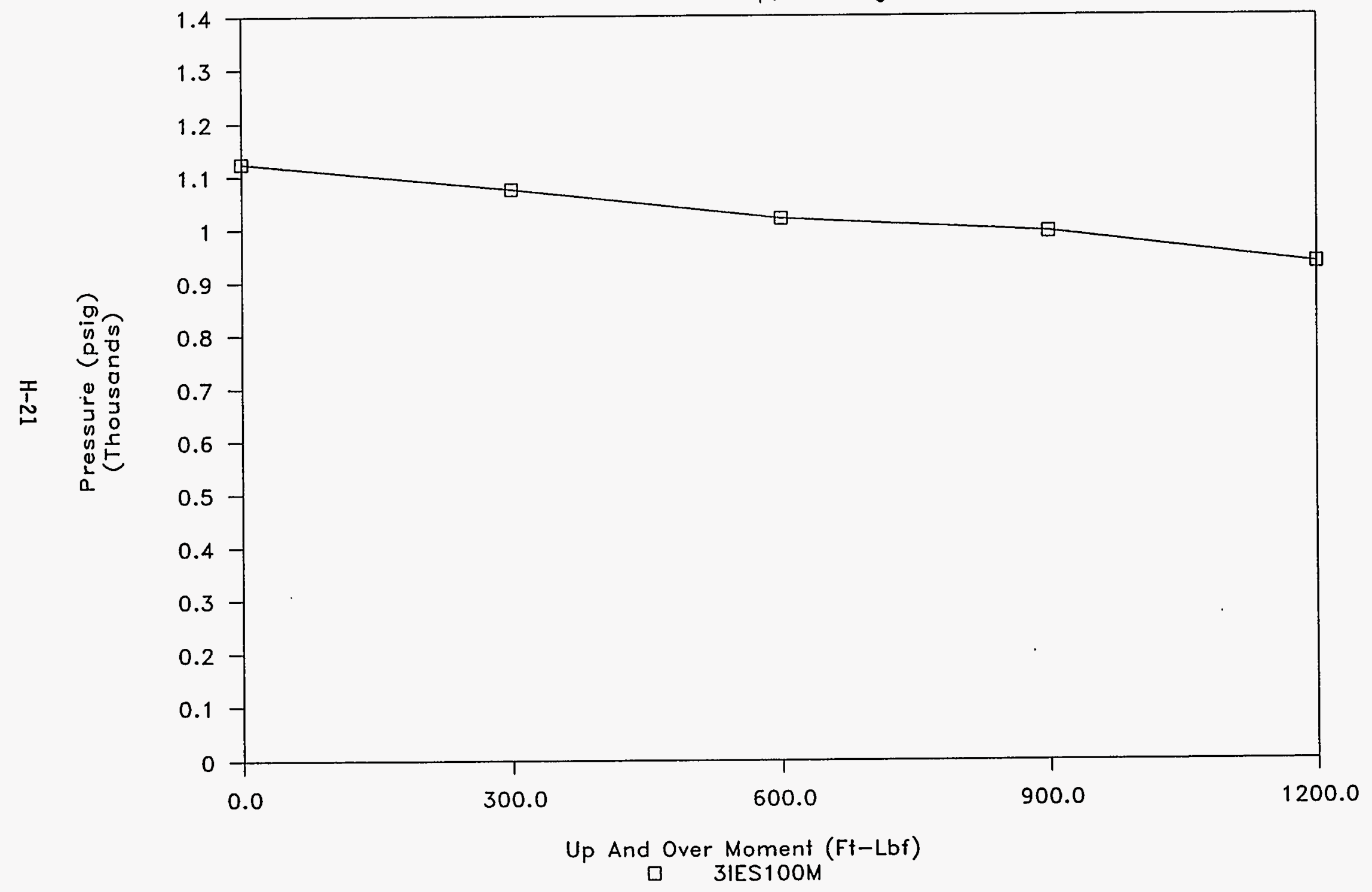


OCTOBER 27,1994

3" ISB CONNECTOR, 70 SH FLUOROSILICONE O-RING, ELEVATED TEMP. (300 DEG. F LEAK TEST - UP AND OVER MOMENT (UPWARD)

CLAMPING TORQUE $=51.7$ FT-LBF

CHARGE PRESSURE $=250$ PSIG

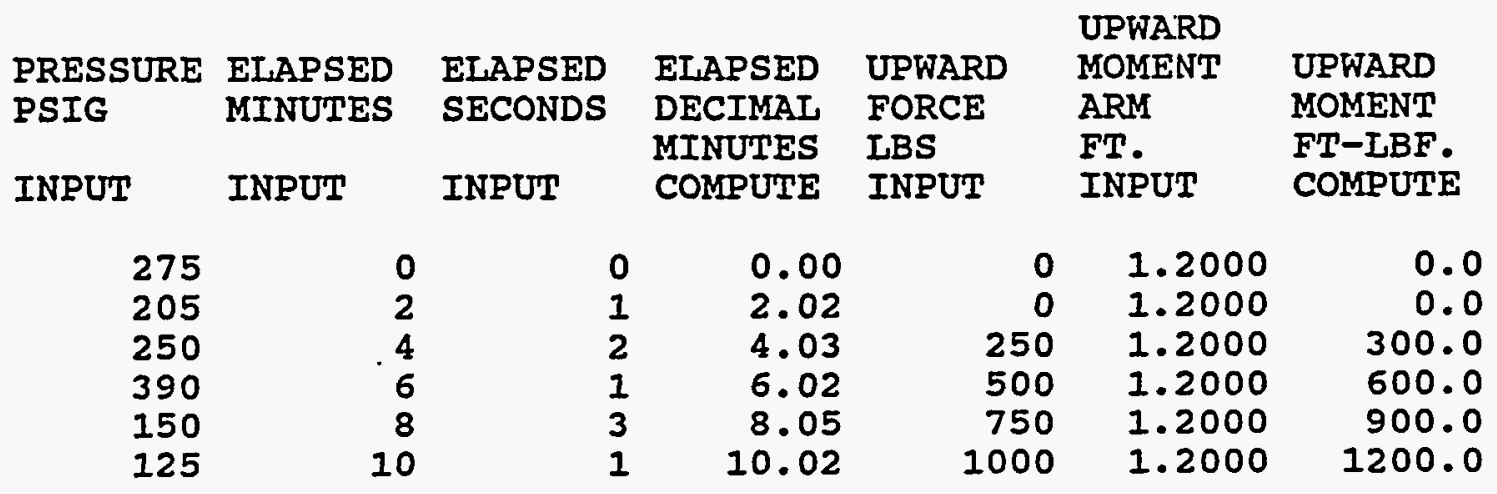

INCREASED PRESSURE TO 500 PSIG

$\begin{array}{ll}540 & 12 \\ 545 & 14 \\ 530 & 16 \\ 455 & 18 \\ 440 & 20\end{array}$

INCREASED PRESSURE TO 750 PSIG

$\begin{array}{llll}800 & 22 & 2 & 22.03 \\ 760 & 24 & 2 & 24.03 \\ 695 & 26 & 1 & 26.02 \\ 660 & 28 & 1 & 28.02 \\ 620 & 30 & 5 & 30.08\end{array}$

INCREASED PRESSURE TO 1000 PSIG

$\begin{array}{rrrrrrr}1125 & 32 & 1 & 32.02 & 0 & 1.2000 & 0.0 \\ 1075 & 34 & 3 & 34.05 & 250 & 1.2000 & 300.0 \\ 1020 & 36 & 3 & 36.05 & 500 & 1.2000 & 600.0 \\ 995 & 38 & 1 & 38.02 & 750 & 1.2000 & 900.0 \\ 935 & 40 & 1 & 40.02 & 1000 & 1.2000 & 1200.0\end{array}$

GRAPH NAME $=3$ IES750M

$\begin{array}{rrr}0 & 1.2000 & 0.0 \\ 250 & 1.2000 & 300.0 \\ 500 & 1.2000 & 600.0 \\ 750 & 1.2000 & 900.0 \\ 1000 & 1.2000 & 1200.0\end{array}$


WHC-SD-WM-TRP-223

Rev. 0

APPENDIX I: GRAPHS OF 3-IN. KALREZ TESTS

I-1 


\section{3" ISB Kalrez O-Ring}

53.9 Ft-Lbf Clamp, Ambient Temp.

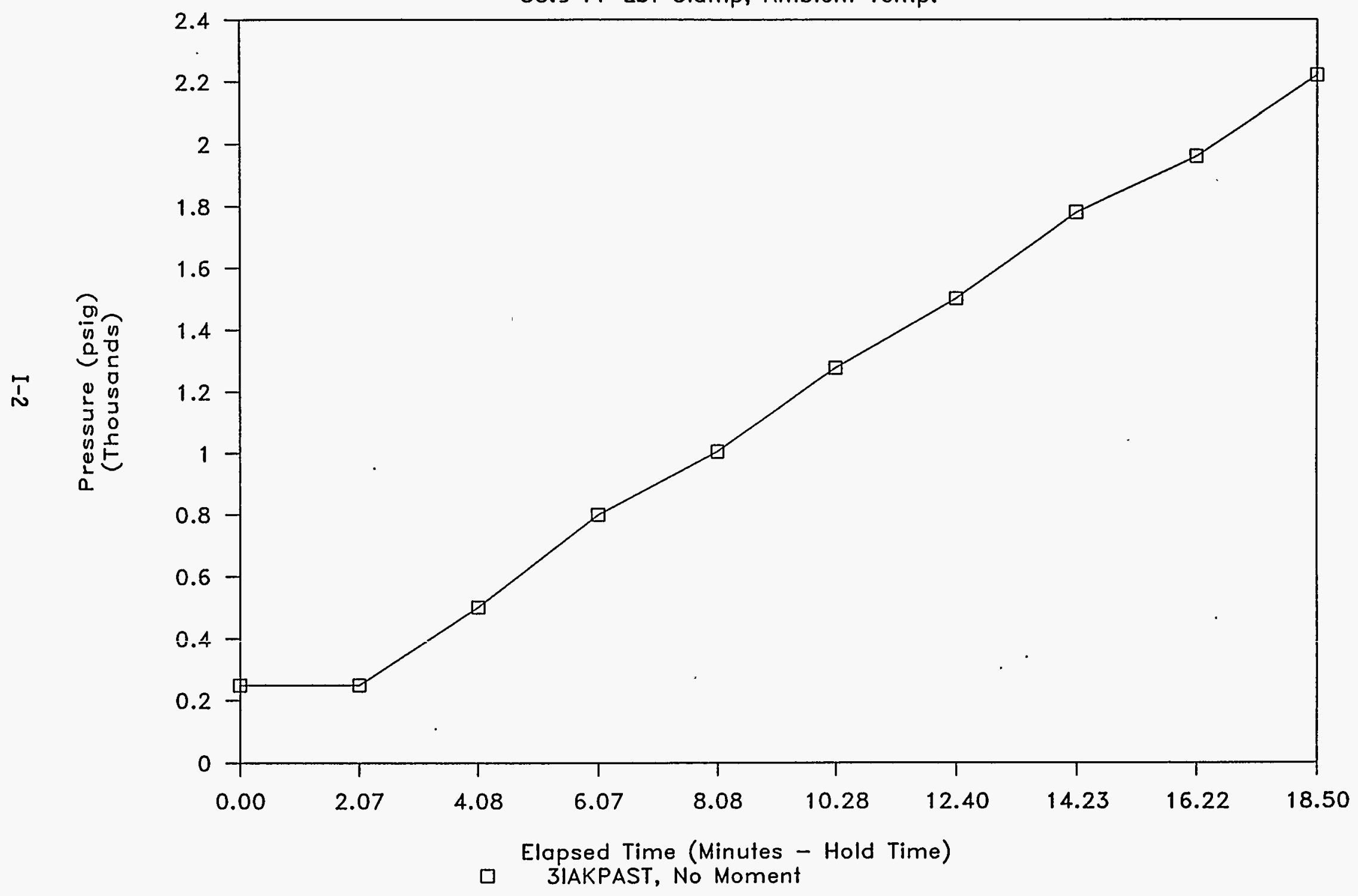




\section{WHC-SD-WM-TRP-223 Rev 0}

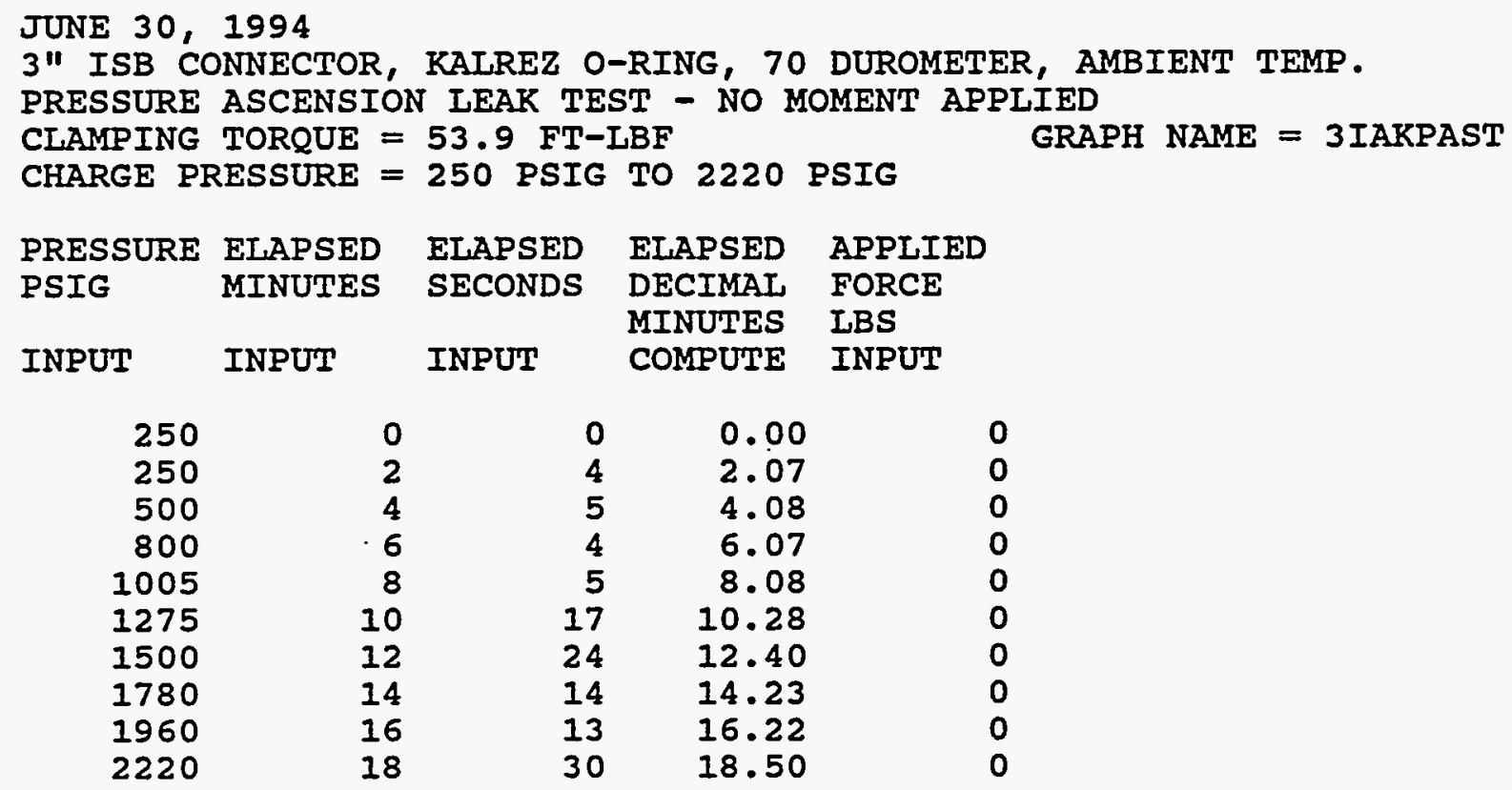


WHC-SD-WM-TRP-223

Rev. 0

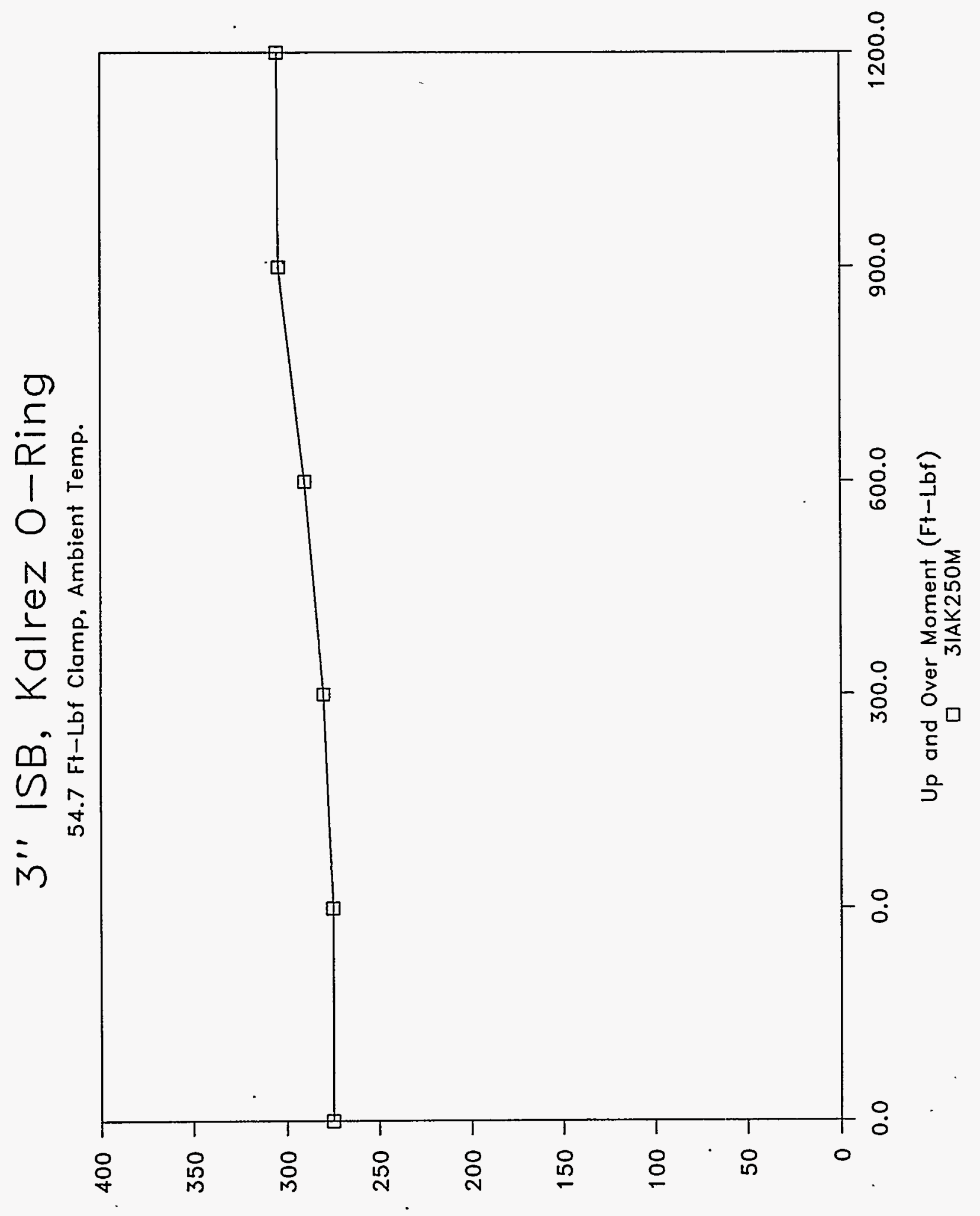

(b!sd) axnssadd 


\section{3" ISB, Kalrez O-Ring}

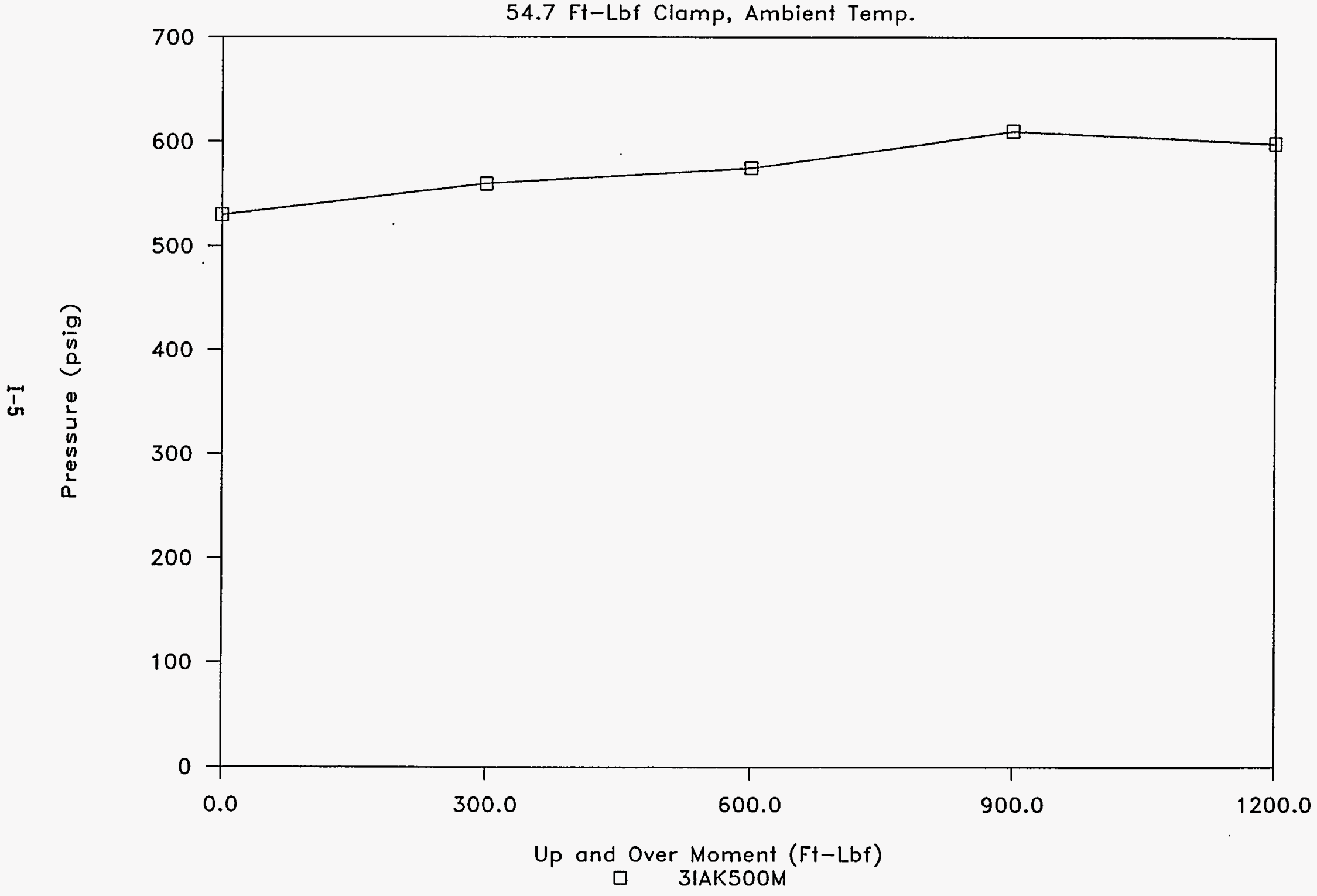


Rev. 0

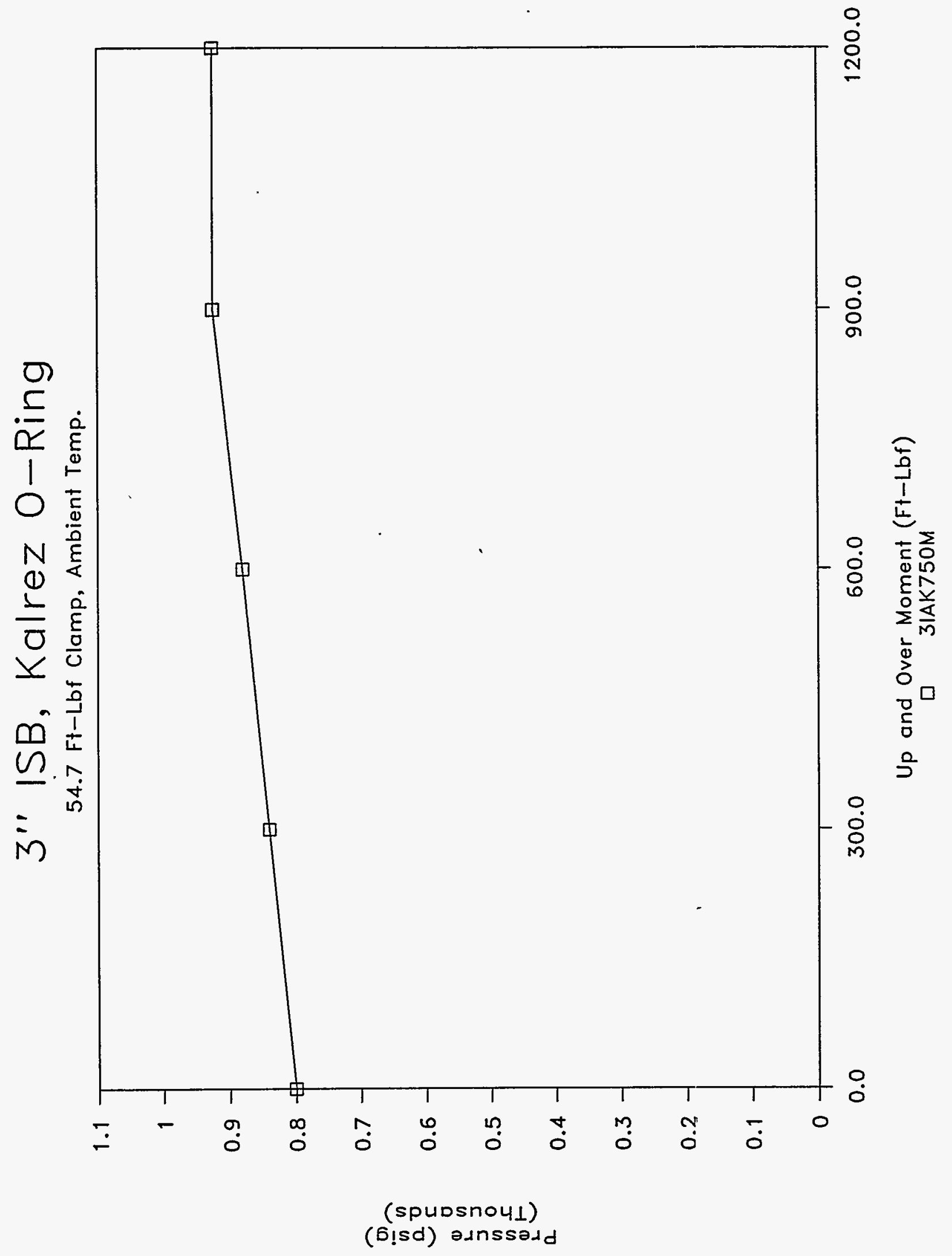




\section{3" ISB, Kalrez O-Ring}

$54.7 \mathrm{Ft}$-Lbf Clamp, Ambient Temp.

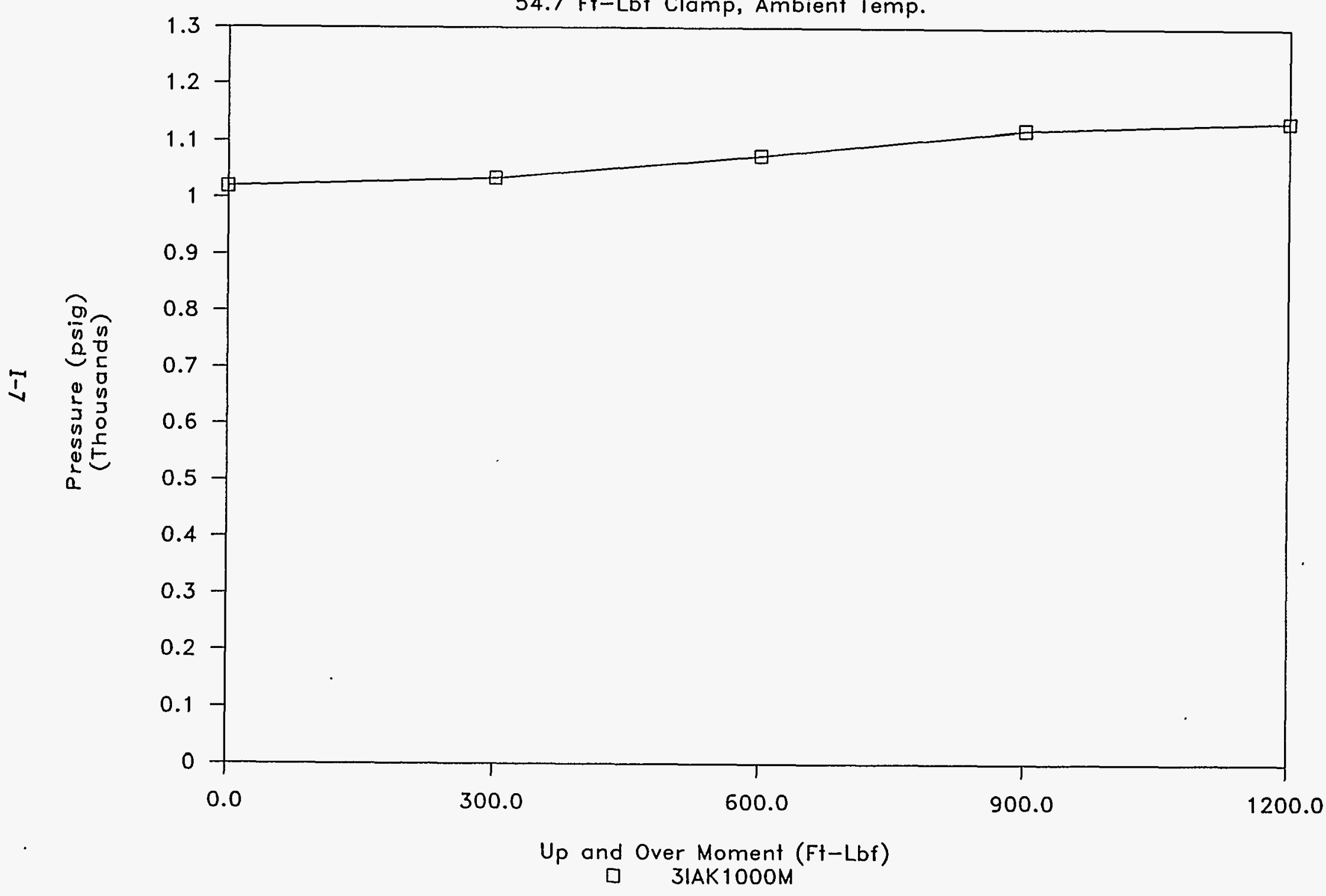




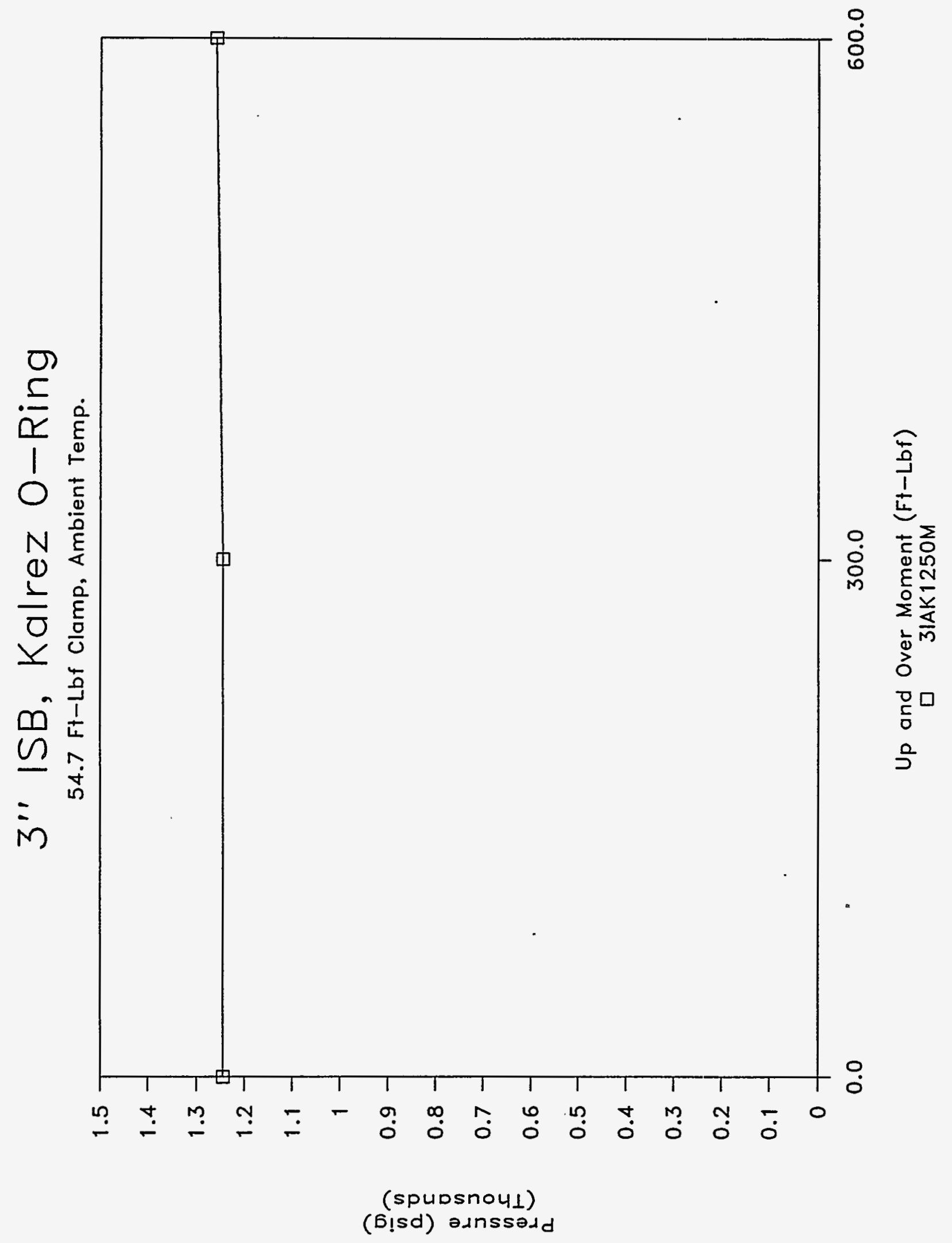


JULY 06, 1994

3" ISB CONNECTOR, KALREZ O-RING, 70 DUROMETER, AMBIENT TEMP. PRESSURE ASCENSION LEAK TEST - UP AND OVER MOMENT (UPWARD ROTATION) CIAMPING TORQUE $=54.7$ FT-IBF GRAPH NAME $=3$ IAK25OM CHARGE PRESSURE $=250$ PSIG

$\begin{array}{lllllll} & & & & & \text { UPWARD } \\ \text { PRESSURE } & \text { ELAPSED } & \text { ELAPSED } & \text { ELAPSED } & \text { UPWARD } & \text { MOMENT } & \text { UPWARD } \\ \text { PSIG } & \text { MINUTES } & \text { SECONDS } & \text { DECIMAL } & \text { FORCE } & \text { ARM } & \text { MOMENT } \\ & & & \text { MINUTES } & \text { LBS } & \text { FT. } & \text { FT-IBBF. } \\ \text { INPUT } & \text { INPUT } & \text { INPUT } & \text { COMPUTE } & \text { INPUT } & \text { INPUT } & \text { COMPUTE }\end{array}$

$\begin{array}{rrrrrrr}275 & 0 & 0 & 0.00 & 0 & 1.2000 & 0.0 \\ 275 & 2 & 2 & 2.03 & 0 & 1.2000 & 0.0 \\ 280 & 4 & 1 & 4.02 & 250 & 1.2000 & 300.0 \\ 290 & 6 & 18 & 6.30 & 500 & 1.2000 & 600.0 \\ 304 & 8 & 6 & 8.10 & 750 & 1.2000 & 900.0 \\ 305 & 10 & 4 & 10.07 & 1000 & 1.2000 & 1200.0\end{array}$

INCREASED PRESSURE TO 500 PSIG

GRAPH NAME $=3$ IAK50OM

$\begin{array}{rrrrrrr}530 & 12 & 4 & 12.07 & 0 & 1.2000 & 0.0 \\ 560 & 14 & 4 & 14.07 & 250 & 1.2000 & 300.0 \\ 575 & 16 & 2 & 16.03 & 500 & 1.2000 & 600.0 \\ 610 & 18 & 3 & 18.05 & 750 & 1.2000 & 900.0 \\ 599 & 20 & 12 & 20.20 & 1000 & 1.2000 & 1200.0\end{array}$

INCREASED PRESSURE TO 750 PSIG

$\begin{array}{rrrr}800 & 22 & 2 & 22.03 \\ 840 & 24 & 2 & 24.03 \\ 880 & 26 & 6 & 26.10 \\ 925 & 28 & 11 & 28.18 \\ 925 & 30 & 9 & 30.15\end{array}$

INCREASED PRESSURE TO 1000 PSIG

$\begin{array}{llrl}1020 & 32 & 3 & 32.05 \\ 1035 & 34 & 3 & 34.05 \\ 1075 & 36 & 13 & 36.22 \\ 1120 & 38 & 2 & 38.03 \\ 1135 & 40 & 5 & 40.08\end{array}$

INCREASED PRESSURE TO 1250 PSIG

$\begin{array}{rrrrrrr}1245 & 42 & 3 & 42.05 & 0 & 1.2000 & 0.0 \\ 1245 & 44 & 3 & 44.05 & 250 & 1.2000 & 300.0 \\ 1260 & 46 & 3 & 46.05 & 500 & 1.2000 & 600.0\end{array}$

GRAPH NAME $=3$ IAK750M

$\begin{array}{rrr}0 & 1.2000 & 0.0 \\ 250 & 1.2000 & 300.0 \\ 500 & 1.2000 & 600.0 \\ 750 & 1.2000 & 900.0 \\ 1000 & 1.2000 & 1200.0\end{array}$

GRAPH NAME $=3$ IAKIOOOM

$\begin{array}{rrr}0 & 1.2000 & 0.0 \\ 250 & 1.2000 & 300.0 \\ 500 & 1.2000 & 600.0 \\ 750 & 1.2000 & 900.0 \\ 1000 & 1.2000 & 1200.0\end{array}$

GRAPH NAME $=3$ IAK1250M

$500 \quad 1.2000 \quad 600.0$ 


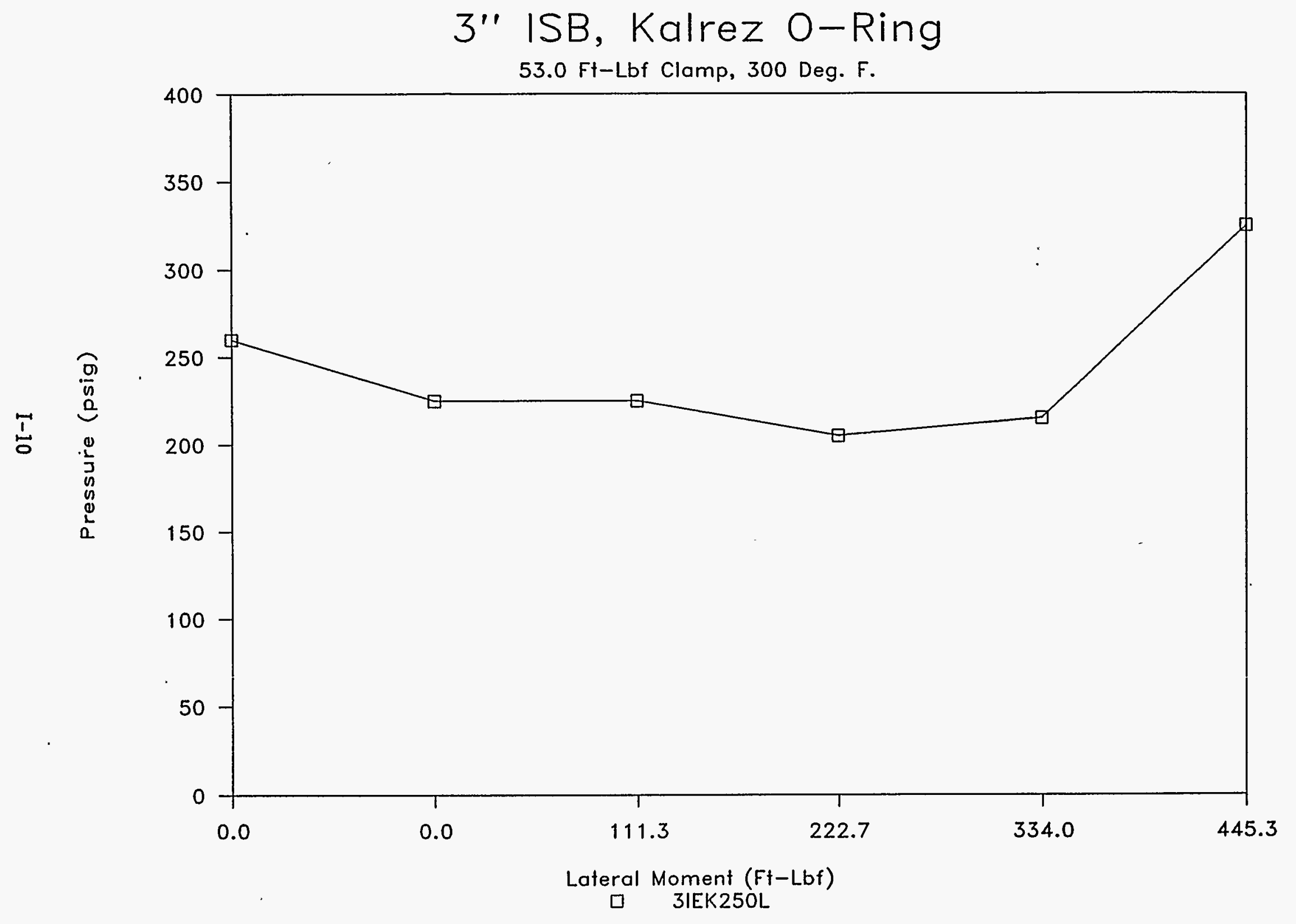


3" ISB, Kalrez O-Ring

53.0 Ft-Lbf Clamp, 300 Deg. F.

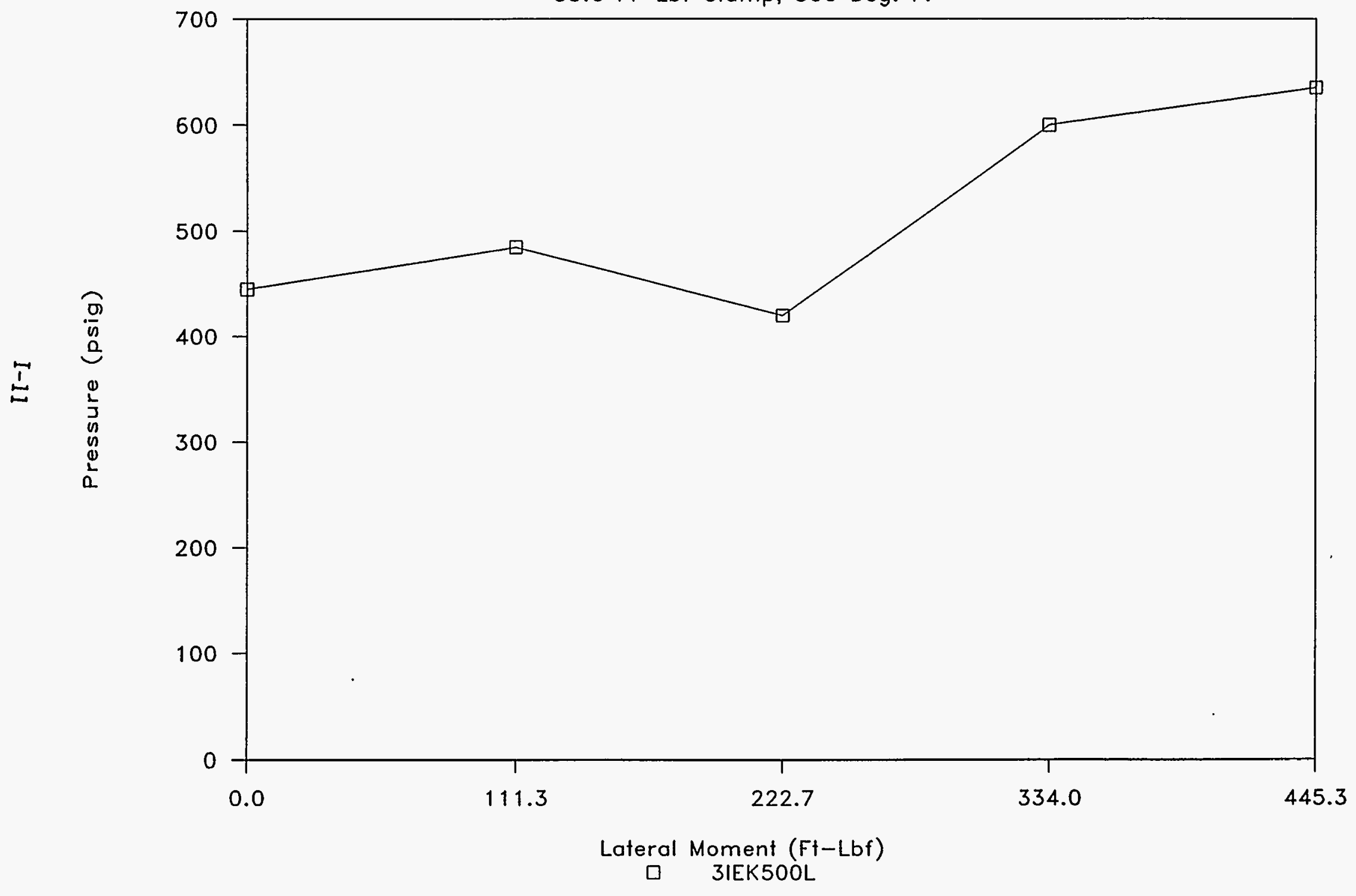




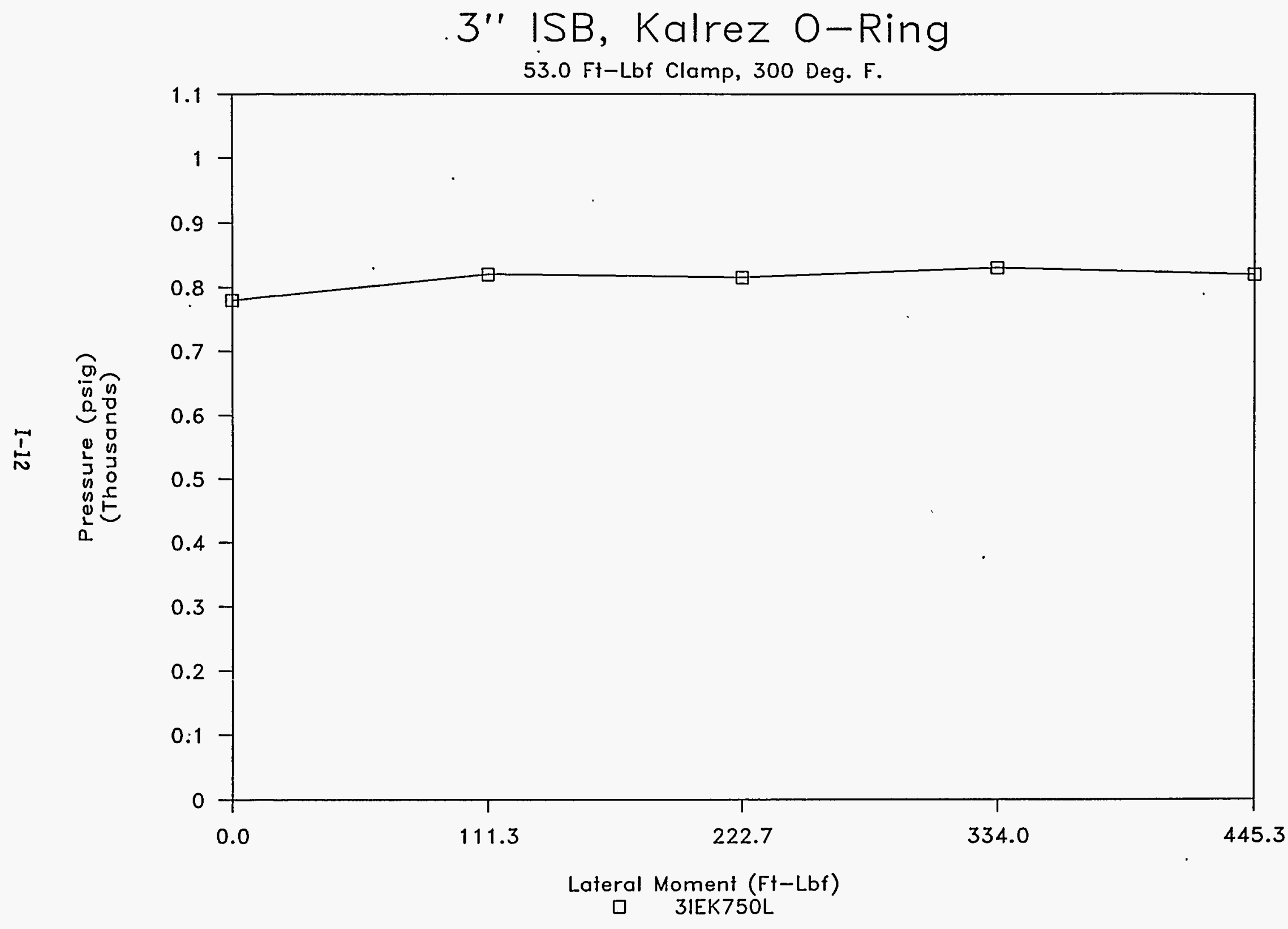


WHC-SD-WM-TRP-223

Rev. 0

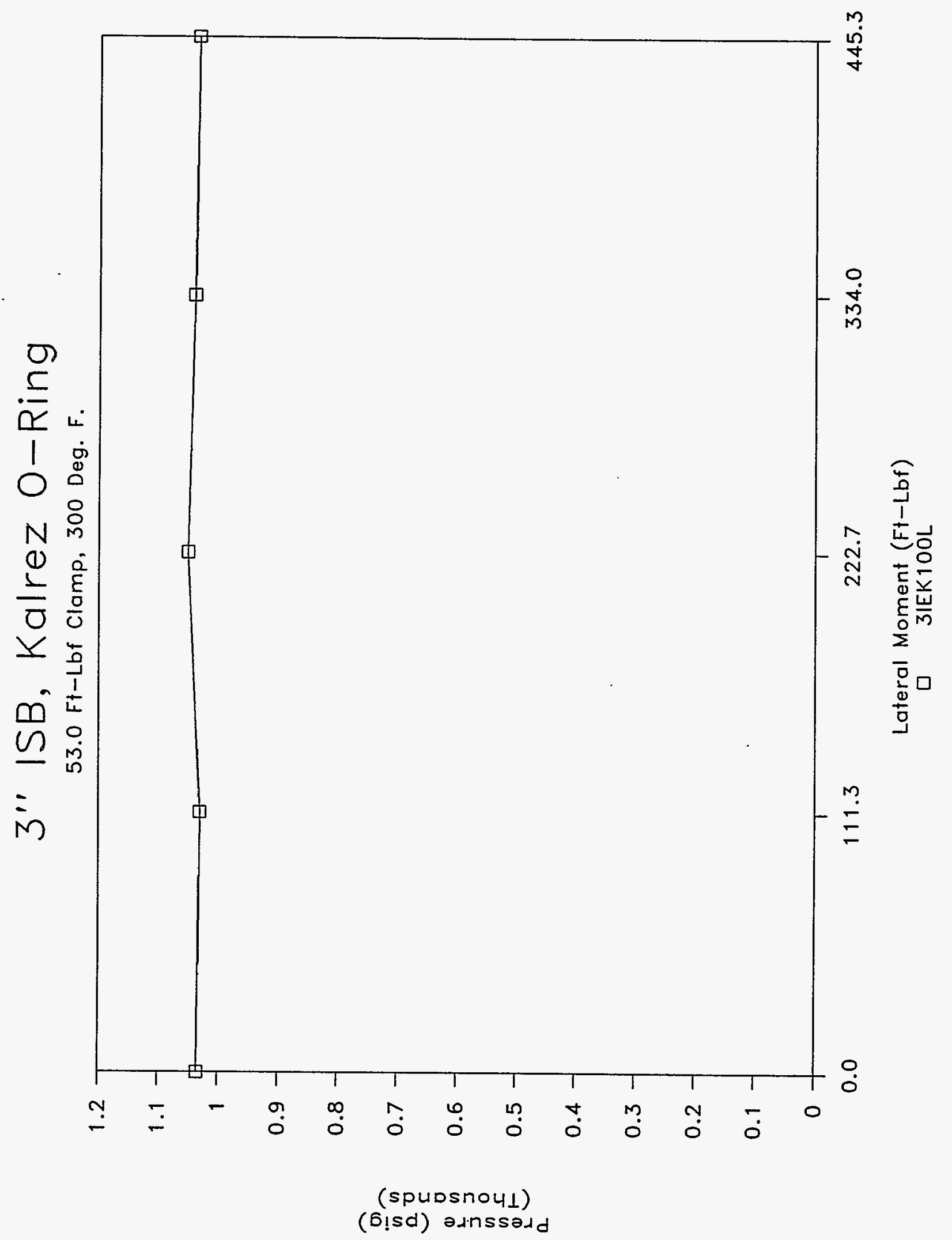


NOVEMBER 03, 1994

3" ISB CONNECTOR, KALREZ O-RING, ELEVATED TEMP. (300 DEG. F) LEAK TEST - LATERAL MOMENT (SIDEWAYS)

CLAMPING TORQUE $=53.0$ FT-LBF

CHARGE PRESSURE $=250$ PSIG

$\begin{array}{lllllll} & & & & & \text { IATERAI } \\ \text { PRESSURE } & \text { ELAPSED } & \text { ELAPSED } & \text { ELAPSED } & \text { LATERAI } & \text { MOMENT } & \text { IAATERAI } \\ \text { PSIG } & \text { MINUTES } & \text { SECONDS } & \text { DECIMAI } & \text { FORCE } & \text { ARM } & \text { MOMENT } \\ & & & \text { MINUTES } & \text { LBS } & \text { FT. } & \text { FT-IBF. } \\ \text { INPUT } & \text { INPUT } & \text { INPUT } & \text { COMPUTE } & \text { INPUT } & \text { INPUT } & \text { COMPUTE }\end{array}$

$\begin{array}{rrrrrrr}260 & 0 & 0 & 0.00 & 0 & 0.4453 & 0.0 \\ 225 & 2 & 1 & 2.02 & 0 & 0.4453 & 0.0 \\ 225 & 4 & 1 & 4.02 & 250 & 0.4453 & 111.3 \\ 205 & 6 & 2 & 6.03 & 500 & 0.4453 & 222.7 \\ 215 & 8 & 1 & 8.02 & 750 & 0.4453 & 334.0 \\ 325 & 10 & 3 & 10.05 & 1000 & 0.4453 & 445.3\end{array}$

INCREASED PRESSURE TO 500 PSIG

GRAPH NAME $=3$ IEK50OI

$\begin{array}{rrrrrrr}445 & 12 & 7 & 12.12 & 0 & 0.4453 & 0.0 \\ 485 & 14 & 1 & 14.02 & 250 & 0.4453 & 111.3 \\ 420 & 16 & 3 & 16.05 & 500 & 0.4453 & 222.7 \\ 600 & 18 & 2 & 18.03 & 750 & 0.4453 & 334.0 \\ 635 & 20 & 1 & 20.02 & 1000 & 0.4453 & 445.3\end{array}$

INCREASED PRESSURE TO 750 PSIG

$\begin{array}{llll}780 & 22 & 0 & 22.00 \\ 820 & 24 & 3 & 24.05 \\ 815 & 26 & 2 & 26.03 \\ 830 & 28 & 0 & 28.00 \\ 820 & 30 & 1 & 30.02\end{array}$

INCREASED PRESSURE TO 1000 PSIG

$\begin{array}{rrrrrrr}1035 & 32 & 1 & 32.02 & 0 & 0.4453 & 0.0 \\ 1030 & 34 & 2 & 34.03 & 250 & 0.4453 & 111.3 \\ 1050 & 36 & 1 & 36.02 & 500 & 0.4453 & 222.7 \\ 1040 & 38 & 3 & 38.05 & 750 & 0.4453 & 334.0 \\ 1035 & 40 & 2 & 40.03 & 1000 & 0.4453 & 445.3\end{array}$

GRAPH NAME $=3$ IEK750L

$\begin{array}{rlr}0 & 0.4453 & 0.0 \\ 250 & 0.4453 & 111.3 \\ 500 & 0.4453 & 222.7 \\ 750 & 0.4453 & 334.0 \\ 1000 & 0.4453 & 445.3\end{array}$




\section{3" ISB, Kalrez O-Ring}

54.2 Ff-Lbf Clamp, 300 Deg. F.

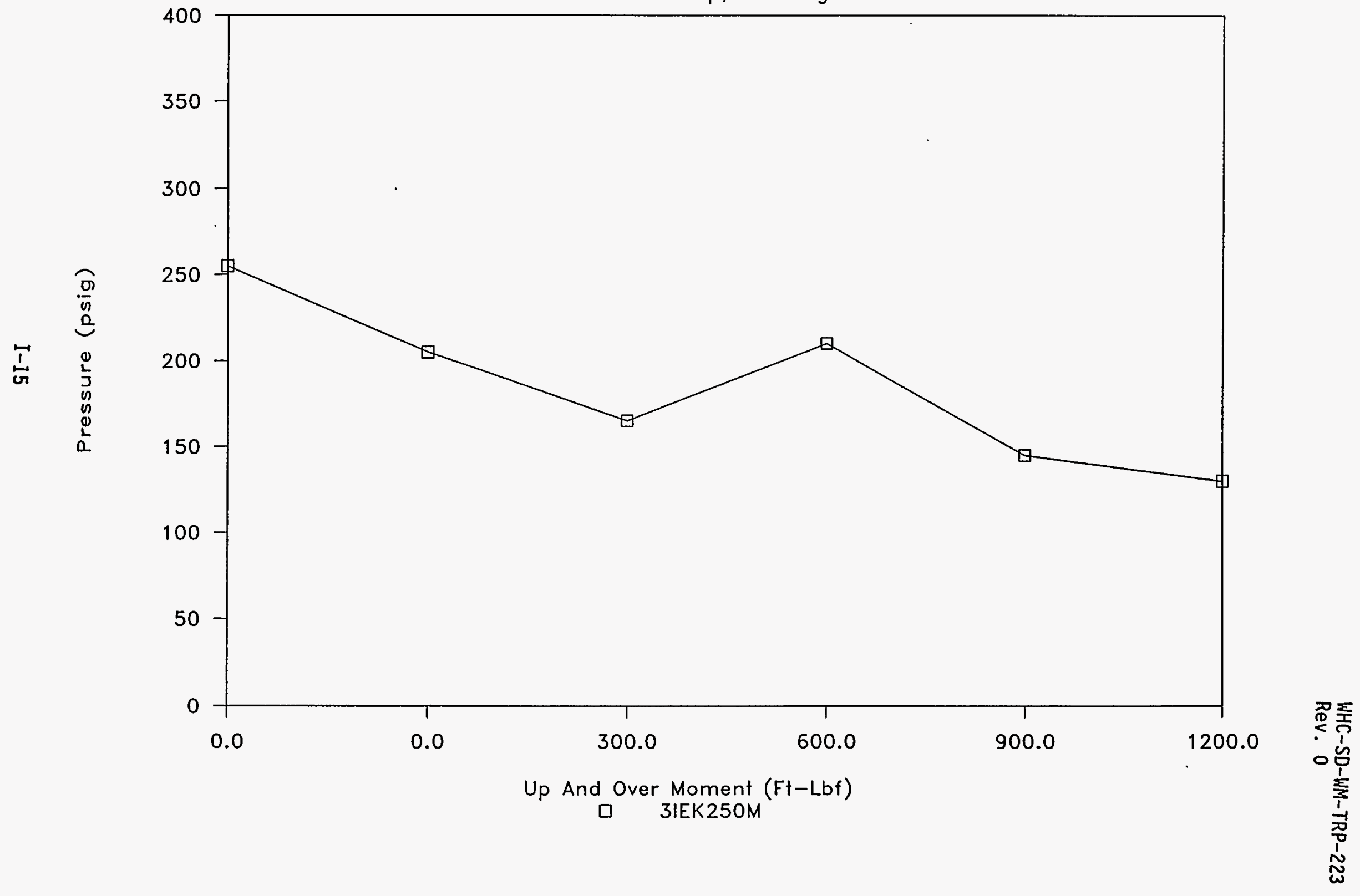




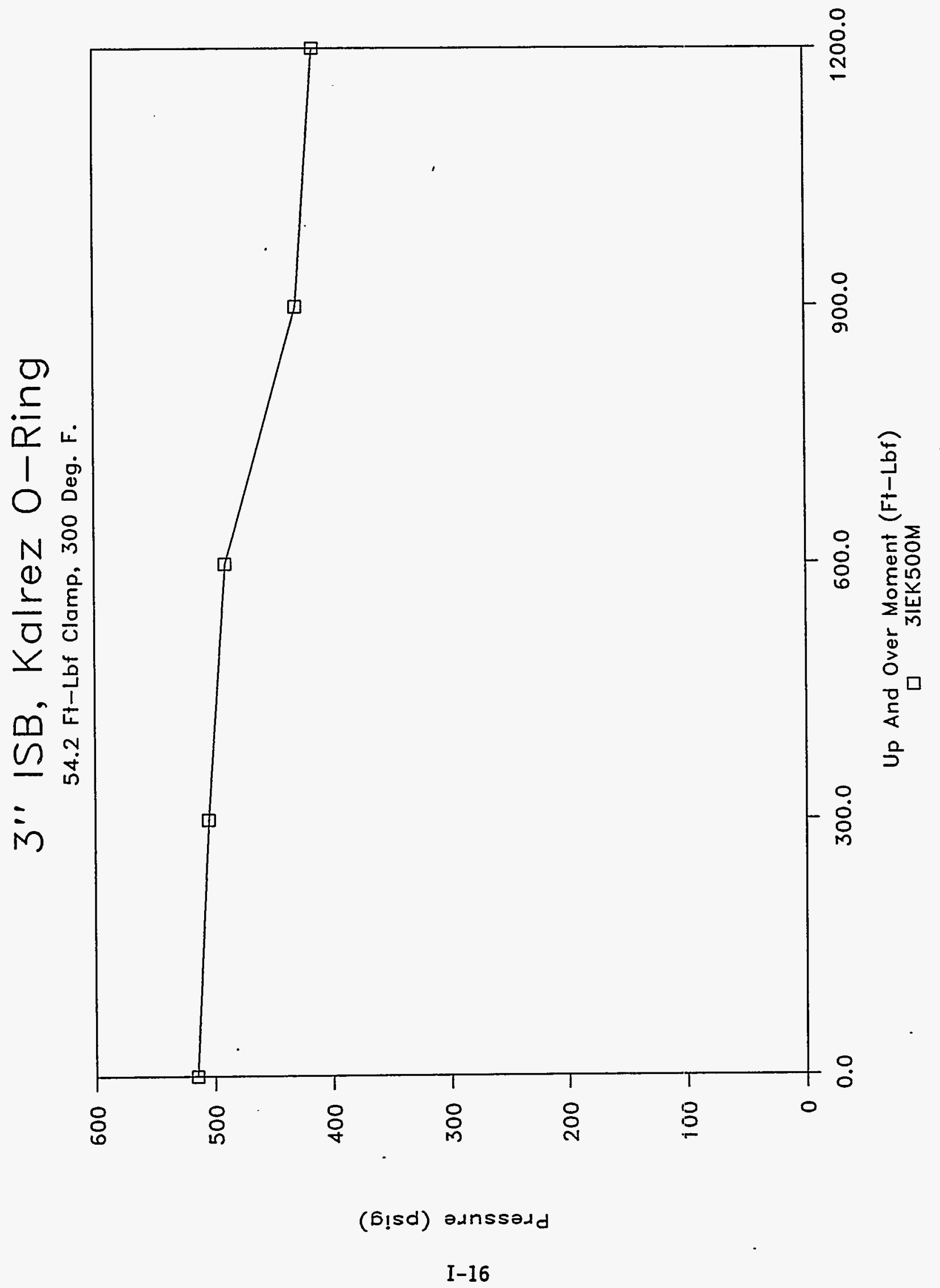


WHC-SD-WM-TRP-223

Rev. 0

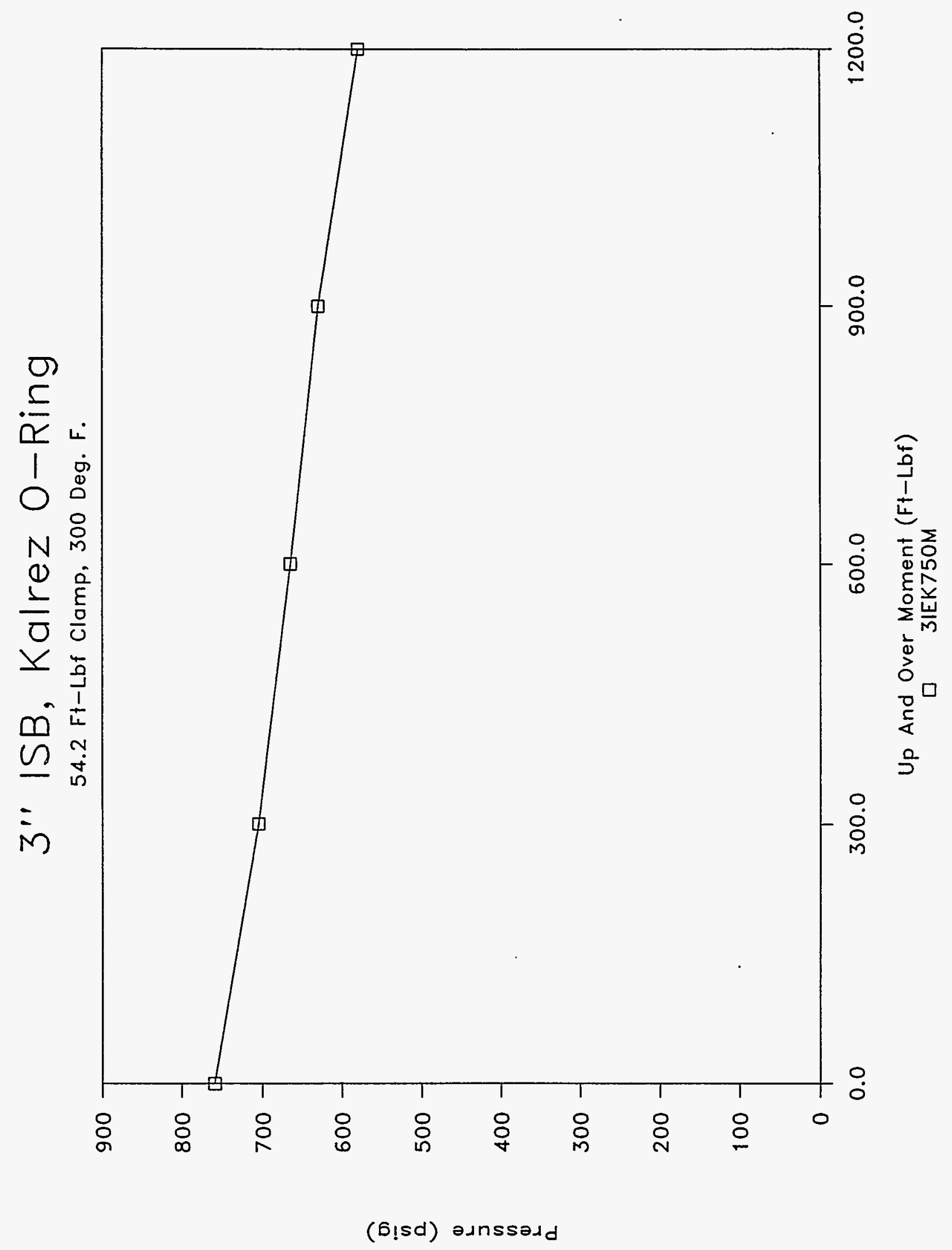




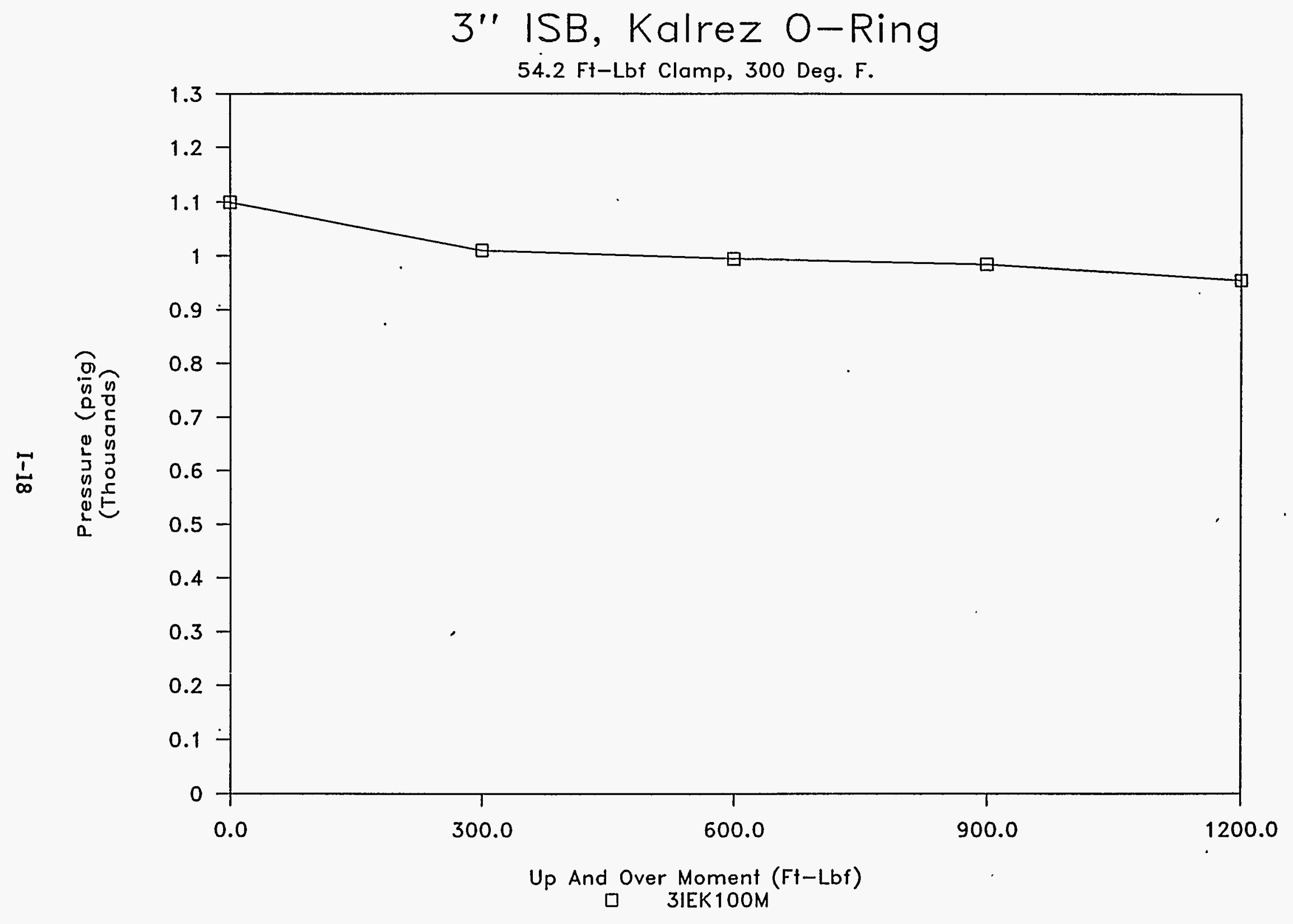


WHC-SD-WM-TRP-223 ReV 0

NOVEMBER 02, 1994

3" ISB CONNECTOR, KAIREZ O-RING, ELEVATED TEMP. (300 DEG. F)

LEAK TEST - UP AND OVER MOMENT (UPWARD)

CLAMPING TORQUE $=54.2$ FT-LBF GRAPH NAME $=3$ IEK25OM

CHARGE PRESSURE $=250$ PSIG

$\begin{array}{lllllll} & & & & & \text { UPWARD } & \\ \text { PRESSURE } & \text { ELAPSED } & \text { ELAPSED } & \text { ELAPSED } & \text { UPWARD } & \text { MOMENT } & \text { UPWARD } \\ \text { PSIG } & \text { MINUTES } & \text { SECONDS } & \text { DECIMAI } & \text { FORCE } & \text { ARM } & \text { MOMENT } \\ & & & \text { MINUTES } & \text { LBS } & \text { FT. } & \text { FT-IBBF. } \\ \text { INPUT } & \text { INPUT } & \text { INPUT } & \text { COMPUTE } & \text { INPUT } & \text { INPUT } & \text { COMPUTE }\end{array}$

$\begin{array}{rrrrrrr}255 & 0 & 0 & 0.00 & 0 & 1.2000 & 0.0 \\ 205 & 2 & 5 & 2.08 & 0 & 1.2000 & 0.0 \\ 165 & 4 & 3 & 4.05 & 250 & 1.2000 & 300.0 \\ 210 & 6 & 1 & 6.02 & 500 & 1.2000 & 600.0 \\ 145 & 8 & 2 & 8.03 & 750 & 1.2000 & 900.0 \\ 130 & 10 & 1 & 10.02 & 1000 & 1.2000 & 1200.0\end{array}$

INCREASED PRESSURE TO 500 PSIG

GRAPH NAME $=3$ IEK50OM

$\begin{array}{rrrrrrr}515 & 12 & 1 & 12.02 & 0 & 1.2000 & 0.0 \\ 505 & 14 & 3 & 14.05 & 250 & 1.2000 & 300.0 \\ 490 & 16 & 2 & 16.03 & 500 & 1.2000 & 600.0 \\ 430 & 18 & 2 & 18.03 & 750 & 1.2000 & 900.0 \\ 415 & 20 & 1 & 20.02 & 1000 & 1.2000 & 1200.0\end{array}$

INCREASED PRESSURE TO 750 PSIG

$\begin{array}{llll}760 & 22 & 3 & 22.05 \\ 705 & 24 & 1 & 24.02 \\ 665 & 26 & 1 & 26.02 \\ 630 & 28 & 2 & 28.03 \\ 580 & 30 & 1 & 30.02\end{array}$

INCREASED PRESSURE TO 1000 PSIG

$\begin{array}{rrrrrrr}1100 & 32 & 2 & 32.03 & 0 & 1.2000 & 0.0 \\ 1010 & 34 & 5 & 34.08 & 250 & 1.2000 & 300.0 \\ 995 & 36 & 1 & 36.02 & 500 & 1.2000 & 600.0 \\ 985 & 38 & 3 & 38.05 & 750 & 1.2000 & 900.0 \\ 955 & 40 & 3 & 40.05 & 1000 & 1.2000 & 1200.0\end{array}$

GRAPH NAME $=3$ IEK750M

$\begin{array}{rrr}0 & 1.2000 & 0.0 \\ 250 & 1.2000 & 300.0 \\ 500 & 1.2000 & 600.0 \\ 750 & 1.2000 & 900.0 \\ 1000 & 1.2000 & 1200.0\end{array}$

GRAPH NAME $=3$ IEK10OM 
WHC-SD-WM-TRP-223

Rev. 0

APPENDIX J: GRAPHS OF 3-IN. VITON TESTS

J-1 


\section{3" ISB Viton O-Ring}

52.8 Fł-Lbf Clamp, Ambient Temp.

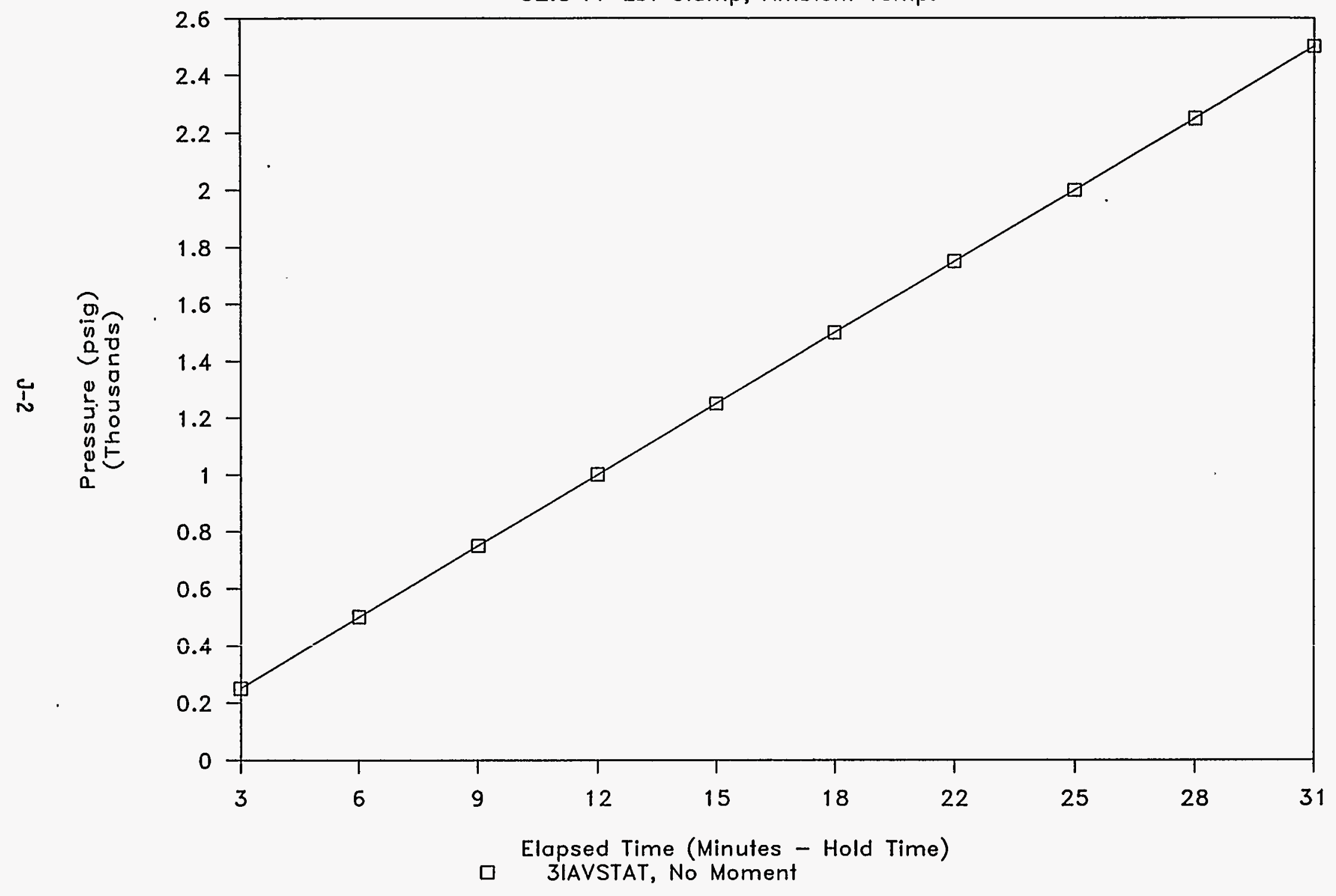


WHC-SD-WM-TRP-223 ReV 0

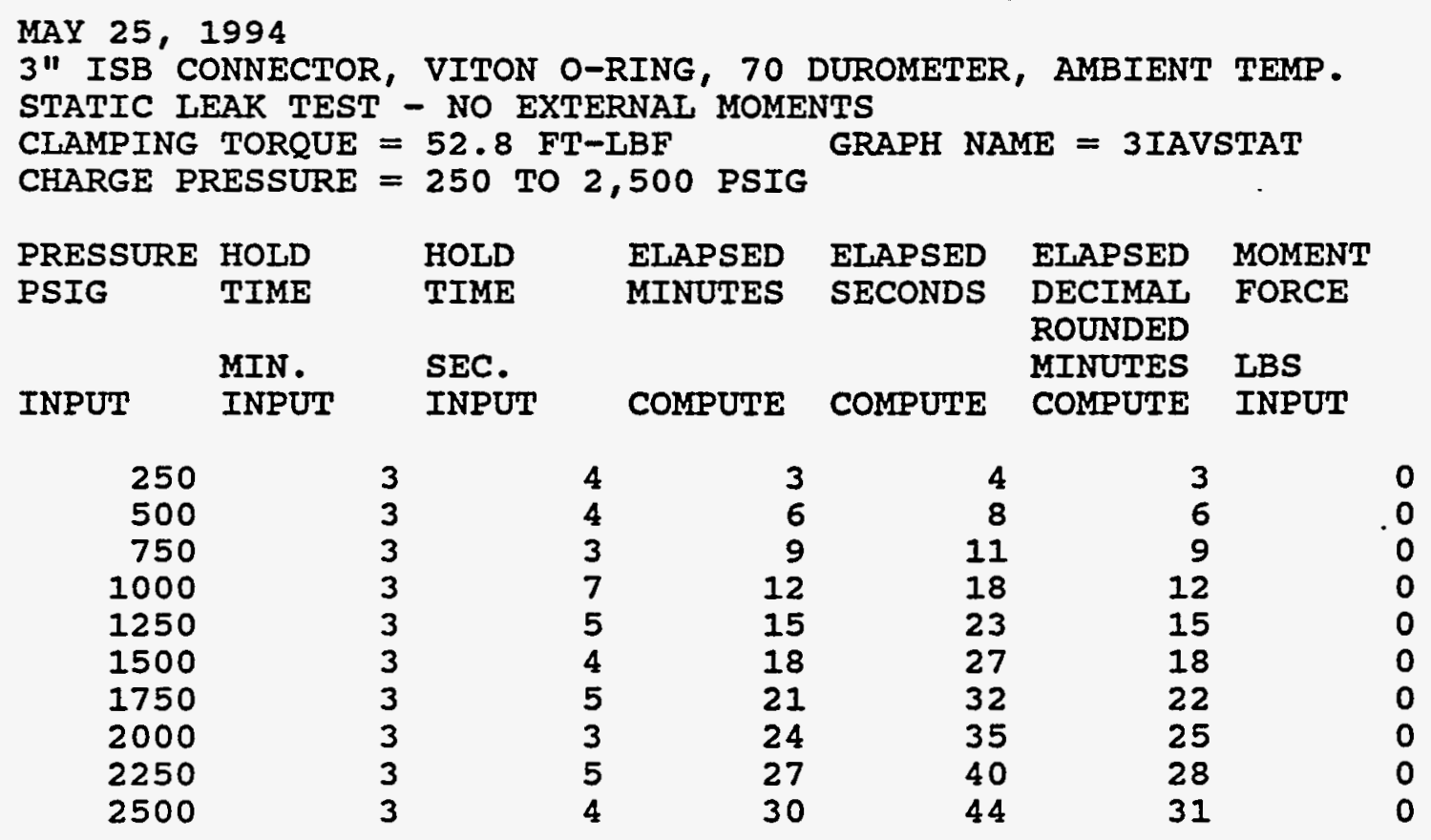


WHC-SD-WM-TRP-223

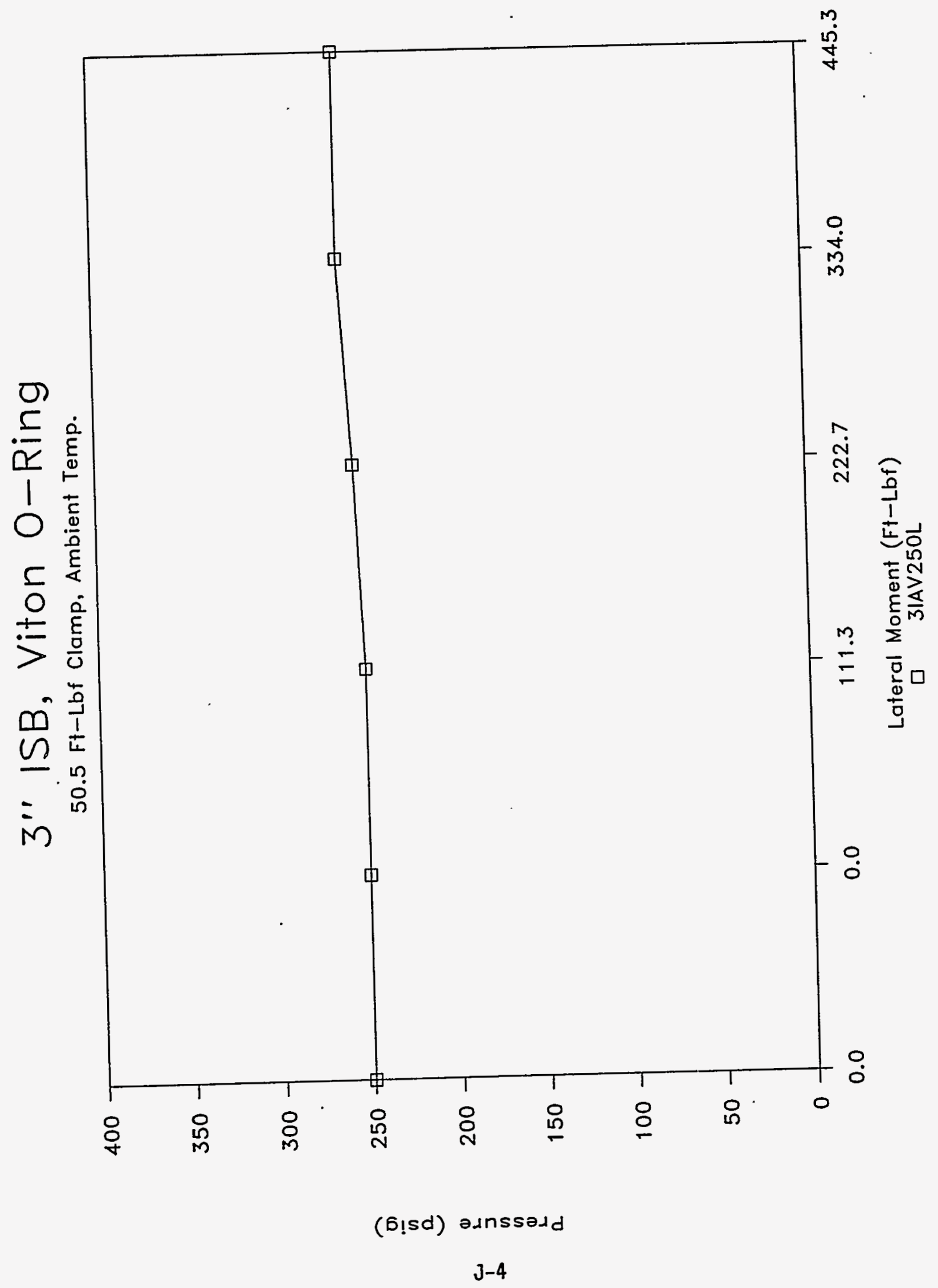




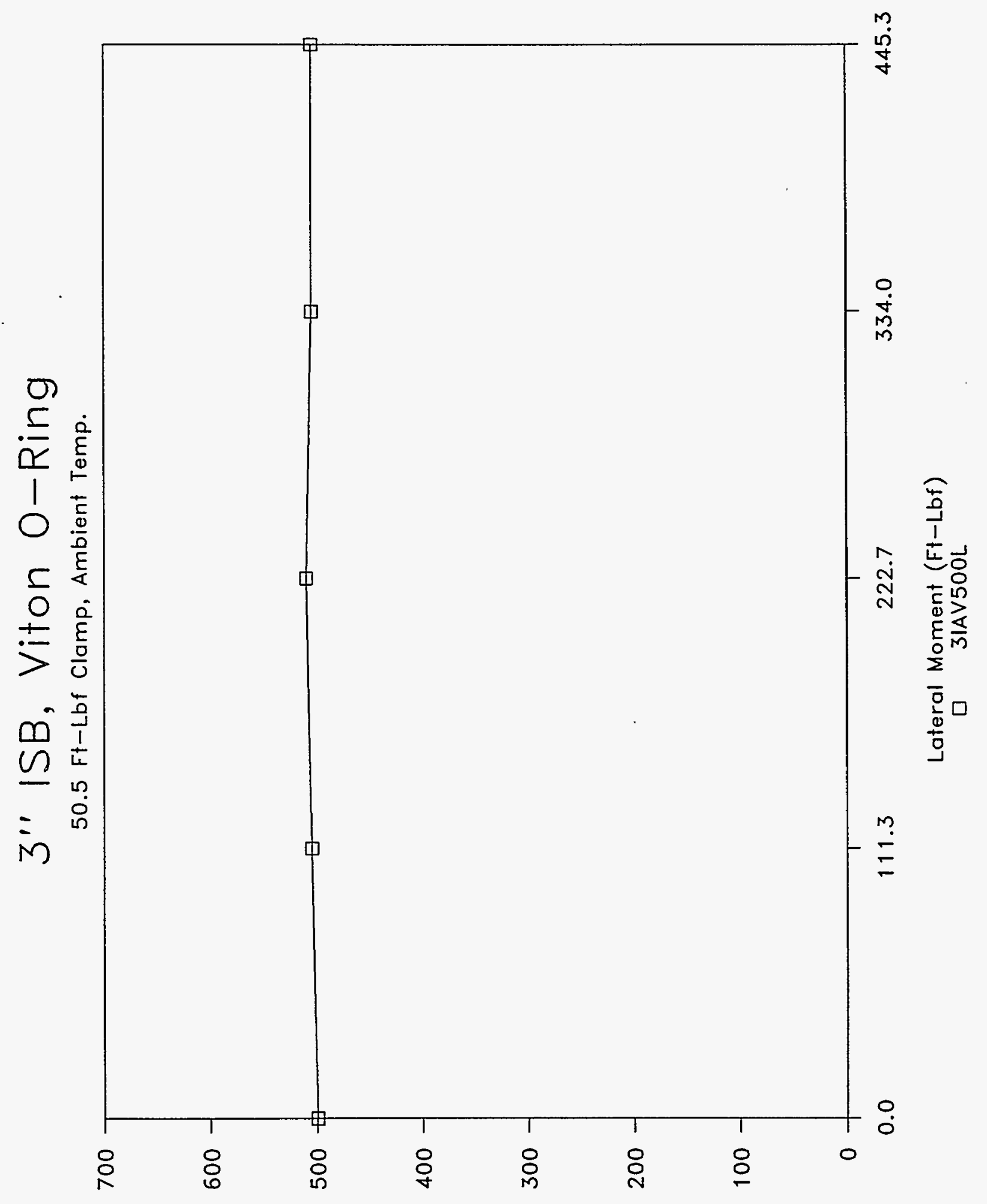

(b!sd) adnssàd 


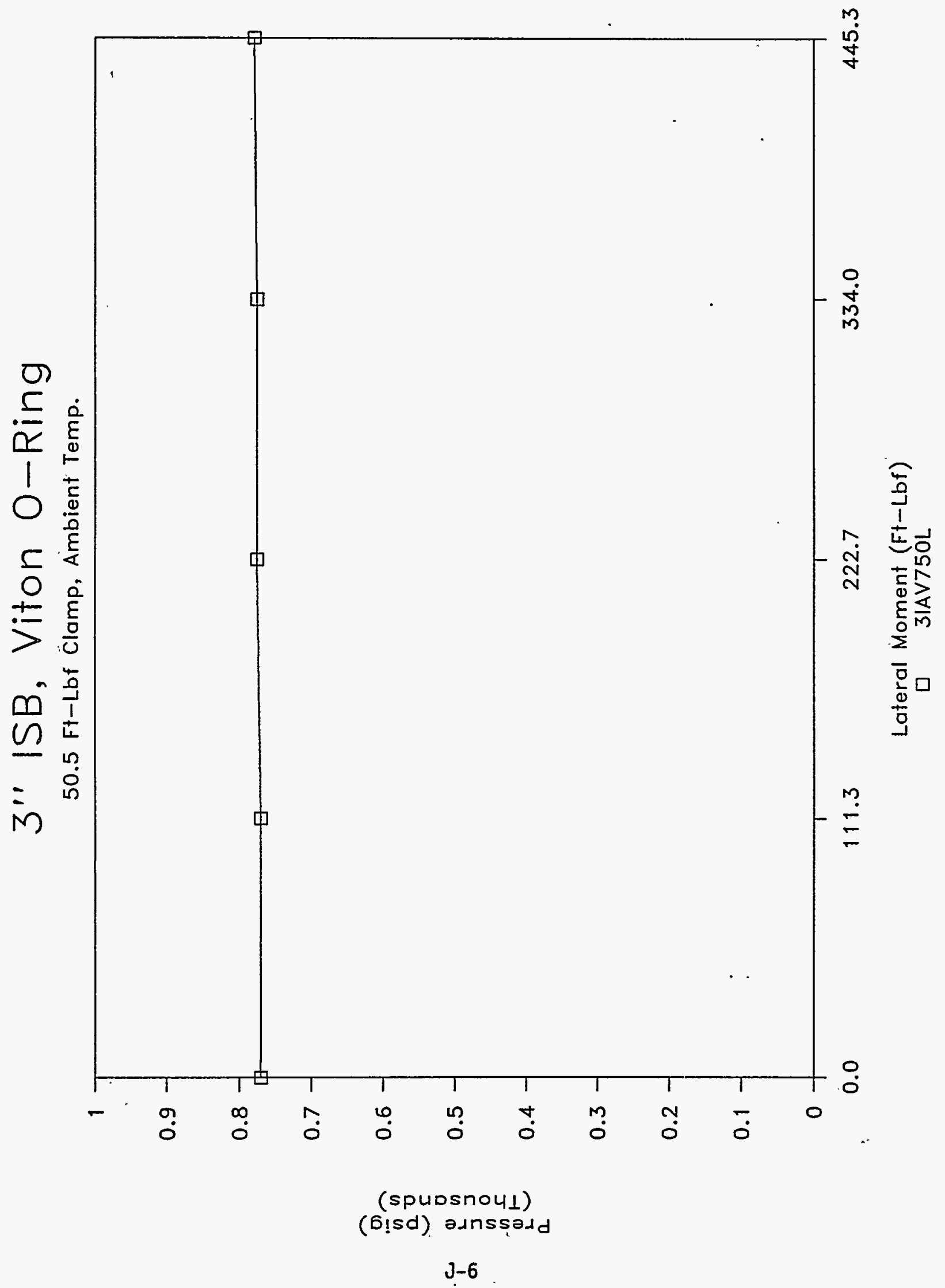


3" ISB, Viton O-Ring

$50.5 \mathrm{Ft}$-Lbf Clamp, Ambient Temp.

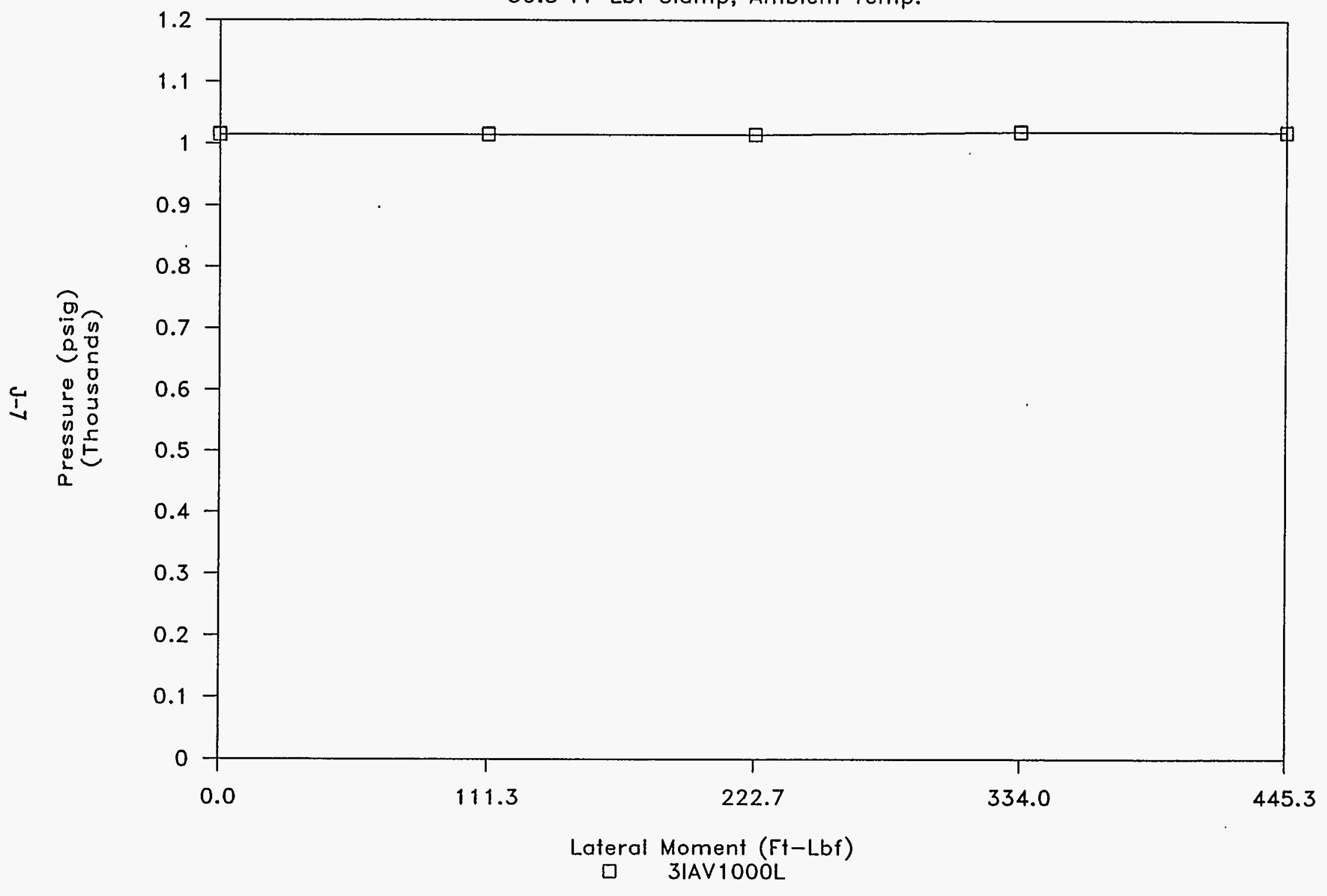


JULY 12,1994

3" ISB CONNECTOR, VITON O-RING, 70 DUROMETER, AMBIENT TEMP. PRESSURE ASCENSION LEAK TEST - IATERAI MOMENT (SIDEWAYS ROTATION) CLAMPING TORQUE $=50.5$ FT-LBF CHARGE PRESSURE $=250$ PSIG

$\begin{array}{lllllll} & & & & & \text { LATERAI } \\ \text { PRESSURE } & \text { ELAAPSED } & \text { ELAPSED } & \text { ELAPSED } & \text { LATERAI } & \text { MOMENT } & \text { LATERAI } \\ \text { PSIG } & \text { MINUTES } & \text { SECONDS } & \text { DECIMAL } & \text { FORCE } & \text { ARM } & \text { MOMENT } \\ & & & \text { MINUTES } & \text { LBS } & \text { FT. } & \text { FT-LBF. } \\ \text { INPUT } & \text { INPUT } & \text { INPUT } & \text { COMPUTE } & \text { INPUT } & \text { INPUT } & \text { COMPUTE }\end{array}$

$\begin{array}{rrrrrrr}250 & 0 & 0 & 0.00 & 0 & 0.4453 & 0.0 \\ 250 & 2 & 1 & 2.02 & 0 & 0.4453 & 0.0 \\ 250 & 4 & 2 & 4.03 & 250 & 0.44 .53 & 111.3 \\ 255 & 6 & 8 & 6.13 & 500 & 0.44 .53 & 222.7 \\ 262 & 8 & 7 & 8.12 & 750 & 0.4453 & 334.0 \\ 262 & 10 & 4 & 10.07 & 1000 & 0.4453 & 445.3\end{array}$

INCREASED PRESSURE TO 500 PSIG

$\begin{array}{rrrrrrr}500 & 12 & 6 & 12.10 & 0 & 0.4453 & 0.0 \\ 505 & 14 & 5 & 14.08 & 250 & 0.4453 & 111.3 \\ 510 & 16 & 4 & 16.07 & 500 & 0.4453 & 222.7 \\ 505 & 18 & 3 & 18.05 & 750 & 0.4453 & 334.0 \\ 505 & 20 & 8 & 20.13 & 1000 & 0.4453 & 445.3\end{array}$

INCREASED PRESSURE TO 750 PSIG GRAPH NAME = 3IAV750I

$\begin{array}{rrrrrrr}770 & 22 & 2 & 22.03 & 0 & 0.4453 & 0.0 \\ 770 & 24 & 3 & 24.05 & 250 & 0.4453 & 111.3 \\ 775 & 26 & 7 & 26.12 & 500 & 0.4453 & 222.7 \\ 775 & 28 & 4 & 28.07 & 750 & 0.4453 & 334.0 \\ 780 & 30 & 15 & 30.25 & 1000 & 0.4453 & 445.3\end{array}$

INCREASED PRESSURE TO 1000 PSIG GRAPH NAME = 3ILAV1000L

$\begin{array}{rrrrrrr}1015 & 32 & 3 & 32.05 & 0 & 0.4453 & 0.0 \\ 1015 & 34 & 4 & 34.07 & 250 & 0.4453 & 111.3 \\ 1015 & 36 & 9 & 36.15 & 500 & 0.4453 & 222.7 \\ 1020 & 38 & 10 & 38.17 & 750 & 0.4453 & 334.0 \\ 1020 & 40 & 8 & 40.13 & 1000 & 0.4453 & 445.3\end{array}$


WHC-SD-WM-TRP-223

Rev. 0

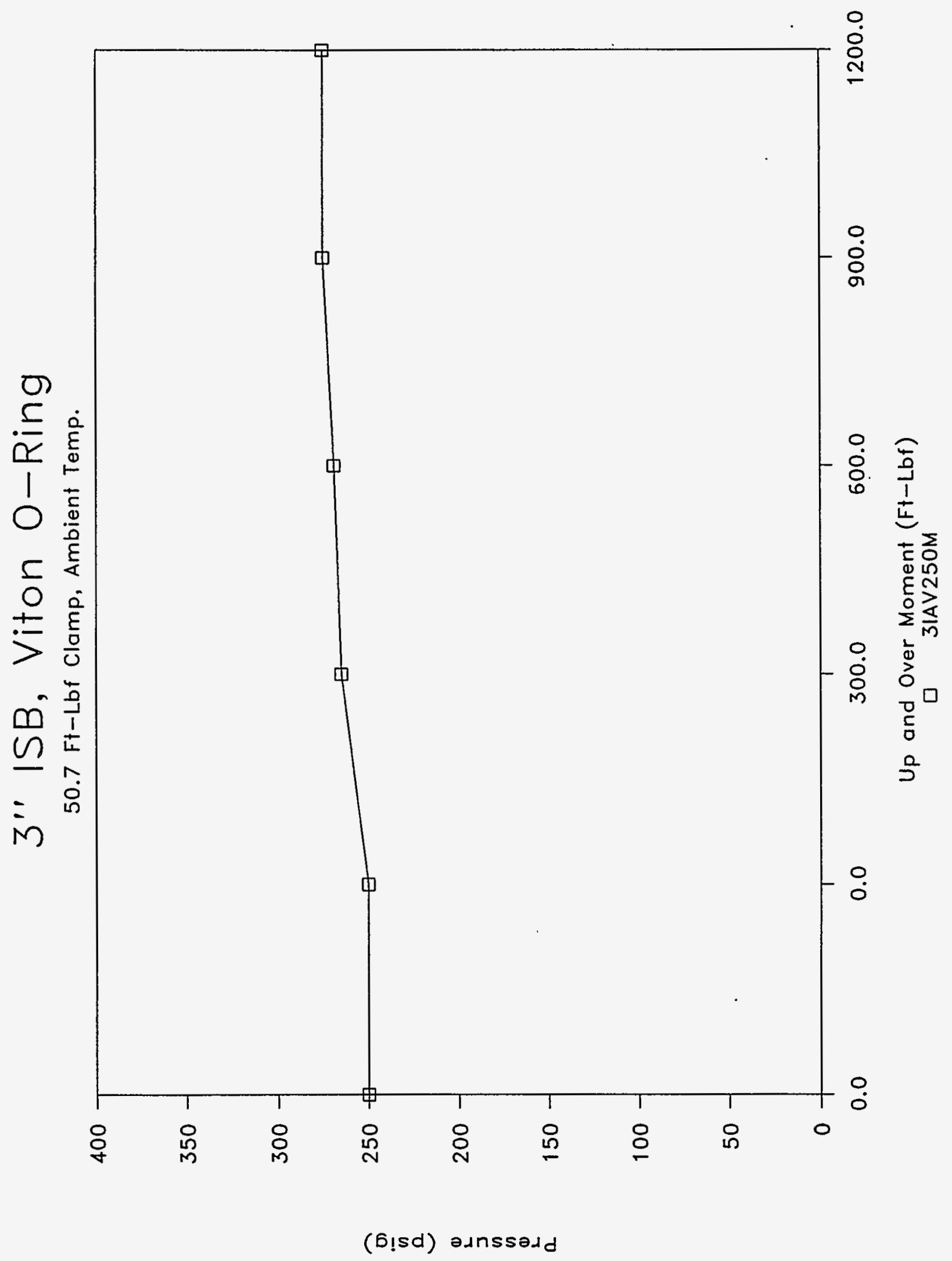

J-9 
WHC-SD-WM-TRP-223

Rev. 0

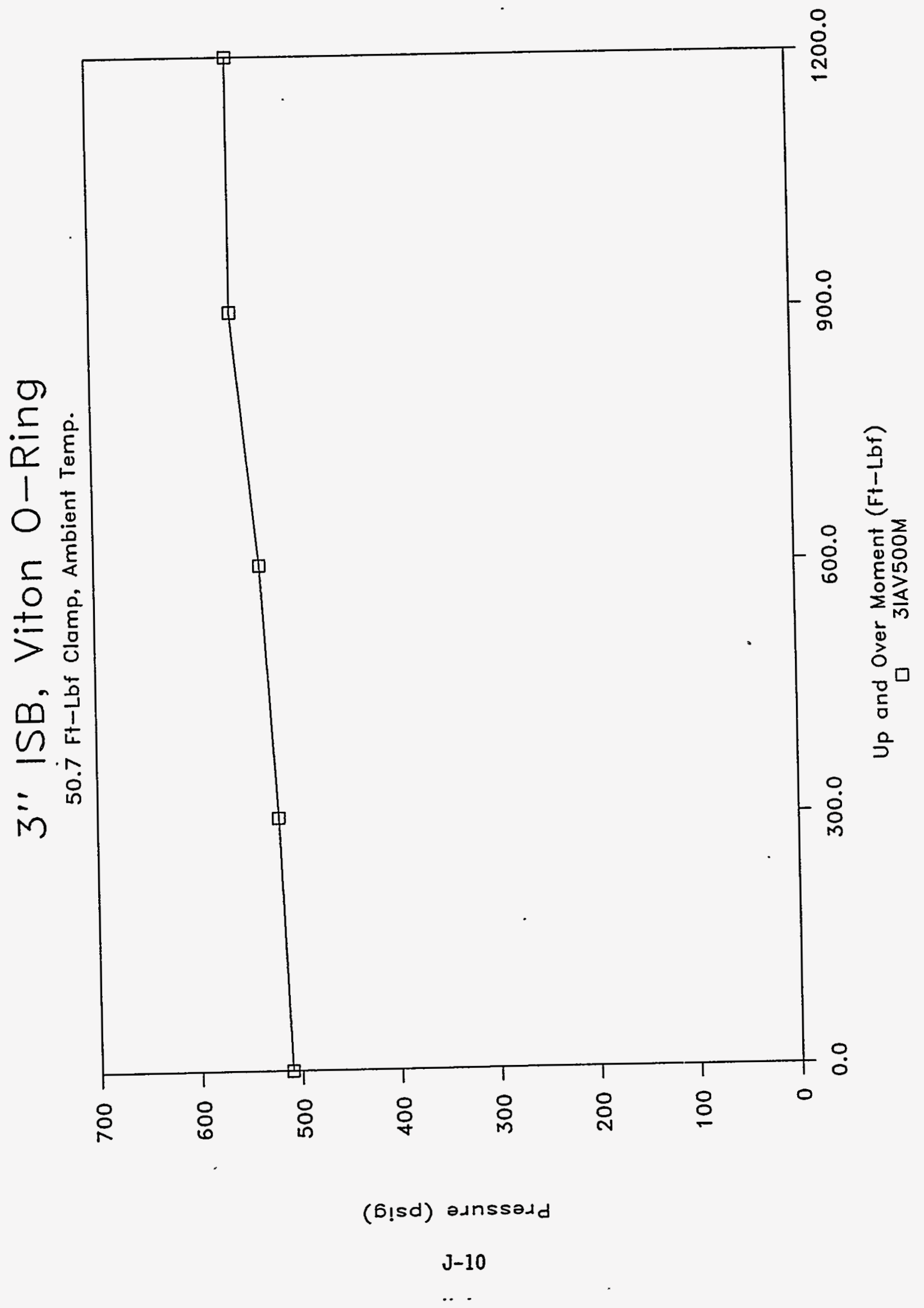


WHC-SD-WM-TRP-223

Rev. 0

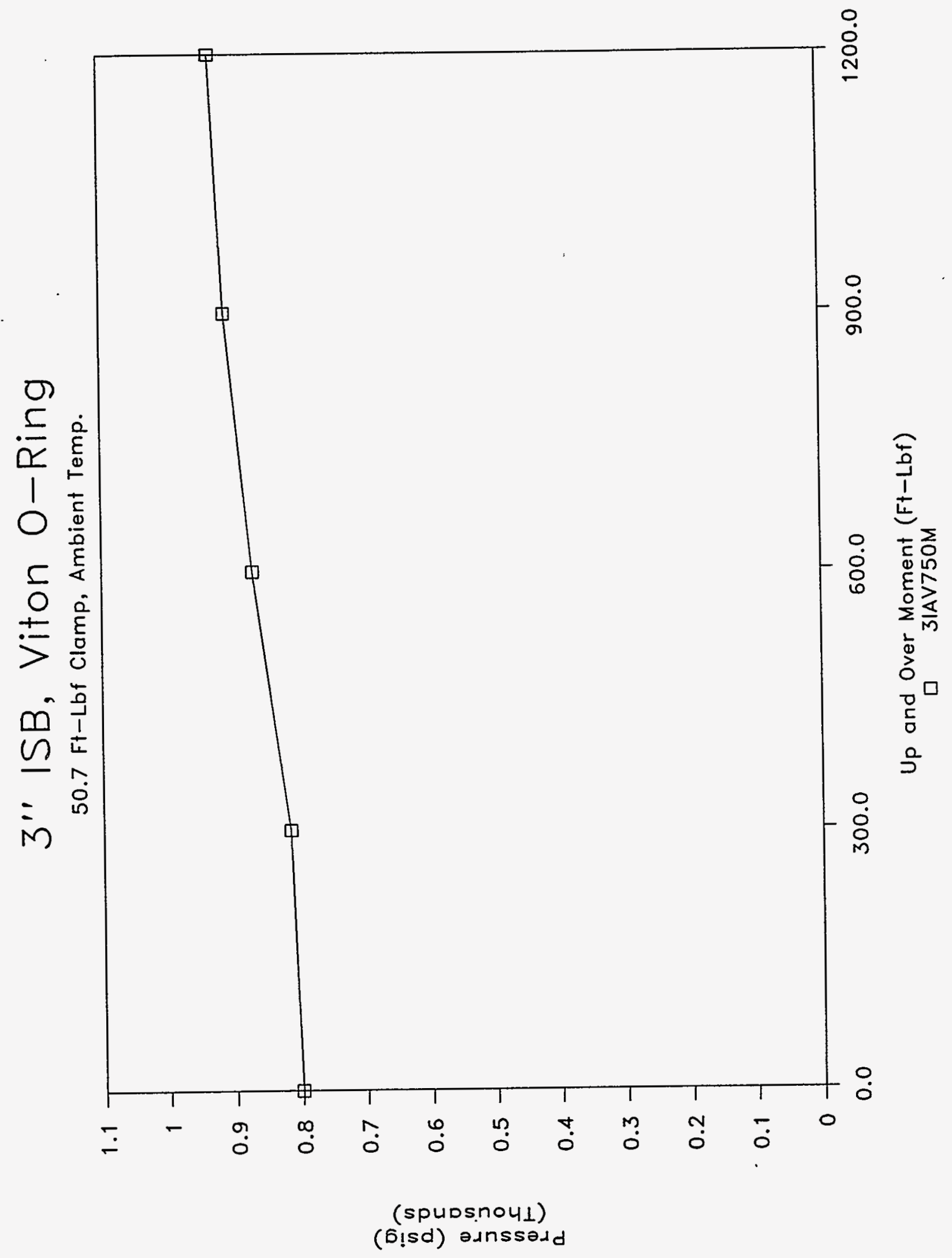

J-11 
WHC-SD-WM-TRP-223

Rev. 0

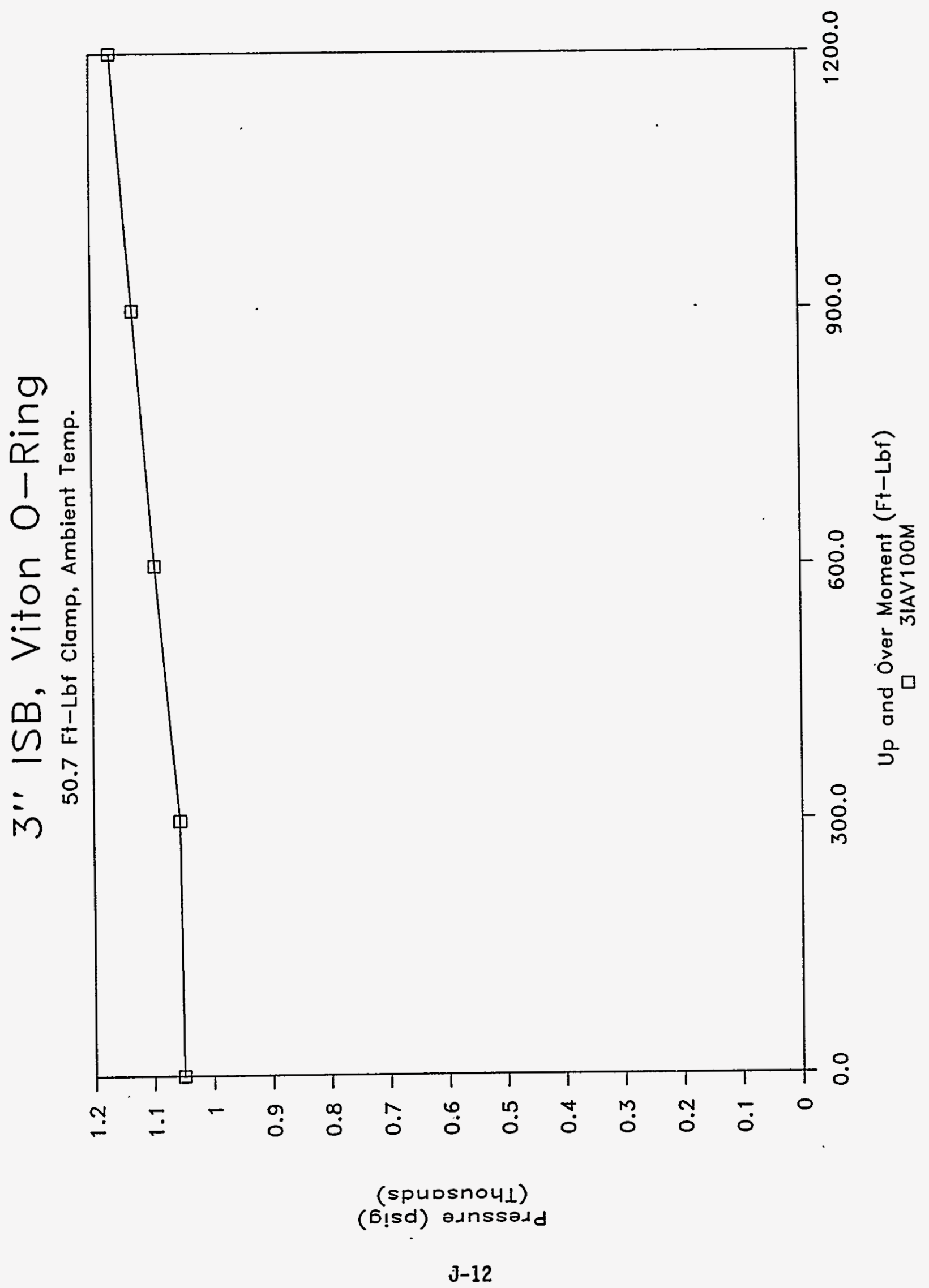


JULY 08,1994

3" ISB CONNECTOR, VITON O-RING, 70 DUROMETER, AMBIENT TEMP.

PRESSURE ASCENSION LEAK TEST - UP AND OVER MOMENT (UPWARD ROTATION)

CLAMPING TORQUE $=50.7 \mathrm{FT}-\mathrm{IBF}$

GRAPH NAME = 3IAV25OM

CHARGE PRESSURE $=250$ PSIG

$\begin{array}{lllllll} & & & & & \text { UPWARD } & \\ \text { PRESSURE } & \text { ELAPSED } & \text { ELAPSED } & \text { ELAPSED } & \text { UPWARD } & \text { MOMENT } & \text { UPWARD } \\ \text { PSIG } & \text { MINUTES } & \text { SECONDS } & \text { DECIMAL } & \text { FORCE } & \text { ARM } & \text { MOMENT } \\ & & & \text { MINUTES } & \text { LBS } & \text { FT. } & \text { FT-LBF. } \\ \text { INPUT } & \text { INPUT } & \text { INPUT } & \text { COMPUTE } & \text { INPUT } & \text { INPUT } & \text { COMPUTE }\end{array}$

$\begin{array}{rrrrrrr}250 & 0 & 0 & 0.00 & 0 & 1.2000 & 0.0 \\ 250 & 2 & 1 & 2.02 & 0 & 1.2000 & 0.0 \\ 265 & 4 & 3 & 4.05 & 250 & 1.2000 & 300.0 \\ 269 & 6 & 2 & 6.03 & 500 & 1.2000 & 600.0 \\ 275 & 8 & 2 & 8.03 & 750 & 1.2000 & 900.0 \\ 275 & 10 & 44 & 10.73 & 1000 & 1.2000 & 1200.0\end{array}$

INCREASED PRESSURE TO 500 PSIG

GRAPH NAME $=3$ IAV50OM

$\begin{array}{rrrrrrr}510 & 12 & 33 & 12.55 & 0 & 1.2000 & 0.0 \\ 520 & 14 & 13 & 14.22 & 250 & 1.2000 & 300.0 \\ 535 & 16 & 13 & 16.22 & 500 & 1.2000 & 600.0 \\ 560 & 18 & 15 & 18.25 & 750 & 1.2000 & 900.0 \\ 560 & 20 & 13 & 20.22 & 1000 & 1.2000 & 1200.0\end{array}$

INCREASED PRESSURE TO 750 PSIG

GRAPH NAME $=3$ IAV750M

$\begin{array}{rrrrrrr}800 & 21 & 35 & 21.58 & 0 & 1.2000 & 0.0 \\ 815 & 23 & 18 & 23.30 & 250 & 1.2000 & 300.0 \\ 870 & 25 & 40 & 25.67 & 500 & 1.2000 & 600.0 \\ 910 & 27 & 22 & 27.37 & 750 & 1.2000 & 900.0 \\ 930 & 29 & 24 & 29.40 & 1000 & 1.2000 & 1200.0\end{array}$

INCREASED PRESSURE TO 1000 PSIG GRAPH NAME = 3IAV100M

$\begin{array}{rrrrrrr}1050 & 31 & 26 & 31.43 & 0 & 1.2000 & 0.0 \\ 1055 & 33 & 15 & 33.25 & 250 & 1.2000 & 300.0 \\ 1095 & 35 & 22 & 35.37 & 500 & 1.2000 & 600.0 \\ 1130 & 37 & 19 & 37.32 & 750 & 1.2000 & 900.0 \\ 1165 & 39 & 27 & 39.45 & 1000 & 1.2000 & 1200.0\end{array}$




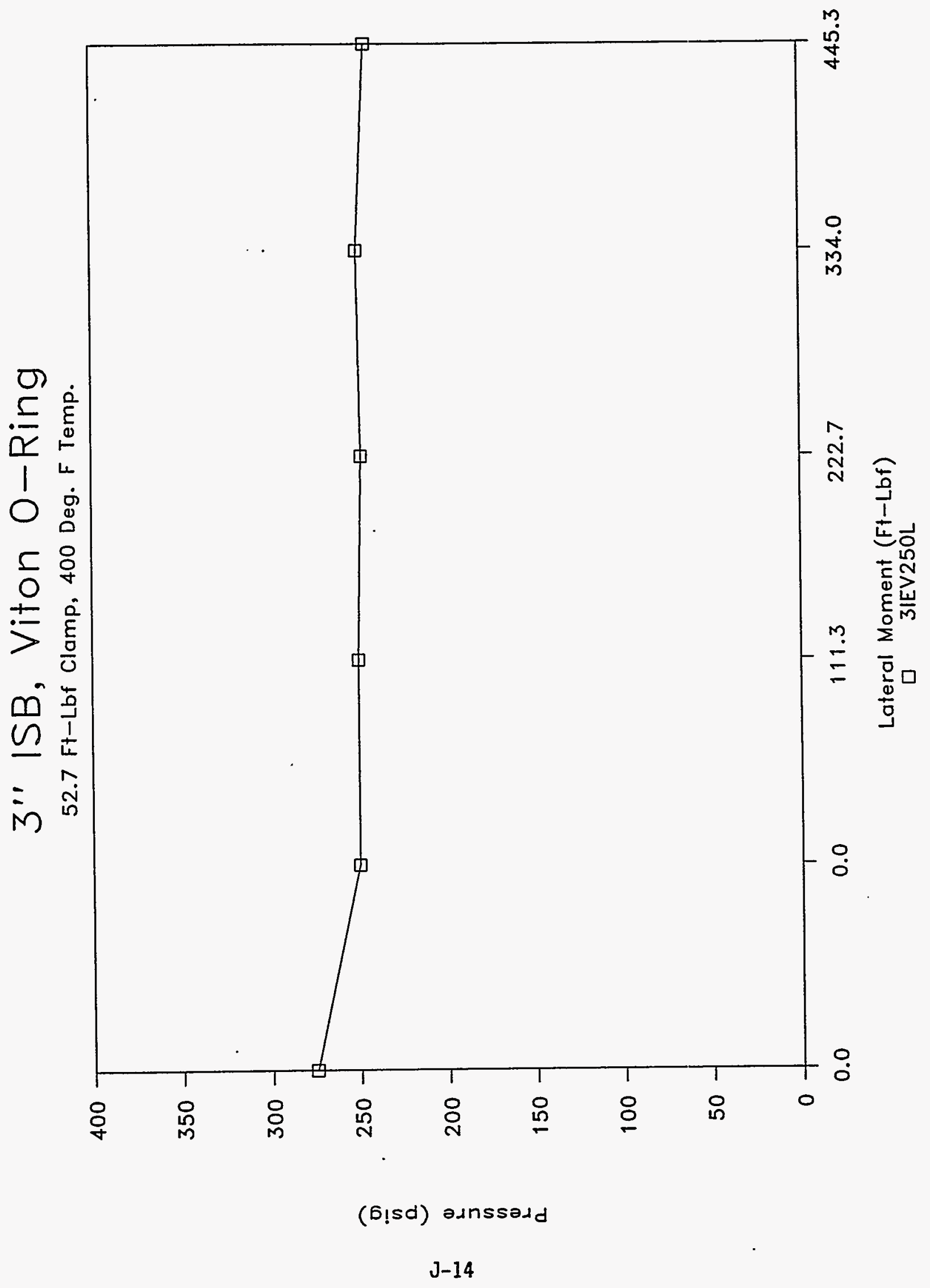


WHC-SD-WM-TRP-223

Rev. 0

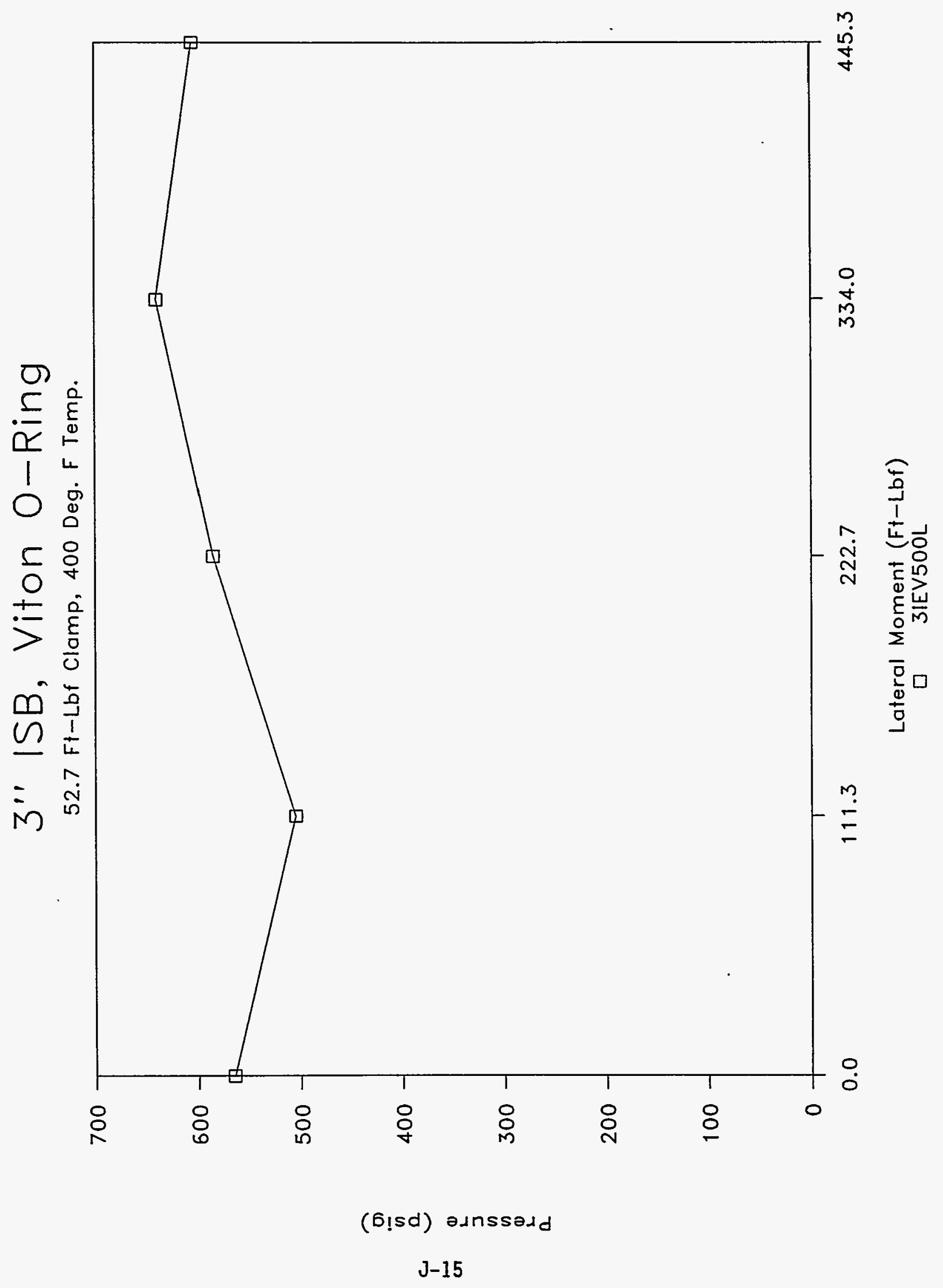


WHC-SD-WM-TRP-223

Rev. 0

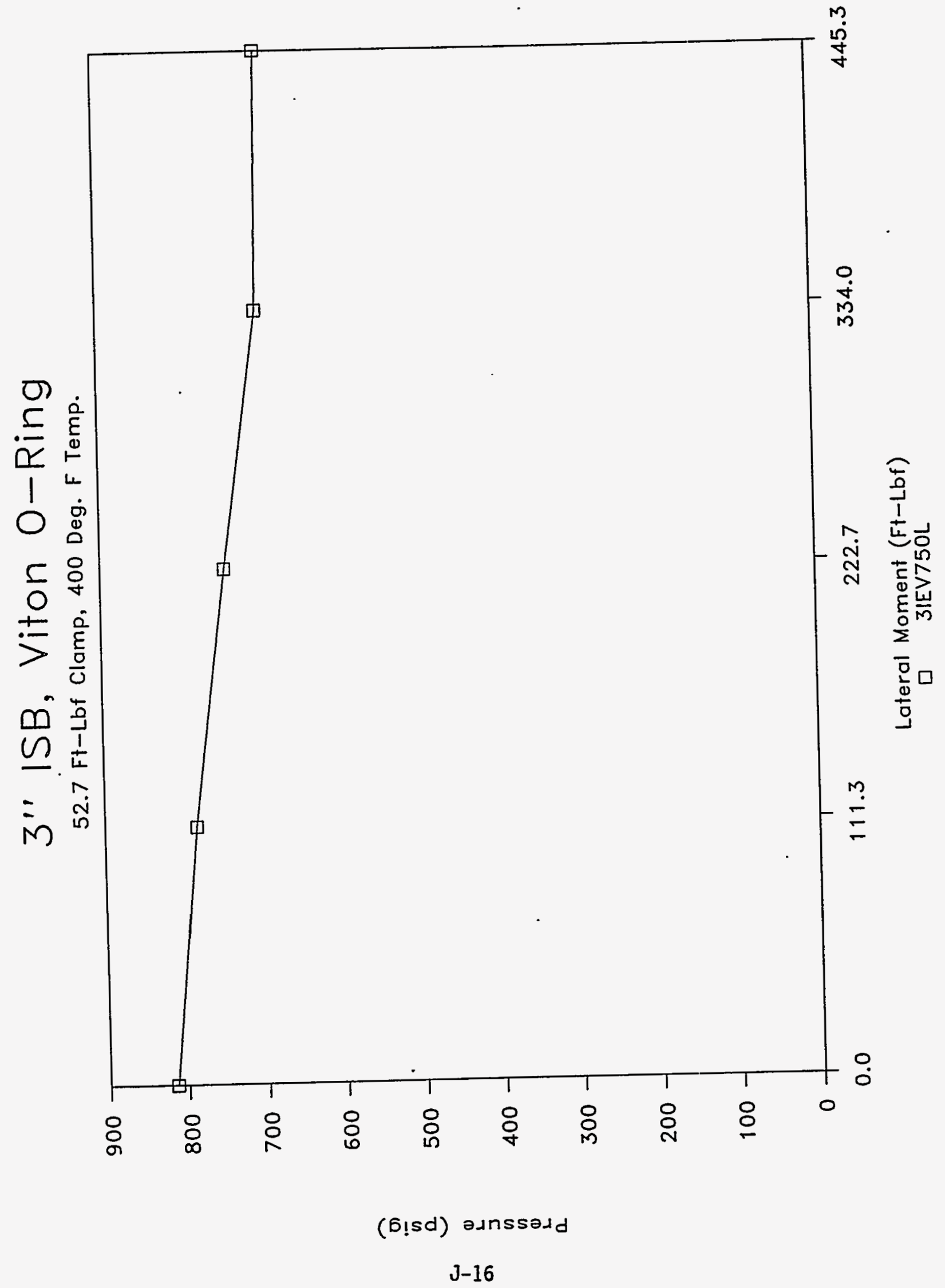


3" ISB, Viton O-Ring

52.7 Ft-Lbf Clamp, 400 Deg. F Temp.

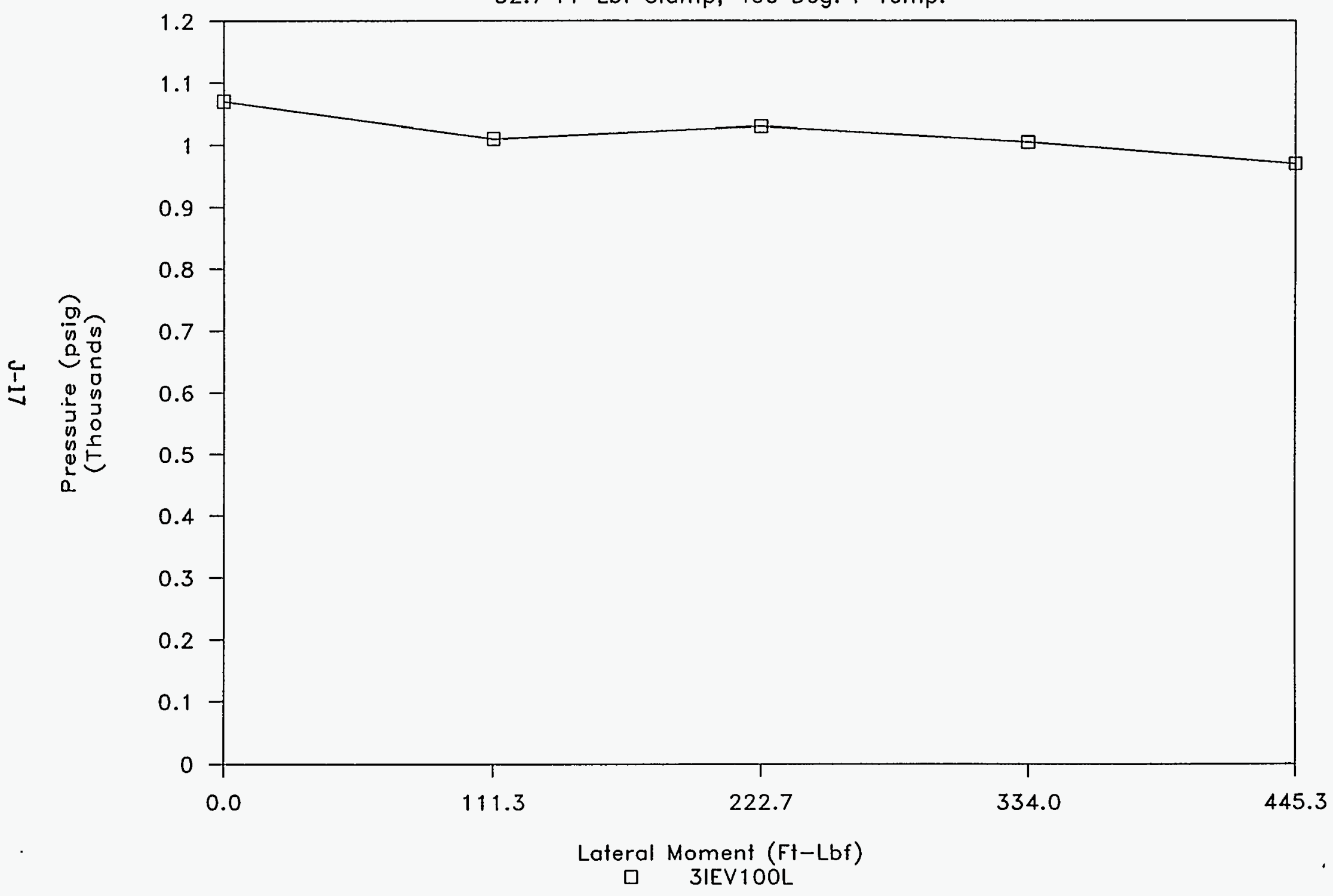


WHC-SD-WM-TRP-223 ReV 0

JULY 13,1994

3" ISB CONNECTOR, VITON O-RING, 70 DUROMETER, ELEVATED TEMP. PRESSURE ASCENSION LEAK TEST - LATERAI MOMENT (SIDEWAYS ROTATION) CLAMPING TORQUE $=52.7 \mathrm{FT}-\mathrm{LBF}$

GRAPH NAME $=3$ IEV250L

CHARGE PRESSURE $=250$ PSIG

$\begin{array}{lllllll} & & & & \text { LATERAL } & \\ \text { PRESSURE } & \text { ELAAPSED } & \text { ELAPSED } & \text { ELAPSED } & \text { IATERAL } & \text { MOMENT } & \text { LATERAI } \\ \text { PSIG } & \text { MINUTES } & \text { SECONDS } & \text { DECIMAI } & \text { FORCE } & \text { ARM } & \text { MOMENT } \\ & & & \text { MINUTES } & \text { LBS } & \text { FT. } & \text { FT-IBF. } \\ \text { INPUT } & \text { INPUT } & \text { INPUT } & \text { COMPUTE } & \text { INPUT } & \text { INPUT } & \text { COMPUTE }\end{array}$

$\begin{array}{rrrrrrr}275 & 0 & 0 & 0.00 & 0 & 0.4453 & 0.0 \\ 250 & 2 & 10 & 2.17 & 0 & 0.4453 & 0.0 \\ 250 & 4 & 9 & 4.15 & 250 & 0.4453 & 111.3 \\ 248 & 6 & 9 & 6.15 & 500 & 0.4453 & 222.7 \\ 250 & 8 & 16 & 8.27 & 750 & 0.4453 & 334.0 \\ 245 & 10 & 4 & 10.07 & 1000 & 0.4453 & 445.3\end{array}$

INCREASED PRESSURE TO 500 PSIG

GRAPH NAME $=3$ IEV50OL

$\begin{array}{rrrrrrr}565 & 12 & 5 & 12.08 & 0 & 0.4453 & 0.0 \\ 505 & 14 & 3 & 14.05 & 250 & 0.4453 & 111.3 \\ 585 & 16 & 2 & 16.03 & 500 & 0.4453 & 222.7 \\ 640 & 18 & 5 & 18.08 & 750 & 0.4453 & 334.0 \\ 605 & 20 & 4 & 20.07 & 1000 & 0.4453 & 445.3\end{array}$

INCREASED PRESSURE TO 750 PSIG GRAPH NAME = 3IEV750L

$\begin{array}{rrrrrrr}815 & 22 & 4 & 22.07 & 0 & 0.4453 & 0.0 \\ 785 & 24 & 10 & 24.17 & 250 & 0.4453 & 111.3 \\ 745 & 26 & 8 & 26.13 & 500 & 0.4453 & 222.7 \\ 700 & 28 & 10 & 28.17 & 750 & 0.4453 & 334.0 \\ 695 & 30 & 8 & 30.13 & 1000 & 0.4453 & 445.3\end{array}$

INCREASED PRESSURE TO 1000 PSIG GRAPH NAME = 3IEV1000I

$\begin{array}{rrrrrrr}1070 & 32 & 7 & 32.12 & 0 & 0.4453 & 0.0 \\ 1010 & 34 & 15 & 34.25 & 250 & 0.4453 & 111.3 \\ 1030 & 36 & 3 & 36.05 & 500 & 0.4453 & 222.7 \\ 1005 & 38 & 13 & 38.22 & 750 & 0.4453 & 334.0 \\ 970 & 40 & 5 & 40.08 & 1000 & 0.4453 & 445.3\end{array}$


WHC-SD-WM-TRP-223

Rev. 0

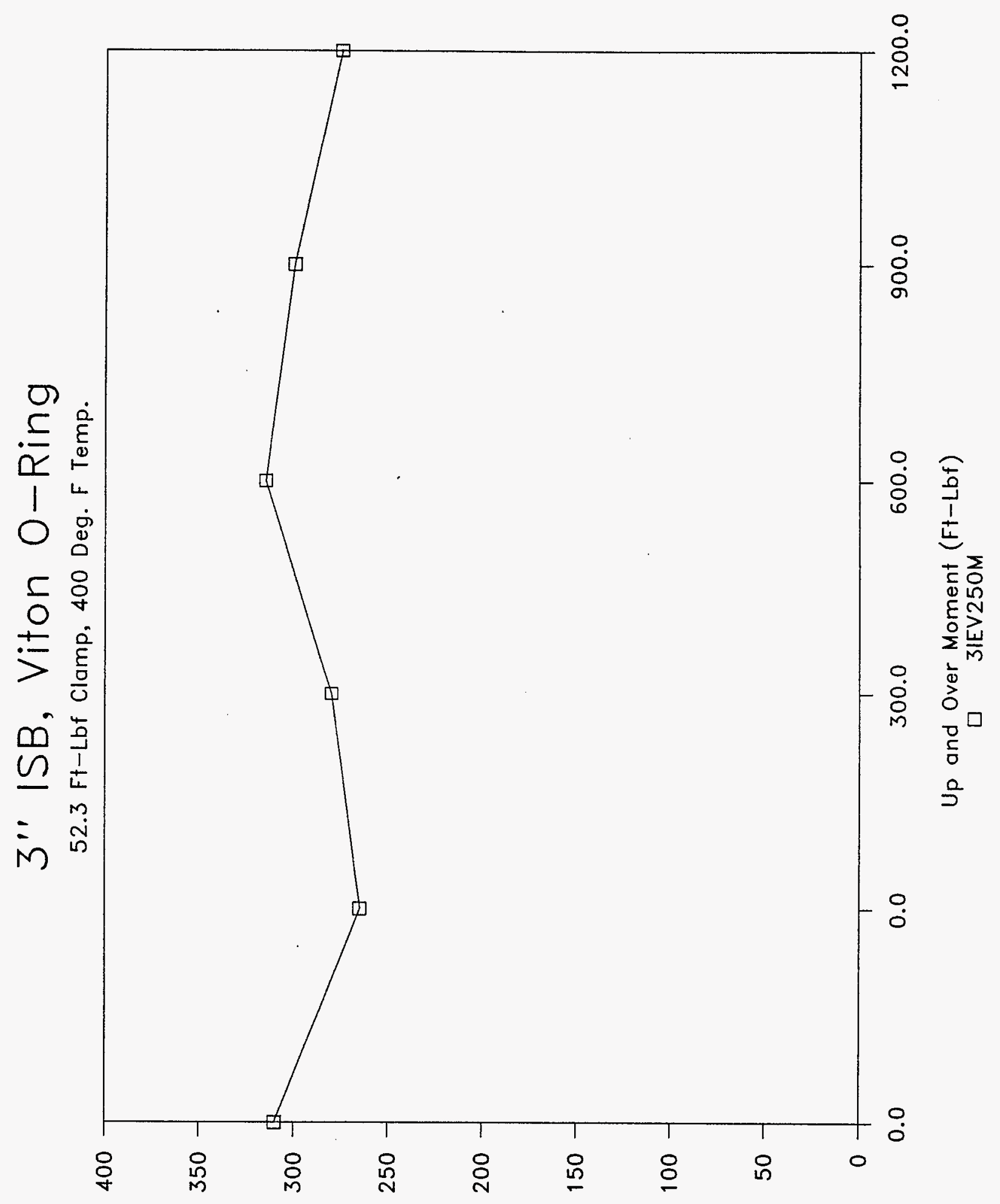

(bisd) asnssadd

J-19 
WHC-SD-WM-TRP-223

Rev. 0

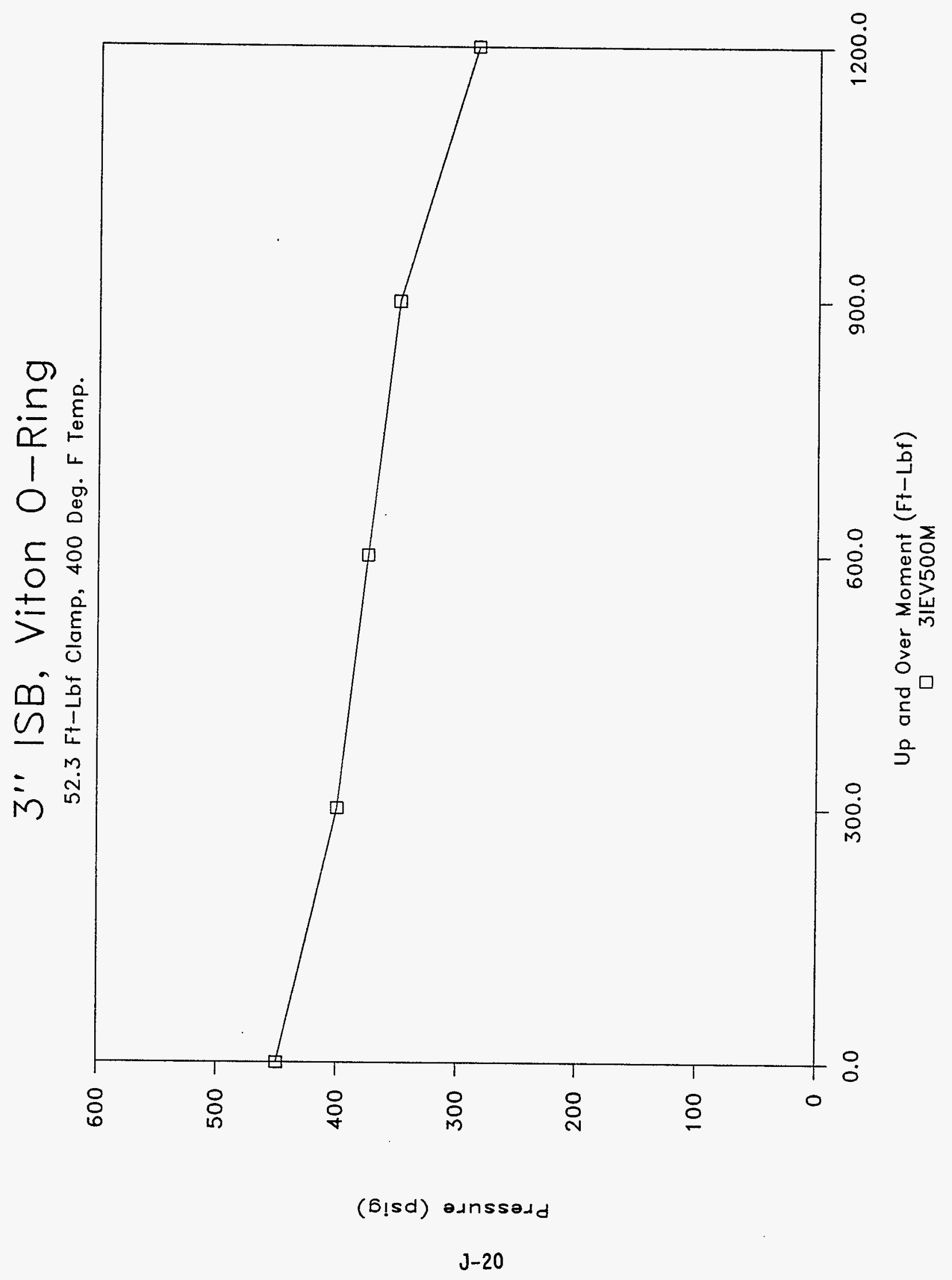


WHC-SD-WM-TRP-223

Rev. 0

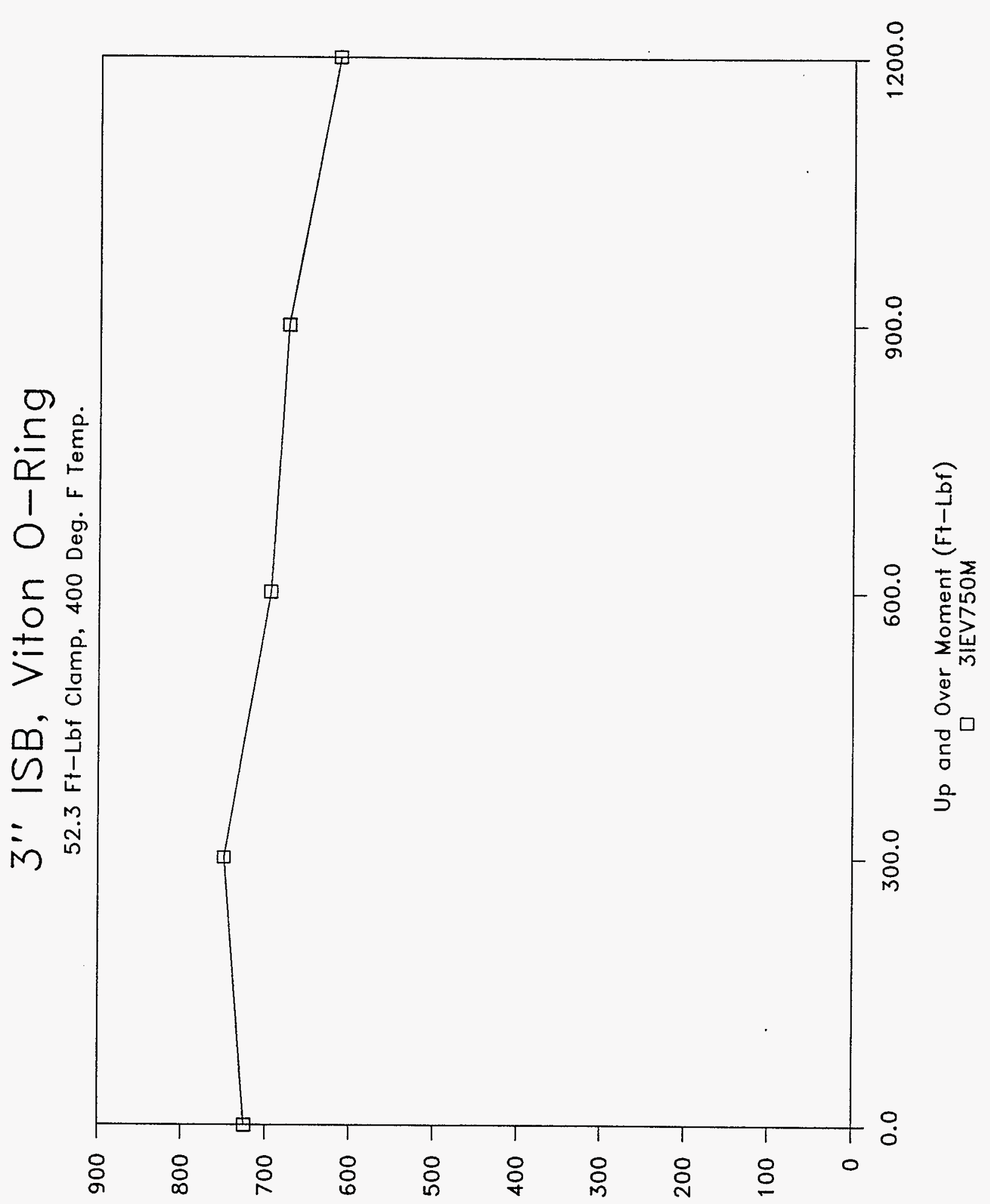

(6!sd) adnssadd 


\section{3" ISB, Viton O-Ring}

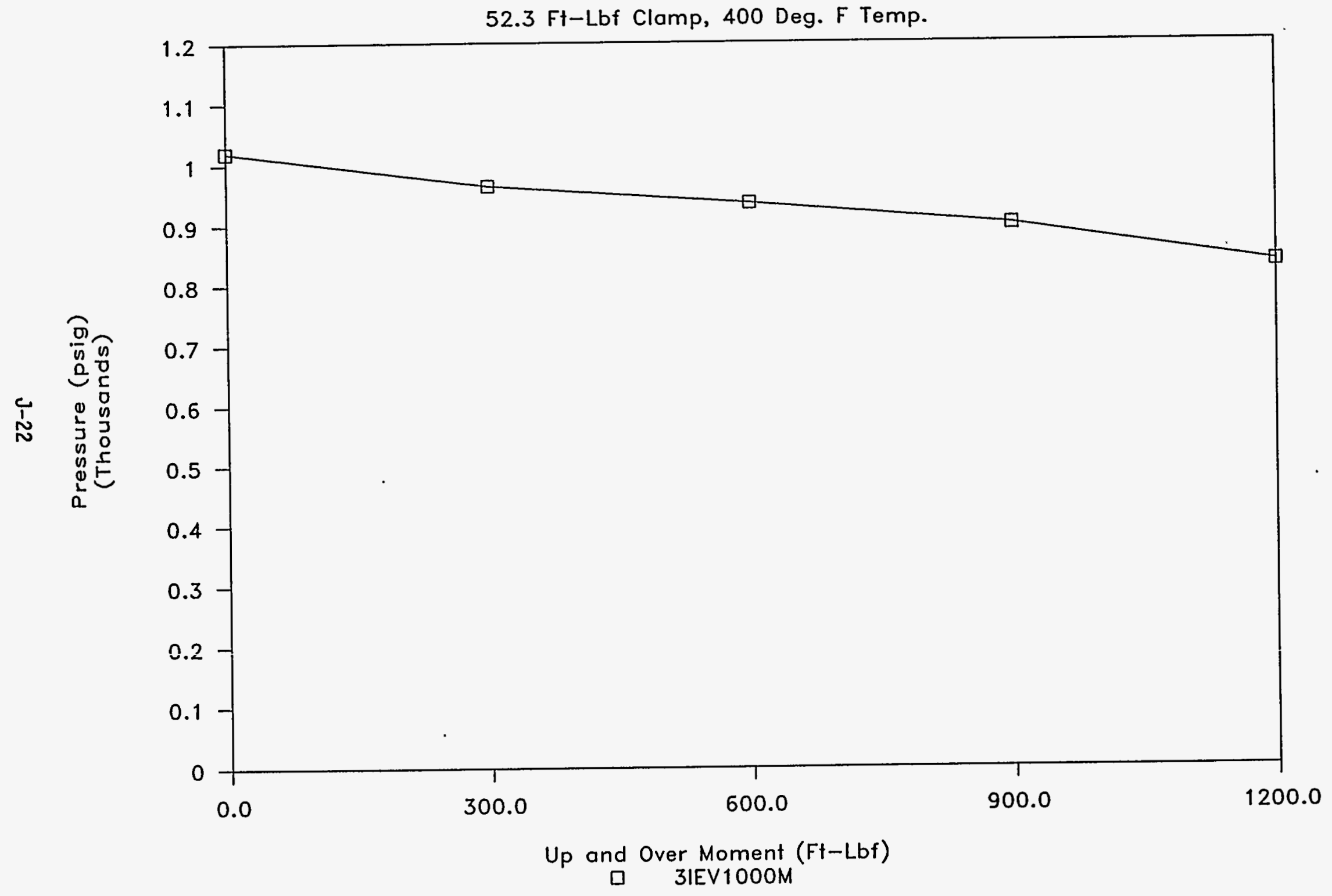


WHC-SD-WM-TRP-223 ReV 0

JULY 12,1994

3" ISB CONNECTOR, VITON O-RING, 70 DUROMETER, ELEVATED TEMP. PRESSURE ASCENSION LEAK TEST - UP AND OVER MOMENT (UPWARD ROTATION)

CLAMPING TORQUE $=52.3$ FT-LBF GRAPH NAME $=3$ IEV250M

CHARGE PRESSURE $=250$ PSIG

TEMPERATURE $=400 \mathrm{Deg} \cdot \mathrm{F}$

$\begin{array}{llllllll} & & & & & & \text { UPWARD } \\ \text { INITIAI } & \text { HOLD } & \text { ELAPSED } & \text { ELAPSED } & \text { ELAPSED } & \text { UPWARD } & \text { MOMENT } & \text { UPWARD } \\ \text { PRESSURE } & \text { PRESSURE } & \text { MINUTES } & \text { SECONDS } & \text { DECIMAL } & \text { FORCE } & \text { ARM } & \text { MOMENT } \\ \text { PSIG } & \text { PSIG } & & & \text { MINUTES } & \text { IBS } & \text { FT. } & \text { FT-IBF. } \\ \text { INPUT } & \text { INPUT } & \text { INPUT } & \text { INPUT } & \text { COMPUTE } & \text { INPUT } & \text { INPUT } & \text { COMPUTE }\end{array}$

$\begin{array}{rrrrrrrr}310 & 310 & 0 & 0 & 0.00 & 0 & 1.2000 & 0.0 \\ 265 & 265 & 2 & 3 & 2.05 & 0 & 1.2000 & 0.0 \\ 300 & 280 & 4 & 3 & 4.05 & 250 & 1.2000 & 300.0 \\ 280 & 315 & 6 & 5 & 6.08 & 500 & 1.2000 & 600.0 \\ 290 & 300 & 8 & 3 & 8.05 & 750 & 1.2000 & 900.0 \\ 275 & 275 & 10 & 5 & 10.08 & 1000 & 1.2000 & 1200.0\end{array}$

INCREASED PRESSURE TO 500 PSIG

GRAPH NAME $=3$ IEV50OM

$\begin{array}{rrrrrrrr}510 & 450 & 12 & 9 & 12.15 & 0 & 1.2000 & 0.0 \\ 360 & 400 & 14 & 8 & 14.13 & 250 & 1.2000 & 300.0 \\ 400 & 375 & 16 & 2 & 16.03 & 500 & 1.2000 & 600.0 \\ 350 & 350 & 18 & 3 & 18.05 & 750 & 1.2000 & 900.0 \\ 320 & 285 & 20 & 3 & 20.05 & 1000 & 1.2000 & 1200.0\end{array}$

INCREASED PRESSURE TO 750 PSIG

GRAPH NAME $=3$ IEV750M

$\begin{array}{rrrrrrrr}760 & 725 & 22 & 2 & 22.03 & 0 & 1.2000 & 0.0 \\ 735 & 750 & 24 & 1 & 24.02 & 250 & 1.2000 & 300.0 \\ 705 & 695 & 26 & 3 & 26.05 & 500 & 1.2000 & 600.0 \\ 685 & 675 & 28 & 3 & 28.05 & 750 & 1.2000 & 900.0 \\ 690 & 615 & 30 & 3 & 30.05 & 1000 & 1.2000 & 1200.0\end{array}$

INCREASED PRESSURE TO 1000 PSIG

GRAPH NAME $=3$ IEV1000M

$\begin{array}{rrrrrrrr}1020 & 1020 & 32 & 5 & 32.08 & 0 & 1.2000 & 0.0 \\ 1020 & 965 & 34 & 4 & 34.07 & 250 & 1.2000 & 300.0 \\ 940 & 935 & 36 & 9 & 36.15 & 500 & 1.2000 & 600.0 \\ 940 & 900 & 38 & 6 & 38.10 & 750 & 1.2000 & 900.0 \\ 855 & 835 & 40 & 4 & 40.07 & 1000 & 1.2000 & 1200.0\end{array}$




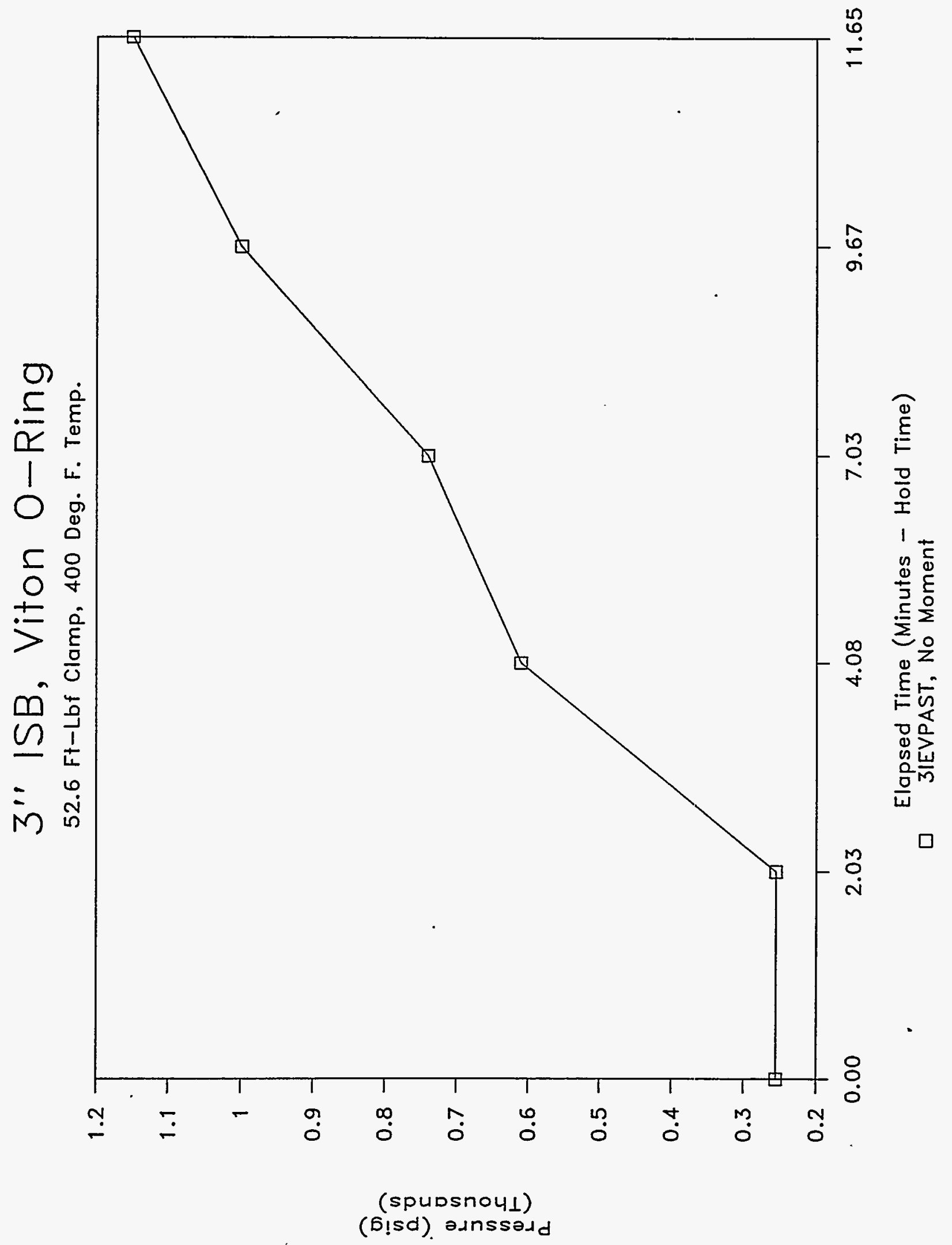


WHC-SD-WM-TRP-223 ReV 0

JULY 11, 1994

3" ISB CONNECTOR, VITON O-RING, 70 DUROMETER, ELEVATED TEMP.

PRESSURE ASCENSION LEAK TEST - NO MOMENT APPLIED

CLAMPING TORQUE $=52.6 \mathrm{FT}-\mathrm{LBF}$

GRAPH NAME $=3$ IEVPAST

CHARGE PRESSURE $=250$ PSIG TO 1150 PSIG

TEMPERATURE $=400 \mathrm{Deg} . \mathrm{F}$

\begin{tabular}{|c|c|c|c|c|}
\hline $\begin{array}{l}\text { PRESSURE } \\
\text { PSIG }\end{array}$ & $\begin{array}{l}\text { EI_APSED } \\
\text { MINUTES }\end{array}$ & $\begin{array}{l}\text { ELAPSED } \\
\text { SECONDS }\end{array}$ & $\begin{array}{l}\text { ELAPSED } \\
\text { DECIMAI } \\
\text { MINUTES }\end{array}$ & $\begin{array}{l}\text { APPLIED } \\
\text { FORCE } \\
\text { LBS }\end{array}$ \\
\hline INPUT & INPUT & INPUT & COMPUTE & INPUT \\
\hline $\begin{array}{r}255 \\
255 \\
610 \\
740 \\
1000\end{array}$ & $\begin{array}{r}0 \\
2 \\
4 \\
7 \\
9\end{array}$ & $\begin{array}{r}0 \\
2 \\
5 \\
2 \\
40 \\
30\end{array}$ & $\begin{array}{r}0.00 \\
2.03 \\
4.08 \\
7.03 \\
9.67\end{array}$ & $\begin{array}{l}0 \\
0 \\
0 \\
0 \\
0 \\
0\end{array}$ \\
\hline
\end{tabular}


WHC-SD-WM-TRP-223

Rev. 0

APPENDIX K: GRAPHS OF 4-IN. FLUOROSILICOINE TESTS

K-1 


\section{4" ISB, 70 SH Fluorosilic one O-Ring}

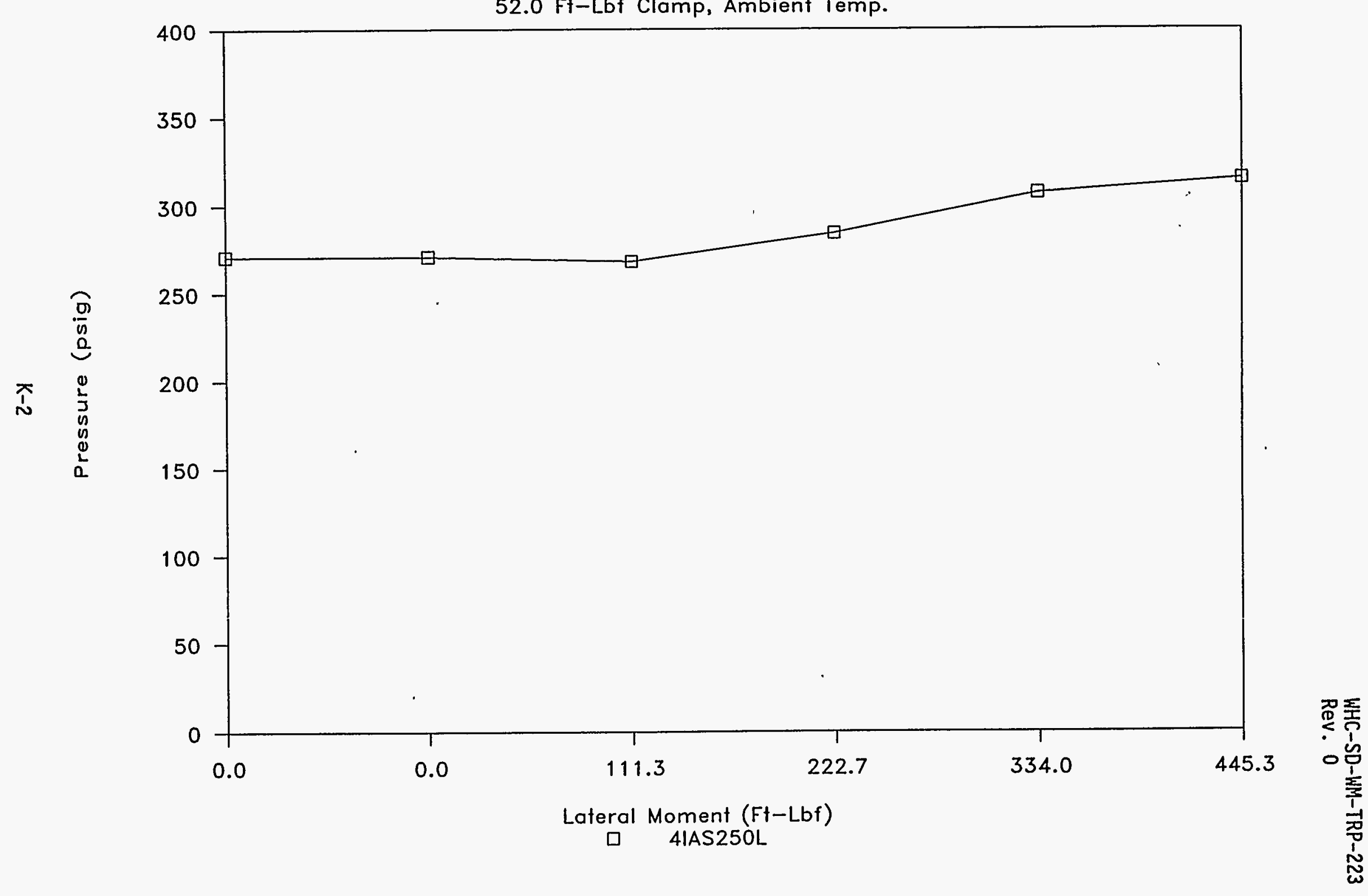


WHC-SD-WM-TRP-223

Rev. 0

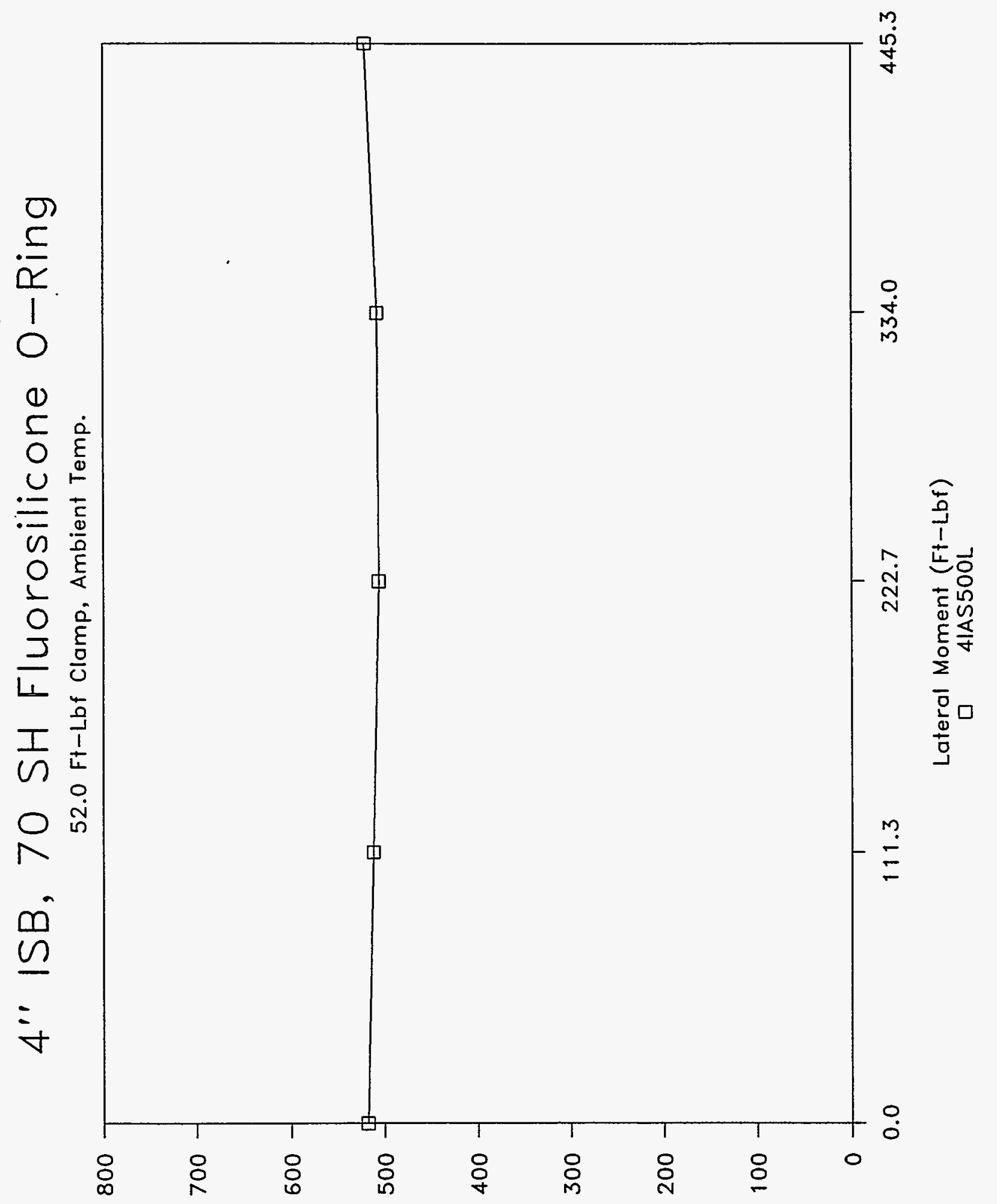

(b!sd) a annssadd

$k-3$ 
WHC-SD-HM-TRP-223

Rev. 0

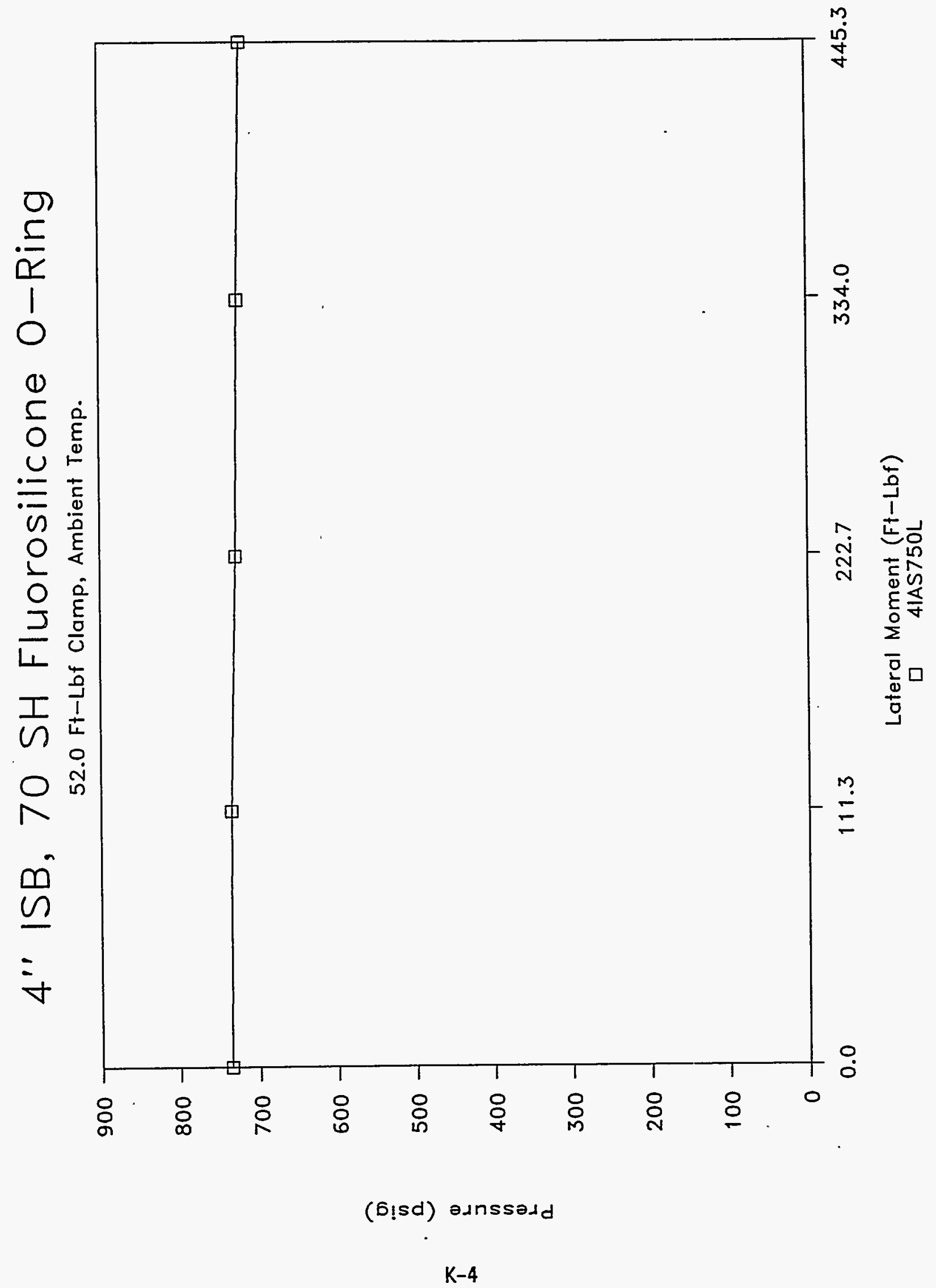


WHC-SD-WM-TRP-223

Rev. 0

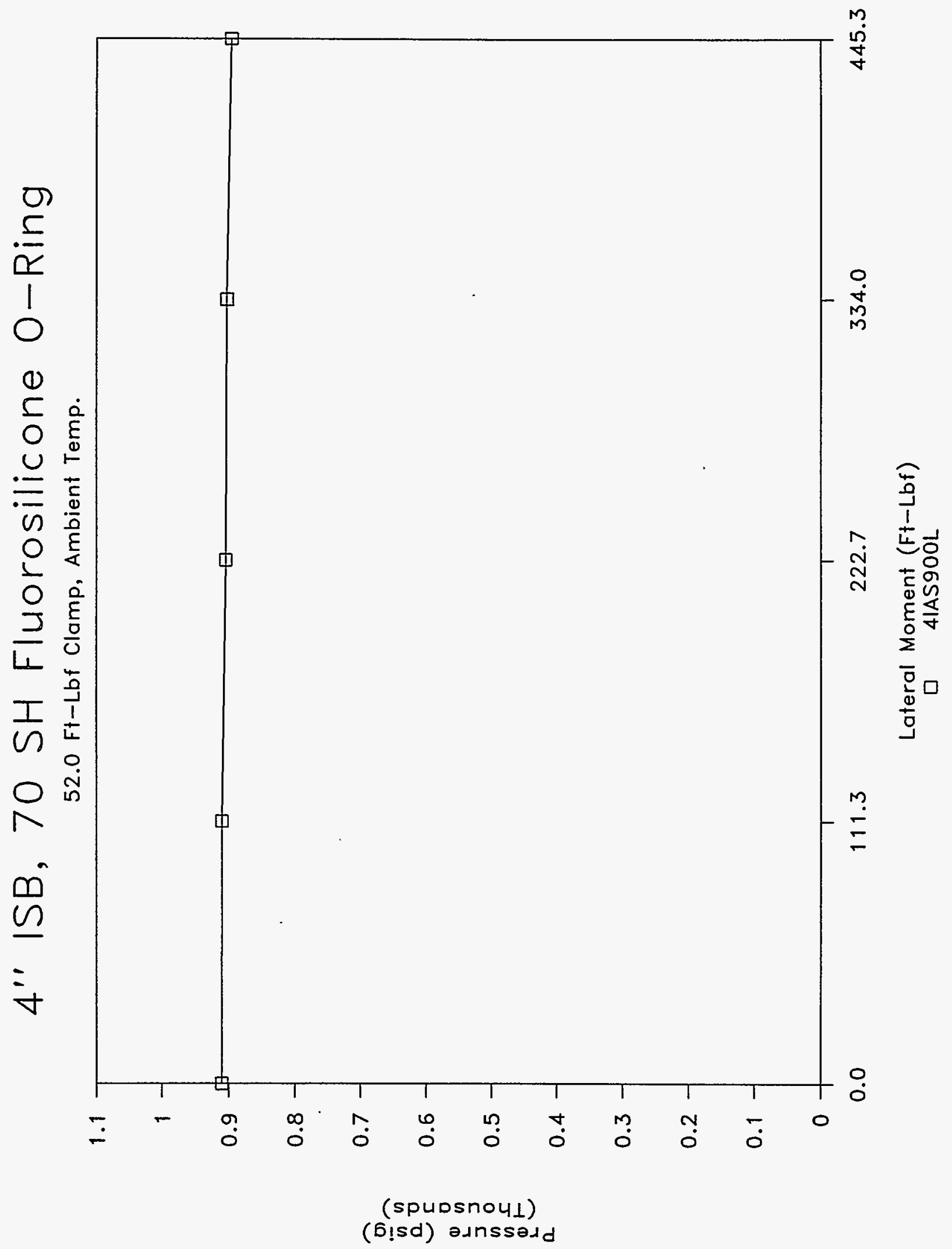

K-5 
JUNE 20, 1994

4" ISB CONNECTOR, 70 SH FLUOROSIIICONE O-RING, AMBI:ENT TEMP. PRESSURE ASCENSION IEAK TEST - ILATERAI MOMENT (SIDIWAYS ROTATION) CLAMPING TORQUE $=52.0$ FT-IBF GRAPH NAME = 4JAS250L

CHARGE PRESSURE $=250$ PSIG

$\begin{array}{lllllll} & & & & \text { LAATERIL } & \\ \text { PRESSURE } & \text { ELAPSED } & \text { ELAPSED } & \text { ELAPSED } & \text { LATERAL } & \text { MOMENI } & \text { LATERAI } \\ \text { PSIG } & \text { MINUTES } & \text { SECONDS } & \text { DECIMAI } & \text { FORCE } & \text { ARM } & \text { MOMENT } \\ & & & \text { MINUTES } & \text { LBS } & \text { FT. } & \text { FT-LBF. } \\ \text { INPUT } & \text { INPUT } & \text { INPUT } & \text { COMPUTE } & \text { INPUT } & \text { INPUT } & \text { COMPUTE }\end{array}$

$\begin{array}{rrrrrrr}271 & 0 & 0 & 0.00 & 0 & 0.4453 & 0.0 \\ 271 & 2 & 1 & 2.02 & 0 & 0.4453 & 0.0 \\ 268 & 5 & 34 & 5.57 & 250 & 0.4453 & 111.3 \\ 284 & 9 & 18 & 9.30 & 500 & 0.4453 & 222.7 \\ 307 & 13 & 8 & 13.13 & 750 & 0.4453 & 334.0 \\ 315 & 16 & 5 & 16.08 & 1000 & 0.4453 & 445.3\end{array}$

INCREASED CHARGE PRESSURE TO 500 PSIG.

GRAPH NAME $=4$ IAS50OI

$\begin{array}{rrrrrrr}518 & 20 & 50 & 20.83 & 0 & 0.4453 & 0.0 \\ 512 & 23 & 3 & 23.05 & 250 & 0.4453 & 111.3 \\ 506 & 25 & 31 & 25.52 & 500 & 0.4453 & 222.7 \\ 508 & 28 & 4 & 28.07 & 750 & 0.4453 & 334.0 \\ 521 & 31 & 5 & 31.08 & 1000 & 0.4453 & 445.3\end{array}$

INCREASED CHARGE PRESSURE TO 750 PSIG.

GRAPH NAME $=4$ IAS750L

737

36

$21 \quad 36.35$

$0 \quad 0.4453$

0.0

RE-PRESURIZED TO 750 PSIG DUE TO LEAK AT VALVE.

$\begin{array}{rrrrrrr}736 & 39 & 52 & 39.87 & 0 & 0.4453 & 0.0 \\ 735 & 42 & 3 & 42.05 & 250 & 0.4453 & 111.3 \\ 728 & 45 & 10 & 45.17 & 500 & 0.4453 & 222.7 \\ 725 & 48 & 8 & 48.13 & 750 & 0.4453 & 334.0 \\ 720 & 51 & 2 & 51.03 & 1000 & 0.4453 & 445.3\end{array}$

INCREASED CHARGE PRESSURE TO 900 PSIG.

GRAPH: NAME = 4IAS900I

$\begin{array}{rrrrrrr}911 & 55 & 50 & 55.83 & 0 & 0.4453 & 0.0 \\ 910 & 58 & 32 & 58.53 & 250 & 0.4 .453 & 111.3 \\ 905 & 61 & 10 & 61.17 & 500 & 0.4453 & 222.7 \\ 903 & 64 & 8 & 64.13 & 750 & 0.4453 & 334.0 \\ 896 & 67 & 39 & 67.65 & 1000 & 0.4453 & 445.3\end{array}$


4" ISB, 70 SH Fluorosilicone O-Ring

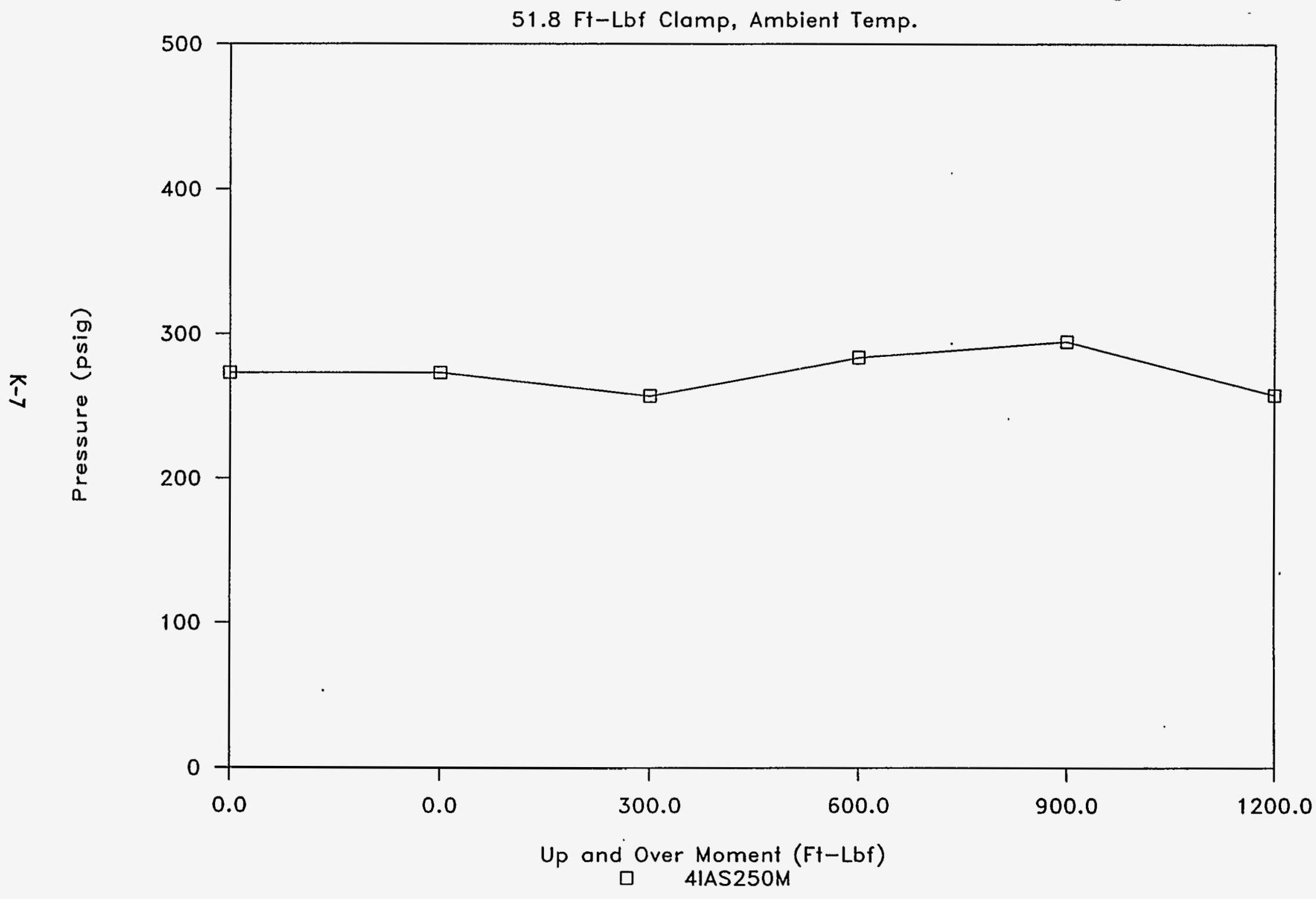




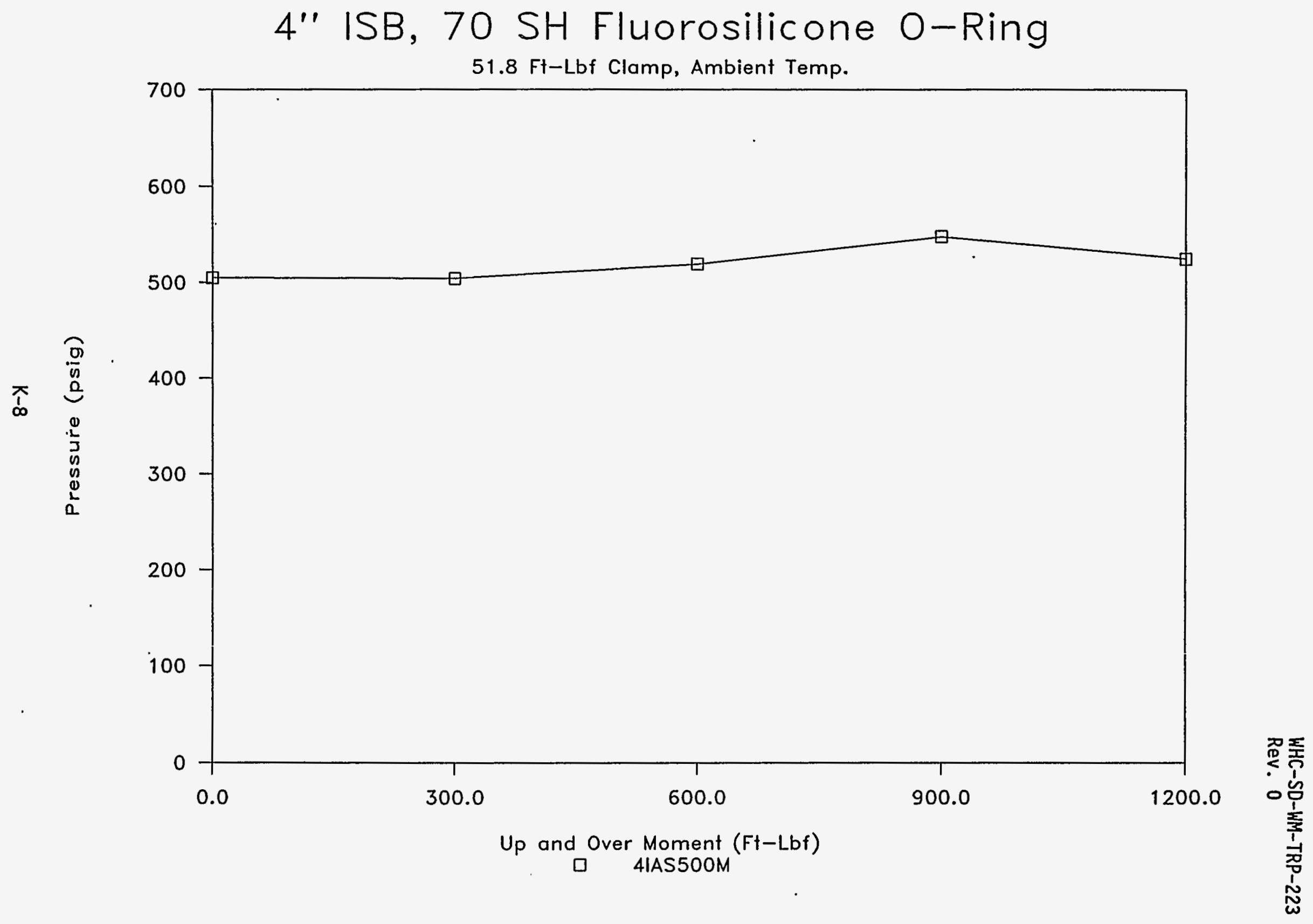




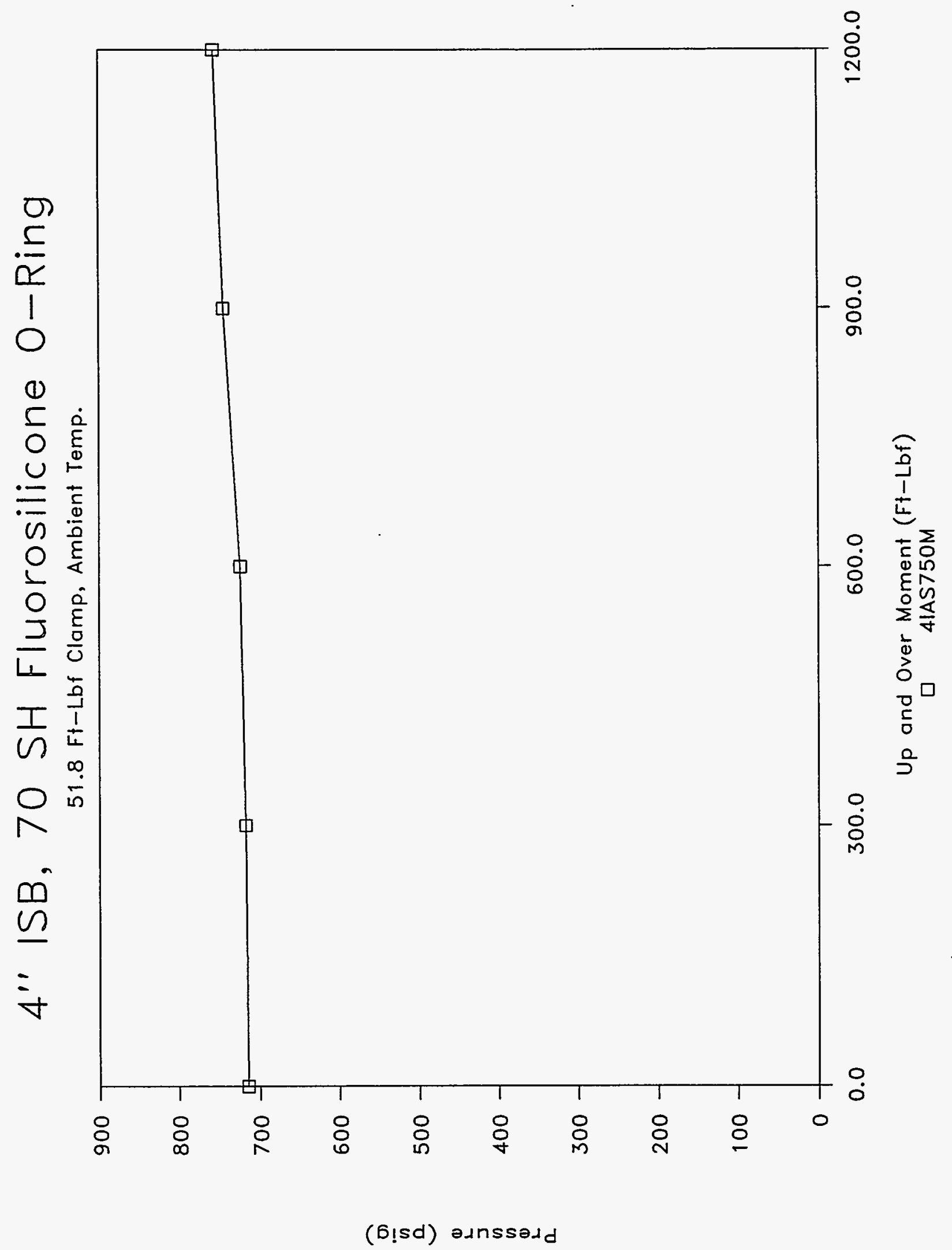




\section{4" ISB, 70 SH Fluorosilicone O-Ring}

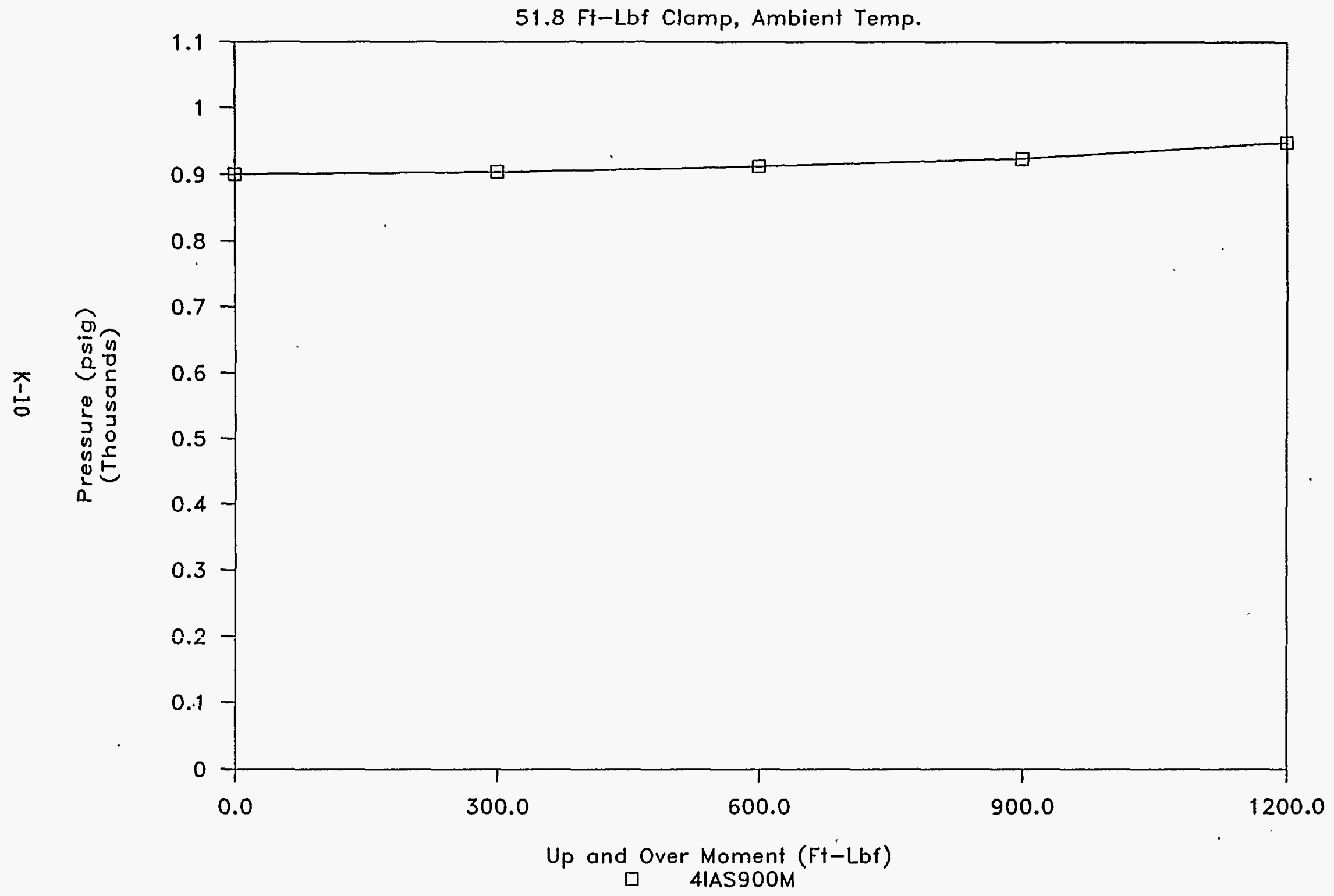


JUNE 16,1994

4" ISB CONNECTOR, $70 \mathrm{SH}$ FLUOROSILICONE O-RING, AMBIENT TEMP. PRESSURE ASCENSION LEAK TEST - UP AND OVER MOMENT (UPWARD ROTATION) CLAAMPING TORQUE $=51.8$ FT-LBF GRAPH NAME $=4$ IAS250M

CHARGE PRESSURE $=250$ PSIG

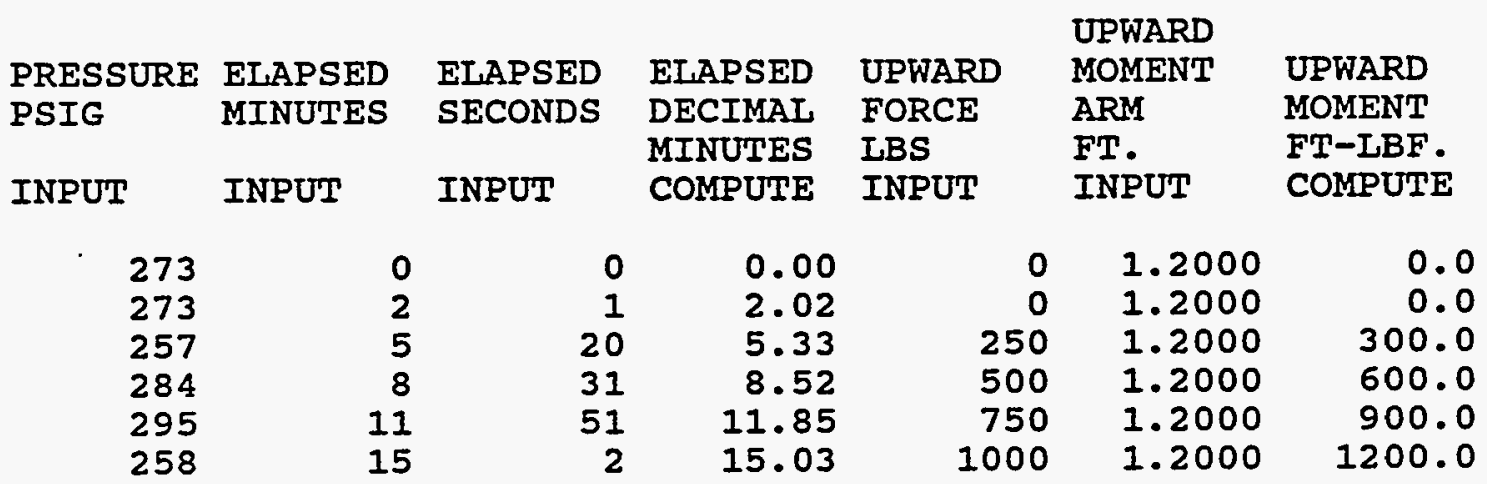

INCREASED CHARGE PRESSURE TO 500 PSIG.

GRAPH NAME $=4$ IAS50OM

$\begin{array}{rrrrrrr}505 & 22 & 3 & 22.05 & 0 & 1.2000 & 0.0 \\ 504 & 25 & 21 & 25.35 & 250 & 1.2000 & 300.0 \\ 519 & 29 & 23 & 29.38 & 500 & 1.2000 & 600.0 \\ 548 & 33 & 3 & 33.05 & 750 & 1.2000 & 900.0 \\ 525 & 35 & 45 & 35.75 & 1000 & 1.2000 & 1200.0\end{array}$

INCREASED CHARGE PRESSURE TO 750 PSIG.

GRAPH NAME $=4$ IAS750M

$\begin{array}{rrrrrrr}715 & 43 & 39 & 43.65 & 0 & 1.2000 & 0.0 \\ 718 & 46 & 12 & 46.20 & 250 & 1.2000 & 300.0 \\ 724 & 49 & 5 & 49.08 & 500 & 1.2000 & 600.0 \\ 745 & 52 & 45 & 52.75 & 750 & 1.2000 & 900.0 \\ 757 & 56 & 30 & 56.50 & 1000 & 1.2000 & 1200.0\end{array}$

INCREASED CHARGE PRESSURE TO 900 PSIG.

$\begin{array}{rrrrrrr}901 & 64 & 3 & 64.05 & 0 & 1.2000 & 0.0 \\ 904 & 68 & 2 & 68.03 & 250 & 1.2000 & 300.0 \\ 912 & 71 & 5 & 71.08 & 500 & 1.2000 & 600.0 \\ 924 & 74 & 20 & 74.33 & 750 & 1.2000 & 900.0 \\ 949 & 78 & 4 & 78.07 & 1000 & 1.2000 & 1200.0\end{array}$


HHC-SD-HM-TRP-223

Rev. 0

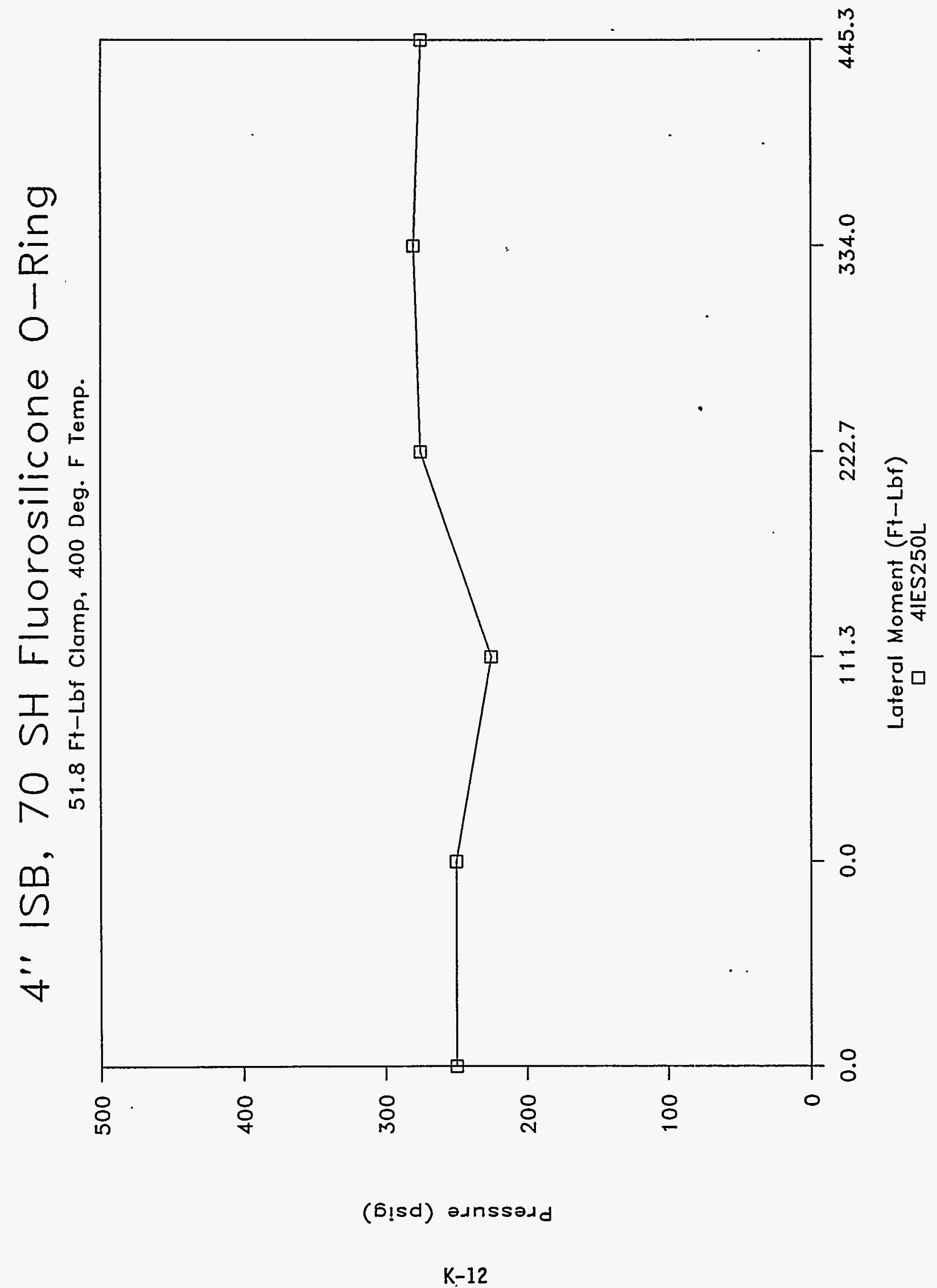


WHC-SD-WM-TRP-223

Rev. 0

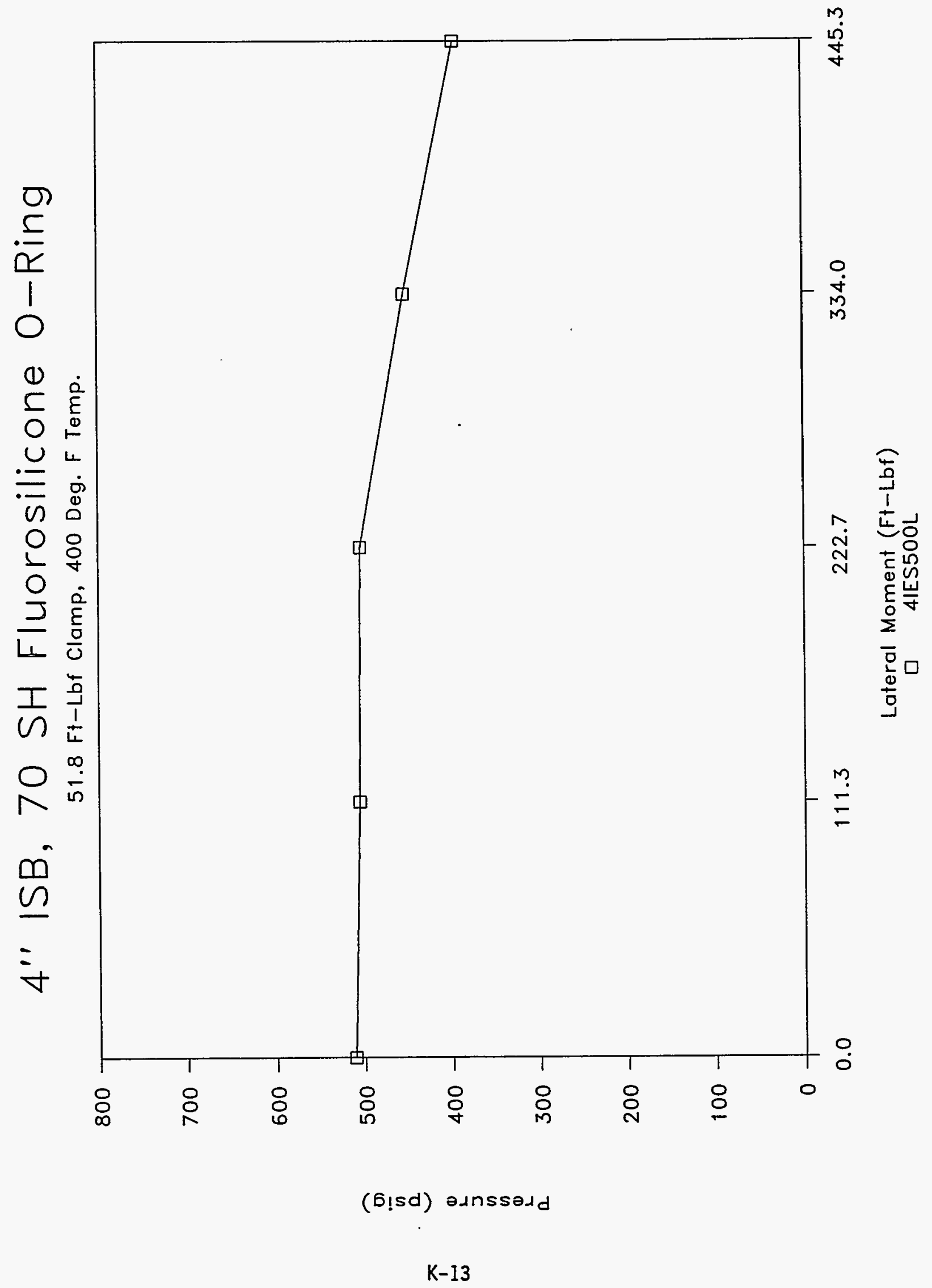


HHC-SD-HM-TRP-223

Rev. 0

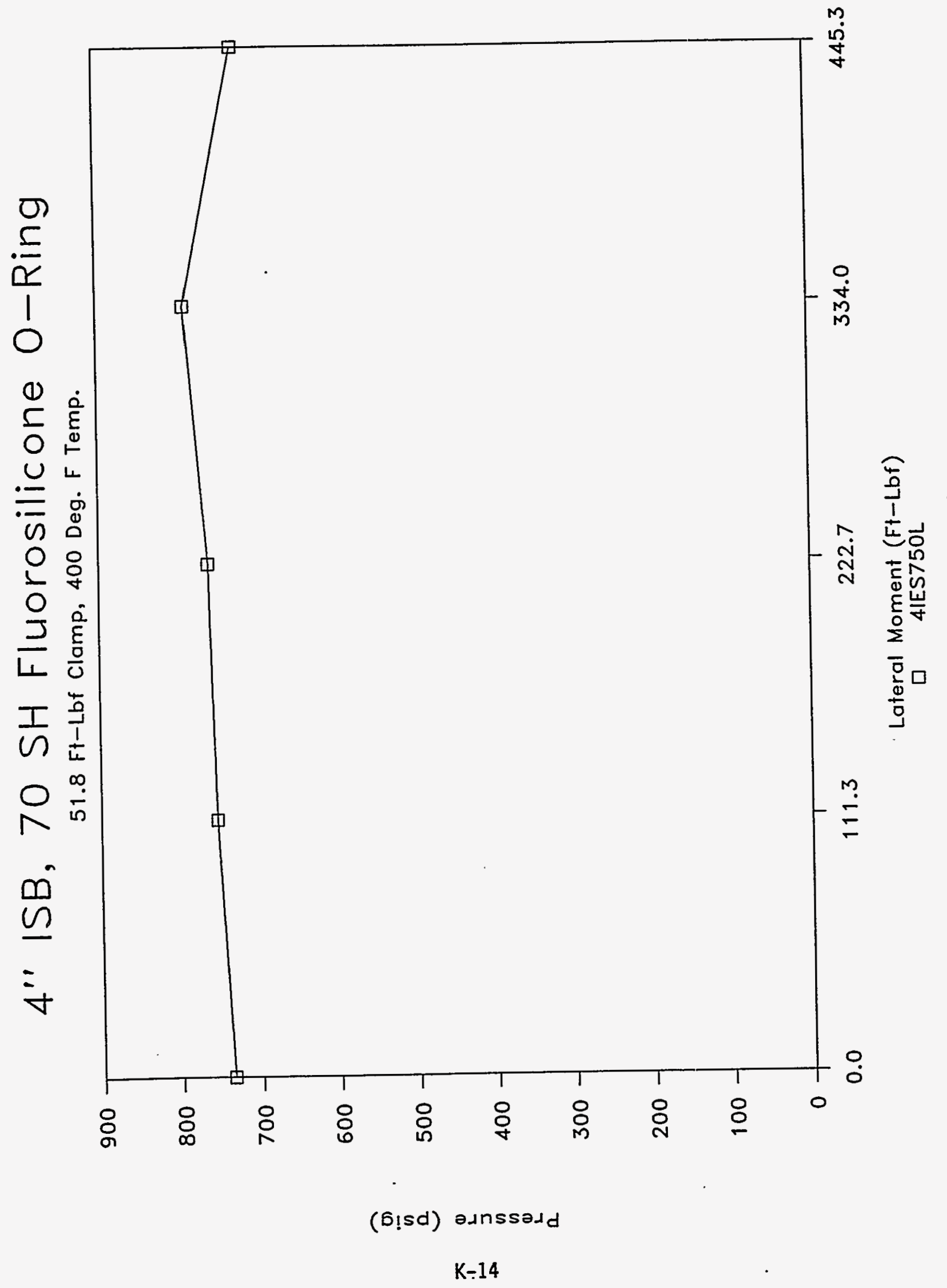


JUNE 20, 1994

4" ISB CONNECTOR, 70 SH FLUOROSILICONE O-RING, ELEVATED TEMP. PRESSURE ASCENSION LEAK TEST - ILATERAL MOMENT.

CILAMPING TORQUE $=51.8 \mathrm{FT}-\mathrm{LBF}$

GRAPH NAME $=4$ IES250L

CHARGE PRESSURE $=250$ PSIG

TEMPERATURE : $400 \mathrm{Deg} . \mathrm{F}$

$\begin{array}{lllllll} & & & & & \text { ILATERAL } \\ \text { PRESSURE } & \text { ELAPSED } & \text { ELAPSED } & \text { ELAPSED } & \text { LATERAI } & \text { MOMENT } & \text { LATERAI } \\ \text { PSIG } & \text { MINUTES } & \text { SECONDS } & \text { DECIMAI } & \text { FORCE } & \text { ARM } & \text { MOMENT } \\ & & & \text { MINUTES } & \text { LBS } & \text { FT. } & \text { FT-IBBF. } \\ \text { INPUT } & \text { INPUT } & \text { INPUT } & \text { COMPUTE } & \text { INPUT } & \text { INPUT } & \text { COMPUTE }\end{array}$

$\begin{array}{rrrrrrr}250 & 0 & 0 & 0.00 & 0 & 0.4453 & 0.0 \\ 250 & 2 & 0 & 2.00 & 0 & 0.4453 & 0.0 \\ 225 & 5 & 21 & 5.35 & 250 & 0.4453 & 111.3 \\ 275 & 8 & 35 & 8.58 & 500 & 0.4453 & 222.7 \\ 280 & 12 & 7 & 12.12 & 750 & 0.4453 & 334.0 \\ 275 & 16 & 45 & 16.75 & 1000 & 0.4453 & 445.3\end{array}$

INCREASED CHARGE PRESSURE TO 500 PSIG.

GRAPH NAME $=4$ IES500L

$\begin{array}{rrrrrrr}511 & 24 & 47 & 24.78 & 0 & 0.4453 & 0.0 \\ 505 & 27 & 2 & 27.03 & 250 & 0.4453 & 111.3 \\ 504 & 29 & 15 & 29.25 & 500 & 0.4453 & 222.7 \\ 453 & 32 & 39 & 32.65 & 750 & 0.4453 & 334.0 \\ 395 & 36 & 3 & 36.05 & 1000 & 0.4453 & 445.3\end{array}$

INCREASED CHARGE PRESSURE TO 750 PSIG.

GRAPH NAME $=4$ IES750L

$\begin{array}{rrrrrrr}736 & 41 & 3 & 41.05 & 0 & 0.4453 & 0.0 \\ 754 & 45 & 37 & 45.62 & 250 & 0.4453 & 111.3 \\ 762 & 48 & 27 & 48.45 & 500 & 0.4453 & 222.7 \\ 790 & 51 & 6 & 51.10 & 750 & 0.4453 & 334.0 \\ 725 & 55 & 13 & 55.22 & 1000 & 0.4453 & 445.3\end{array}$


WHC-SD-WM-TRP-223

Rev. 0

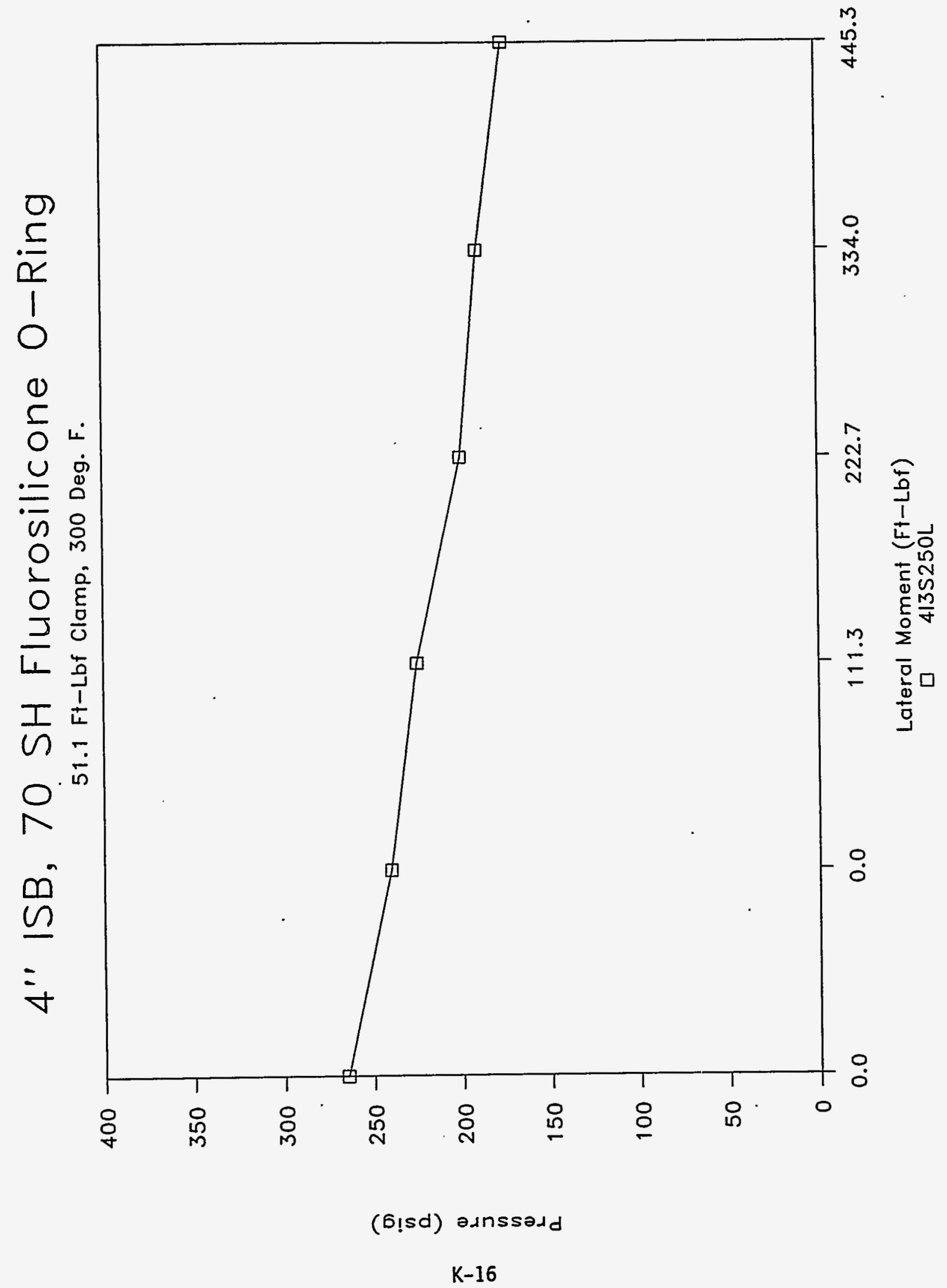


WHC-SD-WM-TRP-223

Rev. 0

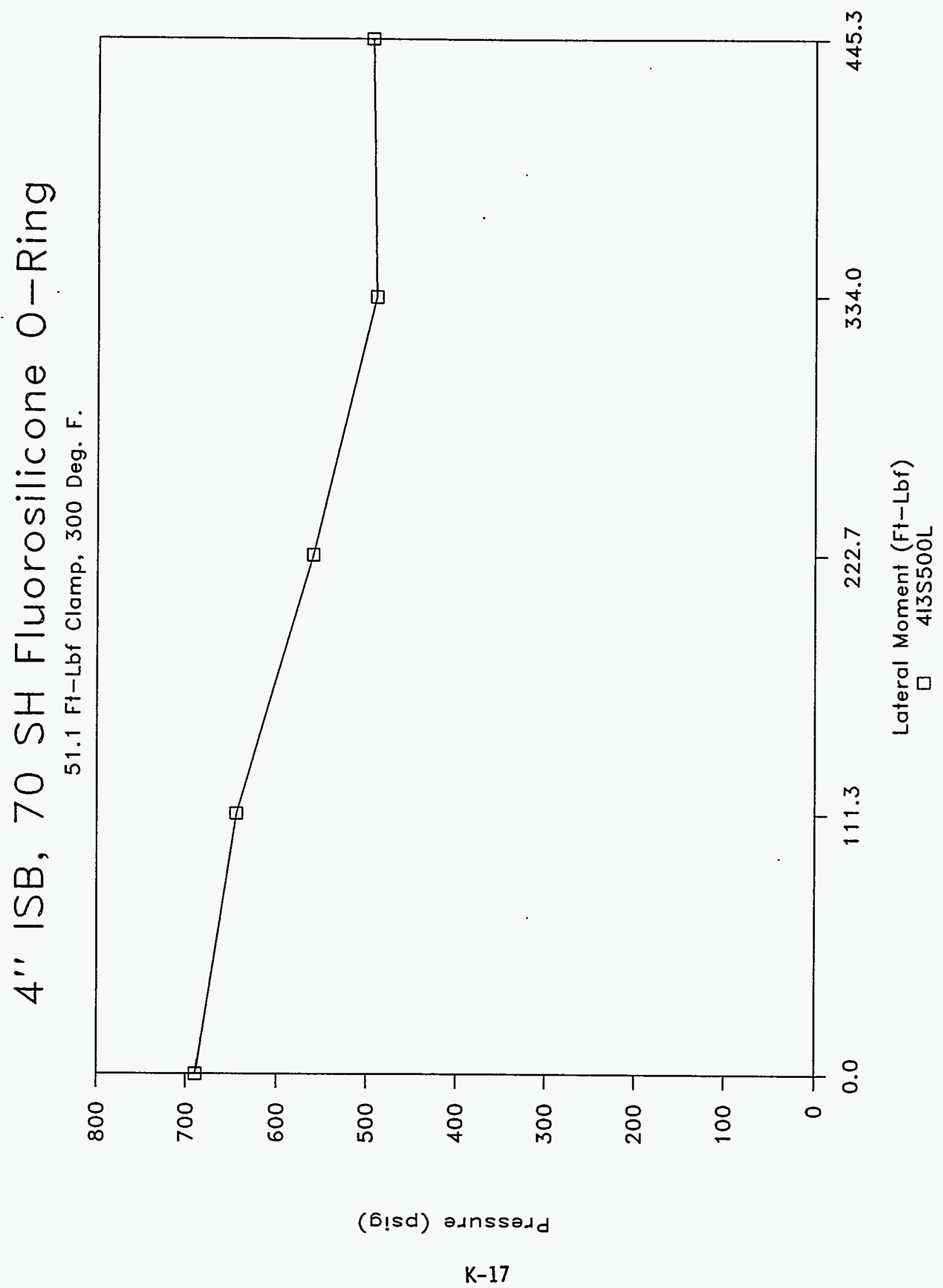


WHC-SD-WM-TRP-223

Rev. 0

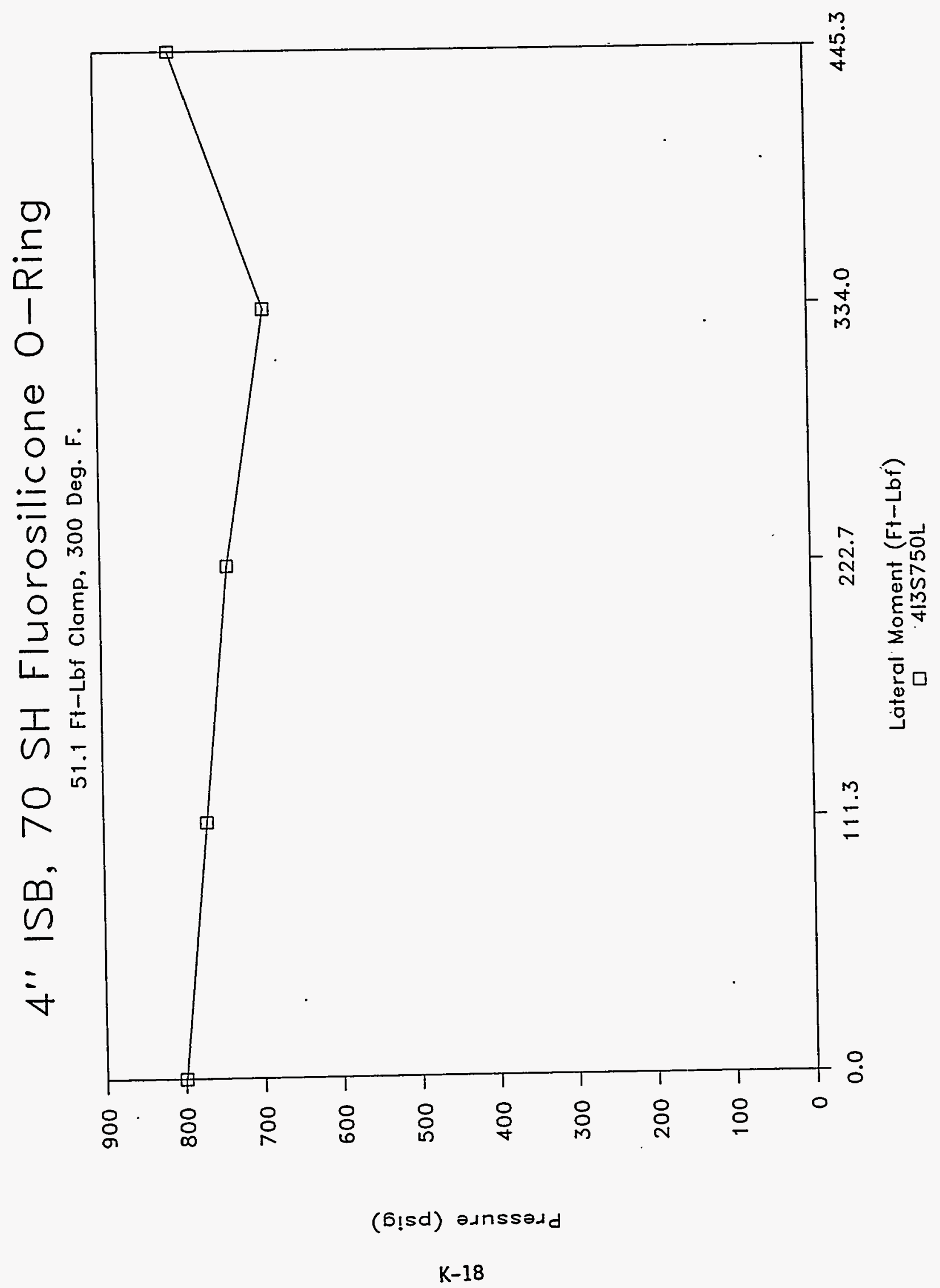




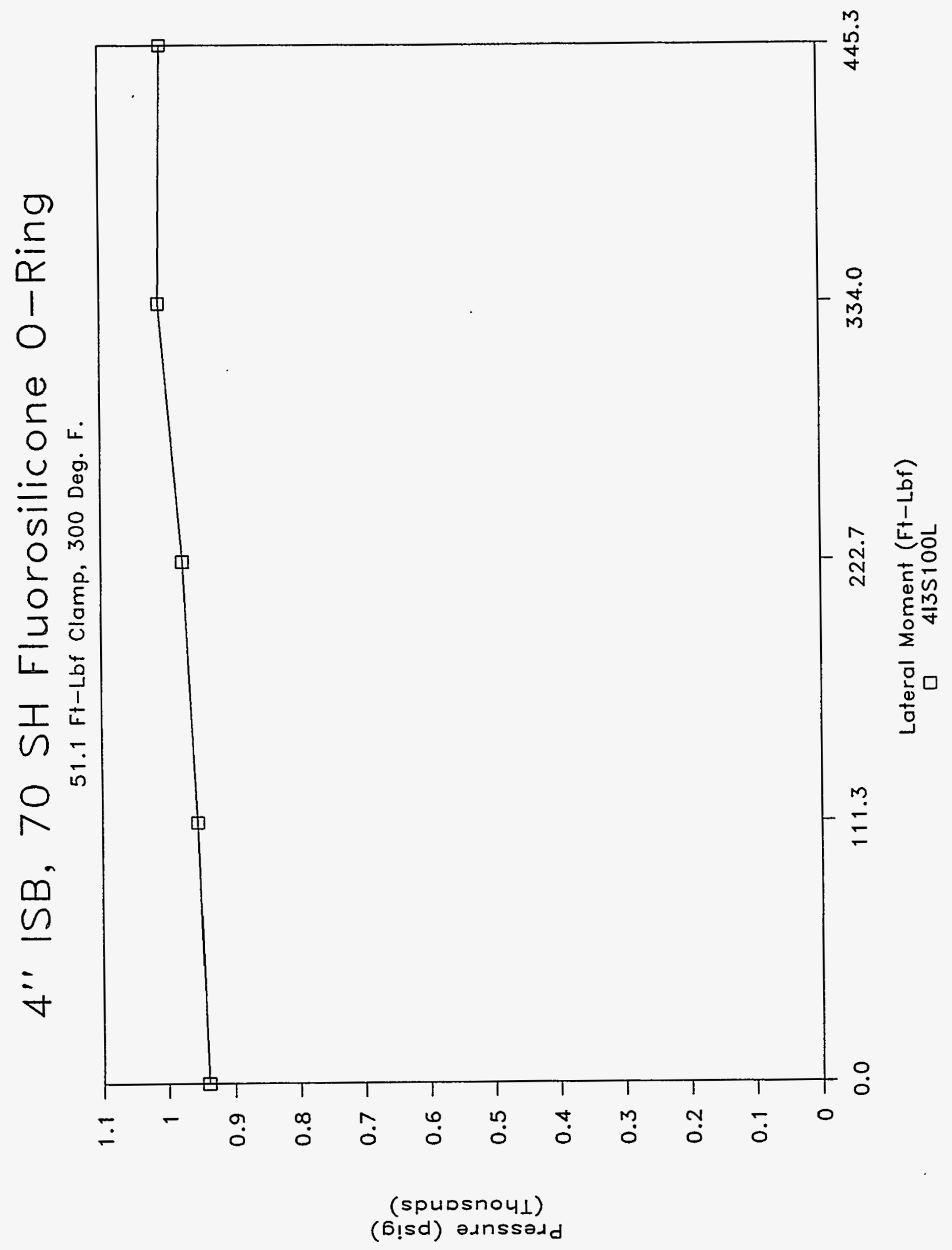

K-19 
OCTOBER 17, 1994

4" ISB CONNECTOR, 70 SH FLUOROSILICONE O-RING, ELEVATED TEMP. (300 DEG. F IEAK TEST - IATERAI MOMENT (SIDEWAYS)

CLAMPING TORQUE $=51.1$ FT-LBF

CHARGE PRESSURE $=250$ PSIG

$\begin{array}{lllllll} & & & & \text { LATERAI } & \\ \text { PRESSURE } & \text { ELAPSED } & \text { ELAPSED } & \text { ELAPSED } & \text { IATERAI } & \text { MOMENT } & \text { LATERAI } \\ \text { PSIG } & \text { MINUTES } & \text { SECONDS } & \text { DECIMAI } & \text { FORCE } & \text { ARM } & \text { MOMENT } \\ & & & \text { MINUTES } & \text { LBS } & \text { FT. } & \text { FT-IBBF. } \\ \text { INPUT } & \text { INPUT } & \text { INPUT } & \text { COMPUTE } & \text { INPUT } & \text { INPUT } & \text { COMPUTE }\end{array}$

$\begin{array}{rrrrrrr}265 & 0 & 0 & 0.00 & 0 & 0.4453 & 0.0 \\ 240 & 2 & 1 & 2.02 & 0 & 0.4453 & 0.0 \\ 225 & 4 & 2 & 4.03 & 250 & 0.4453 & 111.3 \\ 200 & 6 & 1 & 6.02 & 500 & 0.4453 & 222.7 \\ 190 & 8 & 7 & 8.12 & 750 & 0.4453 & 334.0 \\ 175 & 10 & 3 & 10.05 & 1000 & 0.4453 & 445.3\end{array}$

INCREASED PRESSURE TO 500 PSIG

GRAPH NAME $=4 . I 35500 \mathrm{~L}$

$\begin{array}{rrrrrrr}690 & 12 & 4 & 12.07 & 0 & 0.4453 & 0.0 \\ 645 & 14 & 1 & 14.02 & 250 & 0.4453 & 111.3 \\ 560 & 16 & 1 & 16.02 & 500 & 0.44453 & 222.7 \\ 490 & 18 & 1 & 18.02 & 750 & 0.44453 & 334.0 \\ 495 & 20 & 2 & 20.03 & 1000 & 0.44453 & 445.3\end{array}$

INCREASED PRESSURE TO 750 PSIG

$\begin{array}{ll}800 & 22 \\ 770 & 24 \\ 740 & 26 \\ 690 & 28 \\ 805 & 30\end{array}$

$\begin{array}{ll}1 & 22.02 \\ 3 & 24.05 \\ 2 & 26.03 \\ 4 & 28.07 \\ 3 & 30.05\end{array}$

3
GRAPH NAME $=4 I 3 S 250 I$

IATERAL

MOMENT

IBS FT. FT-LBF.

0.4453

0.4453

0.4453

0.4453

0.4453

0.4453

445.3 
WHC-SD-WM-TRP-223

Rev. 0

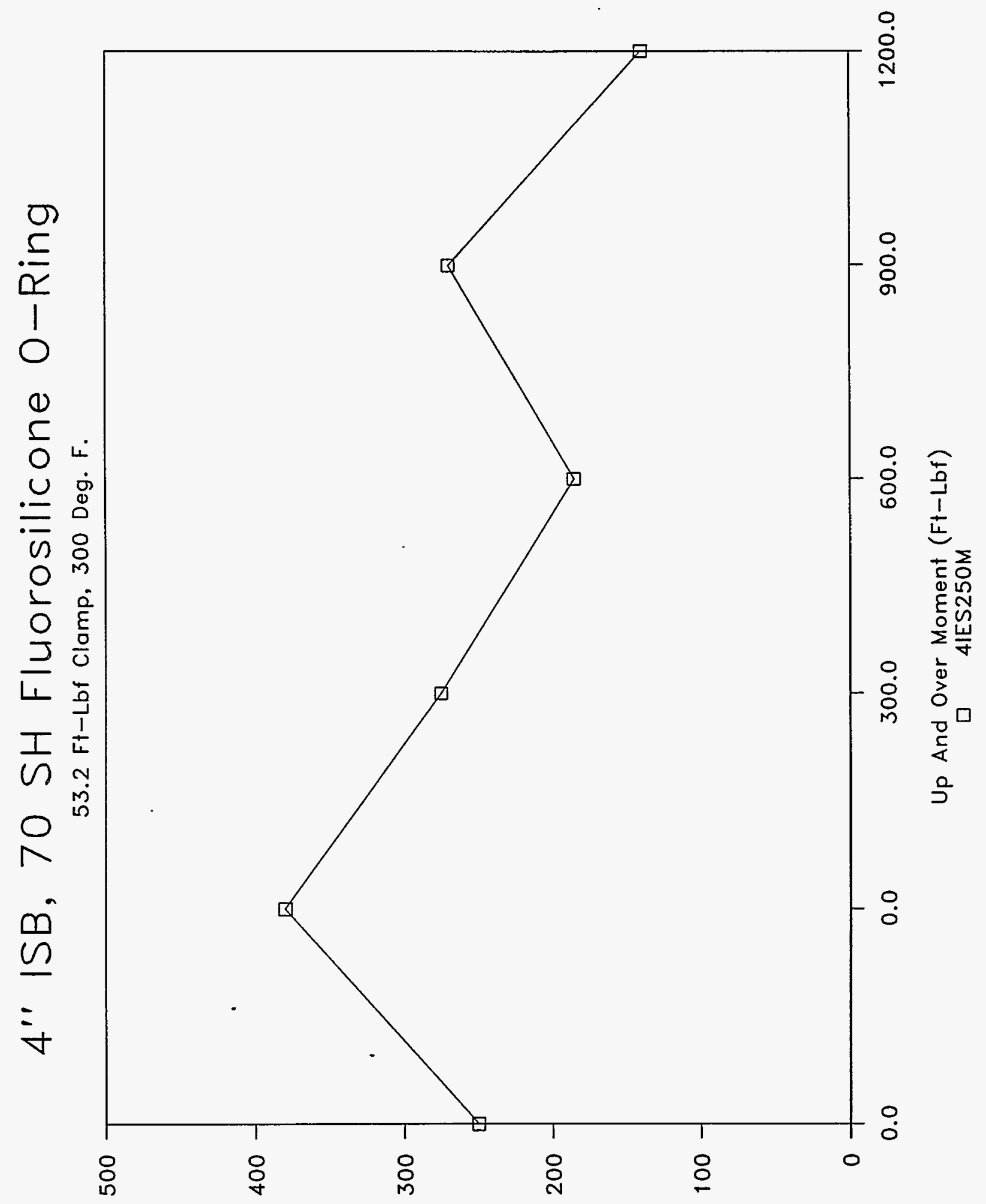

(G!sd) asnssadd 


\section{4" ISB, 70 SH Fluorosilicone O-Ring}

$53.2 \mathrm{Ft}$-Lbf Clamp, 300 Deg. F.

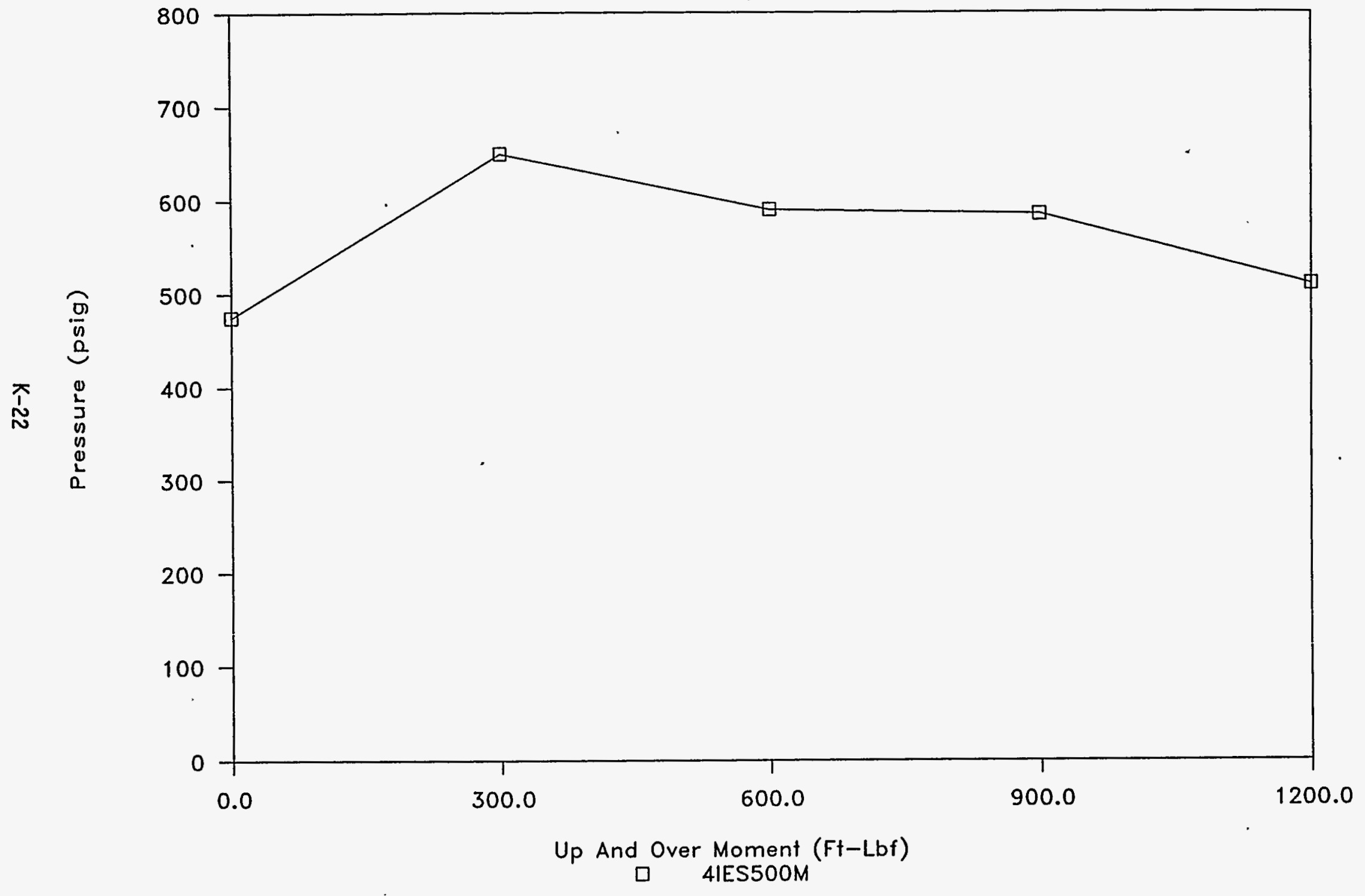


WHC-SD-WM-TRP-223

Rev. 0

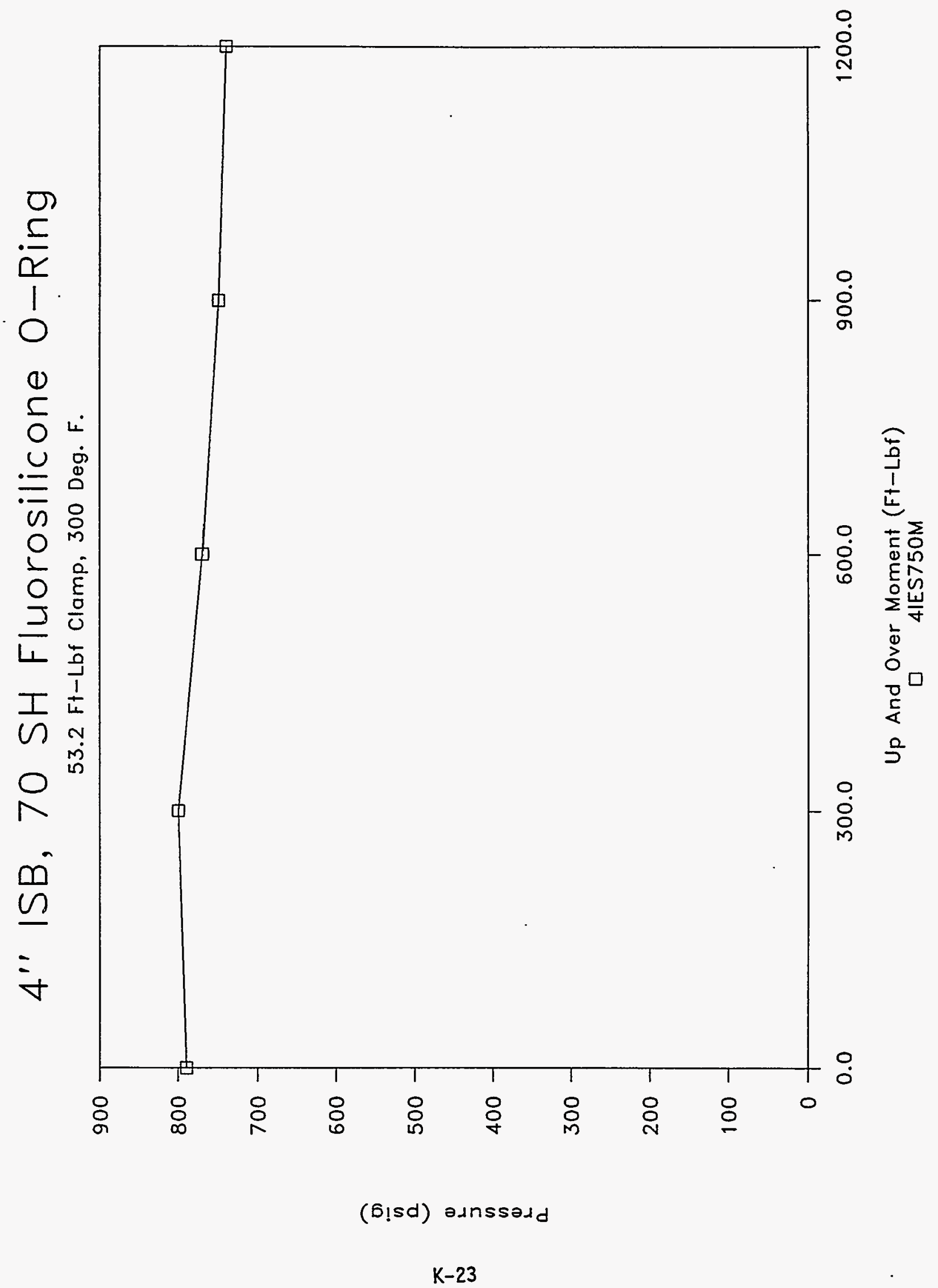




\section{4" ISB, 70 SH Fluorosilicone O-Ring}

$53.2 \mathrm{Ft}$-Lbf Clamp, 300 Deg. F.

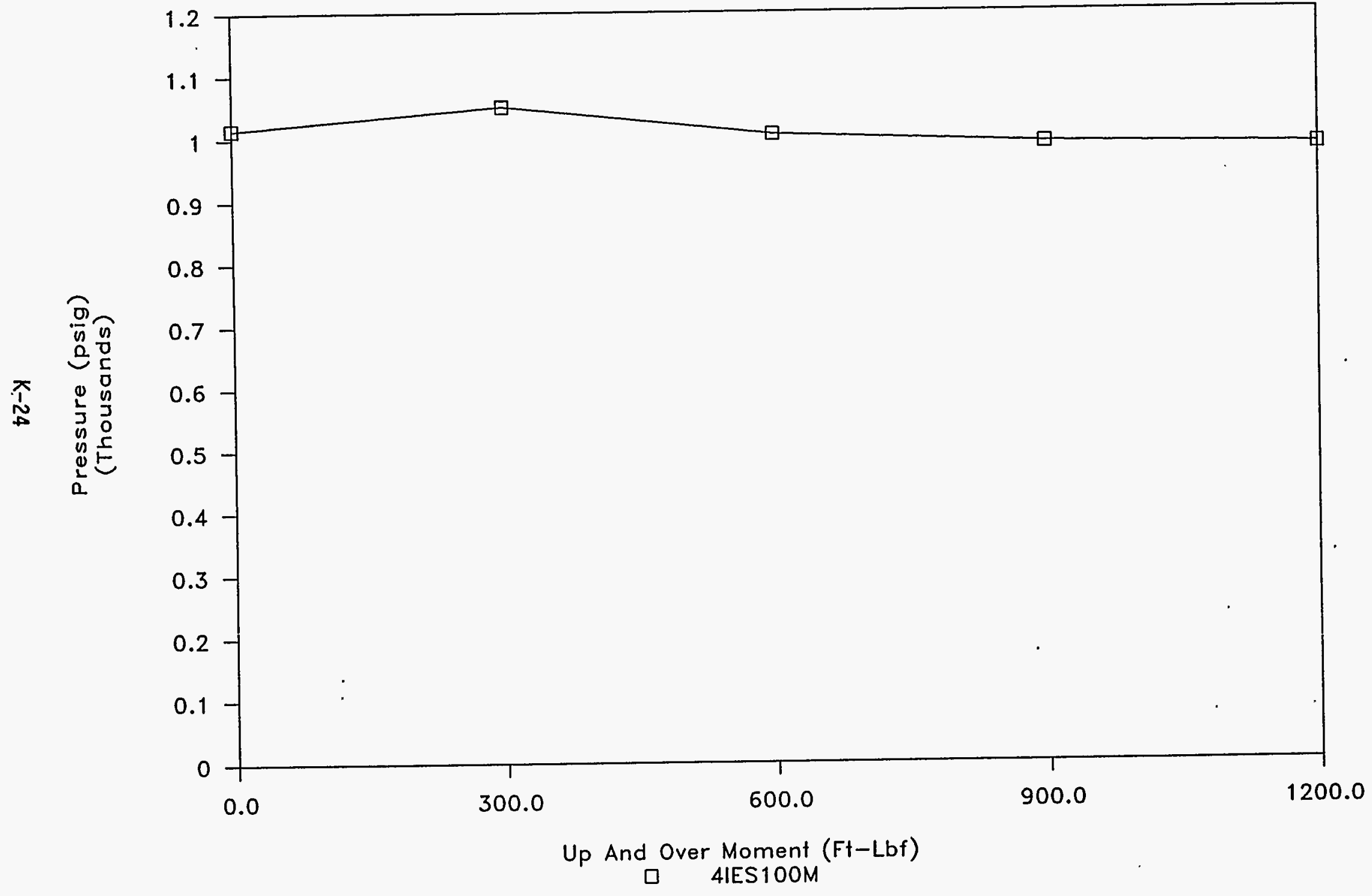


WHC-SD-WM-TRP-223 ReV 0

OCTOBER 12, 1994

4" ISB CONNECTOR, 70 SH FLUOROSIIICONE O-RING, ELEVATED TEMP. (300 DEG. F LEAK TEST - UP AND OVER MOMENT (UPWARD)

CLAMPING TORQUE $=53.2$ FT-IBF

CHARGE PRESSURE $=250$ PSIG

GRAPH NAME $=4$ IES250M

$\begin{array}{lllllll} & & & & & \text { UPWARD } & \\ \text { PRESSURE } & \text { ELAPSED } & \text { ELAPSED } & \text { ELAPSED } & \text { UPWARD } & \text { MOMENT } & \text { UPWARD } \\ \text { PSIG } & \text { MINUTES } & \text { SECONDS } & \text { DECIMAL } & \text { FORCE } & \text { ARM } & \text { MOMENT } \\ & & & \text { MINUTES } & \text { IBS } & \text { FT. } & \text { FT-LBF. } \\ \text { INPUT } & \text { INPUT } & \text { INPUT } & \text { COMPUTE } & \text { INPUT } & \text { INPUT } & \text { COMPUTE }\end{array}$

$\begin{array}{rrrrrrr}250 & 0 & 0 & 0.00 & 0 & 1.2000 & 0.0 \\ 380 & 2 & 1 & 2.02 & 0 & 1.2000 & 0.0 \\ 275 & 4 & 2 & 4.03 & 250 & 1.2000 & 300.0 \\ 185 & 6 & 1 & 6.02 & 500 & 1.2000 & 600.0 \\ 270 & 8 & 3 & 8.05 & 750 & 1.2000 & 900.0 \\ 140 & 10 & 2 & 10.03 & 1000 & 1.2000 & 1200.0\end{array}$

INCREASED PRESSURE TO 500 PSIG

GRAPH NAME $=4$ IES50OM

$\begin{array}{rrrrrrr}475 & 12 & 3 & 12.05 & 0 & 1.2000 & 0.0 \\ 650 & 14 & 4 & 14.07 & 250 & 1.2000 & 300.0 \\ 590 & 16 & 2 & 16.03 & 500 & 1.2000 & 600.0 \\ 585 & 18 & 2 & 18.03 & 750 & 1.2000 & 900.0 \\ 510 & 20 & 1 & 20.02 & 1000 & 1.2000 & 1200.0\end{array}$

INCREASED PRESSURE TO 750 PSIG

$\begin{array}{llll}790 & 22 & 2 & 22.03 \\ 800 & 24 & 1 & 24.02 \\ 770 & 26 & 2 & 26.03 \\ 750 & 28 & 1 & 28.02 \\ 740 & 30 & 3 & 30.05\end{array}$

INCREASED PRESSURE TO 1000 PSIG

$\begin{array}{rl}1015 & 32 \\ 1050 & 34 \\ 1005 & 36 \\ 990 & 38 \\ 985 & 40\end{array}$

GRAPH NAME $=4$ IES750M

$\begin{array}{rrr}0 & 1.2000 & 0.0 \\ 250 & 1.2000 & 300.0 \\ 500 & 1.2000 & 600.0 \\ 750 & 1.2000 & 900.0 \\ 1000 & 1.2000 & 1200.0\end{array}$

GRAPH NAME $=4$ IES100M

$\begin{array}{rrrrr}1 & 32.02 & 0 & 1.2000 & 0.0 \\ 2 & 34.03 & 250 & 1.2000 & 300.0 \\ 2 & 36.03 & 500 & 1.2000 & 600.0 \\ 3 & 38.05 & 750 & 1.2000 & 900.0 \\ 1 & 40.02 & 1000 & 1.2000 & 1200.0\end{array}$


WHC-SD-WM-TRP-223

Rev. 0

APPENDIX L: GRAPHS OF 4-IN. KALREZ TESTS 
WHC-SD-WM-TRP-223

Rev. $O$

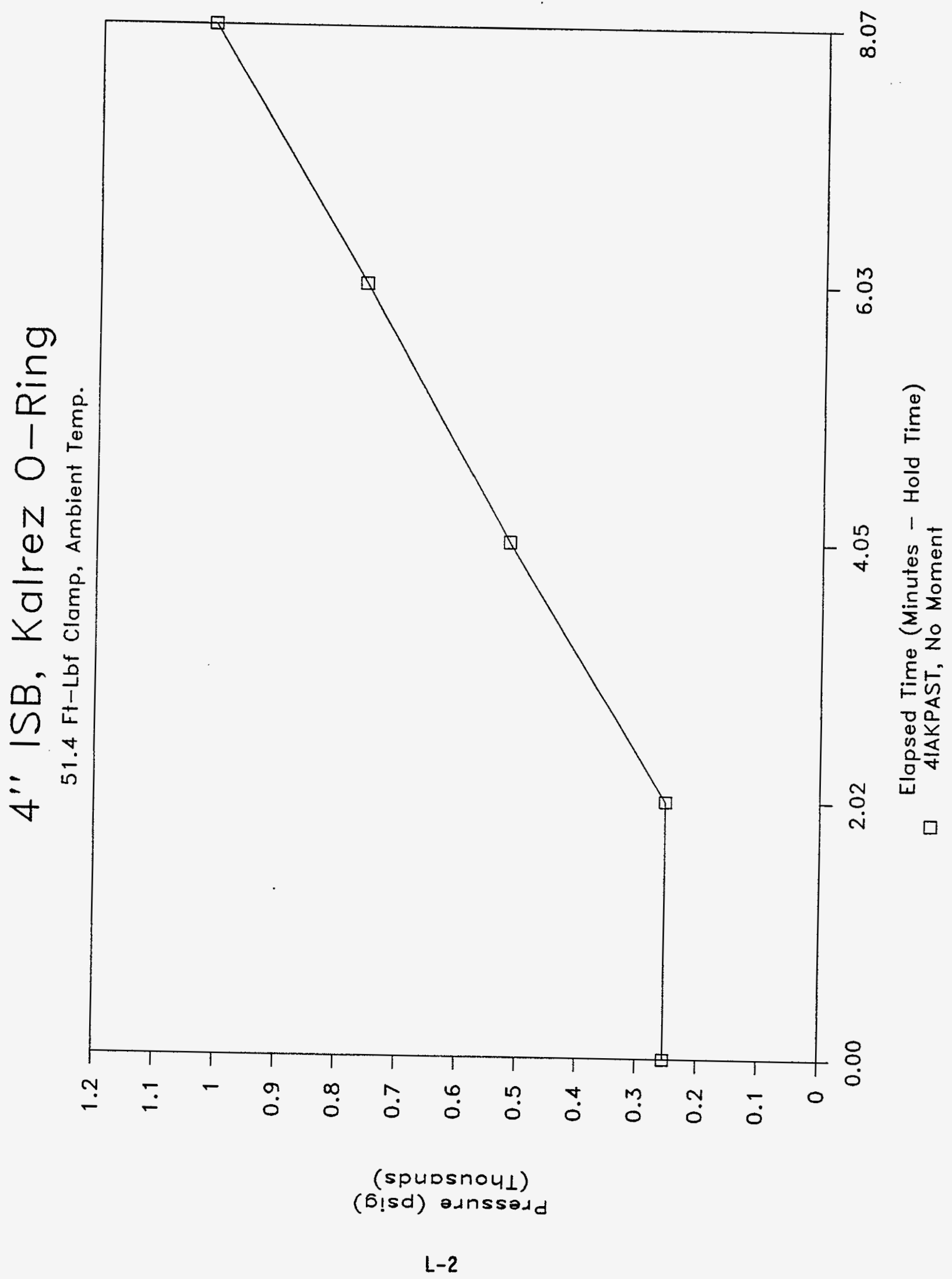


WHC-SD-WM-TRP-223 Rev 0

\begin{tabular}{|c|c|c|c|c|}
\hline $\begin{array}{l}\text { OCTOBER } \\
4 " \text { ISB C } \\
\text { PRESSURE } \\
\text { CLAMPING } \\
\text { CHARGE PI }\end{array}$ & $\begin{array}{l}\text { O6, } 1994 \\
\text { JNNECTOR } \\
\text { ASCENSI } \\
\text { TORQUE } \\
\text { RESSURE }\end{array}$ & $\begin{array}{l}\text { KALREZ } \\
\text { LEAK TI } \\
51.4 \text { FT } \\
\text { GRADUALI }\end{array}$ & $\begin{array}{l}\text {-RING, } 70 \\
\text { ST - NO } \\
\text { LBF } \\
Y \text { INCREME }\end{array}$ & $\begin{array}{l}\text { DUROMET! } \\
\text { OMENTS } \\
\text { GRAPH N } \\
\text { NTED HIGI }\end{array}$ \\
\hline PRESSURE & ELAPSED & ELAPSED & ELAPSED & APPLIED \\
\hline PSIG & MINUTES & SECONDS & $\begin{array}{l}\text { DECIMAL } \\
\text { MINUTES }\end{array}$ & $\begin{array}{l}\text { FORCE } \\
\text { LBS }\end{array}$ \\
\hline INPUT & INPUT & INPUT & COMPUTE & INPUT \\
\hline $\begin{array}{r}255 \\
255 \\
518 \\
760 \\
1015\end{array}$ & & 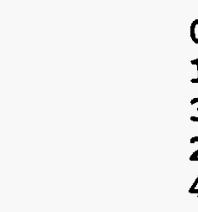 & $\begin{array}{l}0.00 \\
2.02 \\
4.05 \\
6.03 \\
8.07\end{array}$ & \\
\hline
\end{tabular}




\section{4" ISB, Kalrez O-Ring}

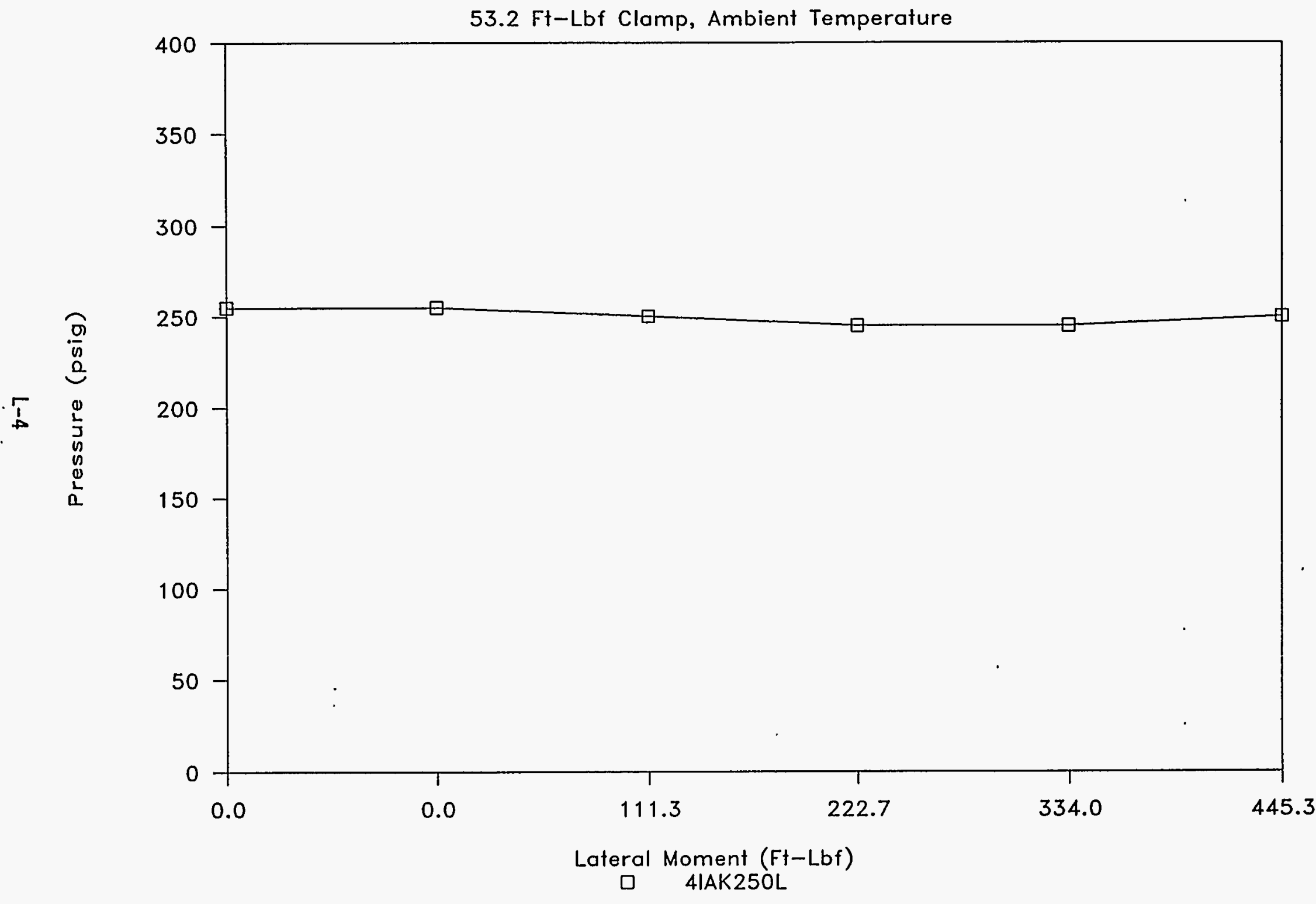




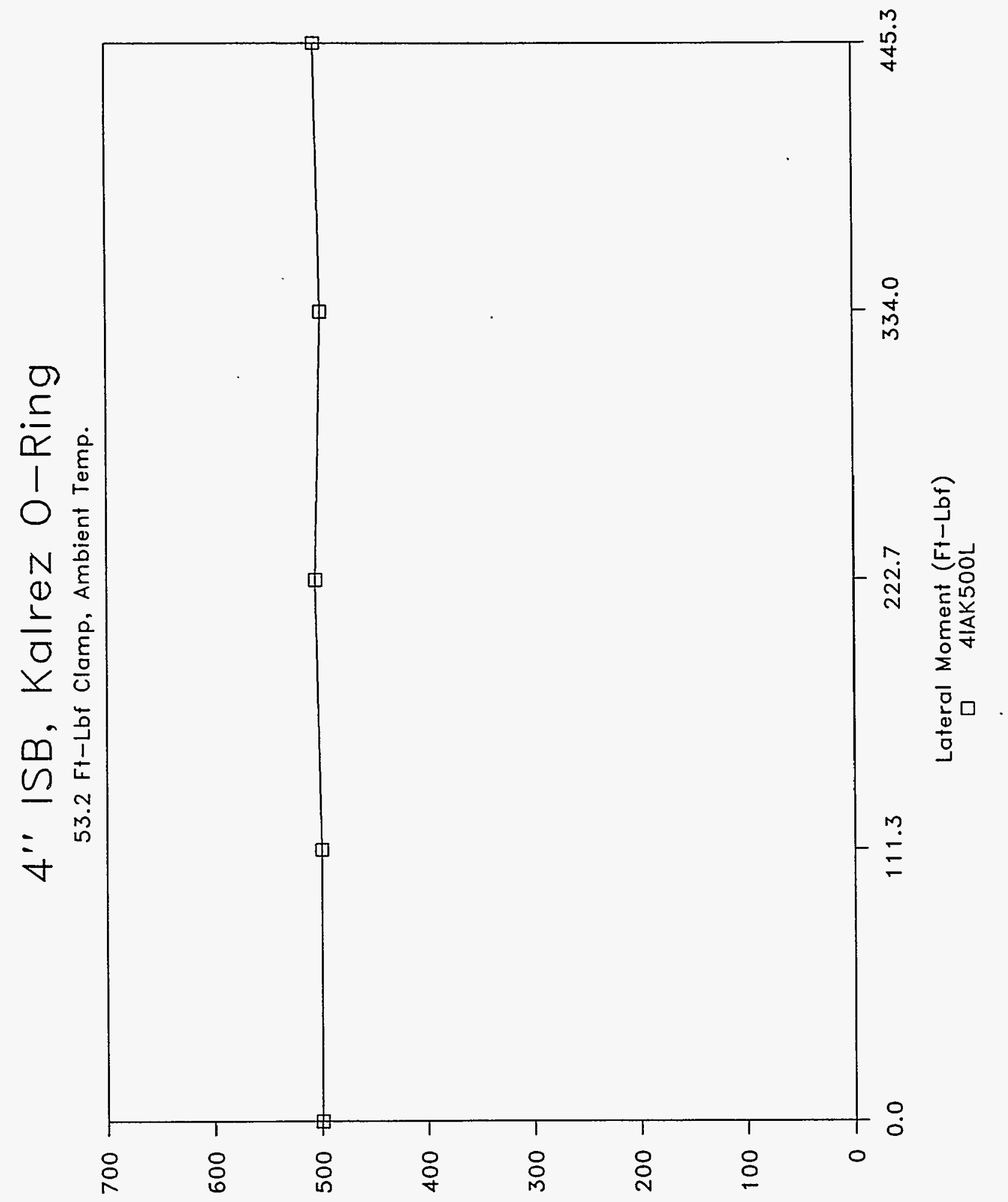

(b!sd) odnssadd 
WHC-SD-WM-TRP-223

Rev. 0

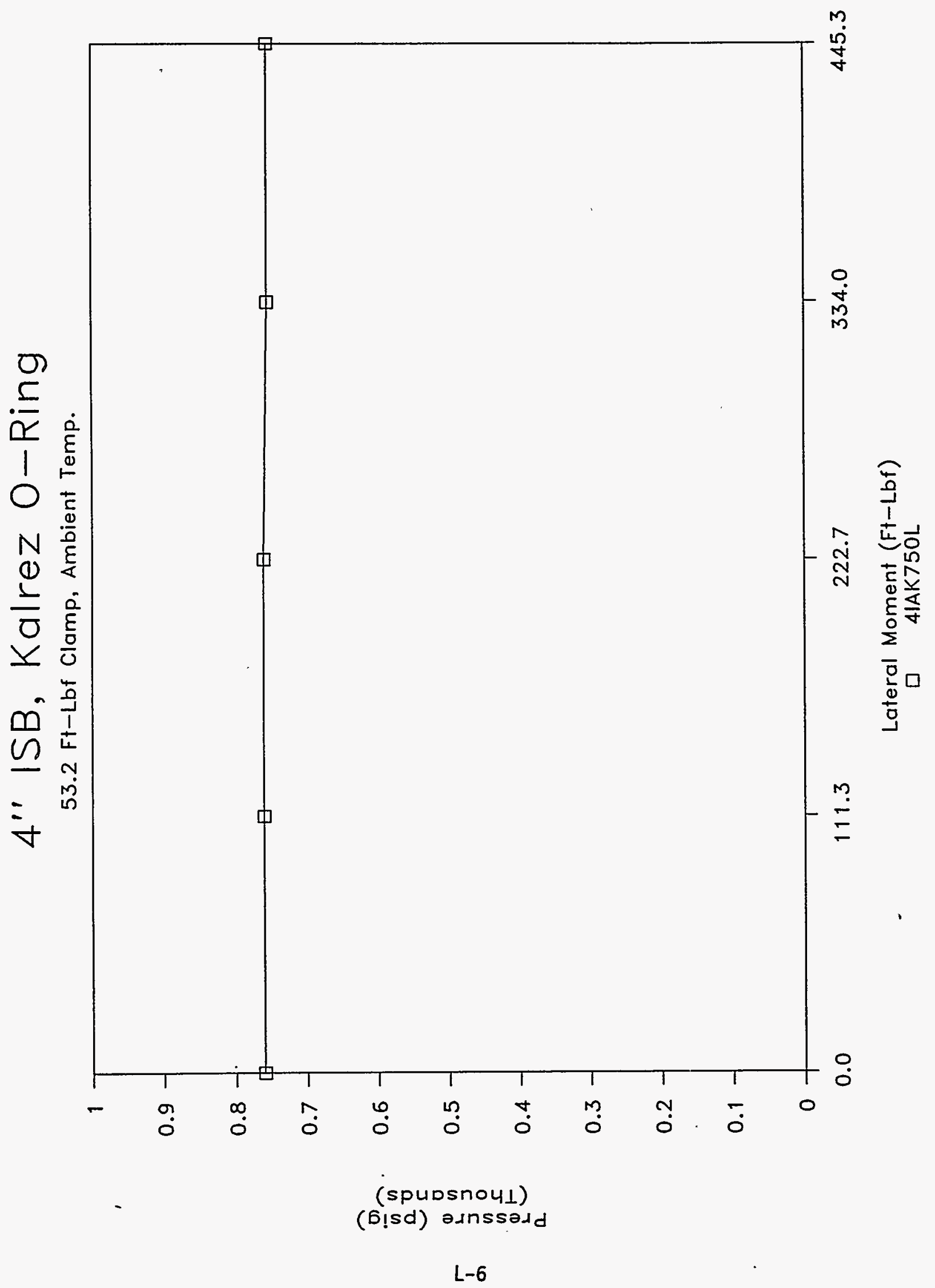


WHC-SD-WM-TRP-223

Rev. 0

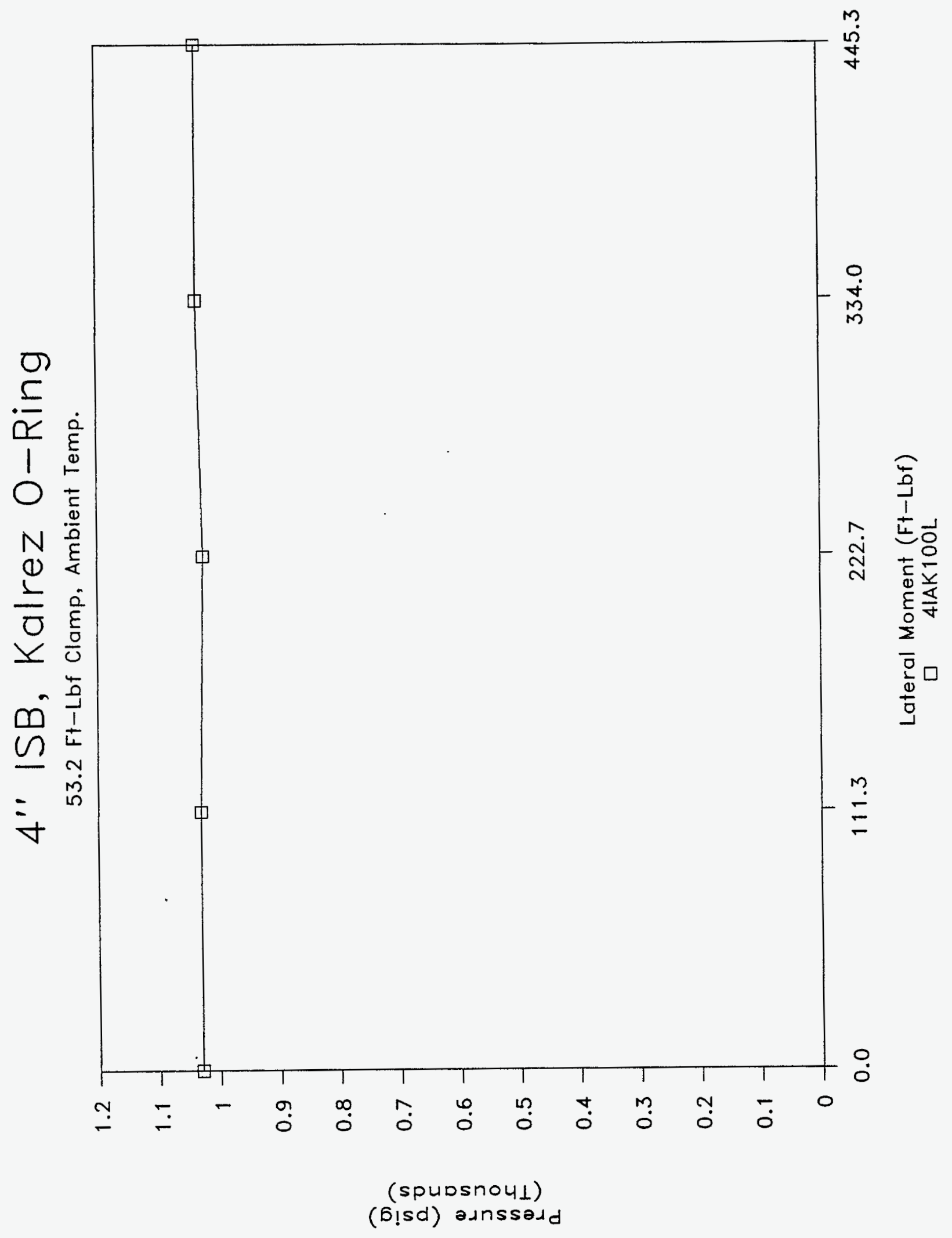

$L-7$ 
OCTOBER 06, 1994

4" ISB CONNECTOR, KALREZ O-RING, 70 DUROMETER, AMBIENT TEMP. LEAK TEST - LATERAL MOMENT (SIDEWAYS ROTATION)

CLAMPING TORQUE $=53.2$ FT-LBF

CHARGE PRESSURE $=250$ PSIG

GRAPH NAME $=4$ IAK250L

$\begin{array}{lllllll} & & & & & \text { LATERAL } \\ \text { PRESSURE } & \text { ELAPSED } & \text { ELAPSED } & \text { ELAPSED } & \text { LATERAL } & \text { MOMENT } & \text { LATERAL } \\ \text { PSIG } & \text { MINUTES } & \text { SECONDS } & \text { DECIMAL } & \text { FORCE } & \text { ARM } & \text { MOMENT } \\ & & & \text { MINUTES } & \text { LBS } & \text { FT. } & \text { FT-LBF. } \\ \text { INPUT } & \text { INPUT } & \text { INPUT } & \text { COMPUTE } & \text { INPUT } & \text { INPUT } & \text { COMPUTE }\end{array}$

$\begin{array}{rrrrrrr}255 & 0 & 0 & 0.00 & 0 & 0.4453 & 0.0 \\ 255 & 2 & 2 & 2.03 & 0 & 0.4453 & 0.0 \\ 250 & 4 & 1 & 4.02 & 250 & 0.4453 & 111.3 \\ 245 & 6 & 3 & 6.05 & 500 & 0.4453 & 222.7 \\ 245 & 8 & 1 & 8.02 & 750 & 0.4453 & 334.0 \\ 250 & 10 & 2 & 10.03 & 1000 & 0.4453 & 445.3\end{array}$

INCREASED PRESSURE TO 500 PSIG

GRAPH NAME $=4$ IAK50OL

$\begin{array}{rrrrrrr}500 & 12 & 3 & 12.05 & 0 & 0.4453 & 0.0 \\ 500 & 14 & 1 & 14.02 & 250 & 0.4453 & 111.3 \\ 505 & 16 & 1 & 16.02 & 500 & 0.4453 & 222.7 \\ 500 & 18 & 2 & 18.03 & 750 & 0.4453 & 334.0 \\ 505 & 20 & 1 & 20.02 & 1000 & 0.4453 & 445.3\end{array}$

INCREASED PRESSURE TO 750 PSIG

GRAPH NAME $=4$ IAK750L

$\begin{array}{rrrrrrr}760 & 22 & 1 & 22.02 & 0 & 0.4453 & 0.0 \\ 760 & 24 & 3 & 24.05 & 250 & 0.4453 & 111.3 \\ 760 & 26 & 1 & 26.02 & 500 & 0.4453 & 222.7 \\ 755 & 28 & 2 & 28.03 & 750 & 0.4453 & 334.0 \\ 755 & 30 & 1 & 30.02 & 1000 & 0.4453 & 445.3\end{array}$

INCREASED PRESSURE TO 1000 PSIG

$\begin{array}{ll}1030 & 32 \\ 1030 & 34 \\ 1025 & 36 \\ 1035 & 38 \\ 1035 & 40\end{array}$

$\begin{array}{ll}2 & 32.03 \\ 1 & 34.02 \\ 3 & 36.05 \\ 1 & 38.02 \\ 1 & 40.02\end{array}$

GRAPH NAME $=4$ IAK10OL

$\begin{array}{rrr}0 & 0.4453 & 0.0 \\ 250 & 0.4453 & 111.3 \\ 500 & 0.4453 & 222.7 \\ 750 & 0.4453 & 334.0 \\ 1000 & 0.4453 & 445.3\end{array}$


WHC-SD-WM-TRP-223

Rev. 0

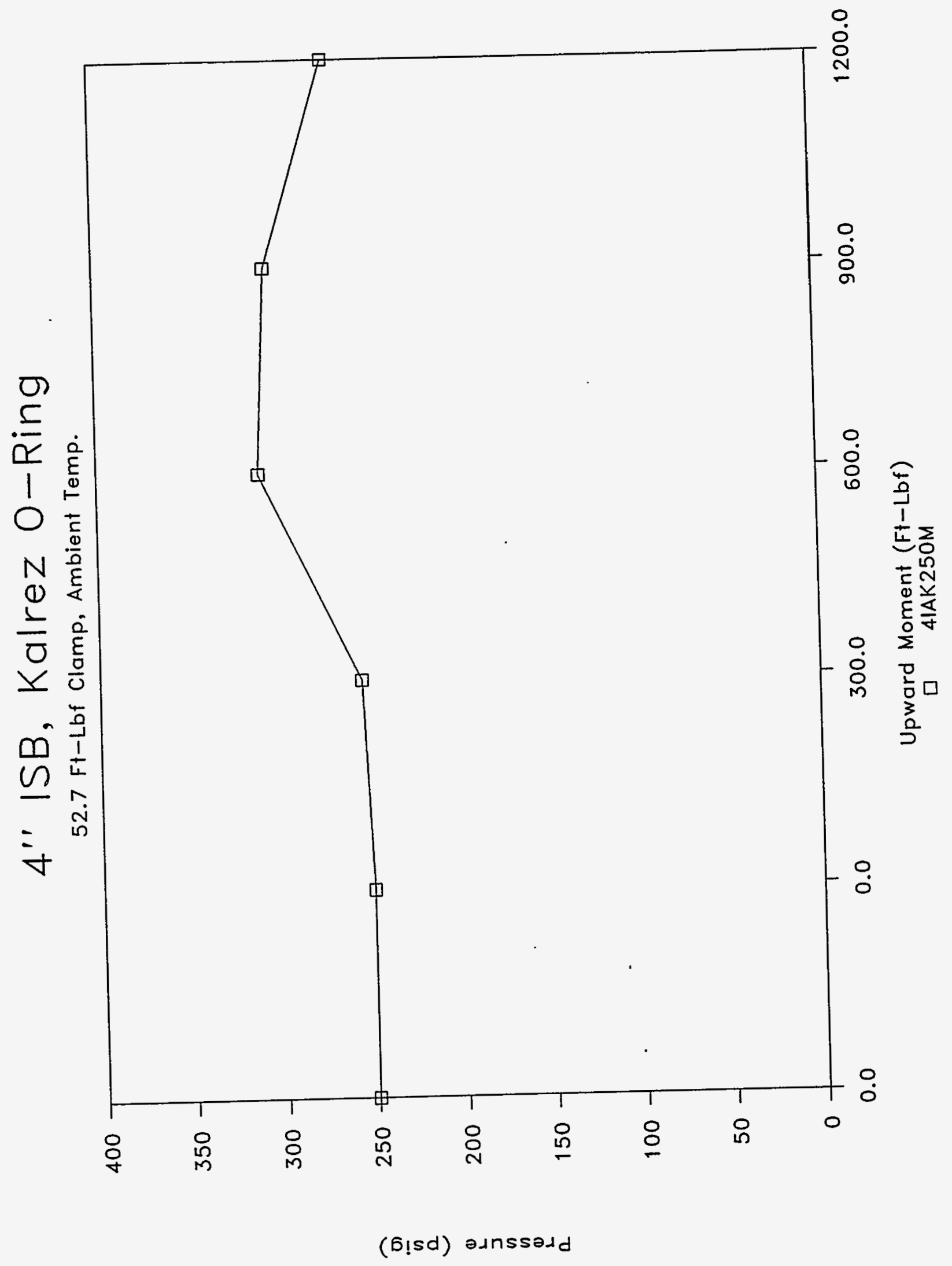

$L-9$ 
HHC-SD-WM-TRP-223

Rev. 0

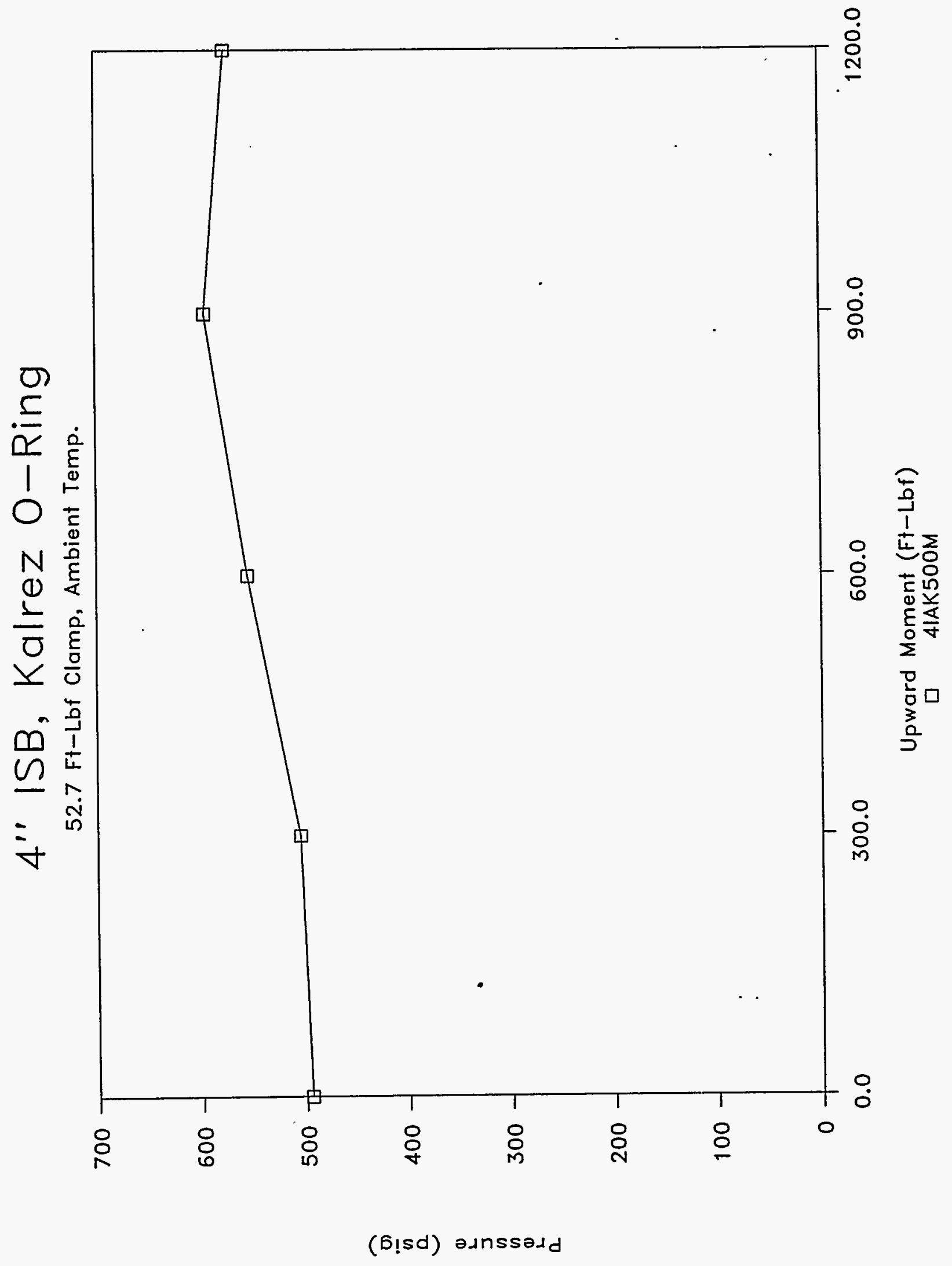


WHC-SD-WM-TRP-223

Rev. 0

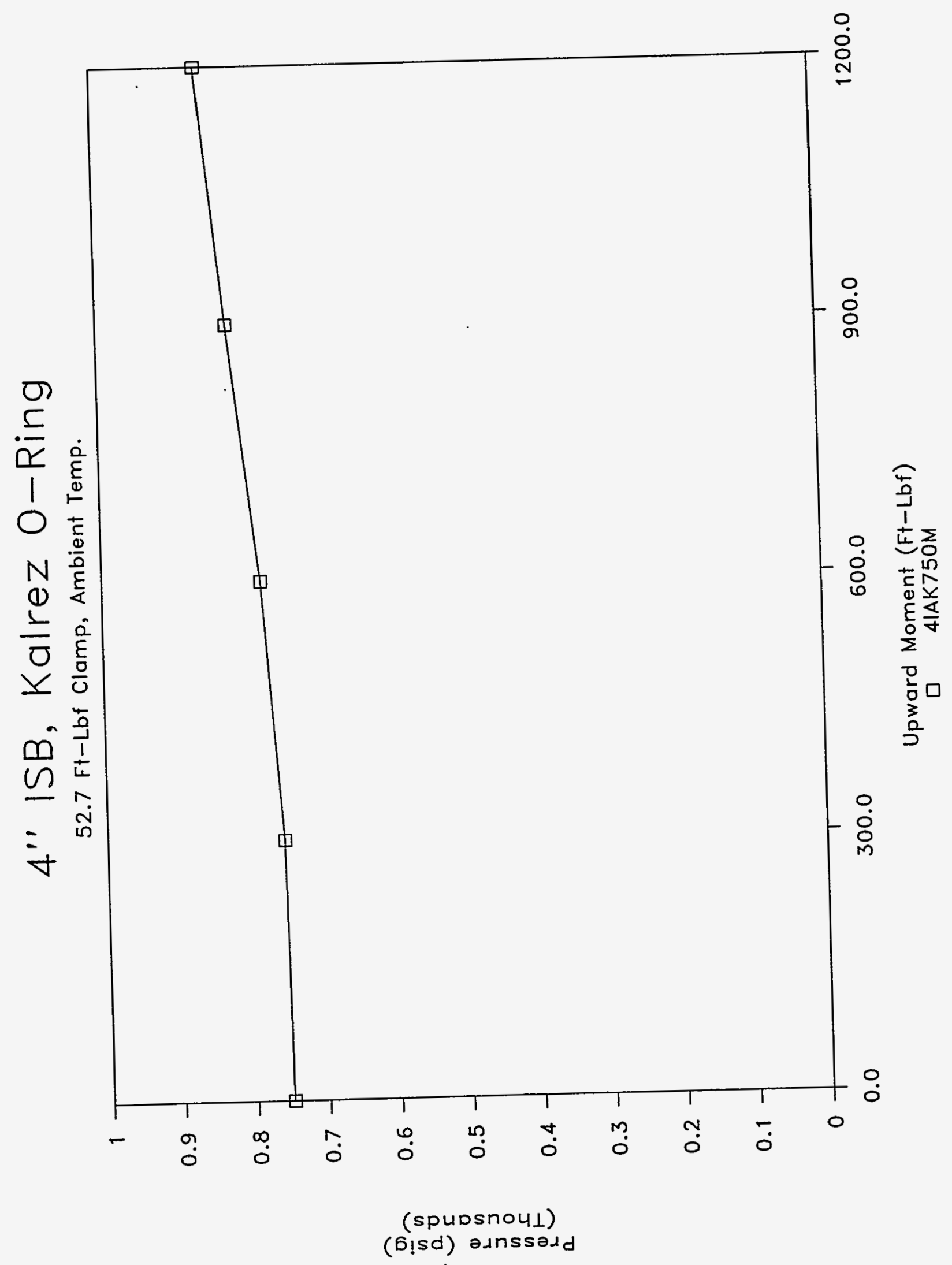


4" ISB, Kalrez O-Ring

52.7 Ff-Lbf Clamp, Ambient Temp.

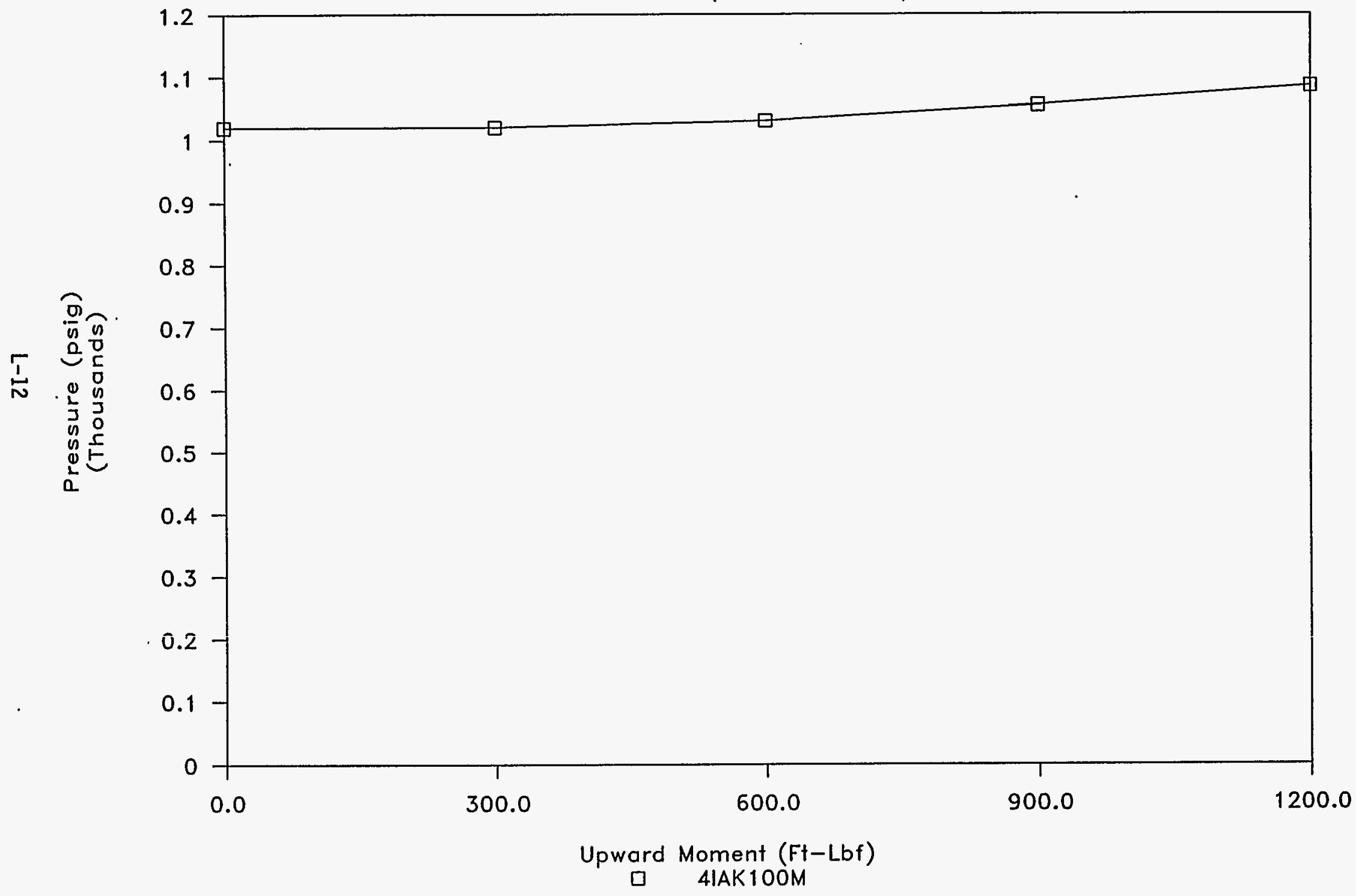


WHC-SD-WM-TRP-223 ReV 0

OCTOBER 06, 1994

4" ISB CONNECTOR, KALREZ O-RING, 70 DUROMETER, AMBIENT TEMP. LEAK TEST - UP AND OVER MOMENT (UPWARD ROTATION)

CLAMPING TORQUE $=52.7$ FT-LBF

CHARGE PRESSURE $=250$ PSIG

GRAPH NAME $=4$ IAK2 $50 M$

\begin{tabular}{|c|c|c|c|}
\hline $\begin{array}{l}\text { PRESSURE } \\
\text { PSIG }\end{array}$ & $\begin{array}{l}\text { ELAPSED } \\
\text { MINUTES }\end{array}$ & $\begin{array}{l}\text { ELAPSED } \\
\text { SECONDS }\end{array}$ & $\begin{array}{l}\text { ELAPSED } \\
\text { DECIMAL } \\
\text { MINUTES }\end{array}$ \\
\hline INPUT & INPUT & INPUT & COMPUTE \\
\hline 250 & 0 & & \\
\hline 250 & 2 & & \\
\hline 255 & 4 & & \\
\hline 310 & 6 & & \\
\hline 30 & 8 & & \\
\hline & 10 & & \\
\hline
\end{tabular}

\begin{tabular}{|c|c|c|}
\hline \multirow{5}{*}{$\begin{array}{l}\text { UPWARD } \\
\text { FORCE } \\
\text { LBS } \\
\text { INPUT }\end{array}$} & UPWARD & \multirow{5}{*}{$\begin{array}{l}\text { UPWARD } \\
\text { MOMENT } \\
\text { FT-LBF. } \\
\text { COMPUTE }\end{array}$} \\
\hline & MOMENT & \\
\hline & ARM & \\
\hline & FT. & \\
\hline & INPUT & \\
\hline 0 & 1.2000 & 0.0 \\
\hline 0 & 1.2000 & 0.0 \\
\hline 250 & 1.2000 & 300.0 \\
\hline 500 & 1.2000 & 600.0 \\
\hline 750 & 1.2000 & 900.0 \\
\hline 1000 & 1.2000 & 1200.0 \\
\hline
\end{tabular}

INCREASED PRESSURE TO 500 PSIG

GRAPH NAME $=4$ IAK50OM

$\begin{array}{rrrrrrr}495 & 12 & 2 & 12.03 & 0 & 1.2000 & 0.0 \\ 505 & 14 & 6 & 14.10 & 250 & 1.2000 & 300.0 \\ 555 & 16 & 2 & 16.03 & 500 & 1.2000 & 600.0 \\ 595 & 18 & 3 & 18.05 & 750 & 1.2000 & 900.0 \\ 575 & 20 & 10 & 20.17 & 1000 & 1.2000 & 1200.0\end{array}$

INCREASED PRESSURE TO 750 PSIG

$\begin{array}{ll}750 & 22 \\ 755 & 24 \\ 780 & 26 \\ 820 & 28 \\ 855 & 30\end{array}$

$\begin{array}{ll}2 & 22.03 \\ 2 & 24.03 \\ 6 & 26.10 \\ 1 & 28.02 \\ 1 & 30.02\end{array}$

INCREASED PRESSURE TO 1000 PSIG

$\begin{array}{ll}1020 & 32 \\ 1020 & 34 \\ 1030 & 36 \\ 1055 & 38 \\ 1085 & 40\end{array}$

GRAPH NAME $=4$ IAK75OM

$\begin{array}{rrr}0 & 1.2000 & 0.0 \\ 250 & 1.2000 & 300.0 \\ 500 & 1.2000 & 600.0 \\ 750 & 1.2000 & 900.0 \\ 1000 & 1.2000 & 1200.0\end{array}$

GRAPH NAME $=4$ IAK10OM

32.03

34.03

36.05

38.03

40.02

0
250
500
750
1000

1.2000

1.2000

1.2000

1.2000

1.2000
0.0 300.0 600.0 900.0 1200.0 
4" ISB, Kalrez O-Ring

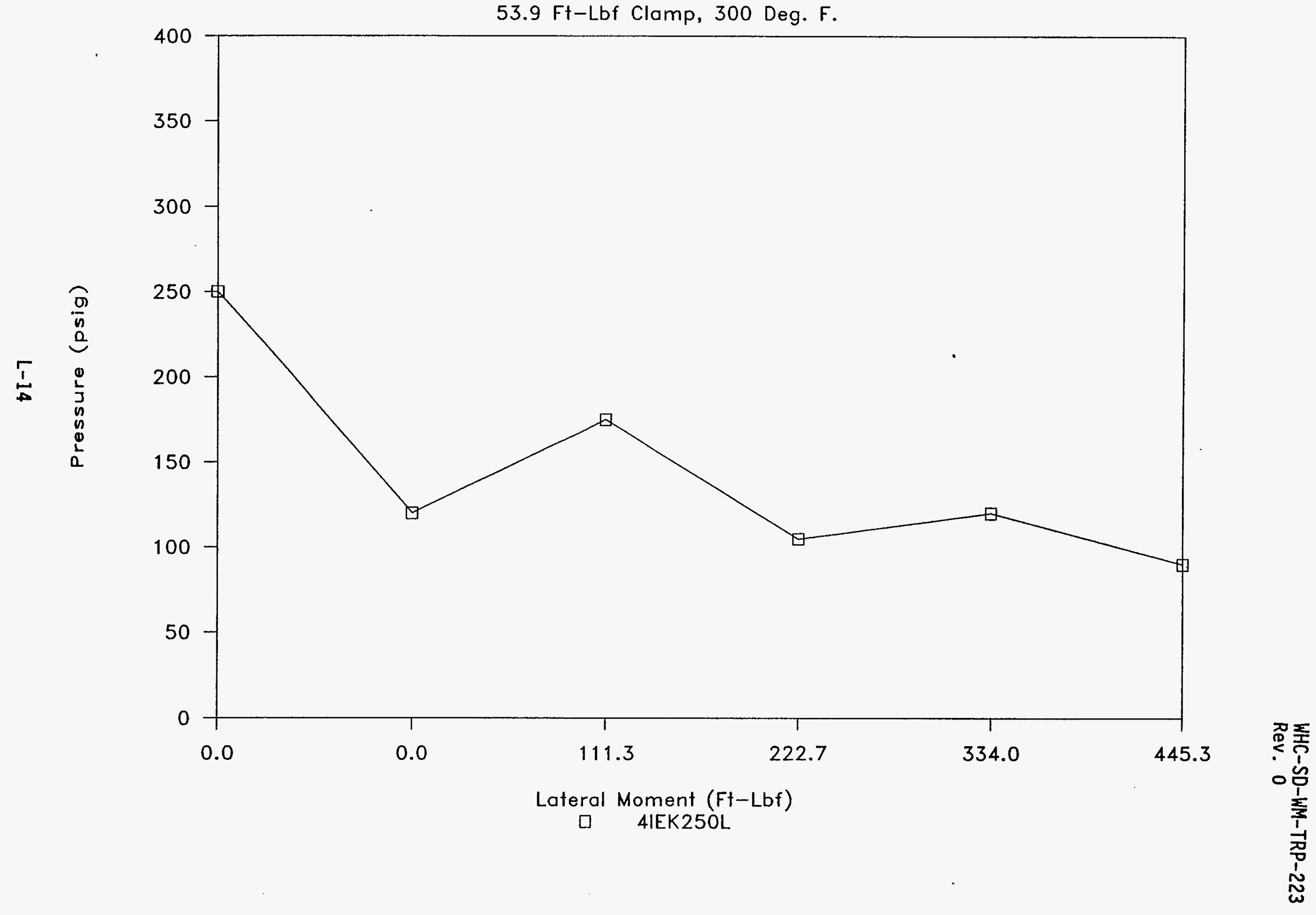


WHC-SD-WM-TRP-223

Rev. 0

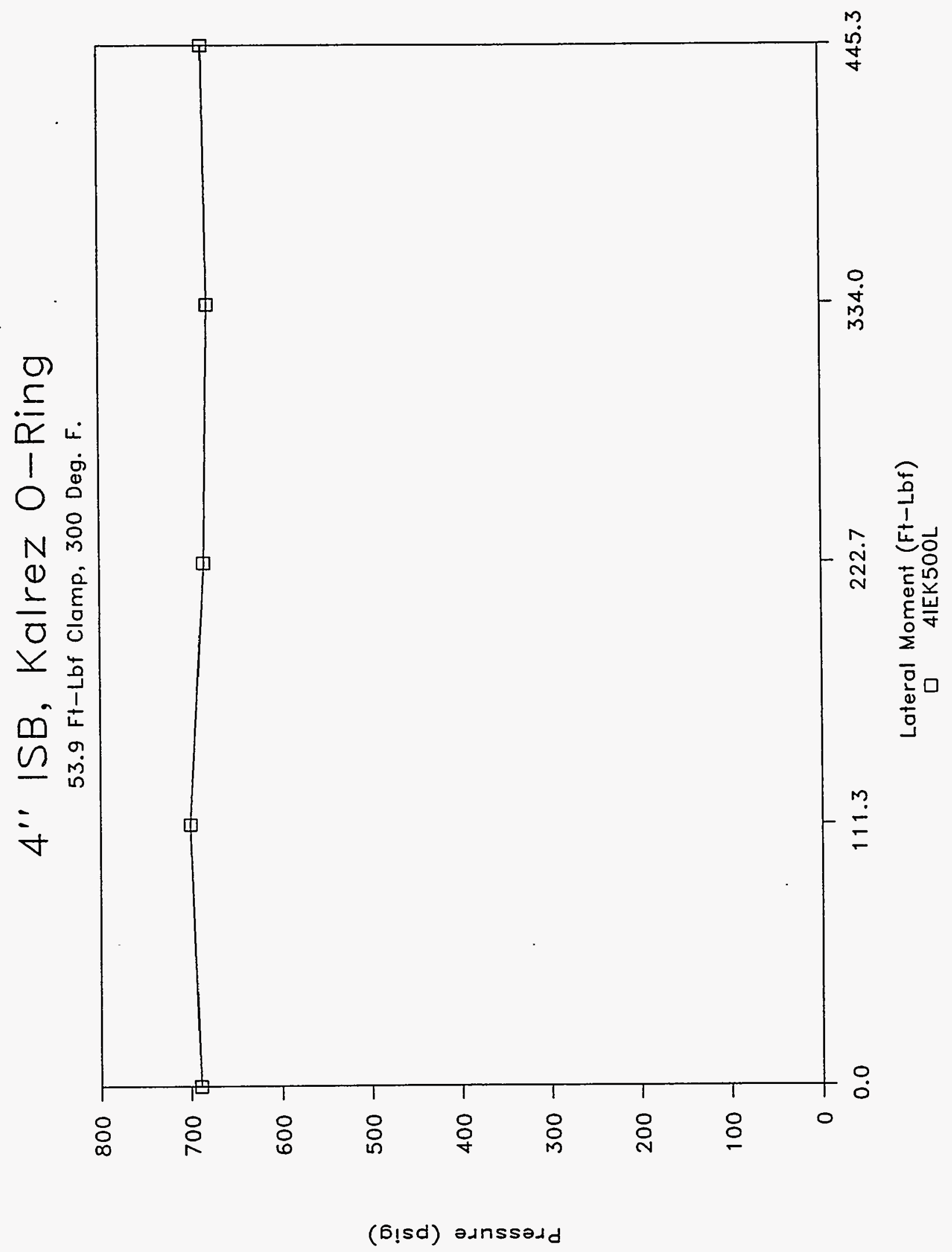

$L-15$ 


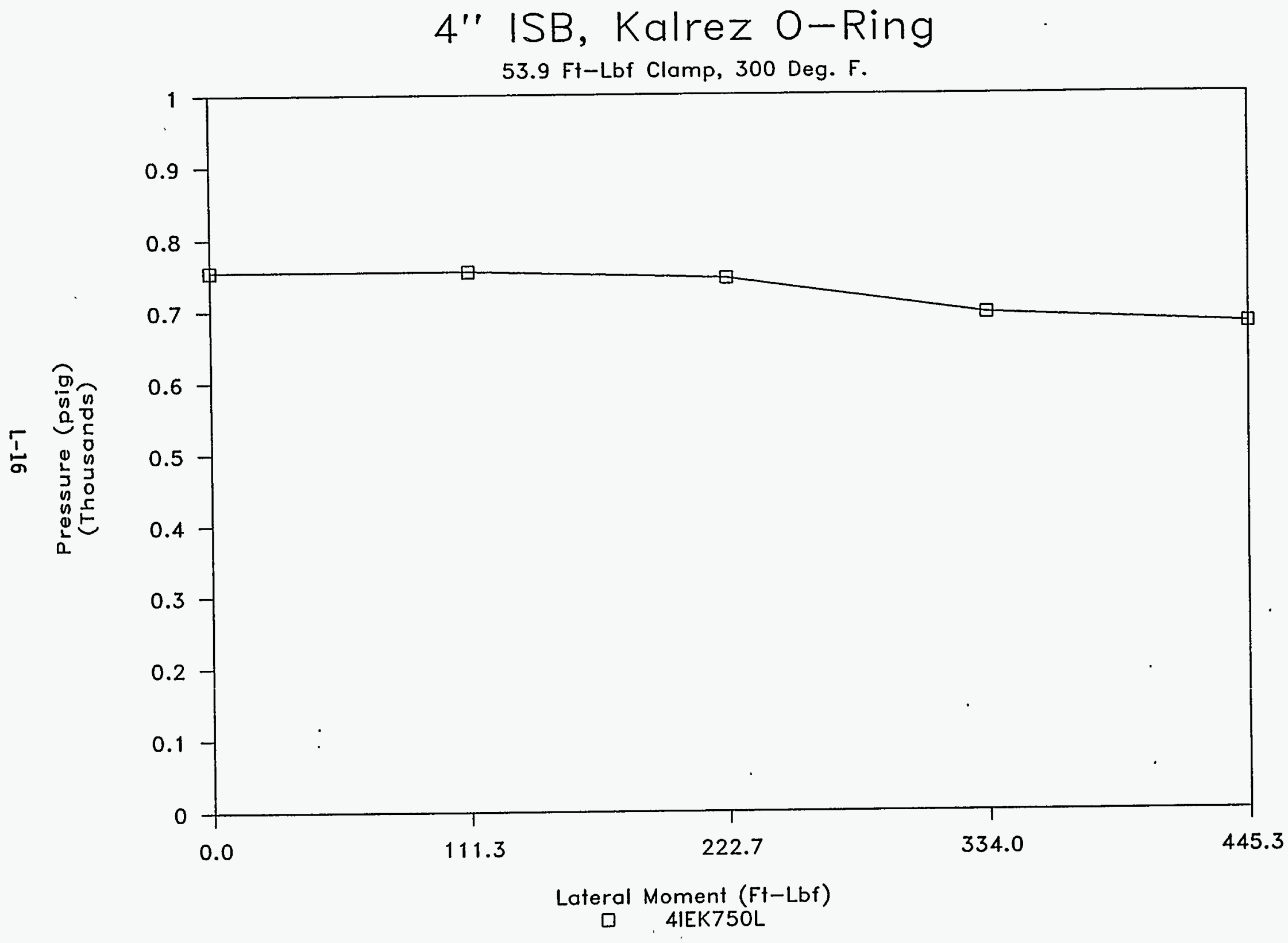


WHC-SD-WM-TRP-223

Rev. 0

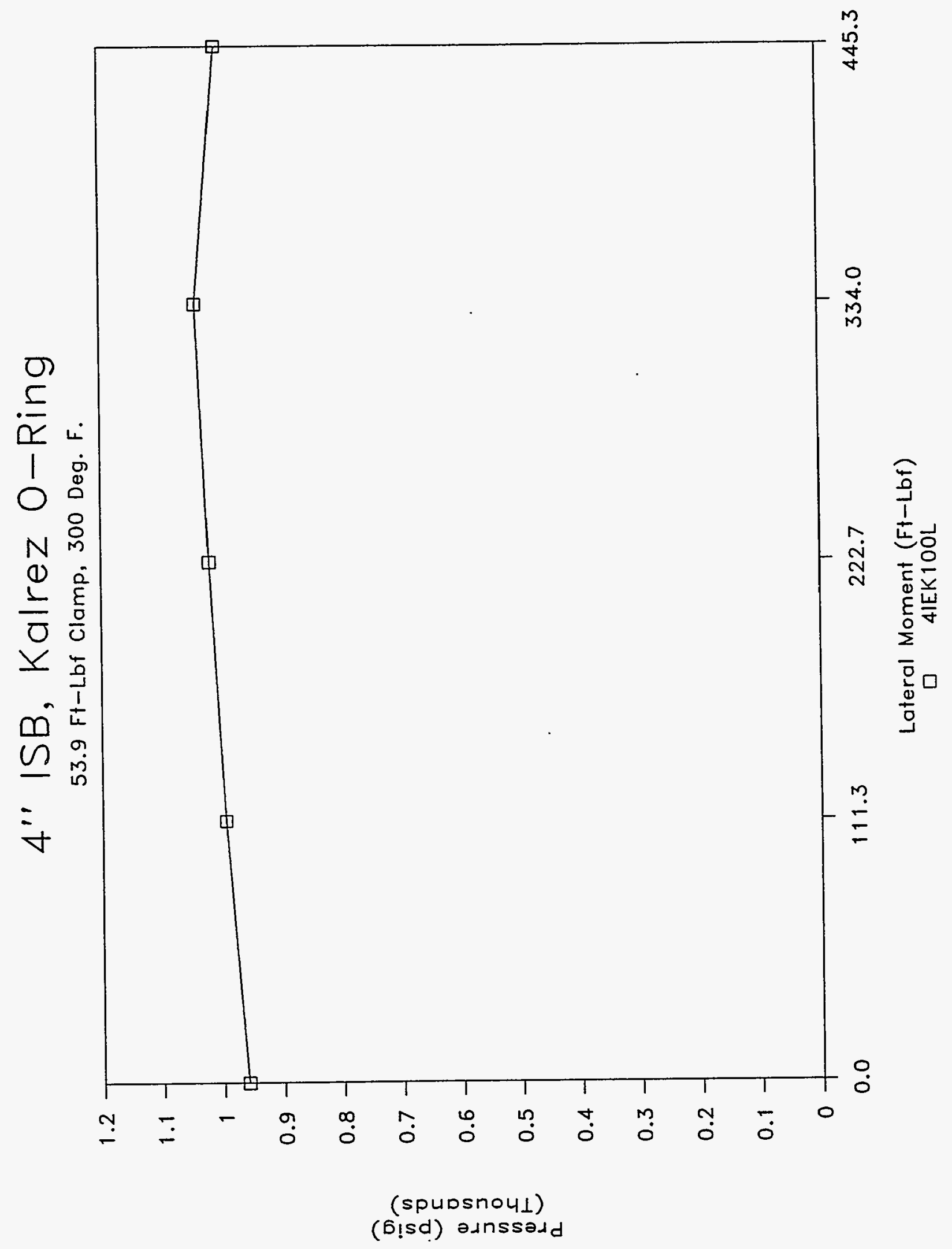

L-17 


\section{WHC-SD-WM-TRP-223 ReV O}

OCTOBER 07, 1994

4" ISB CONNECTOR, KALREZ O-RING, 70 DUROMETER, ELEVATED TEMP. (300 DEG. F IEAK TEST - IATERAL MOMENT (SIDEWAYS)

CLAMPING TORQUE $=53.9 \mathrm{FT}-\mathrm{IBF}$ GRAPH NAME $=4:$ IEK250I

CHARGE PRESSURE $=250$ PSIG

$\begin{array}{lllllll} & & & & & \text { IATEFAL } \\ \text { PRESSURE } & \text { ELAPSED } & \text { ELAPSED } & \text { ELAPSED } & \text { LATERAL } & \text { MOMENT } & \text { LATERAI } \\ \text { PSIG } & \text { MINUTES } & \text { SECONDS } & \text { DECIMAL } & \text { FORCE } & \text { ARM } & \text { MOMENT } \\ & & & \text { MINUTES } & \text { LBS } & \text { FT. } & \text { FT-LBF. } \\ \text { INPUT } & \text { INPUT } & \text { INPUT } & \text { COMPUTE } & \text { INPUT } & \text { INPUI: } & \text { COMPUTE }\end{array}$

$\begin{array}{rrrrrrr}250 & 0 & 0 & 0.00 & 0 & 0.44453 & 0.0 \\ 120 & 2 & 1 & 2.02 & 0 & 0.4453 & 0.0 \\ 175 & 4 & 2 & 4.03 & 250 & 0.4: 453 & 111.3 \\ 105 & 9 & 43 & 9.72 & 500 & 0.44453 & 222.7 \\ 120 & 12 & 0 & 12.00 & 750 & 0.4453 & 334.0 \\ 90 & 14 & 1 & 14.02 & 1000 & 0.44453 & 445.3\end{array}$

INCREASED PRESSURE TO 500 PSIG

GRAPH NAME = 4:IEK50OL

$\begin{array}{rrrrrrr}690 & 16 & 1 & 16.02 & 0 & 0.44453 & 0.0 \\ 700 & 18 & 2 & 18.03 & 250 & 0.44453 & 111.3 \\ 685 & 20 & 2 & 20.03 & 500 & 0.44453 & 222.7 \\ 680 & 22 & 8 & 22.13 & 750 & 0.44453 & 334.0 \\ 685 & 24 & 13 & 24.22 & 1000 & 0.44453 & 445.3\end{array}$

INCREASED PRESSURE TO 750 PSIG

GRAPH NAME = 4:IEK750I

$\begin{array}{rrrrrrr}755 & 26 & 8 & 26.13 & 0 & 0.44453 & 0.0 \\ 755 & 28 & 5 & 28.08 & 250 & 0.44453 & 111.3 \\ 745 & 30 & 3 & 30.05 & 500 & 0.44453 & 222.7 \\ 695 & 32 & 2 & 32.03 & 750 & 0.44453 & 334.0 \\ 680 & 34 & 1 & 34.02 & 1000 & 0.44453 & 445.3\end{array}$

INCREASED PRESSURE TO 1000 PSIG

$\begin{array}{rl}960 & 36 \\ 995 & 38 \\ 1020 & 40 \\ 1040 & 42 \\ 1005 & 44\end{array}$

$\begin{array}{ll}2 & 36.03 \\ 1 & 38.02 \\ 1 & 40.02 \\ 3 & 42.05 \\ 3 & 44.05\end{array}$
GRAPH NAME = 4:IEK100I

$\begin{array}{rlr}0 & 0.4: 453 & 0.0 \\ 250 & 0.4: 453 & 111.3 \\ 500 & 0.4: 453 & 222.7 \\ 750 & 0.4: 453 & 334.0 \\ 1000 & 0.44453 & 445.3\end{array}$


4" ISB, Kalrez O-Ring

54.9 Ft-Lbf Clamp, 300 Deg. F.

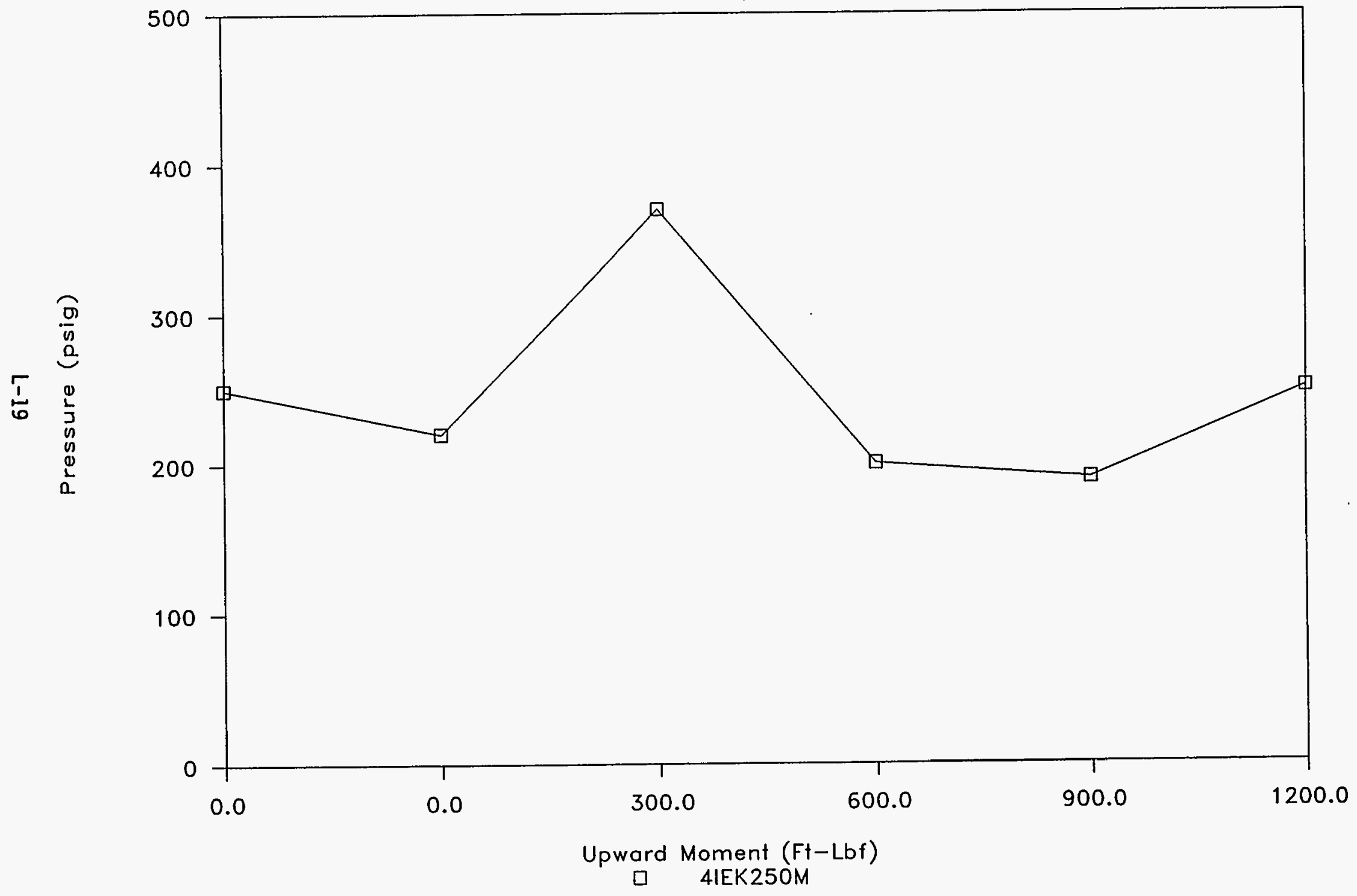


Rev. 0

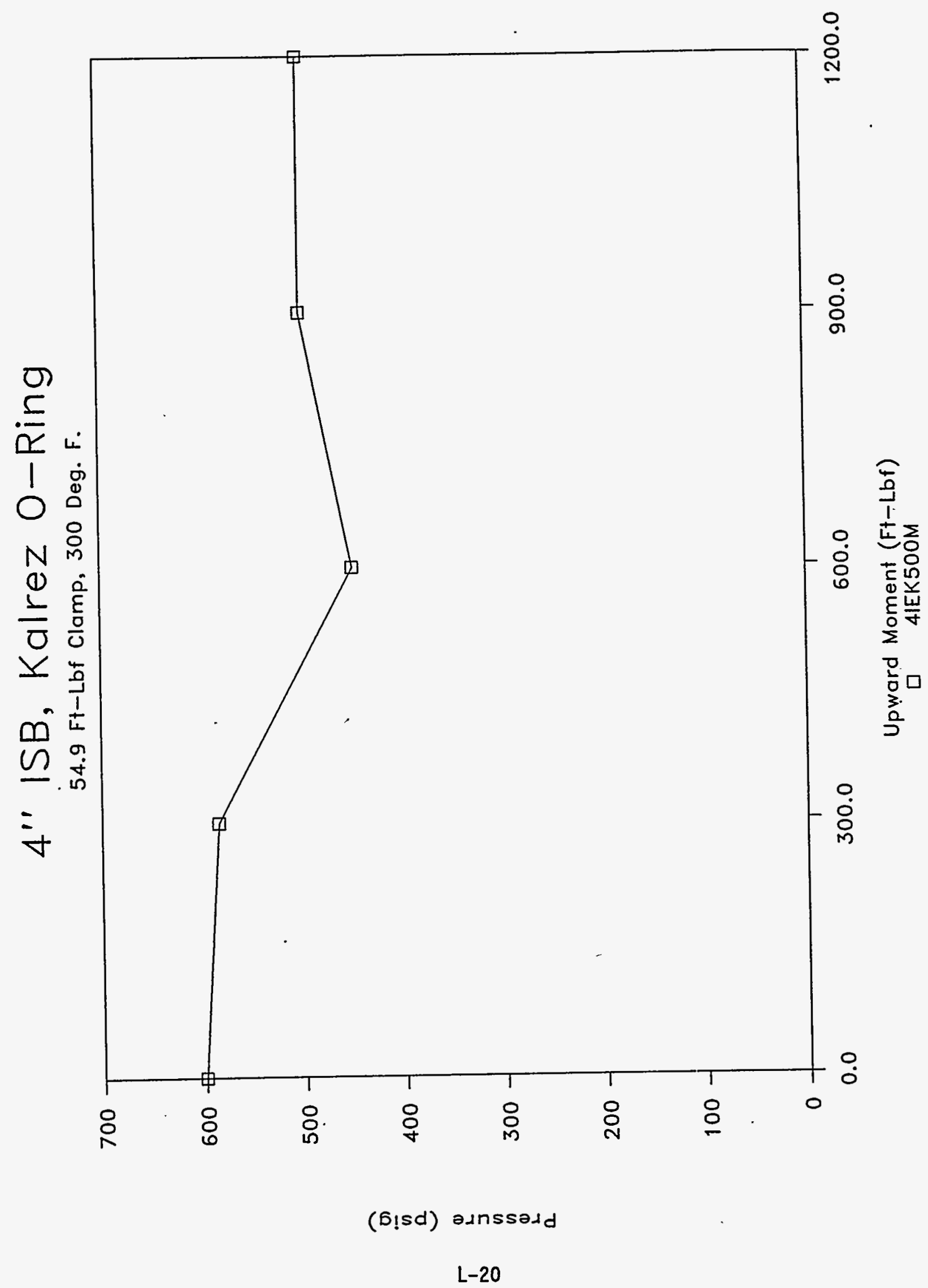


WHC-SD-WM-TRP-223

Rev. 0

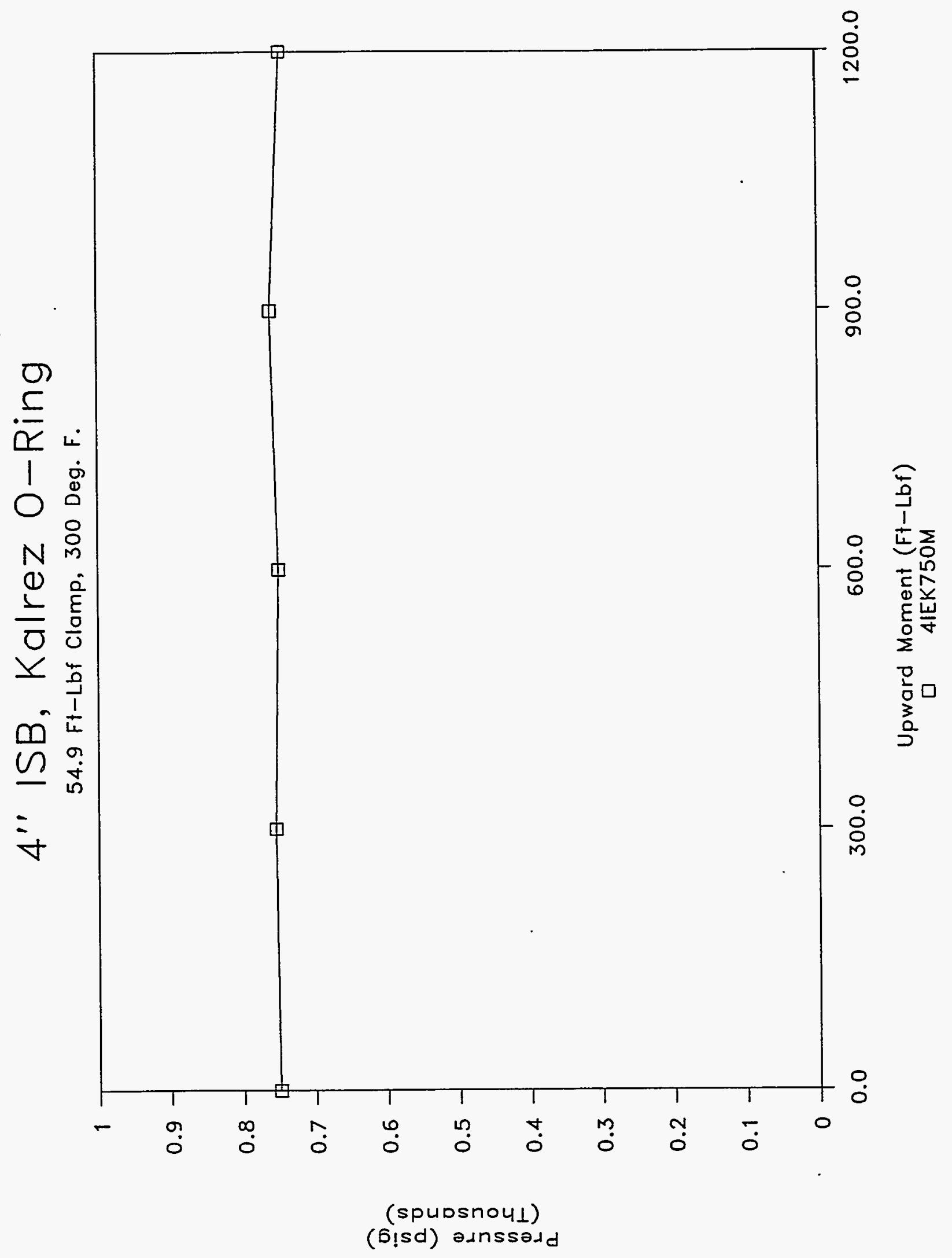




\section{4" ISB, Kalrez O-Ring}

$54.9 \mathrm{Ft}$-Lbf Clamp, 300 Deg. F.

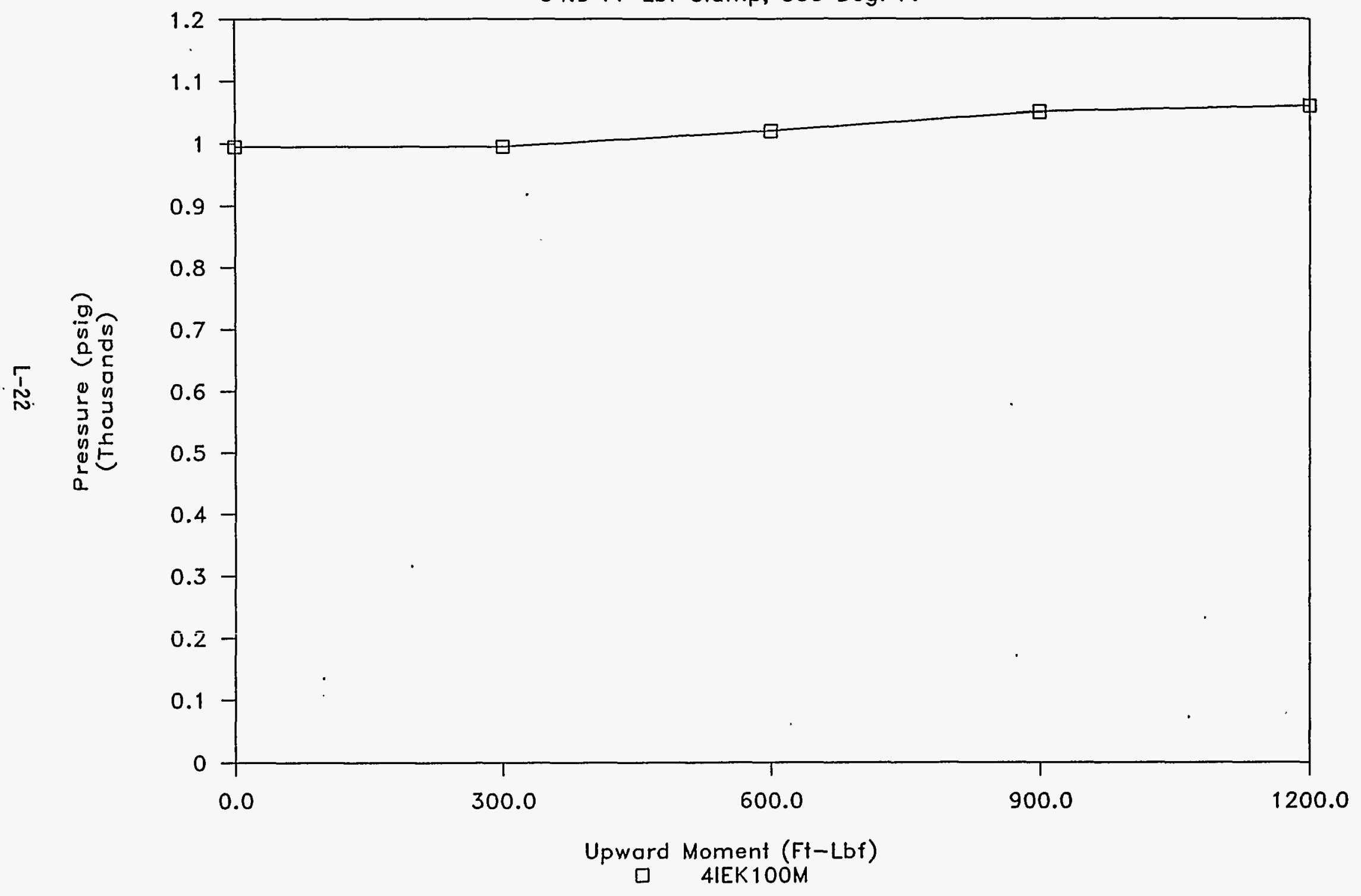


WHC-SD-WM-TRP-223 ReV 0

OCTOBER 07, 1994

4" ISB CONNECTOR, KALREZ O-RING, 70 DUROMETER, ELEVATED TEMP. (300 DEG. F IEAK TEST - UP AND OVER MOMENT (UPWARD ROTATION)

CLAMPING TORQUE $=54.9$ FT-LBF

GRAPH NAME $=4$ IEK250M

CHARGE PRESSURE $=250$ PSIG

\begin{tabular}{|c|c|c|c|c|c|c|}
\hline \multirow{3}{*}{$\begin{array}{l}\text { PRESSURE } \\
\text { PSIG }\end{array}$} & \multirow[b]{2}{*}{ ELAPSED } & \multirow[b]{2}{*}{ ELAPSED } & \multirow[b]{2}{*}{ ELAPSED } & \multicolumn{3}{|c|}{ UPWARD } \\
\hline & & & & UPWARD & MOMENT & UPWARD \\
\hline & MINUTES & SECONDS & DECIMAL & FORCE & ARM & MOMENT \\
\hline NPUT & INPUT & INPUT & $\begin{array}{l}\text { MINUTES } \\
\text { COMPUTE }\end{array}$ & $\begin{array}{l}\text { LBS } \\
\text { INPUT }\end{array}$ & $\begin{array}{l}\text { FT. } \\
\text { INPUT }\end{array}$ & $\begin{array}{l}\text { FT-LBF. } \\
\text { COMPUTE }\end{array}$ \\
\hline 25 & c & 0 & 0.1 & 0 & 1.2000 & 0.0 \\
\hline 220 & 2 & 7 & 2 & 0 & 1.2 & 0.0 \\
\hline 370 & 4 & 3 & 4. & $25 c$ & 1.2 & 300. \\
\hline 200 & $\epsilon$ & 2 & 6.0 & 50 & 1.2 & 600.0 \\
\hline 190 & $\varepsilon$ & 2 & 8.03 & 75 & 1.2000 & 900.0 \\
\hline 250 & 10 & 2 & 10.03 & 1000 & 1.2000 & 1200.0 \\
\hline
\end{tabular}

INCREASED PRESSURE TO 500 PSIG

GRAPH NAME $=4$ IEK50OM

$\begin{array}{rrrrrrr}600 & 12 & 4 & 12.07 & 0 & 1.2000 & 0.0 \\ 585 & 14 & 1 & 14.02 & 250 & 1.2000 & 300.0 \\ 450 & 16 & 1 & 16.02 & 500 & 1.2000 & 600.0 \\ 500 & 18 & 2 & 18.03 & 750 & 1.2000 & 900.0 \\ 500 & 20 & 7 & 20.12 & 1000 & 1.2000 & 1200.0\end{array}$

INCREASED PRESSURE TO 750 PSIG

$\begin{array}{llll}750 & 22 & 3 & 22.05 \\ 755 & 24 & 1 & 24.02 \\ 750 & 26 & 1 & 26.02 \\ 760 & 28 & 3 & 28.05 \\ 745 & 30 & 2 & 30.03\end{array}$

INCREASED PRESSURE TO 1000 PSIG

$\begin{array}{rl}995 & 32 \\ 995 & 34 \\ 1020 & 36 \\ 1050 & 38 \\ 1060 & 40\end{array}$

GRAPH NAME $=4$ IEK750M

$\begin{array}{rrr}0 & 1.2000 & 0.0 \\ 250 & 1.2000 & 300.0 \\ 500 & 1.2000 & 600.0 \\ 750 & 1.2000 & 900.0 \\ 1000 & 1.2000 & 1200.0\end{array}$

GRAPH NAME $=4$ IEK10OM

$\begin{array}{rrr}0 & 1.2000 & 0.0 \\ 250 & 1.2000 & 300.0 \\ 500 & 1.2000 & 600.0 \\ 750 & 1.2000 & 900.0 \\ 1000 & 1.2000 & 1200.0\end{array}$


WHC-SD-WM-TRP-223

Rev. 0

APPENDIX M: GRAPHS OF 4-IN. VITON TESTS

$M-1$ 
WHC-SD-WM-TRP-223

Rev. 0

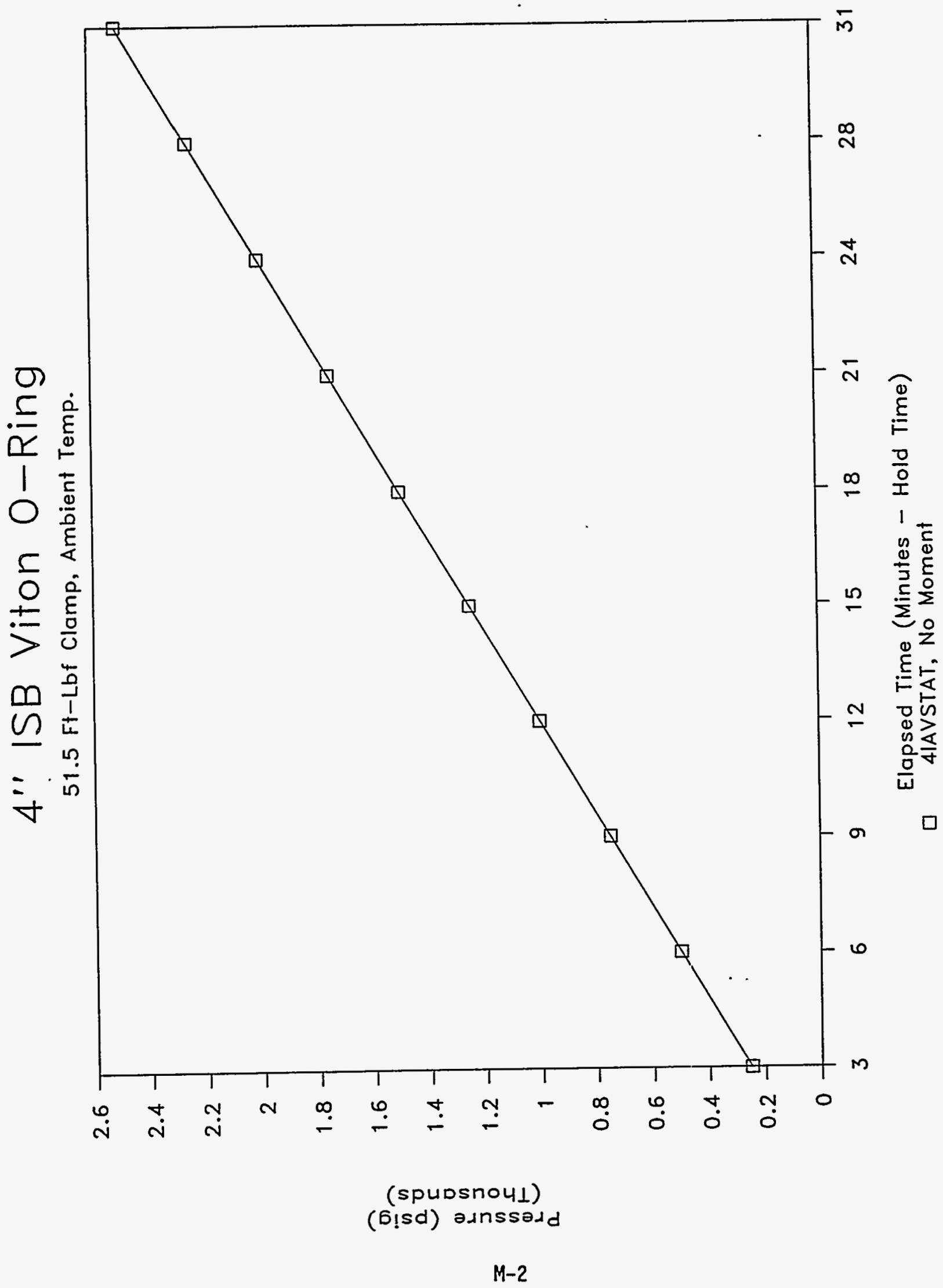


WHC-SD-WM-TRP-223 ReV 0

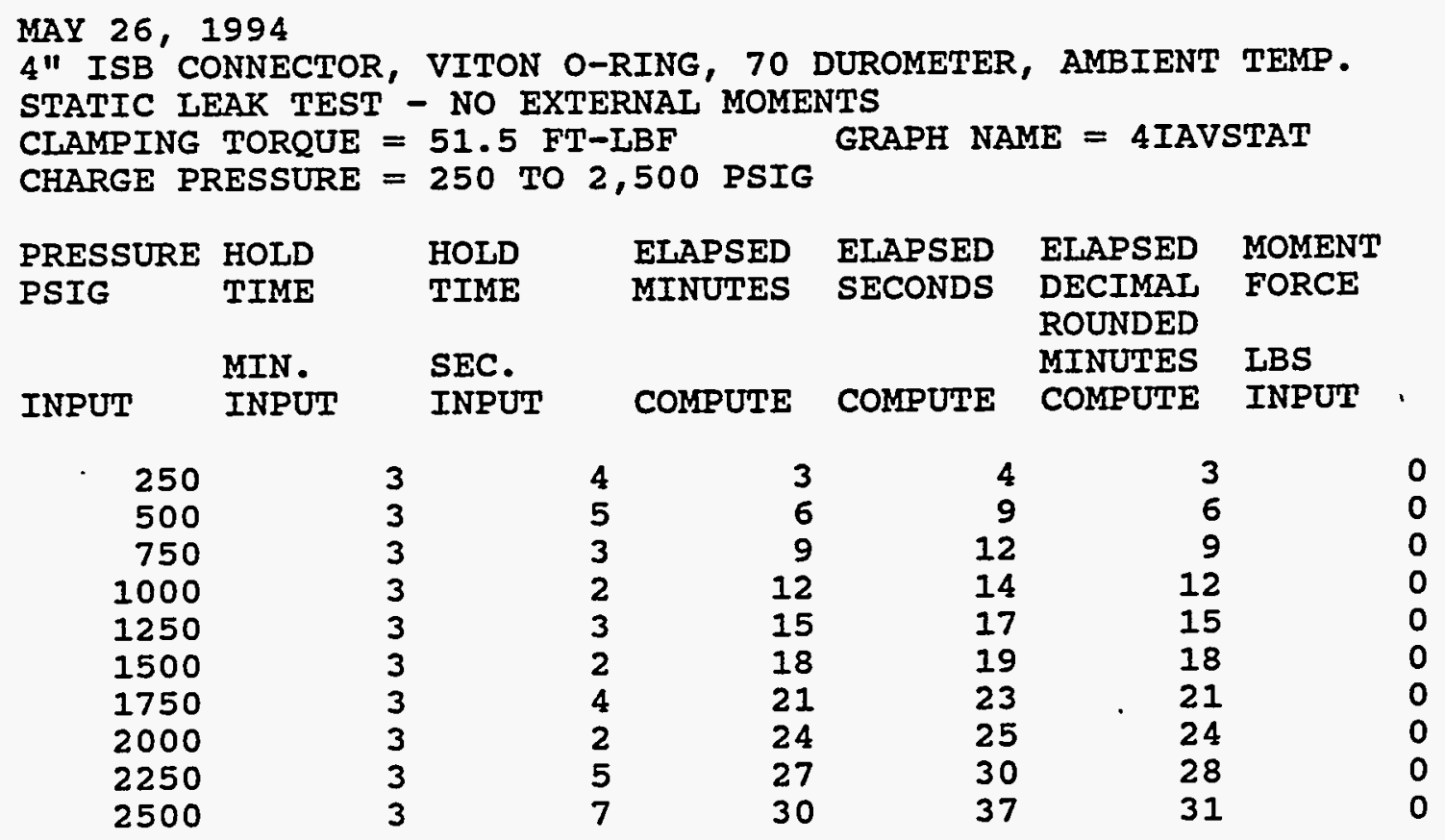


Rev. 0

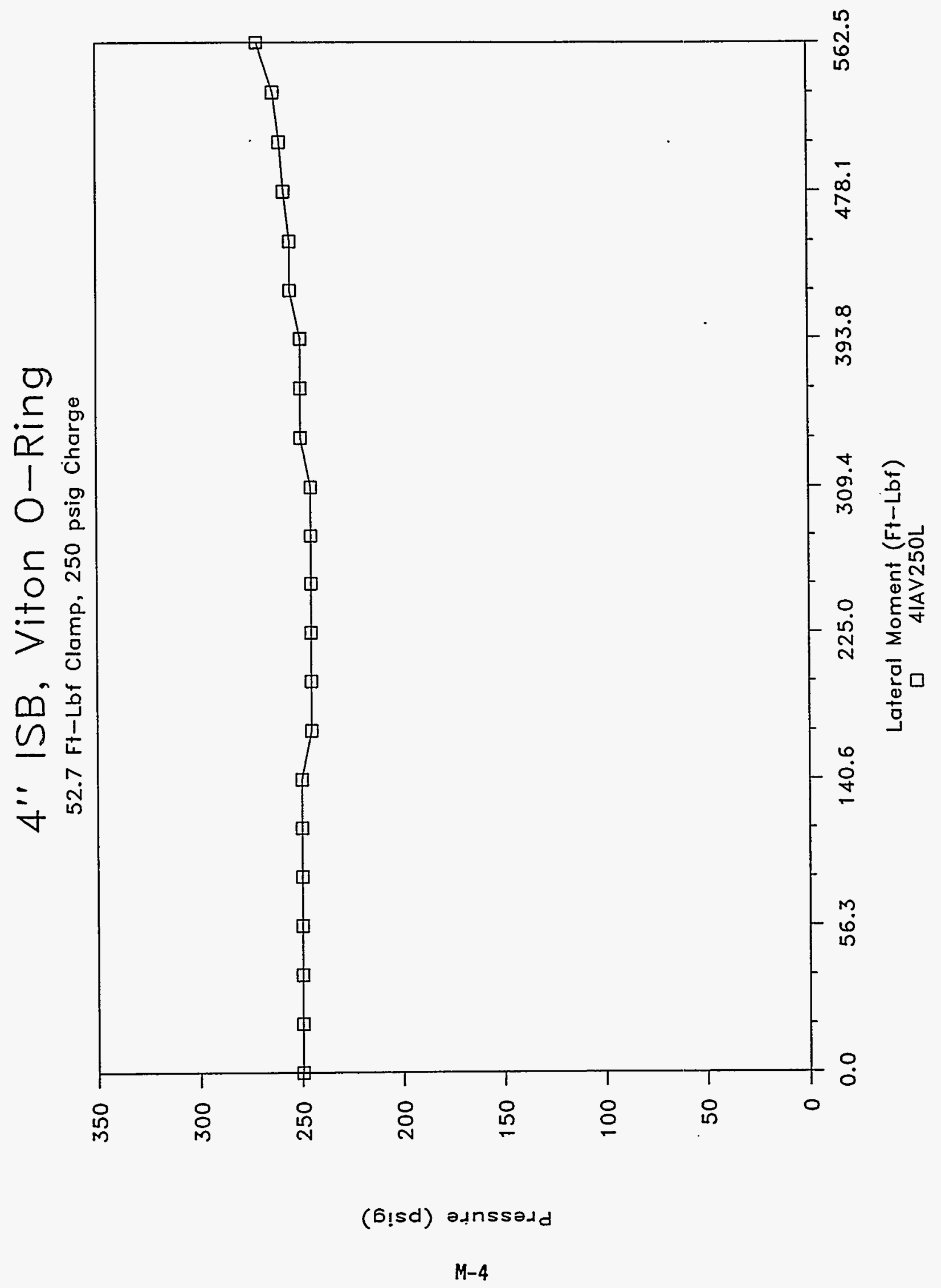


JUNE 01, 1994

4" ISB CONNECTOR, VITON O-RING, 70 DURONETER, AMBIENT TEMP.

LEAX TEST - LATERAL MOMENTS APPLIED (SIDEWAYS ROTATION)

CLAMPING TOROUE $=52.7 \mathrm{FT}-\mathrm{LBF}$ GRAPH NIAME $=4$ IAV25OL

CHARGE PRESSURE $=250$ PSIG

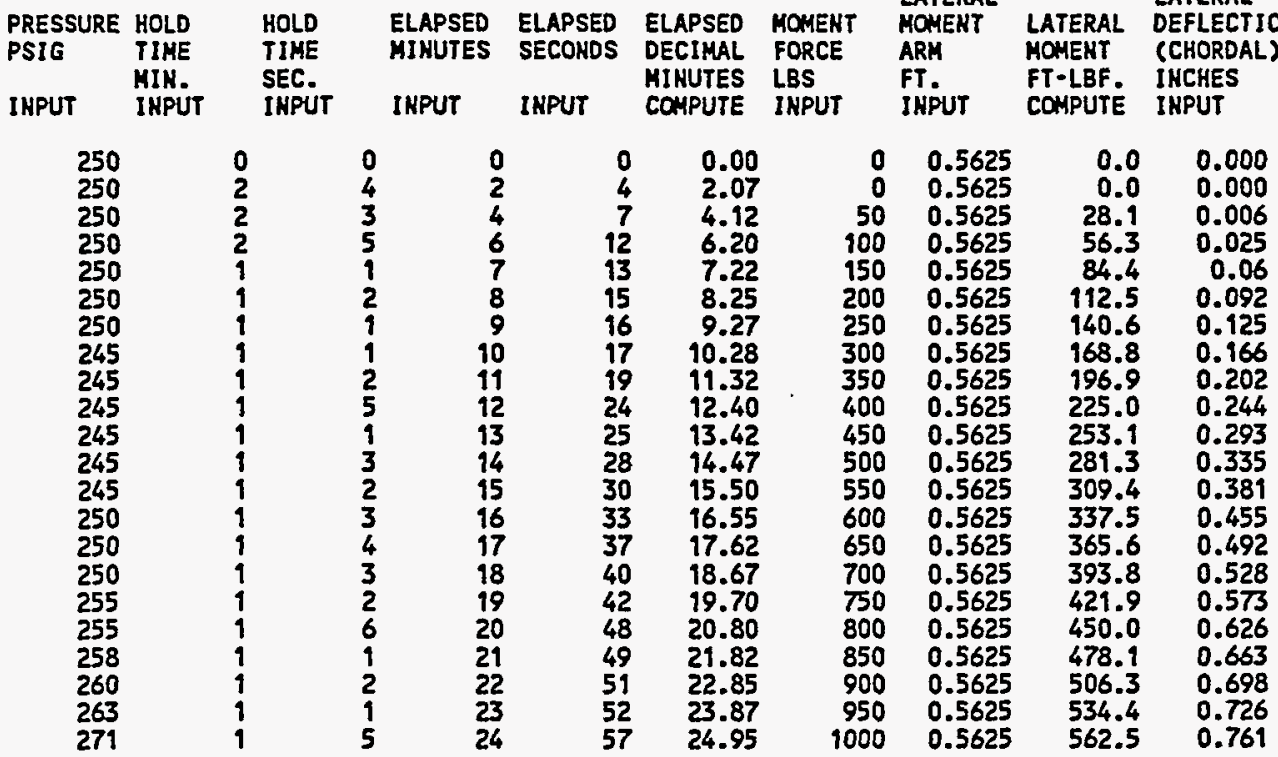


WHC-SD-WM-TRP-223

Rev. 0

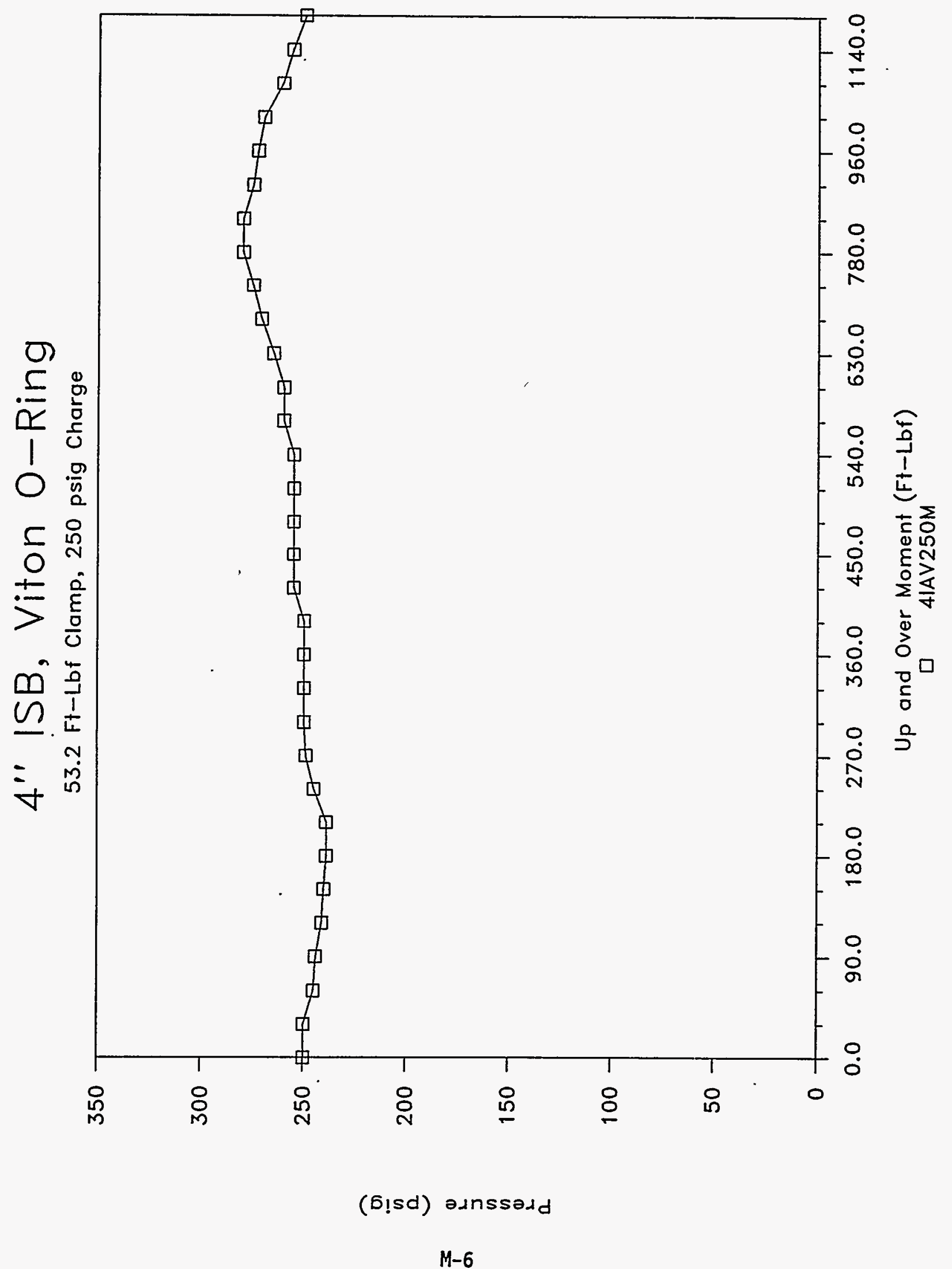




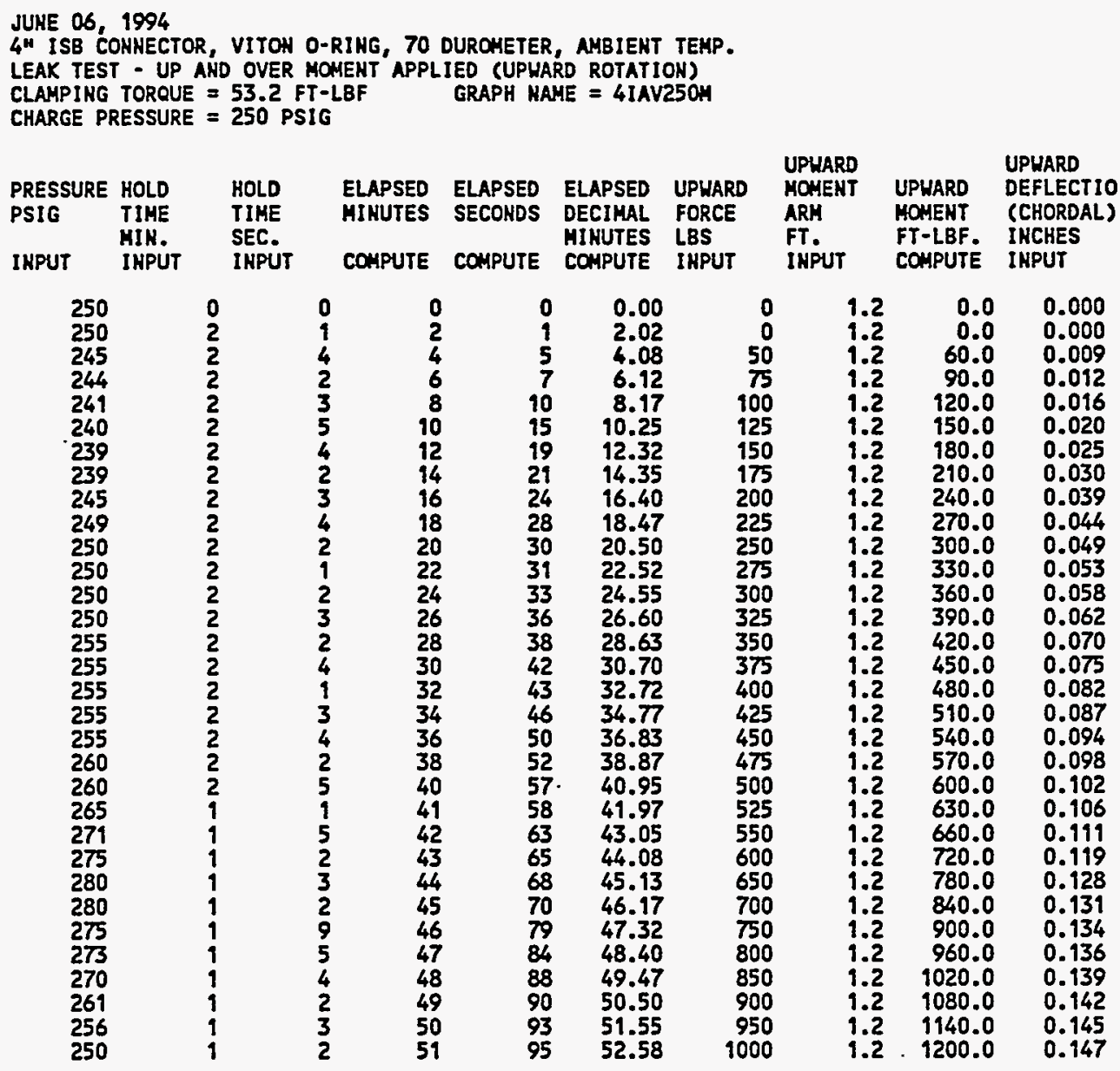


WHC-SD-WM-TRP-223

Rev. 0

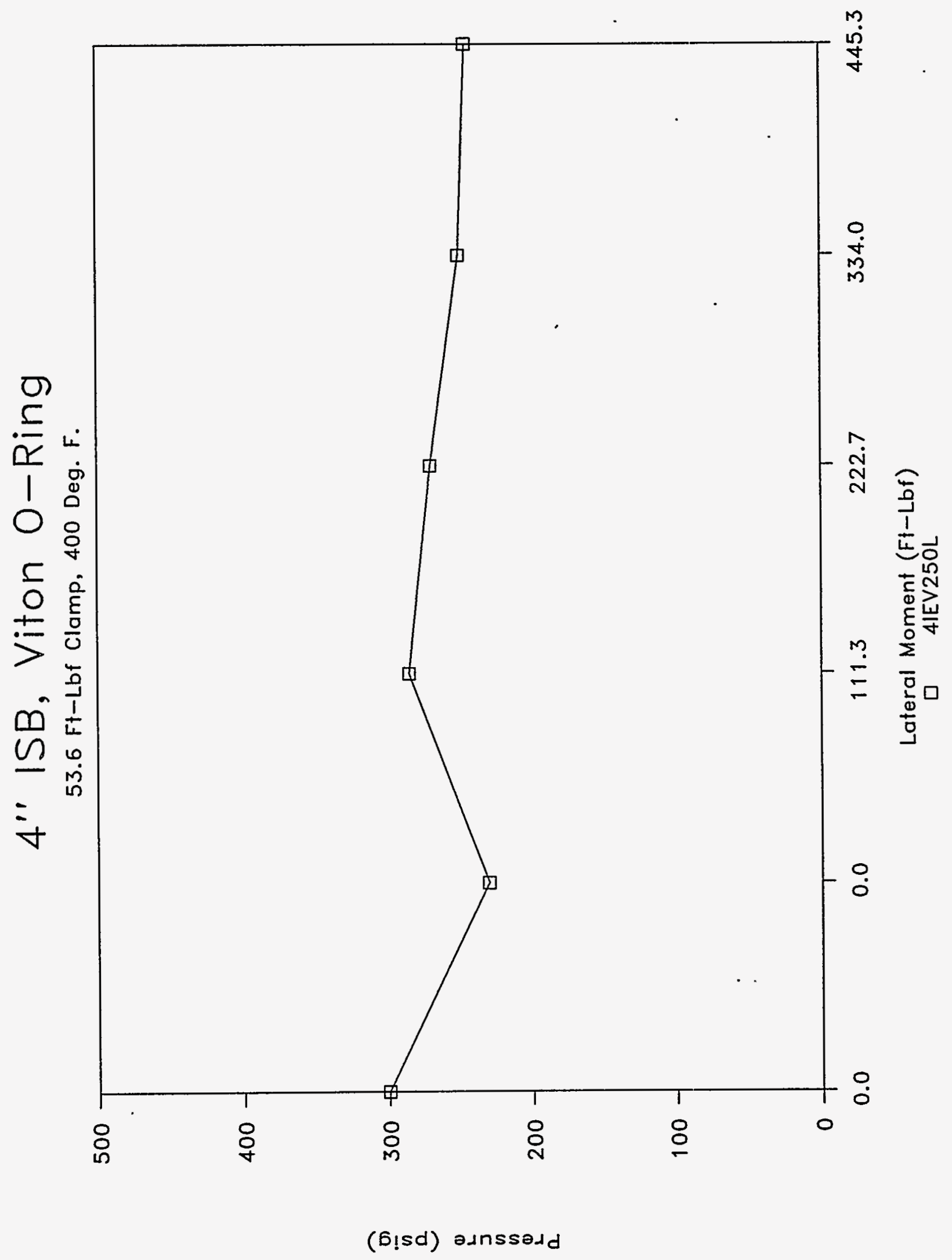

$M-8$ 
WHC-SD-WM-TRP-223

Rev. 0

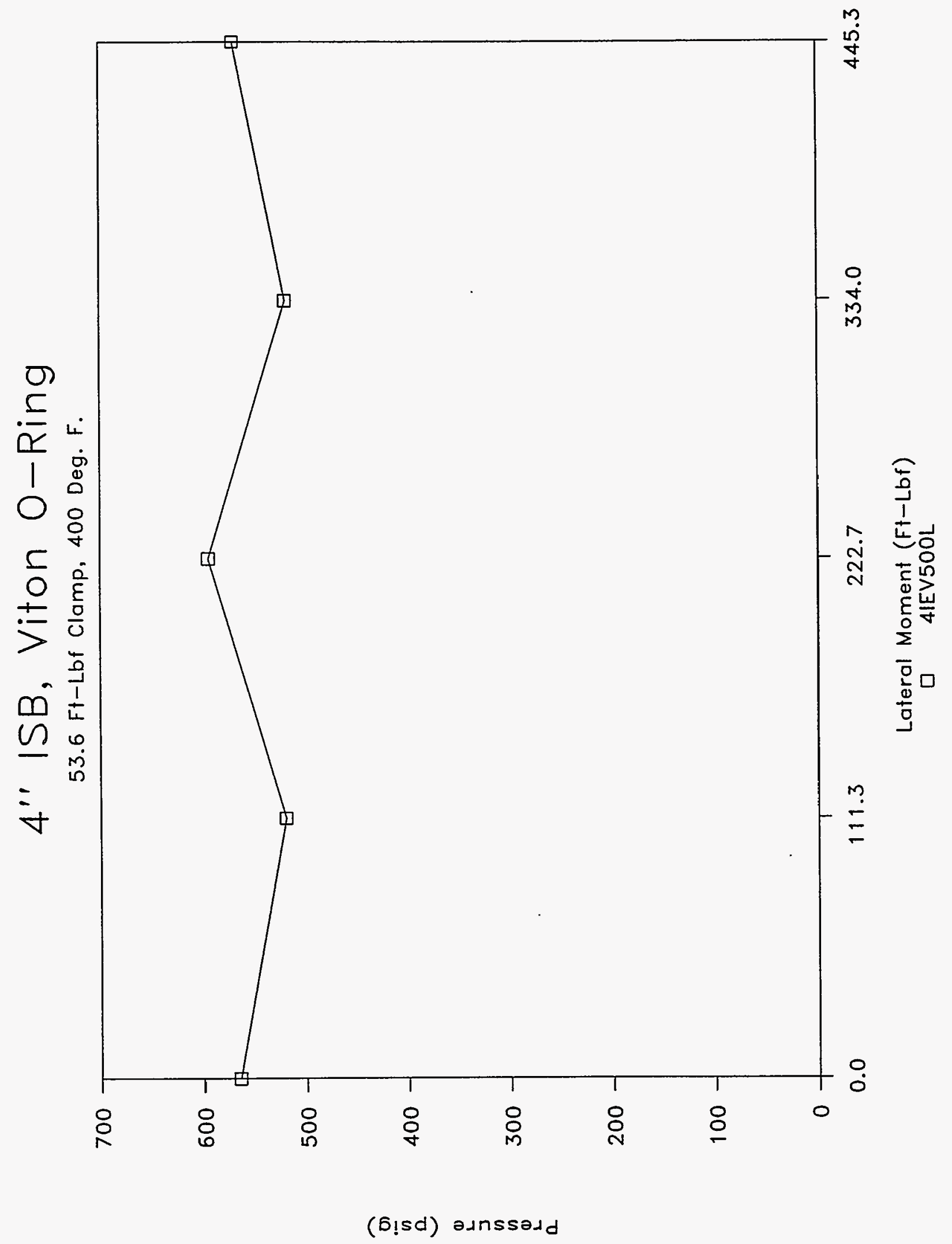


Rev. 0

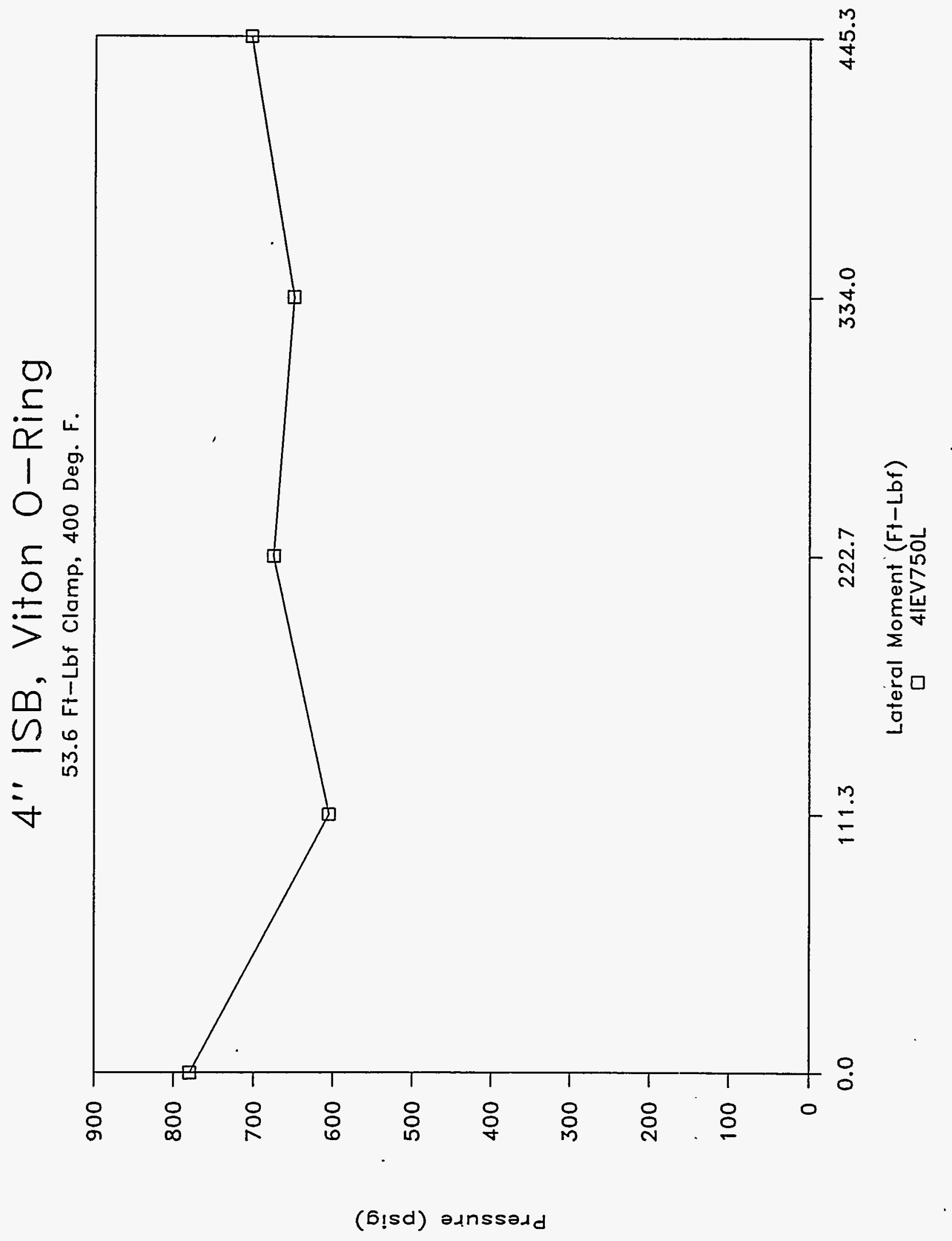

$M-10$ 
WHC-SD-HM-TRP-223

Rev. 0

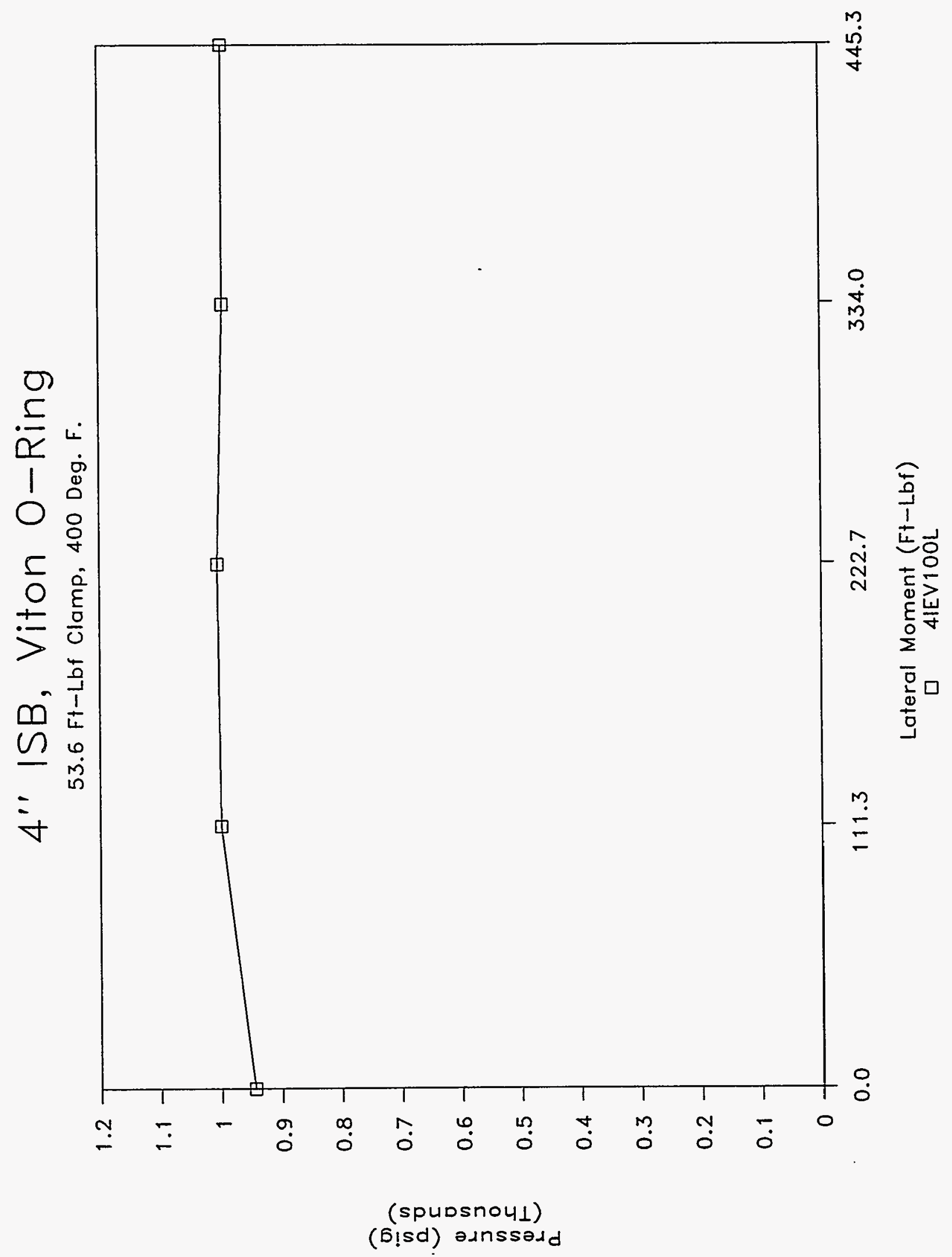

$M-11$ 
WHC-SD-WM-TRP-223 ReV 0

OCTOBER 11, 1994

4" ISB CONNECTOR, VITON O-RING, 70 DUROMETER, ELEVATED TEMP. (400 DEG. F) LEAK TEST - LATERAL MOMENT (SIDEWAYS)

CLAMPING TORQUE $=53.6$ FT-LBF GRAPH NAME $=4$ IEV250I

CHARGE PRESSURE $=250$ PSIG

\begin{tabular}{|c|c|c|c|c|c|c|}
\hline $\begin{array}{l}\text { PRESSURE } \\
\text { PSIG }\end{array}$ & $\begin{array}{l}\text { ELAAPSED } \\
\text { MINUTES }\end{array}$ & $\begin{array}{l}\text { ELAPSED } \\
\text { SECONDS }\end{array}$ & $\begin{array}{l}\text { ELAPSED } \\
\text { DECIMAL } \\
\text { MINUTES }\end{array}$ & $\begin{array}{l}\text { LATERAL } \\
\text { FORCE } \\
\text { LBS }\end{array}$ & $\begin{array}{l}\text { LATERAI } \\
\text { MOMENT } \\
\text { ARM } \\
\text { FT. }\end{array}$ & $\begin{array}{l}\text { IAATERAL } \\
\text { MOMENT } \\
\text { FT-LBF. }\end{array}$ \\
\hline INPUT & INPUT & INPUT & COMPUTE & INPUT & INPUT & COMPUTE \\
\hline $\begin{array}{l}300 \\
230 \\
285 \\
270 \\
250 \\
245\end{array}$ & $\begin{array}{r}0 \\
2 \\
4 \\
6 \\
8 \\
10\end{array}$ & $\begin{array}{l}0 \\
0 \\
9 \\
5 \\
0 \\
2\end{array}$ & $\begin{array}{r}0.00 \\
2.00 \\
4.15 \\
6.08 \\
8.00 \\
10.03\end{array}$ & $\begin{array}{r}0 \\
0 \\
250 \\
500 \\
750 \\
1000\end{array}$ & $\begin{array}{l}0.4453 \\
0.4453 \\
0.4453 \\
0.4453 \\
0.4453 \\
0.4453\end{array}$ & $\begin{array}{r}0.0 \\
0.0 \\
111.3 \\
222.7 \\
334.0 \\
445.3\end{array}$ \\
\hline
\end{tabular}

INCREASED PRESSURE TO 500 PSIG

GRAPH NAME $=4$ IEV50OI

$\begin{array}{rrrrrrr}565 & 12 & 1 & 12.02 & 0 & 0.4453 & 0.0 \\ 520 & 14 & 1 & 14.02 & 250 & 0.4453 & 111.3 \\ 595 & 16 & 7 & 16.12 & 500 & 0.4453 & 222.7 \\ 520 & 18 & 10 & 18.17 & 750 & 0.4453 & 334.0 \\ 570 & 20 & 4 & 20.07 & 1000 & 0.4453 & 445.3\end{array}$

INCREASED PRESSURE TO 750 PSIG

$\begin{array}{llll}780 & 22 & 1 & 22.02 \\ 605 & 24 & 2 & 24.03 \\ 675 & 26 & 3 & 26.05 \\ 650 & 28 & 2 & 28.03 \\ 705 & 30 & 1 & 30.02\end{array}$

INCREASED PRESSURE TO 1000 PSIG

$\begin{array}{rr}945 & 32 \\ 1000 & 34 \\ 1005 & 36 \\ 995 & 38 \\ 995 & 40\end{array}$

GRAPH NAME $=4$ IEV750工

$\begin{array}{rrr}0 & 0.4453 & 0.0 \\ 250 & 0.4453 & 111.3 \\ 500 & 0.4453 & 222.7 \\ 750 & 0.4453 & 334.0 \\ 1000 & 0.4453 & 445.3\end{array}$

GRAPH NAME $=4$ IEV100I

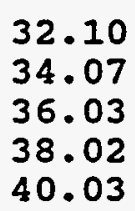

$$
\begin{array}{r}
0 \\
250
\end{array}
$$$$
0.4453
$$$$
0.4453
$$$$
0.4453
$$$$
0.4453
$$$$
0.4453
$$ 


\section{4" ISB, Viton O-Ring}

52.4 Ft-Lbf Clamp, 400 Deg. F.

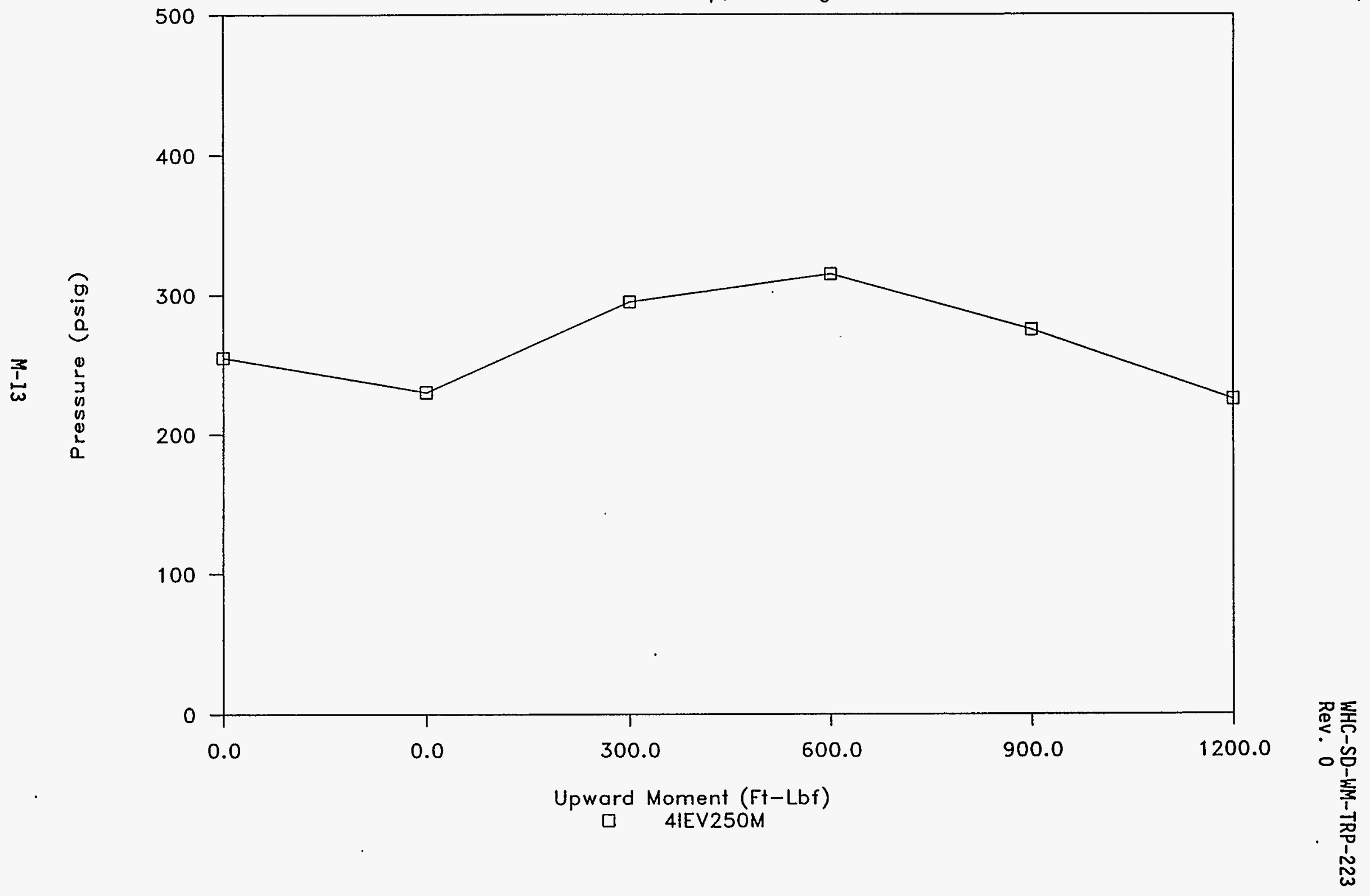


WHC-SD-WM-TRP-223

Rev. 0

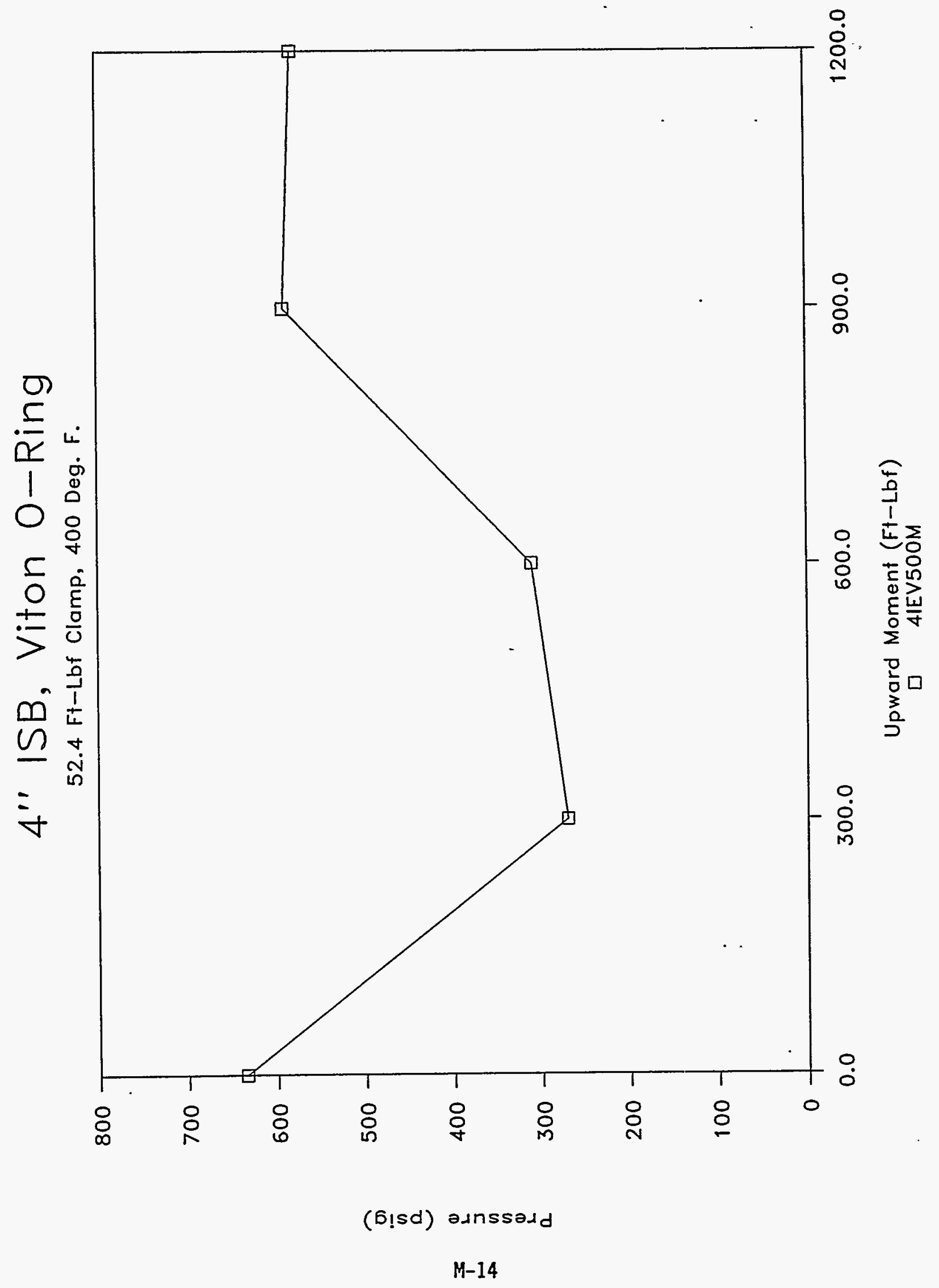


WHC-SD-WM-TRP-223

Rev. 0

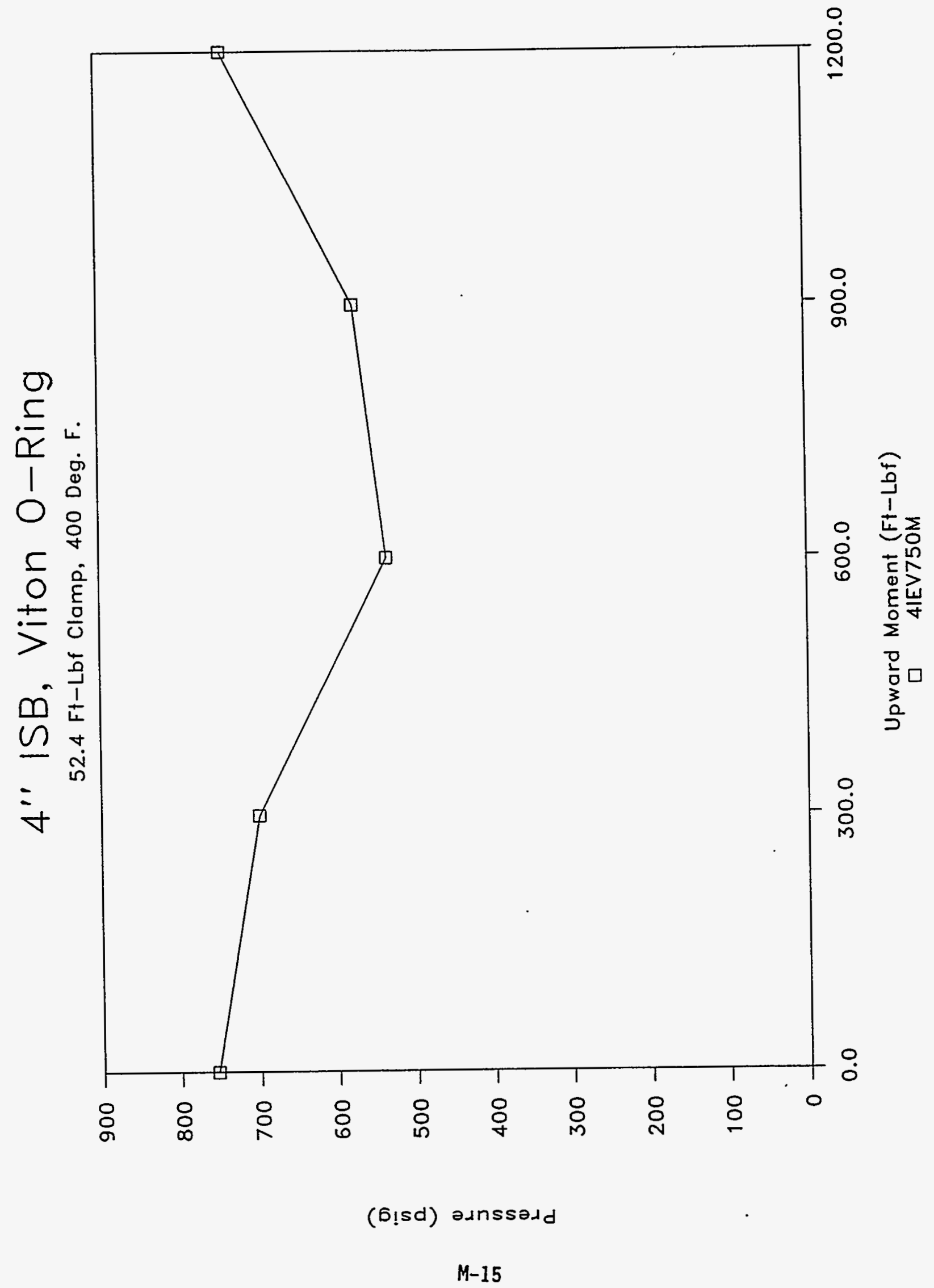


WHC-SD-WM-TRP-223

Rev. 0

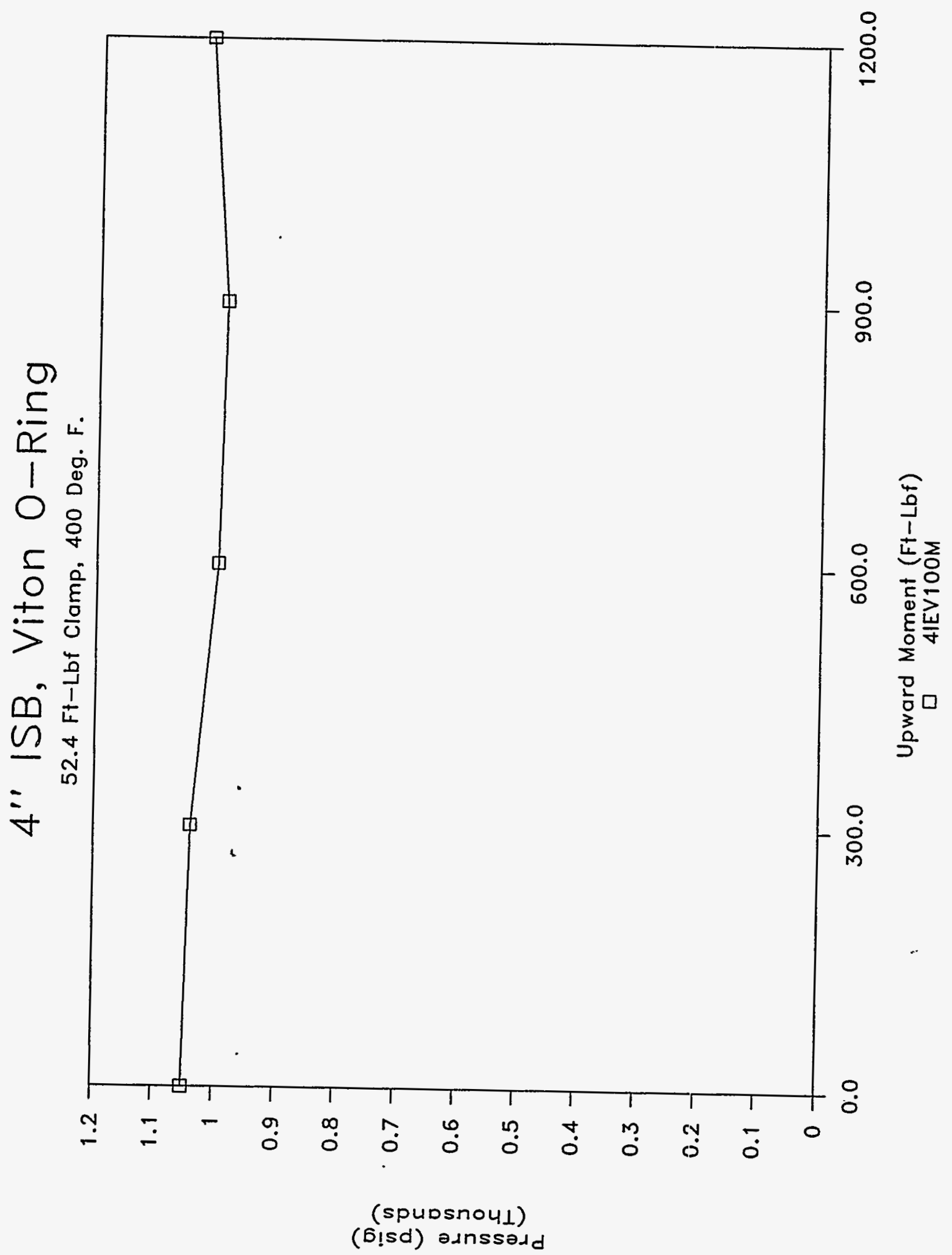

$M-16$ 
WHC-SD-WM-TRP-223 ReV 0

OCTOBER 10,1994

4" ISB CONNECTOR, VITON O-RING, 70 DUROMETER, ELEVATED TEMP. (400 DEG. F) LEAK TEST - UP AND OVER MOMENT (UPWARD)

CLAMPING TORQUE $=52.4 \mathrm{FT}-\mathrm{LBF} \quad$ GRAPH NAME $=4$ IEV250M

CHARGE PRESSURE $=250$ PSIG

$\begin{array}{lllllll} & & & & & \text { UPWARD } \\ \text { PRESSURE } & \text { ELAPSED } & \text { ELAPSED } & \text { ELAPSED } & \text { UPWARD } & \text { MOMENT } & \text { UPWARD } \\ \text { PSIG } & \text { MINUTES } & \text { SECONDS } & \text { DECIMAI } & \text { FORCE } & \text { ARM } & \text { MOMENT } \\ & & & \text { MINUTES } & \text { LBS } & \text { FT. } & \text { FT-LBF. } \\ \text { INPUT } & \text { INPUT } & \text { INPUT } & \text { COMPUTE } & \text { INPUT } & \text { INPUT } & \text { COMPUTE }\end{array}$

$\begin{array}{rrrrrrr}255 & 0 & 0 & 0.00 & 0 & 1.2000 & 0.0 \\ 230 & 2 & 1 & 2.02 & 0 & 1.2000 & 0.0 \\ 295 & 4 & 0 & 4.00 & 250 & 1.2000 & 300.0 \\ 315 & 6 & 3 & 6.05 & 500 & 1.2000 & 600.0 \\ 275 & 8 & 5 & 8.08 & 750 & 1.2000 & 900.0 \\ 225 & 10 & 1 & 10.02 & 1000 & 1.2000 & 1200.0\end{array}$

INCREASED PRESSURE TO 500 PSIG

GRAPH NAME $=4$ IEV50OM

$\begin{array}{rrrrrrr}635 & 12 & 1 & 12.02 & 0 & 1.2000 & 0.0 \\ 270 & 14 & 1 & 14.02 & 250 & 1.2000 & 300.0 \\ 310 & 16 & 5 & 16.08 & 500 & 1.2000 & 600.0 \\ 590 & 18 & 4 & 18.07 & 750 & 1.2000 & 900.0 \\ 580 & 20 & 2 & 20.03 & 1000 & 1.2000 & 1200.0\end{array}$

RELEASED MOMENT LOAD AND ALLOWED TEST FIXTURE TO SET FOR AN EXTENDED PERIOD OF TIME.
550
59
$12 \quad 59.20$
$0 \quad 1.2000$
0.0

INCREASED PRESSURE TO 750 PSIG

$\begin{array}{llll}755 & 61 & 2 & 61.03 \\ 700 & 63 & 5 & 63.08 \\ 535 & 65 & 2 & 65.03 \\ 575 & 67 & 3 & 67.05 \\ 740 & 69 & 2 & 69.03\end{array}$

INCREASED PRESSURE TO 1000 PSIG

$\begin{array}{rrrrrrr}1050 & 71 & 3 & 71.05 & 0 & 1.2000 & 0.0 \\ 1040 & 73 & 15 & 73.25 & 250 & 1.2000 & 300.0 \\ 1000 & 75 & 18 & 75.30 & 500 & 1.2000 & 600.0 \\ 990 & 77 & 10 & 77.17 & 750 & 1.2000 & 900.0 \\ 1020 & 79 & 3 & 79.05 & 1000 & 1.2000 & 1200.0\end{array}$

GRAPH NAME $=4$ IEV750M

$\begin{array}{rrr}0 & 1.2000 & 0.0 \\ 250 & 1.2000 & 300.0 \\ 500 & 1.2000 & 600.0 \\ 750 & 1.2000 & 900.0 \\ 1000 & 1.2000 & 1200.0\end{array}$


4" ISB, Viton O-Ring Upward Deflection

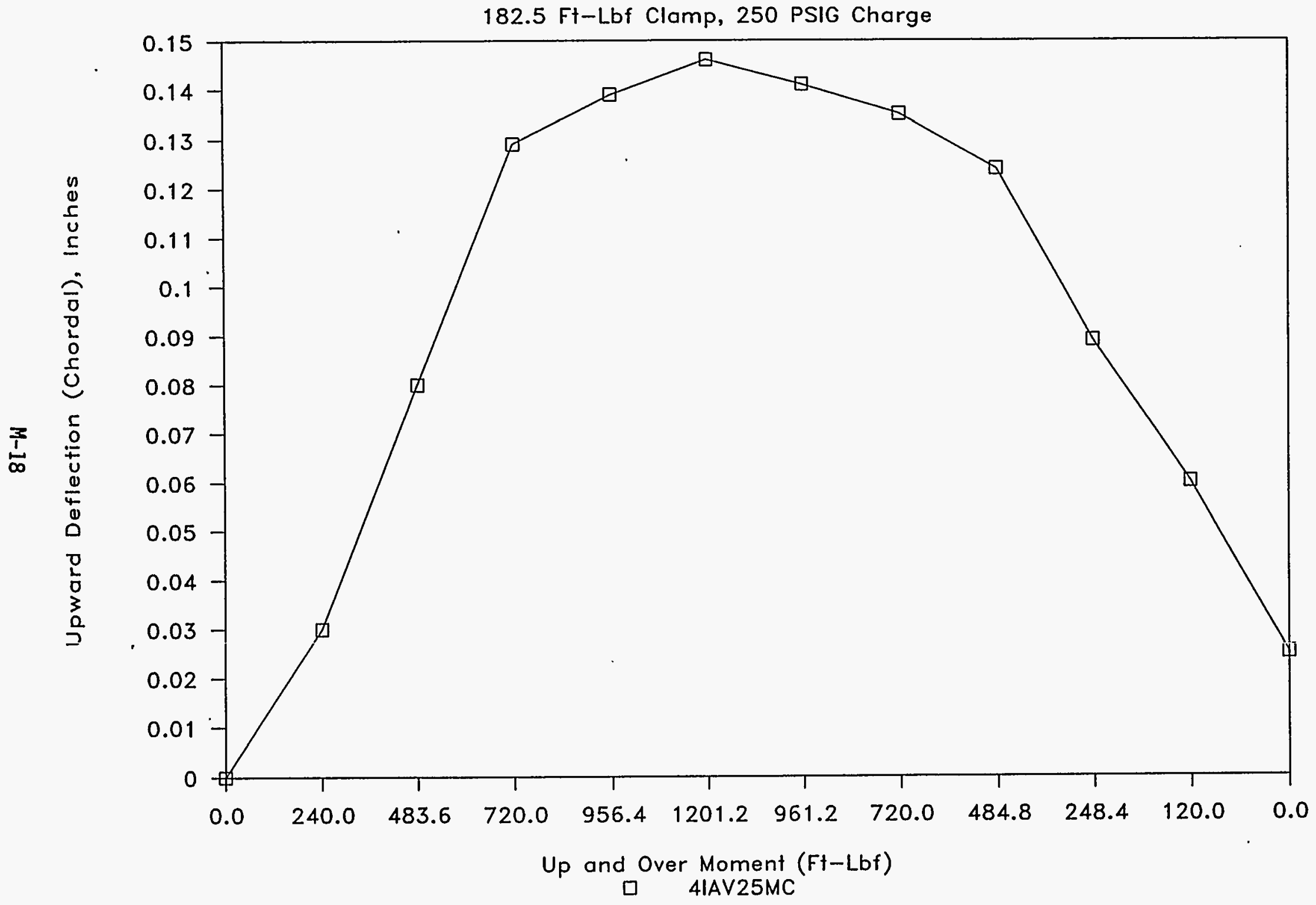




$$
\text { WHC-SD-WM-TRP-223 ReV } 0
$$
INCREASED TORQUE TO 182.5 FT-LBF. PRESSURE INCREASED TO 335 PSIG
AS TORQUE LOAD INCREASED.

\begin{tabular}{|c|c|c|c|c|c|c|c|}
\hline $\begin{array}{l}\text { PRESSURE } \\
\text { PSIG }\end{array}$ & $\begin{array}{l}\text { ELAPSED } \\
\text { MINUTES }\end{array}$ & $\begin{array}{l}\text { ELAPSED } \\
\text { SECONDS }\end{array}$ & $\begin{array}{l}\text { ELAPSED } \\
\text { DECIMAI } \\
\text { MINUTES } \\
\text { COMPUTE }\end{array}$ & $\begin{array}{l}\text { UPWARD } \\
\text { FORCE } \\
\text { LBS } \\
\text { INPUT }\end{array}$ & $\begin{array}{l}\text { UPWARD } \\
\text { MOMENT } \\
\text { ARM } \\
\text { FT. } \\
\text { INPUT }\end{array}$ & $\begin{array}{l}\text { UPWARD } \\
\text { MOMENT } \\
\text { FT-LBF. } \\
\text { COMPUTE }\end{array}$ & $\begin{array}{l}\text { UPWARD } \\
\text { DEFLECTIO } \\
\text { (CHORDAI) } \\
\text { INCHES } \\
\text { INPUT }\end{array}$ \\
\hline $\begin{array}{l}335 \\
335 \\
335 \\
335 \\
335 \\
337 \\
340 \\
340 \\
339 \\
335 \\
340 \\
340\end{array}$ & $\begin{array}{r}0 \\
1 \\
2 \\
3 \\
4 \\
5 \\
6 \\
7 \\
8 \\
9 \\
12 \\
12\end{array}$ & $\begin{array}{r}0 \\
10 \\
10 \\
15 \\
15 \\
15 \\
14 \\
0 \\
0 \\
5 \\
10 \\
50\end{array}$ & $\begin{array}{r}0.00 \\
1.17 \\
2.17 \\
3.25 \\
4.25 \\
5.25 \\
6.23 \\
7.00 \\
8.00 \\
9.08 \\
12.17 \\
12.83\end{array}$ & $\begin{array}{r}0 \\
200 \\
403 \\
600 \\
797 \\
1001 \\
801 \\
600 \\
404 \\
207 \\
100 \\
0\end{array}$ & $\begin{array}{l}1.2000 \\
1.2000 \\
1.2000 \\
1.2000 \\
1.2000 \\
1.2000 \\
1.2000 \\
1.2000 \\
1.2000 \\
1.2000 \\
1.2000 \\
1.2000\end{array}$ & $\begin{array}{r}0.0 \\
240.0 \\
483.6 \\
720.0 \\
956.4 \\
1201.2 \\
961.2 \\
720.0 \\
484.8 \\
248.4 \\
120.0 \\
0.0\end{array}$ & $\begin{array}{l}0.000 \\
0.030 \\
0.080 \\
0.129 \\
0.139 \\
0.146 \\
0.141 \\
0.135 \\
0.124 \\
0.089 \\
0.060 \\
0.025\end{array}$ \\
\hline
\end{tabular}




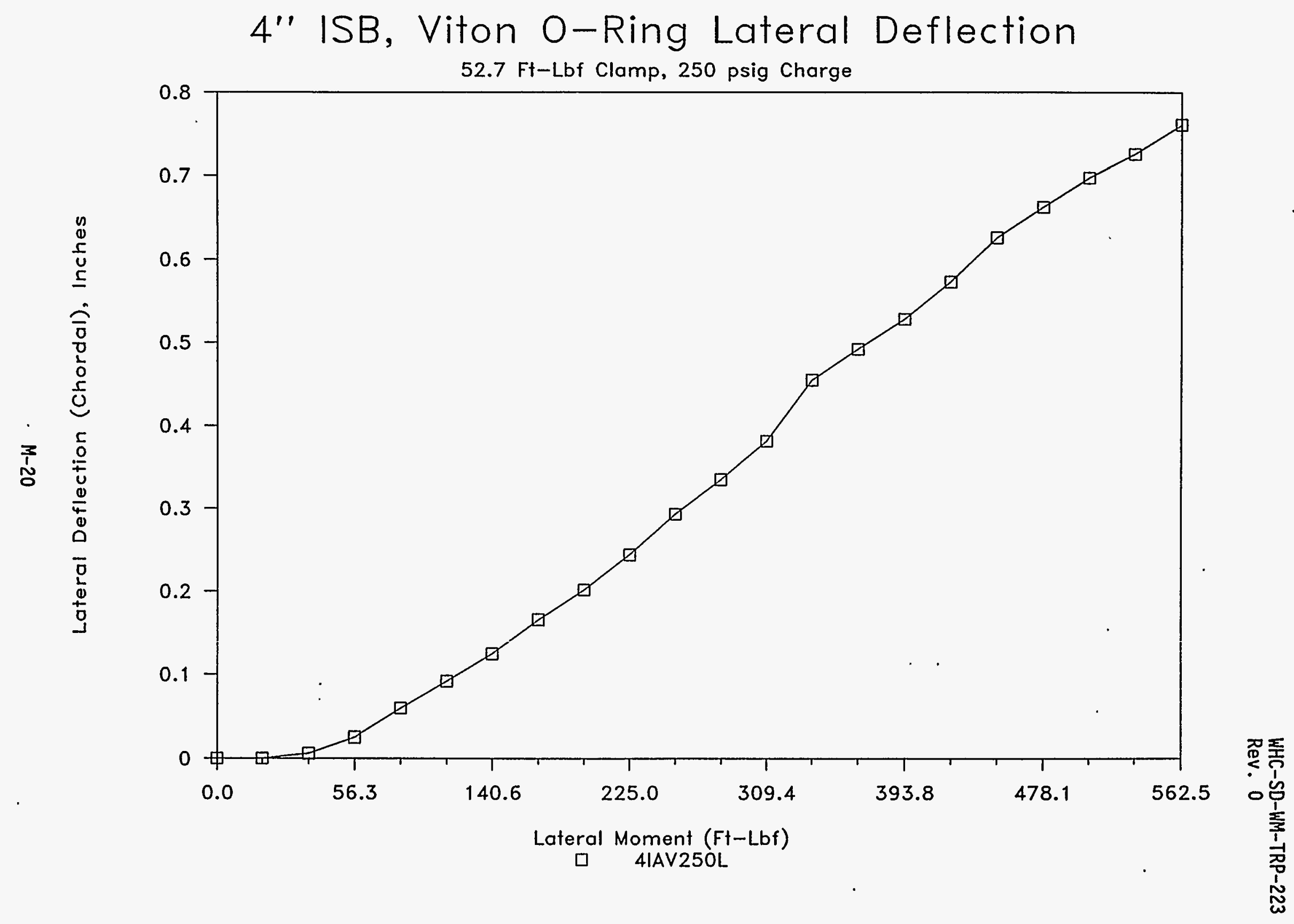


JUNE 08, 1994

4" ISB CONNECTOR, VITON O-RING, 70 DUROMETER, AMBIENT TEMP.

LEAK TEST - UP AND OVER MOMENT APPLIED (UPWARD ROTATION)

CLAMPING TORQUE $=53.6$ FT-LBF GRAPH NAME $=4$ IAV252M

CHARGE PRESSURE $=250$ PSIG

$\begin{array}{llllllll} & & & & \text { UPWARD } & & \text { UPWARD } \\ \text { PRESSURE } & \text { ELAPSED } & \text { ELAPSED } & \text { ELAPSED } & \text { UPWARD } & \text { MOMENT } & \text { UPWARD } & \text { DEFLECTIO } \\ \text { PSIG } & \text { MINUTES } & \text { SECONDS } & \text { DECIMAL } & \text { FORCE } & \text { ARM } & \text { MOMENT } & \text { (CHORDAL) } \\ & & & \text { MINUTES } & \text { IBS } & \text { FT. } & \text { FT-LBF. } & \text { INCHES } \\ \text { INPUT } & \text { INPUT } & \text { INPUT } & \text { COMPUTE } & \text { INPUT } & \text { INPUT } & \text { COMPUTE } & \text { INPUT }\end{array}$

$\begin{array}{rrrrrrrr}250 & 0 & 0 & 0.00 & 0 & 1.2000 & 0.0 & 0.000 \\ 235 & 2 & 0 & 2.00 & 199 & 1.2000 & 238.8 & 0.030 \\ 235 & 4 & 0 & 4.00 & 398 & 1.2000 & 477.6 & 0.080 \\ 260 & 5 & 45 & 5.75 & 606 & 1.2000 & 727.2 & 0.129 \\ 241 & 7 & 7 & 7.12 & 810 & 1.2000 & 972.0 & 0.139 \\ 223 & 8 & 22 & 8.37 & 1002 & 1.2000 & 1202.4 & 0.146 \\ 230 & 10 & 35 & 10.58 & 805 & 1.2000 & 966.0 & 0.141 \\ 245 & 11 & 33 & 11.55 & 602 & 1.2000 & 722.4 & 0.135 \\ 255 & 12 & 28 & 12.47 & 403 & 1.2000 & 483.6 & 0.124 \\ 240 & 13 & 33 & 13.55 & 204 & 1.2000 & 244.8 & 0.099 \\ 220 & 14 & 39 & 14.65 & 0 & 1.2000 & 0.0 & 0.060\end{array}$




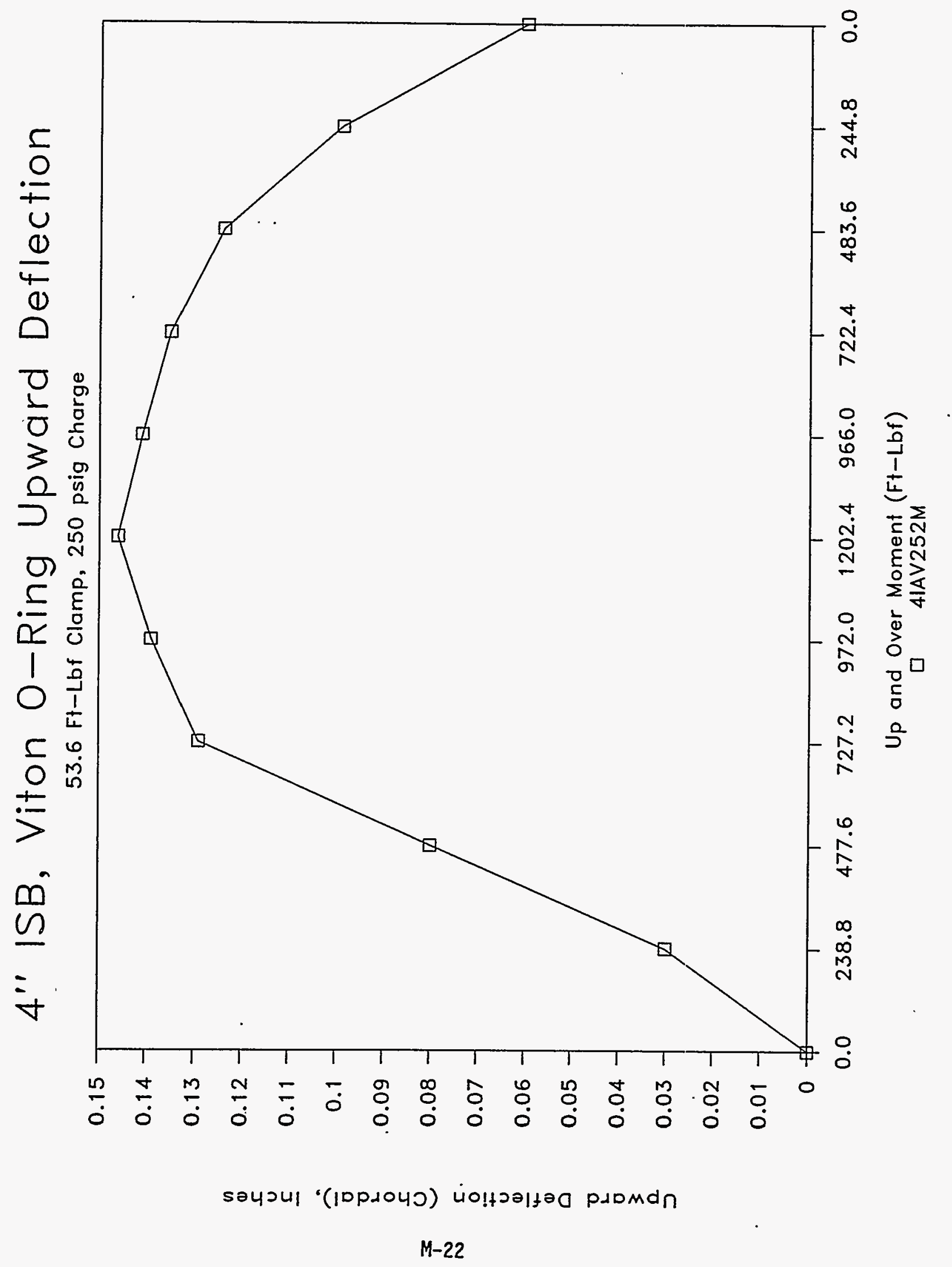


WHC-SD-WM-TRP-223

Rev. 0

APPENDIX N: GRAPHS OF 4-IN. TFE-O-SIL TESTS

$\mathrm{N}-1$ 


\section{4" ISB "TFE-O-SIL" O-Ring \\ 100.0 Ft-Lbf Clamp, (Retest)}

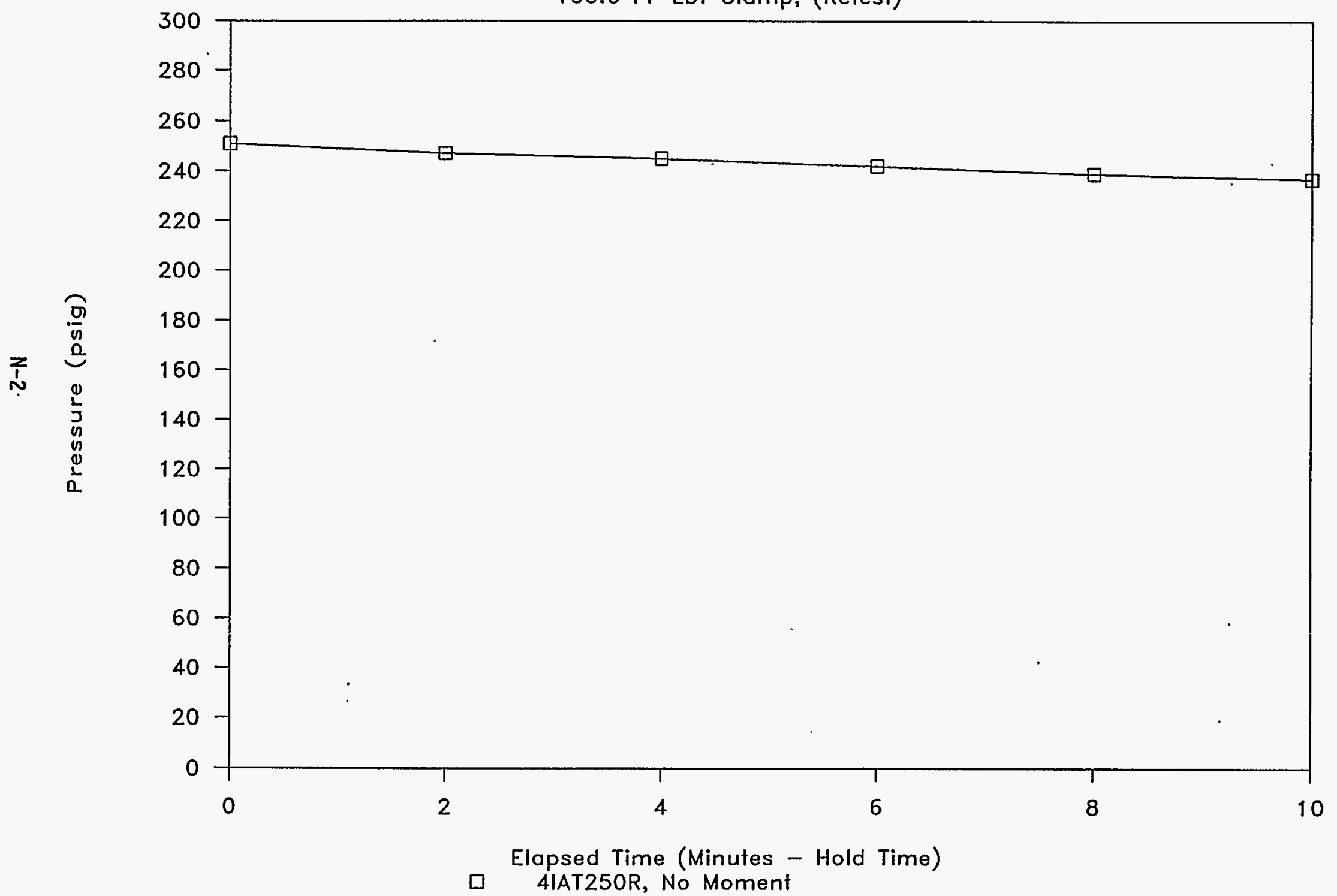


WHC-SD-WM-TRP-223 ReV 0

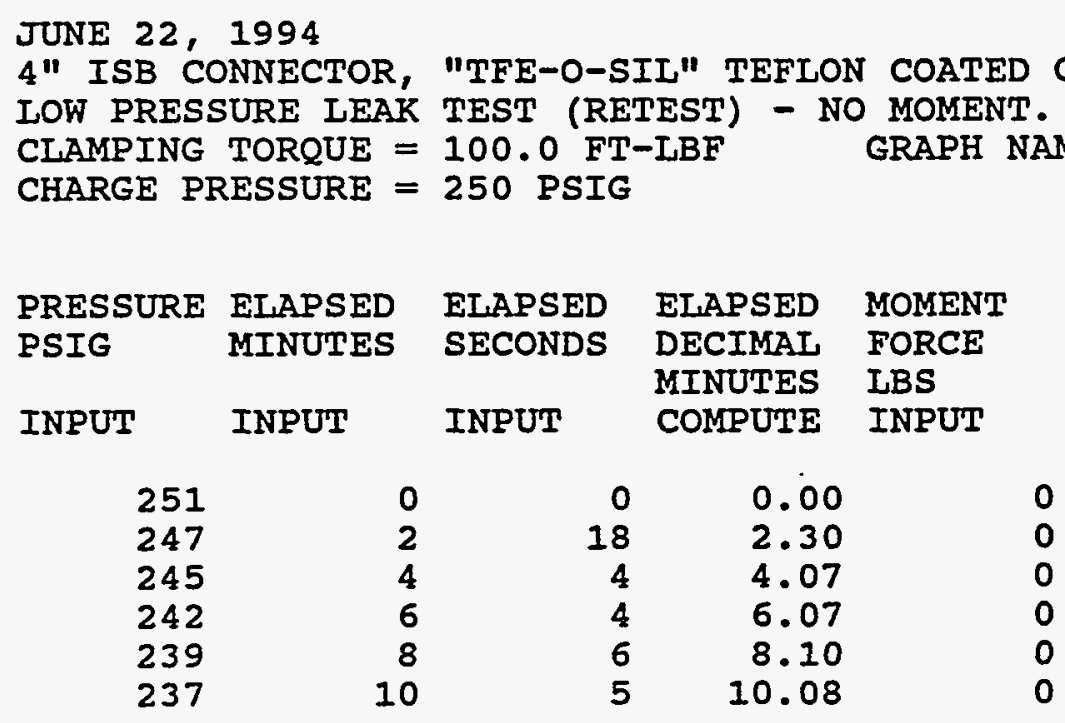


WHC-SD-WM-TRP-223

Rev. 0

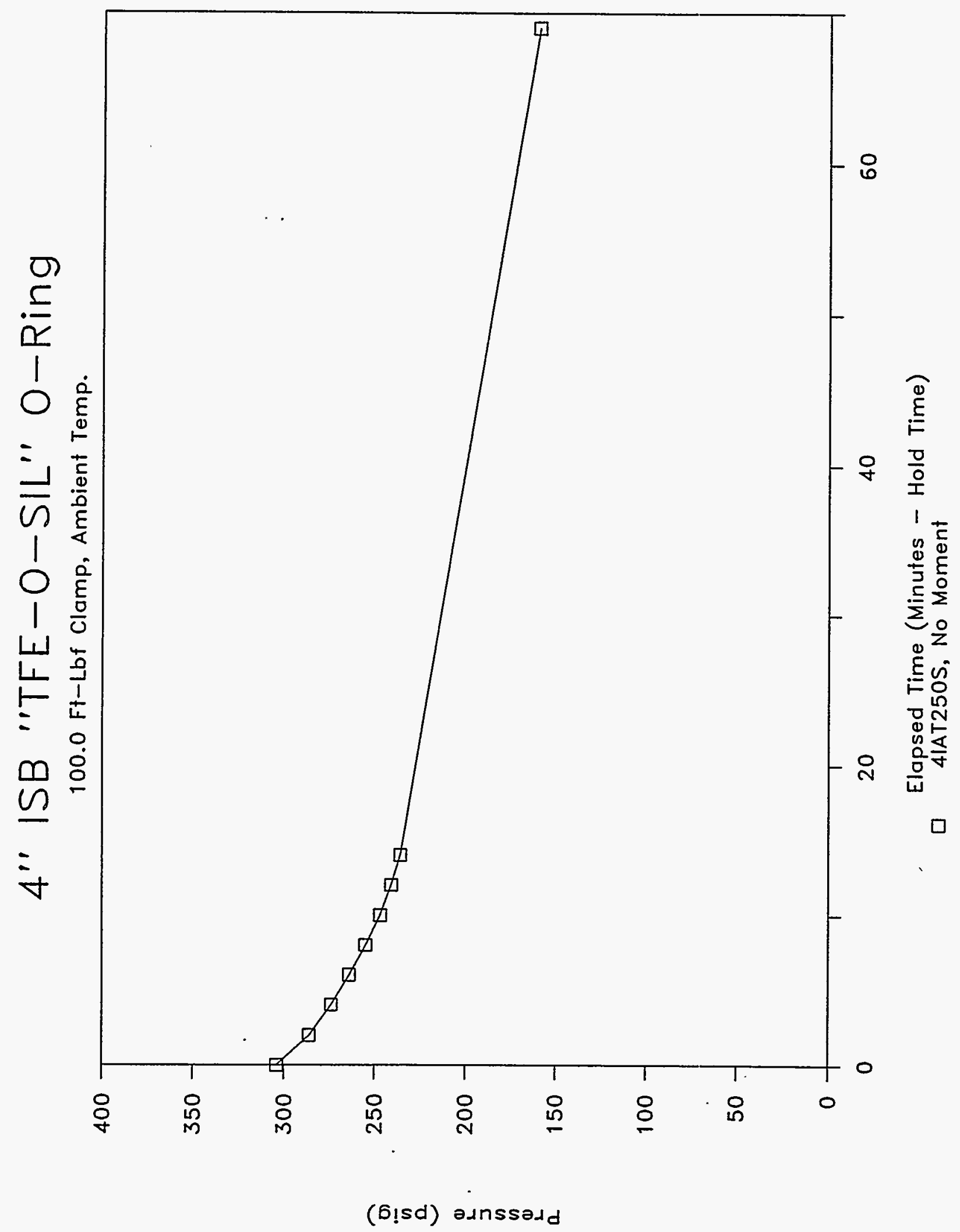

$N-4$ 
4" ISB "TFE-O-SIL" O-Ring

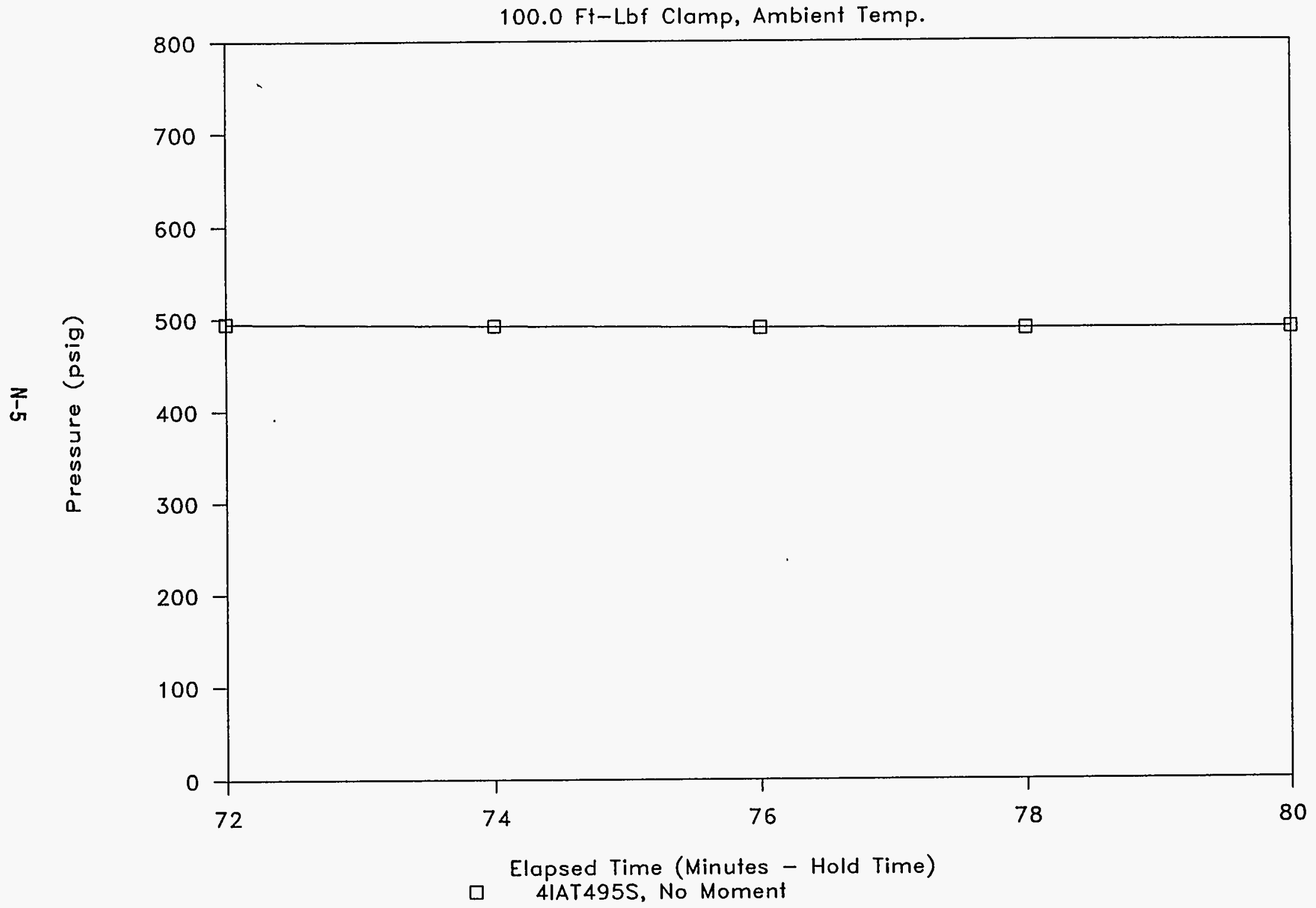




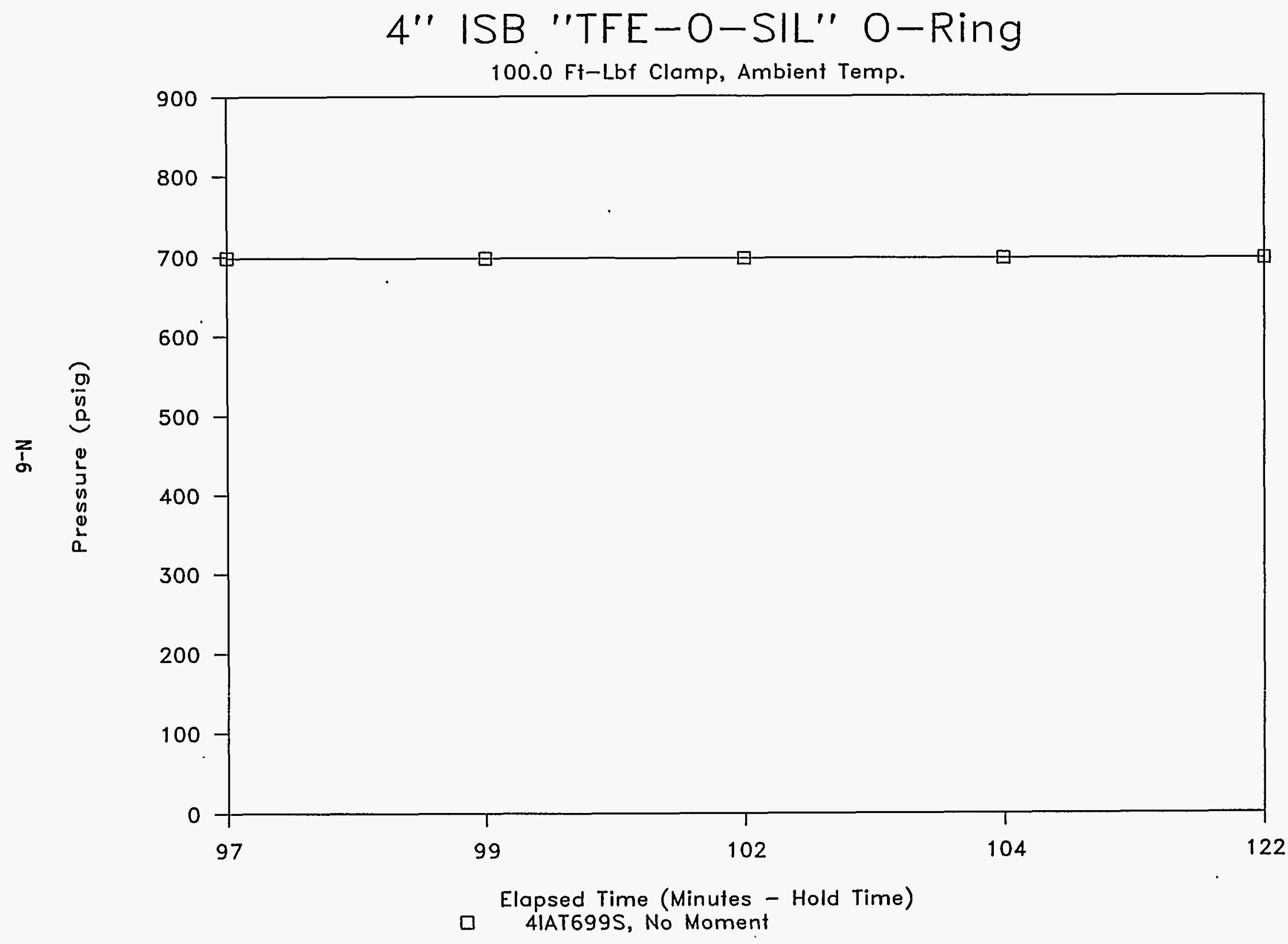


4" ISB "TFE-O-SIL" O-Ring.

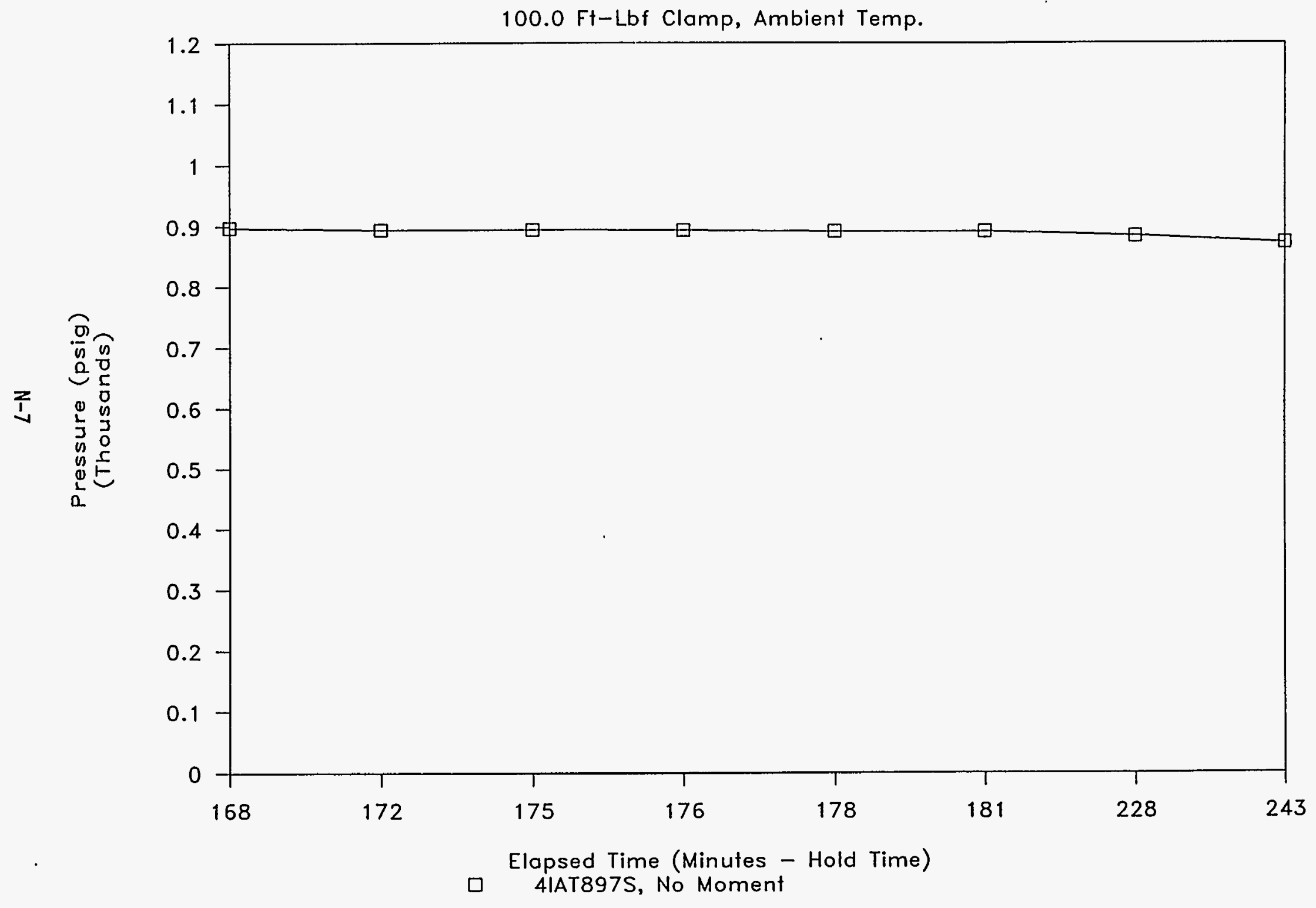


JUNE 21, 1994

4" ISB CONNECTOR, TEFLON COATED SILICONE "TFE-O-SIL" O-RING.

AMBIENT TEMP. / PRESSURE ASCENSION LEAK TEST - NO MOMENT

CLAMPING TORQUE $=100.0 \mathrm{FT}-\mathrm{LBF}$ GRAPH NAME $=4$ IAT25OS

CHARGE PRESSURE $=250$ PSIG

$\begin{array}{lllll}\text { PRESSURE } & \text { ELAPSED } & \text { ELAPSED } & \text { ELAPSED } & \text { MOMENT } \\ \text { PSIG } & \text { MINUTES } & \text { SECONDS } & \text { DECIMAL } & \text { FORCE } \\ & & & \text { MINUTES } & \text { LBS } \\ \text { INPUT } & \text { INPUT } & \text { INPUT } & \text { COMPUTE } & \text { INPUT }\end{array}$

$\begin{array}{rrrrr}304 & 0 & 0 & 0.00 & 0 \\ 286 & 2 & 7 & 2.12 & 0 \\ 274 & 4 & 10 & 4.17 & 0 \\ 264 & 6 & 7 & 6.12 & 0 \\ 255 & 8 & 4 & 8.07 & 0 \\ 247 & 10 & 21 & 10.35 & 0 \\ 241 & 12 & 23 & 12.38 & 0 \\ 236 & 14 & 25 & 14.42 & 0 \\ 160 & 69 & 48 & 69.80 & 0\end{array}$

INCREASED CHARGE PRESSURE TO 495 PSIG.

GRAPH NAME $=4$ IAT495S

$\begin{array}{llrll}495 & 72 & 42 & 72.70 & 0 \\ 492 & 74 & 21 & 74.35 & 0 \\ 490 & 76 & 0 & 76.00 & 0 \\ 489 & 78 & 51 & 78.85 & 0 \\ 489 & 80 & 53 & 80.88 & 0\end{array}$

INCREASED CHARGE PRESSURE TO 699 PSIG. GRAPH NAME = 4IAT699S

$\begin{array}{rrrrr}699 & 97 & 30 & 97.50 & 0 \\ 698 & 99 & 31 & 99.52 & 0 \\ 697 & 102 & 33 & 102.55 & 0 \\ 697 & 104 & 45 & 104.75 & 0 \\ 697 & 122 & 46 & 122.77 & 0\end{array}$

INCREASED CHARGE PRESSURE TO 897 PSIG.

GRAPEI NAME = 4IAT897S

$\begin{array}{llrll}897 & 168 & 50 & 168.83 & 0 \\ 894 & 172 & 1 & 172.02 & 0 \\ 894 & 175 & 29 & 175.48 & 0 \\ 893 & 176 & 52 & 176.87 & 0 \\ 891 & 178 & 59 & 178.98 & 0 \\ 891 & 181 & 7 & 181.12 & 0 \\ 884 & 228 & 43 & 228.72 & 0 \\ 873 & 243 & 50 & 243.83 & 0\end{array}$


4" ISB "TFE-O-SIL" O-Ring

100.0 Ft-Lbf Clamp, 400 Deg. F Temp.

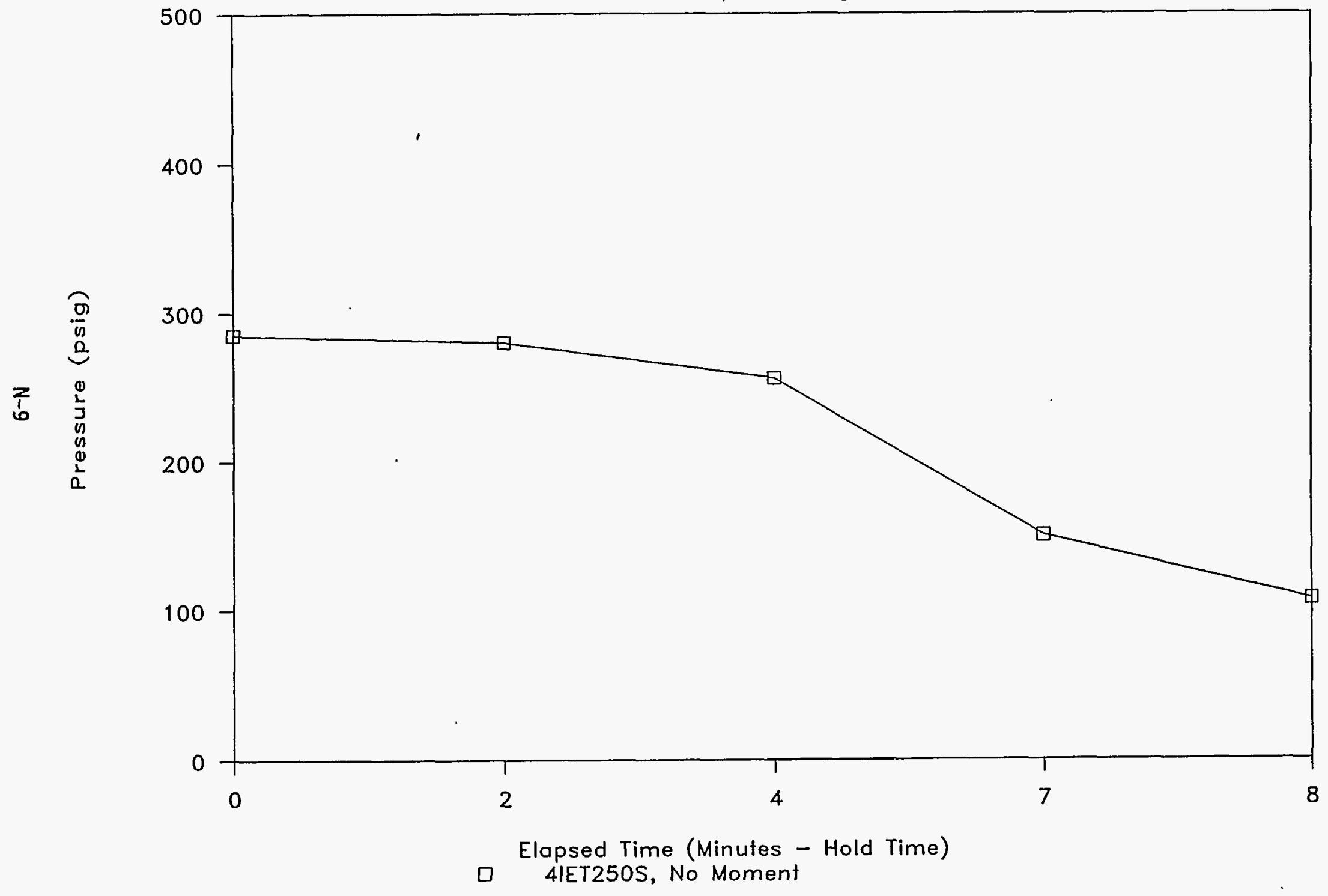




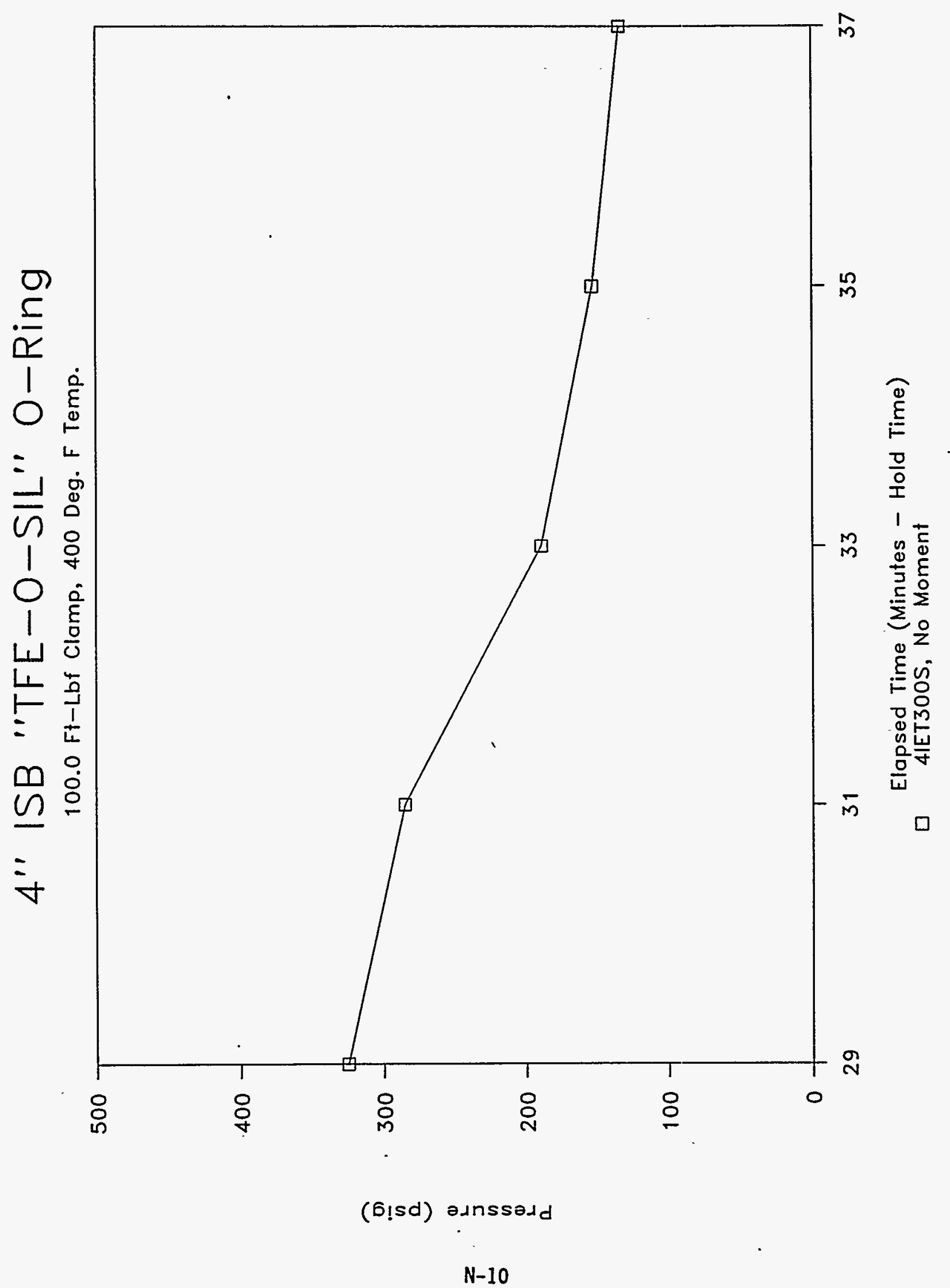


WHC-SD-WM-TRP-223 ReV 0

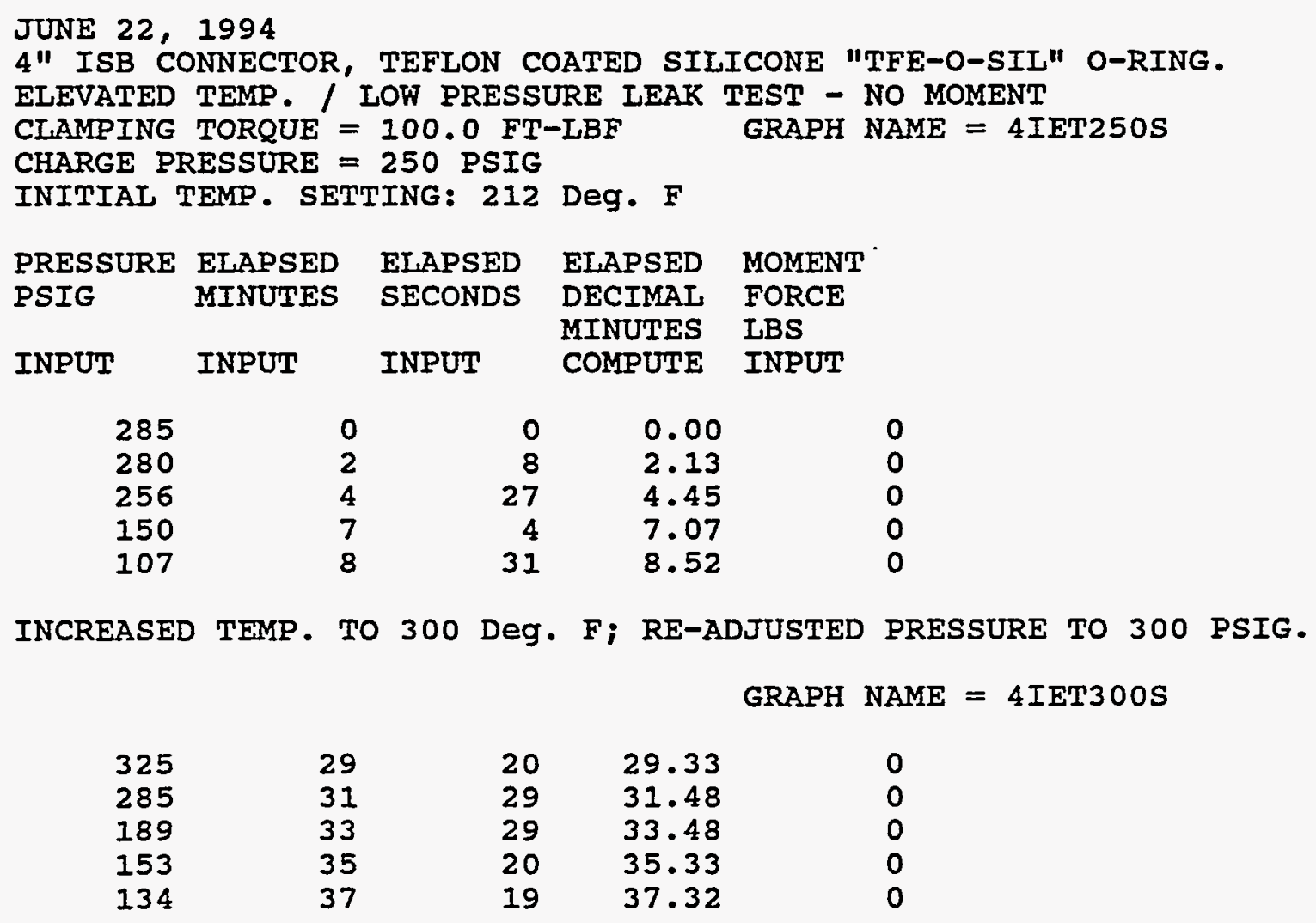


WHC-SD-WM-TRP-223

Rev. 0

APPENDIX O: GRAPHS OF THREE-WAY FLUOROSILICONE TESTS

$0-1$ 


\section{3-Way ISB, Fluorosilicone 70 SH O-Ring}

$50 \mathrm{Ft}$-Lbf Clamp, 68 Deg. F.

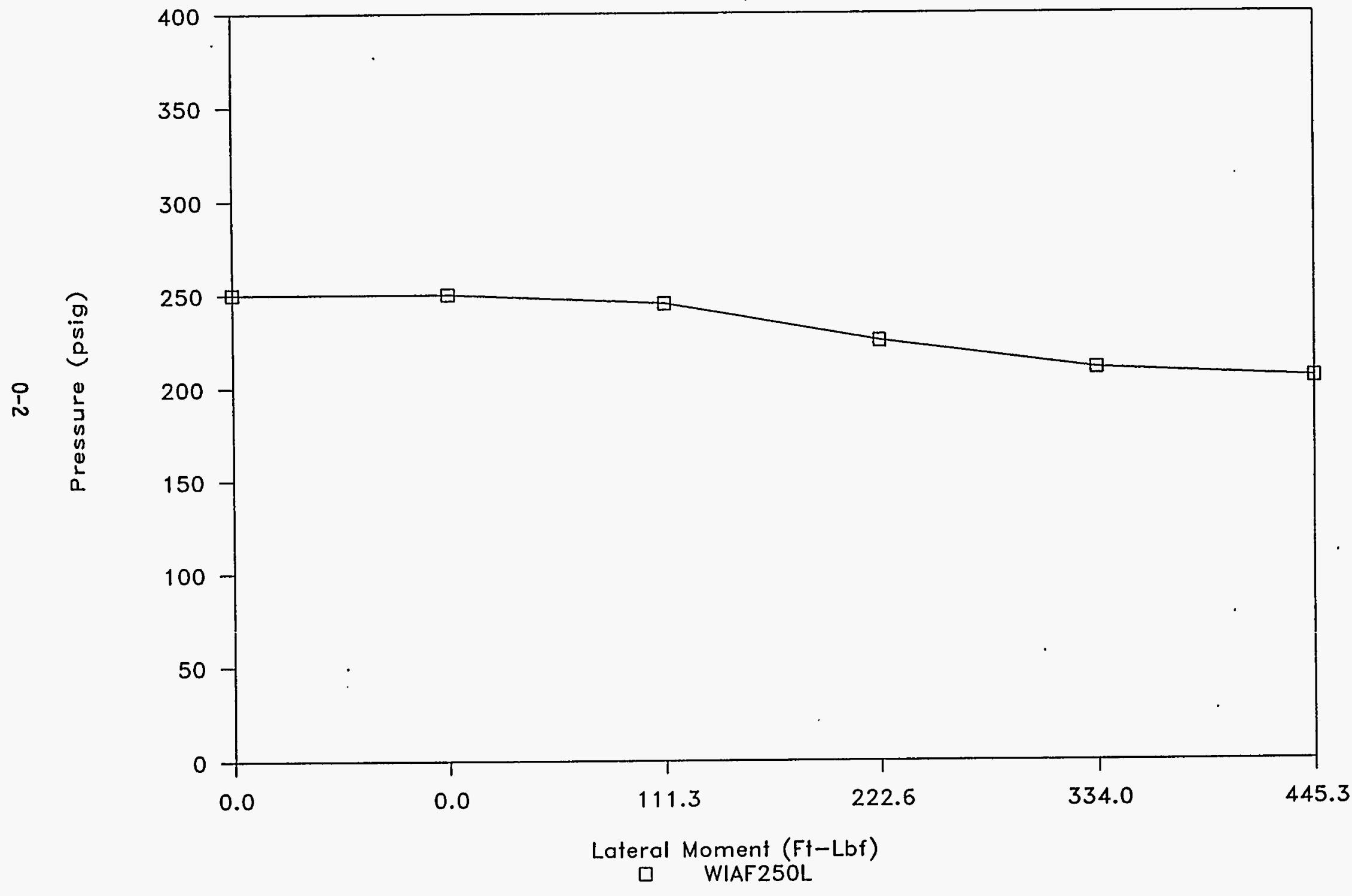


WHC-SD-WM-TRP-223

Rev. 0

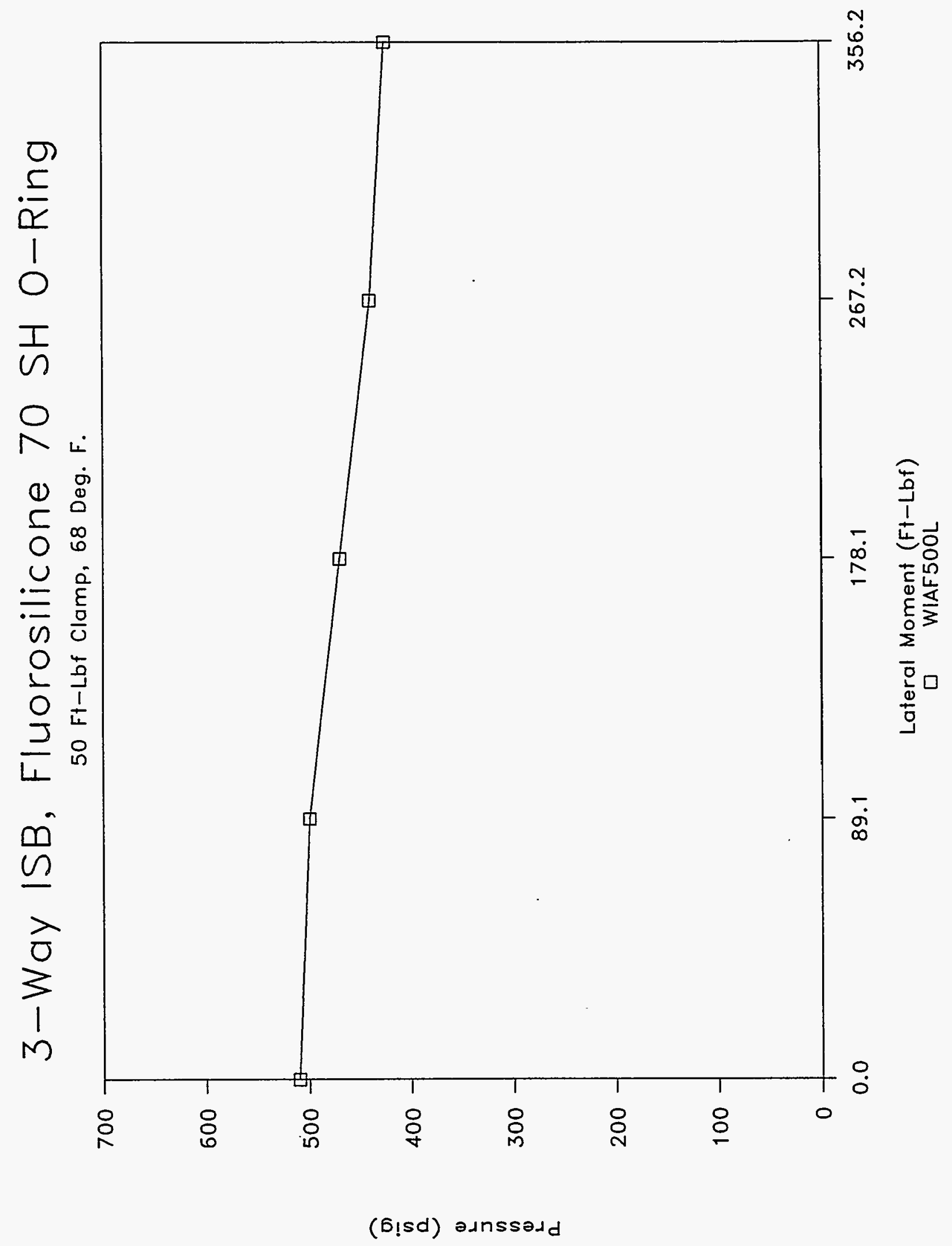

0-3 


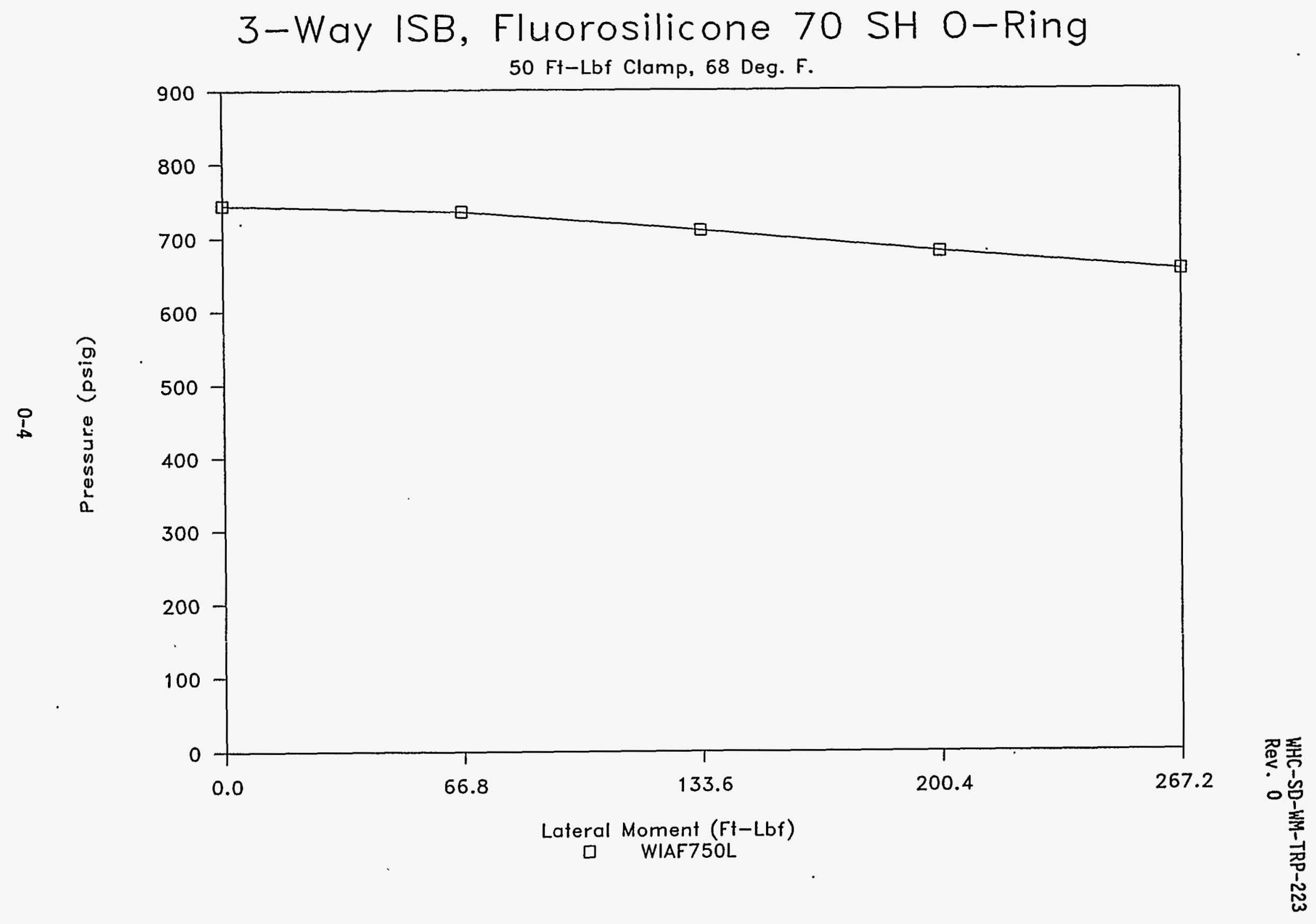




\section{3-Way ISB, Fluorosilicone 70 SH O-Ring}

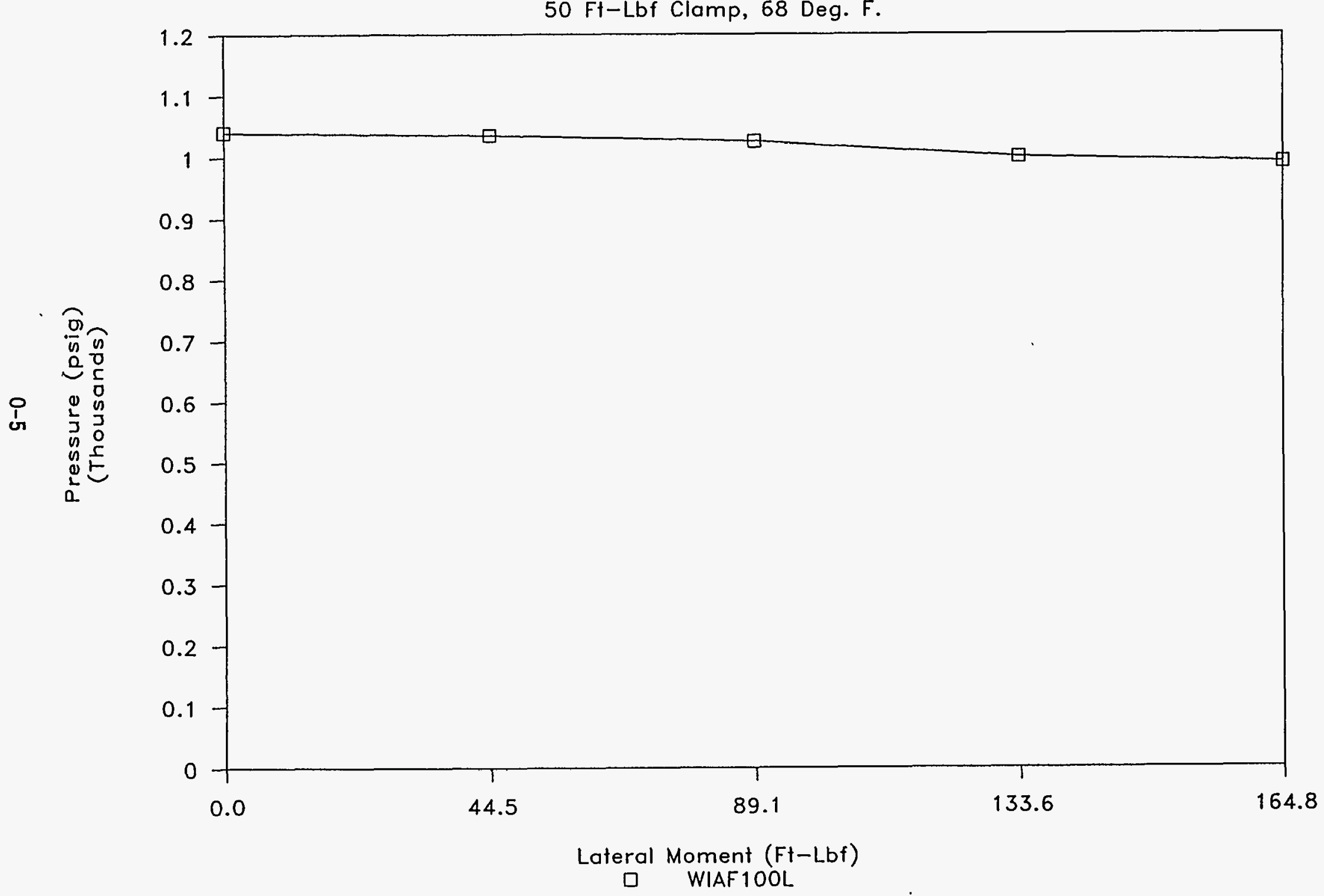


DECEMBER 09, 1994

2" 3-WAY ISB CONNECTOR, FLUOROSILICONE O-RING, 70 DUROMETER, AMBIENT TEM PART \# 2-119 L1120-70, CONQUEST SEAL CO., BATCH 70011, CURE DATE 4989 LEAK TEST - LATERAL MOMENT (SIDEWAYS ROTATION)

CLAMPING TORQUE $=50$ FT-LBF NOMINAI CHARGE PRESSURE $=250$ PSIG

GRAPH NAME = WIAF250L

TEMPERATURE: 6i3 DEG. F.

\begin{tabular}{|c|c|c|c|c|c|c|}
\hline $\begin{array}{l}\text { PRESSURE } \\
\text { PSIG }\end{array}$ & $\begin{array}{l}\text { ELAPSED } \\
\text { MINUTES }\end{array}$ & $\begin{array}{l}\text { ELAPSED } \\
\text { SECONDS }\end{array}$ & $\begin{array}{l}\text { ELAPSED } \\
\text { DECIMAI } \\
\text { MINUTES } \\
\text { COMPUTE }\end{array}$ & $\begin{array}{l}\text { IATERAL } \\
\text { FORCE } \\
\text { IBS } \\
\text { INPUT }\end{array}$ & $\begin{array}{l}\text { LATERAL } \\
\text { MOMEN'I } \\
\text { ARM } \\
\text { FT. } \\
\text { INPUT }\end{array}$ & $\begin{array}{l}\text { LATERAL } \\
\text { MOMENT } \\
\text { FT-IBF. } \\
\text { COMPUTE }\end{array}$ \\
\hline $\begin{array}{l}250 \\
250 \\
245 \\
225 \\
210 \\
205\end{array}$ & $\begin{array}{r}0 \\
2 \\
4 \\
6 \\
8 \\
10\end{array}$ & & $\begin{array}{r}0.00 \\
2.00 \\
4.00 \\
6.00 \\
8.00 \\
10.00\end{array}$ & $\begin{array}{r}0 \\
0 \\
250 \\
500 \\
750 \\
1000\end{array}$ & $\begin{array}{l}0.4453 \\
0.4453 \\
0.4453 \\
0.4453 \\
0.4453 \\
0.4453\end{array}$ & $\begin{array}{r}0.0 \\
0.0 \\
111.3 \\
222.7 \\
334.0 \\
445.3\end{array}$ \\
\hline
\end{tabular}

INCREASED PRESSURE TO 500 PSIG

$\begin{array}{ll}510 & 12 \\ 500 & 14 \\ 470 & 16 \\ 440 & 18 \\ 425 & 20\end{array}$

INCPREASED PRESSURE TO 750 PSIG

745
735
710
680
655

$\begin{array}{ll}0 & 12.00 \\ 0 & 14.00 \\ 0 & 16.00 \\ 0 & 18.00 \\ 0 & 20.00\end{array}$

GRAPH NAME $=$ WIAF50OL

$\begin{array}{rrr}0 & 0.4453 & 0.0 \\ 200 & 0.4453 & 89.1 \\ 400 & 0.4453 & 178.1 \\ 600 & 0.4453 & 267.2 \\ 800 & 0.4453 & 356.2\end{array}$

GRAPH NAME = WIAF750L

$\begin{array}{rrr}0 & 0.4453 & 0.0 \\ 150 & 0.4453 & 66.8 \\ 300 & 0.4453 & 133.6 \\ 450 & 0.4453 & 200.4 \\ 600 & 0.4453 & 267.2\end{array}$

GRAPH NAME = WIAF10OL

INCREASED PRESSURE TO 1000 PSIG

$\begin{array}{rl}1040 & 32 \\ 1035 & 34 \\ 1025 & 36 \\ 1000 & 38 \\ 990 & 40\end{array}$

22.00

24.00

26.00

28.00

30.00

0.4453

267.2

32.00
34.00
36.00
38.00
40.00

0
100
200
300
370

0.4453

0.4453

0.4453

0.4453

0.4453
0.0
44.5
89.1
33.6
64.8 
WHC-SD-WM-TRP-223

Rev. 0

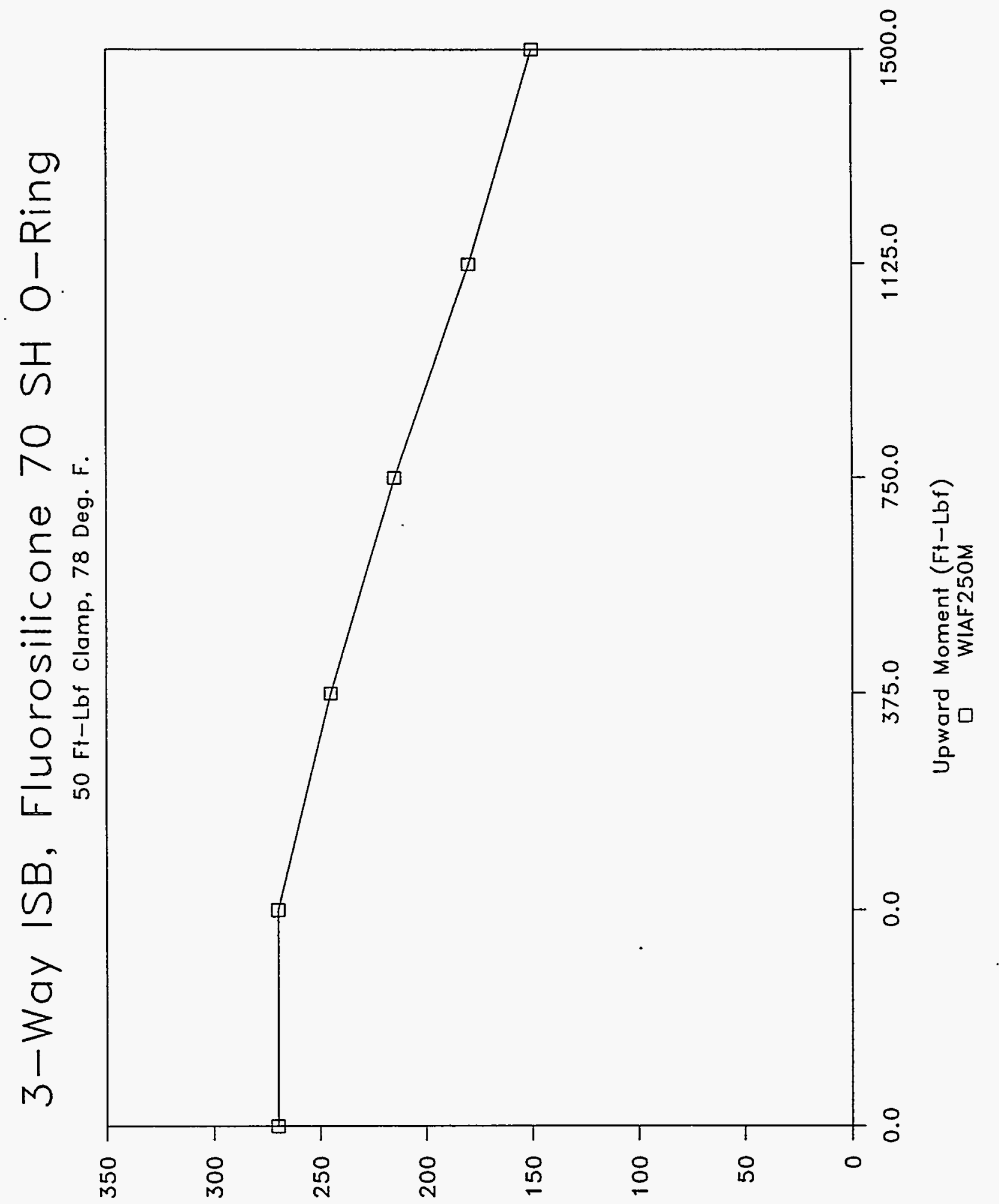

(Gisd) annssadd 


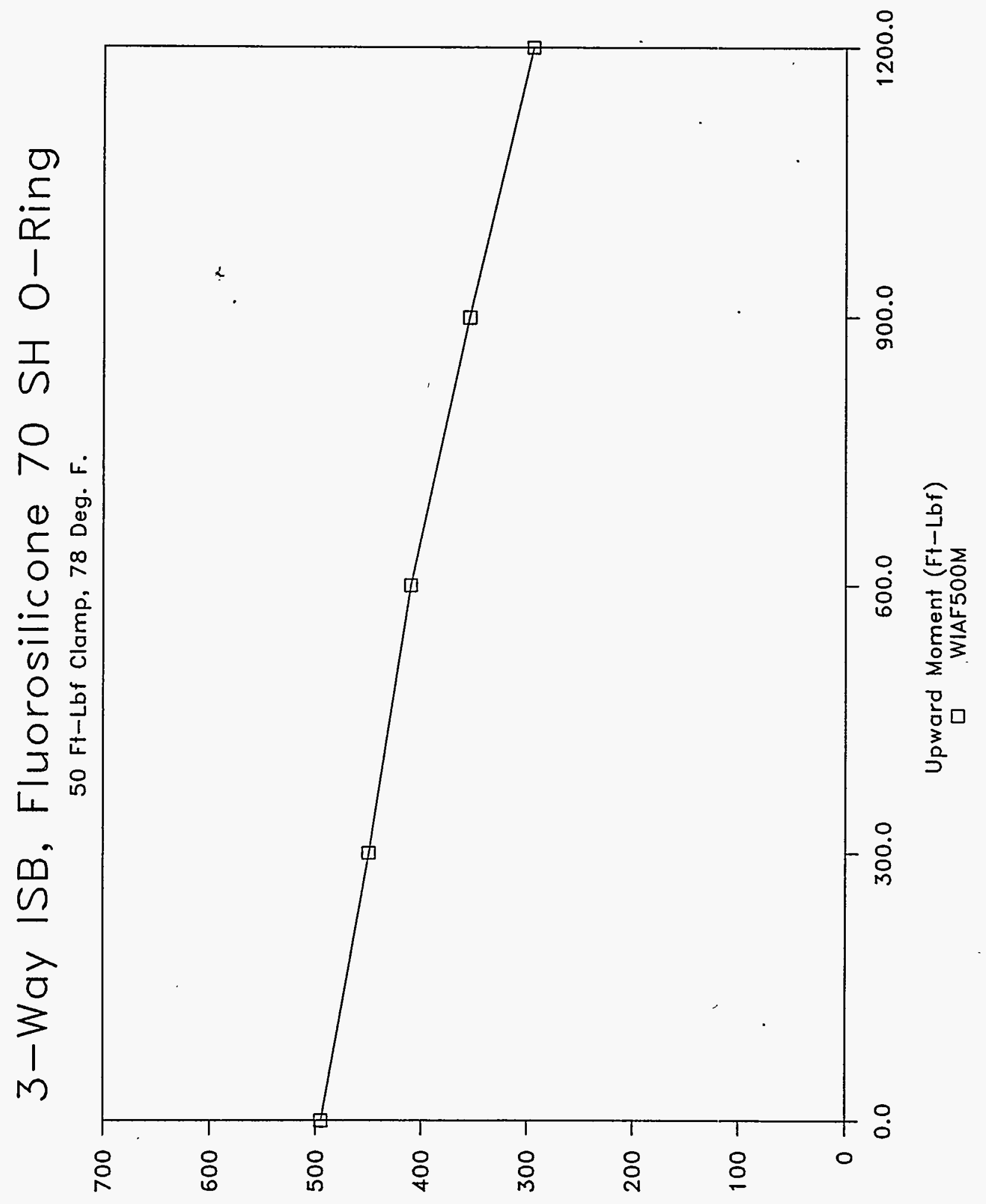

(6!sd) adnssadd 
3-Way ISB, Fluorosilicone 70 SH O-Ring

50 Ft-Lbf Clamp, 78 Deg. F.

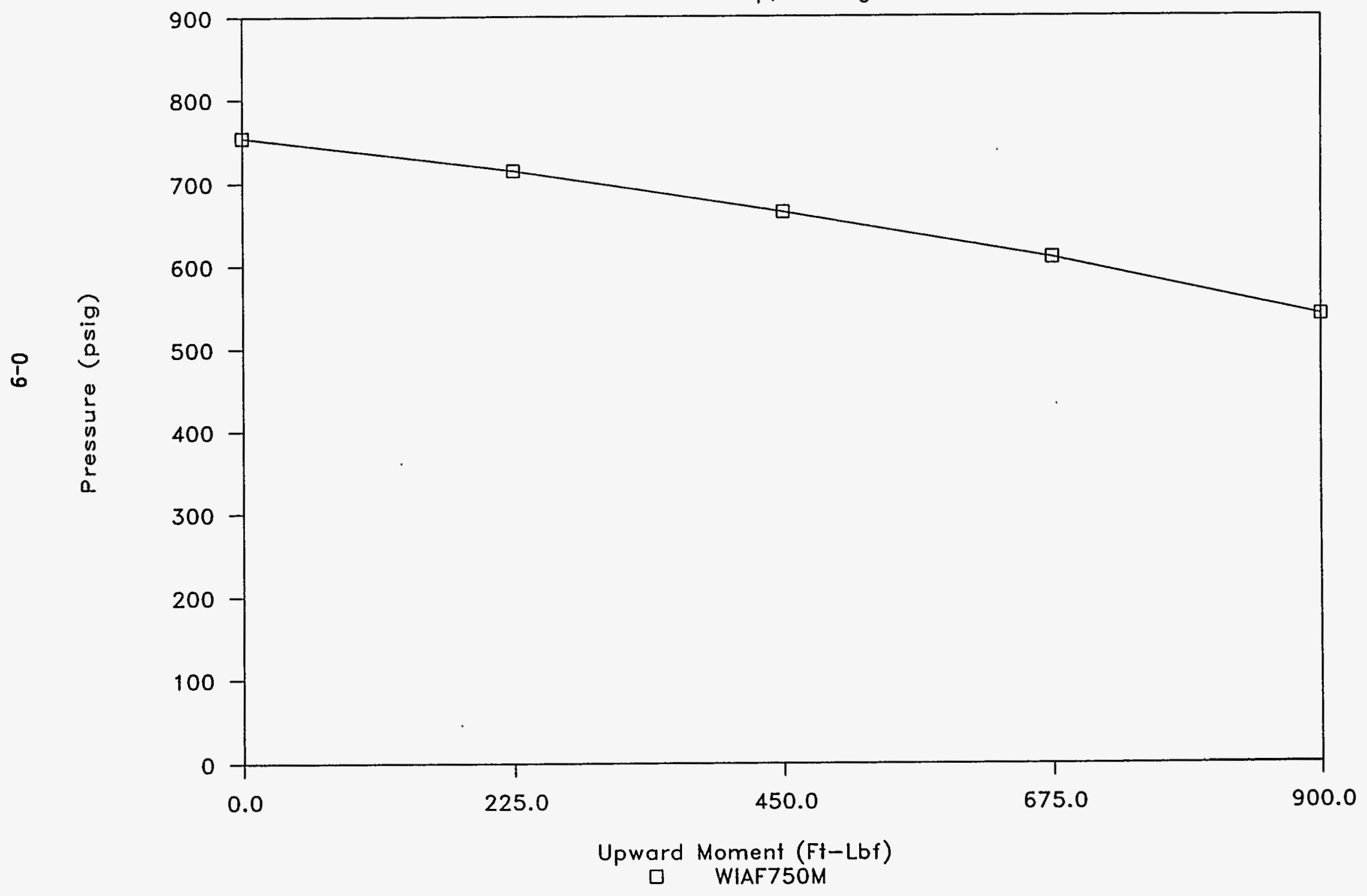




\section{3-Way ISB, Fluorosilicone 70 SH O-Ring}

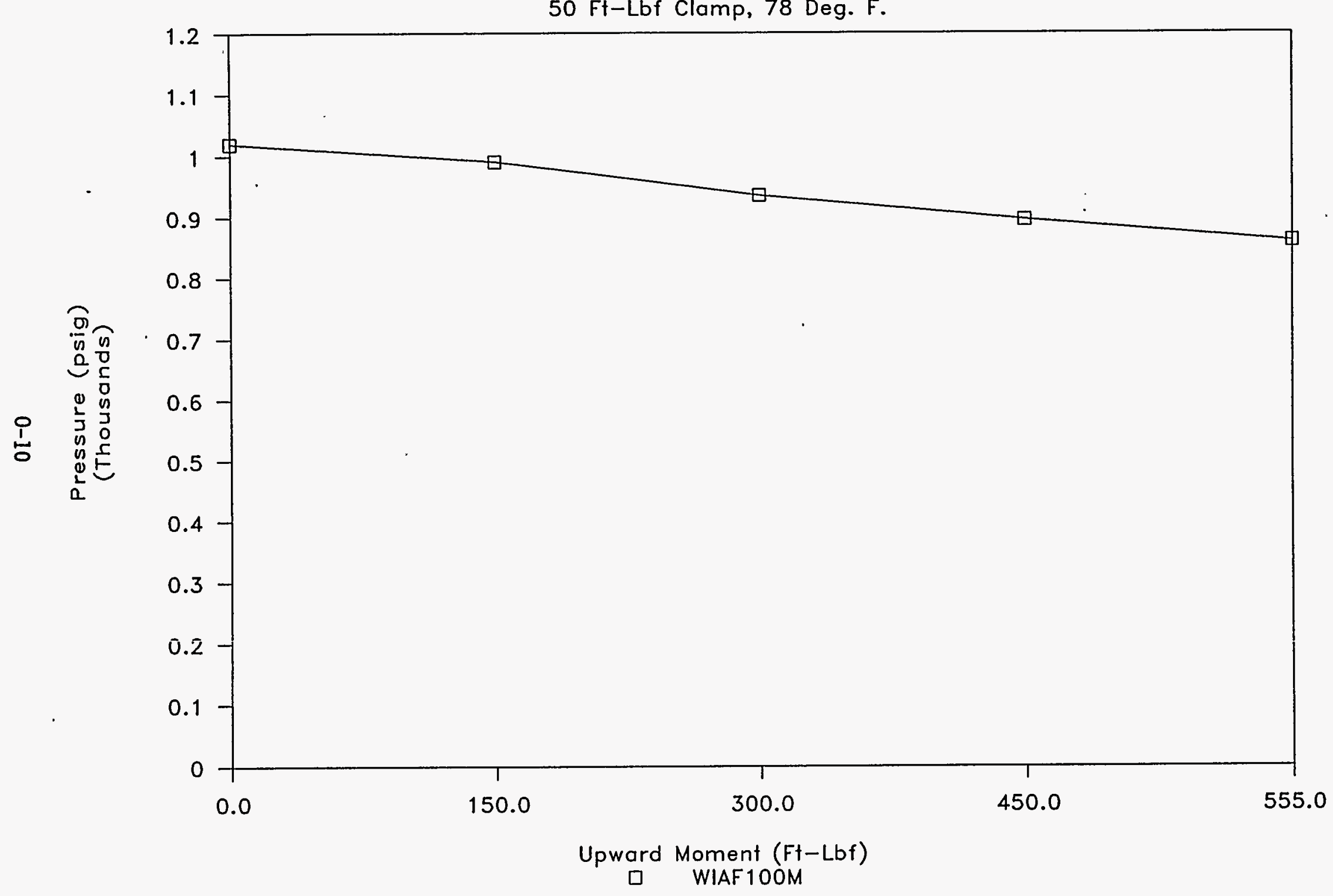


NOVEMBER 29,1994

2" 3-WAY ISB CONNECTOR, FLUOROSILICONE O-RING, 70 DUROMETER, AMBIENT TEM PART \# 2-119 L1120-70, CONQUEST SEAL CO., BATCH 70011, CURE DATE $4 Q 89$ LEAK TEST - UP AND OVER MOMENT (UPWARD ROTATION)

CLAMPING TORQUE $=50$ FT-LBF NOMINAL CHARGE PRESSURE $=250$ PSIG

GRAPH NAME $=$ WIAF25OM

TEMPERATURE: 78 DEG. F.

\begin{tabular}{|c|c|c|c|c|c|c|}
\hline \multirow{3}{*}{$\begin{array}{l}\text { PRESSURE } \\
\text { PSIG }\end{array}$} & \multirow[b]{2}{*}{ ELAPSED } & \multirow[b]{2}{*}{ ELAPSED } & \multirow[b]{2}{*}{ ELAPSED } & \multicolumn{3}{|c|}{ UPWARD } \\
\hline & & & & UPWARD & MOMENT & UPWARD \\
\hline & MINUTES & SECONDS & $\begin{array}{l}\text { DECIMAI } \\
\text { MINUTES }\end{array}$ & $\begin{array}{l}\text { FORCE } \\
\text { IBS }\end{array}$ & $\begin{array}{l}\text { ARM } \\
\text { FT. }\end{array}$ & $\begin{array}{l}\text { MOMENT } \\
\text { FT-LBF. }\end{array}$ \\
\hline NPUT & INPUT & INPUT & COMPUTE & INPUT & INPUT & COMPUTE \\
\hline $\begin{array}{l}270 \\
270\end{array}$ & 0 & r & 0.00 & $\begin{array}{l}0 \\
0\end{array}$ & $\begin{array}{l}1.5000 \\
1.5000\end{array}$ & 0.0 \\
\hline 245 & 4 & & 4.00 & 250 & 1.5000 & 375.0 \\
\hline 215 & 6 & & 6.00 & 500 & 1.5000 & 750.0 \\
\hline 180 & 8 & & 8.00 & 750 & 1.5000 & 1125.0 \\
\hline 150 & 10 & & 10.00 & 1000 & 1.5000 & 1500.0 \\
\hline
\end{tabular}

INCREASED PRESSURE TO 500 PSIG

$\begin{array}{llll}495 & 12 & 0 & 12.00 \\ 450 & 14 & 0 & 14.00 \\ 410 & 16 & 0 & 16.00 \\ 355 & 18 & 0 & 18.00 \\ 295 & 20 & 0 & 20.00\end{array}$

INCREASED PRESSURE TO 750 PSIG

$\begin{array}{llll}755 & 22 & 0 & 22.00 \\ 715 & 24 & 0 & 24.00 \\ 665 & 26 & 0 & 26.00 \\ 610 & 28 & 0 & 28.00 \\ 540 & 30 & 0 & 30.00\end{array}$

INCREASED PRESSURE TO 1000 PSIG

$\begin{array}{rlll}1020 & 32 & 1 & 32.02 \\ 990 & 34 & 0 & 34.00 \\ 935 & 36 & 0 & 36.00 \\ 895 & 38 & 2 & 38.03 \\ 860 & 40 & 0 & 40.00\end{array}$

GRAPH NAME = WIAF50OM

$\begin{array}{rrr}0 & 1.5000 & 0.0 \\ 200 & 1.5000 & 300.0 \\ 400 & 1.5000 & 600.0 \\ 600 & 1.5000 & 900.0 \\ 800 & 1.5000 & 1200.0\end{array}$

GRAPH NAME = WIAF750M

$\begin{array}{rrr}0 & 1.5000 & 0.0 \\ 150 & 1.5000 & 225.0 \\ 300 & 1.5000 & 450.0 \\ 450 & 1.5000 & 675.0 \\ 600 & 1.5000 & 900.0\end{array}$

GRAPH NAME = WIAFIOOM

$\begin{array}{rrr}0 & 1.5000 & 0.0 \\ 100 & 1.5000 & 150.0 \\ 200 & 1.5000 & 300.0 \\ 300 & 1.5000 & 450.0 \\ 370 & 1.5000 & 555.0\end{array}$




\section{3-Way ISB, Fluorosilicone $70 \mathrm{SH}$ O-Ring}

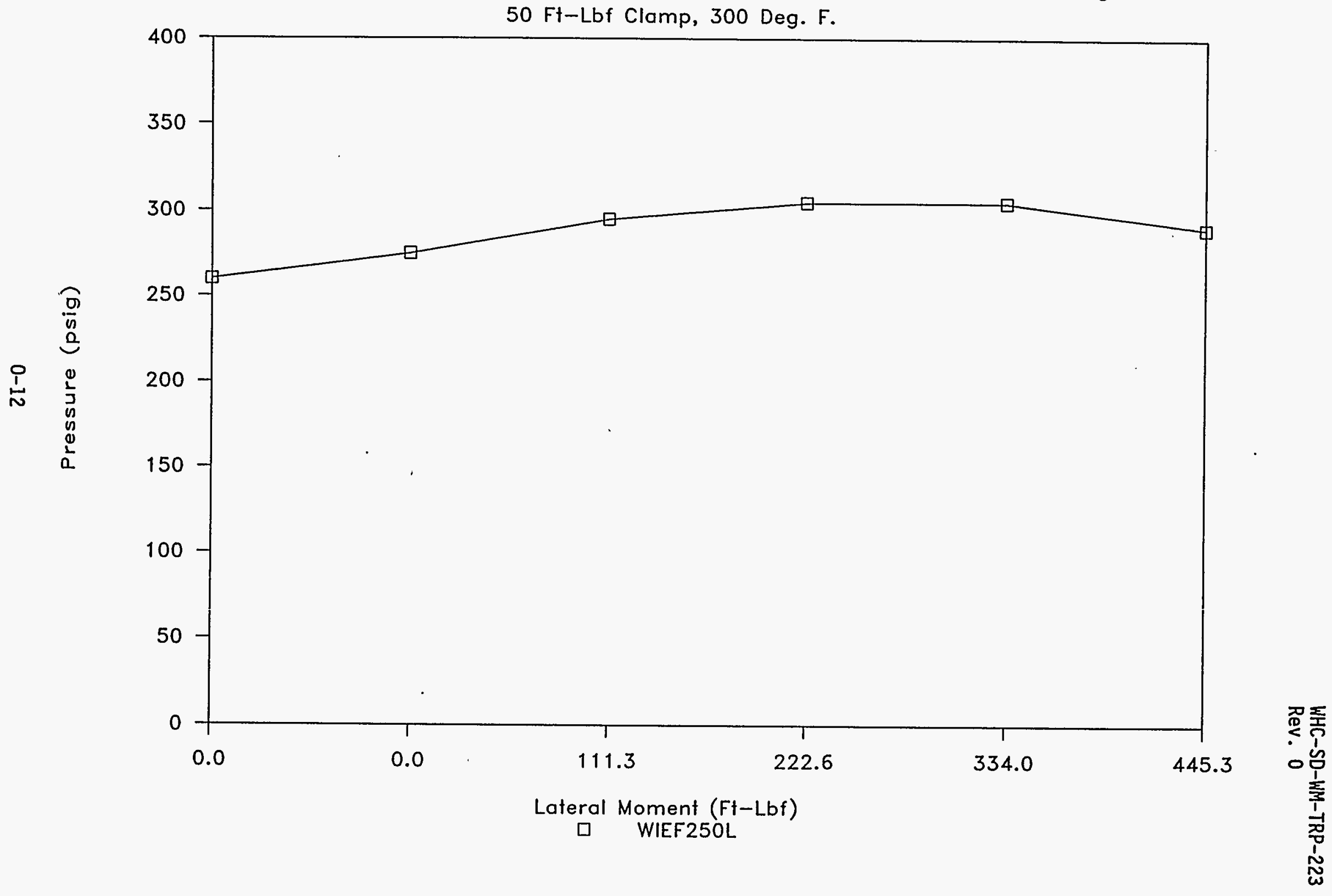


WHC-SD-WM-TRP-223

Rev. 0

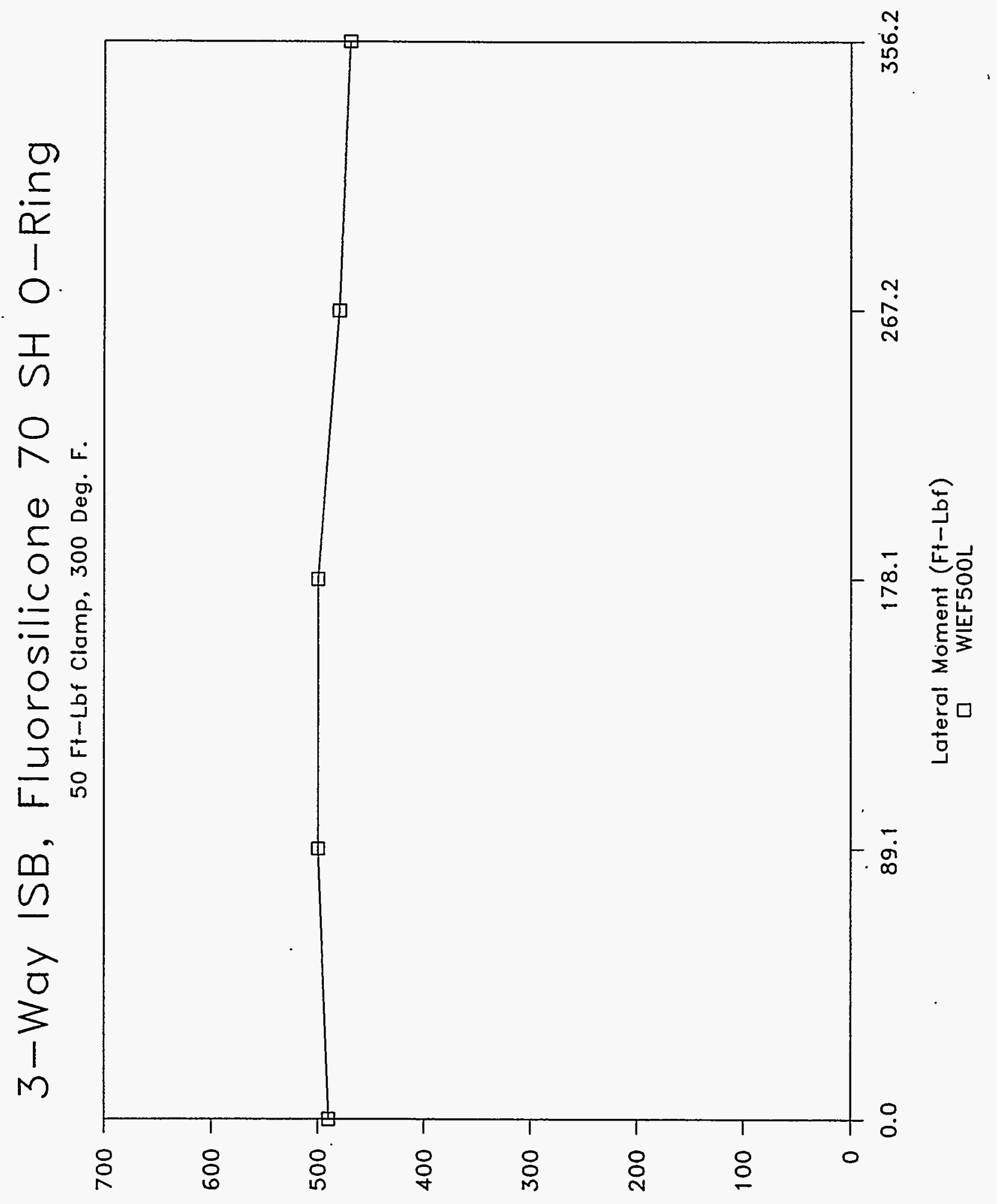

(6!sd) asnssadd 


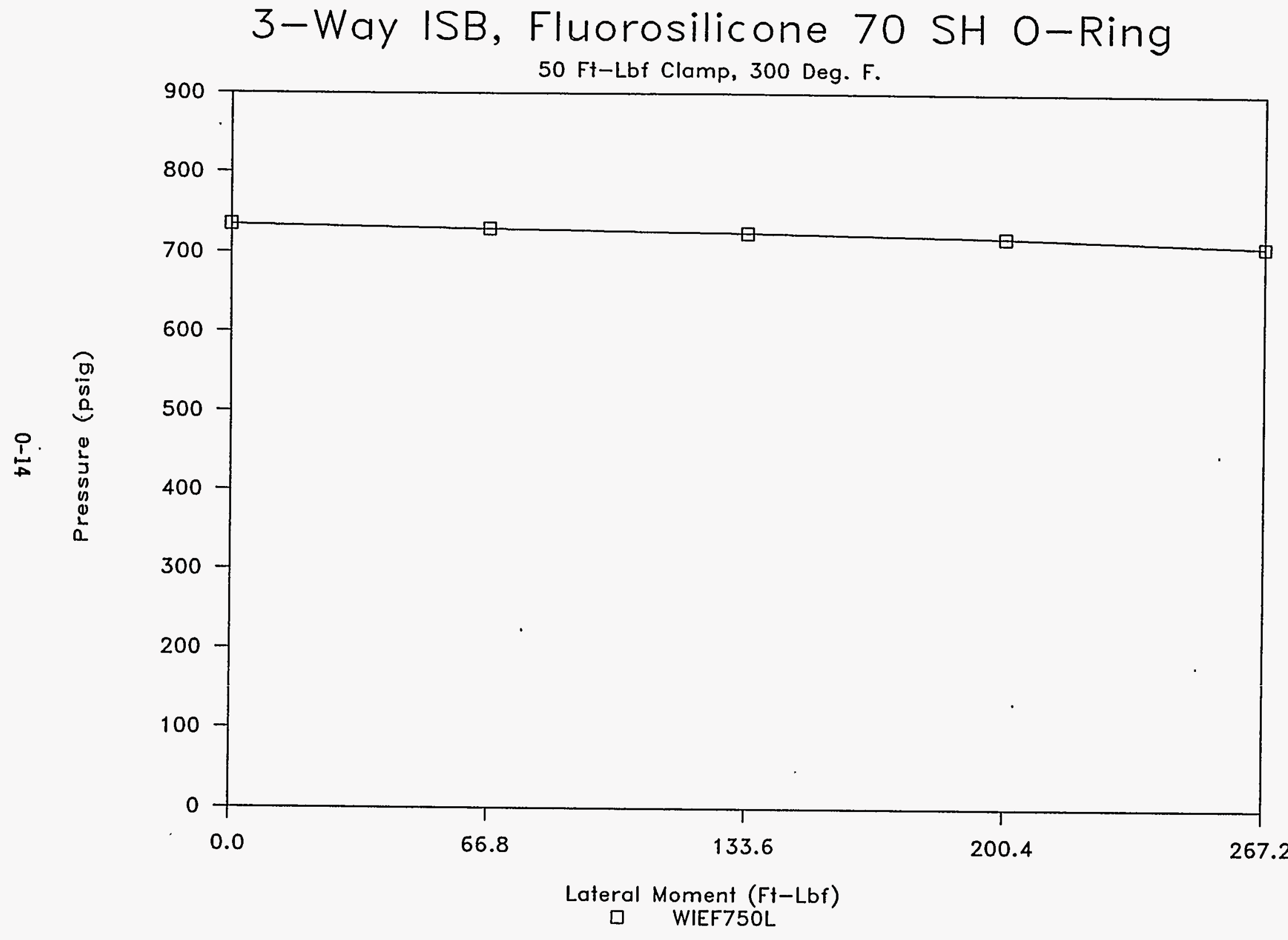




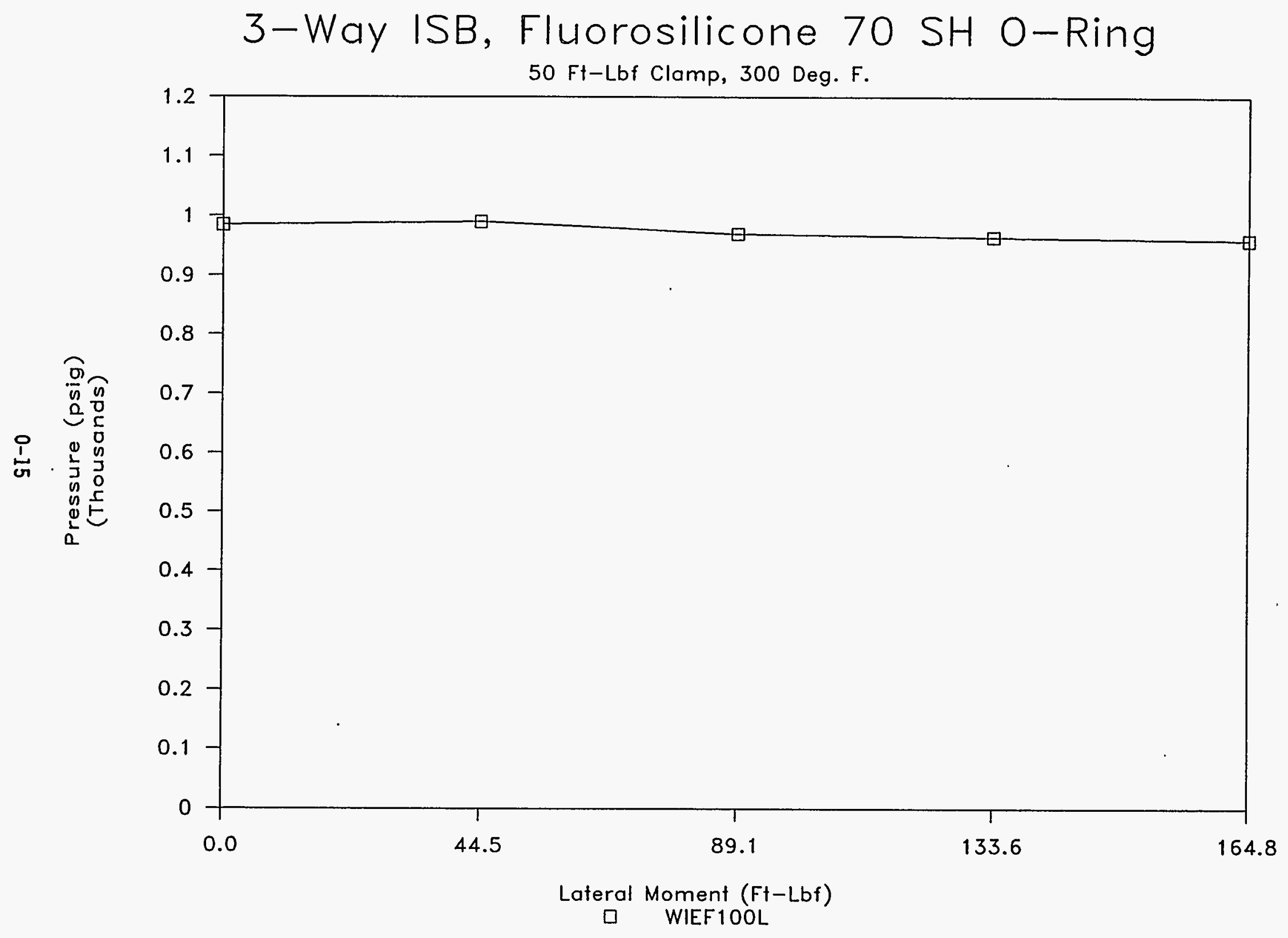


DECEMBER 09, 1994

2" 3-WAY ISB CONNECTOR, FLUOROSILICONE O-RING, 70 DUROMETER, ELEVATED TE PART \# 2-119 L1120-70, CONQUEST SEAI CO., BATCH 701011, CURE DATE $4 Q 89$ LEAK TEST - LATERAL MOMENT (SIDEWAYS ROTATION)

CLAMPING TORQUE $=50$ FT-LBF NOMINAL CHARGE PRESSURE $=250$ PSIG

GRAPE NAME = WIEF250I TEMPERATURE: 3.00 DEG. F.

\begin{tabular}{|c|c|c|c|c|c|c|}
\hline $\begin{array}{l}\text { PRESSURE } \\
\text { PSIG }\end{array}$ & $\begin{array}{l}\text { ELAPSED } \\
\text { MINUTES }\end{array}$ & $\begin{array}{l}\text { ELAPSED } \\
\text { SECONDS }\end{array}$ & $\begin{array}{l}\text { ELAPSED } \\
\text { DECIMAL } \\
\text { MINUTES } \\
\text { COMPUTE }\end{array}$ & $\begin{array}{l}\text { LATERAL } \\
\text { FORCE } \\
\text { LBS } \\
\text { INPUT }\end{array}$ & $\begin{array}{l}\text { IATEFAL } \\
\text { MOMENT } \\
\text { ARM } \\
\text { FT. } \\
\text { INPUI! }\end{array}$ & $\begin{array}{l}\text { LATERAL } \\
\text { MOMENT } \\
\text { FT-LBF. } \\
\text { COMPUTE }\end{array}$ \\
\hline NPUT & INPUT & INPUT & COMPUTE & INPUT & INPUI! & IPUTE \\
\hline $\begin{array}{l}260 \\
275 \\
295 \\
305 \\
305 \\
290\end{array}$ & $\begin{array}{r}0 \\
2 \\
4 \\
6 \\
8 \\
10\end{array}$ & & $\begin{array}{r}0.00 \\
2.00 \\
4.00 \\
6.00 \\
8.00 \\
10.00\end{array}$ & $\begin{array}{r}0 \\
0 \\
250 \\
500 \\
750 \\
1000\end{array}$ & $\begin{array}{l}0.4: 453 \\
0.4: 453 \\
0.4: 453 \\
0.4: 453 \\
0.4: 453 \\
0.4: 453\end{array}$ & $\begin{array}{r}0.0 \\
0.0 \\
111.3 \\
222.7 \\
334.0 \\
445.3\end{array}$ \\
\hline
\end{tabular}

INCREASED PRESSURE TO 500 PSIG

$\begin{array}{llll}490 & 12 & 0 & 12.00 \\ 500 & 14 & 0 & 14.00 \\ 500 & 16 & 0 & 16.00 \\ 480 & 18 & 0 & 18.00 \\ 470 & 20 & 0 & 20.00\end{array}$

INCREASED PRESSURE TO 750 PSIG

$\begin{array}{llll}735 & 22 & 0 & 22.00 \\ 730 & 24 & 0 & 24.00 \\ 725 & 26 & 0 & 26.00 \\ 720 & 28 & 0 & 28.00 \\ 710 & 30 & 0 & 30.00\end{array}$

INCREASED PRESSURE TO 1000 PSIG

$\begin{array}{llll}985 & 32 & 0 & 32.00 \\ 990 & 34 & 0 & 34.00 \\ 970 & 36 & 0 & 36.00 \\ 965 & 38 & 0 & 38.00 \\ 960 & 40 & 0 & 40.00\end{array}$

GRAPH NAME = WIEF50OL

$\begin{array}{rrr}0 & 0.4453 & 0.0 \\ 200 & 0.4453 & 89.1 \\ 400 & 0.4453 & 178.1 \\ 600 & 0.4453 & 267.2 \\ 800 & 0.4453 & 356.2\end{array}$

GRAPH NAME = VIEF750I

$\begin{array}{rrr}0 & 0.4453 & 0.0 \\ 150 & 0.4453 & 66.8 \\ 300 & 0.4453 & 133.6 \\ 450 & 0.4453 & 200.4 \\ 600 & 0.4453 & 267.2\end{array}$

GRAPH NAME = WIEFIOOL

$\begin{array}{rrr}0 & 0.4453 & 0.0 \\ 100 & 0.4453 & 44.5 \\ 200 & 0.4453 & 89.1 \\ 300 & 0.4453 & 133.6 \\ 370 & 0.4453 & 164.8\end{array}$




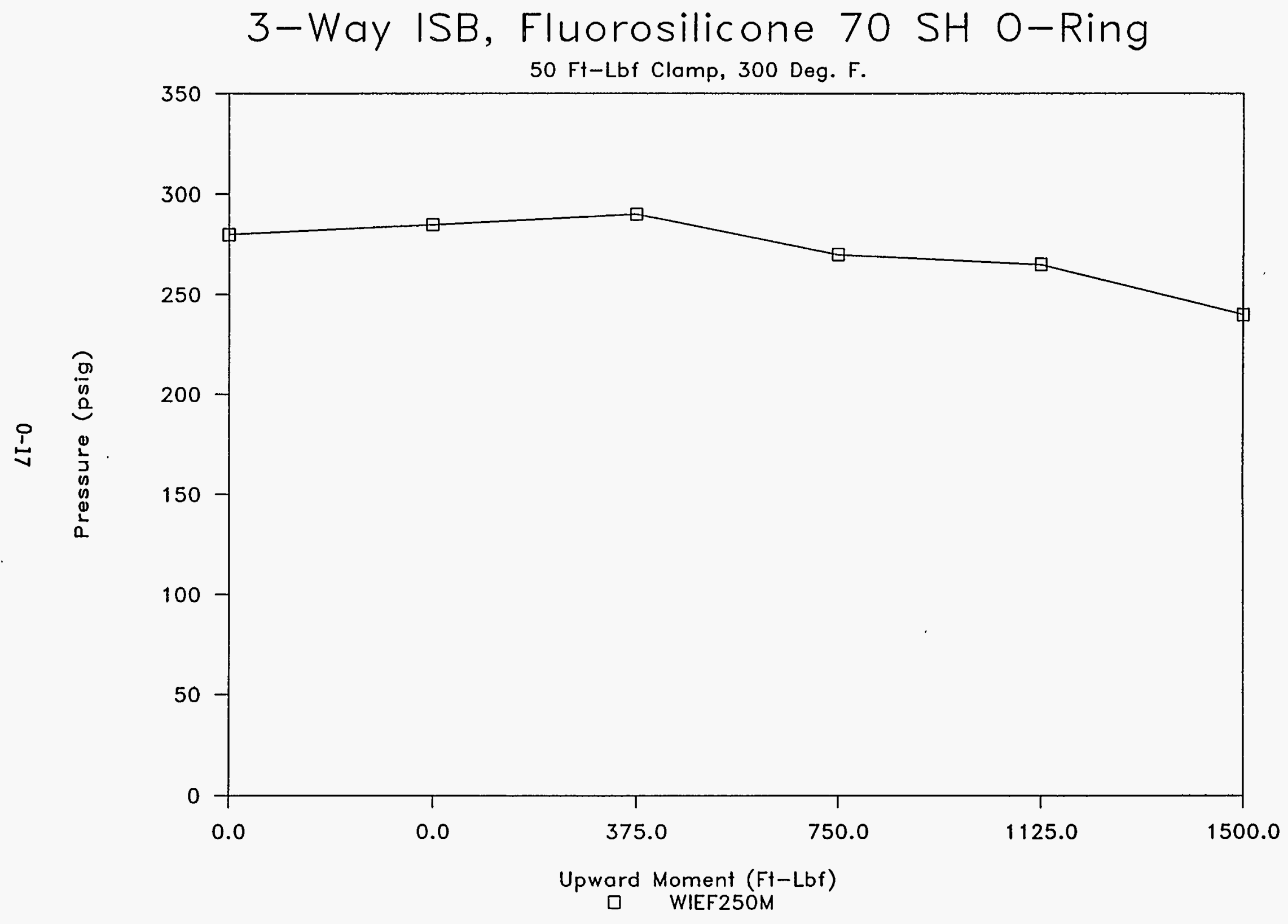




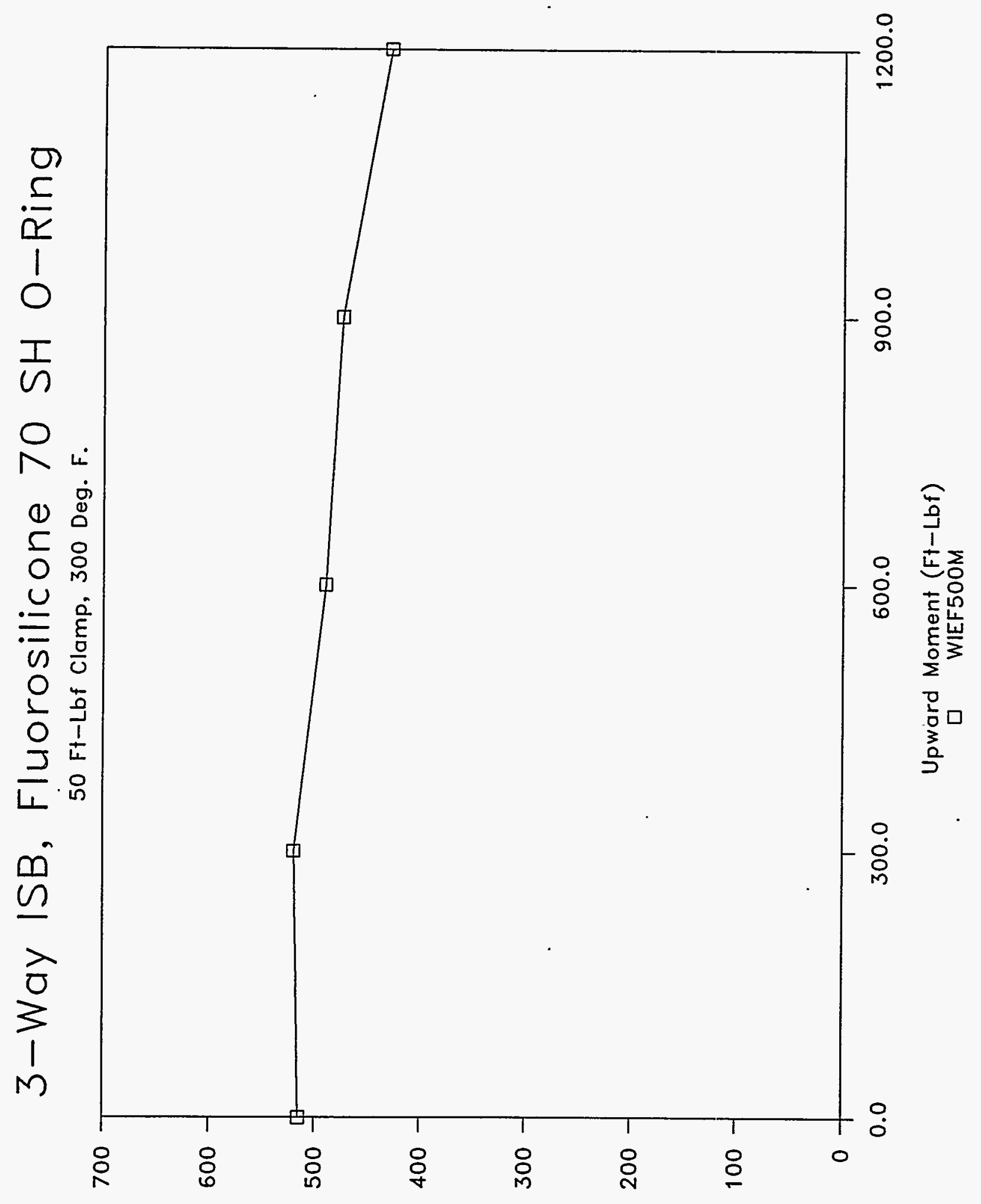

(bisd) asnssasd 
WHC-SD-WM-TRP-223

Rev. 0

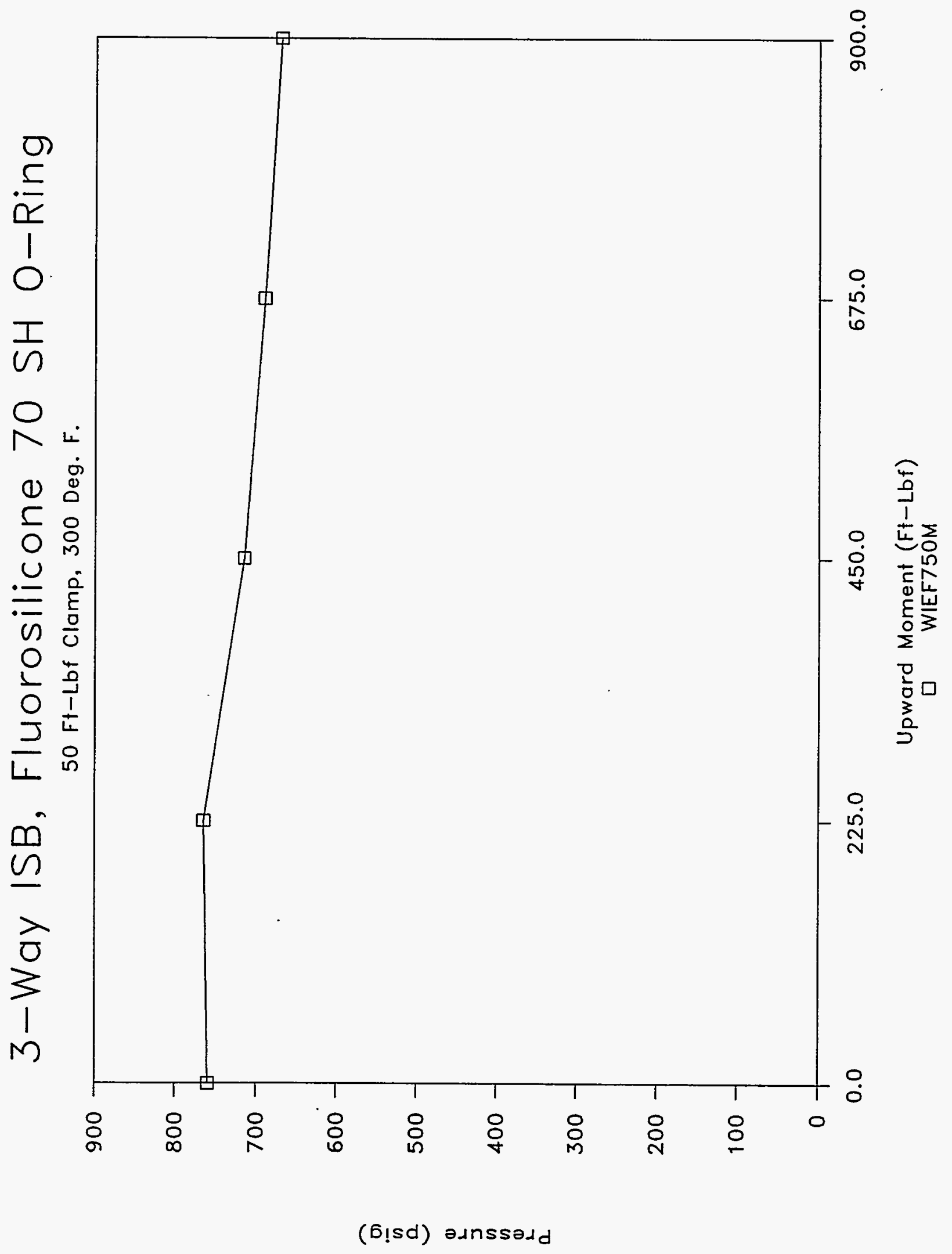


Rev. 0

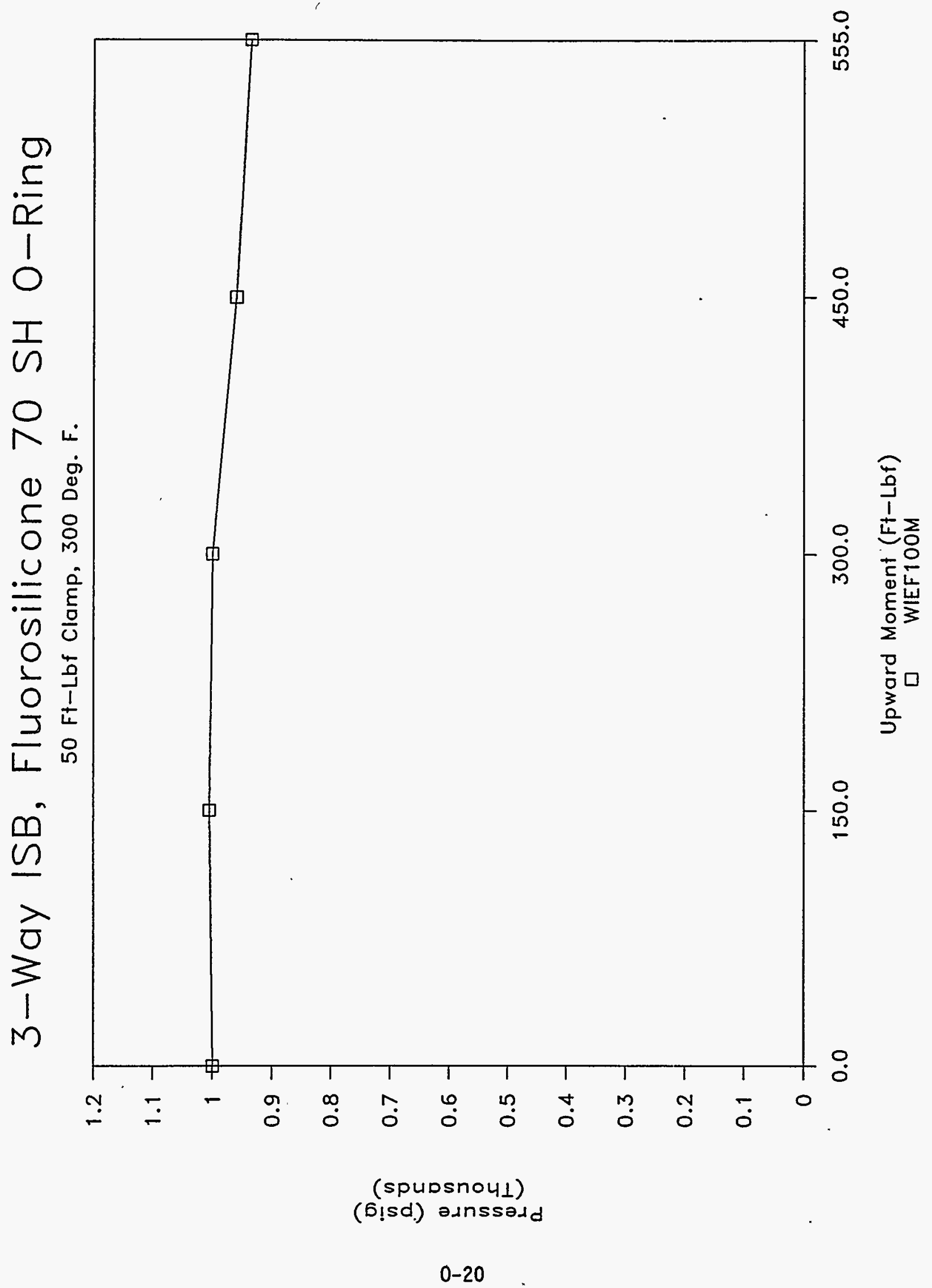


NOVEMBER 29,1994

2" 3-WAY ISB CONNECTOR, FLUOROSIIICONE O-RING, 70 DUROMETER, ELEVATED TE PART \# 2-119 L1120-70, CONQUEST SEAL CO., BATCH 70011, CURE DATE 4 Q89 LEAK TEST - UP AND OVER MOMENT (UPWARD ROTATION)

CLAMPING TORQUE $=50$ FT-LBF NOMINAL CHARGE PRESSURE $=250$ PSIG

GRAPH NAME = WIEF250M

TEMPERATURE: 300 DEG. F.

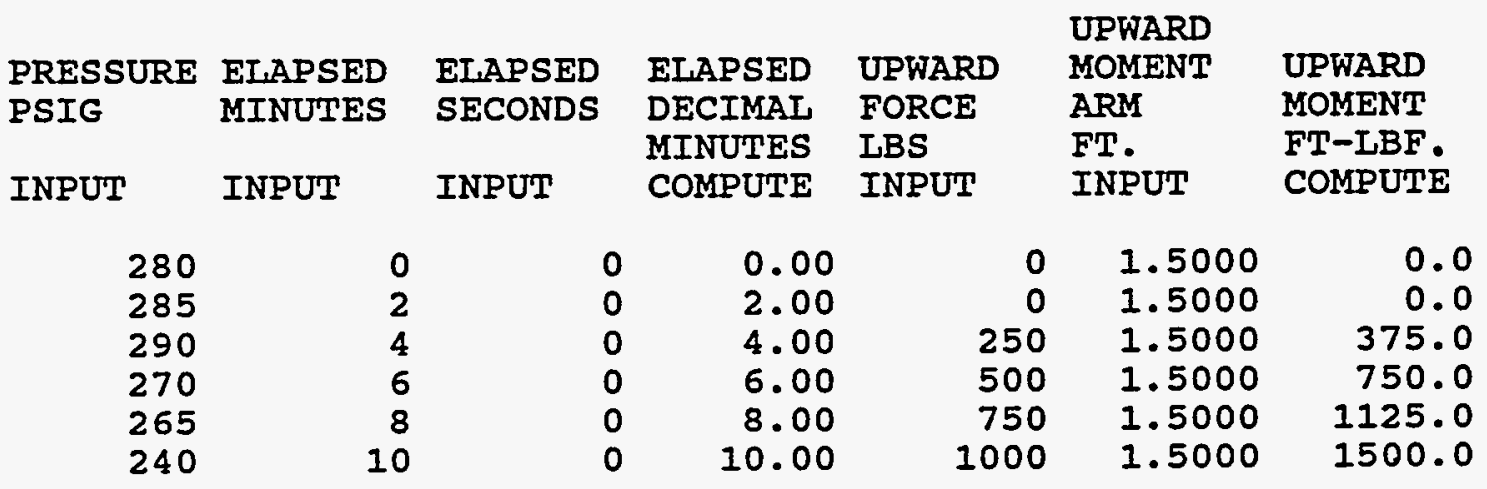

INCREASED PRESSURE TO 500 PSIG

$\begin{array}{llll}515 & 12 & 0 & 12.00 \\ 520 & 14 & 0 & 14.00 \\ 490 & 16 & 0 & 16.00 \\ 475 & 18 & 0 & 18.00 \\ 430 & 20 & 1 & 20.02\end{array}$

INCREASED PRESSURE TO 750 PSIG

$\begin{array}{rrrrrrr}760 & 22 & 0 & 22.00 & 0 & 1.5000 & 0.0 \\ 765 & 24 & 0 & 24.00 & 150 & 1.5000 & 225.0 \\ 715 & 26 & 0 & 26.00 & 300 & 1.5000 & 450.0 \\ 690 & 28 & 0 & 28.00 & 450 & 1.5000 & 675.0 \\ 670 & 30 & 1 & 30.02 & 600 & 1.5000 & 900.0\end{array}$

GRAPH NAME = WIEF50OM

$\begin{array}{rrr}0 & 1.5000 & 0.0 \\ 200 & 1.5000 & 300.0 \\ 400 & 1.5000 & 600.0 \\ 600 & 1.5000 & 900.0 \\ 800 & 1.5000 & 1200.0\end{array}$

GRAPH NAME = WIEF750M

GRAPH NAME = WIEF1OOM

$\begin{array}{rrr}0 & 1.5000 & 0.0 \\ 100 & 1.5000 & 150.0 \\ 200 & 1.5000 & 300.0 \\ 300 & 1.5000 & 450.0 \\ 370 & 1.5000 & 555.0\end{array}$


WHC-SD-WM-TRP-223

Rev. 0

APPENDIX P: GRAPHS OF THREE-WAY KALREZ TESTS

P-1 
HHC-SD-WM-TRP-223

Rev. 0

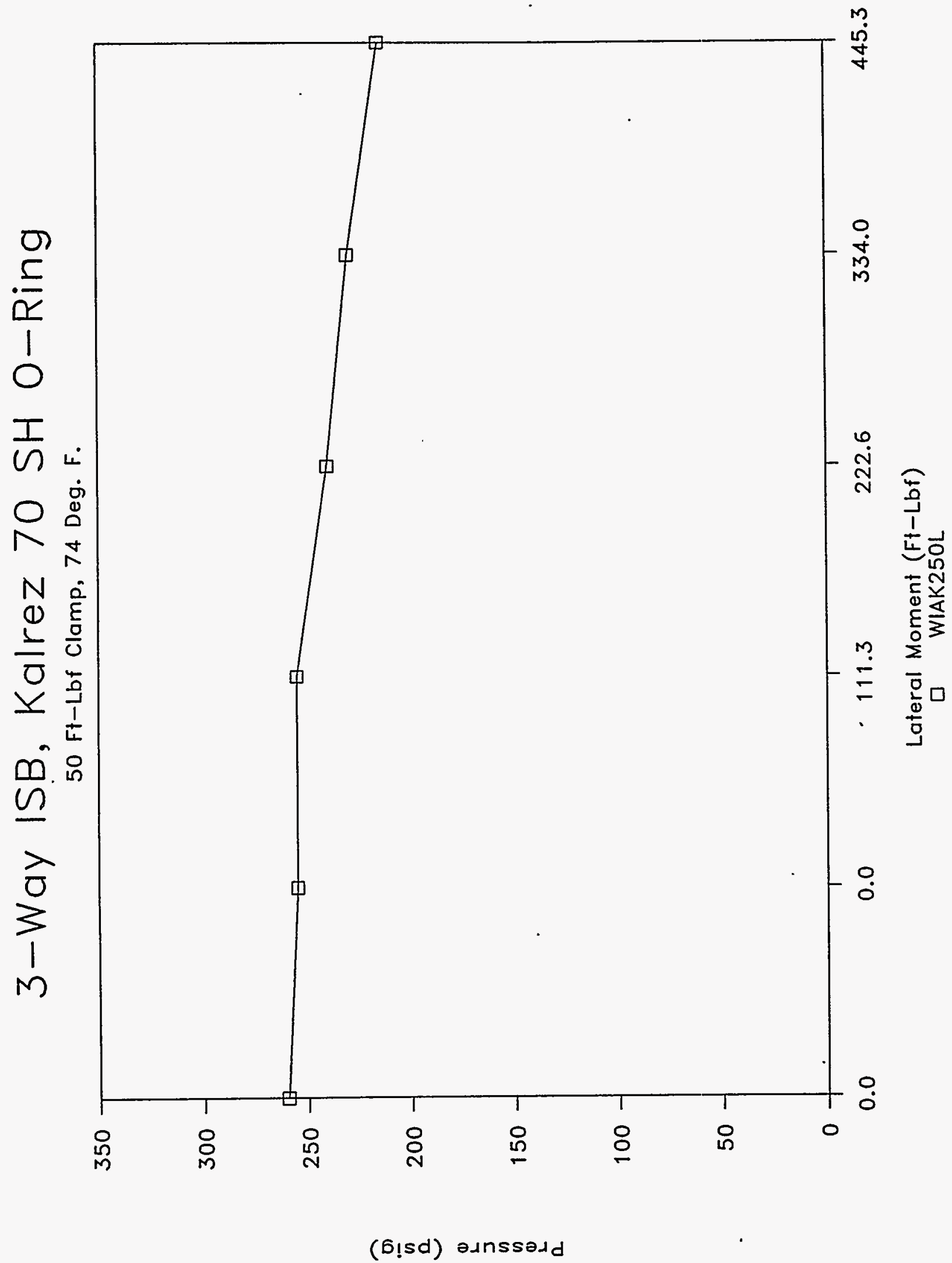

P-2 
3-Way ISB, Kalrez 70 SH O-Ring

$50 \mathrm{Ft}$-Lbf Clamp, 74 Deg. F.

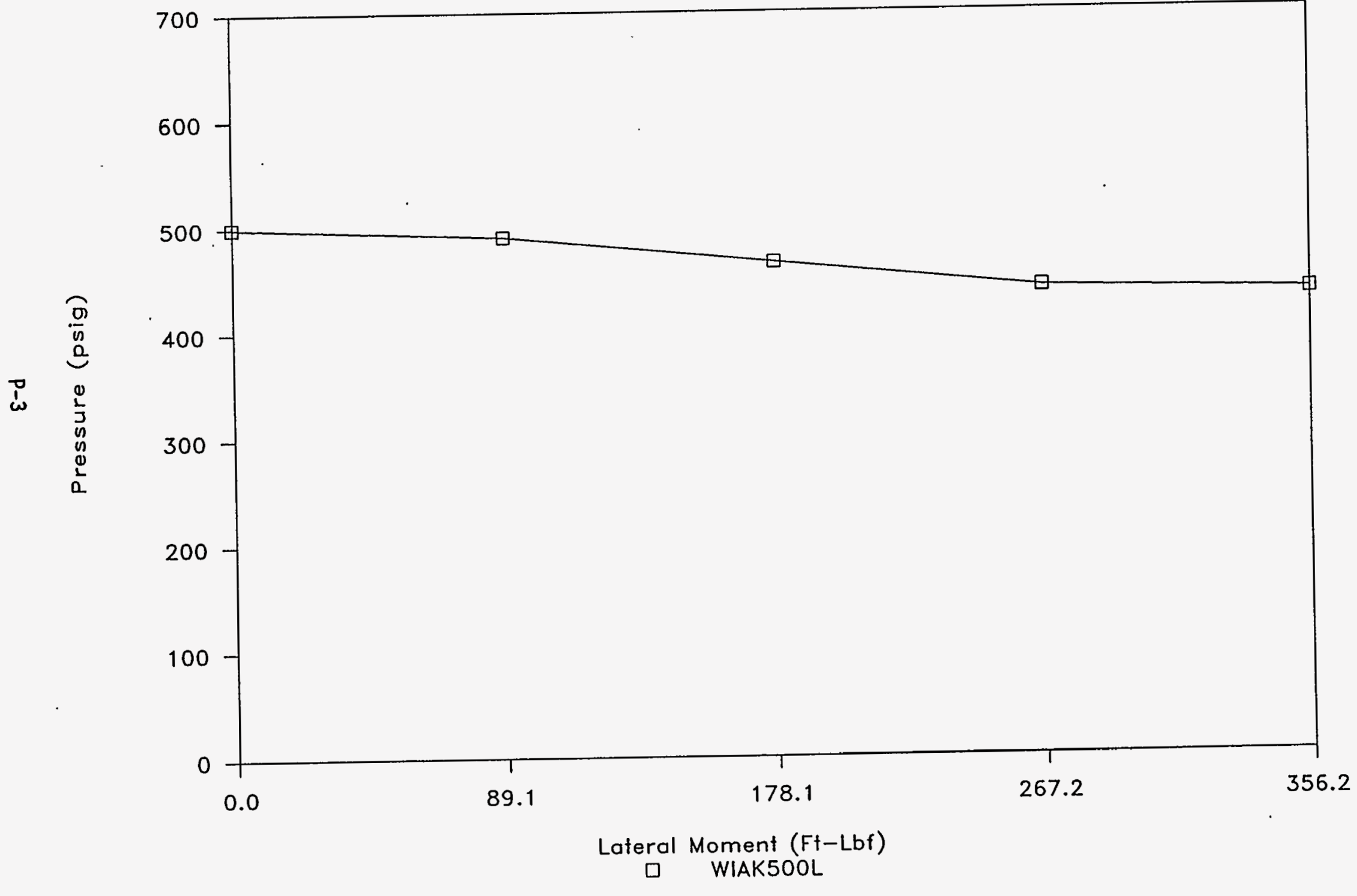




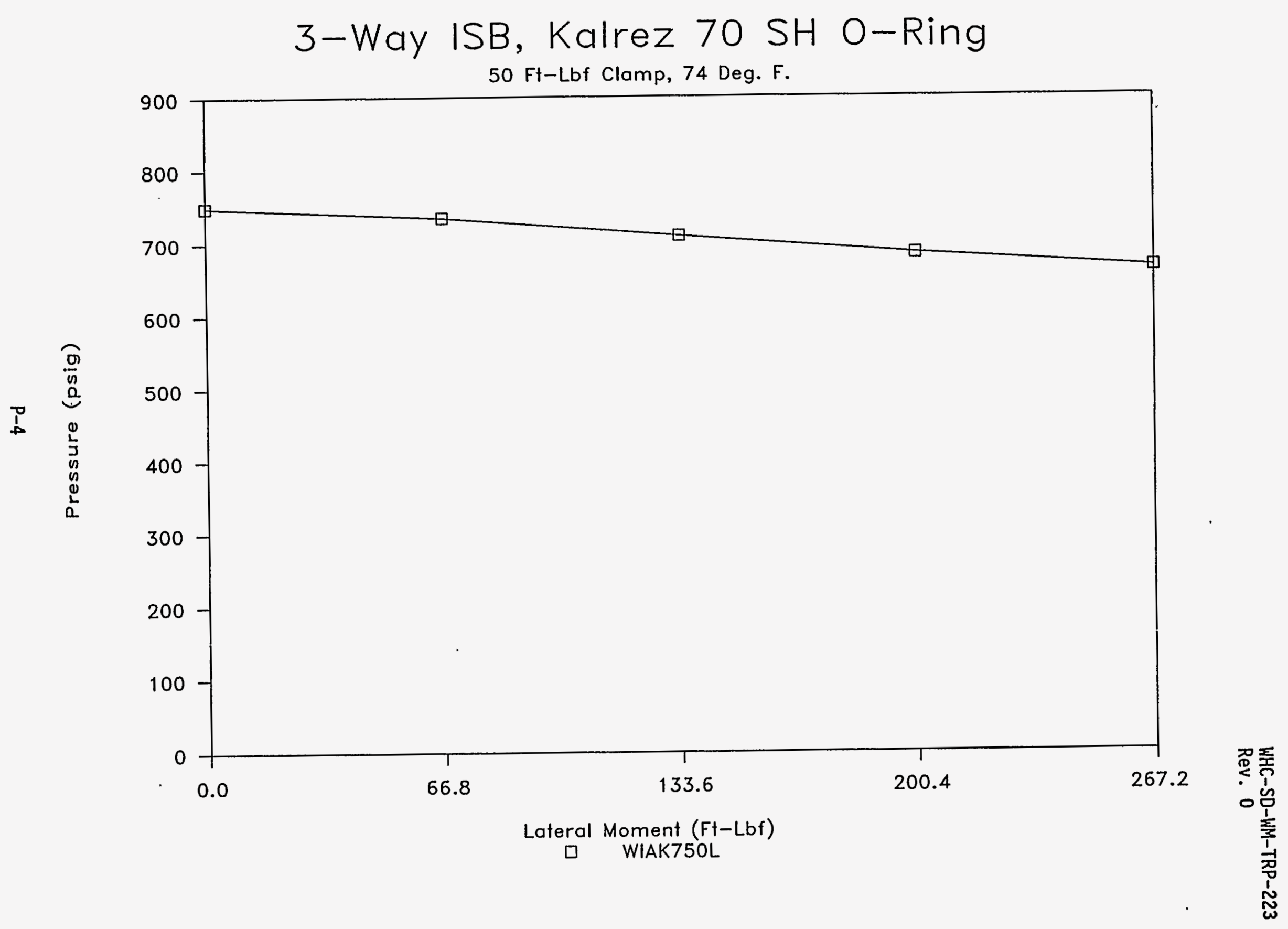




\section{3-Way ISB, Kalrez 70 SH O-Ring}

50 Ft-Lbf Clamp, 74 Deg. F.

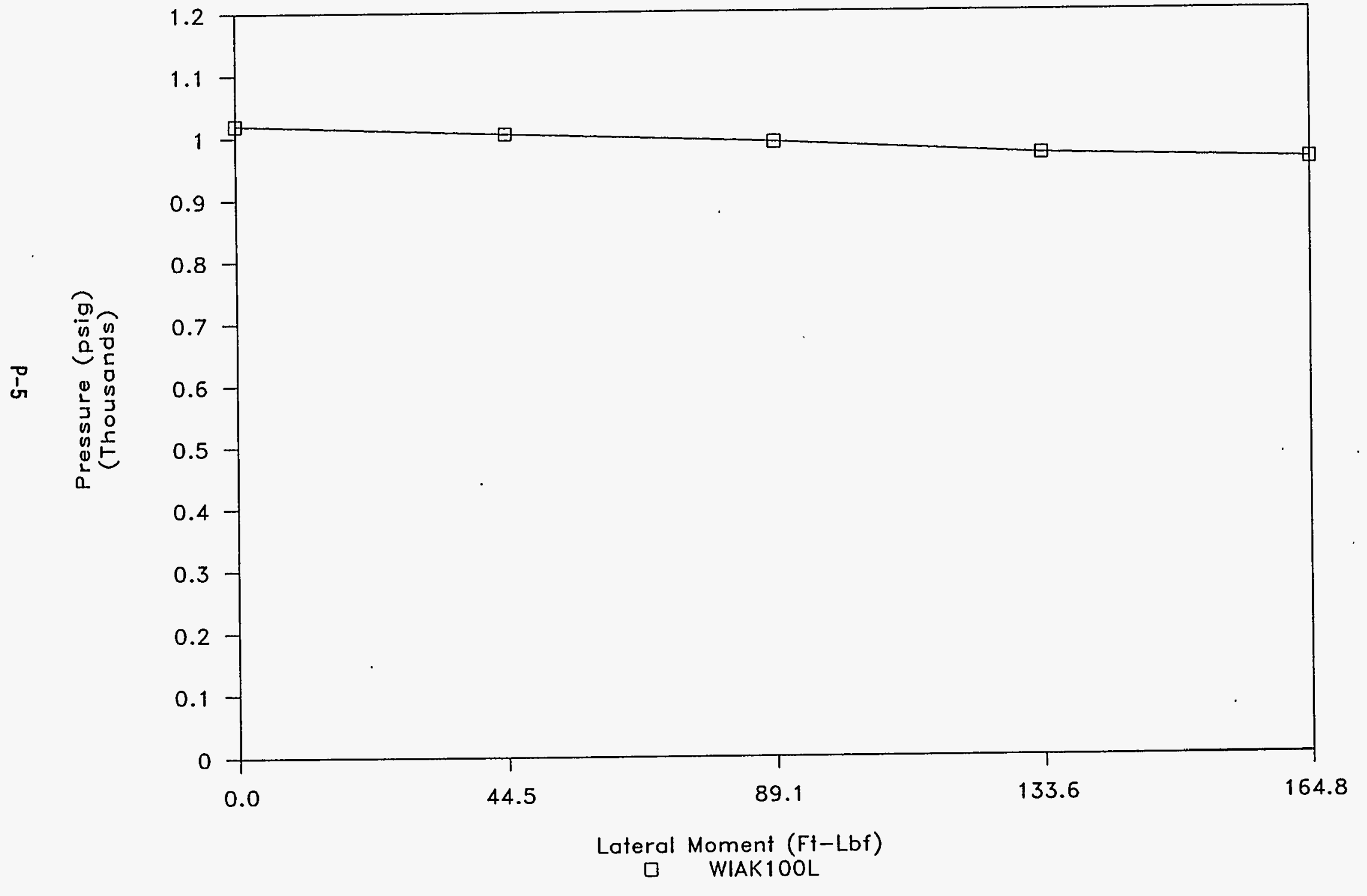


DECEMBER 07, 1994

2" 3-WAY ISB CONNECTOR, KALREZ O-RING, 70 DUROMETIER, AMBIENT TEMP. PART \# AS-568A K\# 119, DUPONT CO., CURE DATE 04/18/94

LEAK TEST - LATERAL MOMENT (SIDEWAYS ROTATION)

CLAMPING TORQUE $=50$ FT-IBF NOMINAI

CHARGE PRESSURE $=250$ PSIG

GRAPH NAME $=$ WIAK250I

TEMPERATURE: 74 DEG. F.

\begin{tabular}{lllllll} 
& & & & LATEIRAL & \\
PRESSURE & ELAPSED & ELAPSED & ELAPSED & LATERAI & MOMEINT & LATERAL \\
PSIG & MINUTES & SECONDS & DECIMAL & FORCE & ARM & MOMENT \\
\multicolumn{1}{c}{} & & & MINUTES & LBS & FT. & FT-LBF. \\
INPUT & INPUT & INPUT & COMPUTE & INPUT & INPUT & COMPUTE
\end{tabular}

$\begin{array}{rrrrrrr}260 & 0 & 0 & 0.00 & 0 & 0.4453 & 0.0 \\ 255 & 2 & 0 & 2.00 & 0 & 0.4453 & 0.0 \\ 255 & 4 & 0 & 4.00 & 250 & 0.4453 & 111.3 \\ 240 & 6 & 0 & 6.00 & 500 & 0.4453 & 222.7 \\ 230 & 8 & 0 & 8.00 & 750 & 0.4453 & 334.0 \\ 215 & 10 & 0 & 10.00 & 1000 & 0.4453 & 445.3\end{array}$

INCREASED PRESSURE TO 500 PSIG

\begin{tabular}{|c|c|c|}
\hline $\begin{array}{r}0 \\
200 \\
400 \\
600 \\
800\end{array}$ & $\begin{array}{l}0.4453 \\
0.4453 \\
0.4453 \\
0.4453 \\
0.4453\end{array}$ & $\begin{array}{r}0.0 \\
89.1 \\
178.1 \\
267.2 \\
356.2\end{array}$ \\
\hline
\end{tabular}

INCREASED PRESSURE TO 750 PSIG

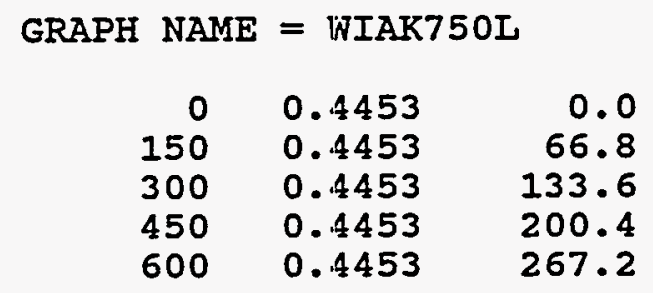

INCREASED PRESSURE TO 1000 PSIG

GRAPH NAME = WIAK1OOL

$\begin{array}{rrrrrrr}1020 & 32 & 0 & 32.00 & 0 & 0.4453 & 0.0 \\ 1005 & 34 & 0 & 34.00 & 100 & 0.4453 & 44.5 \\ 990 & 36 & 0 & 36.00 & 200 & 0.4453 & 89.1 \\ 970 & 38 & 0 & 38.00 & 300 & 0.4453 & 133.6 \\ 960 & 40 & 0 & 40.00 & 370 & 0.4453 & 164.8\end{array}$




\section{3-Way ISB, Kalrez 70 SH O-Ring}

$50 \mathrm{Ft}$-Lbf Clamp, 72 Deg. F.

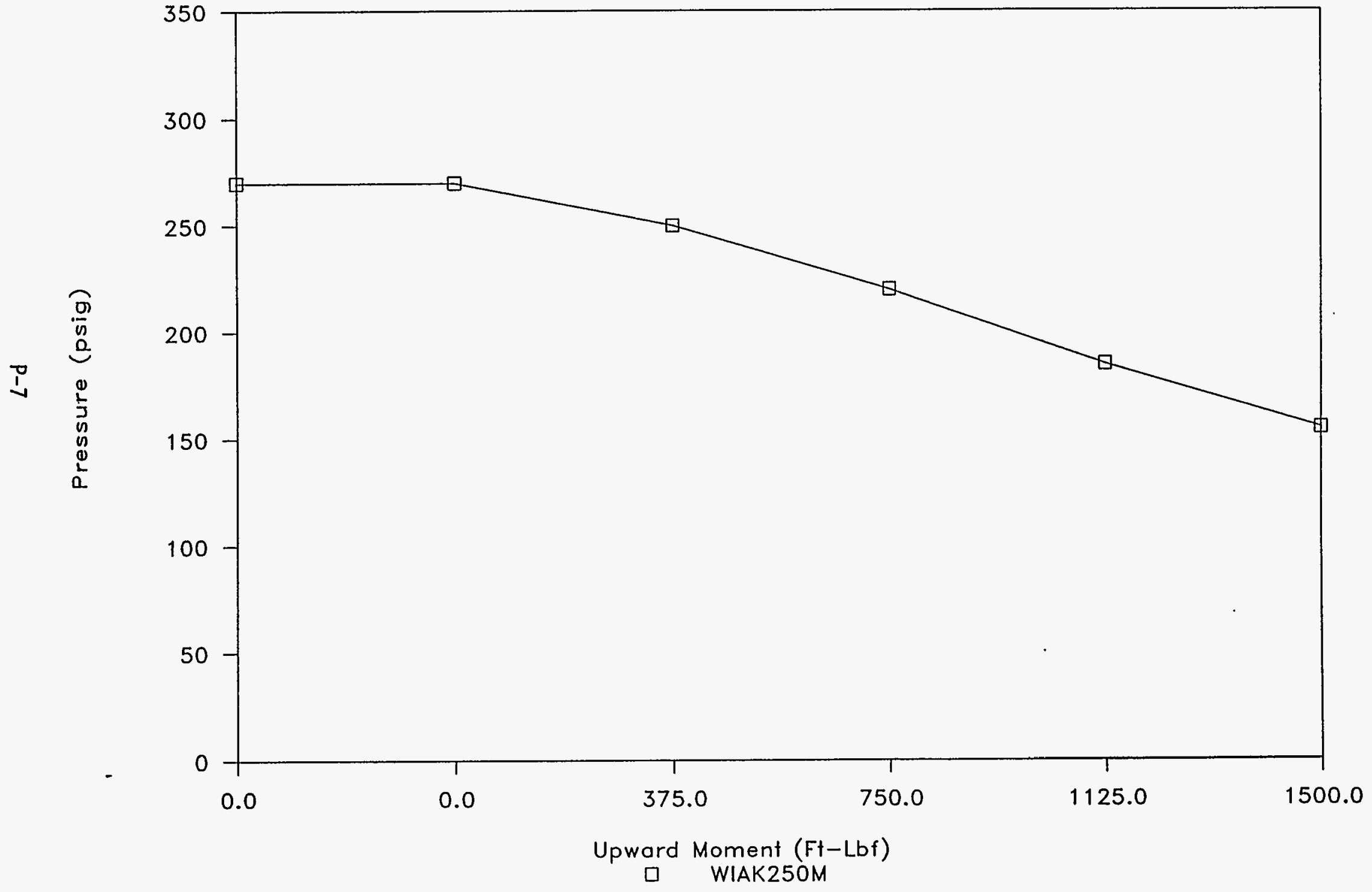


WHC-SD-WM-TRP-223 Rev. 0

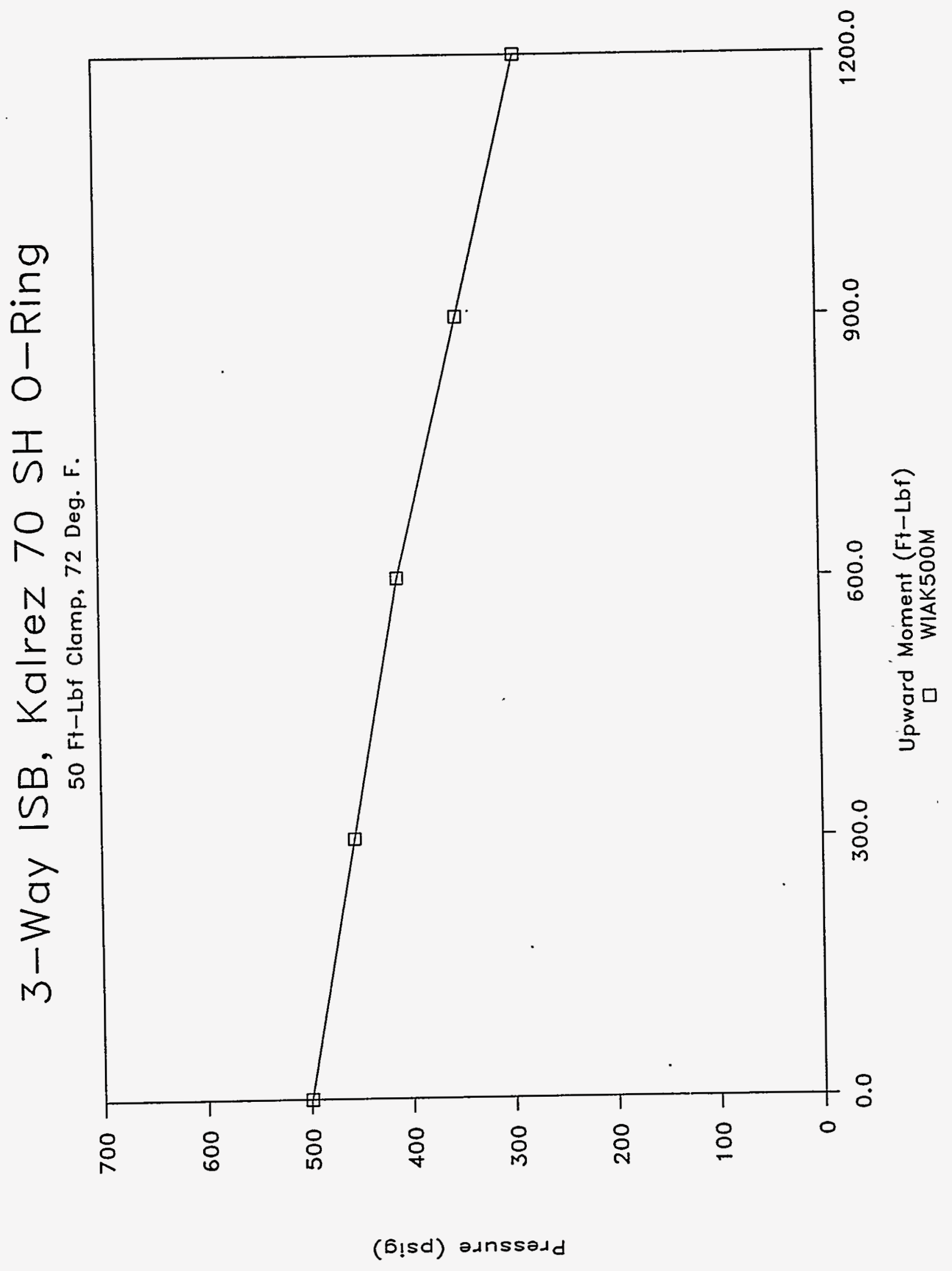

P-8 


\section{3-Way ISB, Kalrez 70 SH O-Ring}

$50 \mathrm{Ft}$-Lbf Clamp, 72 Deg. F.

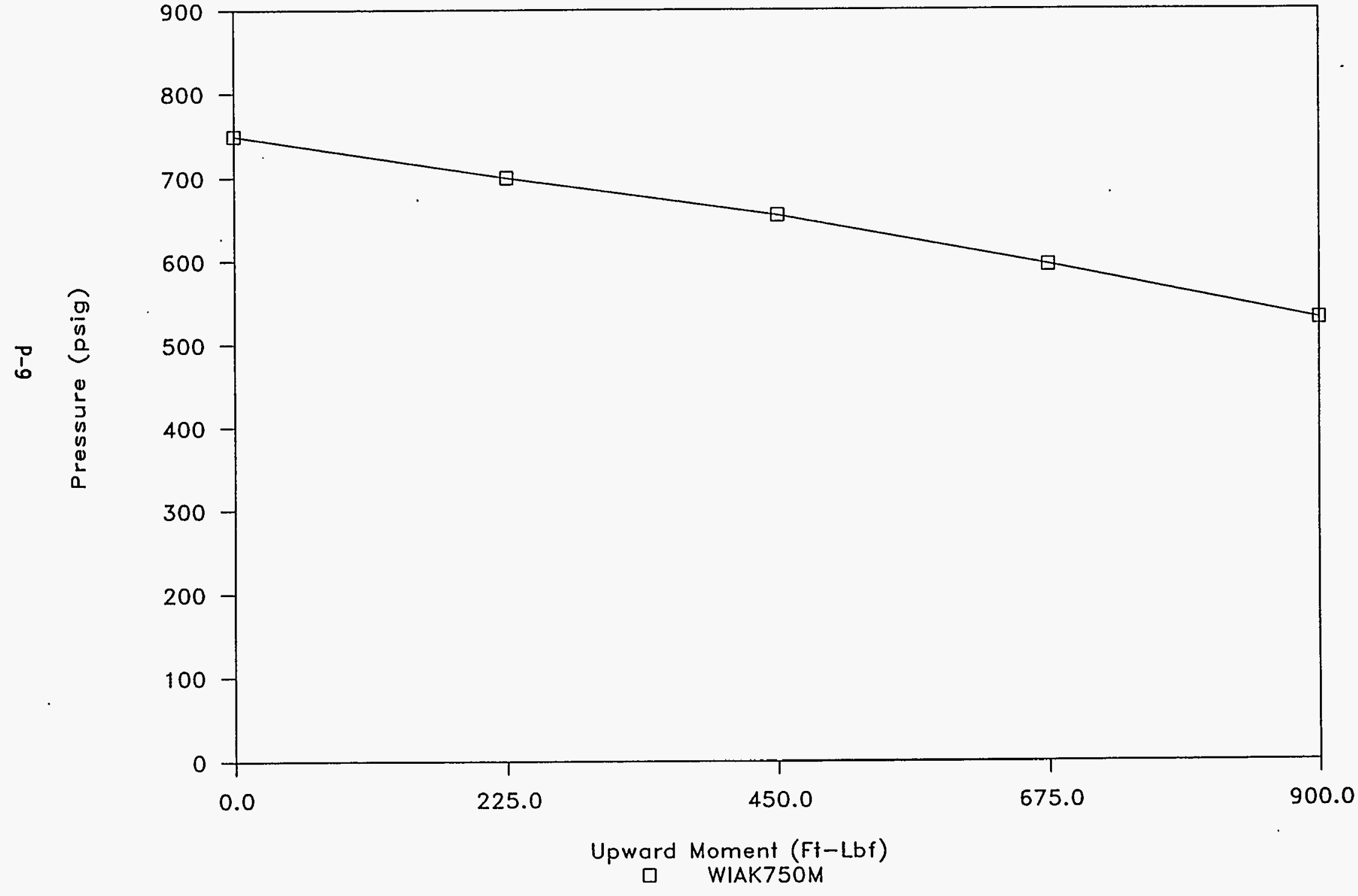


WHC-SD-WM-TRP-223

Rev. 0

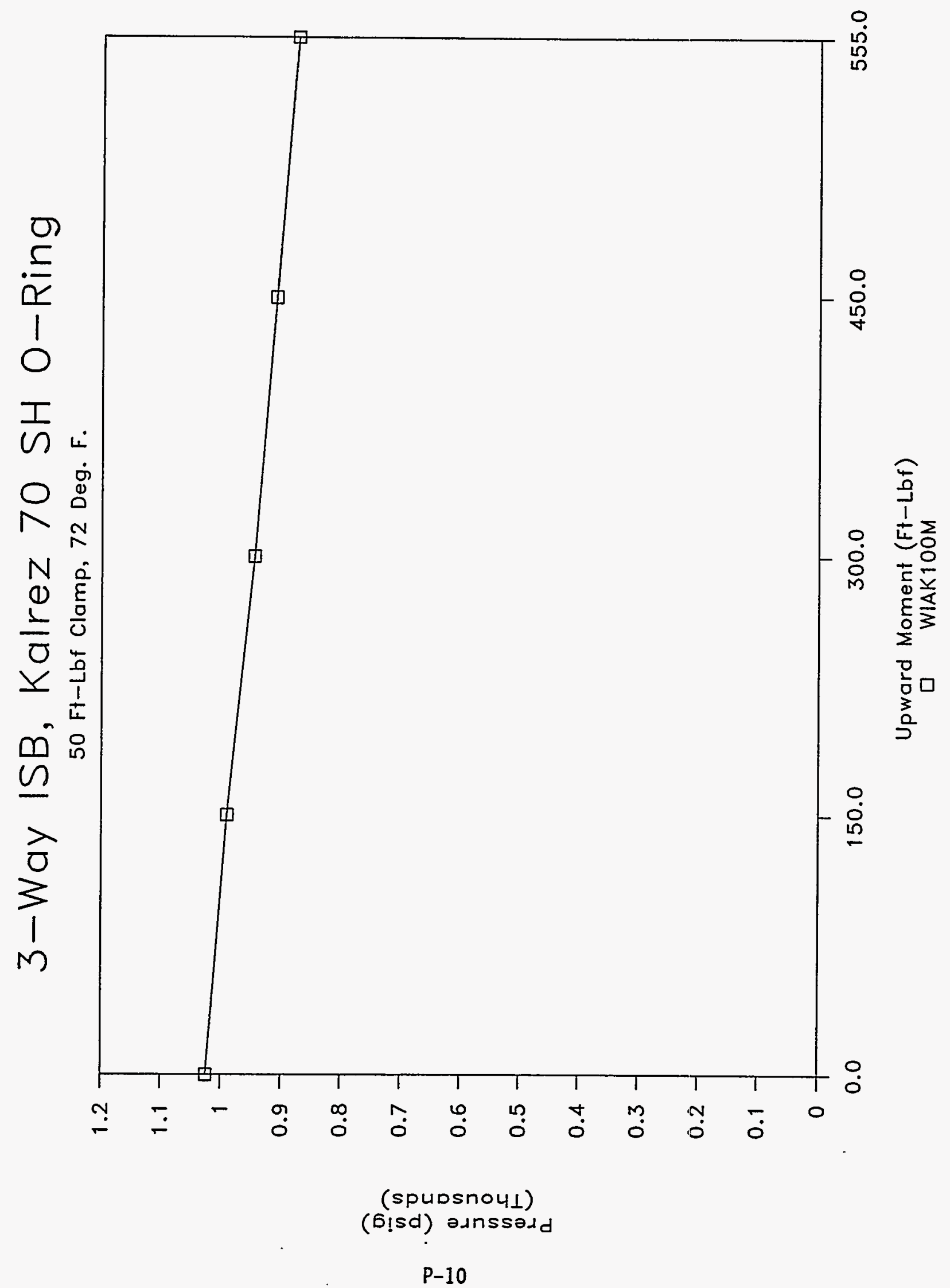


DECEMBER 02, 1994

2" 3-WAY ISB CONNECTOR, KALREZ O-RING, 70 DUROMETER, AMBIENT TEMP. PART \# AS-568A K\# 119, DUPONT CO., CURE DATE 04/18/94

LEAK TEST - UP AND OVER MOMENT (UPWARD ROTATION)

CLAMPING TORQUE $=50$ FT-IBF NOMINAL

CHARGE PRESSURE $=250$ PSIG

GRAPH NAME $=$ WIAK25OM

TEMPERATURE: 72 DEG. F.

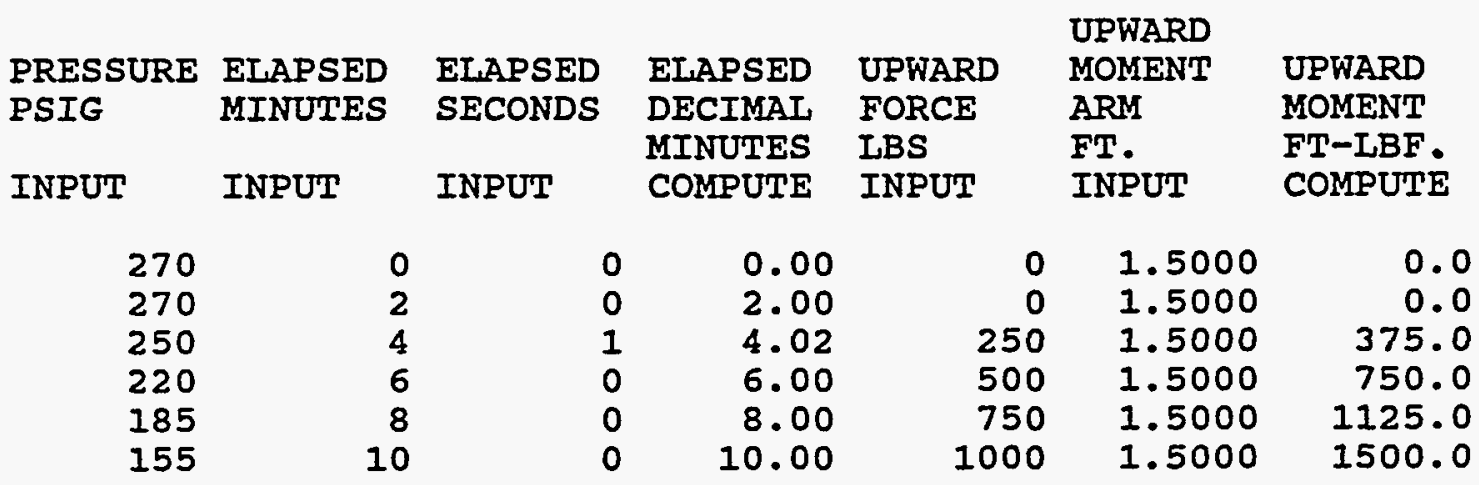

INCREASED PRESSURE TO 500 PSIG

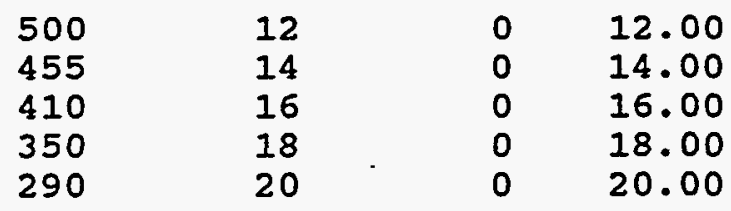

INCREASED PRESSURE TO 750 PSIG

$\begin{array}{ll}750 & 22 \\ 700 & 24 \\ 655 & 26 \\ 595 & 28 \\ 530 & 30\end{array}$

$0 \quad 22.00$

$0 \quad 24.00$

$0 \quad 26.00$

$0 \quad 28.00$

$0 \quad 30.00$
GRAPH NAME = WIAK50OM

$\begin{array}{rrr}0 & 1.5000 & 0.0 \\ 200 & 1.5000 & 300.0 \\ 400 & 1.5000 & 600.0 \\ 600 & 1.5000 & 900.0 \\ 800 & 1.5000 & 1200.0\end{array}$

INCREASED PRESSURE TO 1000 PSIG

$\begin{array}{rr}1025 & 32 \\ 990 & 34 \\ 945 & 36 \\ 910 & 38 \\ 875 & 40\end{array}$

$\begin{array}{ll}0 & 32.00 \\ 1 & 34.02 \\ 1 & 36.02 \\ 0 & 38.00 \\ 2 & 40.03\end{array}$

GRAPH NAME = WIAK10OM

$\begin{array}{rrr}0 & 1.5000 & 0.0 \\ 100 & 1.5000 & 150.0 \\ 200 & 1.5000 & 300.0 \\ 300 & 1.5000 & 450.0 \\ 370 & 1.5000 & 555.0\end{array}$




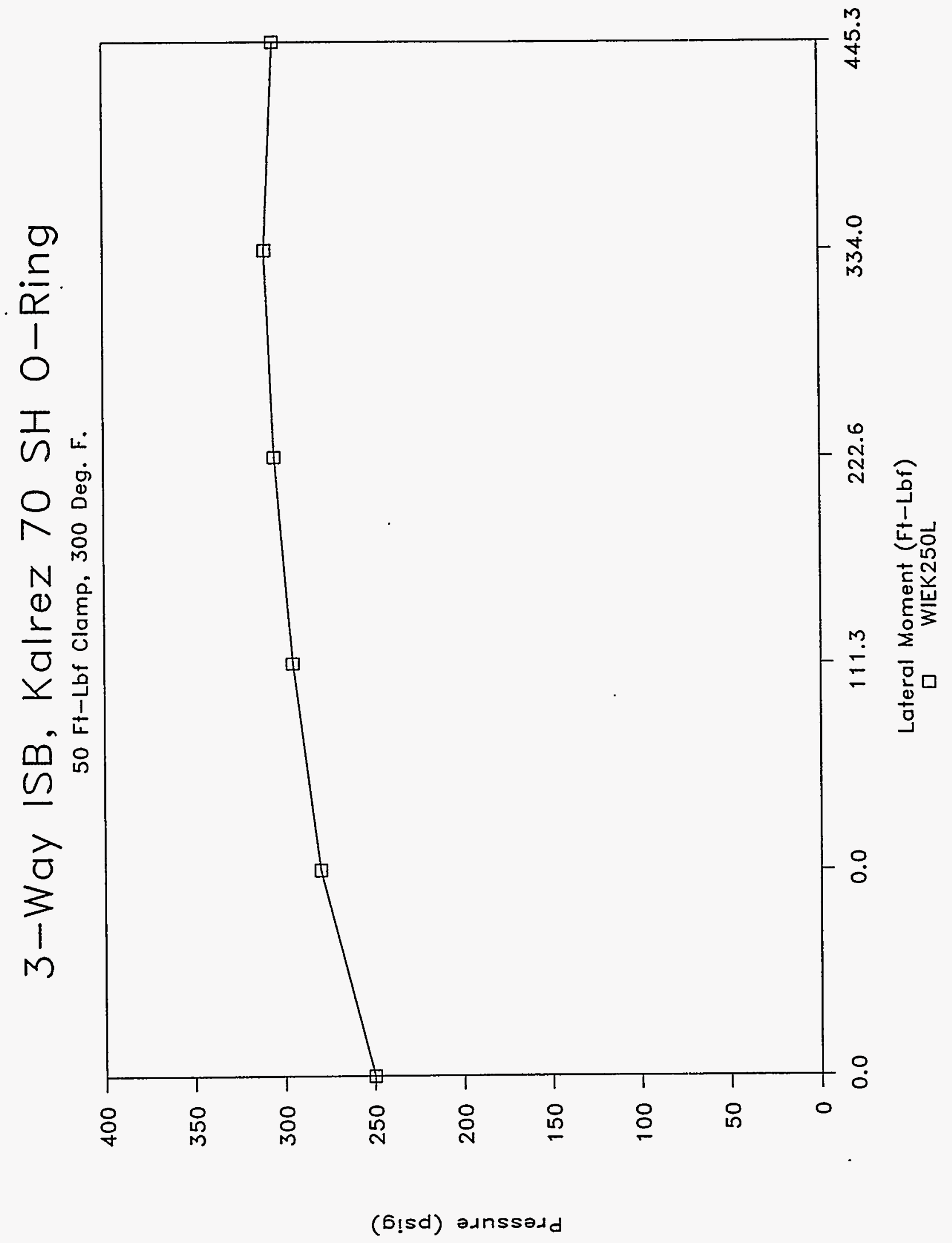




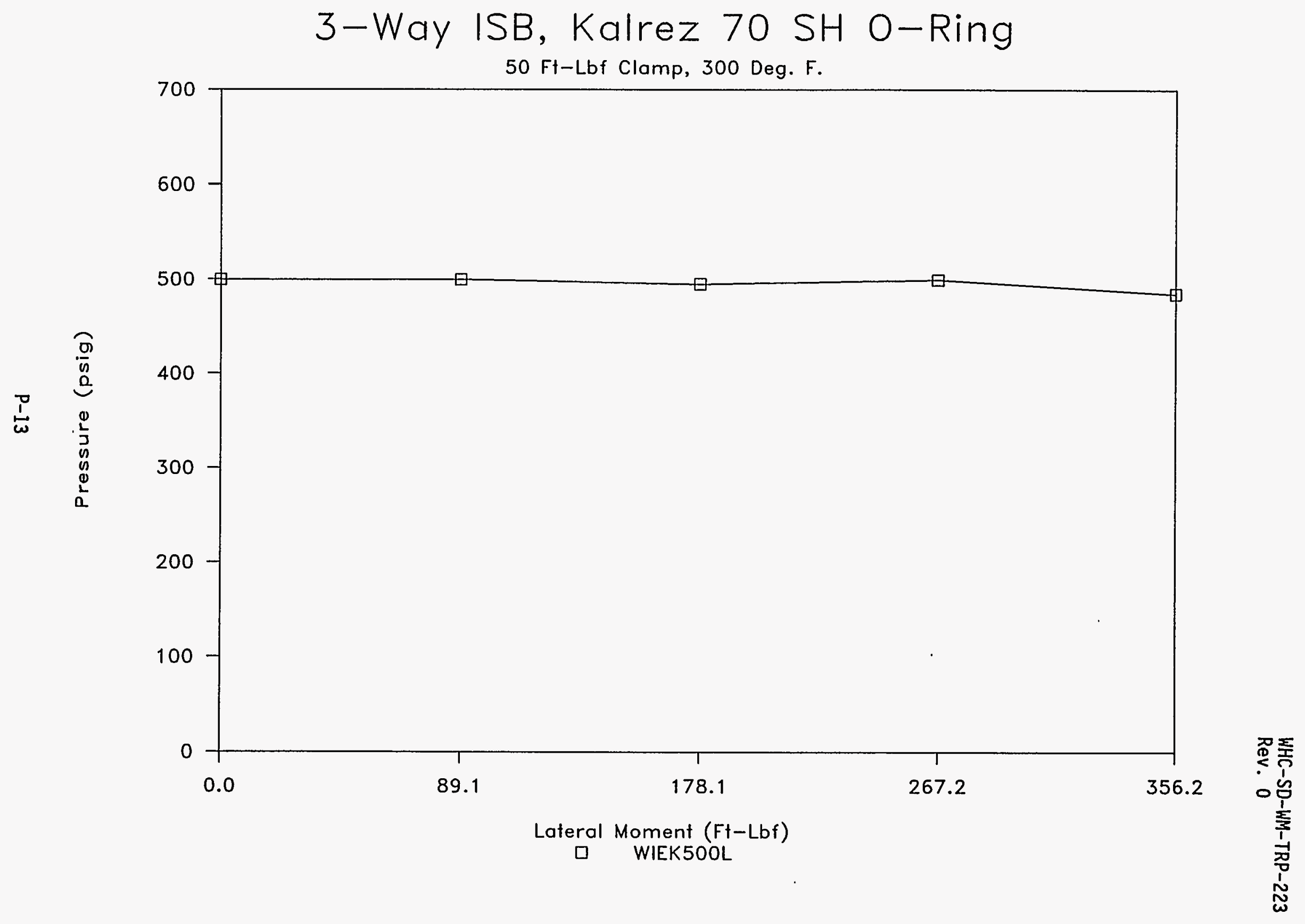


WHC-SD-WM-TRP-223

Rev. 0

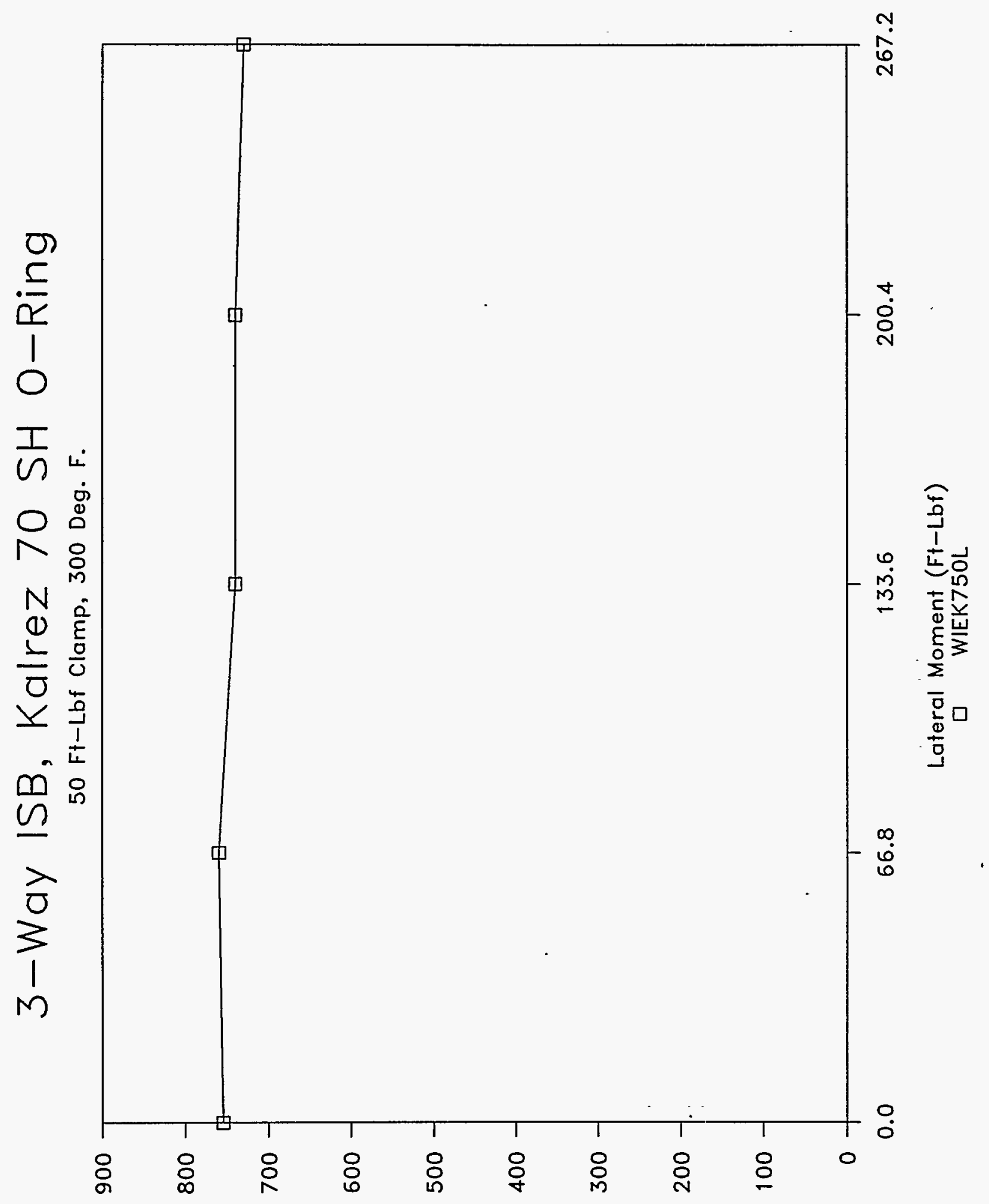

(bisd) annssadd

P-14 


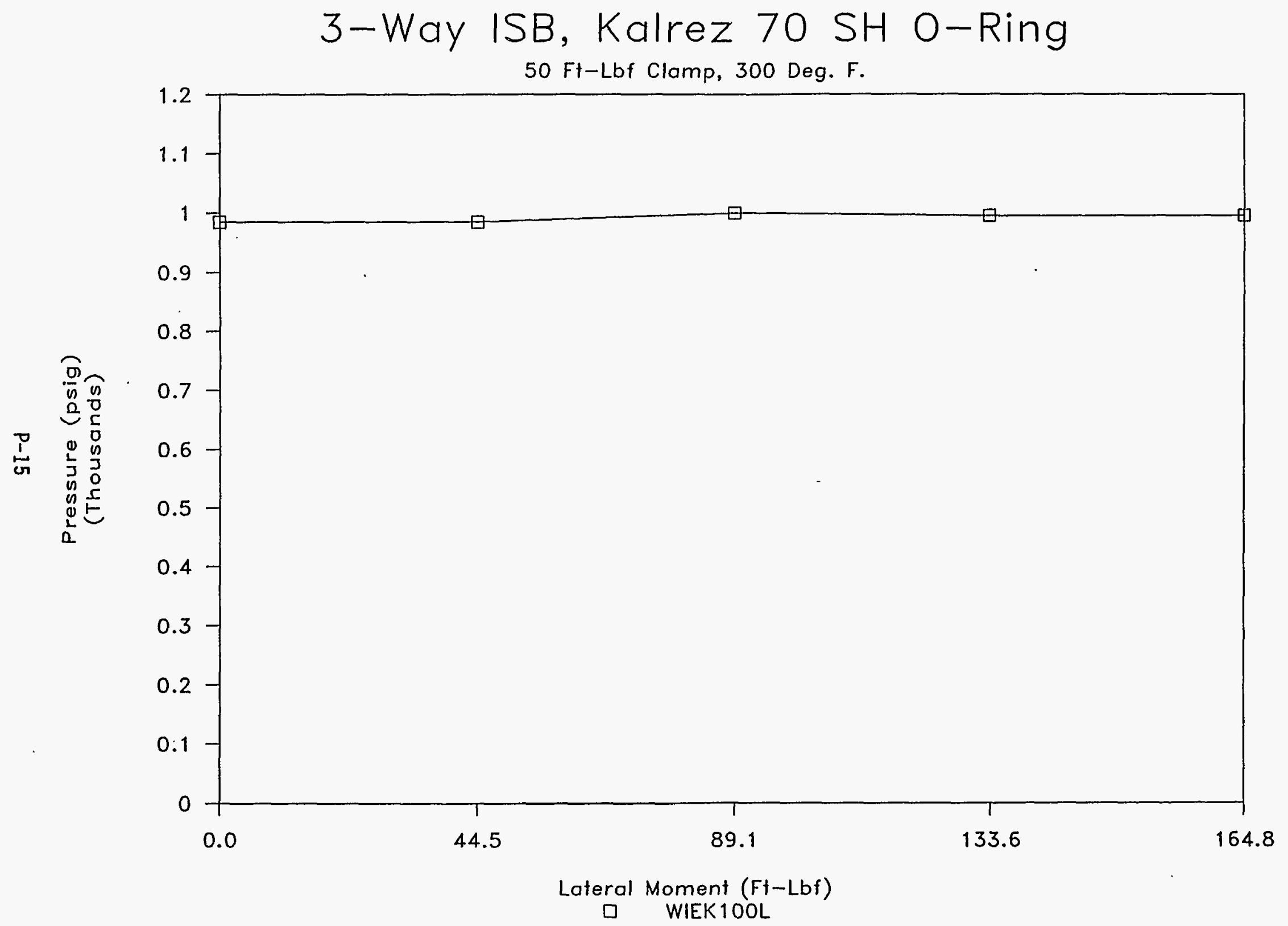


DECEMBER 12, 1994

2" 3-WAY ISB CONNECTOR, . KALREZ O-RING, 70 DUROMETER, ELEVATED TEMP. PART \# AS-568A K\# 119, DUPONT CO., CURE DATE 04/18/94

IEAK TEST - LATERAL MOMENT (SIDEWAYS ROTATION)

CLAMPING TORQUE $=50$ FT-LBF NOMINAL

CHARGE PRESSURE $=250$ PSIG

GRAPH NAME $=$ WIEK250L

TEMPERATURE: 300 DEG. F.

\begin{tabular}{|c|c|c|c|c|c|c|}
\hline $\begin{array}{l}\text { PRESSURE } \\
\text { PSIG }\end{array}$ & $\begin{array}{l}\text { ELAPSED } \\
\text { MINUTES }\end{array}$ & $\begin{array}{l}\text { ELAPSED } \\
\text { SECONDS }\end{array}$ & $\begin{array}{l}\text { ELAPSED } \\
\text { DECIMAI } \\
\text { MINUTES } \\
\text { COMPUTE }\end{array}$ & $\begin{array}{l}\text { IATERAL } \\
\text { FORCE } \\
\text { LBS } \\
\text { INPUT }\end{array}$ & $\begin{array}{l}\text { LATERAL } \\
\text { MOMENT } \\
\text { ARM } \\
\text { FT. } \\
\text { INPUT }\end{array}$ & $\begin{array}{l}\text { IATERAL } \\
\text { MOMENT } \\
\text { FT-LBF. } \\
\text { COMPUTE }\end{array}$ \\
\hline NPUT & INPUT & INPUT & COMPUTE & INPUT & INPU'T & MPUTE \\
\hline $\begin{array}{l}250 \\
280 \\
295 \\
305 \\
310 \\
305\end{array}$ & $\begin{array}{r}0 \\
2 \\
4 \\
6 \\
8 \\
10\end{array}$ & & $\begin{array}{r}0.00 \\
2.00 \\
4.00 \\
6.00 \\
8.00 \\
10.00\end{array}$ & $\begin{array}{r}0 \\
0 \\
250 \\
500 \\
750 \\
1000\end{array}$ & $\begin{array}{l}0.4453 \\
0.4453 \\
0.4453 \\
0.4453 \\
0.4453 \\
0.4453\end{array}$ & $\begin{array}{r}0.0 \\
0.0 \\
111.3 \\
222.7 \\
334.0 \\
445.3\end{array}$ \\
\hline
\end{tabular}

INCREASED PRESSURE TO 500 PSIG

$\begin{array}{llll}500 & 12 & 0 & 12.00 \\ 500 & 14 & 0 & 14.00 \\ 495 & 16 & 0 & 16.00 \\ 500 & 18 & 0 & 18.00 \\ 485 & 20 & 0 & 20.00\end{array}$

INCREASED PRESSURE TO 750 PSIG

$\begin{array}{llll}755 & 22 & 0 & 22.00 \\ 760 & 24 & 0 & 24.00 \\ 740 & 26 & 0 & 26.00 \\ 740 & 28 & 0 & 28.00 \\ 730 & 30 & 0 & 30.00\end{array}$

INCREASED PRESSURE TO 1000 PSIG

$\begin{array}{rr}985 & 32 \\ 985 & 34 \\ 1000 & 36 \\ 995 & 38 \\ 995 & 40\end{array}$

GRAPH NAME = WIEK50OL

$\begin{array}{rrr}0 & 0.4453 & 0.0 \\ 200 & 0.4453 & 89.1 \\ 400 & 0.4453 & 178.1 \\ 600 & 0.4453 & 267.2 \\ 800 & 0.4453 & 356.2\end{array}$

GRAPH NAME = WIEK750L

$\begin{array}{rrr}0 & 0.4453 & 0.0 \\ 150 & 0.4453 & 66.8 \\ 300 & 0.4453 & 133.6 \\ 450 & 0.4453 & 200.4 \\ 600 & 0.4453 & 267.2\end{array}$

GRAPH NAME $=$ WIEKIOOL

$\begin{array}{rrr}0 & 0.4453 & 0.0 \\ 100 & 0.4453 & 44.5 \\ 200 & 0.4453 & 89.1 \\ 300 & 0.4453 & 133.6 \\ 370 & 0.4453 & 164.8\end{array}$


WHC-SD-WM-TRP-223

Rev. 0

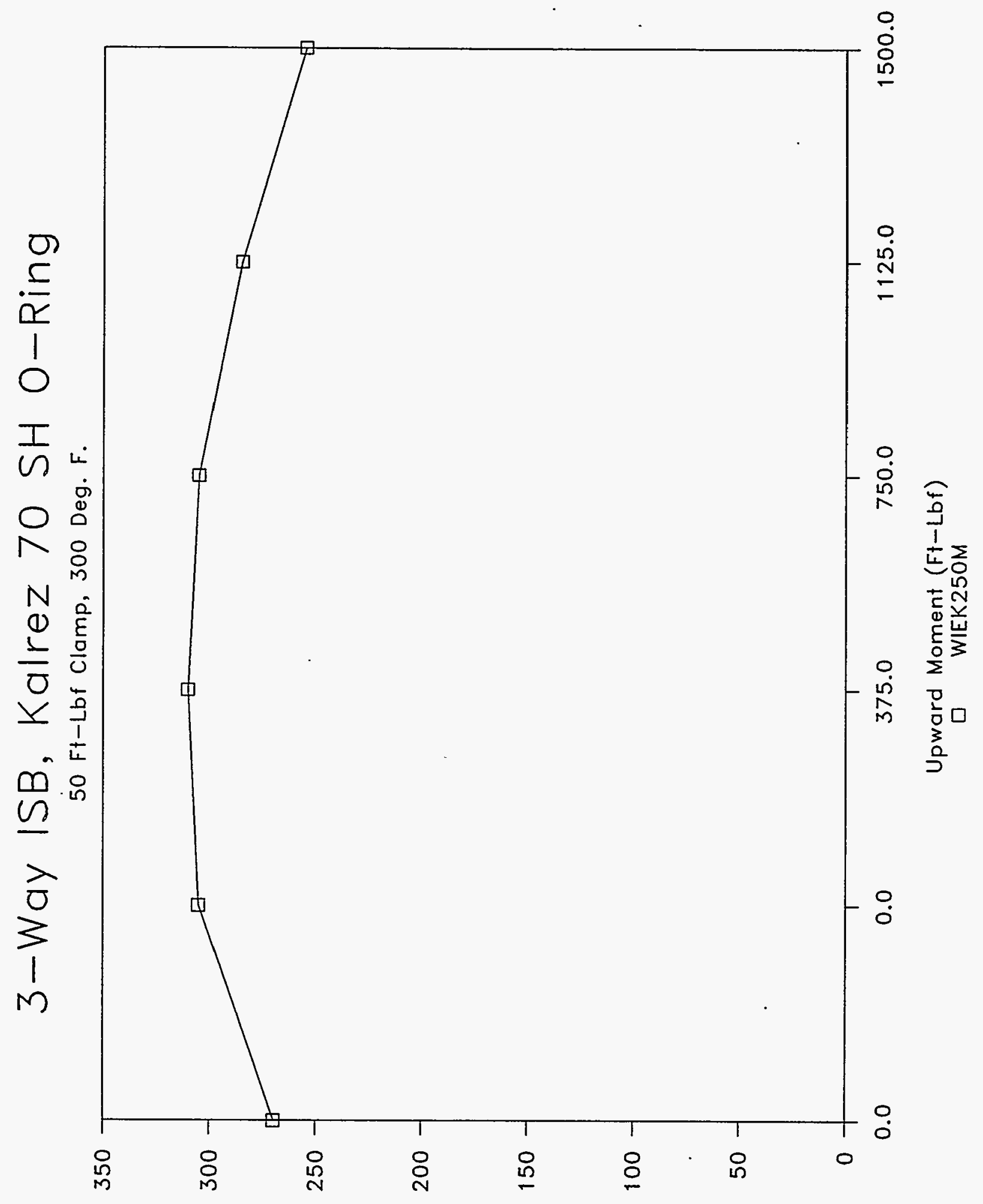

(bisd) ounssadd

P-17 


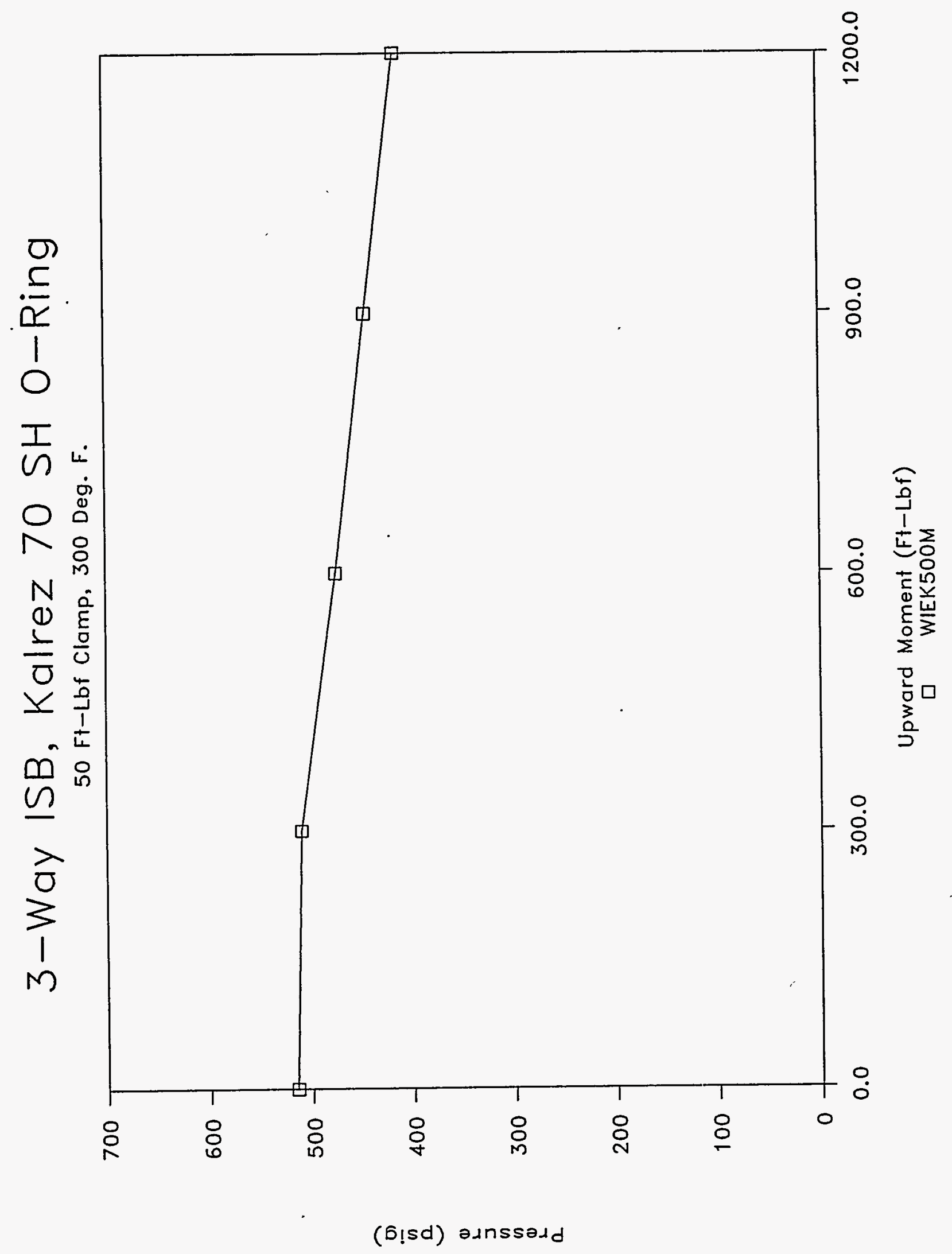




\section{3-Way ISB, Kalrez 70 SH O-Ring}

$50 \mathrm{Ft}$-Lbf Clamp, 300 Deg. F.

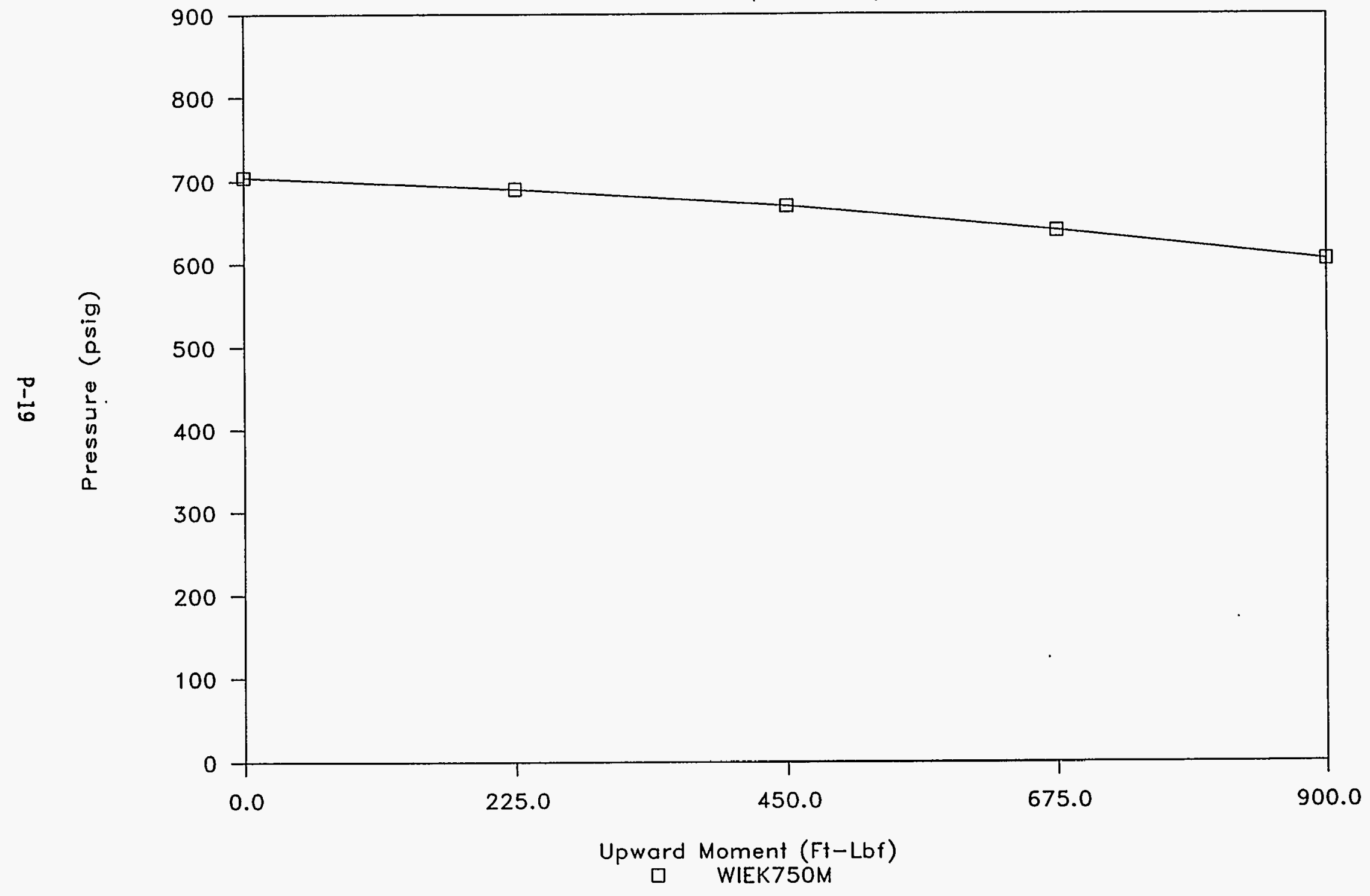


Rev. 0

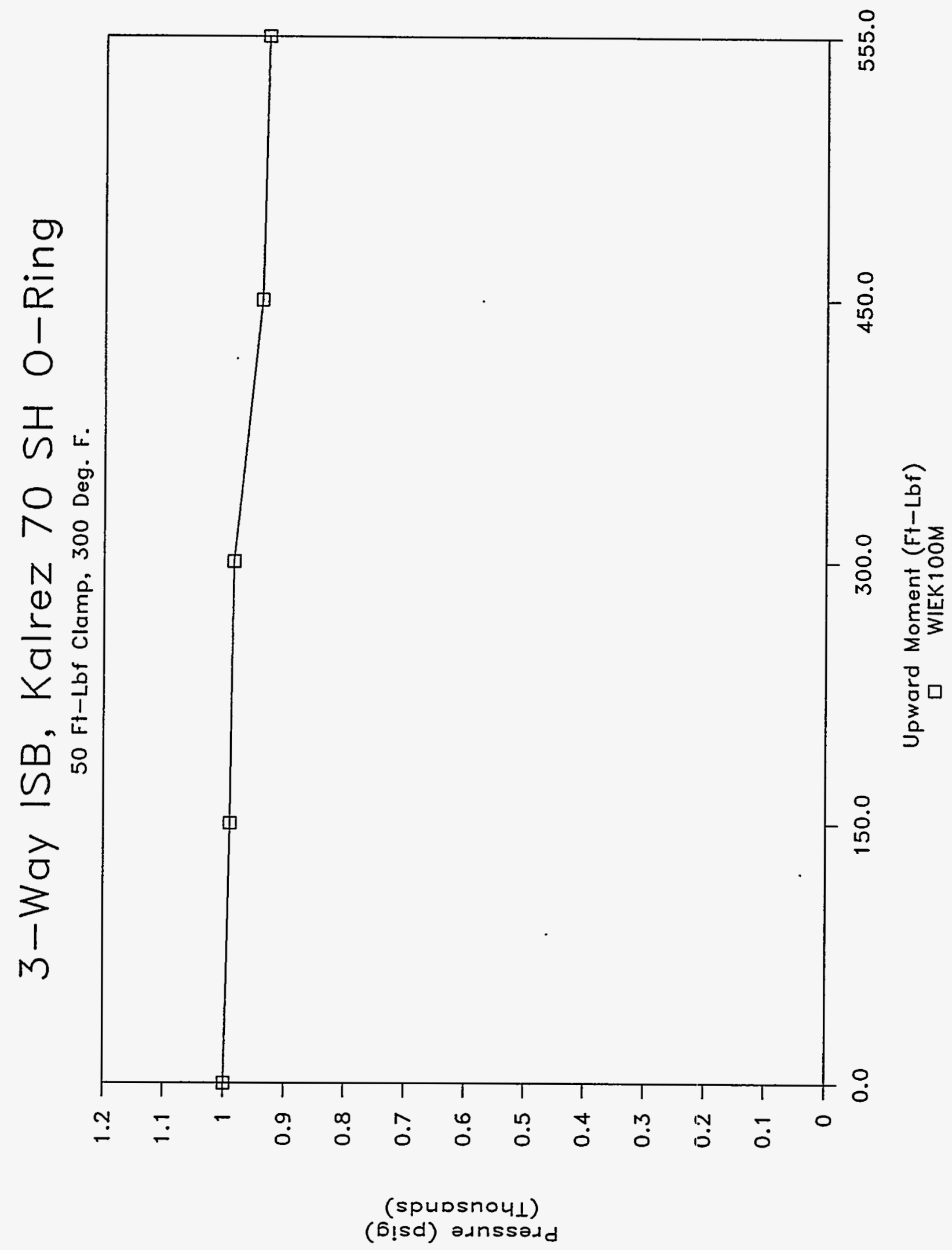

$\mathrm{P}-20$ 
DECEMBER 02, 1994

2" 3-WAY ISB CONNECTOR, KALREZ O-RING, 70 DUROMETER, ELEVATED TEMP. PART \# AS-568A K\# 119, DUPONT CO., CURE DATE 04/18/94

LEAK TEST - UP AND OVER MOMENT (UPWARD ROTATION)

CLAMPING TORQUE $=50$ FT-LBF NOMINAI

CHARGE PRESSURE $=250$ PSIG

GRAPH NAME = WIEK250M

TEMPERATURE: 300 DEG. F.

$\begin{array}{lllllll} & & & & & \text { UPWARD } & \\ \text { PRESSURE } & \text { ELAPSED } & \text { ELAPSED } & \text { ELAPSED } & \text { UPWARD } & \text { MOMENT } & \text { UPWARD } \\ \text { PSIG } & \text { MINUTES } & \text { SECONDS } & \text { DECIMAL } & \text { FORCE } & \text { ARM } & \text { MOMENT } \\ & & & \text { MINUTES } & \text { IBS } & \text { FT. } & \text { FT-IBF. } \\ \text { INPUT } & \text { INPUT } & \text { INPUT } & \text { COMPUTE } & \text { INPUT } & \text { INPUT } & \text { COMPUTE }\end{array}$

$\begin{array}{rrrrrrr}270 & 0 & 0 & 0.00 & 0 & 1.5000 & 0.0 \\ 305 & 2 & 0 & 2.00 & 0 & 1.5000 & 0.0 \\ 310 & 4 & 0 & 4.00 & 250 & 1.5000 & 375.0 \\ 305 & 6 & 0 & 6.00 & 500 & 1.5000 & 750.0 \\ 285 & 8 & 0 & 8.00 & 750 & 1.5000 & 1125.0 \\ 255 & 10 & 0 & 10.00 & 1000 & 1.5000 & 1500.0\end{array}$

INCREASED PRESSURE TO 500 PSIG

GRAPH NAME = WIEK50OM

$\begin{array}{rrrrrrr}515 & 12 & 0 & 12.00 & 0 & 1.5000 & 0.0 \\ 510 & 14 & 0 & 14.00 & 200 & 1.5000 & 300.0 \\ 475 & 16 & 0 & 16.00 & 400 & 1.5000 & 600.0 \\ 445 & 18 & 0 & 18.00 & 600 & 1.5000 & 900.0 \\ 415 & 20 & 0 & 20.00 & 800 & 1.5000 & 1200.0\end{array}$

INCREASED PRESSURE TO 750 PSIG

GRAPH NAME $=$ WIEK750M

$\begin{array}{rrrrrrr}705 & 22 & 0 & 22.00 & 0 & 1.5000 & 0.0 \\ 690 & 24 & 0 & 24.00 & 150 & 1.5000 & 225.0 \\ 670 & 26 & 0 & 26.00 & 300 & 1.5000 & 450.0 \\ 640 & 28 & 0 & 28.00 & 450 & 1.5000 & 675.0 \\ 605 & 30 & 0 & 30.00 & 600 & 1.5000 & 900.0\end{array}$

INCREASED PRESSURE TO 1000 PSIG

\begin{tabular}{|c|c|c|c|c|c|c|}
\hline $\begin{array}{r}1000 \\
990 \\
985 \\
940 \\
930\end{array}$ & $\begin{array}{l}32 \\
34 \\
36 \\
38 \\
40\end{array}$ & $\begin{array}{l}0 \\
0 \\
0 \\
0 \\
0\end{array}$ & $\begin{array}{l}32.00 \\
34.00 \\
36.00 \\
38.00 \\
40.00\end{array}$ & $\begin{array}{r}0 \\
100 \\
200 \\
300 \\
370\end{array}$ & $\begin{array}{l}1.5000 \\
1.5000 \\
1.5000 \\
1.5000 \\
1.5000\end{array}$ & $\begin{array}{r}0.0 \\
150.0 \\
300.0 \\
450.0 \\
555.0\end{array}$ \\
\hline
\end{tabular}


WHC-SD-WM-TRP-223

Rev. 0

APPENDIX Q: GRAPHS OF THREE-WAY VITON TESTS 


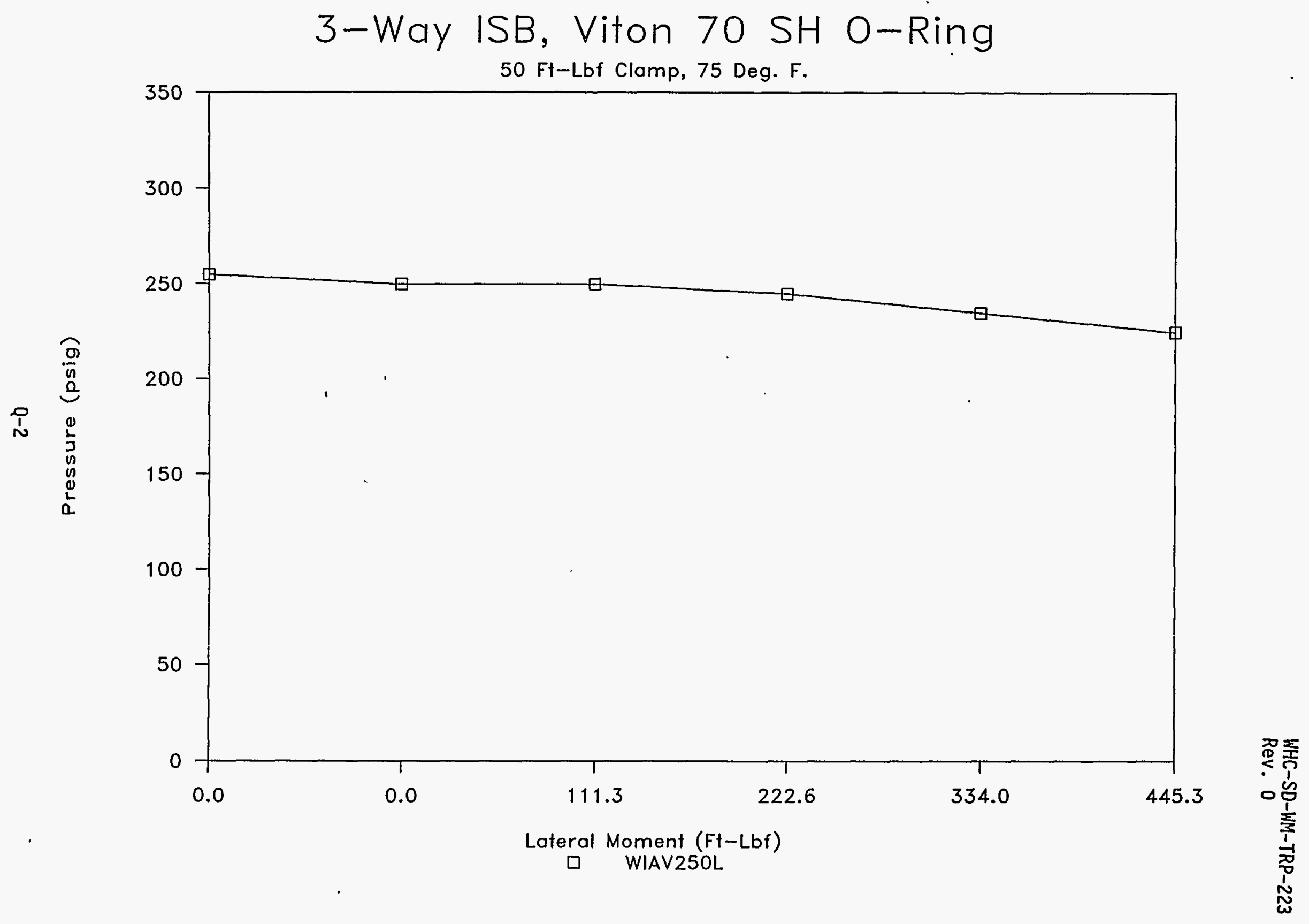


WHC-SD-WM-TRP-223

Rev. 0

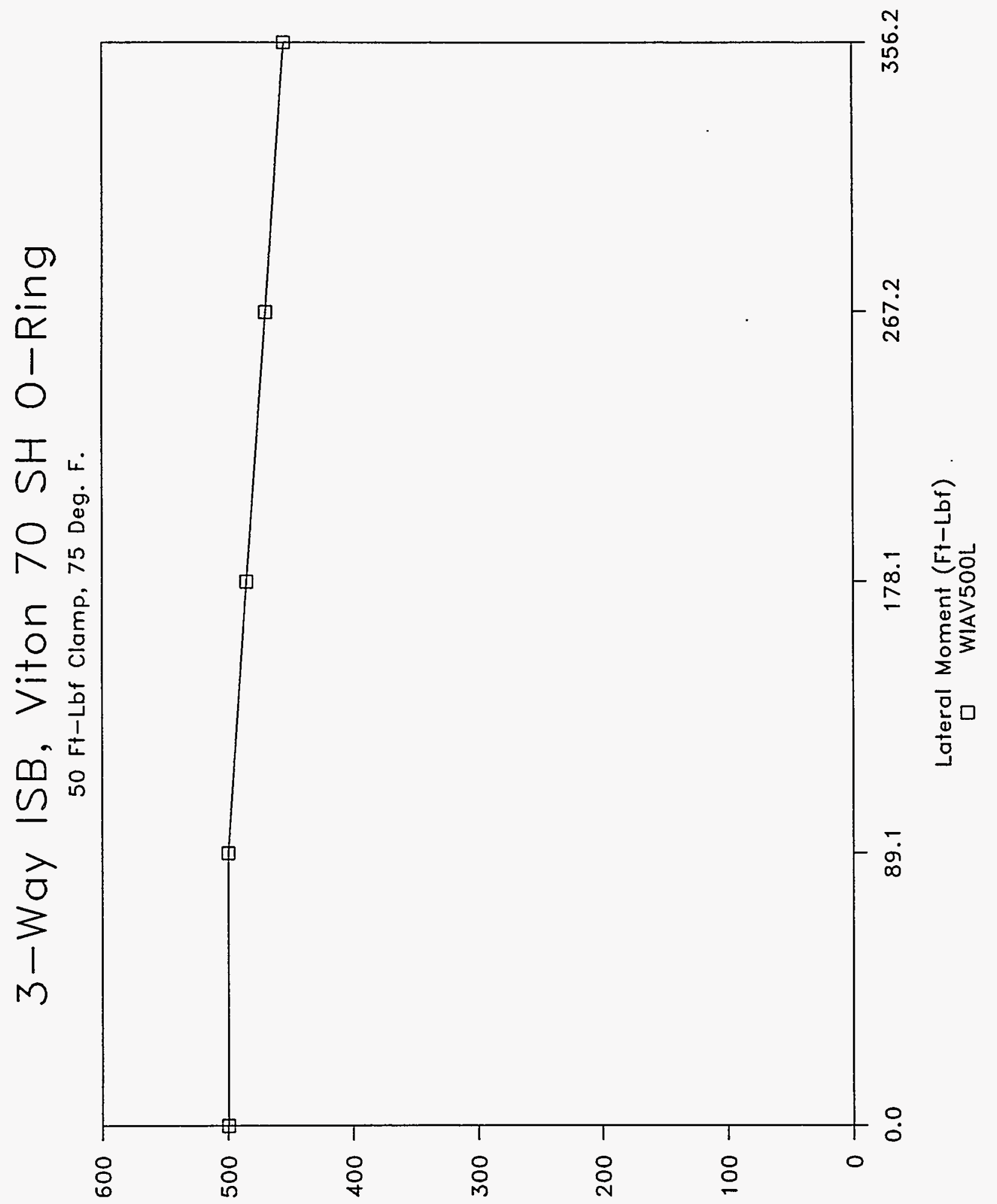

(b!sd) asnssadd 


\section{3-Way ISB, Viton $70 \mathrm{SH} \mathrm{O-Ring}$}

$50 \mathrm{Ft}$-Lbf Clamp, 75 Deg. F.

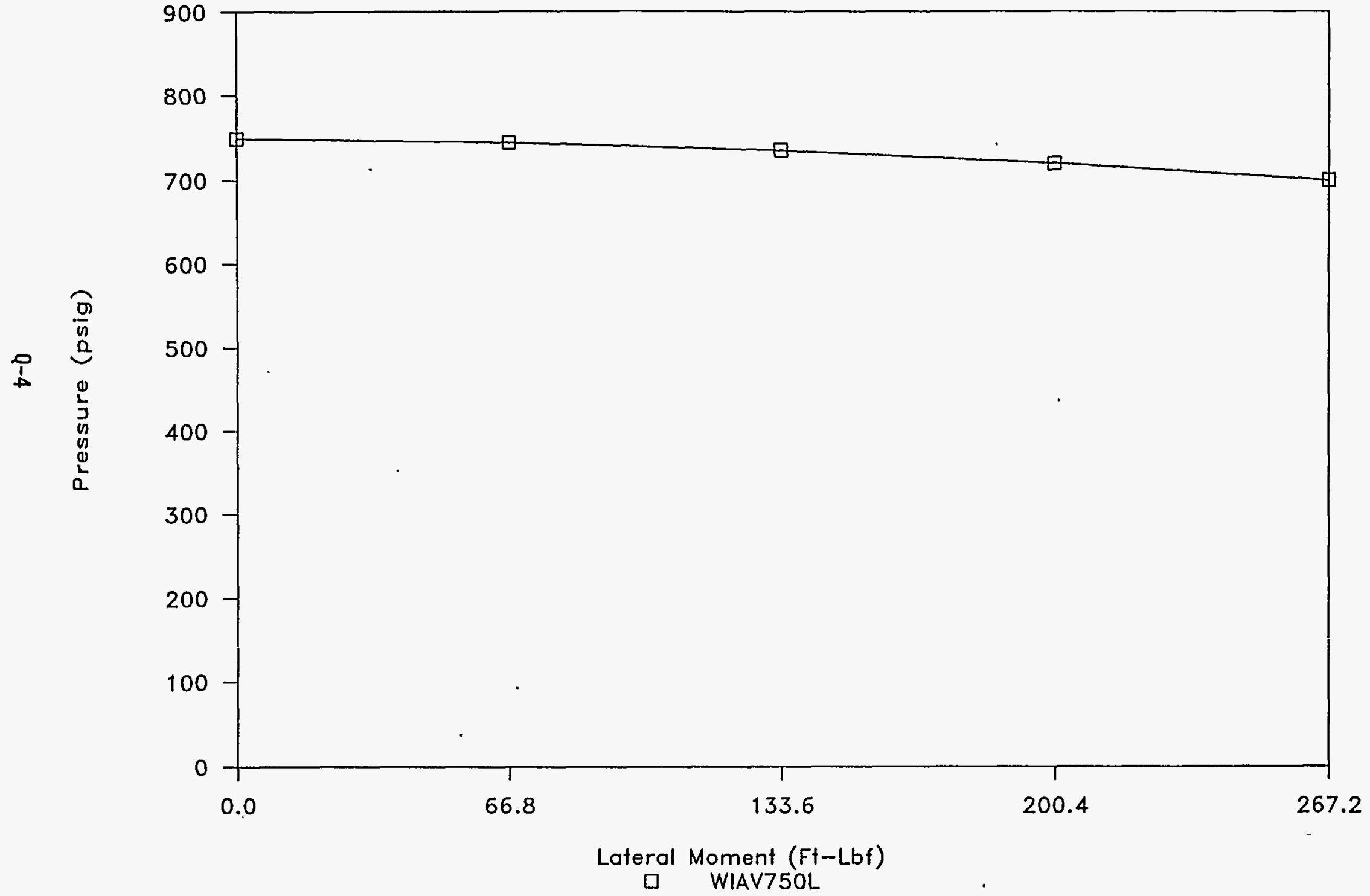


WHC-SD-WM-TRP-223

Rev. 0

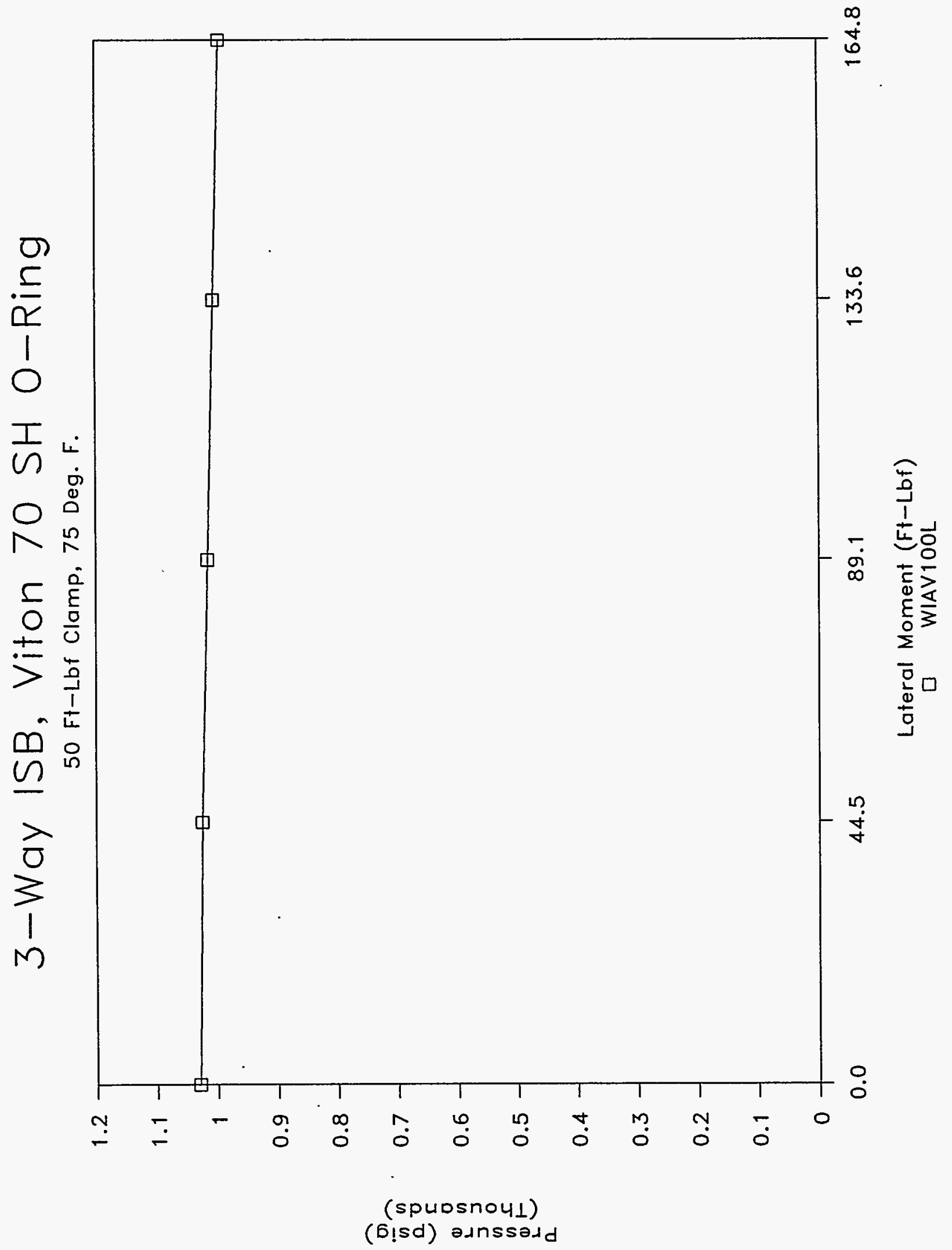


DECEMBER 06, 1994

2" 3-WAY ISB CONNECTOR, VITON O-RING, 70 DUROMETER, AMBIENT TEMP. PART \# 2-142 V884-75, PARKER SEAL CO., BATCH 3119B6, CURE DATE 3093 LEAK TEST - I.ATERAL MOMENT (SIDEWAYS ROTATION)

CLAMPING TORQUE $=50$ FT-LBF NOMINAI

CHARGE PRESSURE $=250$ PSIG

GRAPH NAME = WIAV250I

TEMPERATURE: 75 DEG. F.

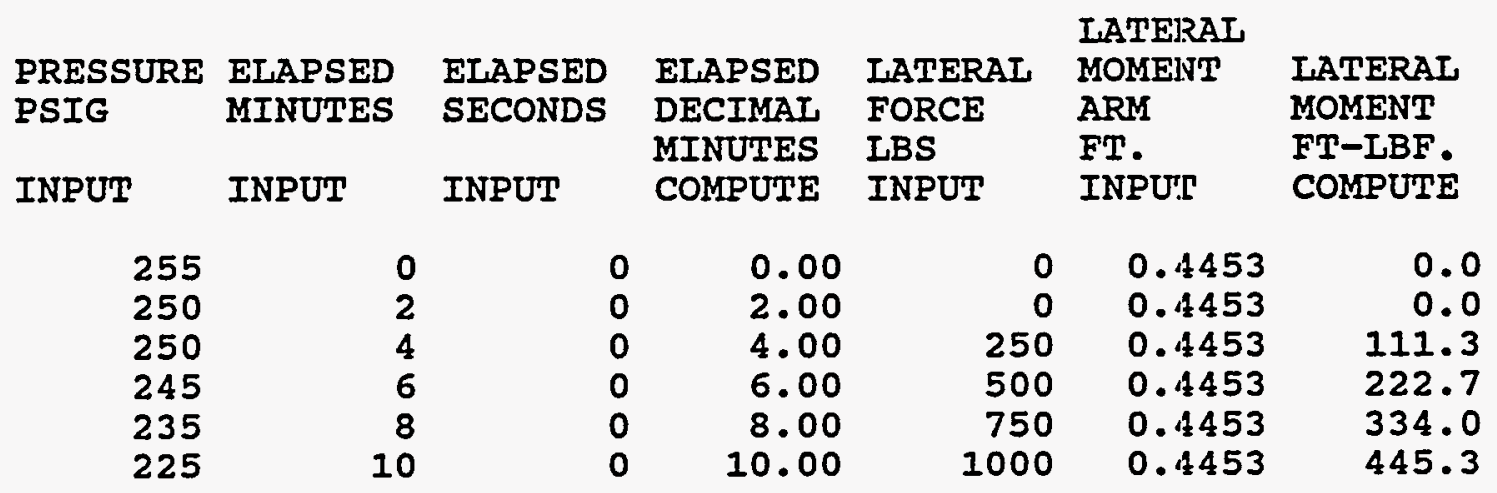

INCREASED PRESSURE TO 500 PSIG

$\begin{array}{llll}500 & 12 & 0 & 12.00 \\ 500 & 14 & 0 & 14.00 \\ 485 & 16 & 0 & 16.00 \\ 470 & 18 & 0 & 18.00 \\ 455 & 20 & 0 & 20.00\end{array}$

INCREASED PRESSURE TO 750 PSIG

$\begin{array}{llll}750 & 22 & 0 & 22.00 \\ 745 & 24 & 0 & 24.00 \\ 735 & 26 & 0 & 26.00 \\ 720 & 28 & 0 & 28.00 \\ 700 & 30 & 0 & 30.00\end{array}$

INCREASED PRESSURE TO 1000 PSIG

$\begin{array}{rlll}1030 & 32 & 0 & 32.00 \\ 1025 & 34 & 0 & 34.00 \\ 1015 & 36 & 0 & 36.00 \\ 1005 & 38 & 0 & 38.00 \\ 995 & 40 & 0 & 40.00\end{array}$

GRAPH NAME = WIAV50OI

$\begin{array}{rrr}0 & 0.4453 & 0.0 \\ 200 & 0.4453 & 89.1 \\ 400 & 0.4453 & 178.1 \\ 600 & 0.4453 & 267.2 \\ 800 & 0.4453 & 356.2\end{array}$

GRAPH NAME = WIAV750L

$\begin{array}{rrr}0 & 0.4453 & 0.0 \\ 150 & 0.4453 & 66.8 \\ 300 & 0.4453 & 133.6 \\ 450 & 0.4453 & 200.4 \\ 600 & 0.4453 & 267.2\end{array}$

GRAPH NAME = WIAV10OL

$\begin{array}{rrr}0 & 0.4453 & 0.0 \\ 100 & 0.4453 & 44.5 \\ 200 & 0.4453 & 89.1 \\ 300 & 0.4453 & 133.6 \\ 370 & 0.4453 & 164.8\end{array}$


WHC-SD-WM-TRP-223

Rev. 0

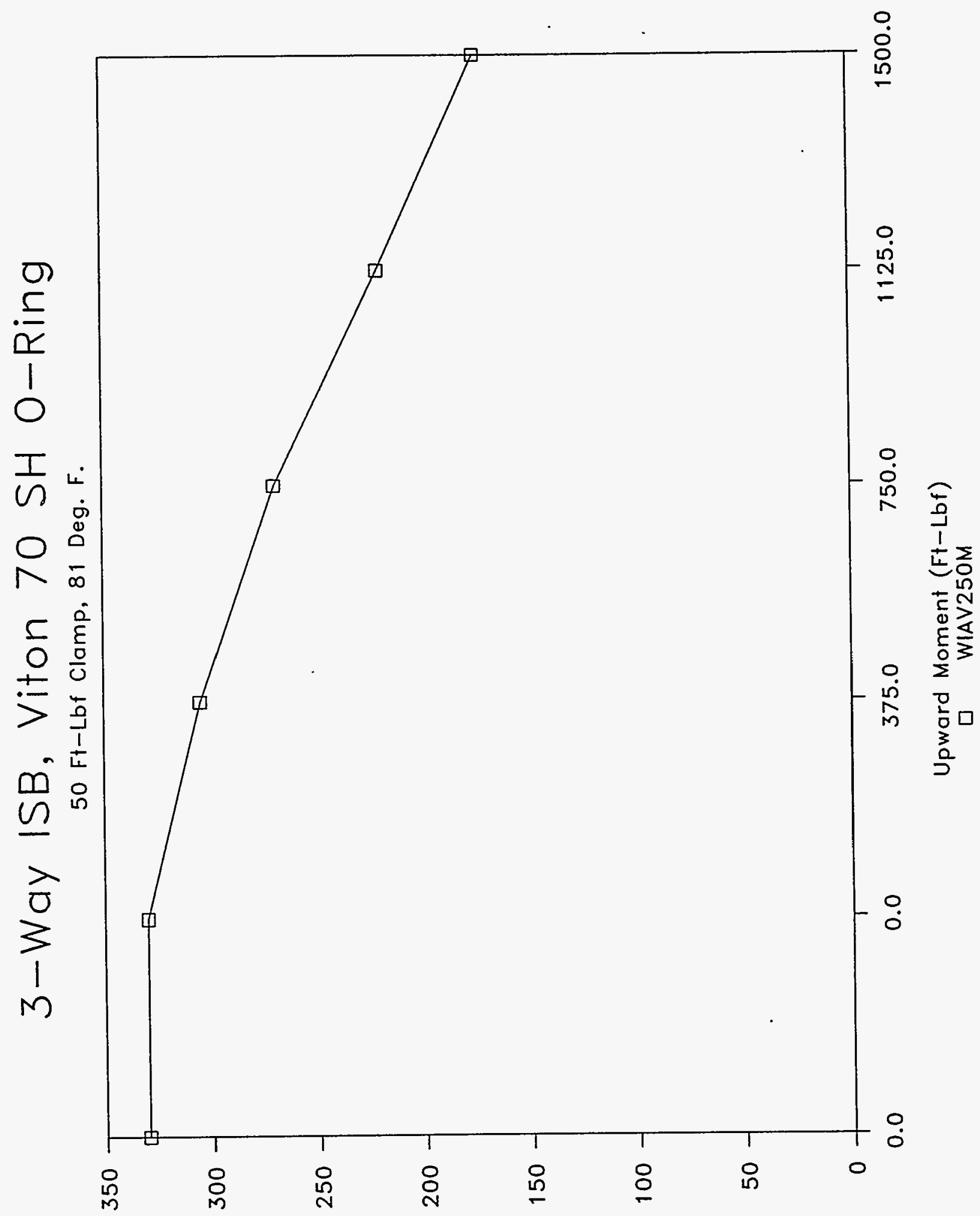

(b!̣d) ounssodd

Q-7 
Rev. 0

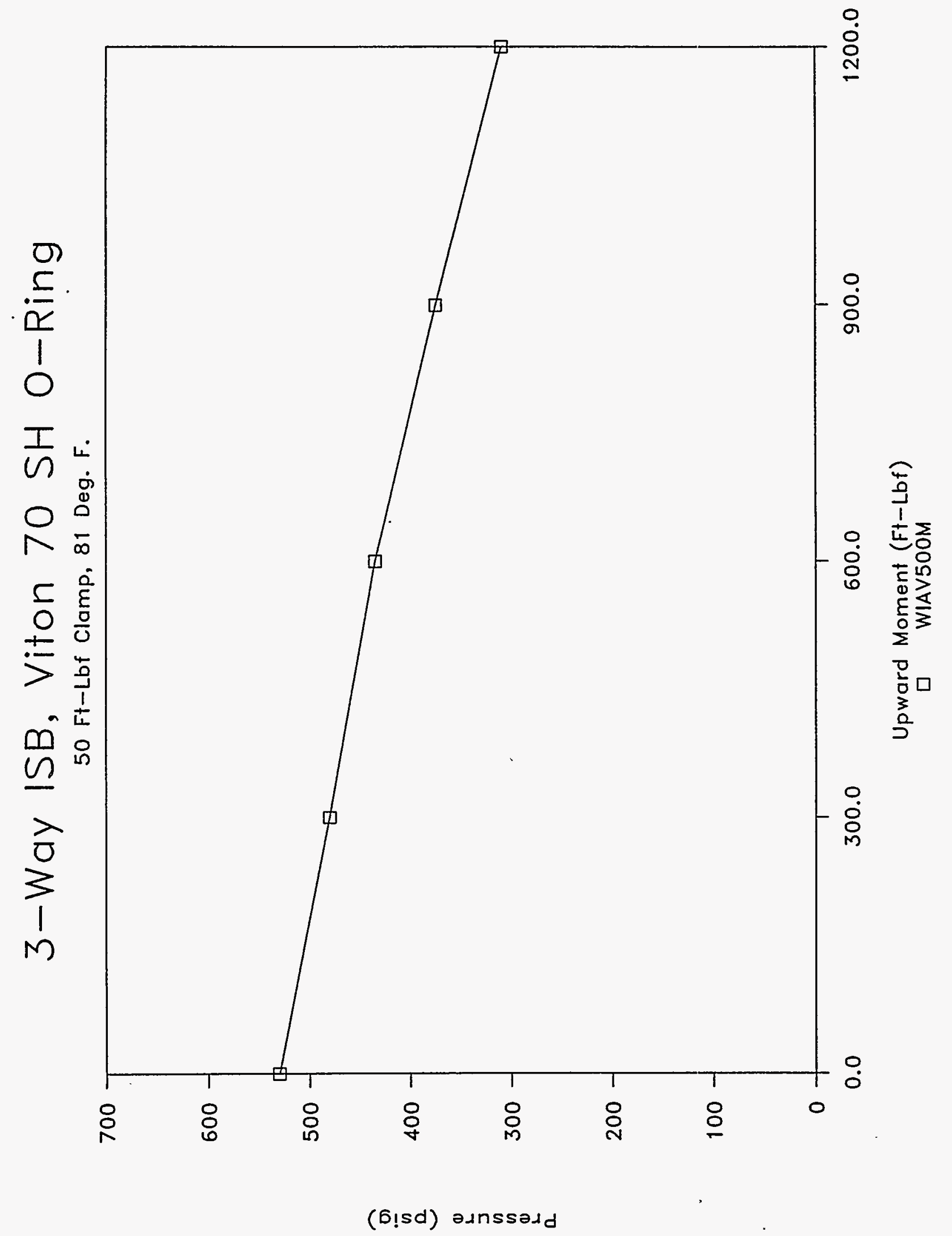


WHC-SD-WM-TRP-223

Rev. 0

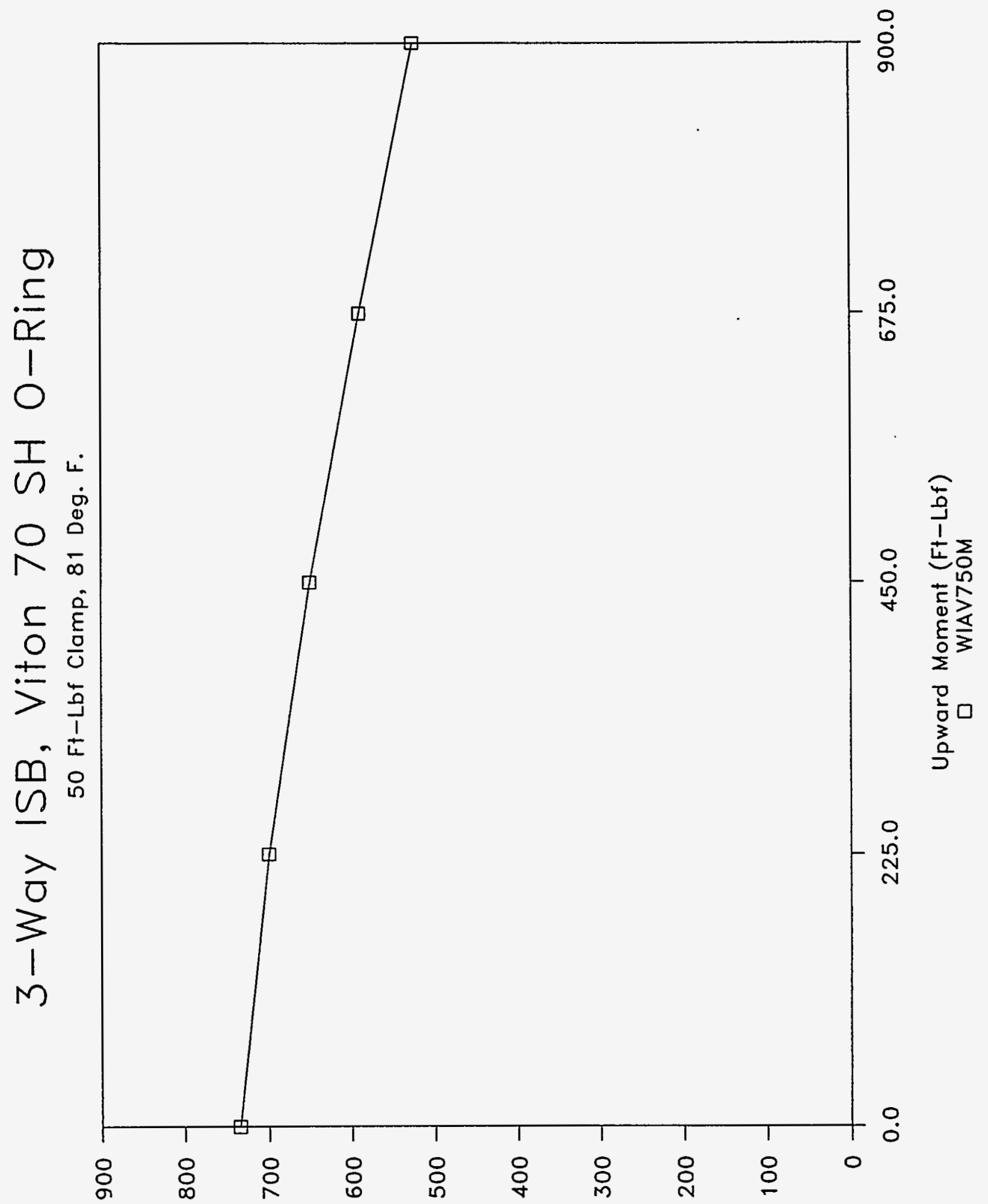

(bisd) osinssadd 
WHC-SD-WM-TRP-223

Rev. 0

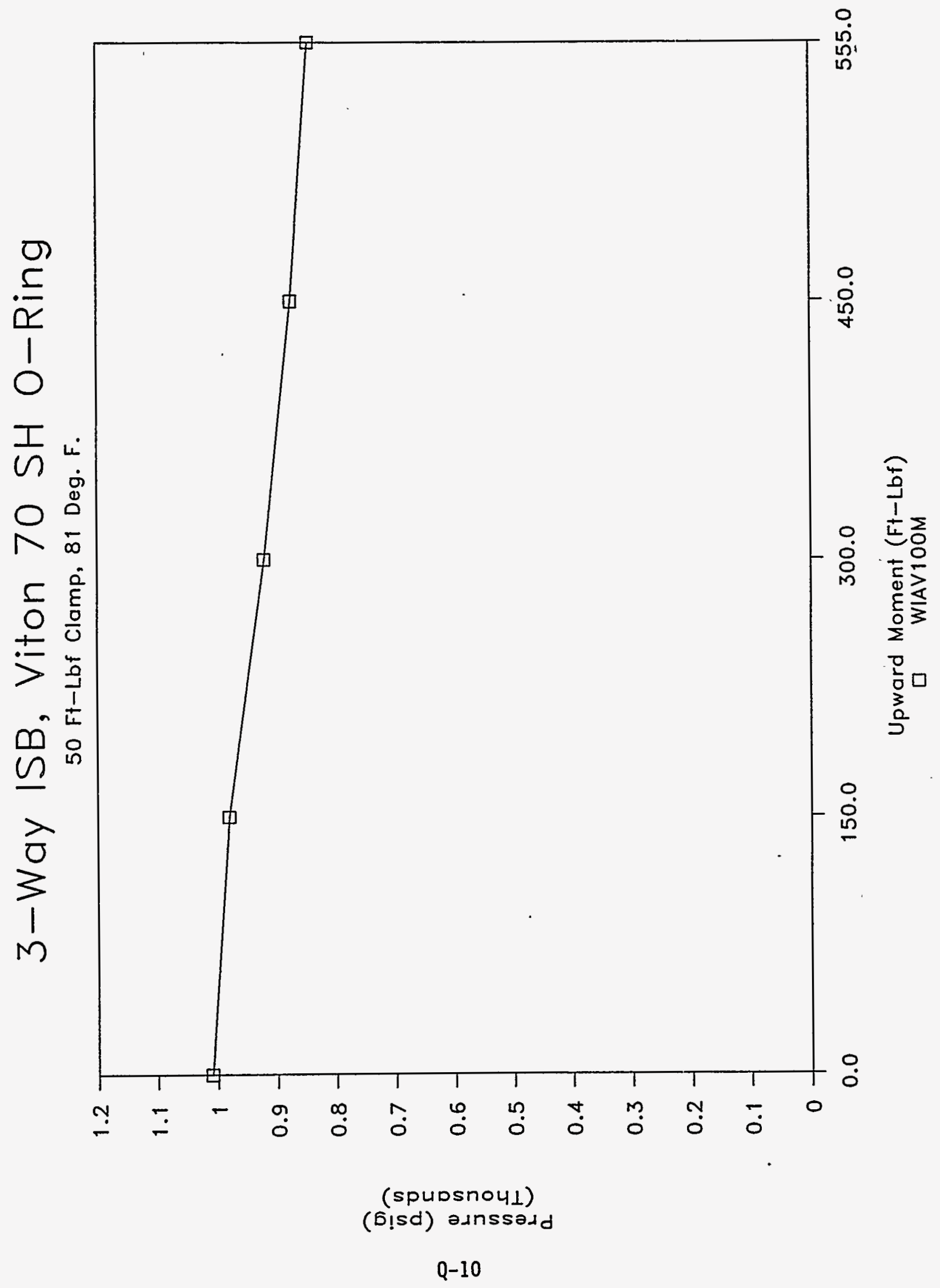


NOVEMBER 28,1994

2" 3-WAY ISB CONNECTOR, VITON O-RING, 70 DUROMETER, AMBIENT TEMP.

PART \# 2-142 V884-75, PARKER SEAL CO,, BATCH 311986, CURE DATE 3293 LEAK TEST - UP AND OVER MOMENT (UPWARD ROTATION)

CLAMPING TORQUE $=50$ FT-LBF NOMINAL CHARGE PRESSURE $=250$ PSIG

GRAPH NAME = WIAV250M TEMPERATURE: 81 DEG. F.

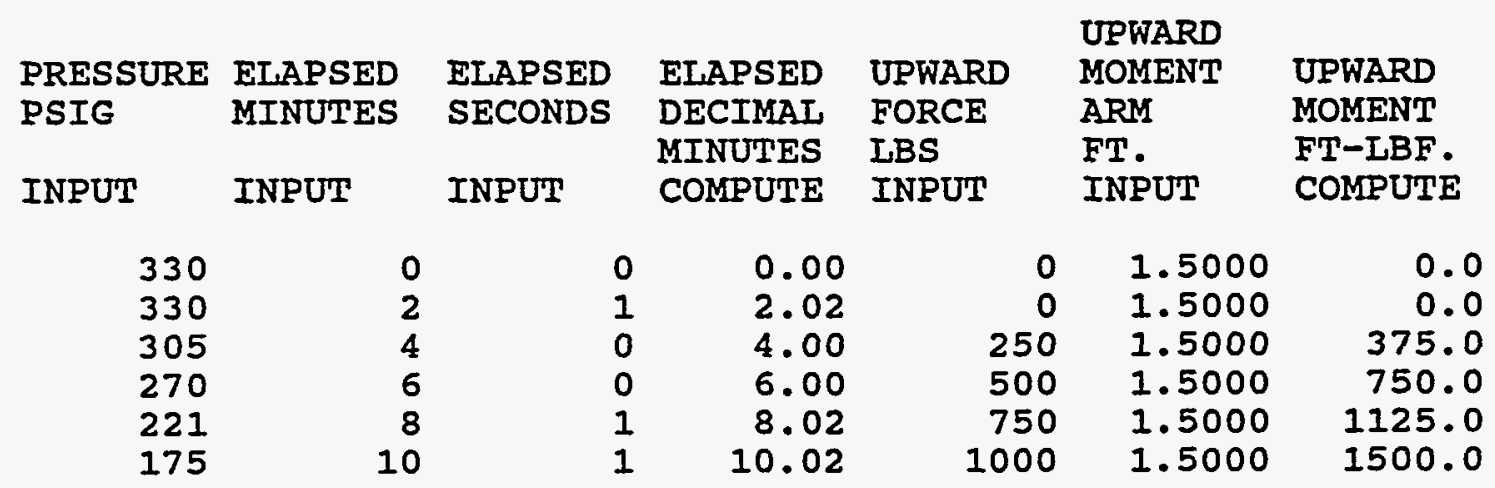

INCREASED PRESSURE TO 500 PSIG

$\begin{array}{ll}530 & 12 \\ 480 & 14 \\ 435 & 16 \\ 375 & 18 \\ 310 & 20\end{array}$

$\begin{array}{ll}1 & 12.02 \\ 0 & 14.00 \\ 0 & 16.00 \\ 0 & 18.00 \\ 0 & 20.00\end{array}$

INCREASED PRESSURE TO 750 PSIG

$\begin{array}{ll}735 & 22 \\ 700 & 24 \\ 650 & 26 \\ 590 & 28 \\ 525 & 30\end{array}$

$\begin{array}{ll}0 & 22.00 \\ 0 & 24.00 \\ 0 & 26.00 \\ 0 & 28.00 \\ 1 & 30.02\end{array}$

$1 \quad 30.02$
INCREASED PRESSURE TO 1000 PSIG

$\begin{array}{rr}1010 & 32 \\ 980 & 34 \\ 920 & 36 \\ 875 & 38 \\ 845 & 40\end{array}$

GRAPH NAME = WIAV5OOM

$\begin{array}{rrr}0 & 1.5000 & 0.0 \\ 200 & 1.5000 & 300.0 \\ 400 & 1.5000 & 600.0 \\ 600 & 1.5000 & 900.0 \\ 800 & 1.5000 & 1200.0\end{array}$

GRAPH NAME $=$ WIAV750M

$\begin{array}{rrr}0 & 1.5000 & 0.0 \\ 150 & 1.5000 & 225.0 \\ 300 & 1.5000 & 450.0 \\ 450 & 1.5000 & 675.0 \\ 600 & 1.5000 & 900.0\end{array}$

GRAPH NAME = WIAV10OM

$\begin{array}{rrr}0 & 1.5000 & 0.0 \\ 100 & 1.5000 & 150.0 \\ 200 & 1.5000 & 300.0 \\ 300 & 1.5000 & 450.0 \\ 370 & 1.5000 & 555.0\end{array}$


WHC-SD-WM-TRP-223

Rev. 0

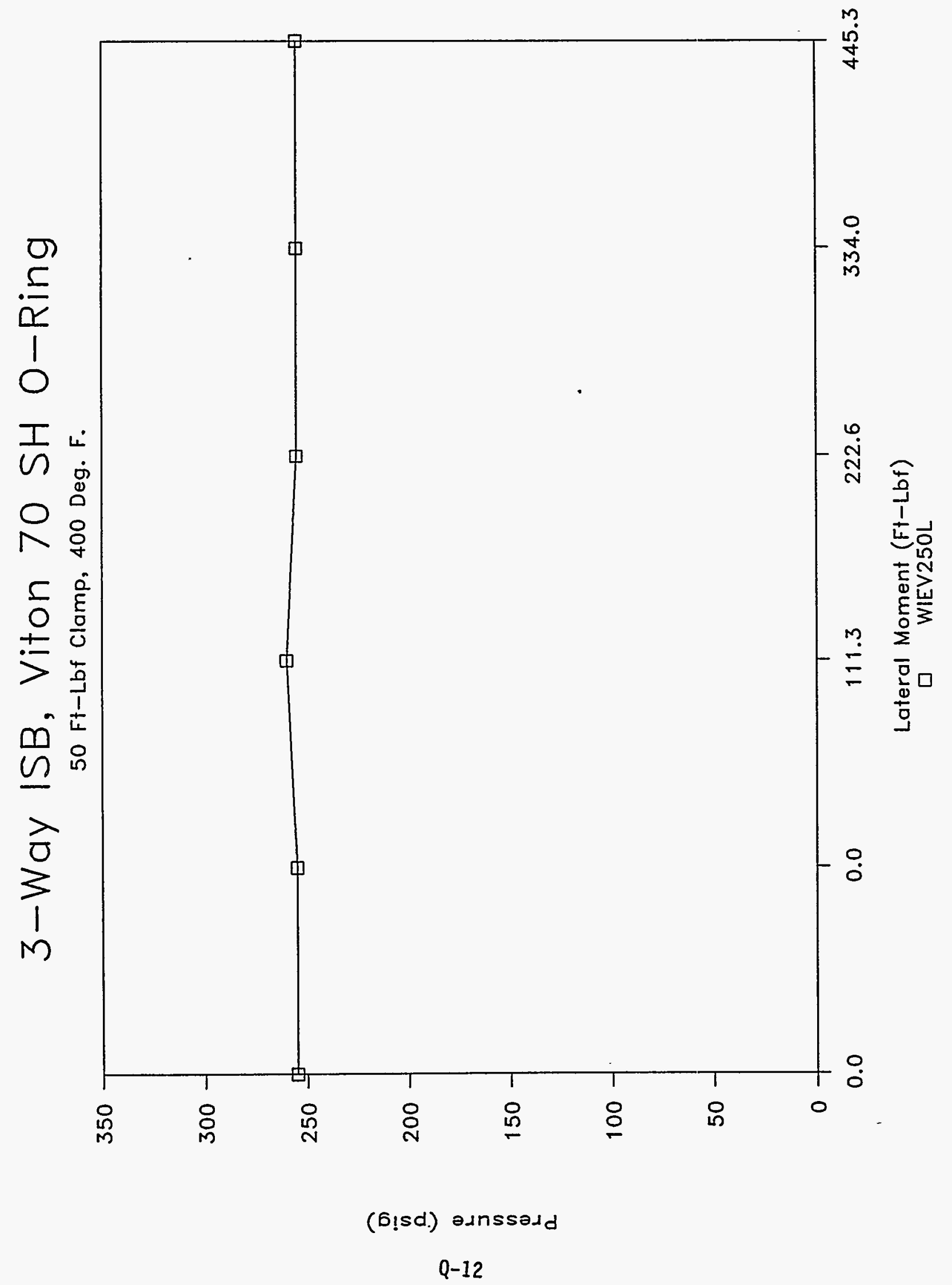


3-Way ISB, Viton $70 \mathrm{SH} \mathrm{O-Ring}$

$50 \mathrm{Ft}$-Lbf Clamp, 400 Deg. F.

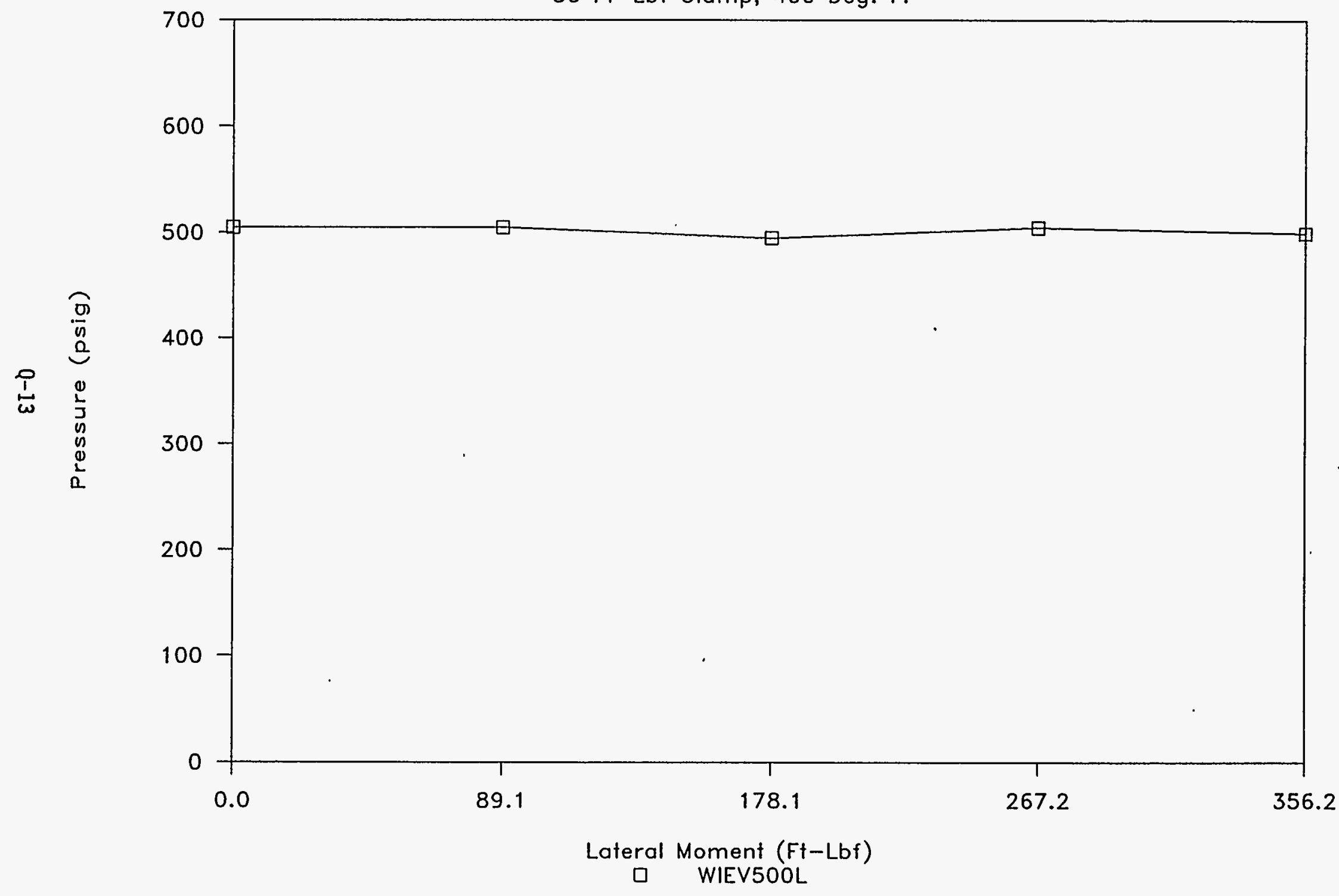




\section{3-Way ISB, Viton 70 SH O-Ring}

$50 \mathrm{Ff}$-Lbf Clamp, $400 \mathrm{Deg}$. F.

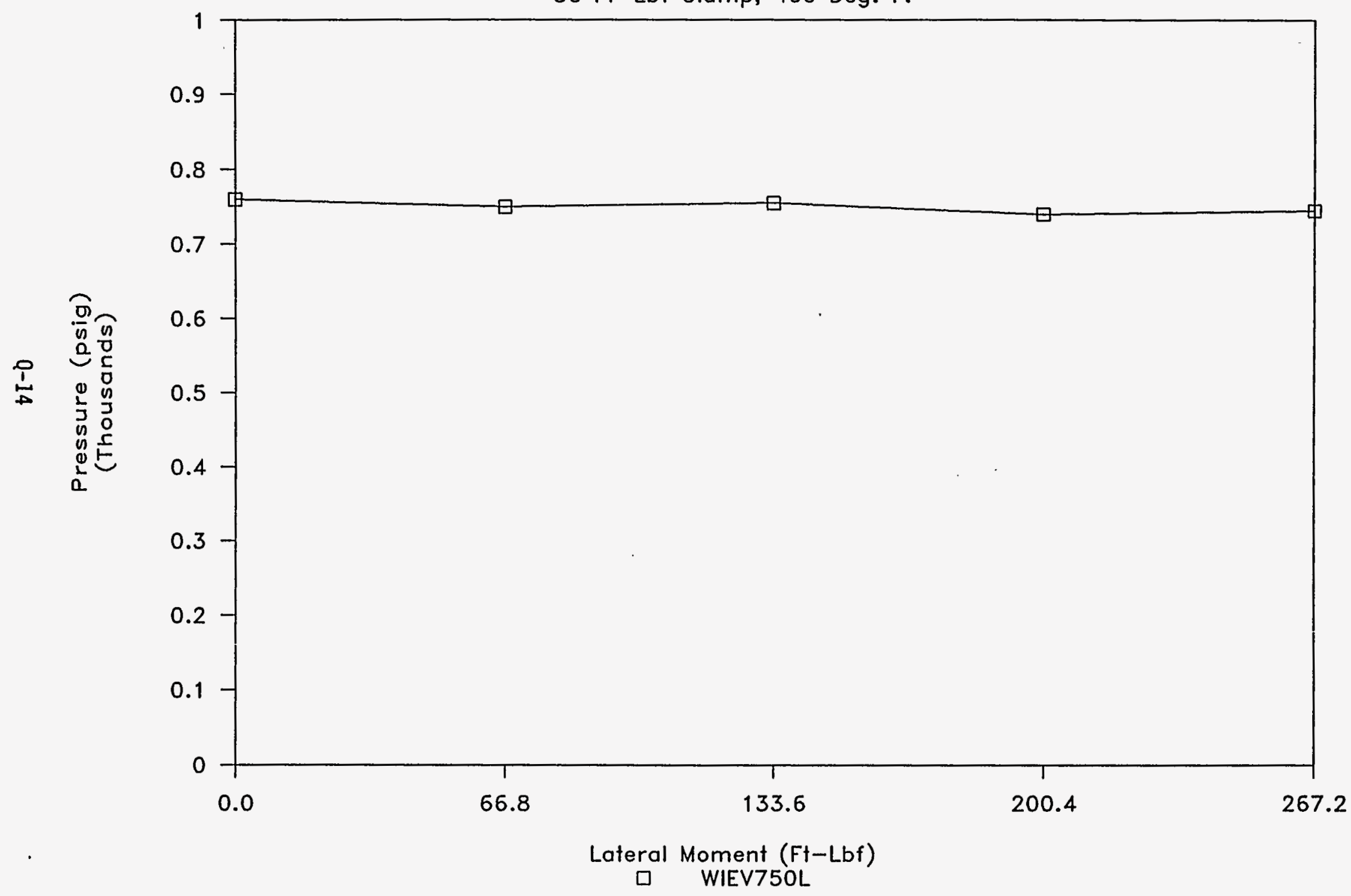




\section{3-Way ISB, Viton $70 \mathrm{SH} \mathrm{O-Ring}$}

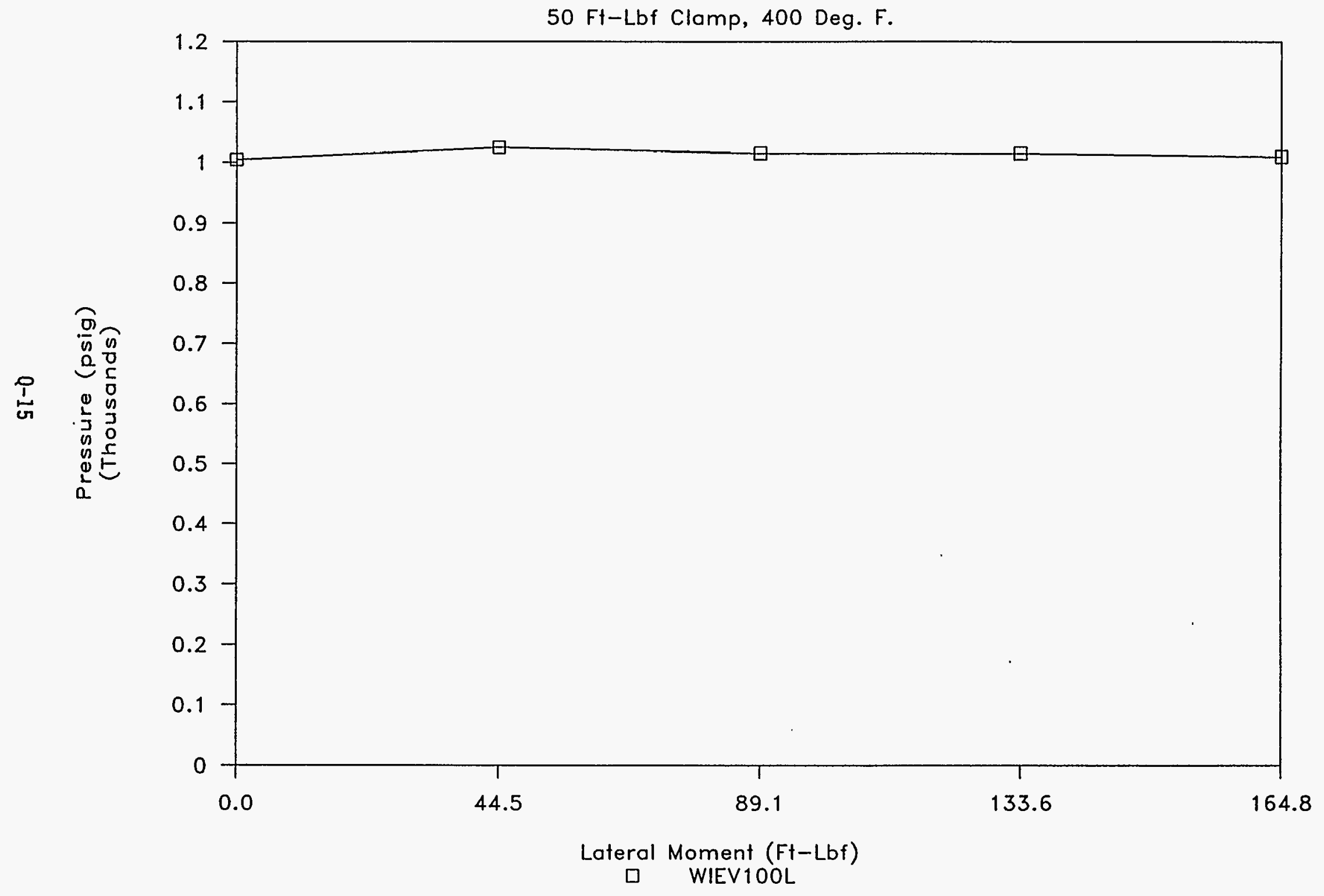


NOVEMBER 23,1994

2" 3-WAY ISB CONNECTOR, VITON O-RING, 70 DUROMETER, ELEVATED TEMP. PART \# 2-142 V884-75, PARKER SEAL CO., BATCH 311986, CURE DATE 3Q93 LEAK TEST - I.ATERAI MOMENT (SIDEWAYS ROTATION)

CLAMPING TORQUE $=50$ FT-LBF NOMINAL

CHARGE PRESSURE $=250$ PSIG

GRAPH NAME $=$ WIEV250L

TEMPERATURE: 400 DEG. F.

\begin{tabular}{|c|c|c|c|c|c|c|}
\hline $\begin{array}{l}\text { PRESSURE } \\
\text { PSIG }\end{array}$ & $\begin{array}{l}\text { ELAPSED } \\
\text { MINUTES }\end{array}$ & $\begin{array}{l}\text { ELAPSED } \\
\text { SECONDS }\end{array}$ & $\begin{array}{l}\text { ELAPSED } \\
\text { DECIMAL } \\
\text { MINUTES } \\
\text { COMPUTE }\end{array}$ & $\begin{array}{l}\text { LATERAL } \\
\text { FORCE } \\
\text { LBS } \\
\text { INPUT }\end{array}$ & $\begin{array}{l}\text { IATEIRAL } \\
\text { MOMENT } \\
\text { ARM } \\
\text { FT. } \\
\text { INPUT: }\end{array}$ & $\begin{array}{l}\text { LATERAL } \\
\text { MOMENT } \\
\text { FT-IBF. } \\
\text { COMPUTE }\end{array}$ \\
\hline $\begin{array}{l}255 \\
255 \\
260 \\
255 \\
255 \\
255\end{array}$ & $\begin{array}{r}0 \\
2 \\
4 \\
6 \\
8 \\
10\end{array}$ & $\frac{1}{2}$ & $\begin{array}{r}0.00 \\
2.02 \\
4.03 \\
6.02 \\
8.02 \\
10.03\end{array}$ & $\begin{array}{r}0 \\
0 \\
250 \\
500 \\
750 \\
1000\end{array}$ & $\begin{array}{l}0.4453 \\
0.4453 \\
0.4453 \\
0.4453 \\
0.4453 \\
0.4453\end{array}$ & $\begin{array}{r}0.0 \\
0.0 \\
111.3 \\
222.7 \\
334.0 \\
445.3\end{array}$ \\
\hline
\end{tabular}

INCREASED PRESSURE TO 500 PSIG

$\begin{array}{llll}505 & 12 & 2 & 12.03 \\ 505 & 14 & 1 & 14.02 \\ 495 & 16 & 2 & 16.03 \\ 505 & 18 & 3 & 18.05 \\ 500 & 20 & 2 & 20.03\end{array}$

INCREASED PRESSURE TO 750 PSIG

$\begin{array}{llll}760 & 22 & 1 & 22.02 \\ 750 & 24 & 1 & 24.02 \\ 755 & 26 & 4 & 26.07 \\ 740 & 28 & 2 & 28.03 \\ 745 & 30 & 1 & 30.02\end{array}$

INCREASED PRESSURE TO 1000 PSIG

$\begin{array}{llll}1005 & 32 & 2 & 32.03 \\ 1025 & 34 & 1 & 34.02 \\ 1015 & 36 & 1 & 36.02 \\ 1015 & 38 & 7 & 38.12 \\ 1010 & 40 & 2 & 40.03\end{array}$

GRAPH NAME = WIEV5OOL

$\begin{array}{rrr}0 & 0.4453 & 0.0 \\ 200 & 0.4453 & 89.1 \\ 400 & 0.4453 & 178.1 \\ 600 & 0.4453 & 267.2 \\ 800 & 0.4453 & 356.2\end{array}$

GRAPH NAME $=$ WIEV750I

$\begin{array}{rrr}0 & 0.4453 & 0.0 \\ 150 & 0.4453 & 66.8 \\ 300 & 0.4453 & 133.6 \\ 450 & 0.4453 & 200.4 \\ 600 & 0.4453 & 267.2\end{array}$

GRAPH NAME $=$ WIEV10OL

$\begin{array}{rrr}0 & 0.4453 & 0.0 \\ 100 & 0.4453 & 44.5 \\ 200 & 0.4453 & 89.1 \\ 300 & 0.4453 & 133.6 \\ 370 & 0.4453 & 164.8\end{array}$




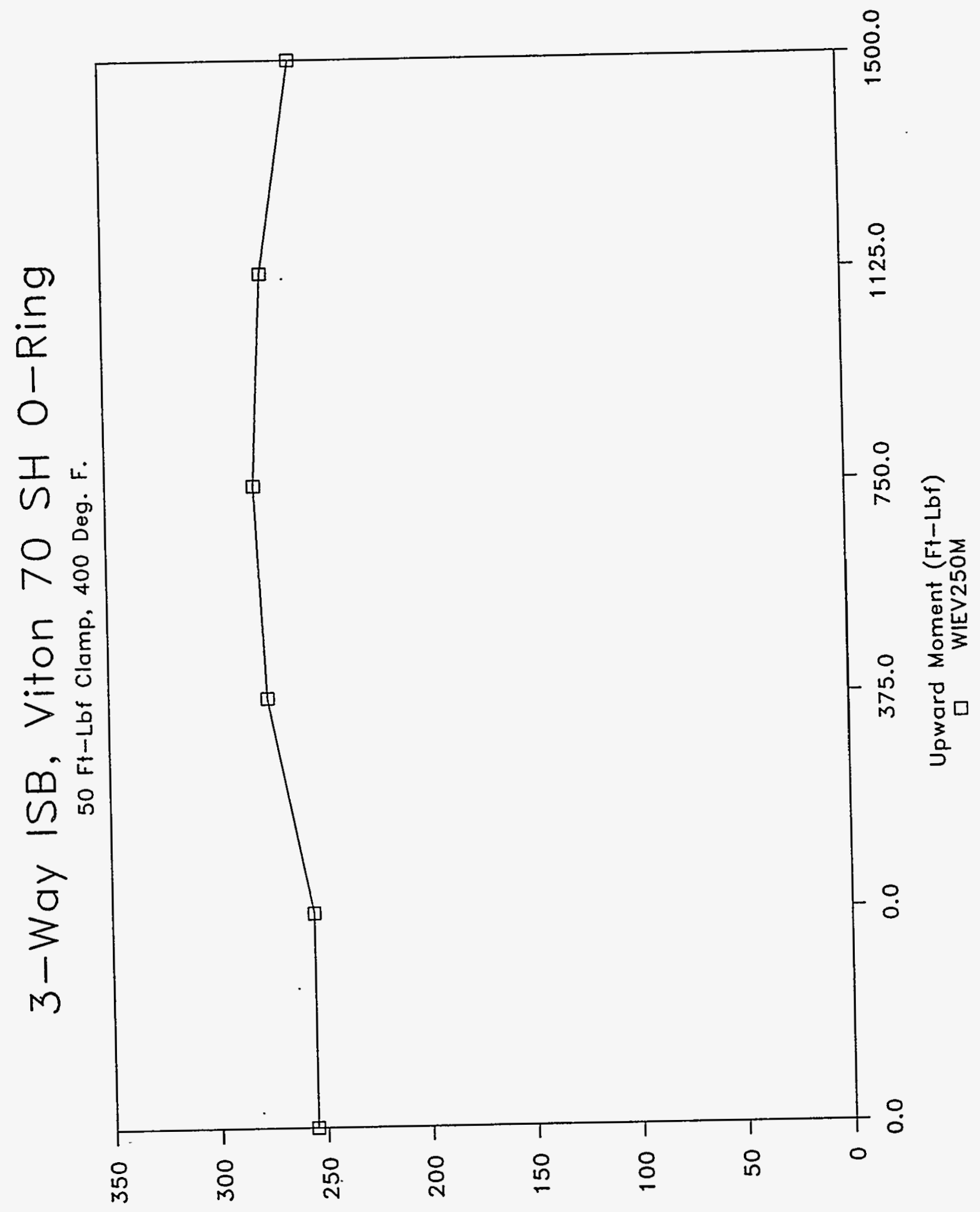

(bisd) adnssadd 
WHC-SD-WM-TRP-223

Rev. 0

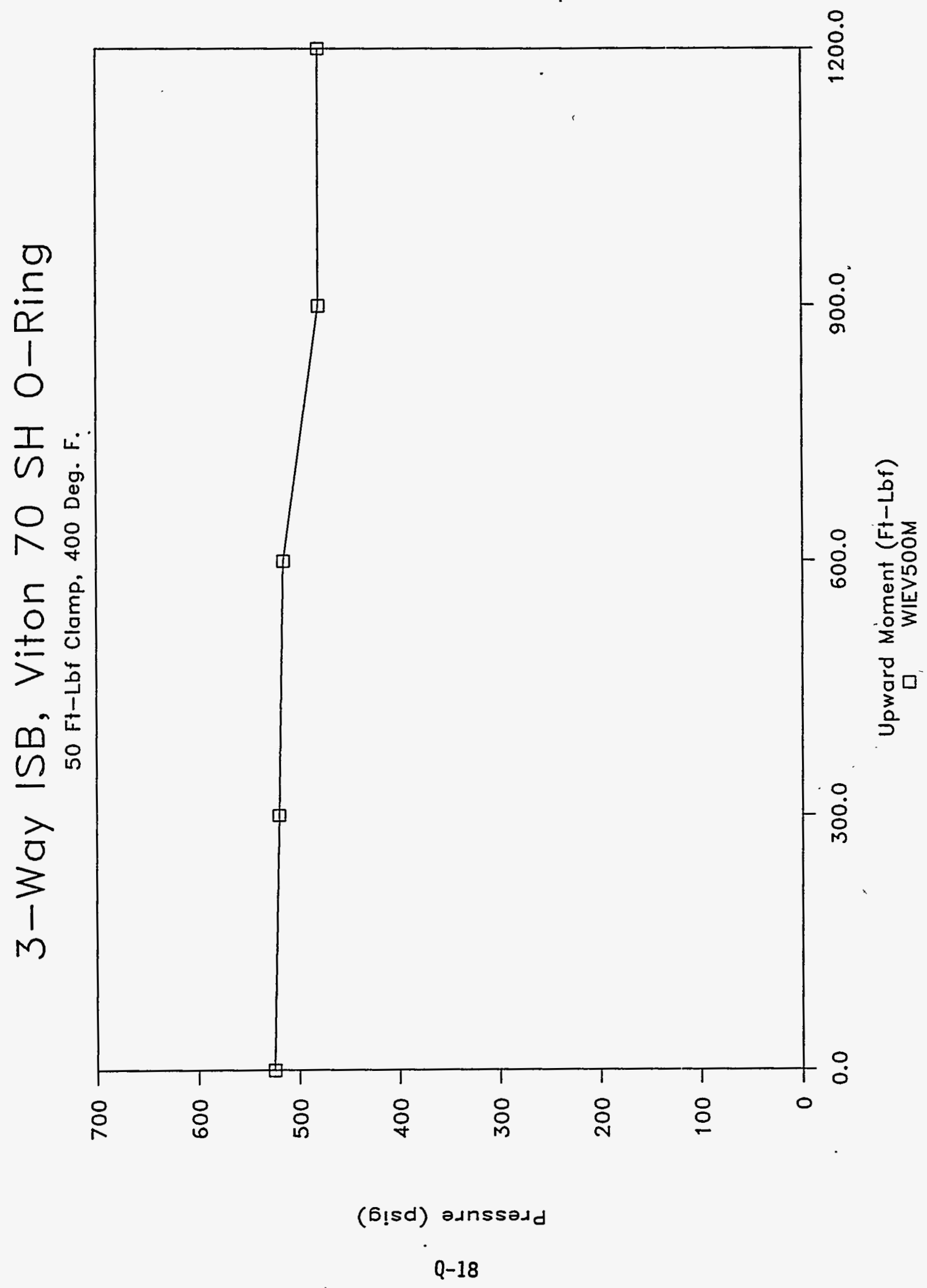




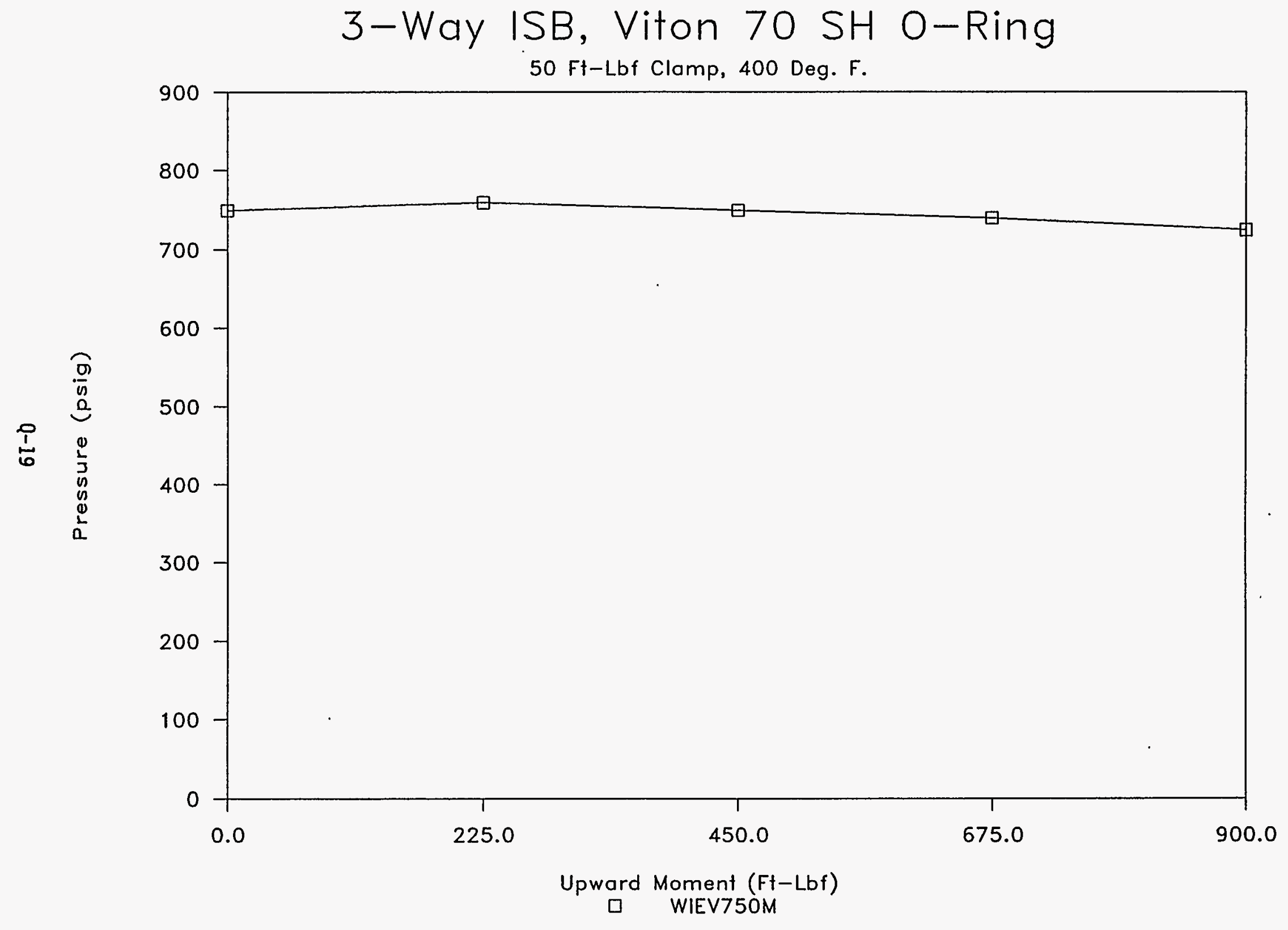




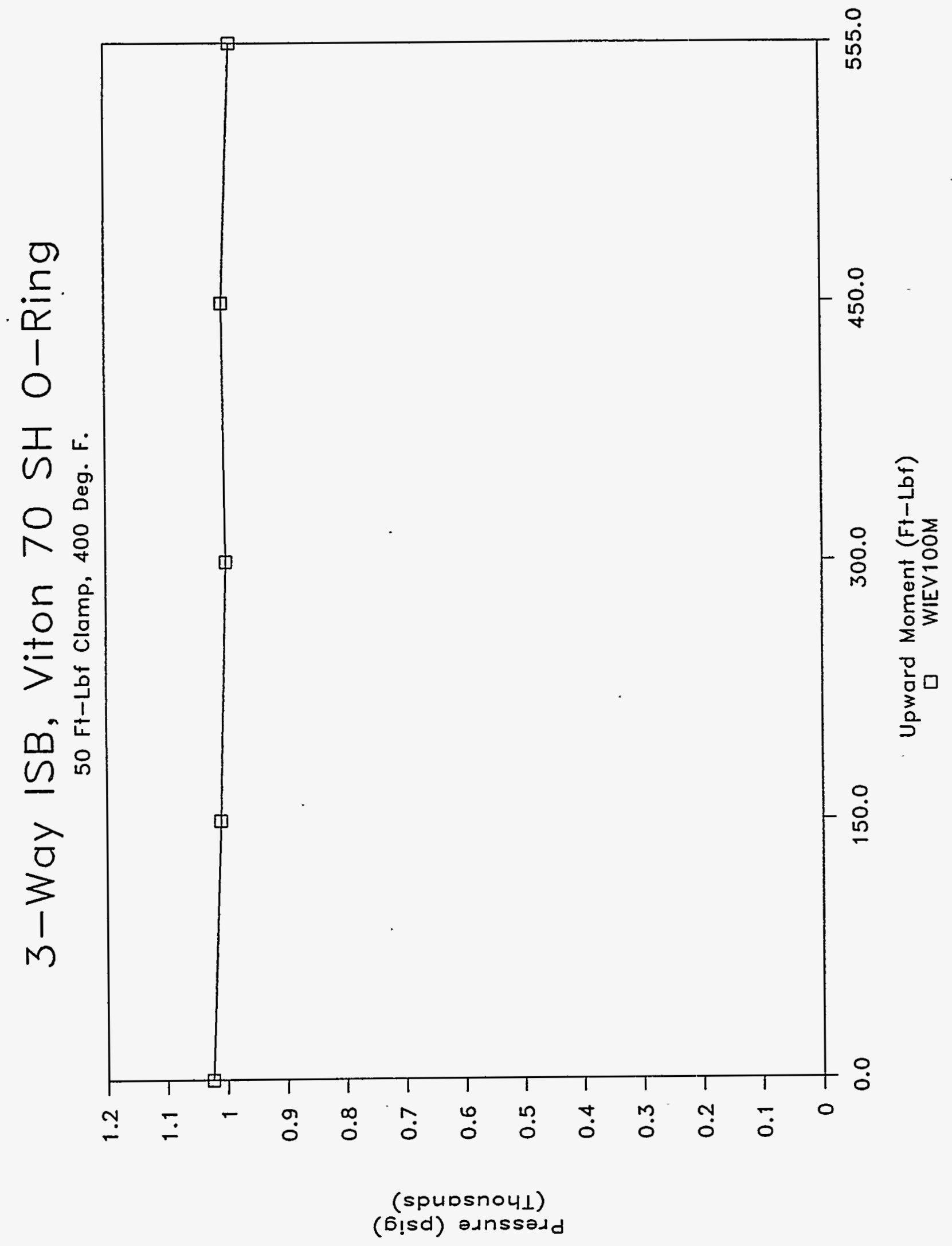


NOVEMBER 28,1994

2" 3-WAY ISB CONNECTOR, VITON O-RING, 70 DUROMETER, ELEVATED TEMP. PART \# 2-142 V884-75, PARKER SEAL CO., BATCH 311986, CURE DATE 3093 LEAK TEST - UP AND OVER MOMENT (UPWARD ROTATION)

CIAMPING TORQUE $=50$ FT-LBF NOMINAL GRAPH NAME $=$ WIEV25OM CHARGE PRESSURE $=250$ PSIG

TEMPERATURE: 400 DEG. F.

\begin{tabular}{|c|c|c|c|c|c|c|}
\hline $\begin{array}{l}\text { PRESSURE } \\
\text { PSIG }\end{array}$ & $\begin{array}{l}\text { ELAPSED } \\
\text { MINUTES }\end{array}$ & $\begin{array}{l}\text { ELAPSED } \\
\text { SECONDS }\end{array}$ & $\begin{array}{l}\text { ELAPSED } \\
\text { DECIMAL } \\
\text { MINUTES } \\
\text { COMPUTTE }\end{array}$ & $\begin{array}{l}\text { UPWARD } \\
\text { FORCE } \\
\text { LBS } \\
\text { INPUT }\end{array}$ & $\begin{array}{l}\text { UPWARD } \\
\text { MOMENT } \\
\text { ARM } \\
\text { FT. } \\
\text { INPUT }\end{array}$ & $\begin{array}{l}\text { UPWARD } \\
\text { MOMENT } \\
\text { FT-LBF. } \\
\text { COMPUTE }\end{array}$ \\
\hline NPUT & INPUT & INPUT & COMPUTE & INPUI & INEUI & COMTUID \\
\hline $\begin{array}{l}255 \\
255 \\
275 \\
280 \\
275 \\
260\end{array}$ & $\begin{array}{r}5 \\
2 \\
18\end{array}$ & & $\begin{array}{r}0.00 \\
2.02 \\
4.03 \\
6.08 \\
8.07 \\
10.10\end{array}$ & $\begin{array}{r}0 \\
0 \\
250 \\
500 \\
750 \\
1000\end{array}$ & $\begin{array}{l}1.5000 \\
1.5000 \\
1.5000 \\
1.5000 \\
1.5000 \\
1.5000\end{array}$ & $\begin{array}{r}0.0 \\
0.0 \\
375.0 \\
750.0 \\
1125.0 \\
1500.0\end{array}$ \\
\hline
\end{tabular}

INCREASED PRESSURE TO 500 PSIG

$\begin{array}{ll}525 & 12 \\ 520 & 14 \\ 515 & 16 \\ 480 & 18 \\ 480 & 20\end{array}$

$\begin{array}{ll}1 & 12.02 \\ 1 & 14.02 \\ 1 & 16.02 \\ 2 & 18.03 \\ 1 & 20.02\end{array}$

INCREASED PRESSURE TO 750 PSIG

$\begin{array}{llll}750 & 22 & 3 & 22.05 \\ 760 & 24 & 1 & 24.02 \\ 750 & 26 & 1 & 26.02 \\ 740 & 28 & 1 & 28.02 \\ 725 & 30 & 1 & 30.02\end{array}$

INCREASED PRESSURE TO 1000 PSIG

$\begin{array}{rr}1025 & 32 \\ 1010 & 34 \\ 1000 & 36 \\ 1005 & 38 \\ 990 & 40\end{array}$

GRAPH NAME = WIEV50OM

$\begin{array}{rrr}0 & 1.5000 & 0.0 \\ 200 & 1.5000 & 300.0 \\ 400 & 1.5000 & 600.0 \\ 600 & 1.5000 & 900.0 \\ 800 & 1.5000 & 1200.0\end{array}$

GRAPH NAME $=$ WIEV750M

$\begin{array}{rrr}0 & 1.5000 & 0.0 \\ 150 & 1.5000 & 225.0 \\ 300 & 1.5000 & 450.0 \\ 450 & 1.5000 & 675.0 \\ 600 & 1.5000 & 900.0\end{array}$

GRAPH NAME $=$ WIEVIOOM

$\begin{array}{rrr}0 & 1.5000 & 0.0 \\ 100 & 1.5000 & 150.0 \\ 200 & 1.5000 & 300.0 \\ 300 & 1.5000 & 450.0 \\ 370 & 1.5000 & 555.0\end{array}$


WHC-SD-WM-TRP-223

Rev. 0

APPENDIX R: GRAPHS OF THREE-WAY SILICONE TESTS

R-1 
WHC-SD-WM-TRP-223

Rev. 0

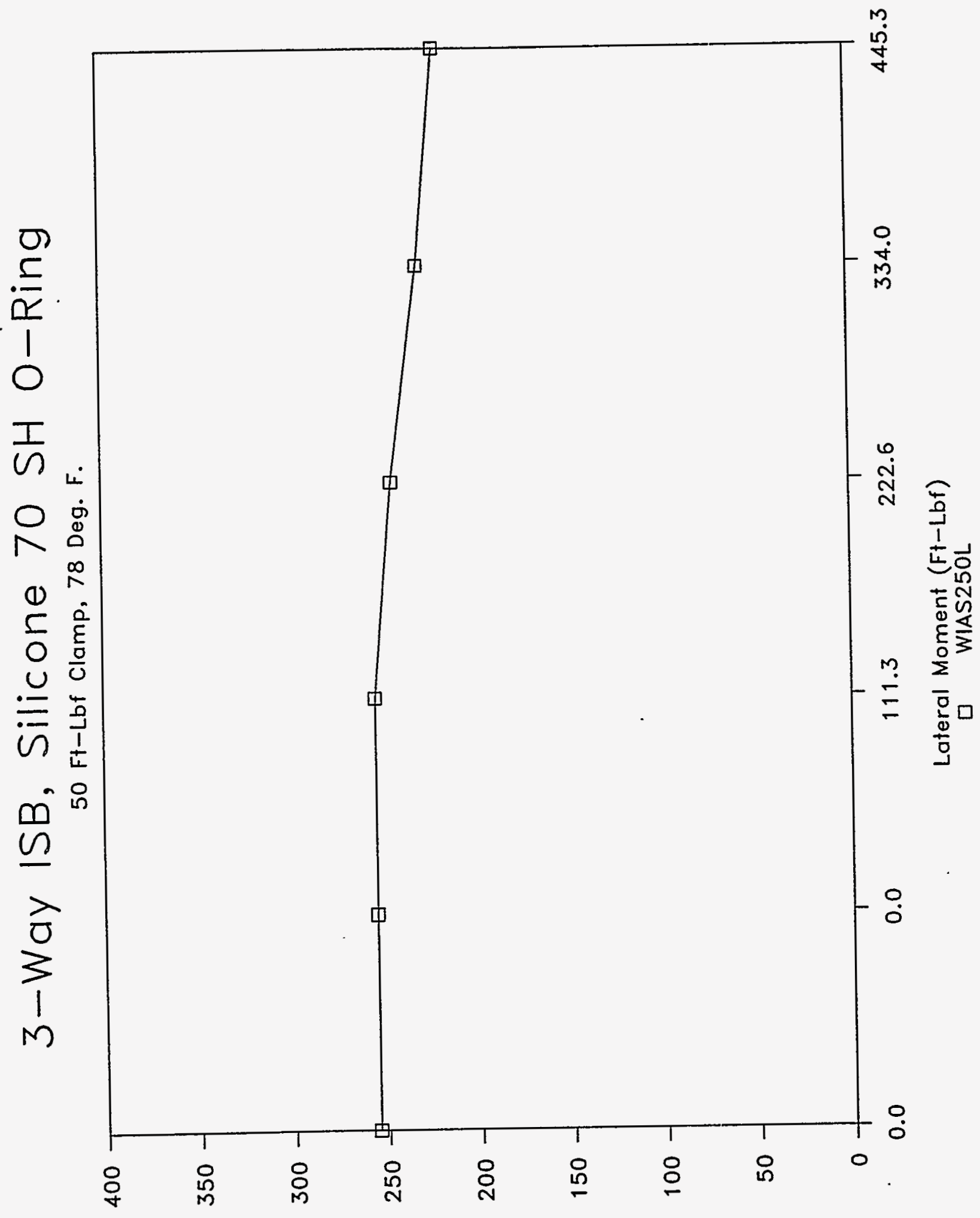

(bisd) aunssadd

$R-2$ 
WHC-SD-WM-TRP-223

Rev. 0

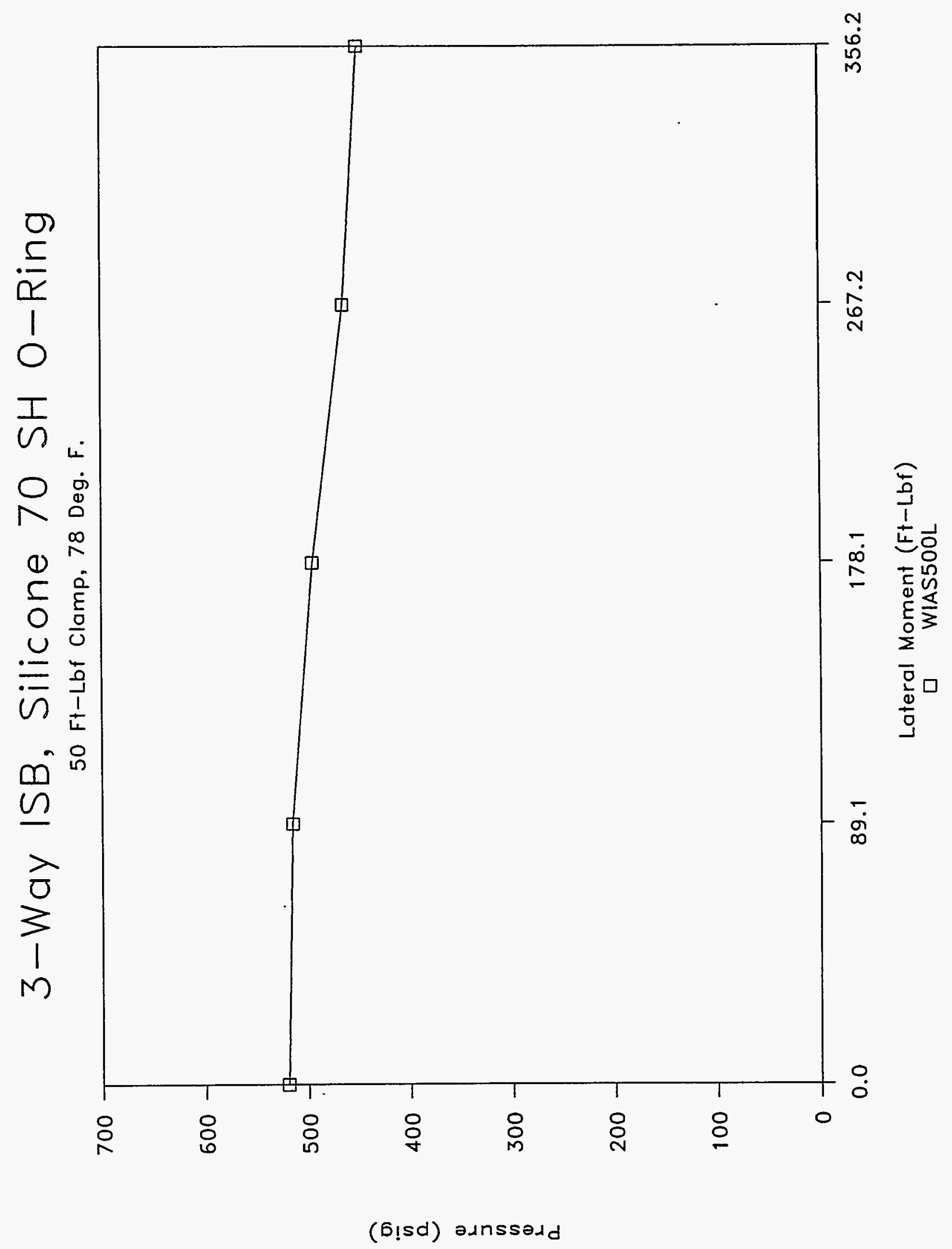

R-3 


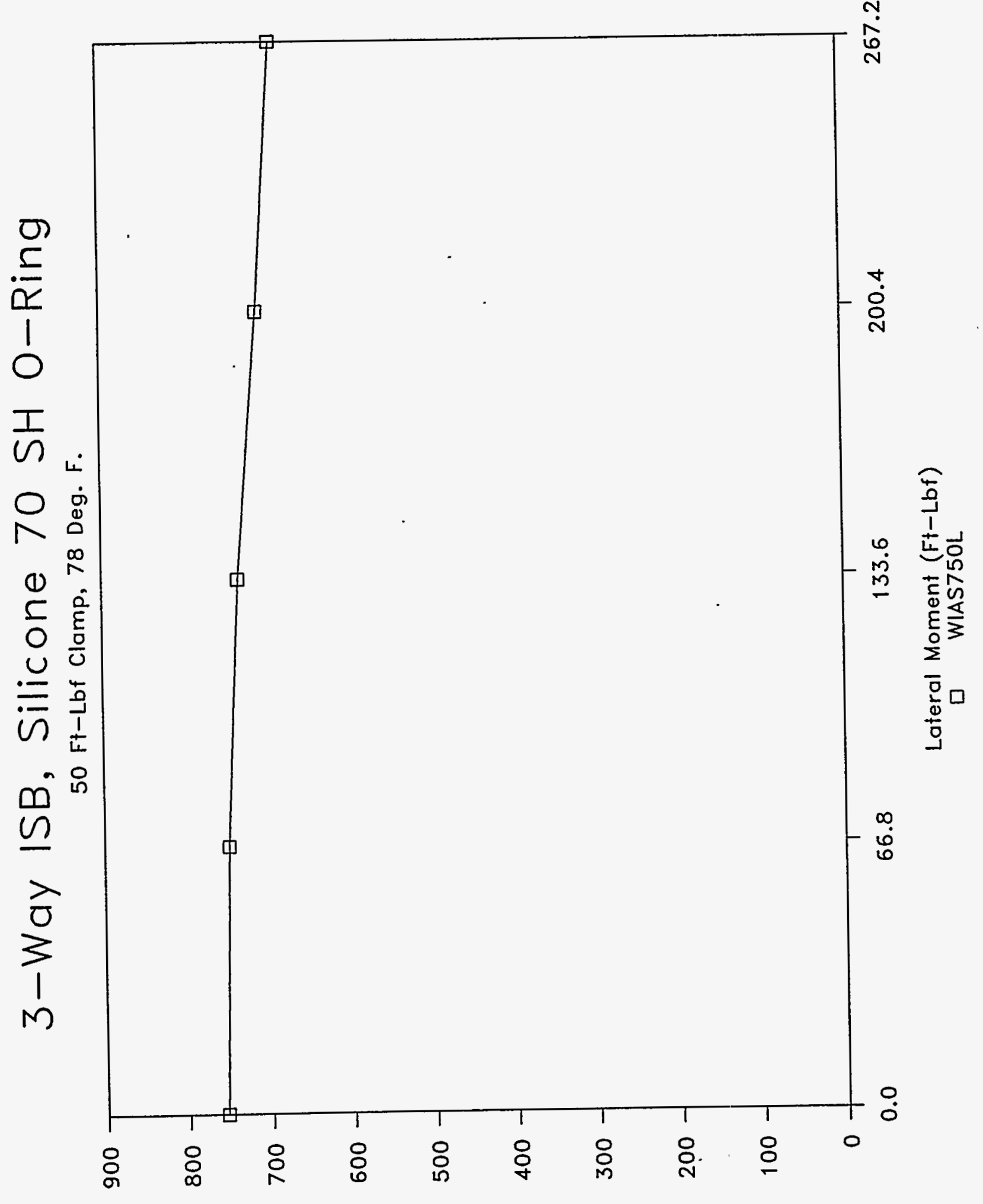

(b!sd) adnssadd 
WHC-SD-WM-TRP-223

Rev. 0

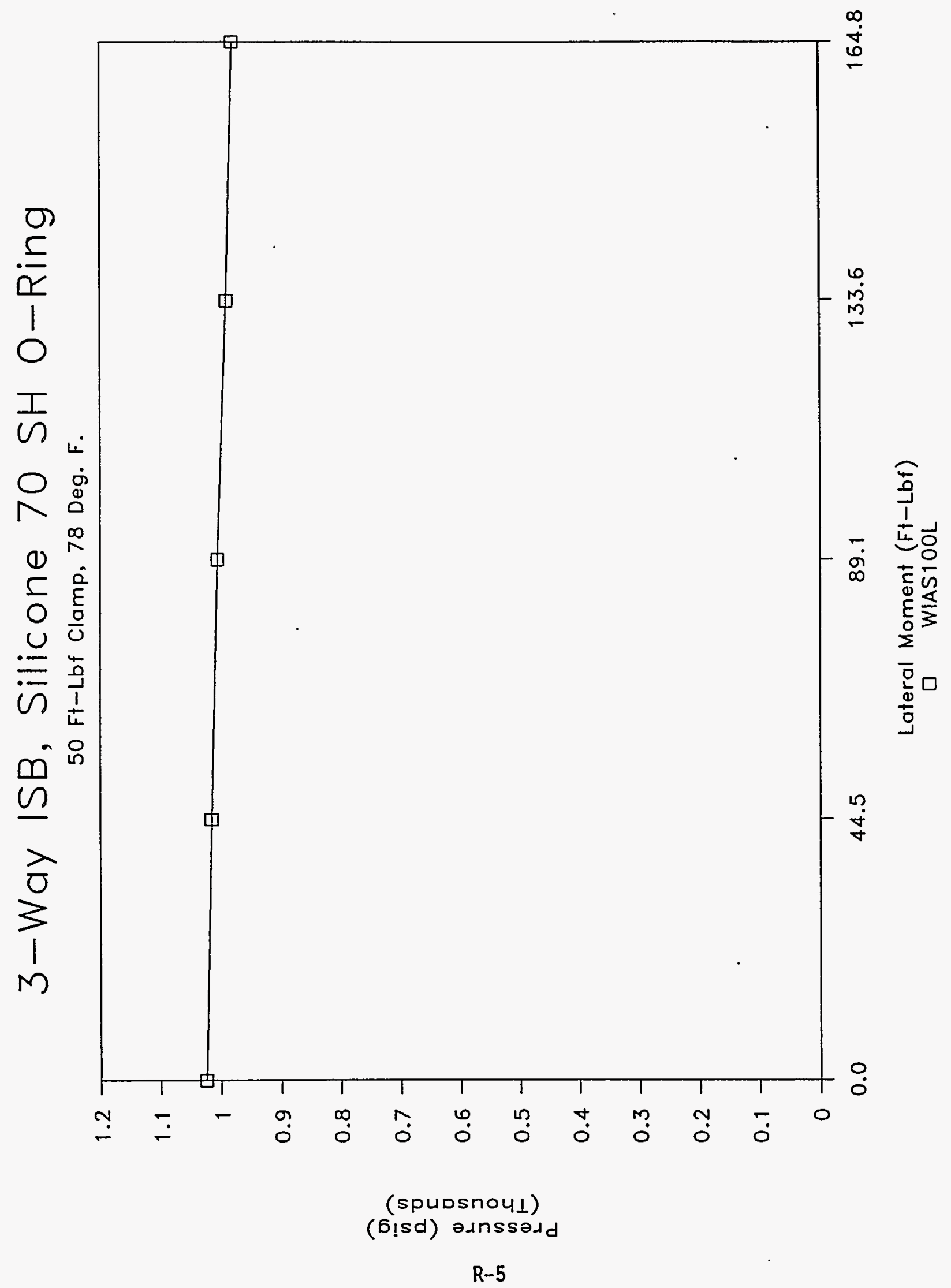


DECEMBER 12,1994

2" 3-WAY ISB CONNECTOR, SILICONE O-RING, 70 DUROMETER, AMBIENT TEMP. PART \# 2-119 5604, PARKER SEAL CO., BATCH \# 75561, CURE DATE 3093 LEAK TEST - LATERAI MOMENT (SIDEWAYS ROTATION)

CLAMPING TORQUE $=50$ FT-LBF NOMINAL CHARGE PRESSURE $=250$ PSIG

GRAPH NAME = WIAS250L

TEMPERATURE: 78 DEG. F.

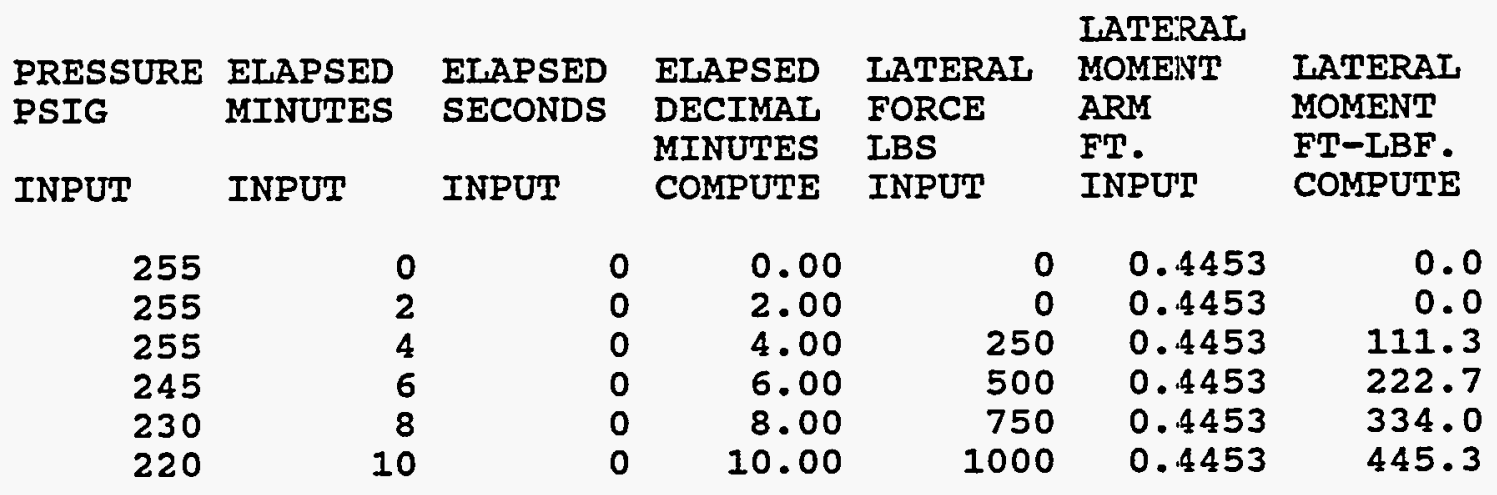

INCREASED PRESSURE TO 500 PSIG

$\begin{array}{ll}520 & 12 \\ 515 & 14 \\ 495 & 16 \\ 465 & 19 \\ 450 & 21\end{array}$

INCREASED PRESSURE TO 750 PSIG

$\begin{array}{ll}755 & 23 \\ 750 & 25 \\ 735 & 27 \\ 710 & 29 \\ 690 & 31\end{array}$

INCREASED PRESSURE TO 1000 PSIG

$\begin{array}{rl}1025 & 33 \\ 1015 & 35 \\ 1005 & 37 \\ 990 & 39 \\ 980 & 41\end{array}$

GRAPH NAME = WIAS50OL

$\begin{array}{rrr}0 & 0.4453 & 0.0 \\ 200 & 0.4453 & 89.1 \\ 400 & 0.4453 & 178.1 \\ 600 & 0.4453 & 267.2 \\ 800 & 0.4453 & 356.2\end{array}$

23.00

25.00

27.00

29.00

31.00
GRAPH NAME = WIAS750L

$\begin{array}{rrr}0 & 0.4453 & 0.0 \\ 150 & 0.4453 & 66.8 \\ 300 & 0.4453 & 133.6 \\ 450 & 0.4453 & 200.4 \\ 600 & 0.4453 & 267.2\end{array}$

GRAPH NAME = WIAS10OL

$\begin{array}{rrr}0 & 0.4453 & 0.0 \\ 100 & 0.4453 & 44.5 \\ 200 & 0.4453 & 89.1 \\ 300 & 0.4453 & 133.6 \\ 370 & 0.4453 & 164.8\end{array}$


WHC-SD-WM-TRP-223

Rev. 0

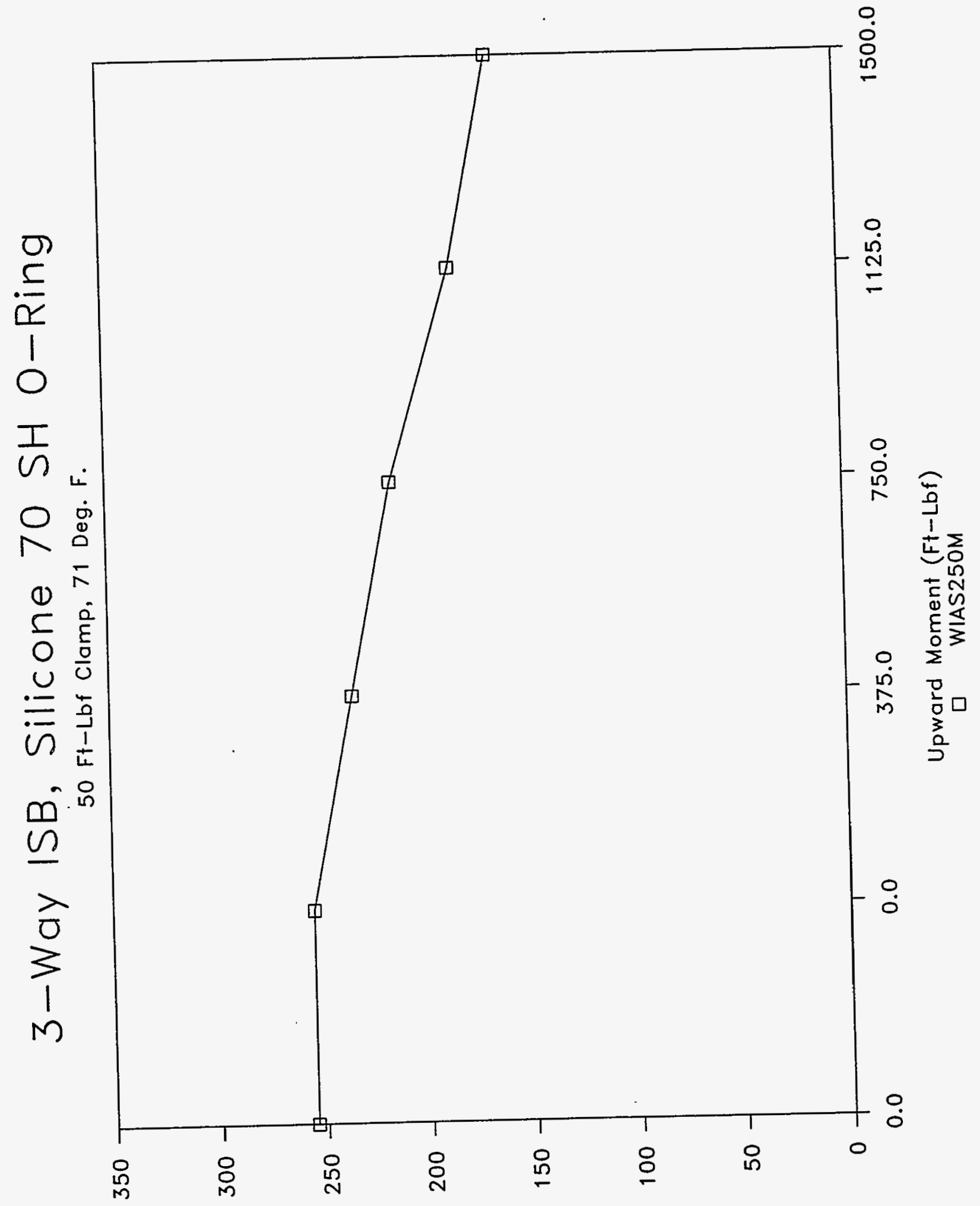

(b!sd) adnssadd

$R-7$ 


\section{3-Way ISB, Silicone 70 SH O-Ring}

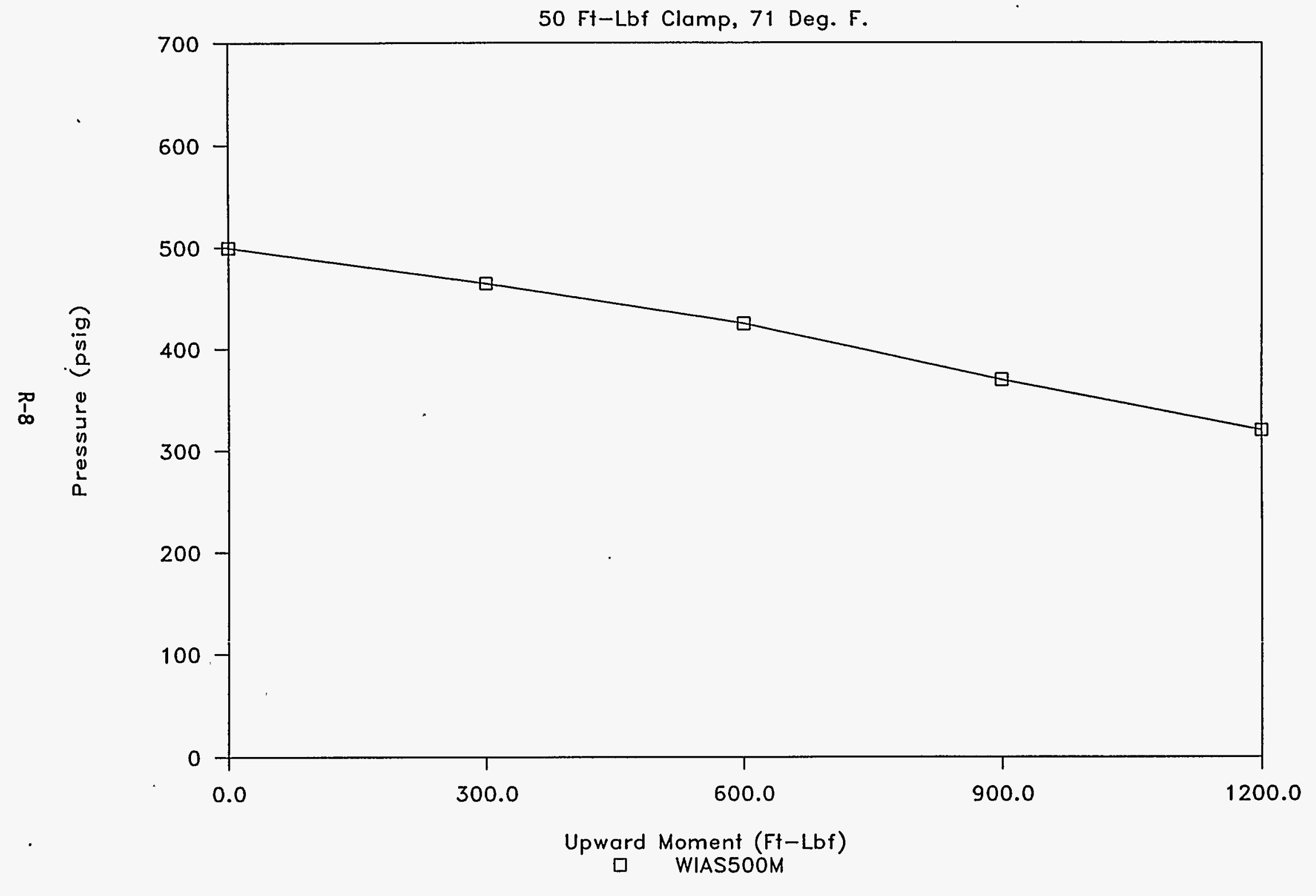




\section{3-Way ISB, Silicone 70 SH O-Ring}

$50 \mathrm{Ft}$-Lbf Clamp, 71 Deg. F.

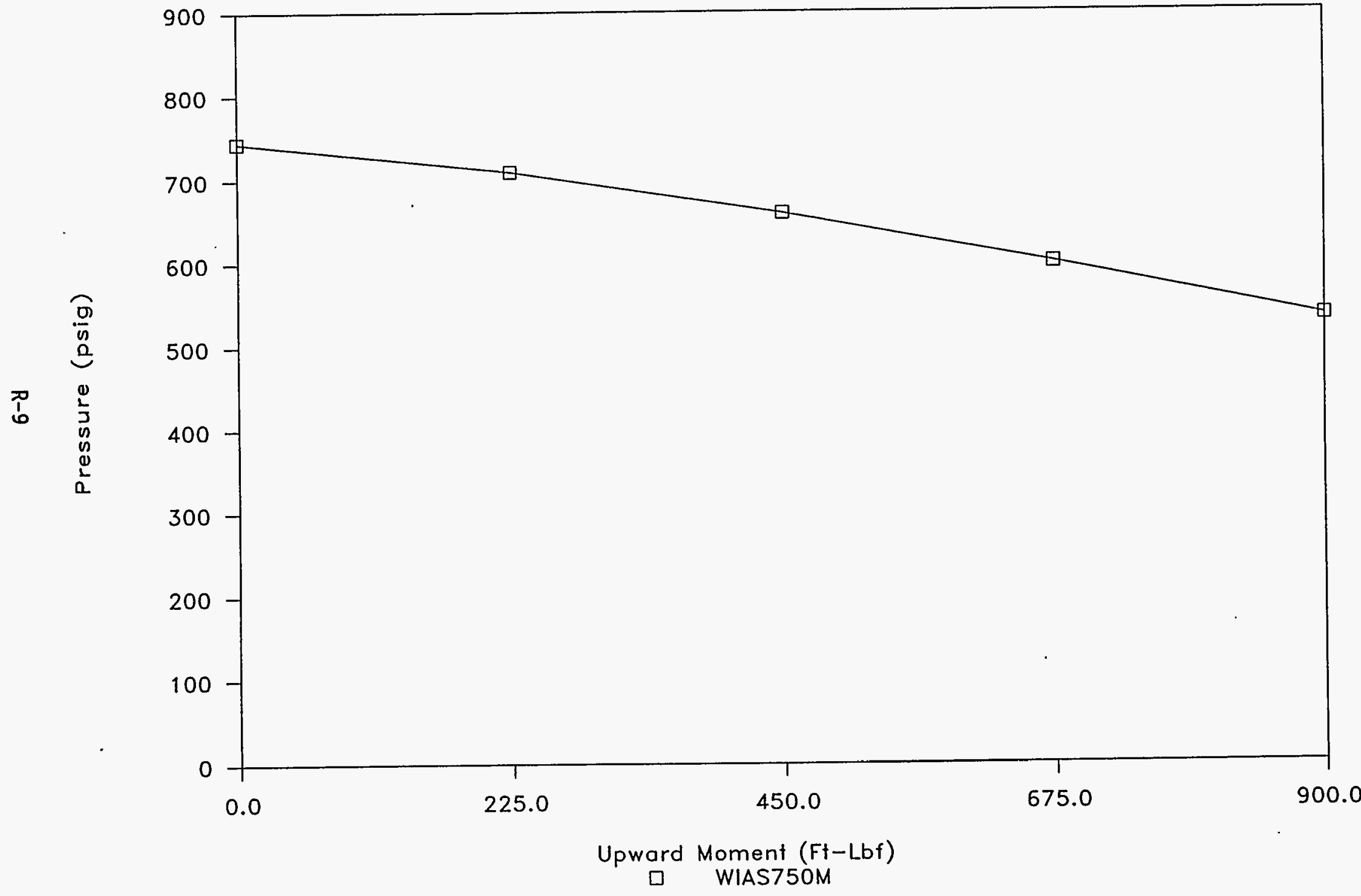


WHC-SD-WM-TRP-223

Rev. 0

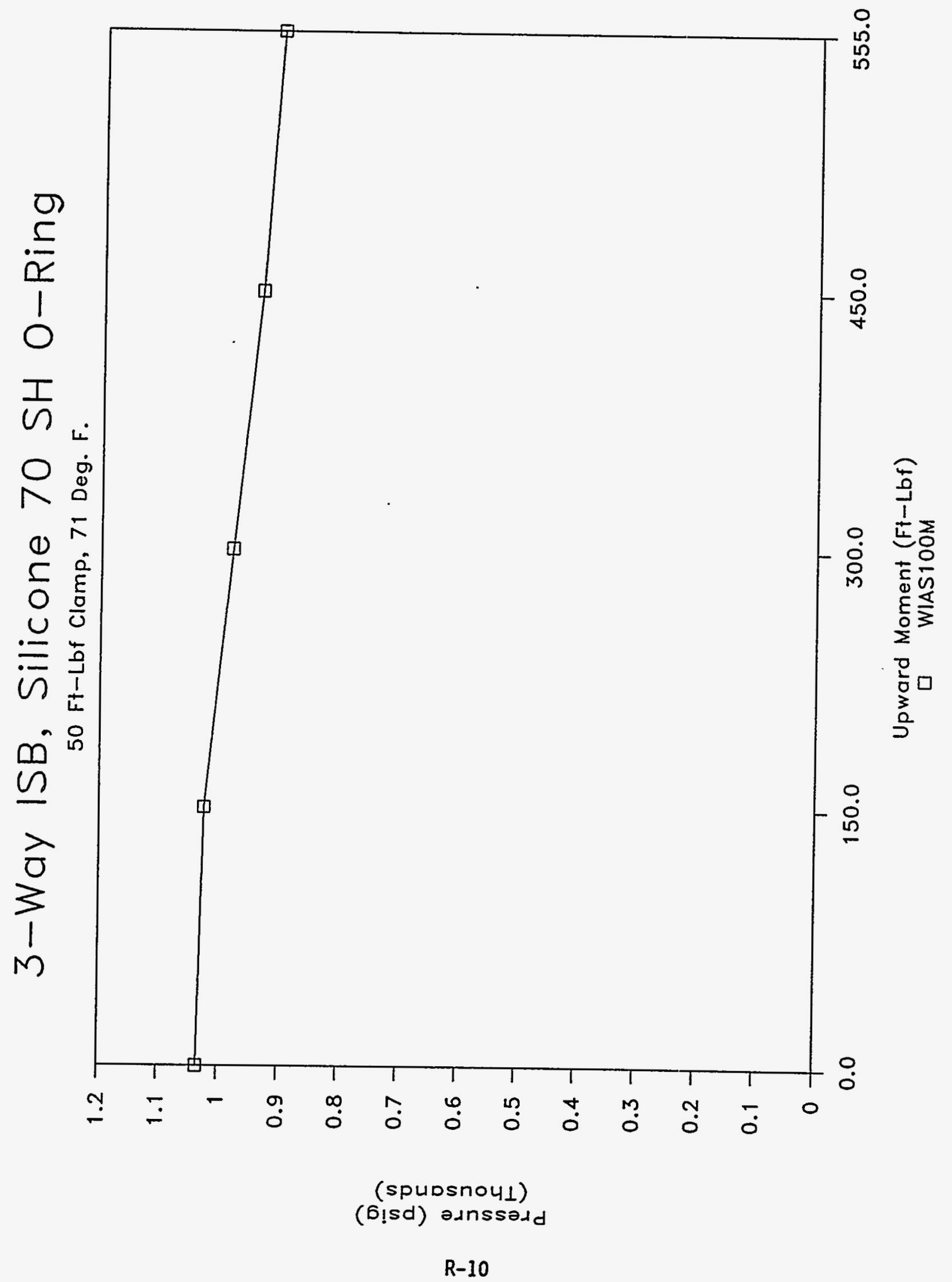


DECEMBER 05, 1994

2" 3-WAY ISB CONNECTOR, SILICONE O-RING, 70 DUROMETER, AMBIENT TEMP. PART \# 2-119 5604, PARKER SEAL CO., BATCH \# 75561, CURE DATE 3 Q93 LEAK TEST - UP AND OVER MOMENT (UPWARD ROTATION)

CLAMPING TORQUE $=50$ FT-LBF NOMINAL CHARGE PRESSURE $=250$ PSIG

GRAPH NAME = WIAS250M

TEMPERATURE: 71 DEG. F.

INCREASED PRESSURE TO 500 PSIG

$\begin{array}{llll}500 & 12 & 0 & 12.00 \\ 465 & 14 & 0 & 14.00 \\ 425 & 16 & 0 & 16.00 \\ 370 & 18 & 0 & 18.00 \\ 320 & 20 & 0 & 20.00\end{array}$

INCREASED PRESSURE TO 750 PSIG

$\begin{array}{llll}745 & 22 & 0 & 22.00 \\ 710 & 24 & 0 & 24.00 \\ 660 & 26 & 0 & 26.00 \\ 600 & 28 & 0 & 28.00 \\ 535 & 30 & 0 & 30.00\end{array}$

INCREASED PRESSURE TO 1000 PSIG

$\begin{array}{rrrrrrr}1035 & 32 & 0 & 32.00 & 0 & 1.5000 & 0.0 \\ 1025 & 34 & 0 & 34.00 & 100 & 1.5000 & 150.0 \\ 980 & 36 & 0 & 36.00 & 200 & 1.5000 & 300.0 \\ 935 & 38 & 0 & 38.00 & 300 & 1.5000 & 450.0 \\ 905 & 40 & 0 & 40.00 & 370 & 1.5000 & 555.0\end{array}$

GRAPH NAME = WIAS50OM

$\begin{array}{rrr}0 & 1.5000 & 0.0 \\ 200 & 1.5000 & 300.0 \\ 400 & 1.5000 & 600.0 \\ 600 & 1.5000 & 900.0 \\ 800 & 1.5000 & 1200.0\end{array}$

GRAPH NAME $=$ WIAS750M

$\begin{array}{rrr}0 & 1.5000 & 0.0 \\ 150 & 1.5000 & 225.0 \\ 300 & 1.5000 & 450.0 \\ 450 & 1.5000 & 675.0 \\ 600 & 1.5000 & 900.0\end{array}$

GRAPH NAME $=$ WIAS10OM 


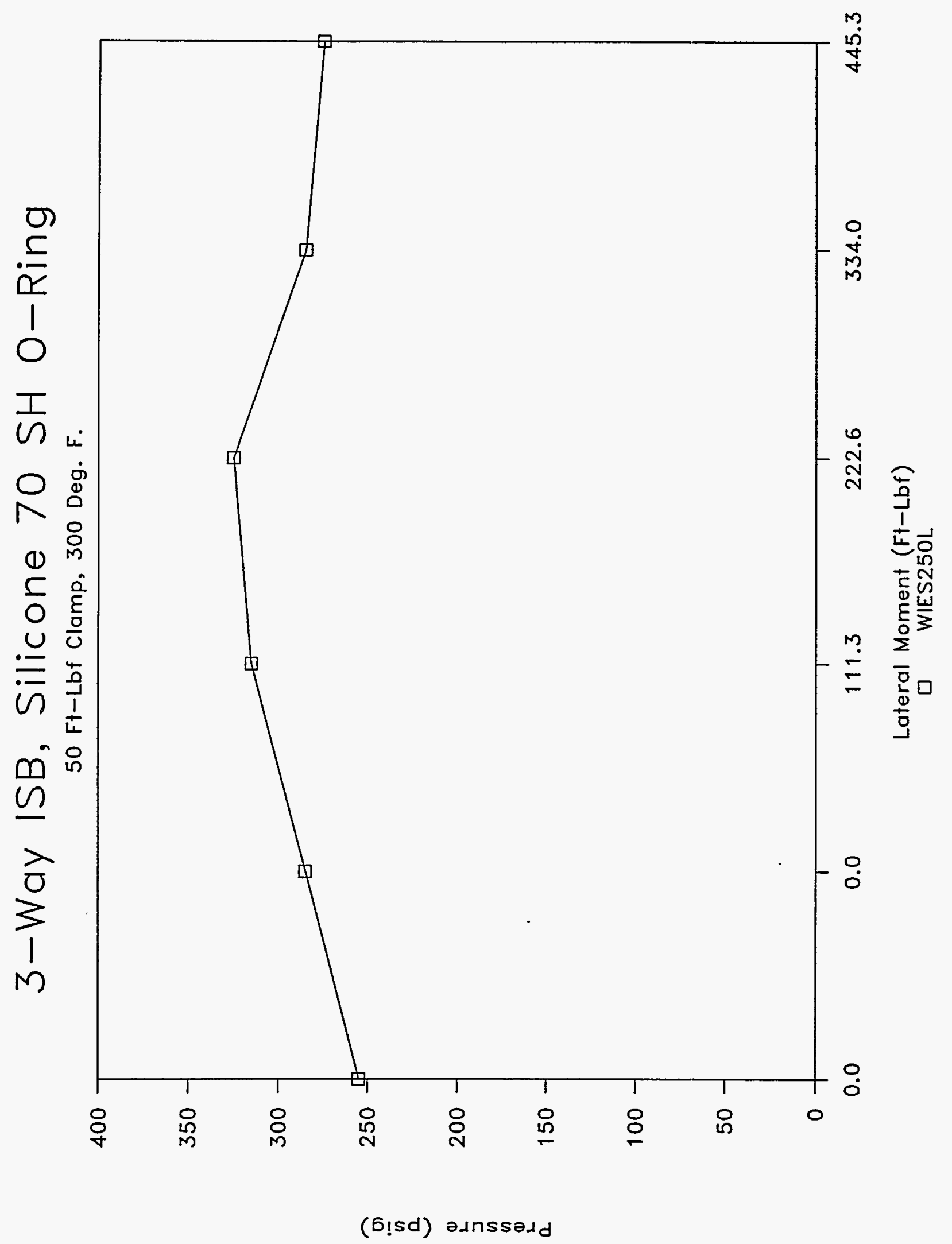

$\mathrm{R}-12$ 
WHC-SD-WM-TRP-223

Rev. 0

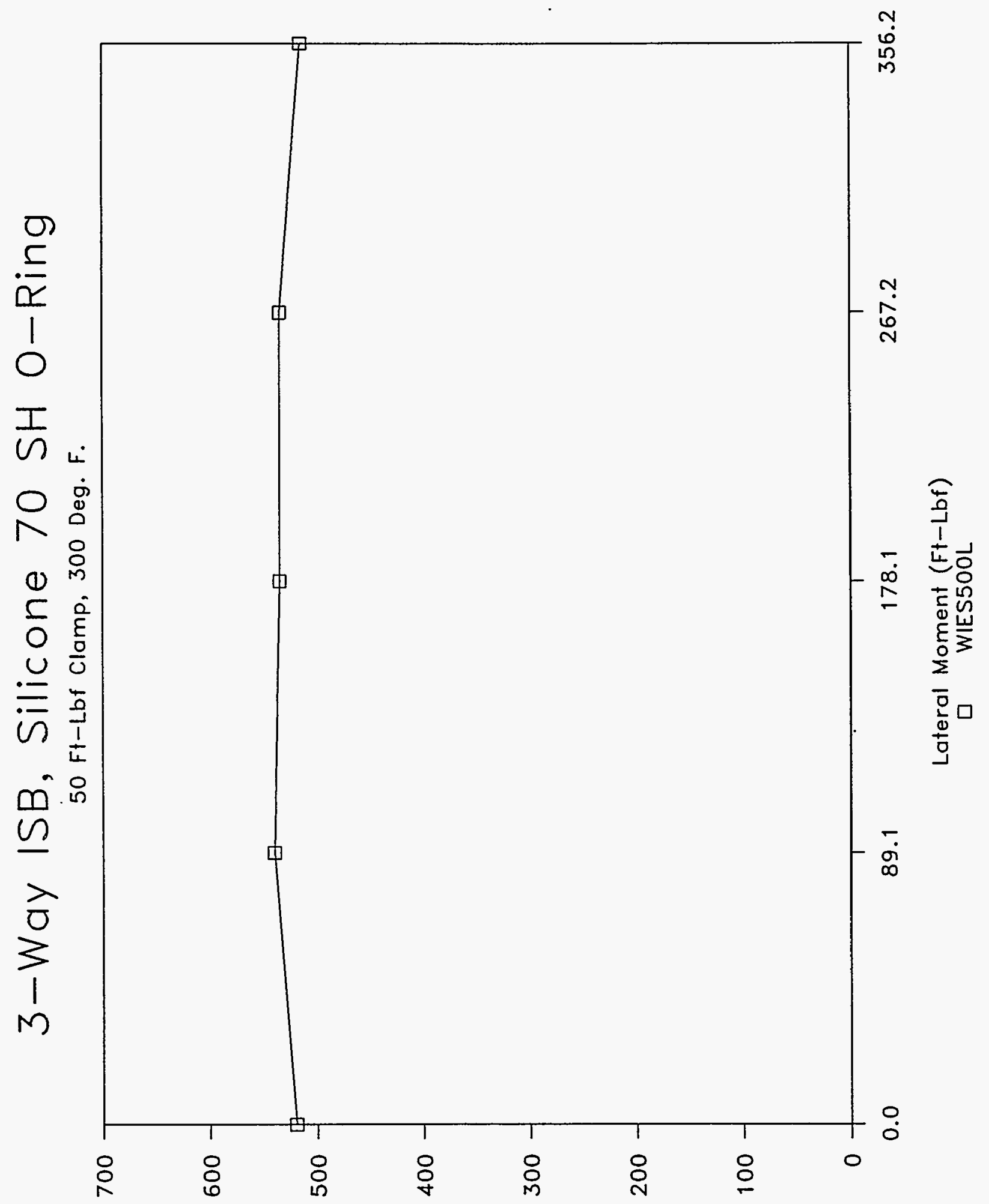

(b!sd) adnssadd 
Rev. 0

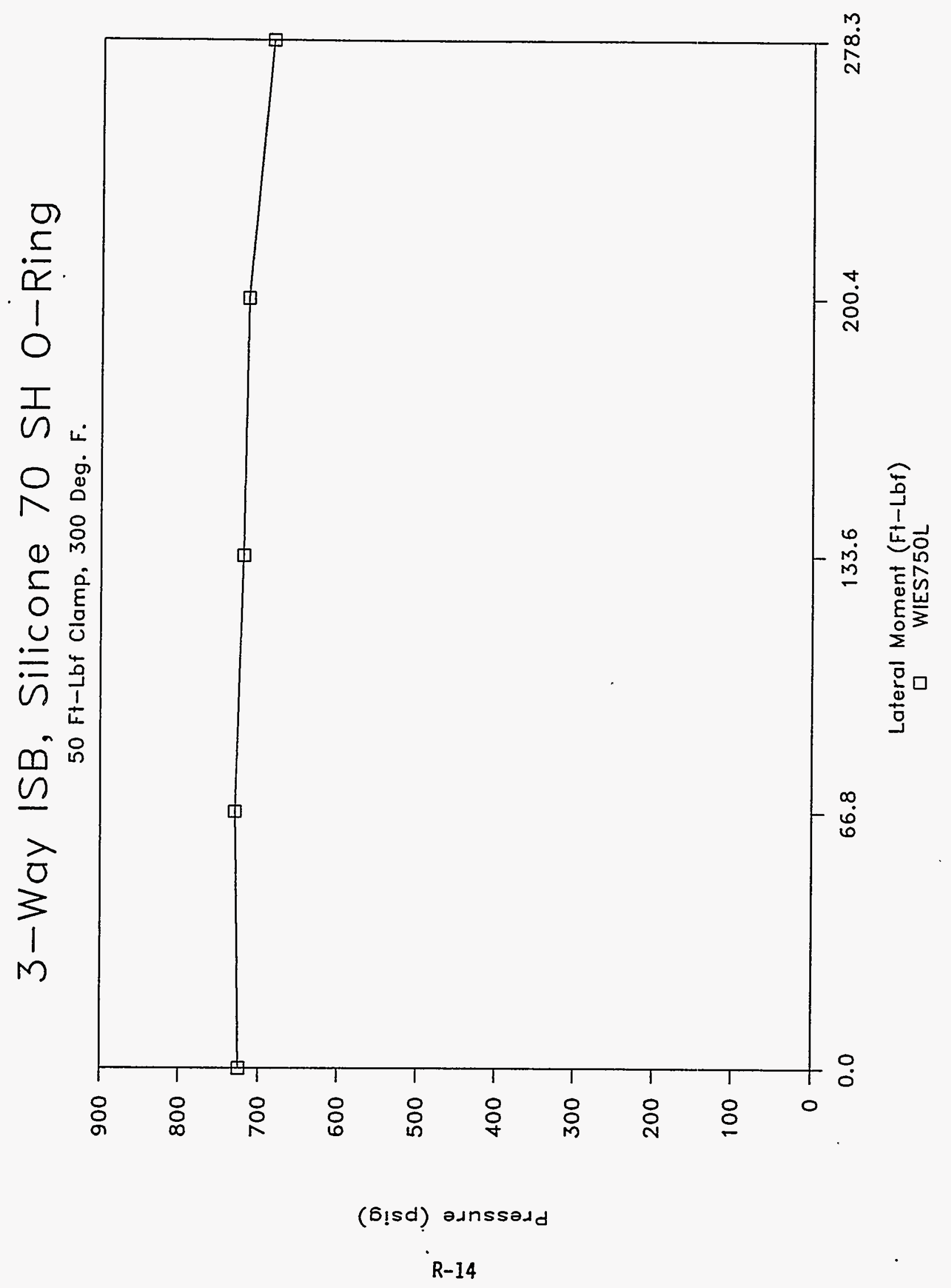


WHC-SD-WM-TRP-223

Rev. 0

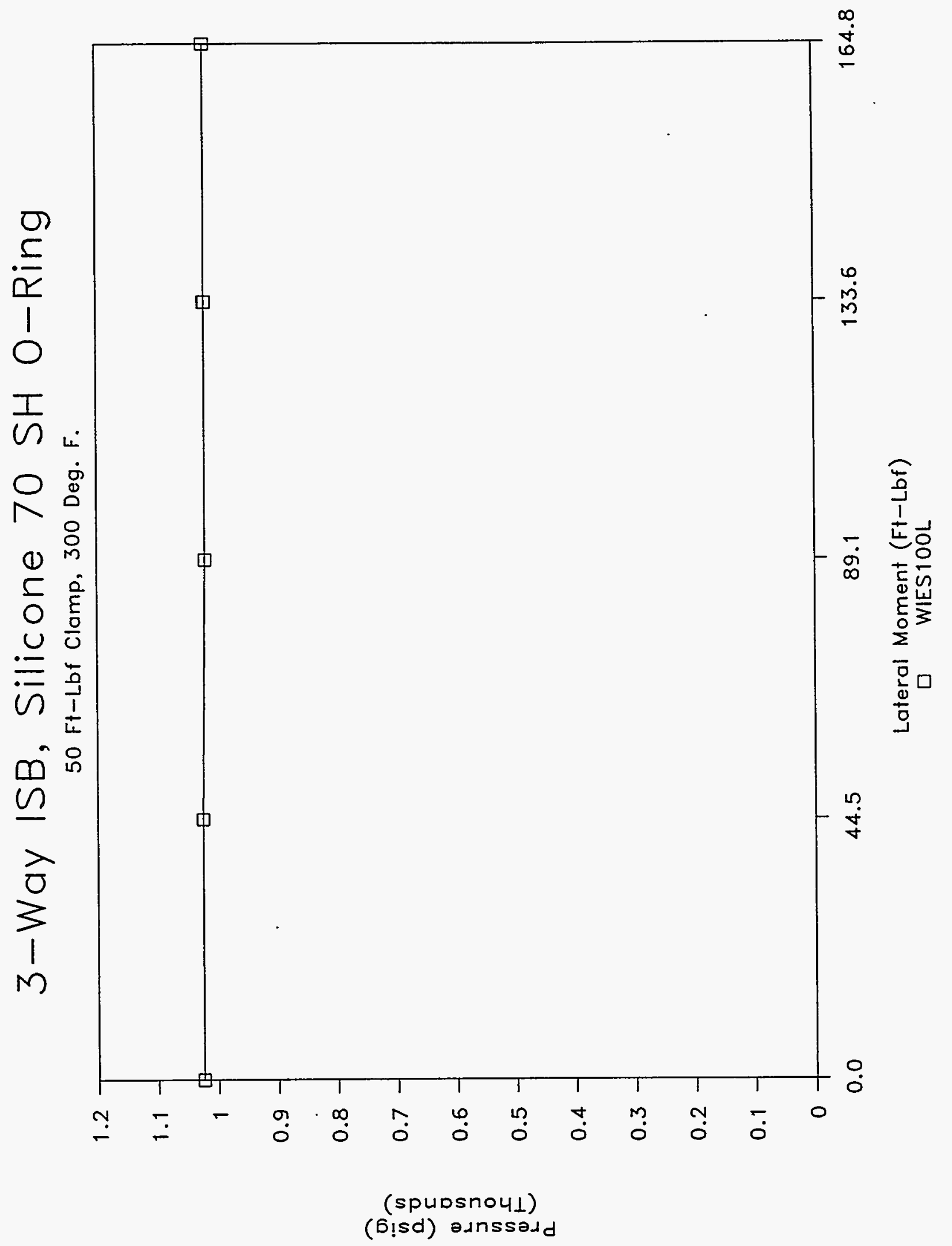


DECEMBER 12,1994

2" 3-WAY ISB CONNECTOR, SILICONE O-RING, 70 DUROMETER, ELEVATED TEMP. PART \# 2-119 5604, PARKER SEAL CO., BATCH \# 75561, CURE DATE $3 Q 93$ LEAK TEST - LATERAI MOMENT (SIDEWAYS ROTATION)

CLAMPING TORQUE $=50$ FT-IBF NOMINAL CHARGE PRESSURE $=250$ PSIG

GRAPH NAME $=$ WIES250I

TEMPERATURE: 300 DEG. F.

$\begin{array}{lllllll} & & & & & \\ \text { PRESSURE } & \text { ELAPSED } & \text { ELAPSED } & \text { ELAPSED } & \text { LATERAL } & \text { MOMENAT } & \text { IATERAL } \\ \text { PSIG } & \text { MINUTES } & \text { SECONDS } & \text { DECIMAL } & \text { FORCE } & \text { ARM } & \text { MOMENT } \\ & & & \text { MINUTES } & \text { IBS } & \text { FT. } & \text { FT-LBF. } \\ \text { INPUT } & \text { INPUT } & \text { INPUT } & \text { COMPUTE } & \text { INPUT } & \text { INPU! } & \text { COMPUTE }\end{array}$

$\begin{array}{rrrrrrr}255 & 0 & 0 & 0.00 & 0 & 0.44453 & 0.0 \\ 285 & 2 & 0 & 2.00 & 0 & 0.44453 & 0.0 \\ 315 & 4 & 0 & 4.00 & 250 & 0.44453 & 111.3 \\ 325 & 6 & 0 & 6.00 & 500 & 0.4: 453 & 222.7 \\ 285 & 8 & 0 & 8.00 & 750 & 0.44453 & 334.0 \\ 275 & 10 & 0 & 10.00 & 1000 & 0.4: 453 & 445.3\end{array}$

INCREASED PRESSURE TO 500 PSIG

GRAPH NAME = WIES50OL

$\begin{array}{rrrrrrr}520 & 12 & 0 & 12.00 & 0 & 0.4: 453 & 0.0 \\ 540 & 14 & 0 & 14.00 & 200 & 0.4: 453 & 89.1 \\ 535 & 16 & 0 & 16.00 & 400 & 0.4: 453 & 178.1 \\ 535 & 18 & 0 & 18.00 & 600 & 0.4: 453 & 267.2 \\ 515 & 20 & 0 & 20.00 & 800 & 0.4: 453 & 356.2\end{array}$

INCREASED PRESSURE TO 750 PSIG

GRAPH NAME = WIES750I

$\begin{array}{rrrrrrr}725 & 22 & 0 & 22.00 & 0 & 0.4: 453 & 0.0 \\ 730 & 24 & 0 & 24.00 & 150 & 0.4: 453 & 66.8 \\ 720 & 26 & 0 & 26.00 & 300 & 0.4: 453 & 133.6 \\ 715 & 28 & 0 & 28.00 & 450 & 0.4: 453 & 200.4 \\ 685 & 30 & 0 & 30.00 & 625 & 0.44453 & 278.3\end{array}$

INCREASED PRÉSSURE TO 1000 PSIG

$\begin{array}{ll}1025 & 32 \\ 1025 & 34 \\ 1020 & 36 \\ 1020 & 38 \\ 1020 & 40\end{array}$

GRAPH NAME $=$ VIIES100L

$\begin{array}{rrr}0 & 0.4453 & 0.0 \\ 100 & 0.4453 & 44.5 \\ 200 & 0.4453 & 89.1 \\ 300 & 0.4453 & 133.6 \\ 370 & 0.4453 & 164.8\end{array}$


WHC-SD-WM-TRP-223

Rev. 0

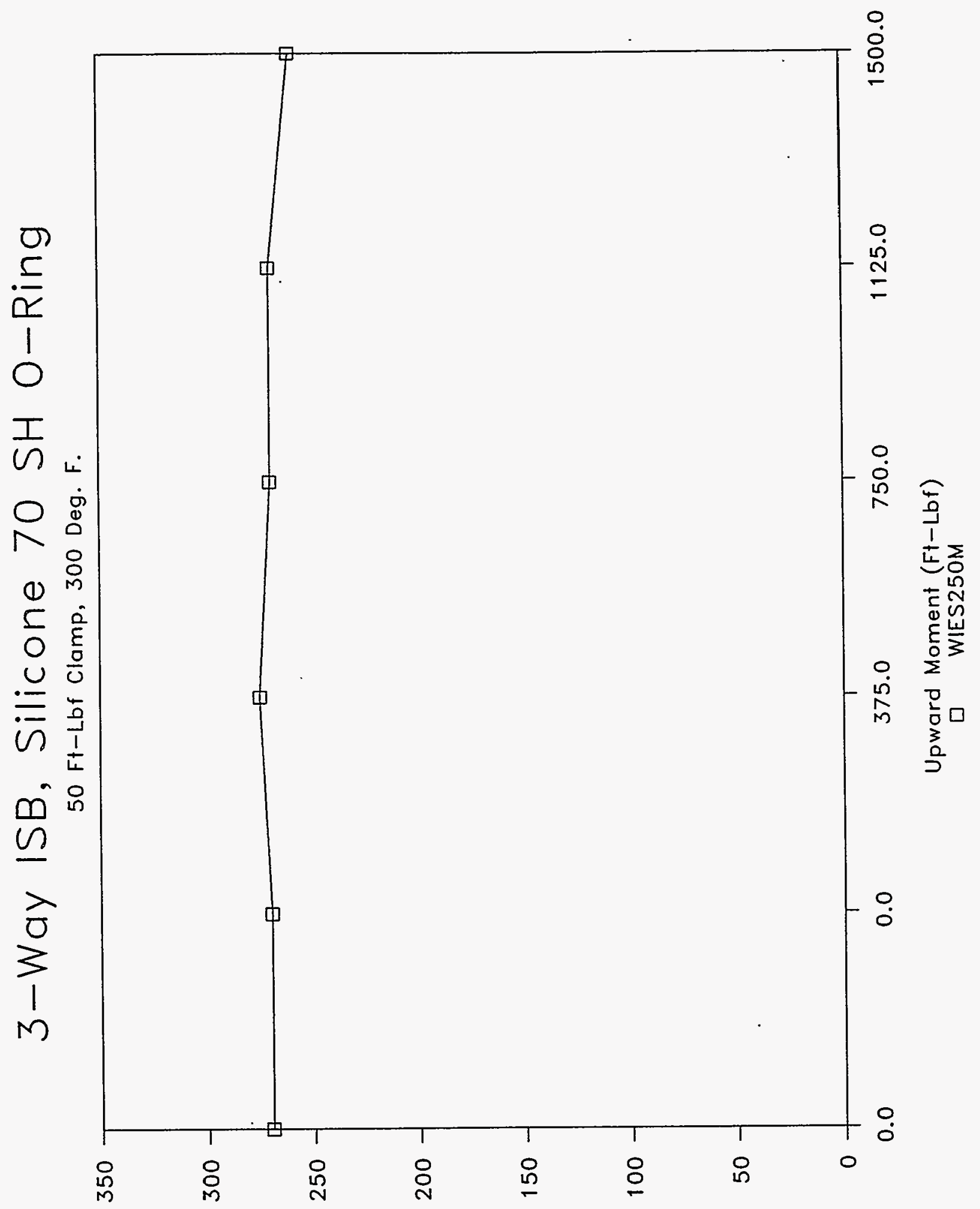

(b!sd) aגnssadd

$R$ X-17

Per telecener. 


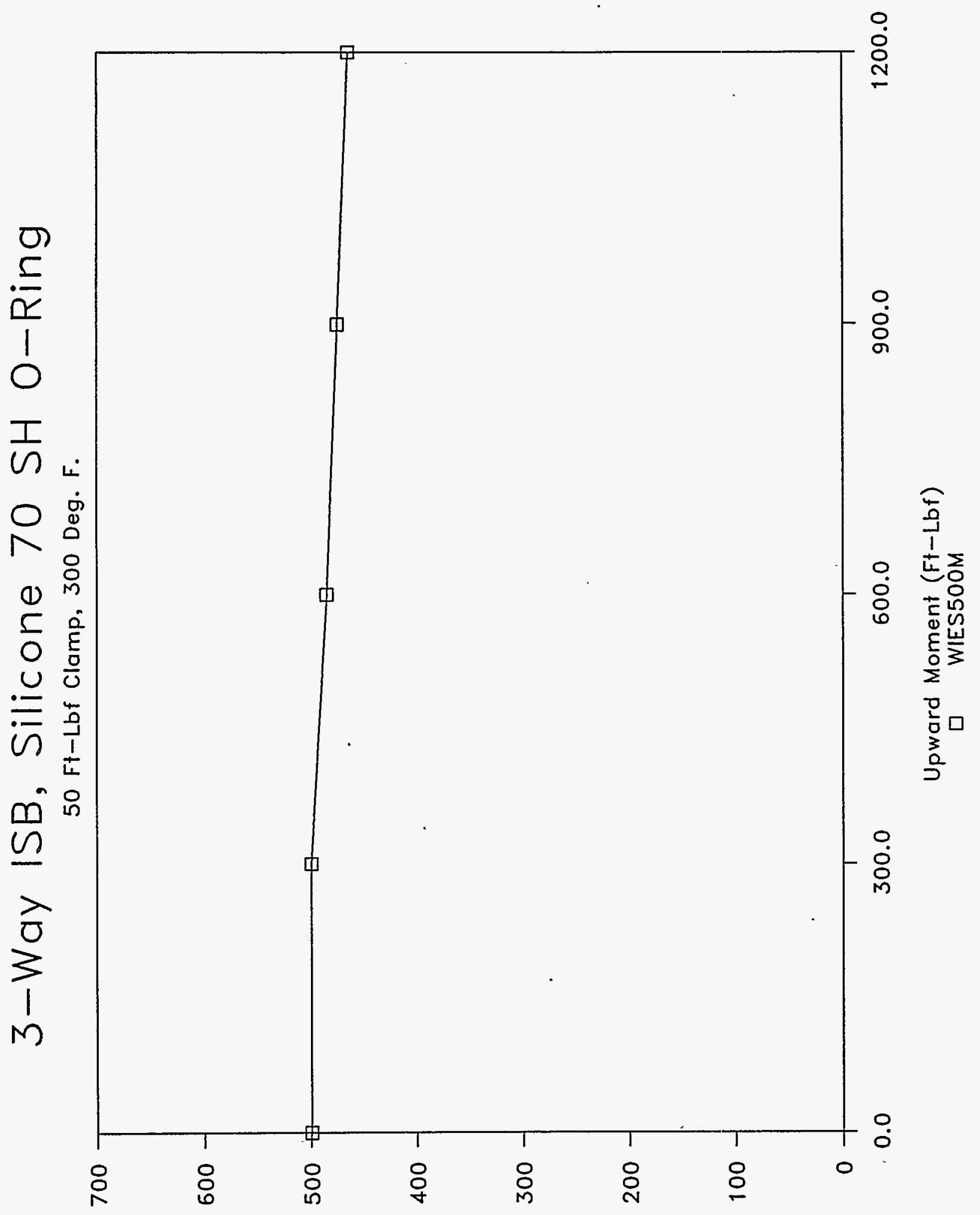

(b!sd) asnssadd 
WHC-SD-WM-TRP-223

Rev. 0

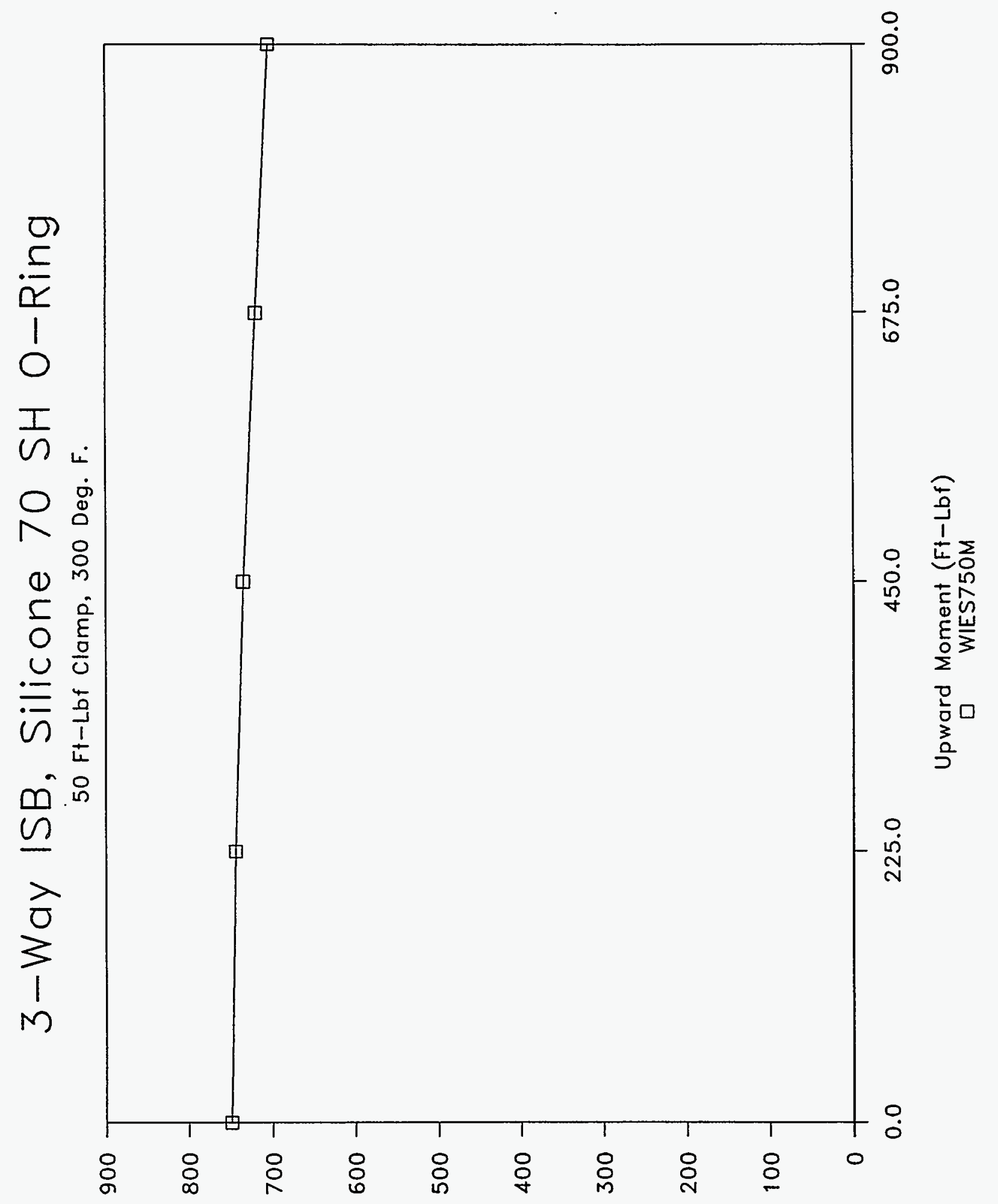

(bisd) adnssadd

$R-19$ 


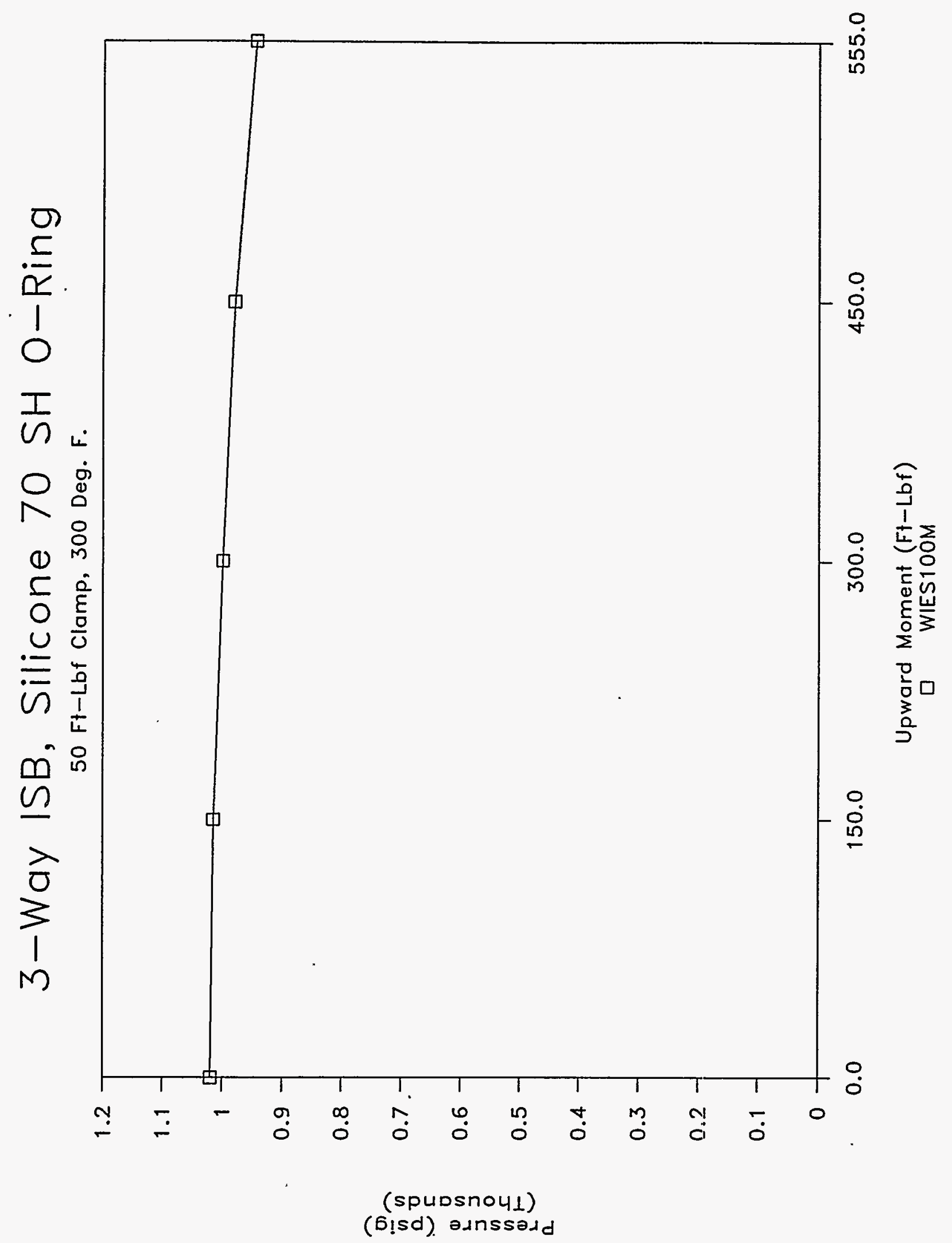


DECEMBER 06, 1994

2" 3-WAY ISB CONNECTOR, SILICONE O-RING, 70 DUROMETER, ELEVATED TEMP. PART \# 2-119 5604, PARKER SEAL CO., BATCH \# 75561, CURE DATE 3093 IEAK TEST - UP AND OVER MOMENT (UPWARD ROTATION)

CLAMPING TORQUE $=50$ FT-LBF NOMINAI GRAPH NAME $=$ WIES25OM

CHARGE PRESSURE $=250$ PSIG

TEMPERATURE: 300 DEG. F.

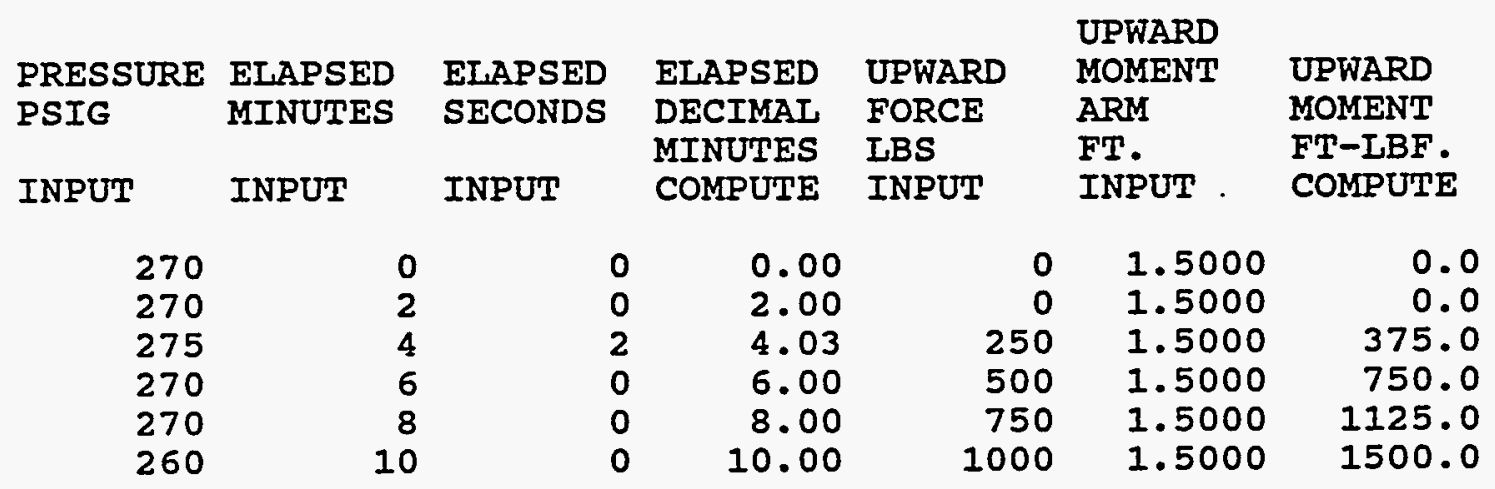

INCREASED PRESSURE TO 500 PSIG

$\begin{array}{llll}500 & 12 & 0 & 12.00 \\ 500 & 14 & 0 & 14.00 \\ 485 & 16 & 0 & 16.00 \\ 475 & 18 & 0 & 18.00 \\ 465 & 20 & 0 & 20.00\end{array}$

INCREASED PRESSURE TO 750 PSIG

$\begin{array}{llll}750 & 22 & 0 & 22.00 \\ 745 & 24 & 0 & 24.00 \\ 735 & 26 & 0 & 26.00 \\ 720 & 28 & 0 & 28.00 \\ 705 & 30 & 0 & 30.00\end{array}$

INCREASED PRESSURE TO 1000 PSIG

$\begin{array}{rr}1020 & 32 \\ 1015 & 34 \\ 1000 & 36 \\ 980 & 38 \\ 945 & 40\end{array}$

GRAPH NAME = WIES50OM

$\begin{array}{rrr}0 & 1.5000 & 0.0 \\ 200 & 1.5000 & 300.0 \\ 400 & 1.5000 & 600.0 \\ 600 & 1.5000 & 900.0 \\ 800 & 1.5000 & 1200.0\end{array}$

GRAPH NAME = WIES750M

$\begin{array}{rrr}0 & 1.5000 & 0.0 \\ 150 & 1.5000 & 225.0 \\ 300 & 1.5000 & 450.0 \\ 450 & 1.5000 & 675.0 \\ 600 & 1.5000 & 900.0\end{array}$

GRAPH NAME = WIESIOOM

$\begin{array}{rrr}0 & 1.5000 & 0.0 \\ 100 & 1.5000 & 150.0 \\ 200 & 1.5000 & 300.0 \\ 300 & 1.5000 & 450.0 \\ 370 & 1.5000 & 555.0\end{array}$


WHC-SD-WM-TRP-223

Rev. 0

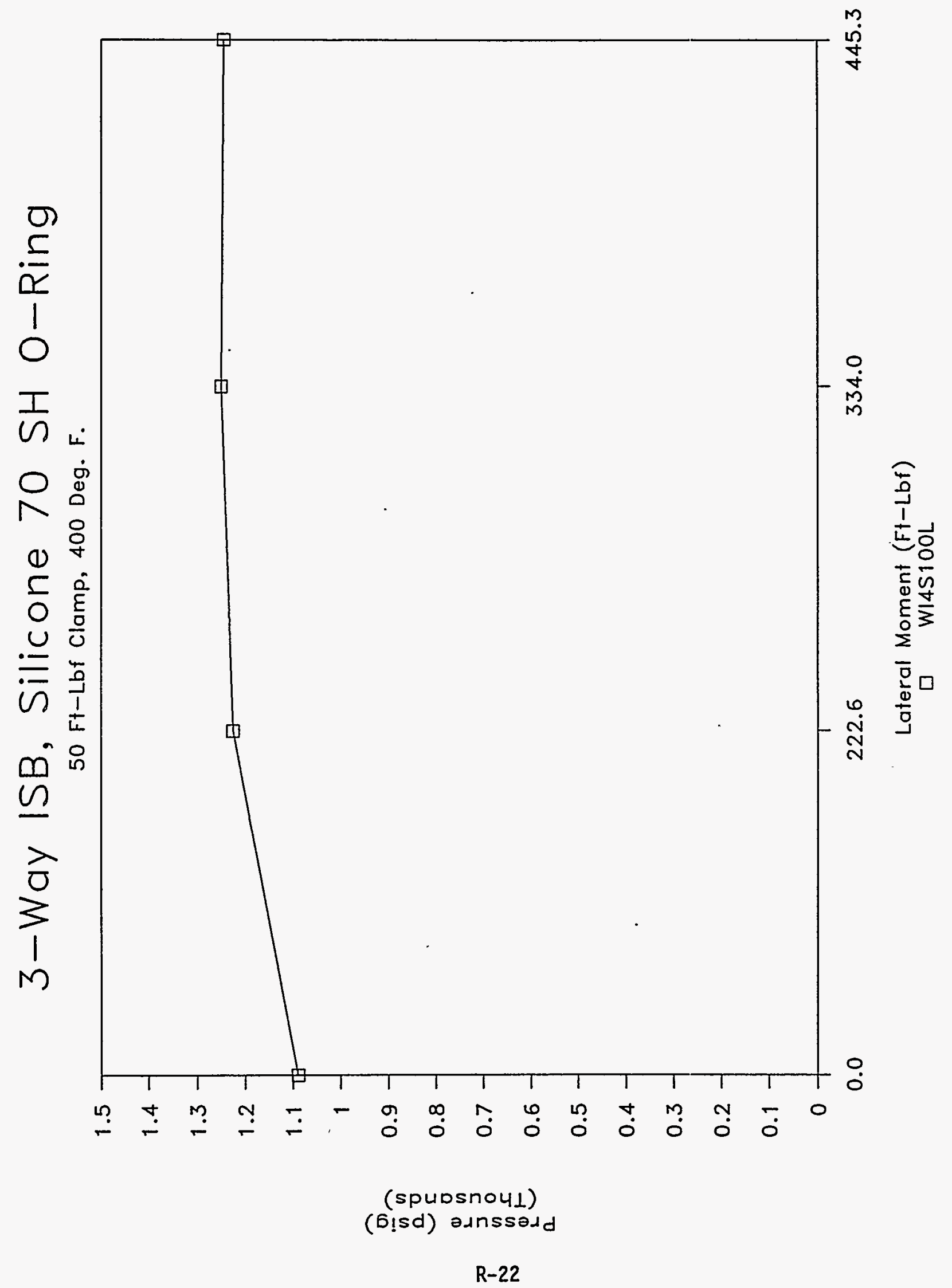


WHC-SD-WM-TRP-223

Rev. 0

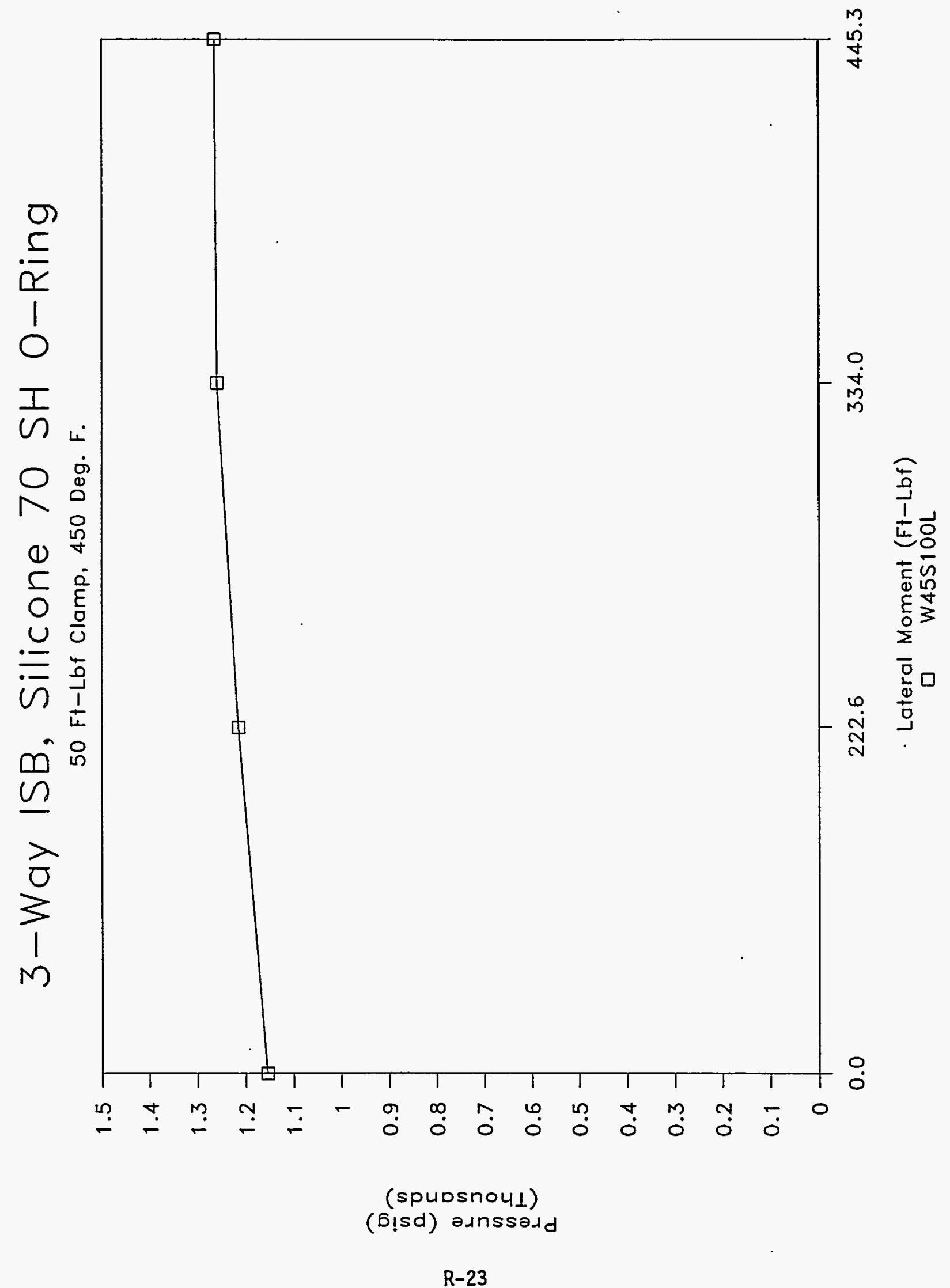




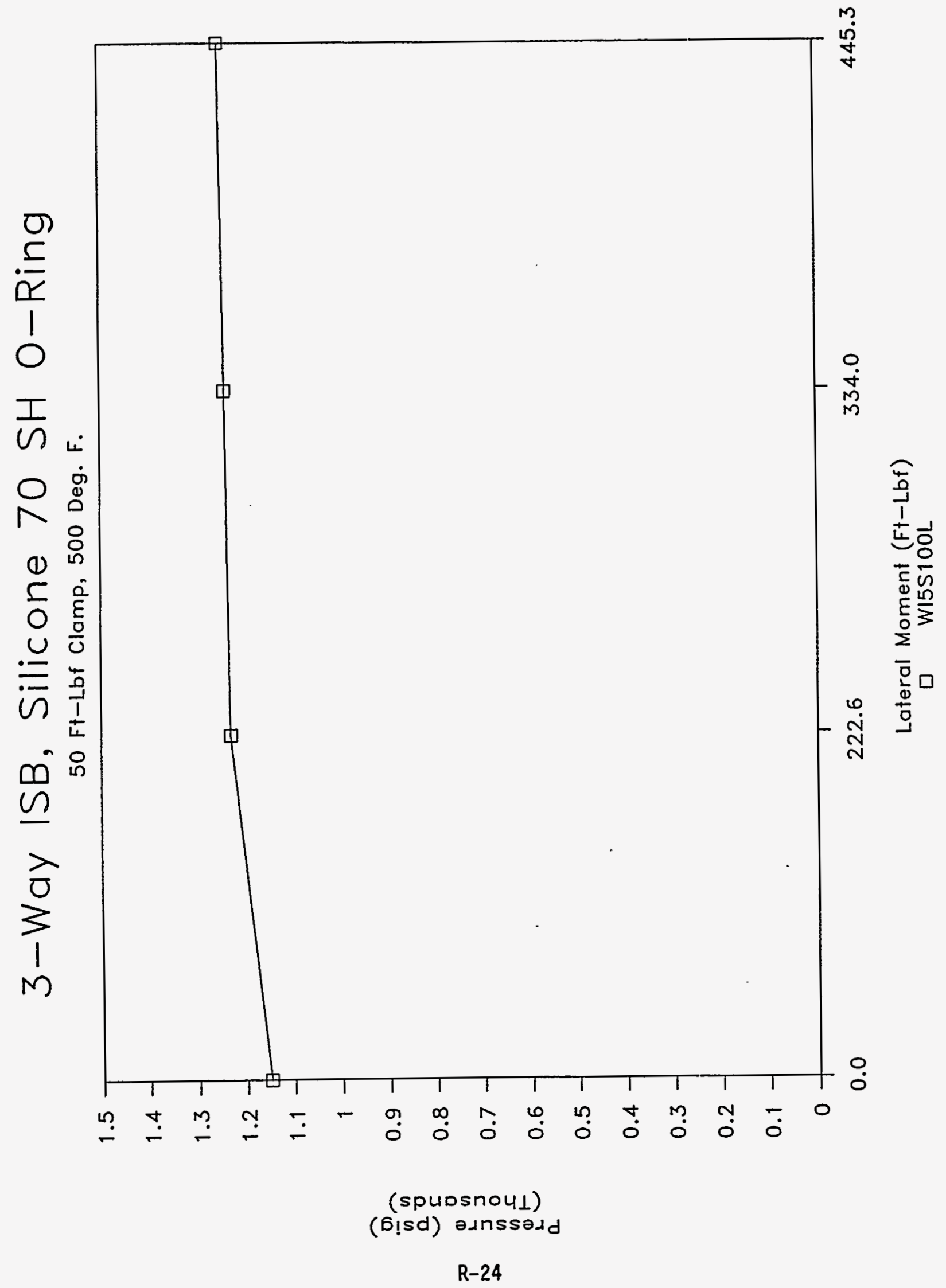


DECEMBER 12,1994

2" 3-WAY ISB CONNECTOR, SILICONE O-RING, 70 DUROMETER, ELEVATED TEMP. PART \# 2-119 5604, PARKER SEAL CO., BATCH \# 75561, CURE DATE 3093

LEAK TEST - ILATERAL MOMENT (SIDEWAYS ROTATION)

CLAMPING TORQUE $=50$ FT-LBF NOMINAL

Note: Following comments added on Dec. 15, 1994 by Ed S. Ruff:

The following series of tests was performed at the end of the official" test program sequence. A deliberate attempt was made to cause an o-ring failure, and subsequent leak, in the 3-way ISB connector that was on the stand from the previous test.

The lateral moment load was increased to the max. test stand capacity of 1,000 Ibf. Ultimately, the pressure was increased to approx. 1,250 psig and the temperature was elevated to $500 \mathrm{deg}$. F.

In spite of all this, the 3-way ISB connector, with silicone o-rings installed, did not leak or lose pressure.

MOMENT LOAD INCREASED TO 1000 LBS.

NO GRAPH
960
42
$0 \quad 42.00$
1000
0.4453
445.3

INCREASED TEMP. TO 400 DEG. F; PRESSURE AT 1000 PSIG.

GRAPH NAME = WI4S10OL

$\begin{array}{rrrrrrr}1090 & 44 & 0 & 44.00 & 0 & 0.4453 & 0.0 \\ 1225 & 46 & 0 & 46.00 & 500 & 0.4453 & 222.7 \\ 1250 & 48 & 0 & 48.00 & 750 & 0.4453 & 334.0 \\ 1245 & 50 & 0 & 50.00 & 1000 & 0.4453 & 445.3\end{array}$

INCREASED TEMP. TO 450 DEG. F; PRESSURE AT 1000 PSIG.

GRAPH NAME $=$ W45S100I

$\begin{array}{rrrrrrr}1155 & 52 & 0 & 52.00 & 0 & 0.4453 & 0.0 \\ 1215 & 54 & 0 & 54.00 & 500 & 0.4453 & 222.7 \\ 1260 & 56 & 0 & 56.00 & 750 & 0.4453 & 334.0 \\ 1265 & 58 & 0 & 58.00 & 1000 & 0.4453 & 445.3\end{array}$

INCREASED TEMP. TO 500 DEG. F; PRESSURE AT 1000 PSIG.

GRAPH NAME = WI5S100L

1150

$59 \quad 0 \quad 59.00$

$0 \quad 0.4453$

0.0 
WHC-SD-WM-TRP-2:3 ReV 0

$\begin{array}{rrrrrrr}1230 & 61 & 0 & 61.00 & 500 & 0.4453 & 222.7 \\ 1240 & 63 & 0 & 63.00 & 750 & 0.4453 & 334.0 \\ 1250 & 65 & 0 & 65.00 & 1000 & 0.4453 & 445.3\end{array}$


WHC-SD-WM-TRP-223

Rev. 0

APPENDIX S: GRAPHS OF THREE-WAY EPDM TESTS

S-1 
WHC-SD-WM-TRP-223

Rev. 0

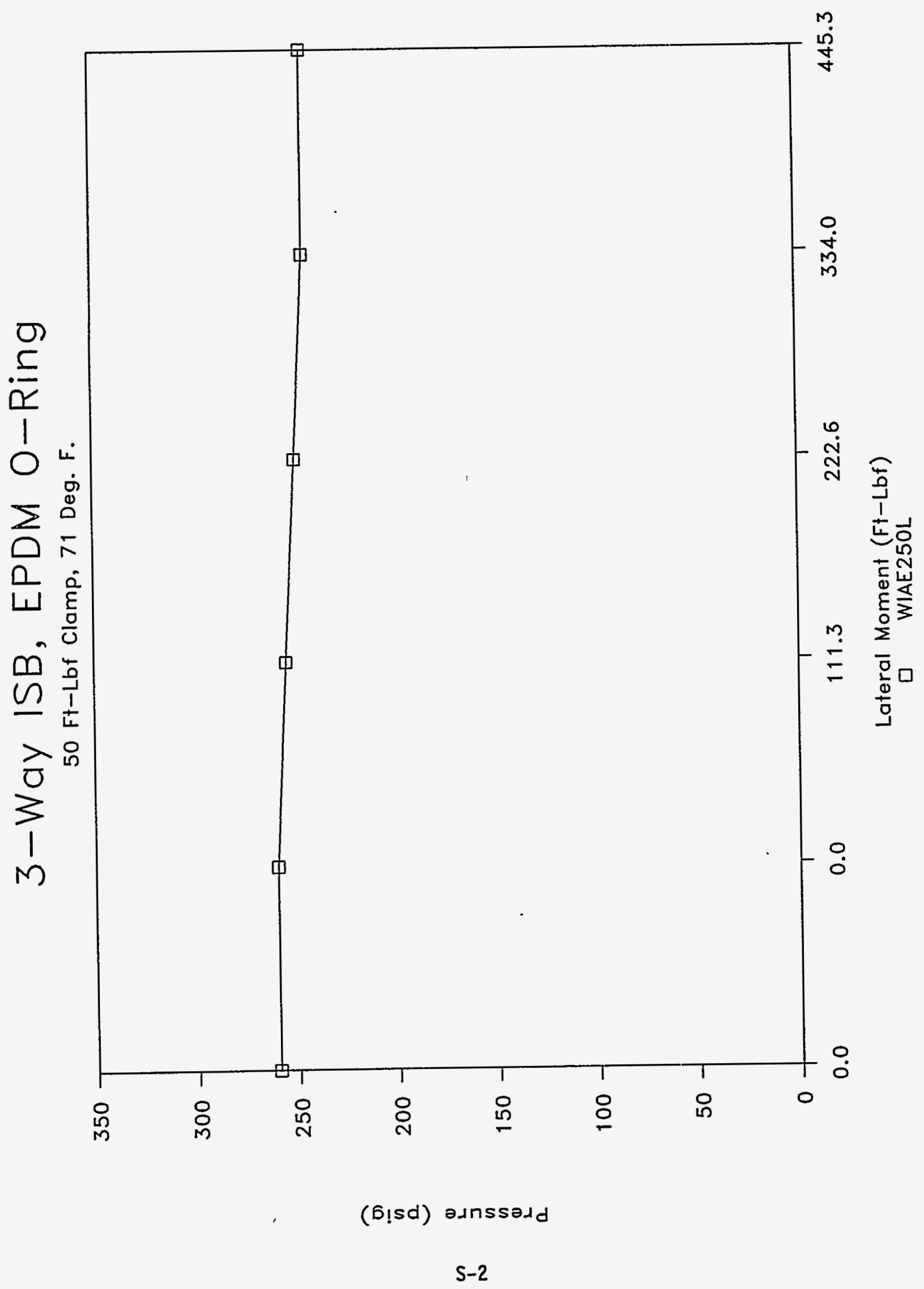


WHC-SD-WM-TRP-223

Rev. 0

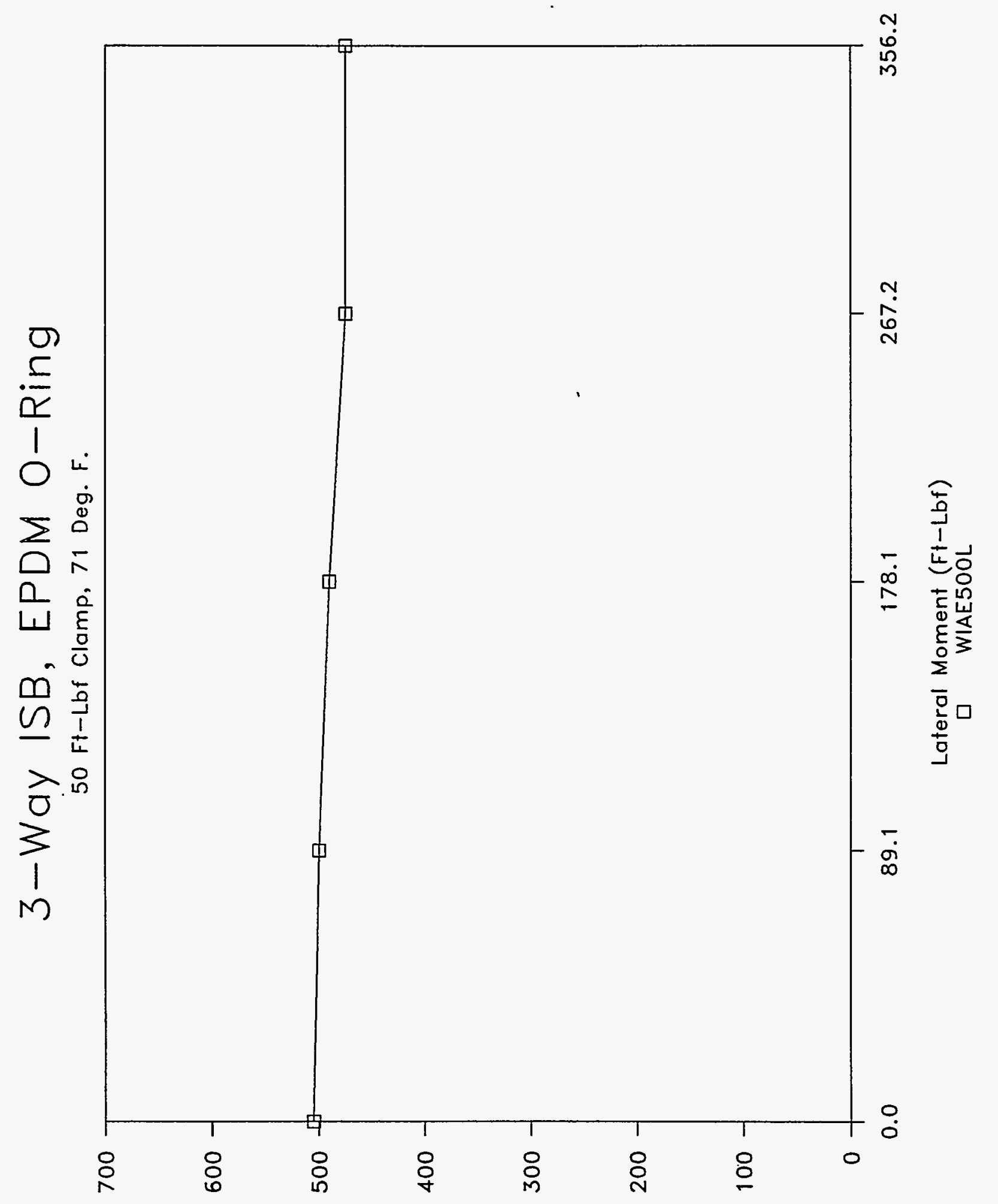

(b!sd) adinssadd 


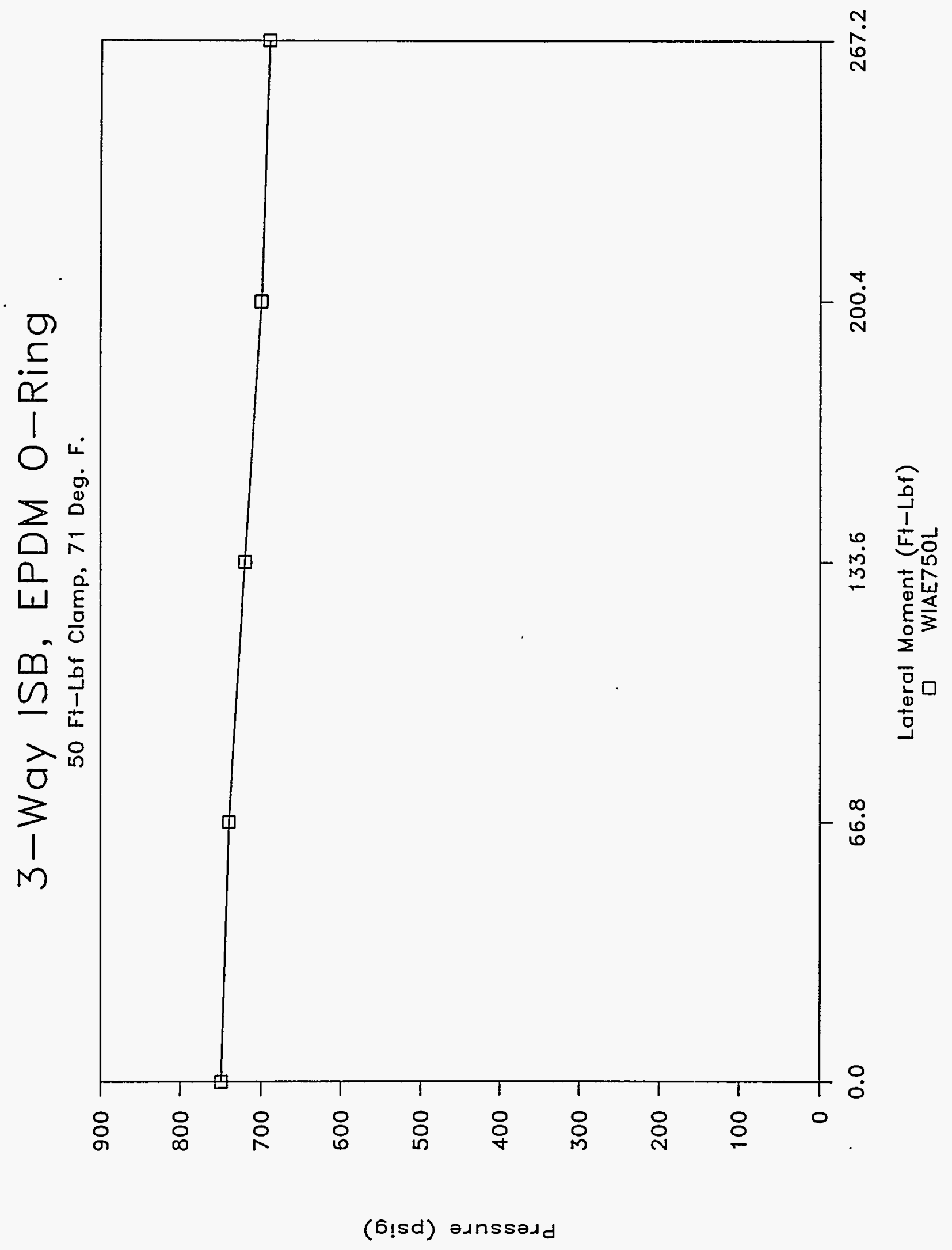


WHC-SD-WM-TRP-223

Rev. 0

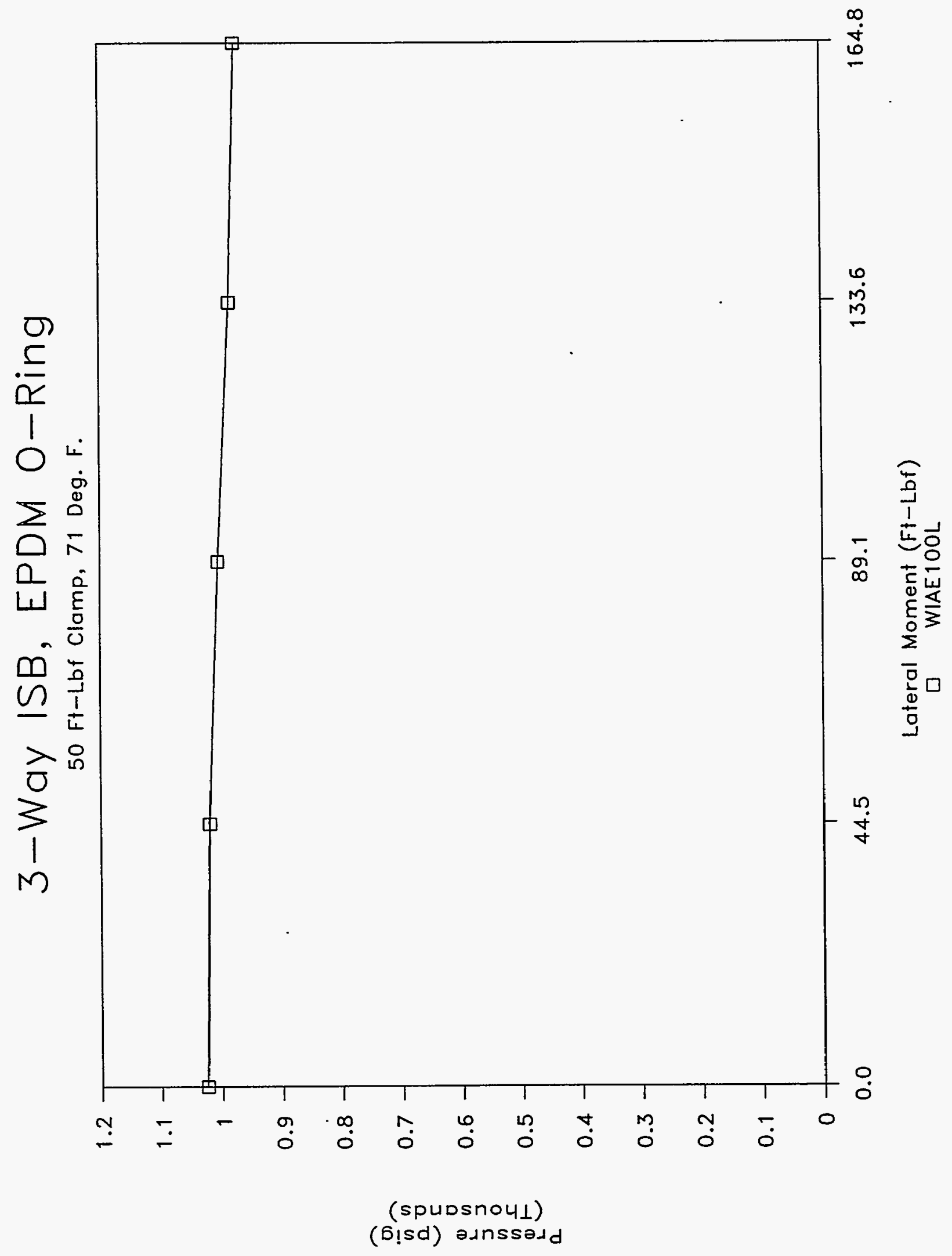

S-5 
DECEMBER 07, 1994

2" 3-WAY ISB CONNECTOR, EPDM O-RING, 70 DUROMETER, AMBIENT TEMP. PART \# 2-119 E893-80, PARKER SEAL CO., BATCH \# 315989, CURE DATE 3294 LEAK TEST - LATERAL MOMENT (SIDEWAYS ROTATION)

CLAMPING TORQUE $=50$ FT-LBF NOMINAL CHARGE PRESSURE $=250$ PSIG GRAPH NAME = WIAE250L TEMPERATURE: 71 DEG. F.

\begin{tabular}{|c|c|c|c|c|c|c|}
\hline $\begin{array}{l}\text { PRESSURE } \\
\text { PSIG }\end{array}$ & $\begin{array}{l}\text { ELAPSED } \\
\text { MINUTES }\end{array}$ & $\begin{array}{l}\text { ELAPSED } \\
\text { SECONDS }\end{array}$ & $\begin{array}{l}\text { ELAPSED } \\
\text { DECIMAL } \\
\text { MINUTES } \\
\text { COMPUTE }\end{array}$ & $\begin{array}{l}\text { IATERAL } \\
\text { FORCE } \\
\text { LBS } \\
\text { INPUT }\end{array}$ & $\begin{array}{l}\text { LATERAL } \\
\text { MOMENT } \\
\text { ARM } \\
\text { FT. }\end{array}$ & $\begin{array}{l}\text { LATERAL } \\
\text { MOMENT } \\
\text { FT-IBF. } \\
\text { COMPUTE }\end{array}$ \\
\hline NPUT & INPUT & INPUT & OMPUTE & UT & NPUT & CUMFUTE \\
\hline $\begin{array}{l}260 \\
260 \\
255 \\
250 \\
245 \\
245\end{array}$ & $\begin{array}{l}0 \\
2 \\
4 \\
6 \\
8\end{array}$ & & $\begin{array}{r}0.00 \\
2.00 \\
4.00 \\
6.00 \\
8.00 \\
10.00\end{array}$ & $\begin{array}{r}0 \\
0 \\
250 \\
500 \\
750 \\
1000\end{array}$ & $\begin{array}{l}0.4453 \\
0.4453 \\
0.4453 \\
0.4453 \\
0.4453 \\
0.4453\end{array}$ & $\begin{array}{r}0.0 \\
0.0 \\
111.3 \\
222.7 \\
334.0 \\
445.3\end{array}$ \\
\hline
\end{tabular}

INCREASED PRESSURE TO 500 PSIG

$\begin{array}{llll}505 & 12 & 0 & 12.00 \\ 500 & 14 & 0 & 14.00 \\ 490 & 16 & 0 & 16.00 \\ 475 & 18 & 0 & 18.00 \\ 475 & 20 & 0 & 20.00\end{array}$

INCREASED PRESSURE TO 750 PSIG

$\begin{array}{llll}750 & 22 & 0 & 22.00 \\ 740 & 24 & 0 & 24.00 \\ 720 & 26 & 0 & 26.00 \\ 700 & 28 & 0 & 28.00 \\ 690 & 30 & 0 & 30.00\end{array}$

INCREASED PRESSURE TO 1000 PSIG

1025
1020
1005
985
975

$\begin{array}{ll}0 & 32.00 \\ 0 & 34.00 \\ 0 & 36.00 \\ 0 & 38.00 \\ 0 & 40.00\end{array}$
GRAPH NAME = WIAE50OI

$\begin{array}{rrr}0 & 0.4453 & 0.0 \\ 200 & 0.4453 & 89.1 \\ 400 & 0.4453 & 178.1 \\ 600 & 0.4453 & 267.2 \\ 800 & 0.4453 & 356.2\end{array}$

GRAPH NAME = WIAE750I

$\begin{array}{rrr}0 & 0.4453 & 0.0 \\ 150 & 0.4453 & 66.8 \\ 300 & 0.4453 & 133.6 \\ 450 & 0.4453 & 200.4 \\ 600 & 0.4453 & 267.2\end{array}$

GRAPH NAME $=$ WIAE10OL

$\begin{array}{rrr}0 & 0.4453 & 0.0 \\ 100 & 0.4453 & 44.5 \\ 200 & 0.4453 & 89.1 \\ 300 & 0.4453 & 133.6 \\ 370 & 0.4453 & 164.8\end{array}$




\section{3-Way ISB, EPDM O-Ring}

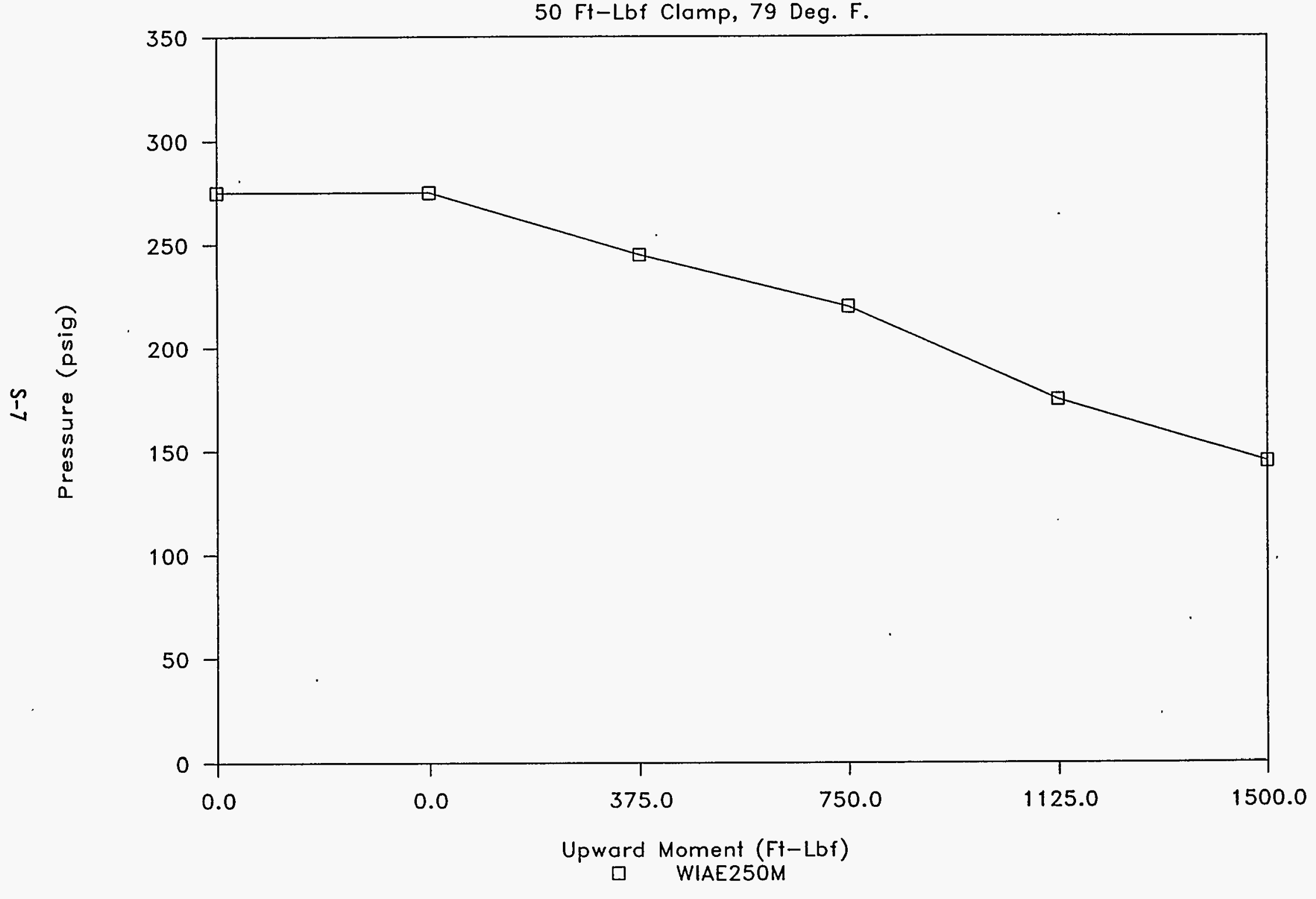


Rev. 0

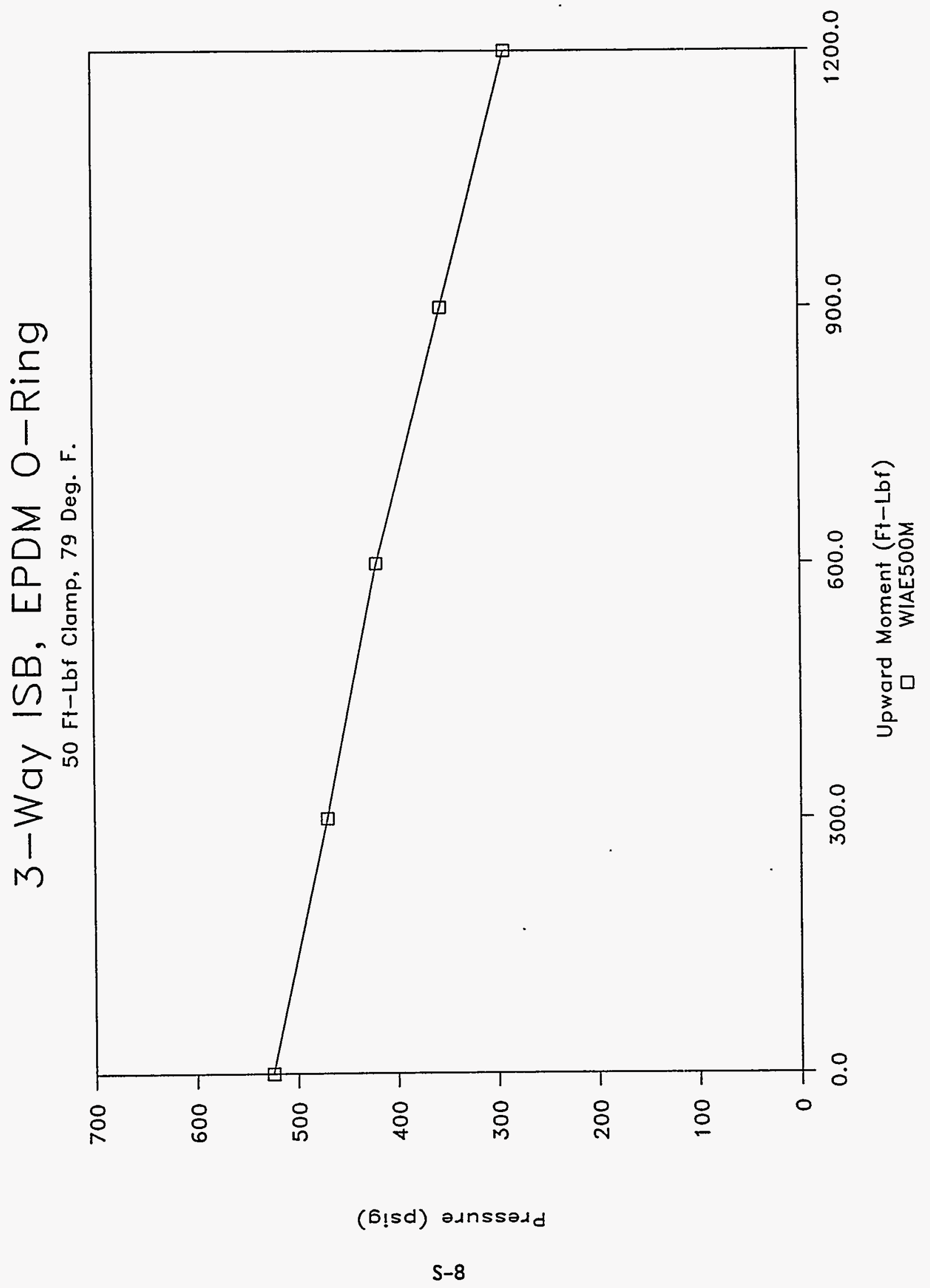


WHC-SD-WM-TRP-223

Rev. 0

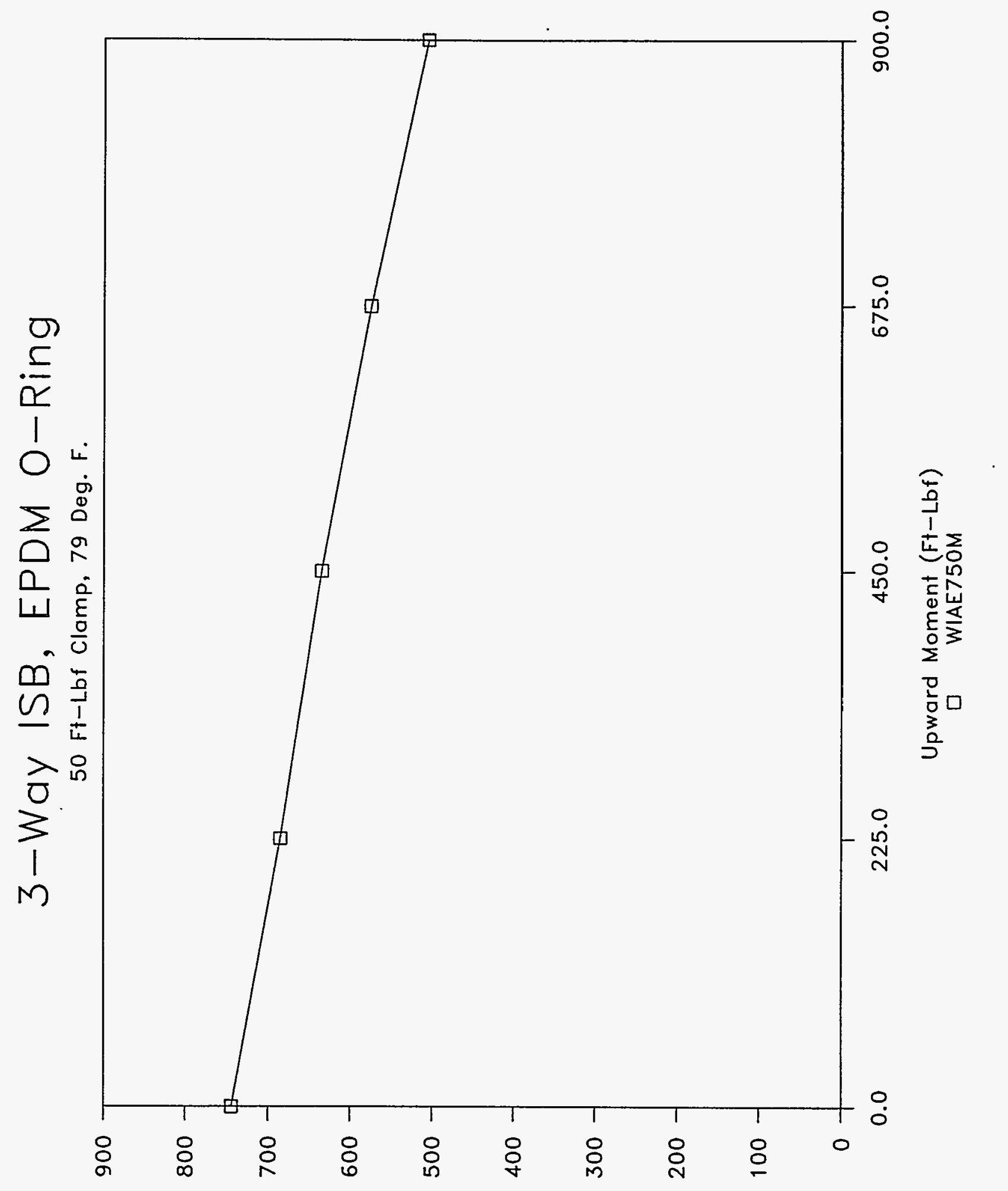

(bisd) annssead 
WHC-SD-WM-TRP-223

Rev. 0

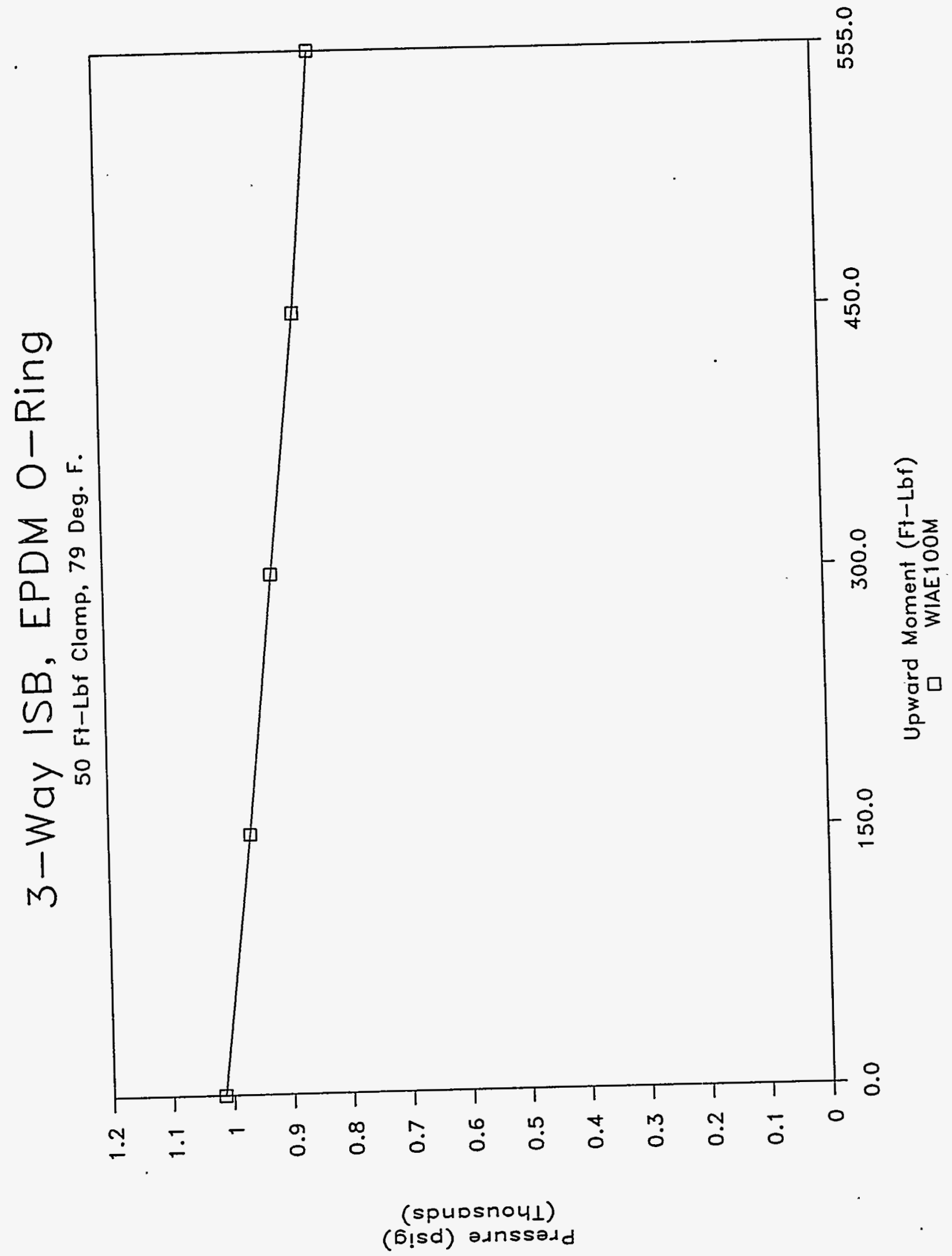


DECEMBER 02, 1994

2" 3-WAY ISB CONNECTOR, EPDM O-RING, 70 DUROMETER, AMBIENT TEMP. PART \# 2-119 E893-80, PARKER SEAL CO., BATCH \# 315989, CURE DATE $3 Q 94$ LEAK TEST - UP AND OVER MOMENT (UPWARD ROTATION)

CLAMPING TORQUE $=50$ FT-IBF NOMINAL CHARGE PRESSURE $=250$ PSIG

GRAPH NAME = WIAE250M

TEMPERATURE: 79 DEG. F.

\begin{tabular}{|c|c|c|c|c|c|c|}
\hline $\begin{array}{l}\text { PRESSURE } \\
\text { PSIG }\end{array}$ & $\begin{array}{l}\text { ELAPSED } \\
\text { MINUTES }\end{array}$ & $\begin{array}{l}\text { ELAPSED } \\
\text { SECONDS }\end{array}$ & $\begin{array}{l}\text { ELAPSED } \\
\text { DECIMAI } \\
\text { MINUTES } \\
\text { COMPUTE }\end{array}$ & $\begin{array}{l}\text { UPWARD } \\
\text { FORCE } \\
\text { LBS } \\
\text { INPUT }\end{array}$ & $\begin{array}{l}\text { UPWARD } \\
\text { MOMENT } \\
\text { ARM } \\
\text { FT. } \\
\text { INPUT }\end{array}$ & $\begin{array}{l}\text { UPWARD } \\
\text { MOMENT } \\
\text { FT-LBF. } \\
\text { COMPUTE }\end{array}$ \\
\hline 5 & INPUT & INPUI & COMPULE & INPUT & INPUI & COINF UID \\
\hline $\begin{array}{l}275 \\
275 \\
245 \\
220 \\
175 \\
145\end{array}$ & $\begin{array}{r}0 \\
2 \\
4 \\
6 \\
8 \\
10\end{array}$ & & $\begin{array}{r}0.00 \\
2.00 \\
4.00 \\
6.00 \\
8.00 \\
10.00\end{array}$ & $\begin{array}{r}0 \\
0 \\
250 \\
500 \\
750 \\
1000\end{array}$ & $\begin{array}{l}1.5000 \\
1.5000 \\
1.5000 \\
1.5000 \\
1.5000 \\
1.5000\end{array}$ & $\begin{array}{r}0.0 \\
0.0 \\
375.0 \\
750.0 \\
1125.0 \\
1500.0\end{array}$ \\
\hline
\end{tabular}

INCREASED PRESSURE TO 500 PSIG

$\begin{array}{llll}525 & 12 & 0 & 12.00 \\ 470 & 14 & 0 & 14.00 \\ 420 & 16 & 0 & 16.00 \\ 355 & 18 & 0 & 18.00 \\ 290 & 20 & 0 & 20.00\end{array}$

INCREASED PRESSURE TO 750 PSIG

$\begin{array}{llll}745 & 22 & 0 & 22.00 \\ 685 & 24 & 0 & 24.00 \\ 635 & 26 & 0 & 26.00 \\ 575 & 28 & 0 & 28.00 \\ 505 & 30 & 0 & 30.00\end{array}$

INCREASED PRESSURE TO 1000 PSIG

$\begin{array}{rlll}1015 & 32 & 0 & 32.00 \\ 965 & 34 & 0 & 34.00 \\ 920 & 36 & 0 & 36.00 \\ 875 & 38 & 0 & 38.00 \\ 840 & 40 & 0 & 40.00\end{array}$

GRAPH NAME = WIAE50OM

$\begin{array}{rrr}0 & 1.5000 & 0.0 \\ 200 & 1.5000 & 300.0 \\ 400 & 1.5000 & 600.0 \\ 600 & 1.5000 & 900.0 \\ 800 & 1.5000 & 1200.0\end{array}$

GRAPH NAME = WIAE750M

$\begin{array}{rrr}0 & 1.5000 & 0.0 \\ 150 & 1.5000 & 225.0 \\ 300 & 1.5000 & 450.0 \\ 450 & 1.5000 & 675.0 \\ 600 & 1.5000 & 900.0\end{array}$

GRAPH NAME = WIAE10OM

$\begin{array}{rrr}0 & 1.5000 & 0.0 \\ 100 & 1.5000 & 150.0 \\ 200 & 1.5000 & 300.0 \\ 300 & 1.5000 & 450.0 \\ 370 & 1.5000 & 555.0\end{array}$


WHC-SD-WM-TRP-223

Rev. 0

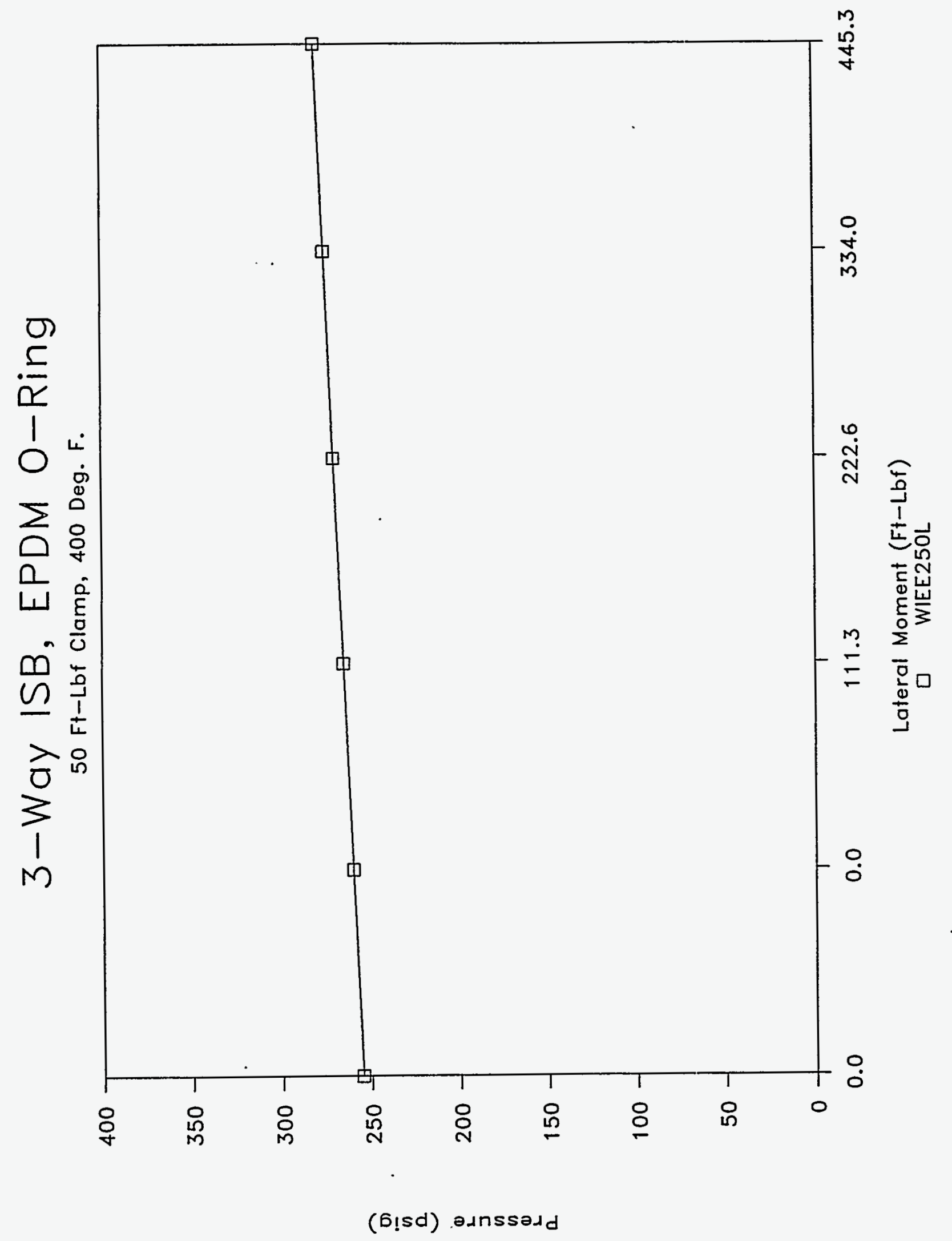

$S-12$ 


\section{3-Way ISB, EPDM O-Ring}

$50 \mathrm{Ft}-$ Lbf Clamp, 400 Deg. F.

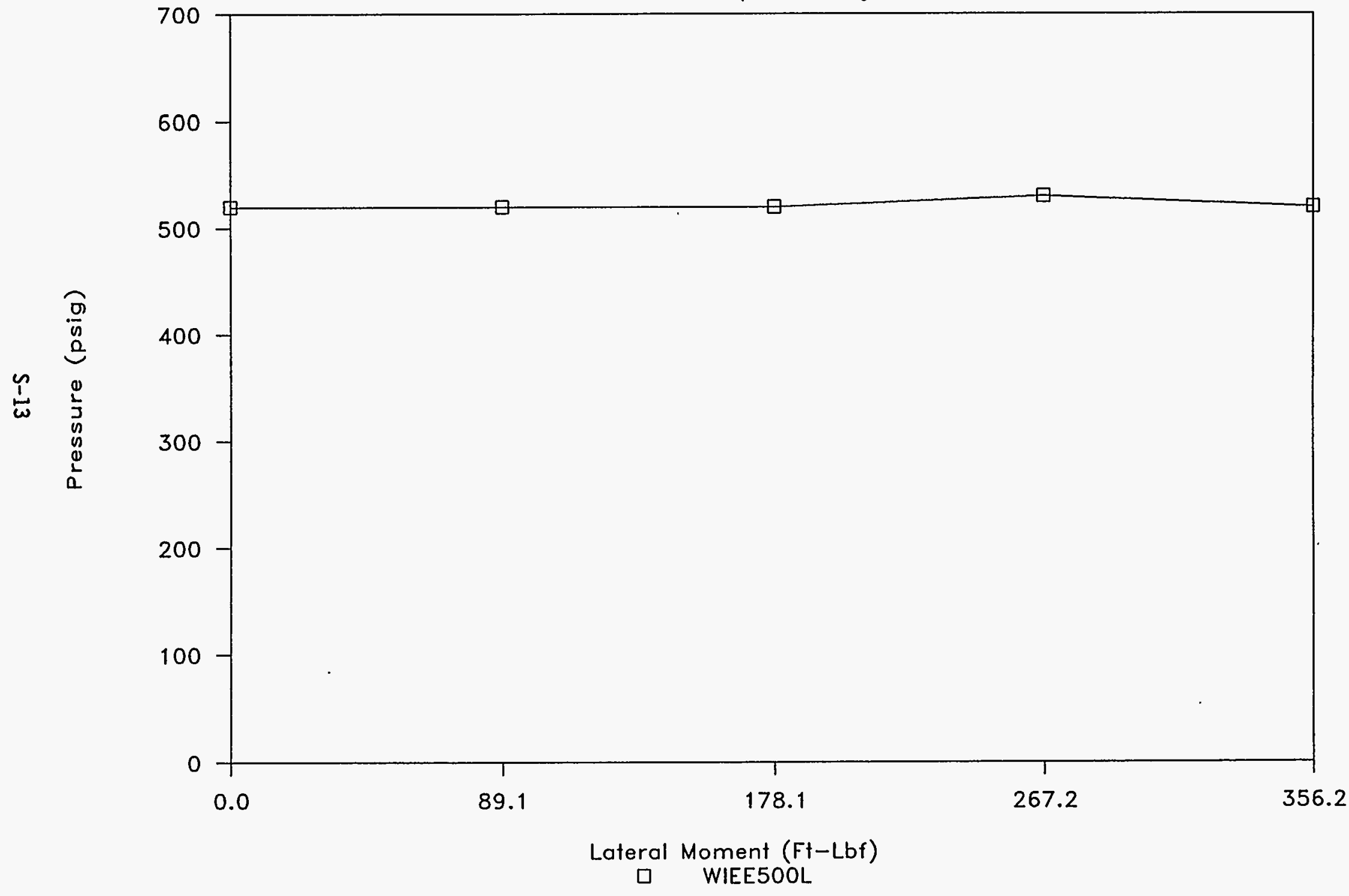




\section{3-Way ISB, EPDM O-Ring}

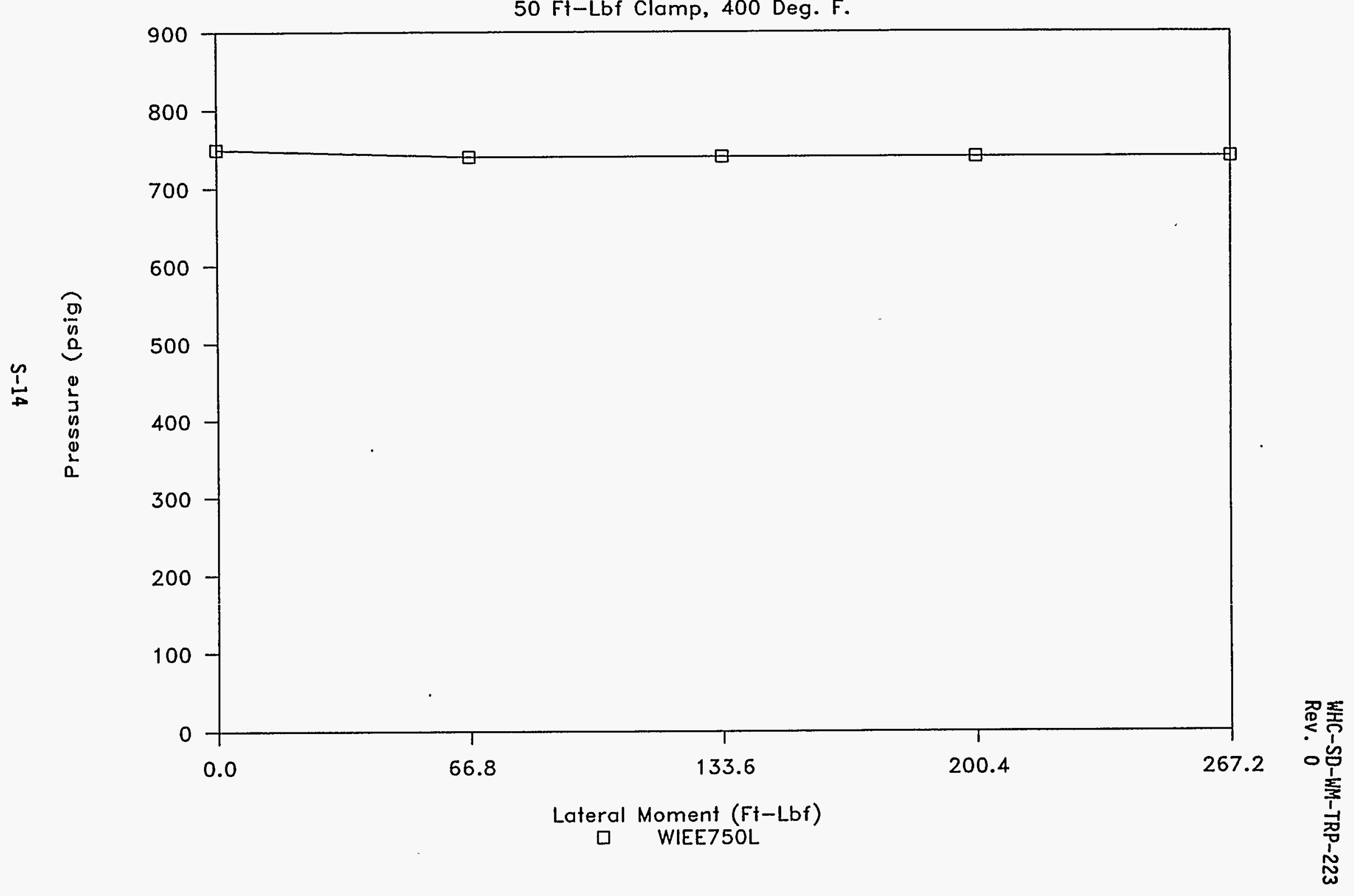


WHC-SD-WM-TRP-223

Rev. 0

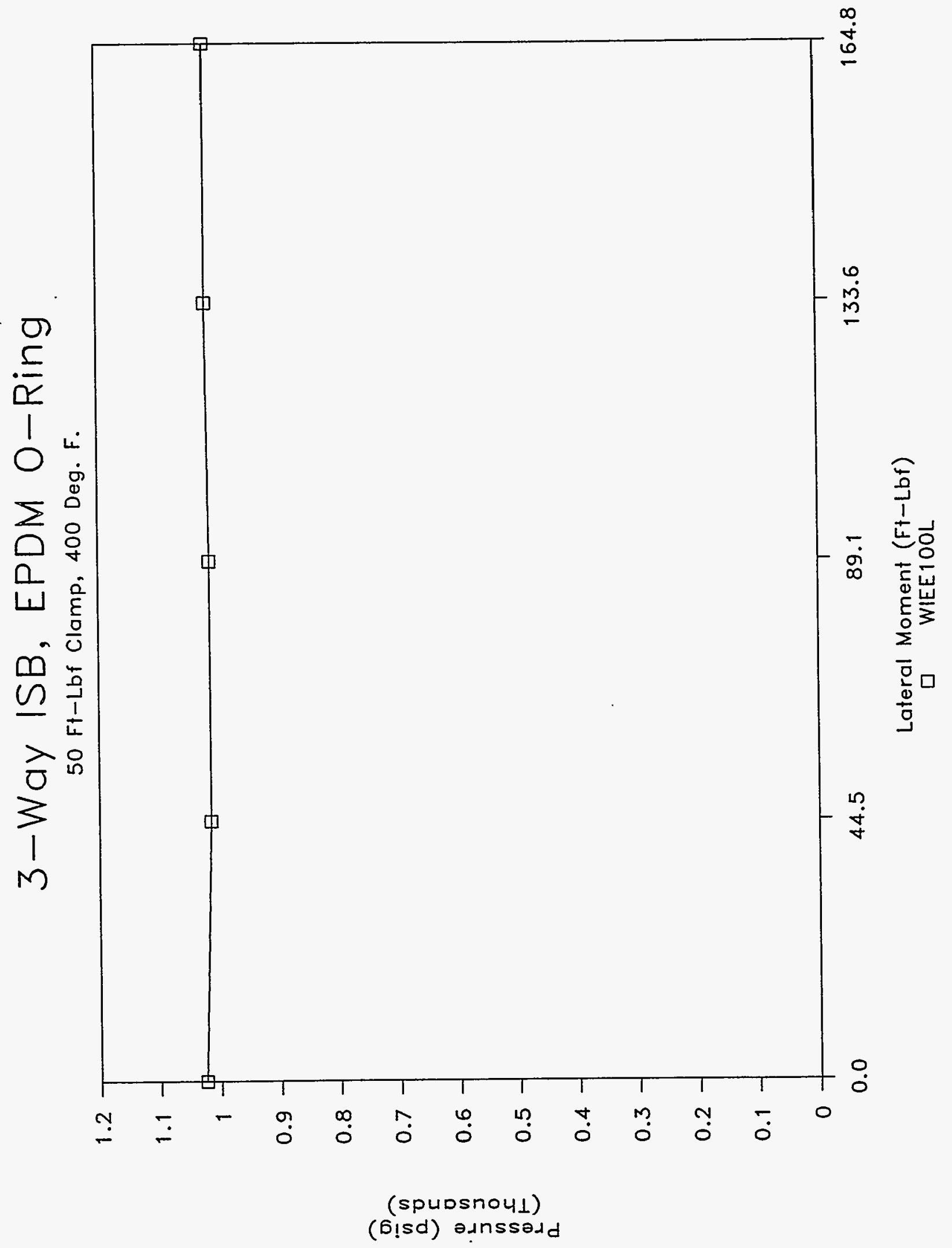

$S-15$ 
DECEMBER 07, 1994

2" 3-WAY ISB CONNECTOR, EPDM O-RING, 70 DUROMETER, ELEVATED TEMP. PART \# 2-119 E893-80, PARKER SEAL CO., BATCH \# 315989, CURE DATE 3294 LEAK TEST - I.ATERAL MOMENT (SIDEWAYS ROTATION)

CLAMPING TORQUE $=50$ FT-LBF NOMINAL CHARGE PRESSURE $=250$ PSIG
GRAPH: NAME = WIEE250L TEMPERATURE: 400 DEG. F.

\begin{tabular}{|c|c|c|c|c|c|c|}
\hline $\begin{array}{l}\text { PRESSURE } \\
\text { PSIG }\end{array}$ & $\begin{array}{l}\text { ELAAPSED } \\
\text { MINUTES }\end{array}$ & $\begin{array}{l}\text { ELAPSED } \\
\text { SECONDS }\end{array}$ & $\begin{array}{l}\text { ELAPSED } \\
\text { DECIMAL } \\
\text { MINUTES } \\
\text { COMPUTE }\end{array}$ & $\begin{array}{l}\text { LATERAL } \\
\text { FORCE } \\
\text { LBS } \\
\text { INPUT }\end{array}$ & $\begin{array}{l}\text { LATERAI } \\
\text { MOMENT } \\
\text { ARM } \\
\text { FT. } \\
\text { INPUT }\end{array}$ & $\begin{array}{l}\text { IATERAL } \\
\text { MOMENT } \\
\text { FT-LBF. } \\
\text { COMPUTE }\end{array}$ \\
\hline $\begin{array}{l}255 \\
260 \\
265 \\
270 \\
275 \\
280\end{array}$ & $\begin{array}{r}0 \\
2 \\
4 \\
6 \\
8 \\
10\end{array}$ & & $\begin{array}{r}0.00 \\
2.00 \\
4.00 \\
6.00 \\
8.00 \\
10.00\end{array}$ & $\begin{array}{r}0 \\
0 \\
250 \\
500 \\
750 \\
1000\end{array}$ & $\begin{array}{l}0.4453 \\
0.4453 \\
0.4453 \\
0.4453 \\
0.4453 \\
0.4453\end{array}$ & $\begin{array}{r}0.0 \\
0.0 \\
111.3 \\
222.7 \\
334.0 \\
445.3\end{array}$ \\
\hline
\end{tabular}

INCREASED PRESSURE TO 500 PSIG

$\begin{array}{llll}520 & 12 & 0 & 12.00 \\ 520 & 14 & 0 & 14.00 \\ 520 & 16 & 0 & 16.00 \\ 530 & 18 & 0 & 18.00 \\ 520 & 20 & 0 & 20.00\end{array}$

INCREASED PRESSURE TO 750 PSIG

$\begin{array}{llll}750 & 22 & 0 & 22.00 \\ 740 & 24 & 0 & 24.00 \\ 740 & 26 & 0 & 26.00 \\ 740 & 28 & 0 & 28.00 \\ 740 & 30 & 0 & 30.00\end{array}$

INCREASED PRESSURE TO 1000 PSIG

$\begin{array}{ll}1025 & 32 \\ 1015 & 34 \\ 1015 & 36 \\ 1020 & 38 \\ 1020 & 40\end{array}$

GRAPH NAME = WIEE500ั

$\begin{array}{rrr}0 & 0.4453 & 0.0 \\ 200 & 0.4453 & 89.1 \\ 400 & 0.4453 & 178.1 \\ 600 & 0.4453 & 267.2 \\ 800 & 0.4453 & 356.2\end{array}$

GRAPH NAME = WIEE750I

$\begin{array}{rrr}0 & 0.4453 & 0.0 \\ 150 & 0.4453 & 66.8 \\ 300 & 0.4453 & 133.6 \\ 450 & 0.4453 & 200.4 \\ 600 & 0.4453 & 267.2\end{array}$

GRAPH NAME = WIEE10OL

$\begin{array}{rrr}0 & 0.4453 & 0.0 \\ 100 & 0.4453 & 44.5 \\ 200 & 0.4453 & 89.1 \\ 300 & 0.4453 & 133.6 \\ 370 & 0.4453 & 164.8\end{array}$


WHC-SD-WM-TRP-223

Rev. 0

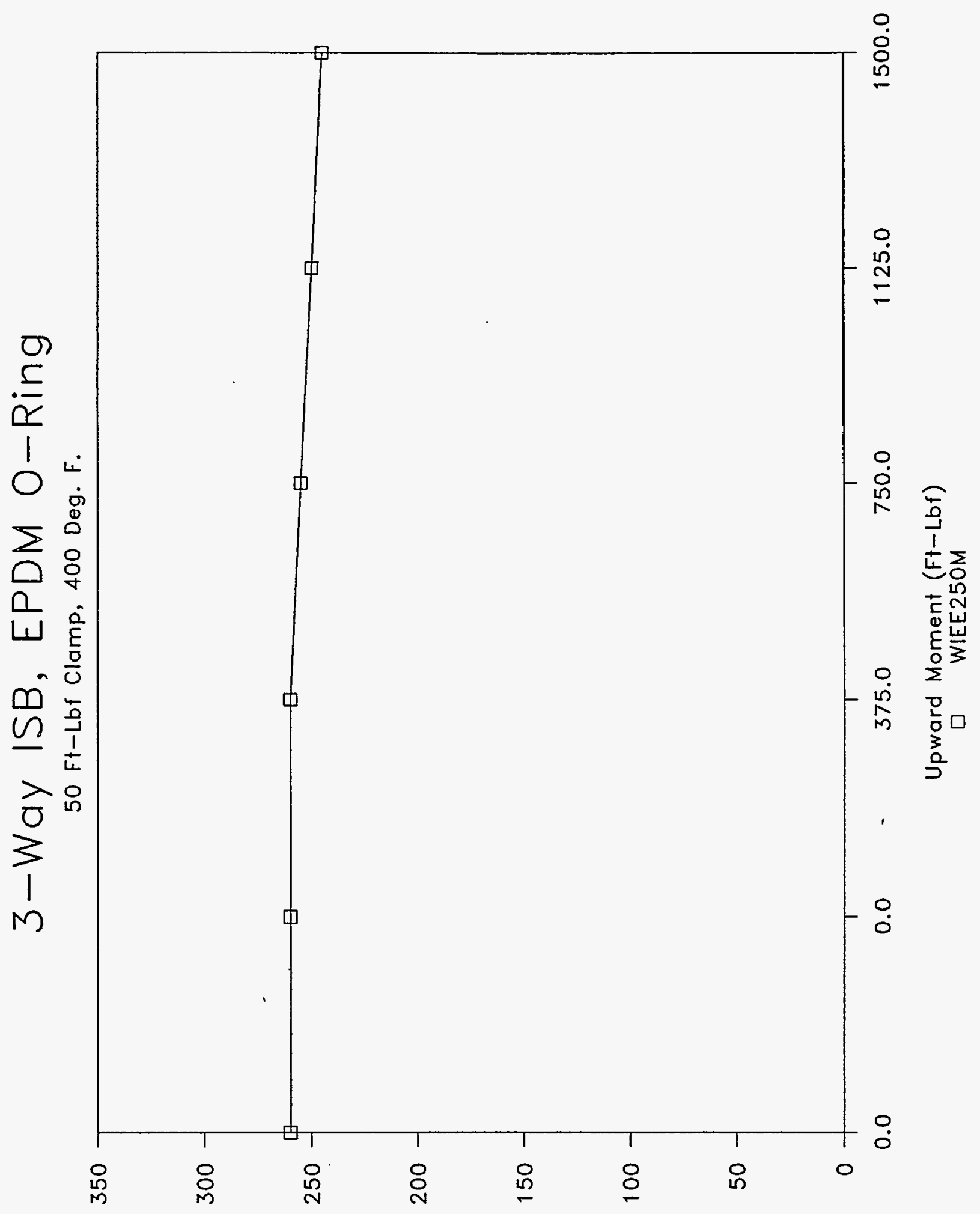

(6!sd) asnssadd 


\section{3-Way ISB, EPDM O-Ring \\ $50 \mathrm{Ft}$-Lbf Clamp, 400 Deg. F.}

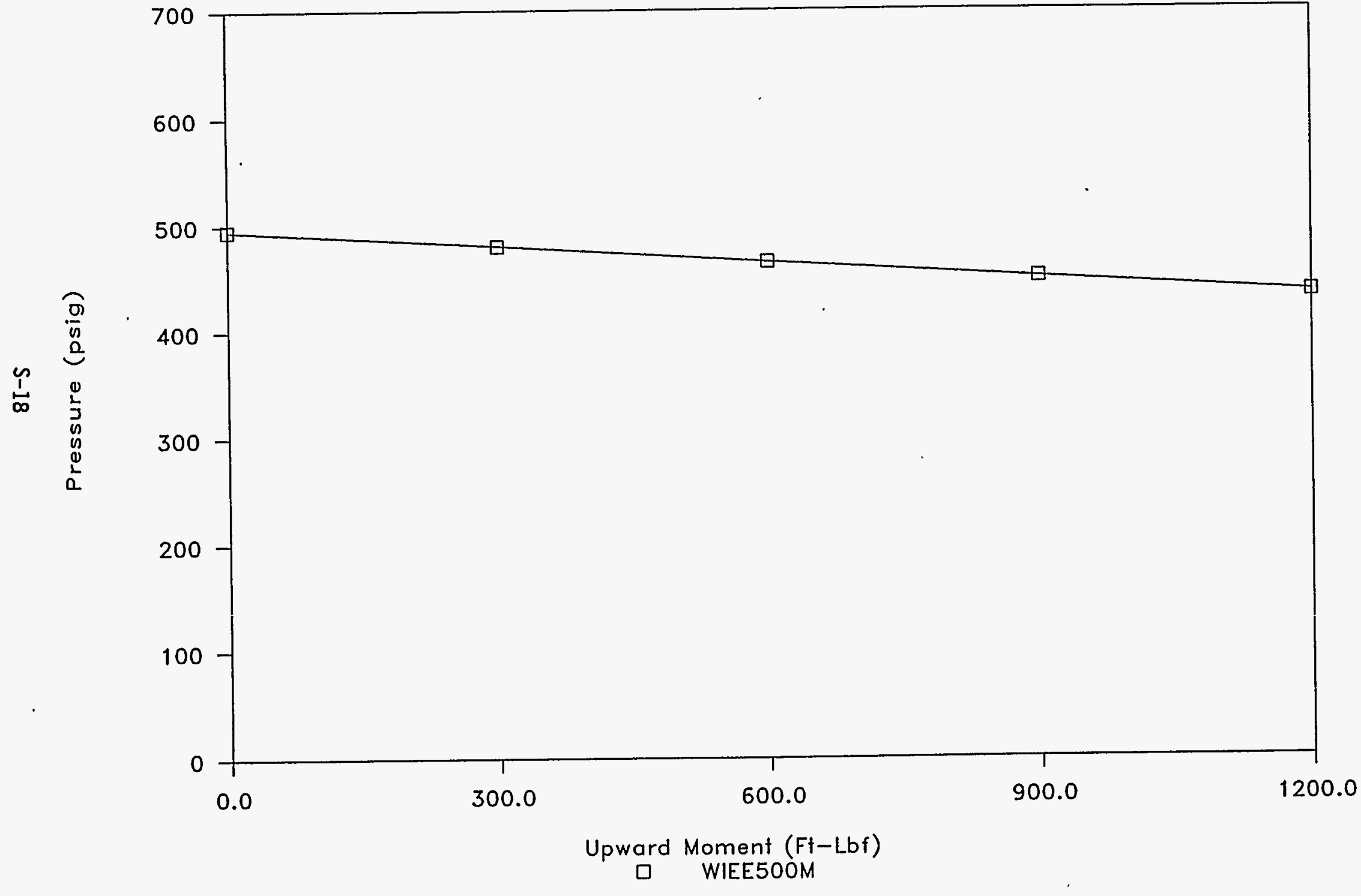


WHC-SD-WM-TRP-223

Rev. 0

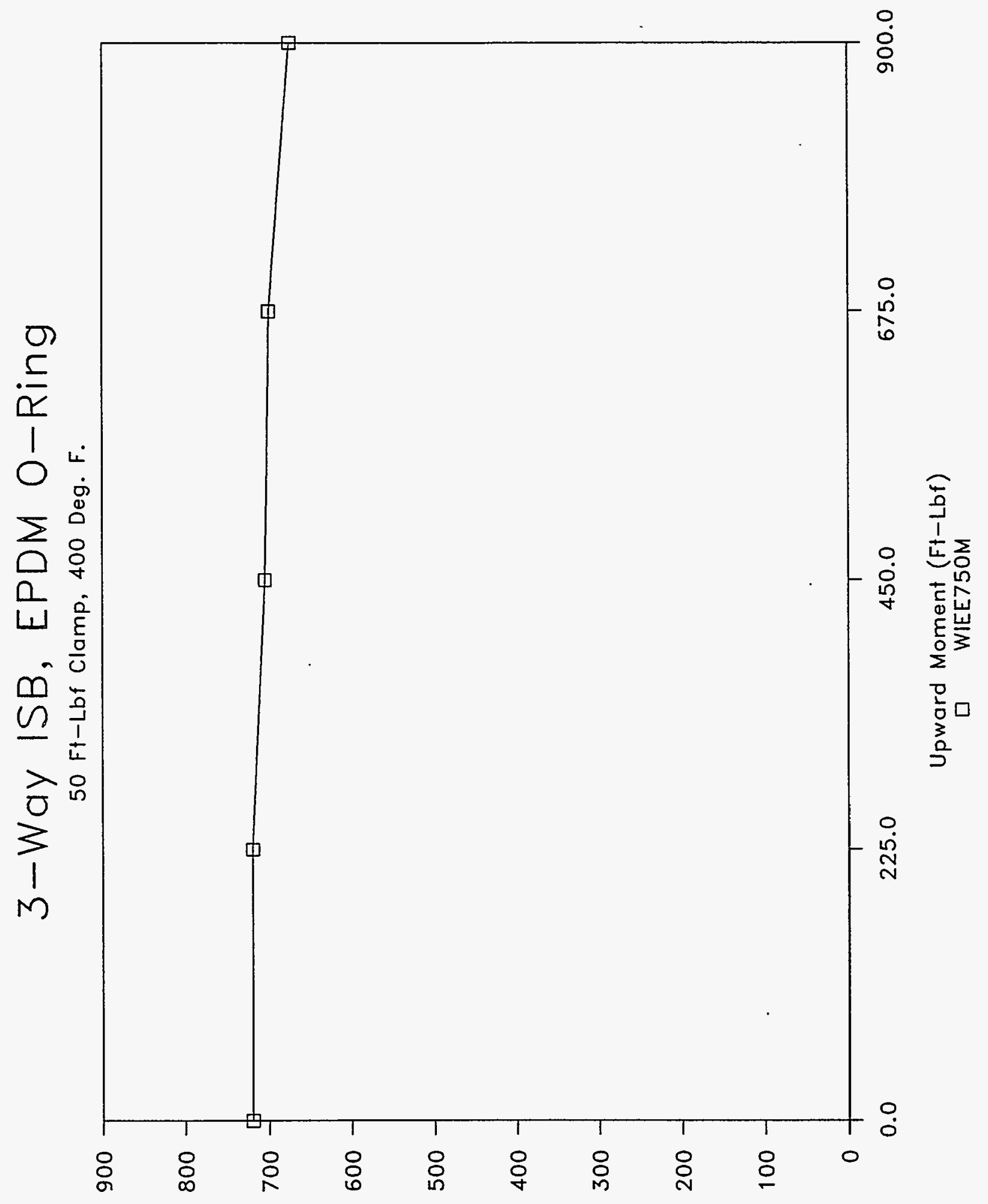

(b!sd) adnssadd 
WHC-SD-HM-TRP-223

Rev. 0

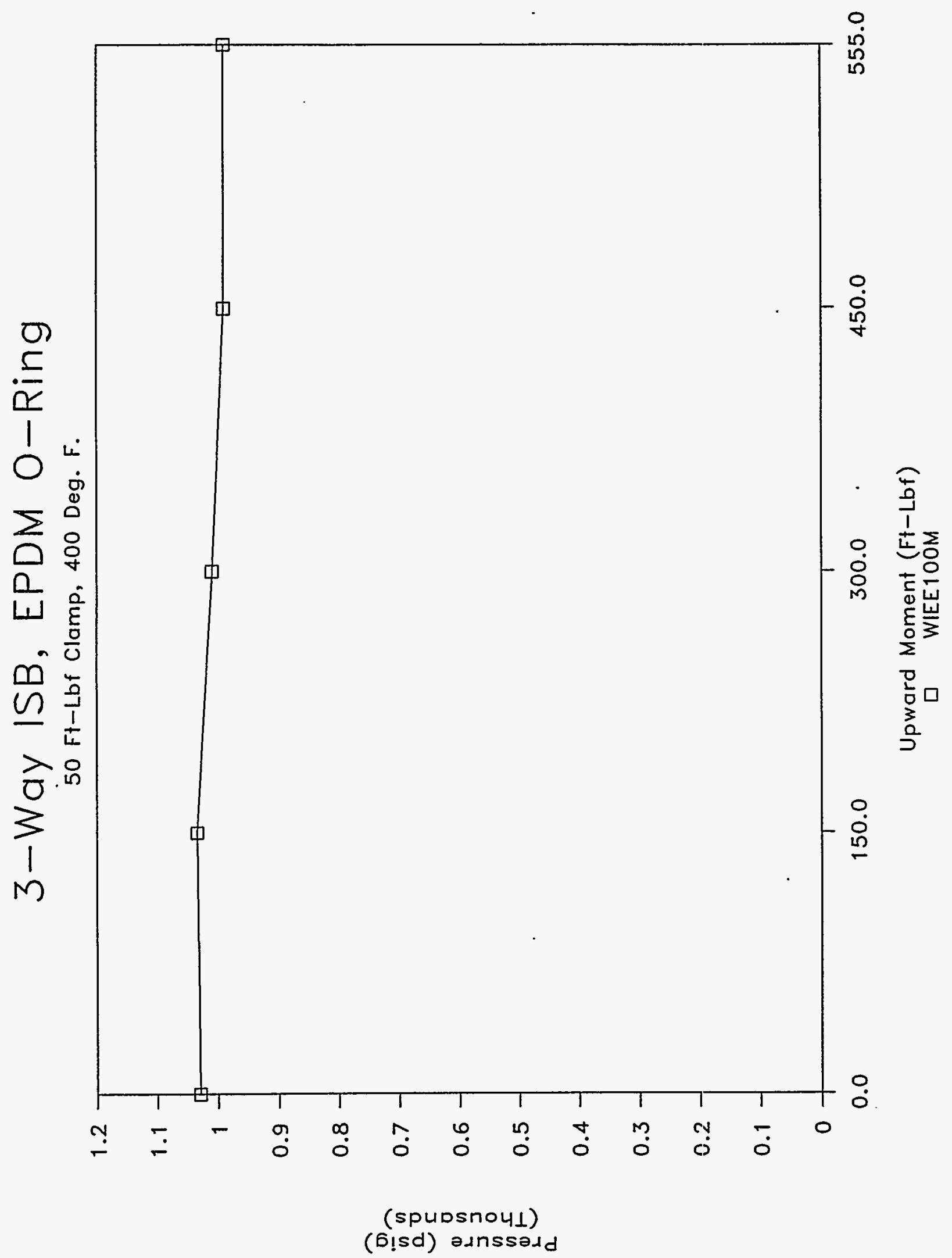


DECEMBER 05, 1994

2" 3-WAY ISB CONNECTOR, EPDM O-RING, 70 DUROMETER, ELEVATED TEMP.

PART \# 2-119 E893-80, PARKER SEAL CO., BATCH \# 315989, CURE DATE 3994 LEAK TEST - UP AND OVER MOMENT (UPWARD ROTATION)

CLAMPING TORQUE $=50$ FT-LBF NOMINAL GRAPH NAME = WIEE25OM

CHARGE PRESSURE $=250$ PSIG

TEMPERATURE: 400 DEG. F.

\begin{tabular}{|c|c|c|c|c|c|c|}
\hline $\begin{array}{l}\text { PRESSURE } \\
\text { PSIG }\end{array}$ & $\begin{array}{l}\text { ELAPSED } \\
\text { MINUTES }\end{array}$ & $\begin{array}{l}\text { ELAPSED } \\
\text { SECONDS } \\
\text { INPUT }\end{array}$ & $\begin{array}{l}\text { ELAPSED } \\
\text { DECIMAL } \\
\text { MINUTES } \\
\text { COMPUTE }\end{array}$ & $\begin{array}{l}\text { UPWARD } \\
\text { FORCE } \\
\text { IBS } \\
\text { INPUT }\end{array}$ & $\begin{array}{l}\text { UPWARD } \\
\text { MOMENT } \\
\text { ARM } \\
\text { FT. } \\
\text { INPUT }\end{array}$ & $\begin{array}{l}\text { UPWARD } \\
\text { MOMENT } \\
\text { FT-LBF. } \\
\text { COMPUTE }\end{array}$ \\
\hline $\begin{array}{l}260 \\
260 \\
260 \\
255 \\
250 \\
245\end{array}$ & $\begin{array}{r}0 \\
2 \\
4 \\
6 \\
8 \\
10\end{array}$ & 0 & $\begin{array}{r}0.00 \\
2.00 \\
4.00 \\
6.00 \\
8.00 \\
10.00\end{array}$ & $\begin{array}{r}0 \\
0 \\
250 \\
500 \\
750 \\
1000\end{array}$ & $\begin{array}{l}1.5000 \\
1.5000 \\
1.5000 \\
1.5000 \\
1.5000 \\
1.5000\end{array}$ & $\begin{array}{r}0.0 \\
0.0 \\
375.0 \\
750.0 \\
1125.0 \\
1500.0\end{array}$ \\
\hline
\end{tabular}

INCREASED PRESSURE TO 500 PSIG

$\begin{array}{llll}495 & 12 & 0 & 12.00 \\ 480 & 14 & 0 & 14.00 \\ 465 & 16 & 0 & 16.00 \\ 450 & 18 & 0 & 18.00 \\ 435 & 20 & 0 & 20.00\end{array}$

INCREASED PRESSURE TO 750 PSIG

$\begin{array}{llll}720 & 22 & 0 & 22.00 \\ 720 & 24 & 0 & 24.00 \\ 705 & 26 & 0 & 26.00 \\ 700 & 28 & 0 & 28.00 \\ 675 & 30 & 0 & 30.00\end{array}$

INCREASED PRESSURE TO 1000 PSIG

$\begin{array}{rlll}1030 & 32 & 0 & 32.00 \\ 1035 & 34 & 0 & 34.00 \\ 1010 & 36 & 0 & 36.00 \\ 990 & 38 & 0 & 38.00 \\ 990 & 40 & 0 & 40.00\end{array}$

GRAPH NAME = WIEE50OM

$\begin{array}{rrr}0 & 1.5000 & 0.0 \\ 200 & 1.5000 & 300.0 \\ 400 & 1.5000 & 600.0 \\ 600 & 1.5000 & 900.0 \\ 800 & 1.5000 & 1200.0\end{array}$

GRAPH NAME = WIEE750M

$\begin{array}{rrr}0 & 1.5000 & 0.0 \\ 150 & 1.5000 & 225.0 \\ 300 & 1.5000 & 450.0 \\ 450 & 1.5000 & 675.0 \\ 600 & 1.5000 & 900.0\end{array}$

GRAPH NAME = WIEE10OM

$\begin{array}{rrr}0 & 1.5000 & 0.0 \\ 100 & 1.5000 & 150.0 \\ 200 & 1.5000 & 300.0 \\ 300 & 1.5000 & 450.0 \\ 370 & 1.5000 & 555.0\end{array}$


WHC-SD-WM-TRP-223

Rev. 0

APPENDIX T: GRAPHS OF THREE-WAY CONVENTIONAL TEFI.ON GASKET TESTS 


\section{3-Way, 2" Conventional Teflon Gasket}

25.7 Ft-Lbf Clamp, 250 psig Charge

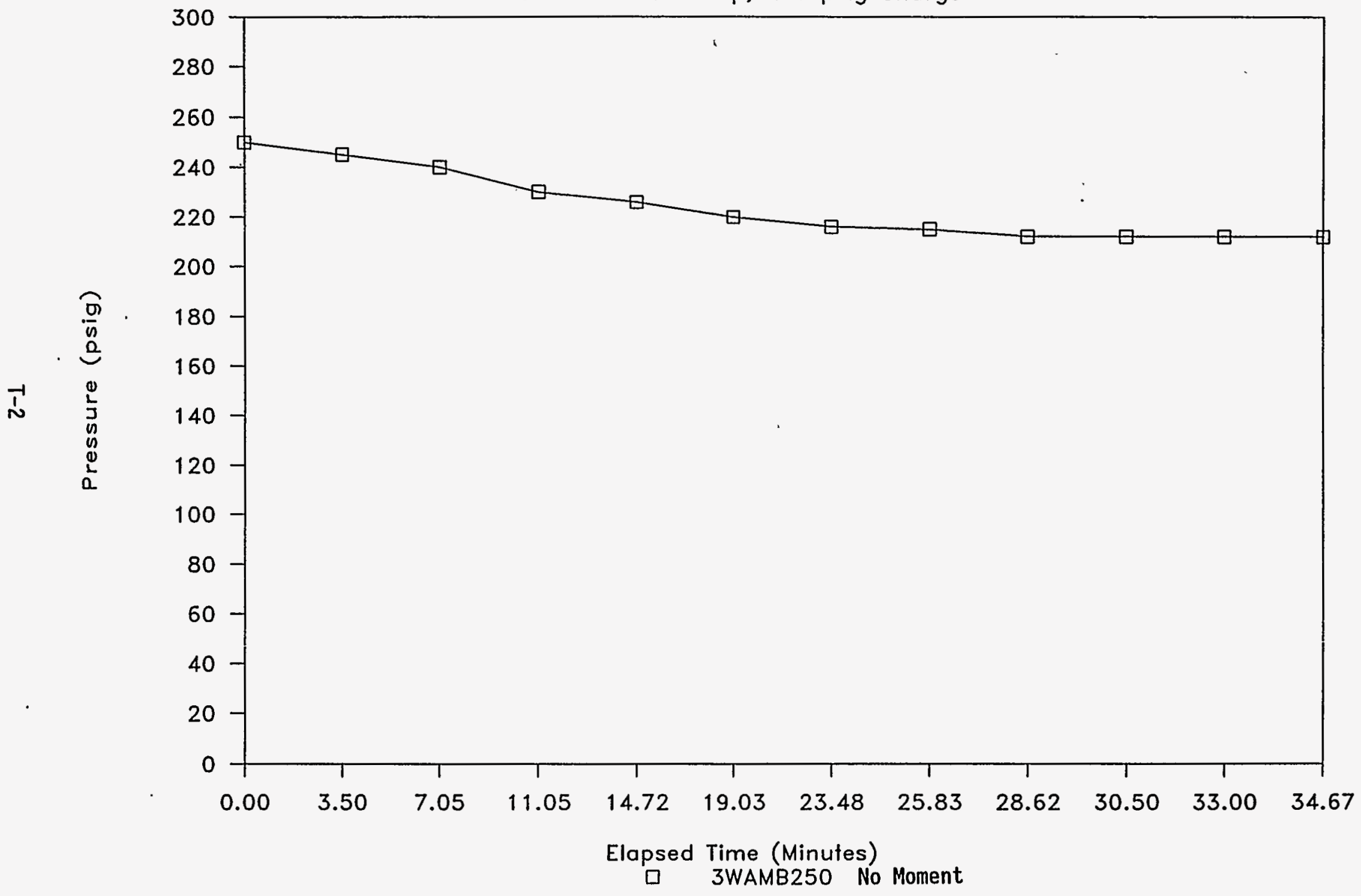


WHC-SD-WM-TRP-223 ReV 0

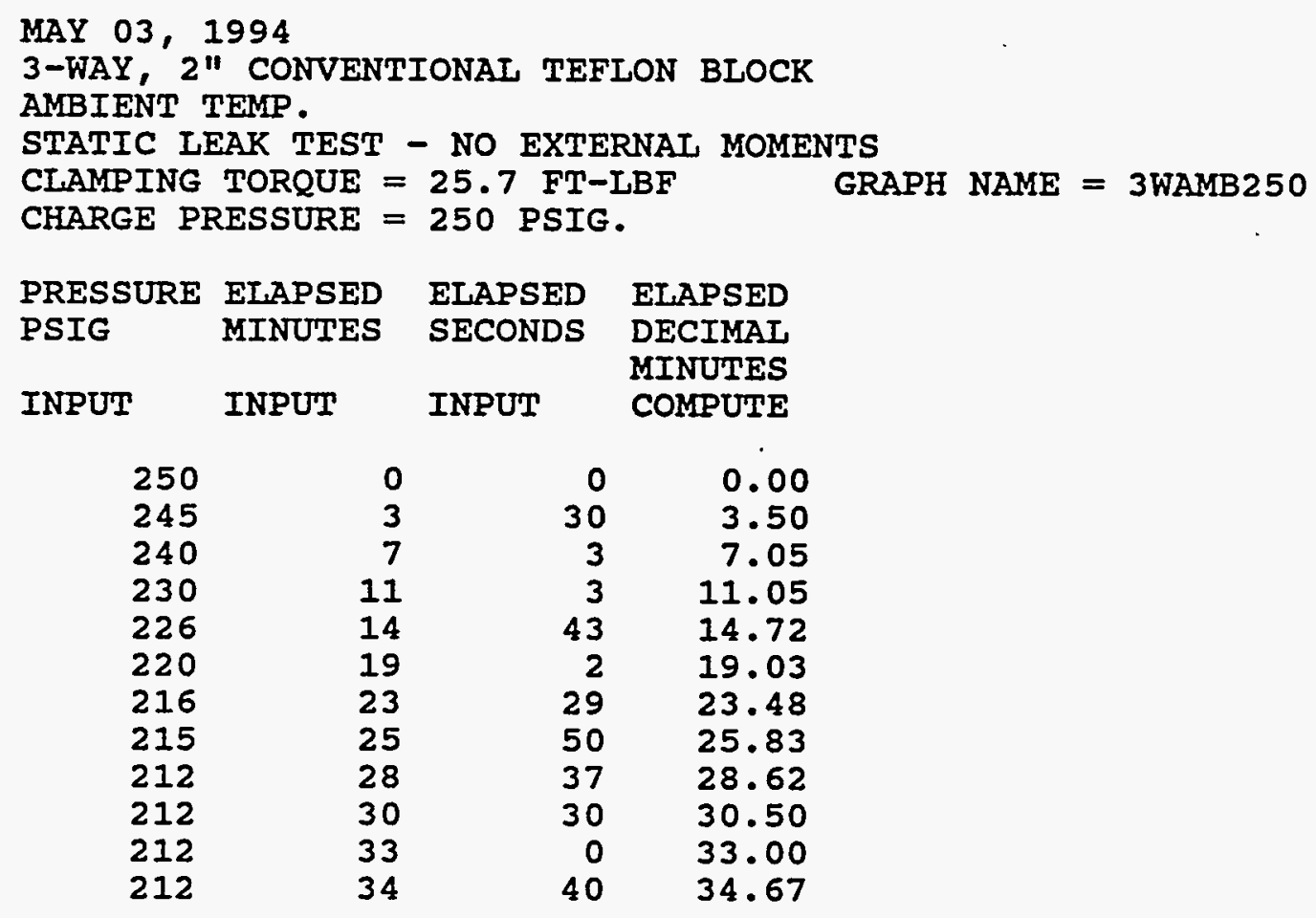




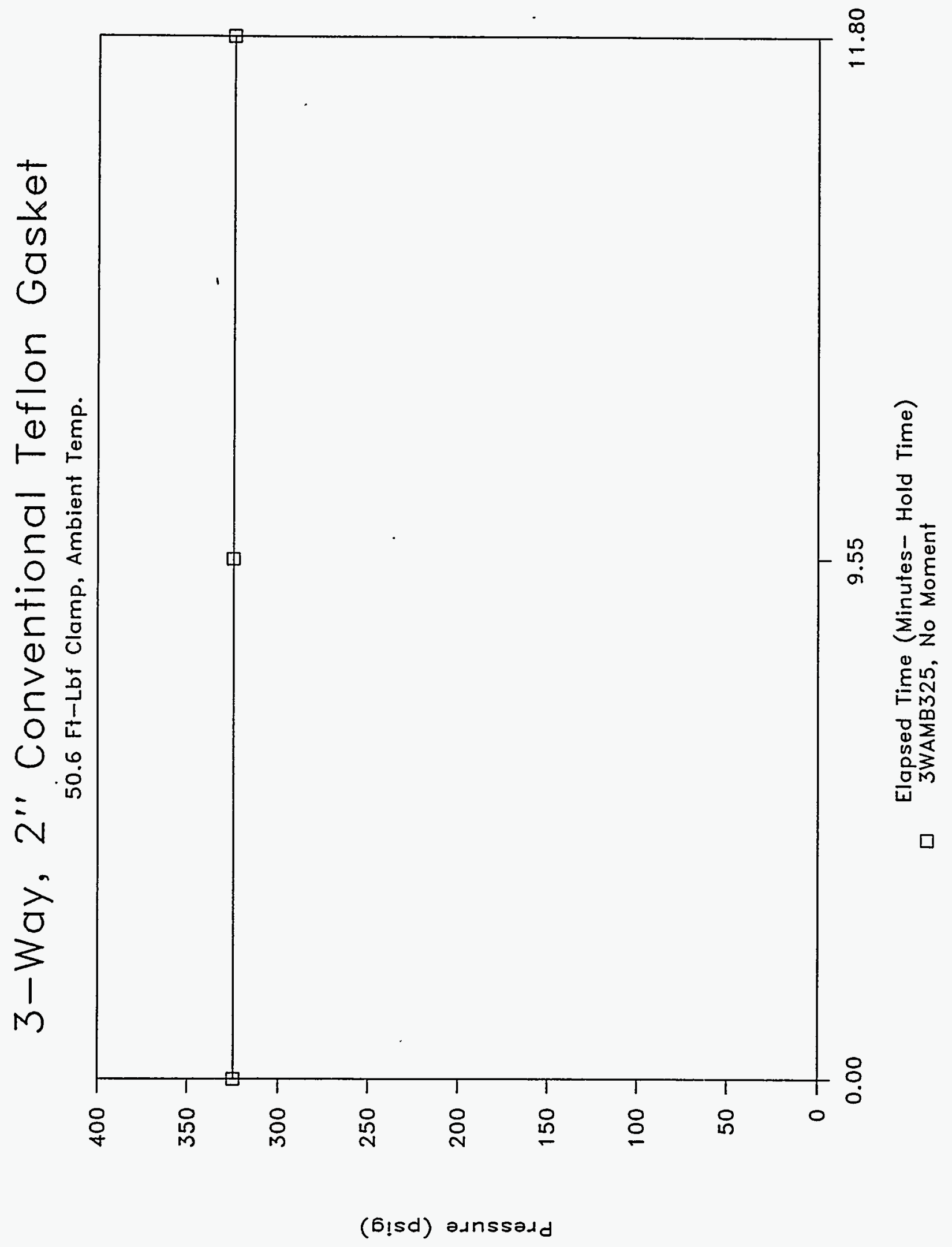


WHC-SD-WM-TRP-223 ReV 0

MAY 03, 1994

3-WAY, $2 "$ CONVENTIONAL TEFLON BLOCK

AMBIENT TEMP.

STATIC IEAK TEST - NO EXTERNAL MOMENTS

CLAMPING TORQUE $=50.6$ FT-LBF

CHARGE PRESSURE $=325$ PSIG.

GRAPH NAME $=3$ WAMB325

\begin{tabular}{|c|c|c|c|}
\hline $\begin{array}{l}\text { PRESSURE } \\
\text { PSIG }\end{array}$ & $\begin{array}{l}\text { ELAPSED } \\
\text { MINUTES }\end{array}$ & $\begin{array}{l}\text { ELAPSED } \\
\text { SECONDS }\end{array}$ & $\begin{array}{l}\text { ELAPSED } \\
\text { DECIMAL } \\
\text { MINUTES }\end{array}$ \\
\hline NPUT & INPUT & INPUT & COMPUTE \\
\hline $\begin{array}{l}32 \\
32 \\
32\end{array}$ & $1:$ & $\begin{array}{r}0 \\
33 \\
48\end{array}$ & $\begin{array}{r}0.00 \\
9.55 \\
11.80\end{array}$ \\
\hline
\end{tabular}




\section{3-Way, 2" Conventional Teflon Gasket}

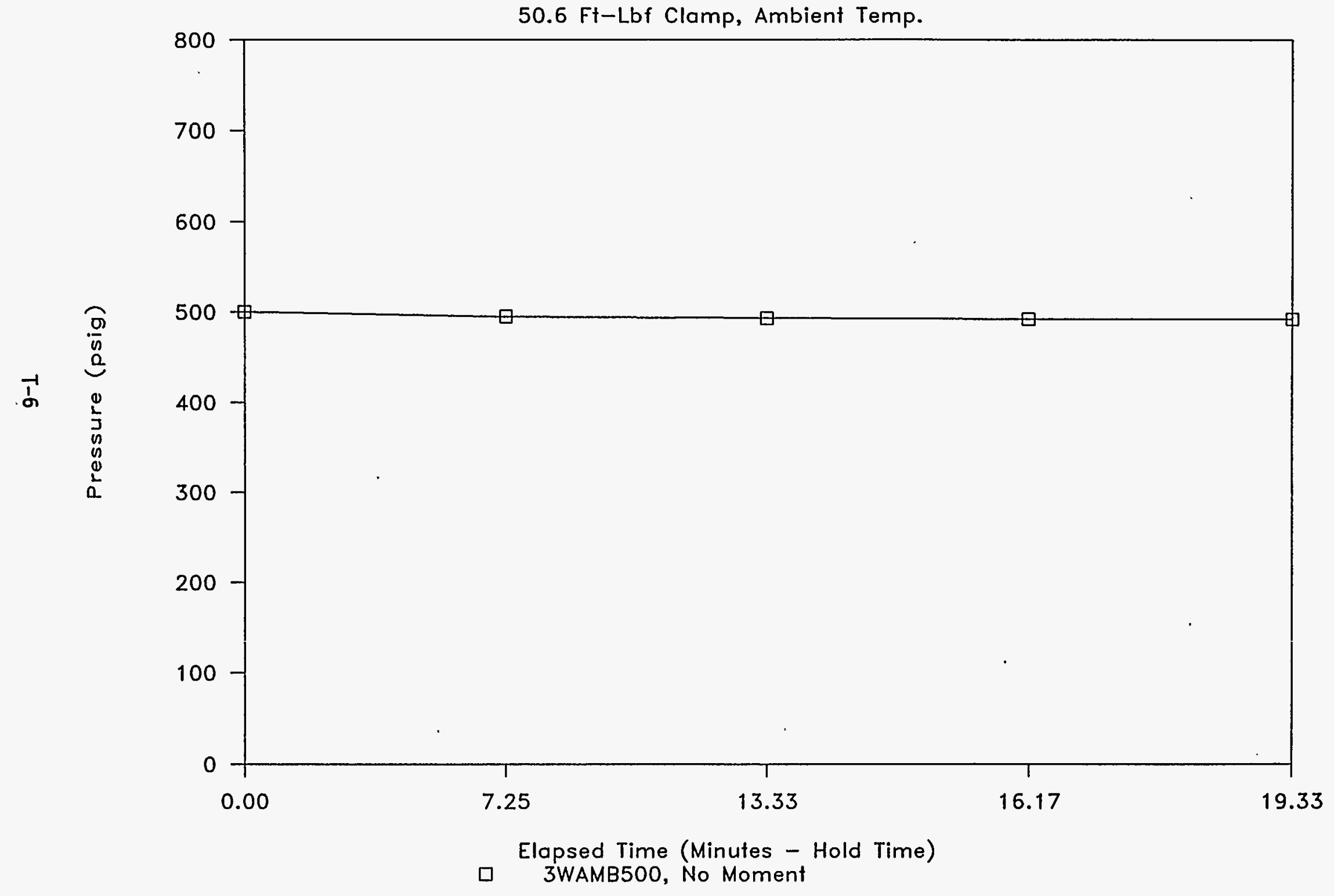


WHC-SD-WM-TRP-223 ReV 0

MAY 03, 1994

3-WAY, 2" CONVENTIONAL TEFLON BLOCK

AMBIENT TEMP.

STATIC LEAK TEST - NO EXTERNAL MOMENTS

CLAMPING TORQUE $=50.6 \mathrm{FT}-\mathrm{LBF}$

CHARGE PRESSURE $=500$ PSIG.

GRAPH NAME $=3$ WAMB 500

\begin{tabular}{|c|c|c|c|}
\hline $\begin{array}{l}\text { PRESSURE } \\
\text { PSIG }\end{array}$ & $\begin{array}{l}\text { ELAPSED } \\
\text { MINUTES }\end{array}$ & $\begin{array}{l}\text { ELAPSED } \\
\text { SECONDS }\end{array}$ & $\begin{array}{l}\text { ELAPSED } \\
\text { DECIMAI } \\
\text { MINUTES }\end{array}$ \\
\hline INPUT & INPUT & INPUT & COMPUTE \\
\hline $\begin{array}{l}500 \\
495 \\
493 \\
492 \\
492\end{array}$ & $\begin{array}{r}0 \\
7 \\
13 \\
16 \\
19\end{array}$ & $\begin{array}{r}0 \\
15 \\
20 \\
10 \\
20\end{array}$ & $\begin{array}{r}0.00 \\
7.25 \\
13.33 \\
16.17 \\
19.33\end{array}$ \\
\hline
\end{tabular}




\section{3-Way, 2" Conventional Teflon Gasket}

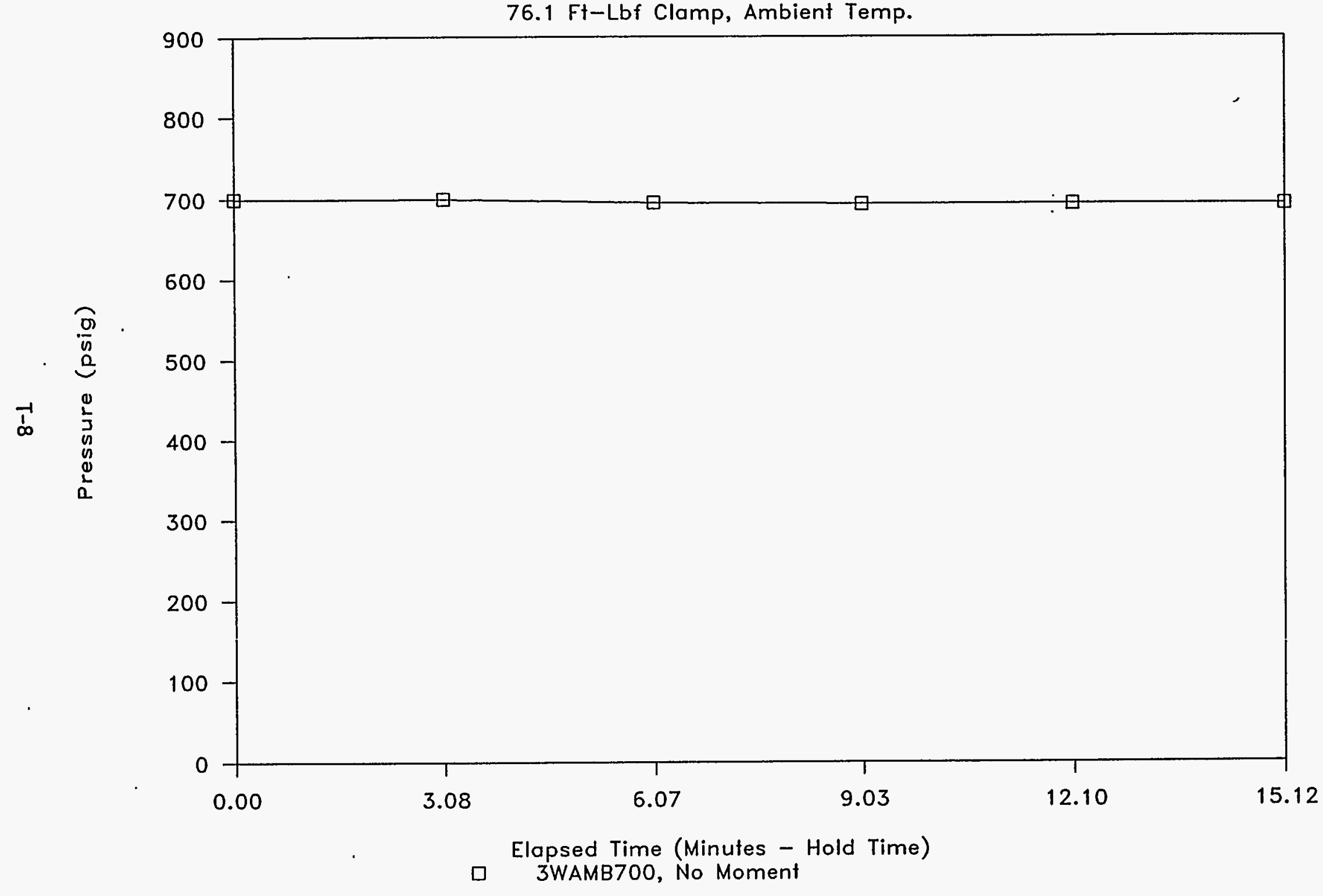


WHC-SD-WM-TRP-223 ReV 0

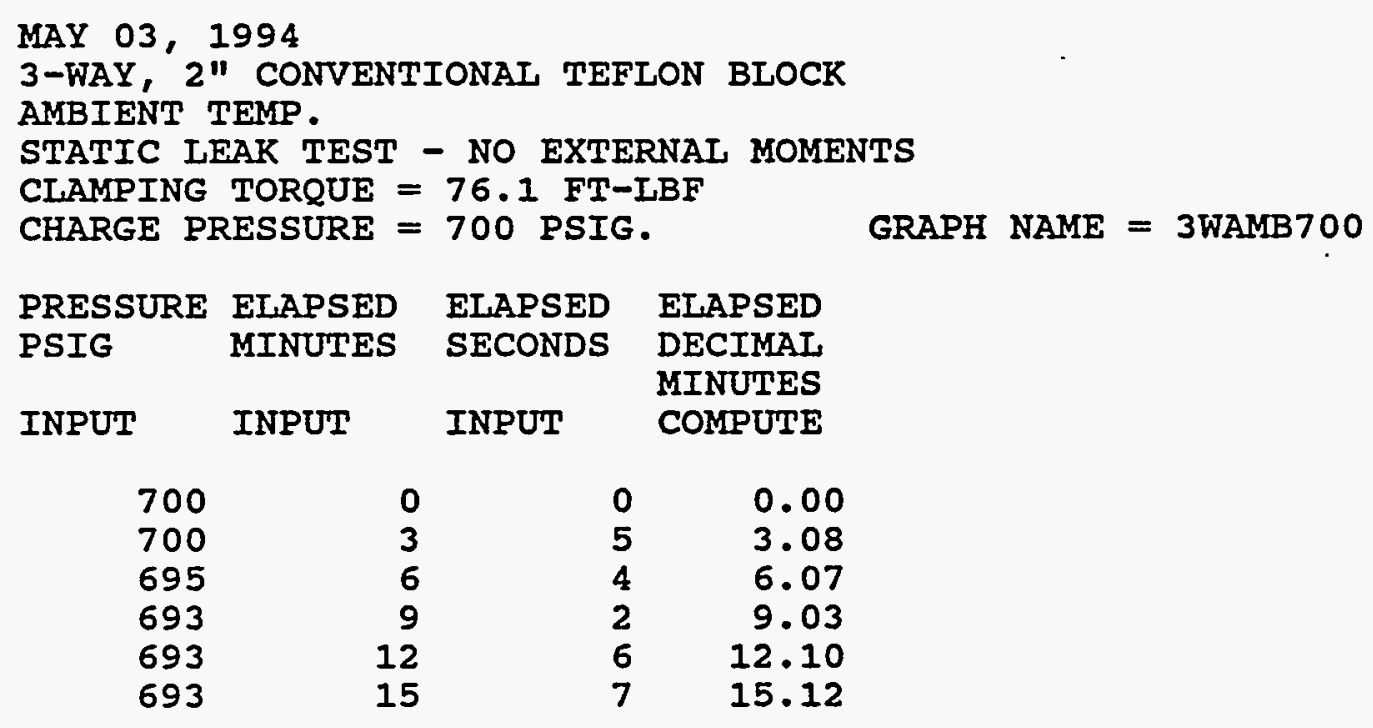


WHC-SD-WM-TRP-223

Rev. 0

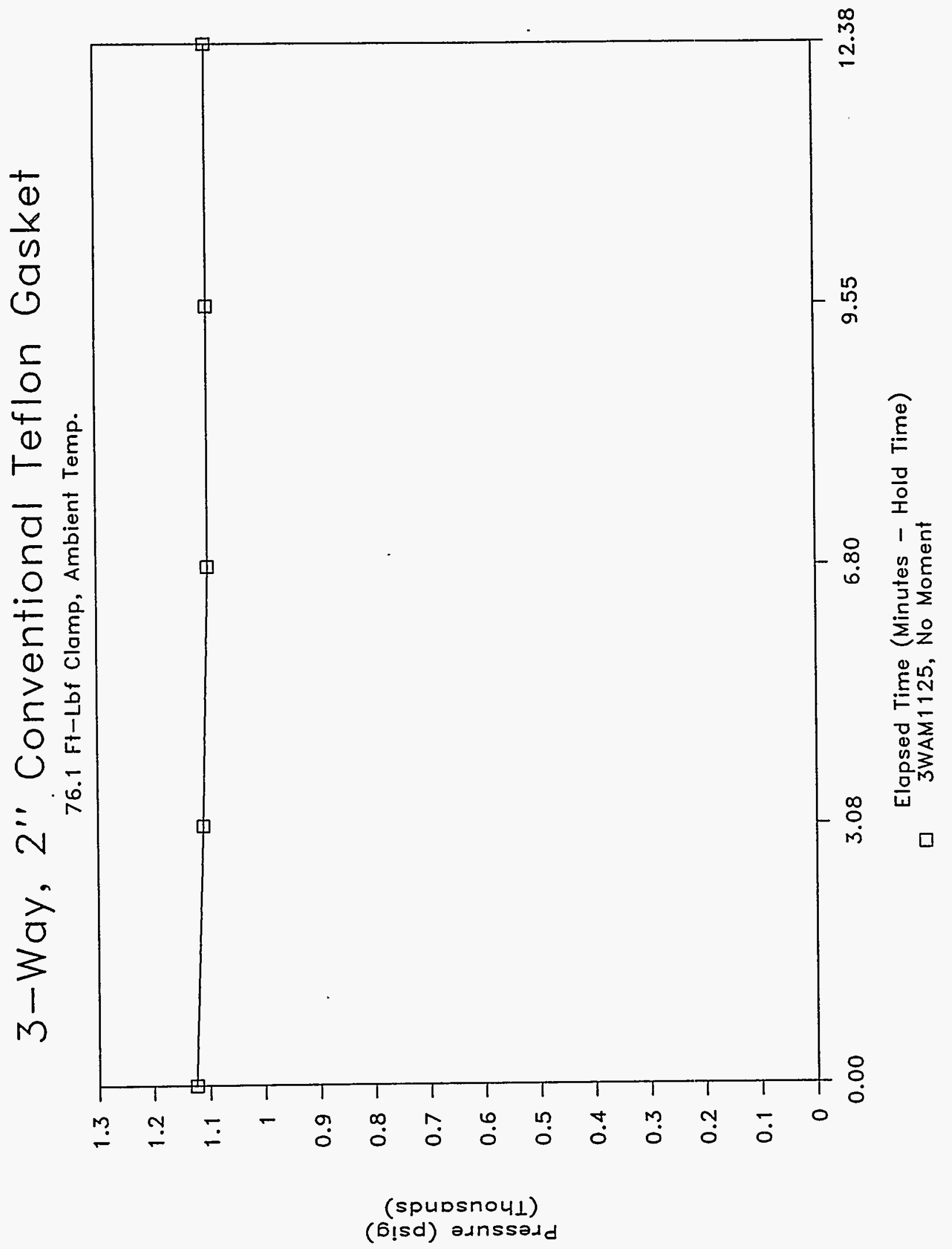


WHC-SD-WM-TRP-223 Rev 0

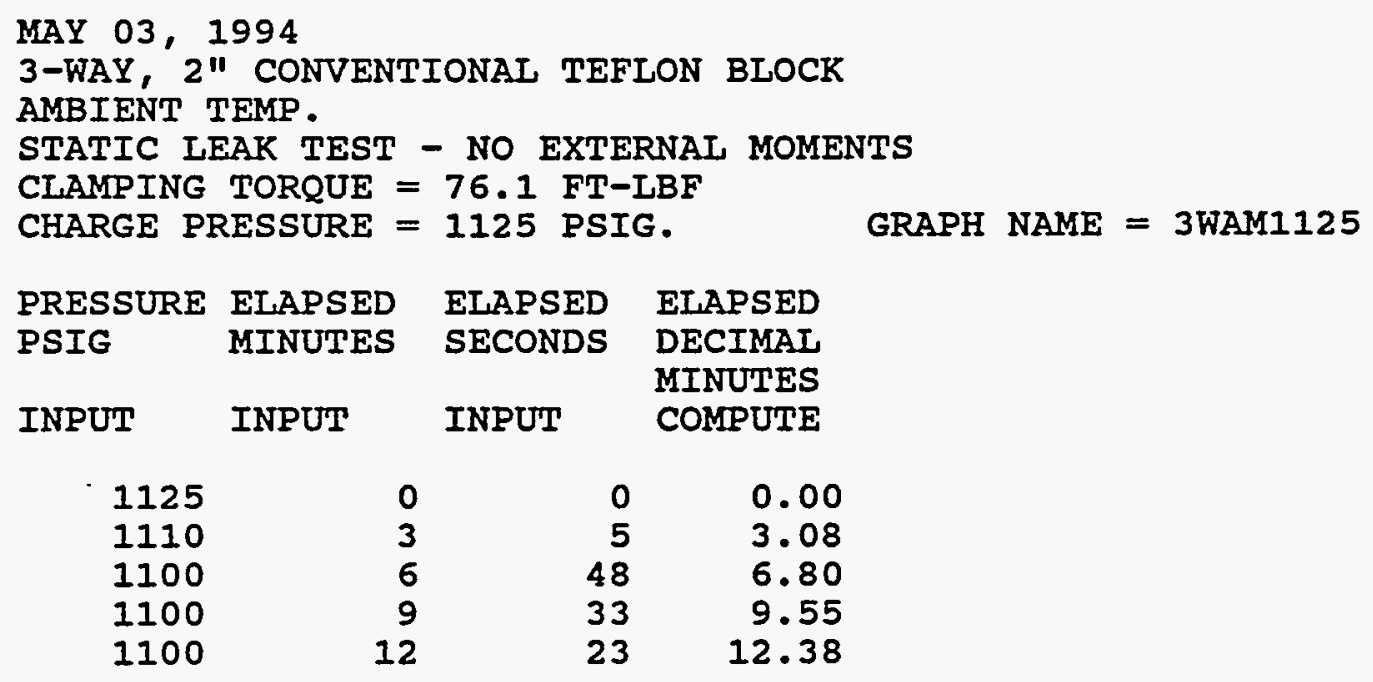




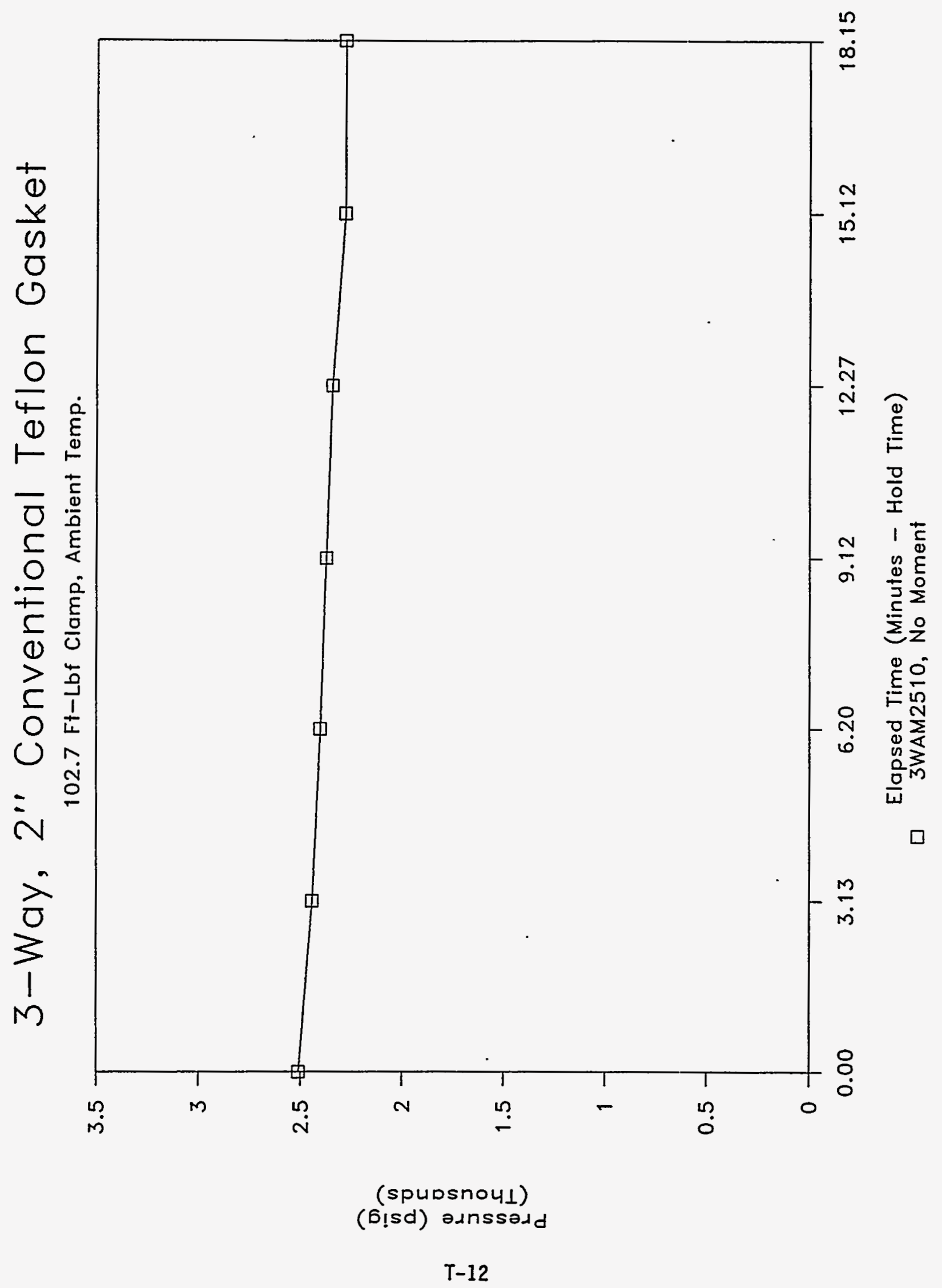


WHC-SD-WM-TRP-223 ReV 0

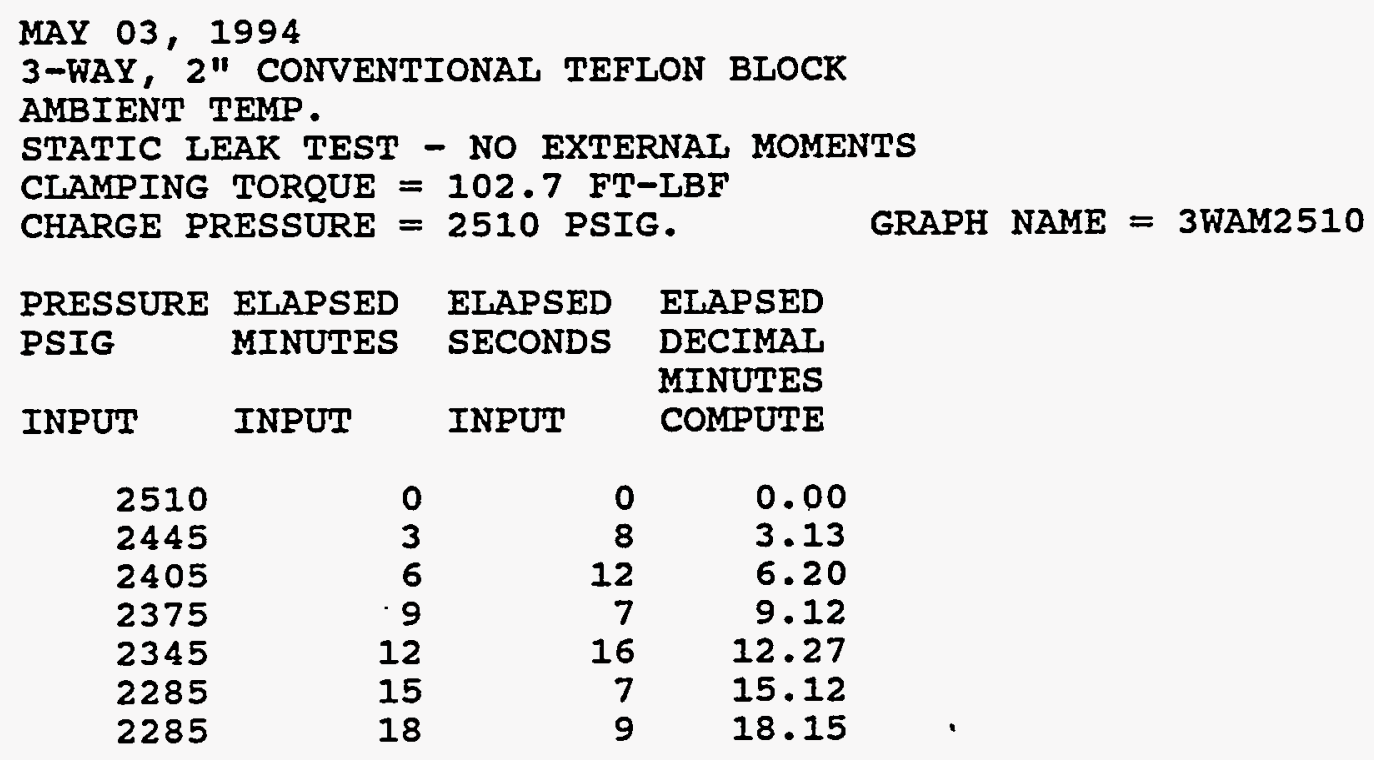




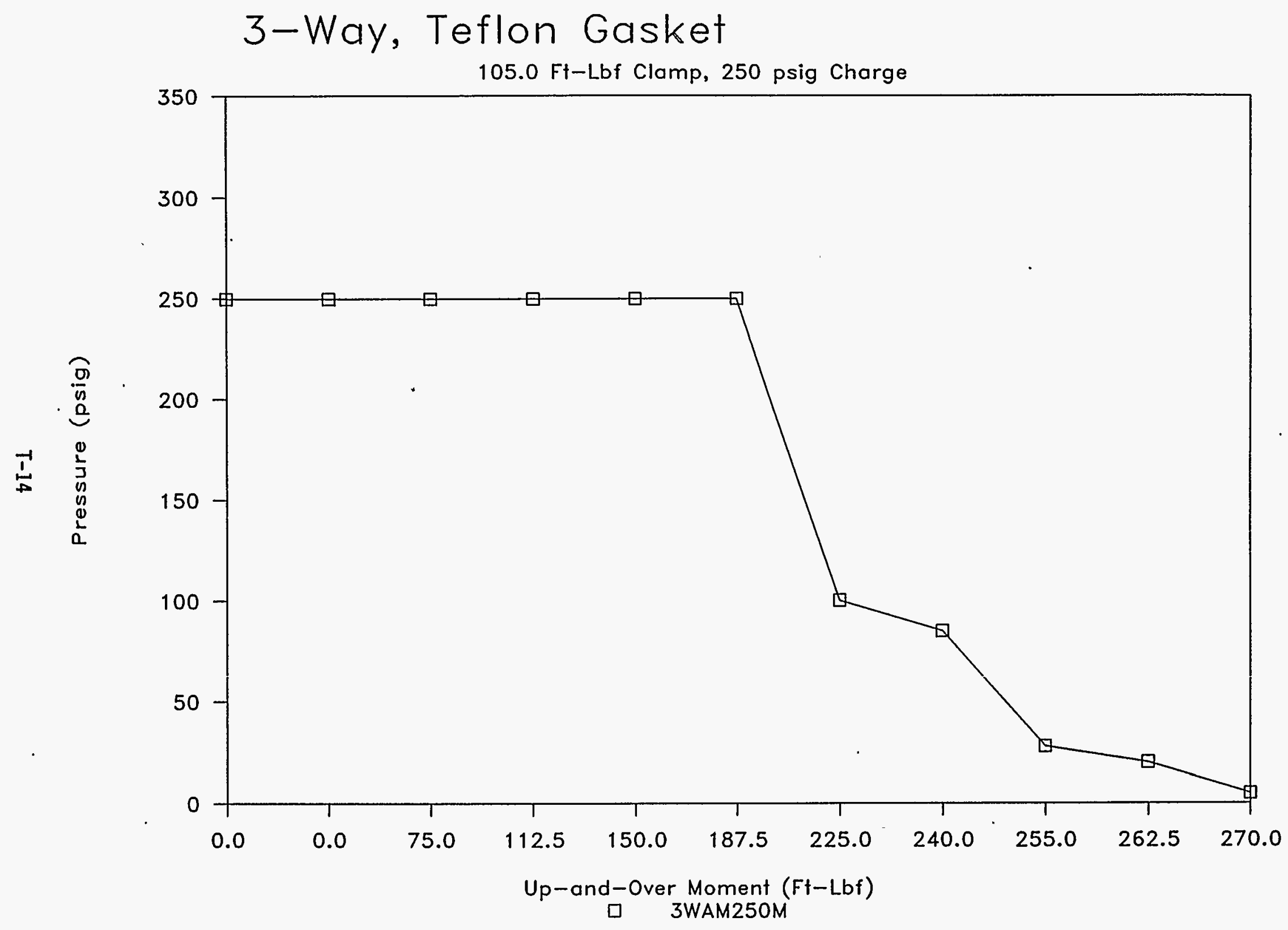


WHC-SD-WM-TRP-223 ReV 0

MAY 24, 1994

3-WAY CONNECTOR, CONVENTIONAL, 100\% TEFLON GASKET, AMBIENT TEMP. LEAK TEST - UP AND OVER MOMENTS APPLIED

CLAMPING TORQUE $=105.0$ FT-LBF

CHARGE PRESSURE $=250$ PSIG

$\begin{array}{llllllll} & & & & & \\ \text { PRESSURE } & \text { ELAPSED } & \text { ELAPSED } & \text { ELAPSED } & \text { MOMENT } & \text { MOMENT } & \text { UP\&OVR } & \text { DEFIRDCTIO } \\ \text { PSIG } & \text { MINUTES } & \text { SECONDS } & \text { DECIMAL } & \text { FORCE } & \text { ARM } & \text { MOMENT } & \text { (CHORDAL) } \\ & & & \text { MINUTES } & \text { LBS } & \text { FT. } & \text { FT-IBF. } & \text { INCHES } \\ \text { INPUT } & \text { INPUT } & \text { INPUT } & \text { COMPUTE } & \text { INPUT } & \text { INPUT } & \text { COMPUTE } & \text { INPUT }\end{array}$

$\begin{array}{rrrrrrrr}250 & 0 & 0 & 0.00 & 0 & 1.5 & 0.0 & 0.000 \\ 250 & 2 & 3 & 2.05 & 0 & 1.5 & 0.0 & 0.000 \\ 250 & 4 & 20 & 4.33 & 50 & 1.5 & 75.0 & 0.006 \\ 250 & 6 & 16 & 6.27 & 75 & 1.5 & 112.5 & 0.010 \\ 250 & 8 & 18 & 8.30 & 100 & 1.5 & 150.0 & 0.015 \\ 250 & 10 & 20 & 10.33 & 125 & 1.5 & 187.5 & 0.021 \\ 100 & 13 & 18 & 13.30 & 150 & 1.5 & 225.0 & 0.029 \\ 85 & 16 & 15 & 16.25 & 160 & 1.5 & 240.0 & 0.032 \\ 28 & 19 & 16 & 19.27 & 170 & 1.5 & 255.0 & 0.035 \\ 20 & 22 & 13 & 22.22 & 175 & 1.5 & 262.5 & 0.037 \\ 5 & 25 & 15 & 25.25 & 180 & 1.5 & 270.0 & 0.039\end{array}$




\section{2", 3-Way, Teflon Gasket, Ambient Temp .}

100.2 Ft-Lbf Clamp, 250 psig Charge

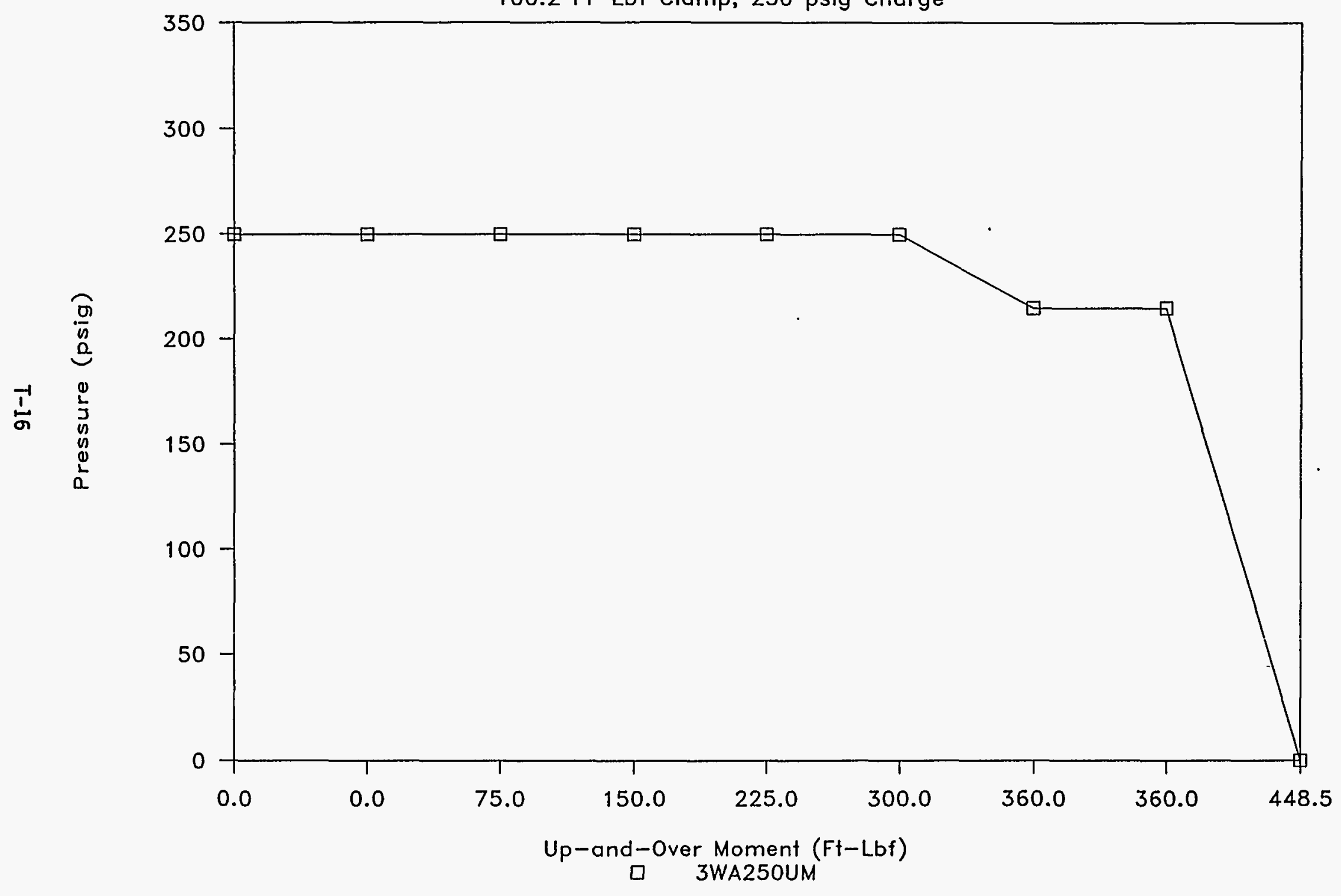


WHC-SD-WM-TRP-223 ReV 0

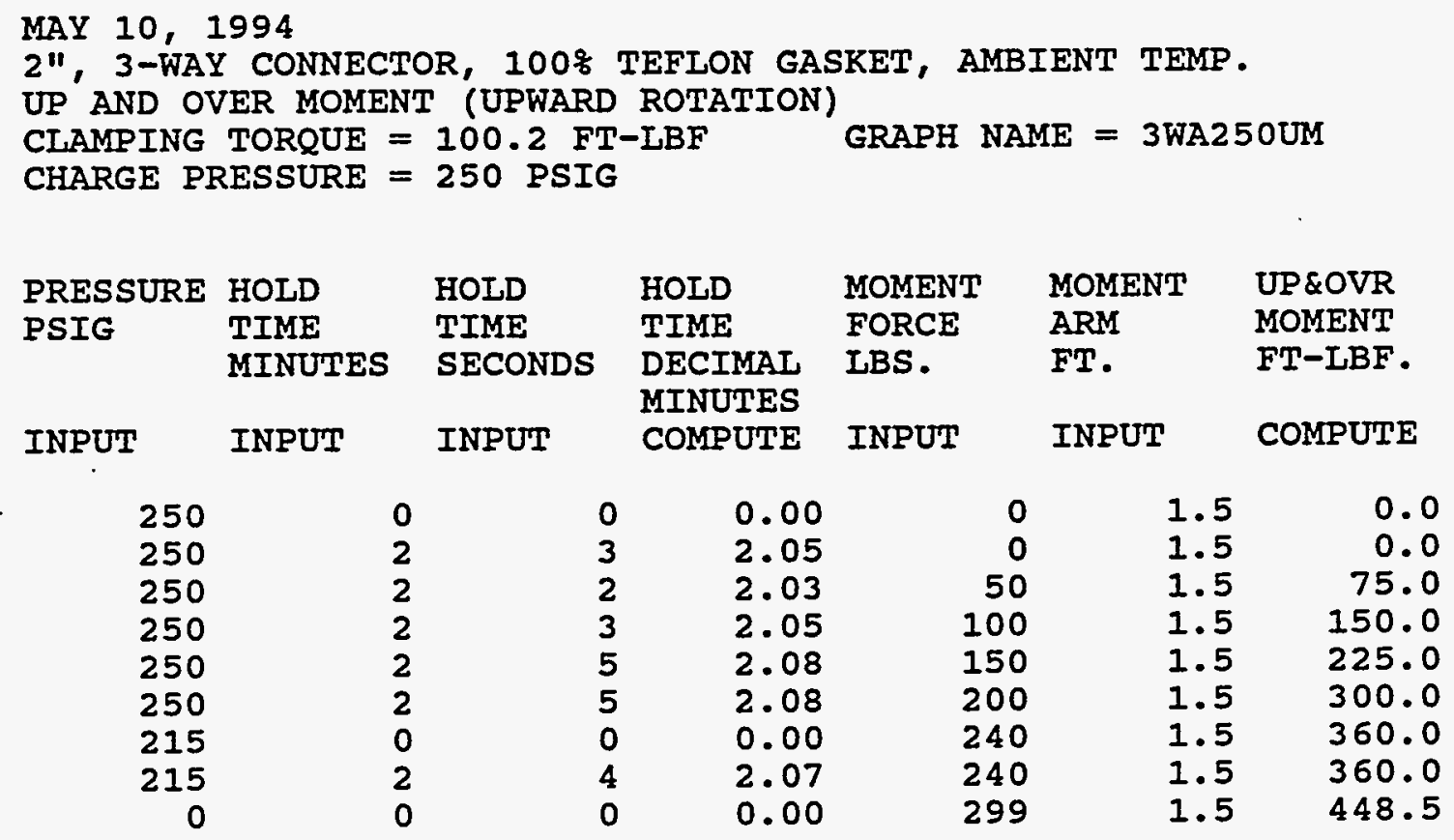




\section{2", 3-Way, Teflon Gasket, 212 Deg. F}

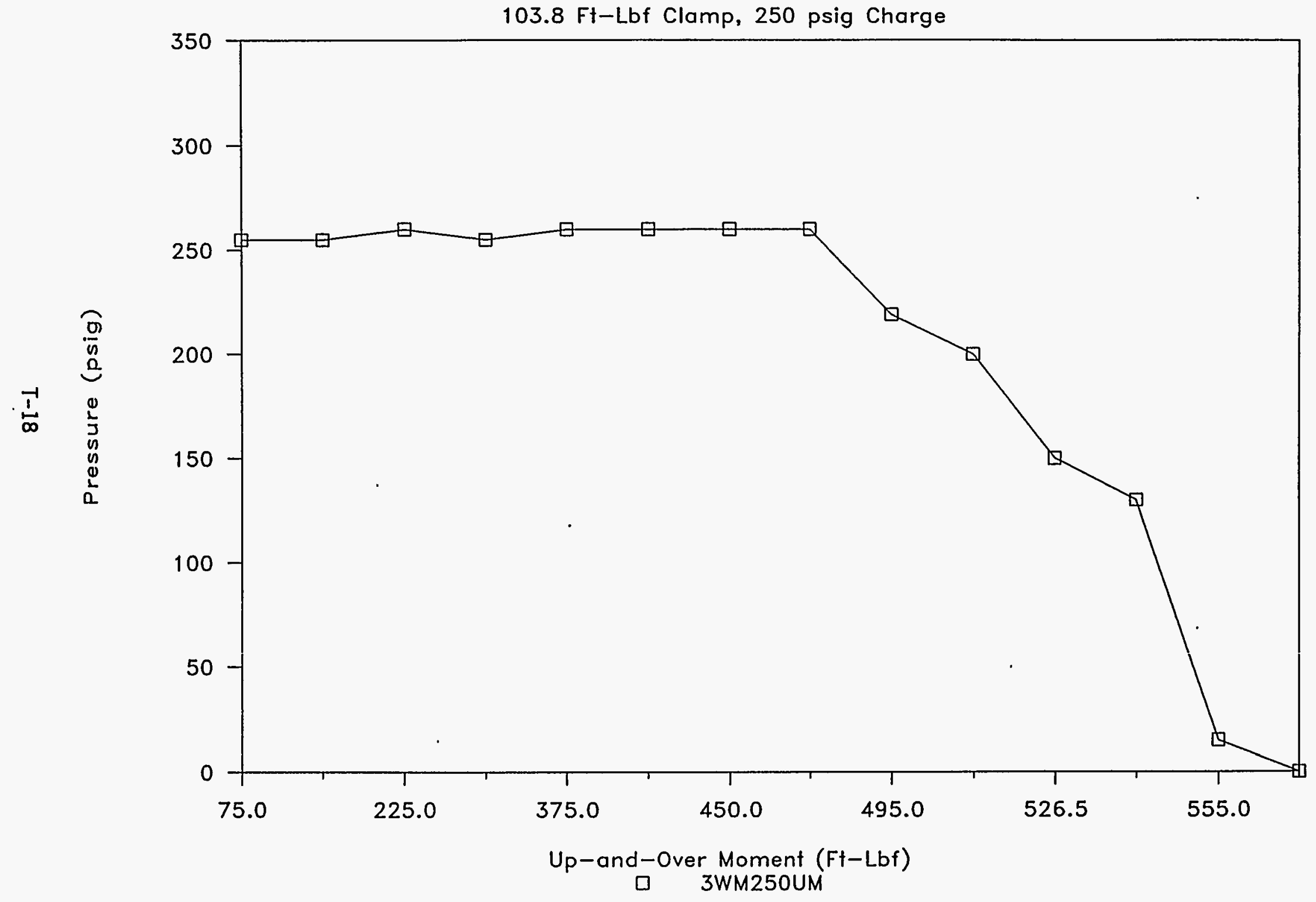


WHC-SD-WM-TRP-223 ReV 0

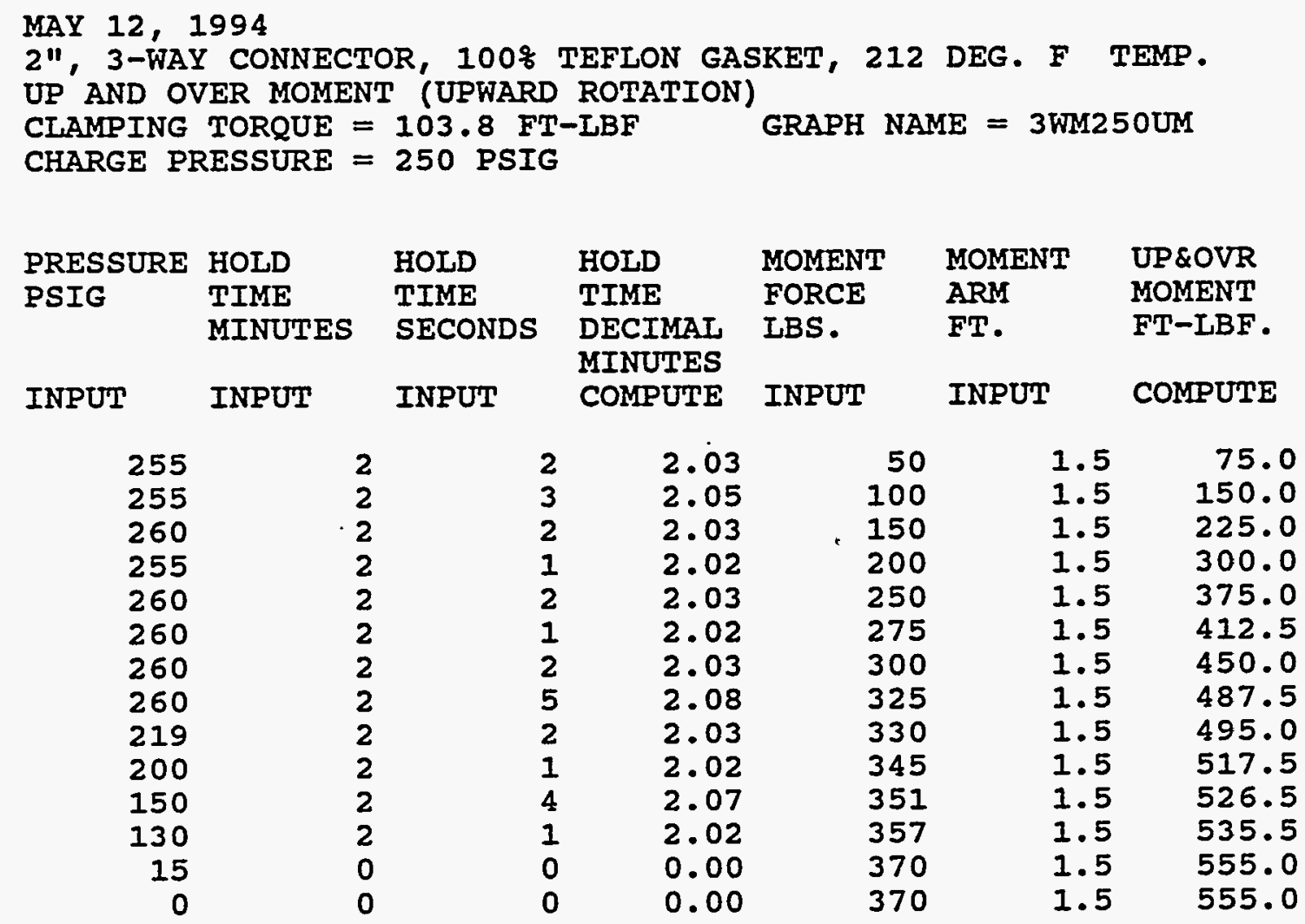




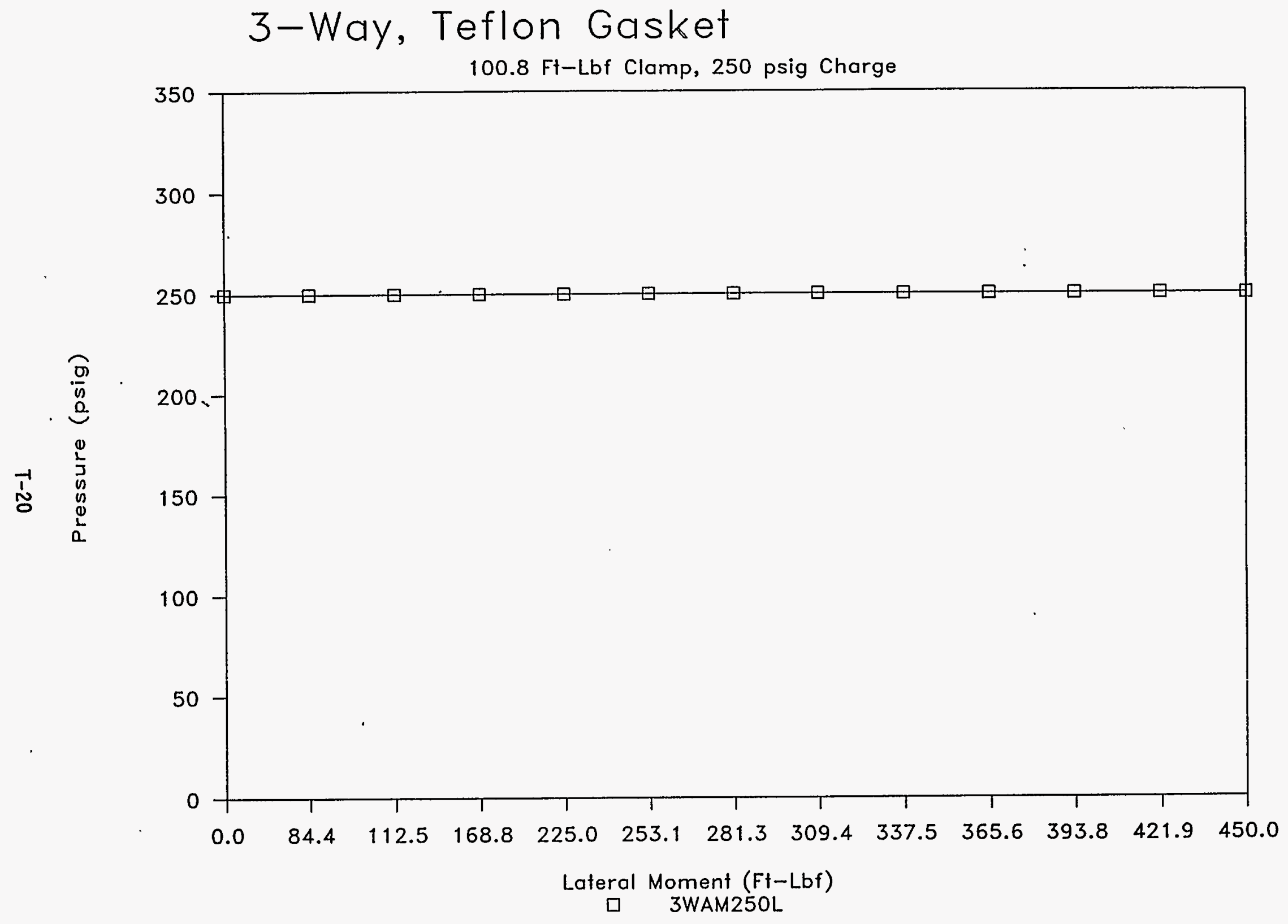


HHC-SD-WM-TRP-223

Rev. 0

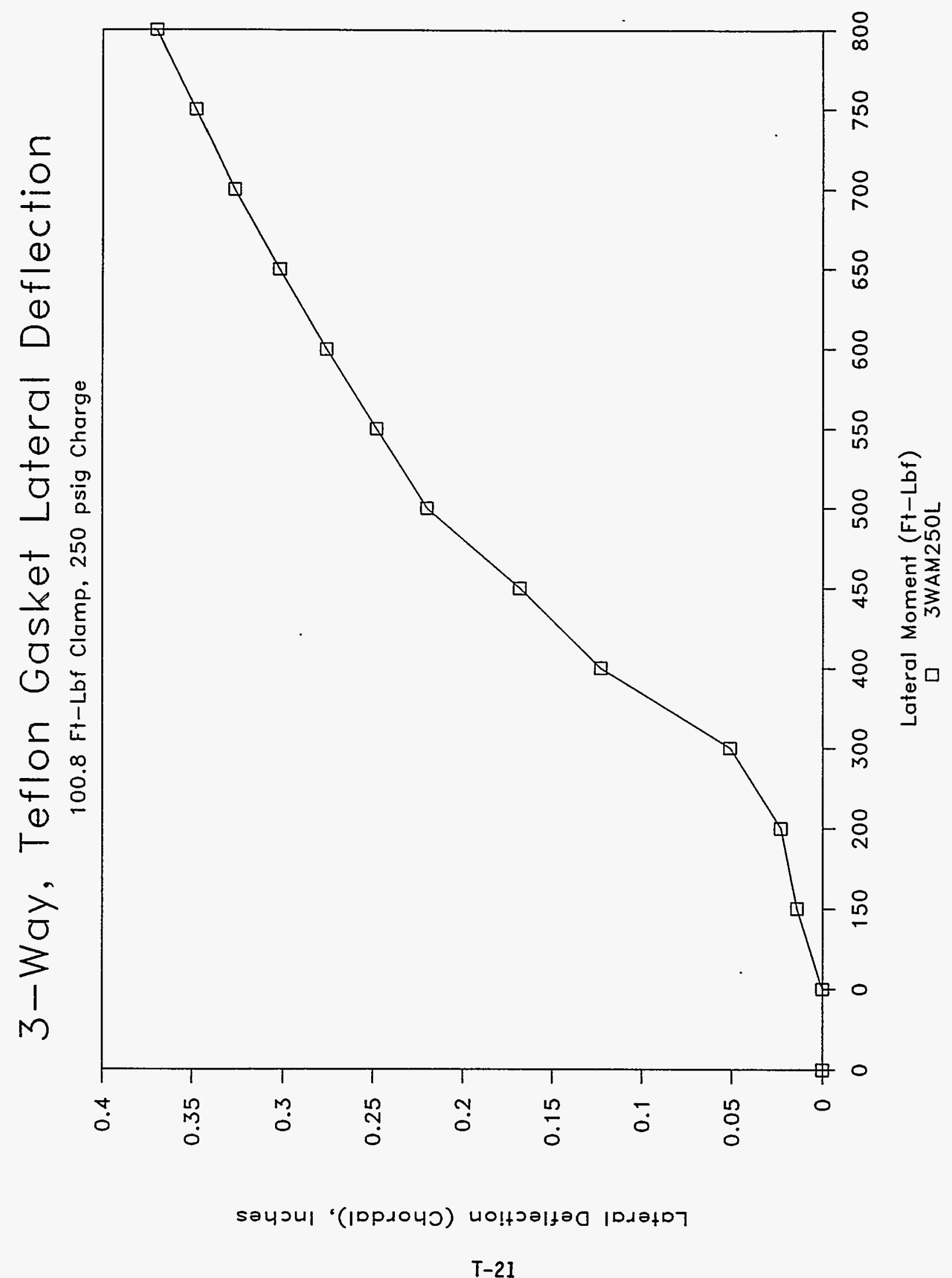


WHC-SD-WM-TRP-223 ReV 0

MAY 20,1994

3-WAY CONNECTOR, CONVENTIONAL, $100 \%$ TEFLON GASKET, AMBIENT TEMP. LEAK TEST - LATERAL EXTERNAL MOMENTS APPLIED

CLAMPING TORQUE $=100.8 \mathrm{FT}-\mathrm{LBF} \quad$ GRAPH NAME $=3$ WAM250L

CHARGE PRESSURE $=250$ PSIG

$\begin{array}{llllllll}\text { PRESSURE } & \text { ELAPSED } & \text { ELAAPSED } & \text { ELAPSED } & \text { LATERAL } & \text { MOMENT } & \text { IAATERAI } & \text { DEFFLECTIO } \\ \text { PSIG } & \text { MINUTES } & \text { SECONDS } & \text { DECIMAL } & \text { FORCE } & \text { ARM } & \text { MOMENT } & \text { (CHORDAL) } \\ & & & \text { MINUTES } & \text { IBS } & \text { FT. } & \text { FT-LBF. } & \text { INCHES } \\ \text { INPUT } & \text { INPUT } & \text { INPUT } & \text { COMPUTE } & \text { INPUT } & \text { INPUT } & \text { COMPUTE } & \text { INPUT }\end{array}$

$\begin{array}{rrrrrrrr}250 & 0 & 0 & 0.00 & 0 & 0.5625 & 0.0 & 0.000 \\ 250 & 2 & 7 & 2.12 & 0 & 0.5625 & 0.0 & 0.000 \\ 250 & 4 & 50 & 4.83 & 150 & 0.5625 & 84.4 & 0.014 \\ 250 & 6 & 56 & 6.93 & 200 & 0.5625 & 112.5 & 0.023 \\ 250 & 10 & 26 & 10.43 & 300 & 0.5625 & 168.8 & 0.051 \\ 250 & 11 & 56 & 11.93 & 400 & 0.5625 & 225.0 & 0.123 \\ 244 & 16 & 0 & 16.00 & 450 & 0.5625 & 253.1 & 0.168 \\ 244 & 21 & 0 & 21.00 & 500 & 0.5625 & 281.3 & 0.220 \\ 244 & 26 & 45 & 26.75 & 550 & 0.5625 & 309.4 & 0.248 \\ 241 & 34 & 0 & 34.00 & 600 & 0.5625 & 337.5 & 0.276 \\ 241 & 41 & 50 & 41.83 & 650 & 0.5625 & 365.6 & 0.302 \\ 240 & 49 & 0 & 49.00 & 700 & 0.5625 & 393.8 & 0.327 \\ 240 & 53 & 30 & 53.50 & 750 & 0.5625 & 421.9 & 0.348 \\ 240 & 57 & 0 & 57.00 & 800 & 0.5625 & 450.0 & 0.370\end{array}$




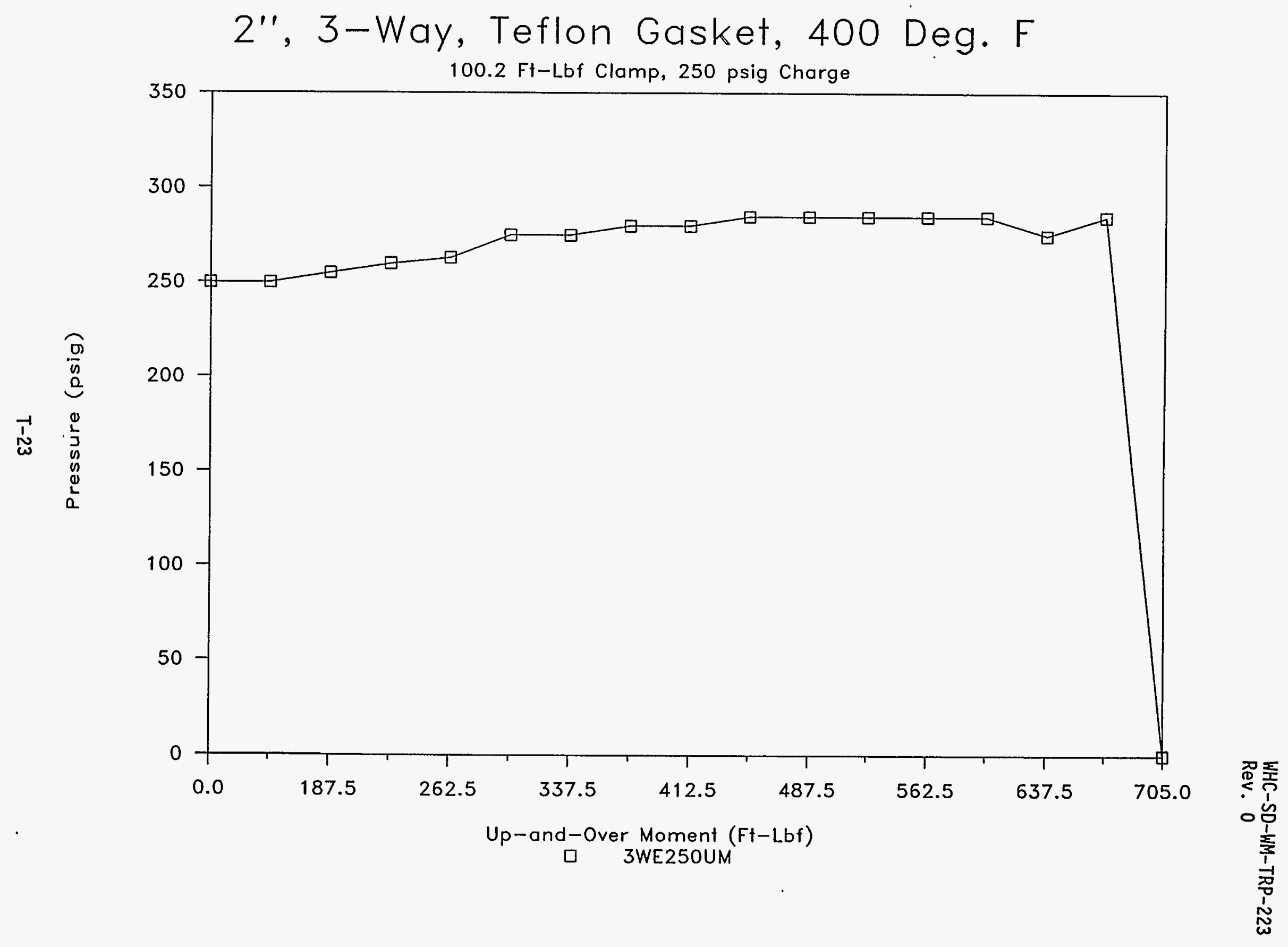


MAX 12,1994

2", 3-WAY CONNECTOR, 100\% TEFLON GASKET, 400 DEG. F TEMP. UP AND OVER MOMENT (UPWARD ROTATION)
CIAMPING TORQUE $=102.3 \mathrm{FT}-\mathrm{IBF}$
GRAPH NAME $=3$ WE250UM

CHARGE PRESSURE $=250$ PSIG

\begin{tabular}{|c|c|c|c|c|c|c|}
\hline $\begin{array}{l}\text { PRESSURE } \\
\text { PSIG }\end{array}$ & $\begin{array}{l}\text { HOLD } \\
\text { TIME } \\
\text { MINUTES }\end{array}$ & $\begin{array}{l}\text { HOLD } \\
\text { TIME } \\
\text { SECONDS }\end{array}$ & $\begin{array}{l}\text { HOLD } \\
\text { TIME } \\
\text { DECIMAL } \\
\text { MINUTES } \\
\text { COMPUTE }\end{array}$ & $\begin{array}{l}\text { MOMENT } \\
\text { FORCE } \\
\text { LBS. } \\
\text { INPUT }\end{array}$ & $\begin{array}{l}\text { MOMENTL } \\
\text { ARM } \\
\text { FT. }\end{array}$ & $\begin{array}{l}\text { UP\&OVR } \\
\text { MOMENT } \\
\text { FT-IBF. } \\
\text { COMPUTE }\end{array}$ \\
\hline $\begin{array}{l}250 \\
250 \\
255 \\
260 \\
263 \\
275 \\
275 \\
280 \\
280 \\
285 \\
285 \\
285 \\
285 \\
285 \\
275 \\
285 \\
0\end{array}$ & $\begin{array}{r}2 \\
2 \\
2 \\
2 \\
2 \\
2 \\
2 \\
2 \\
2 \\
2 \\
2 \\
2 \\
2 \\
42 \\
2 \\
2 \\
0\end{array}$ & $\begin{array}{r}4 \\
5 \\
3 \\
9 \\
7 \\
5 \\
3 \\
2 \\
11 \\
10 \\
3 \\
11 \\
1 \\
58 \\
2 \\
15 \\
0\end{array}$ & $\begin{array}{r}2.07 \\
2.08 \\
2.05 \\
2.15 \\
2.12 \\
2.08 \\
2.05 \\
2.03 \\
2.18 \\
2.17 \\
2.05 \\
2.18 \\
2.02 \\
42.97 \\
2.03 \\
2.25 \\
0.00\end{array}$ & $\begin{array}{l}0 \\
100 \\
125 \\
150 \\
175 \\
200 \\
225 \\
250 \\
275 \\
300 \\
325 \\
350 \\
375 \\
400 \\
425 \\
450 \\
470\end{array}$ & $\begin{array}{l}1.5 \\
1.5 \\
1.5 \\
1.5 \\
1.5 \\
1.5 \\
1.5 \\
1.5 \\
1.5 \\
1.5 \\
1.5 \\
1.5 \\
1.5 \\
1.5 \\
1.5 \\
1.5 \\
1.5\end{array}$ & $\begin{array}{r}0.0 \\
150.0 \\
187.5 \\
225.0 \\
262.5 \\
300.0 \\
337.5 \\
375.0 \\
412.5 \\
450.0 \\
487.5 \\
525.0 \\
562.5 \\
600.0 \\
637.5 \\
675.0 \\
705.0\end{array}$ \\
\hline
\end{tabular}

NOTE: THE LAST READING OF "O" WAS ACTUALLY THE POINT WHERE THE TEFLON SEAL TOTALLY FAILED. PRESSURE LOSS WAS SUDIEN AND COMPLETE. 


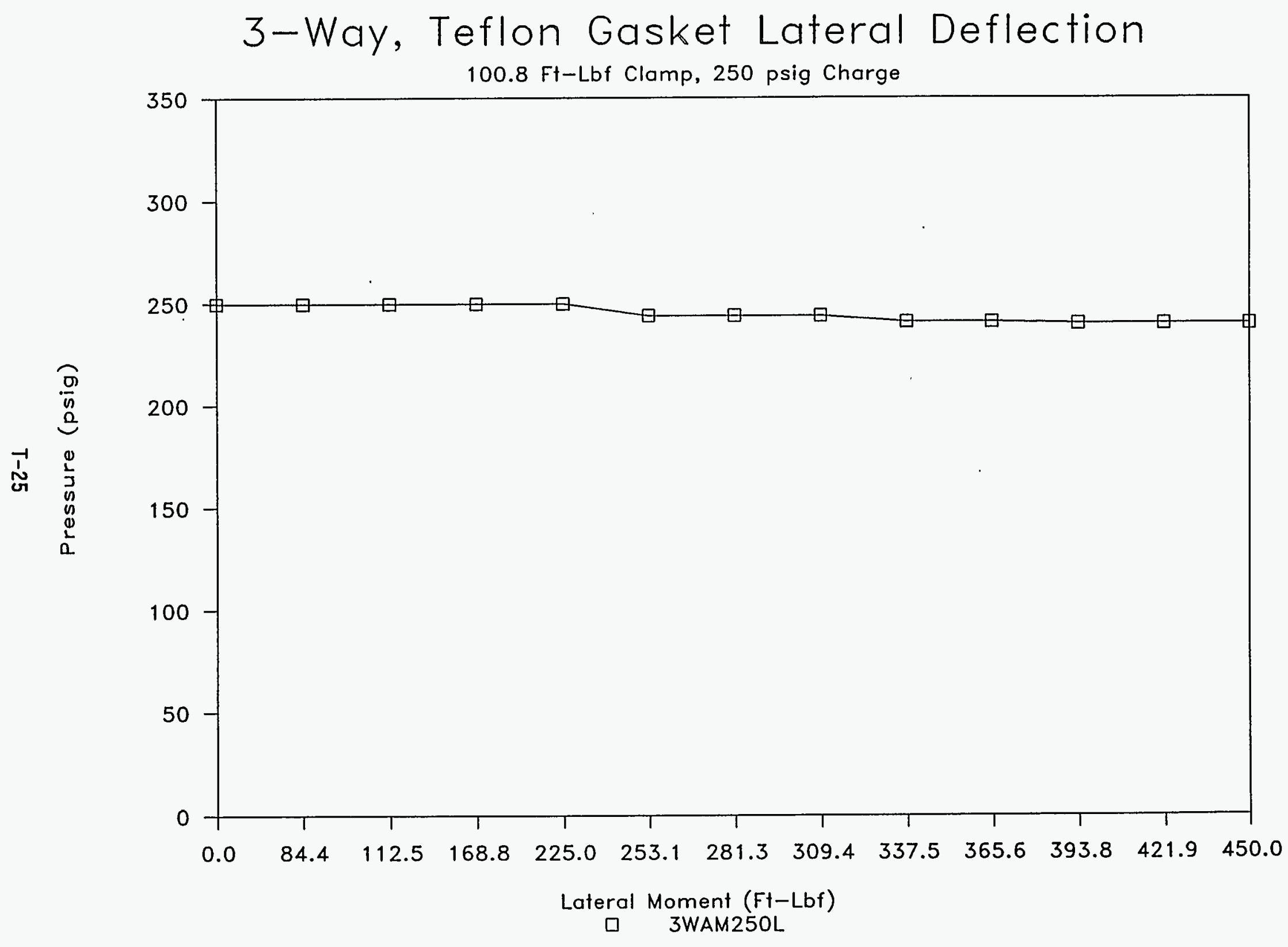


MAY 05, 1994

3-WAY, 2" CONVENTIONAL TEFLON BLOCK

AMBIENT TEMP. THERMAI CYCLE

STATIC LEAK TEST - NO EXTERNAL MOMENTS

CLAMPING TORQUE $=100.7$ FT-LBF

CHARGE PRESSURE $=250$ PSIG.

GRAPH NAME $=3$ WA250-1

\begin{tabular}{|c|c|c|c|}
\hline $\begin{array}{l}\text { PRESSURE } \\
\text { PSIG }\end{array}$ & $\begin{array}{l}\text { ELAPSED } \\
\text { MINUTES }\end{array}$ & $\begin{array}{l}\text { ELAPSED } \\
\text { SECONDS }\end{array}$ & $\begin{array}{l}\text { ELAPSED } \\
\text { DECIMAI } \\
\text { MINUTES }\end{array}$ \\
\hline INPUT & INPUT & INPUT & COMPUTE \\
\hline $\begin{array}{l}255 \\
250 \\
250 \\
250 \\
250\end{array}$ & $\begin{array}{l}0 \\
2 \\
4 \\
6 \\
8\end{array}$ & 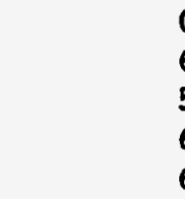 & $\begin{array}{l}0.00 \\
2.10 \\
4.08 \\
6.10 \\
8.10\end{array}$ \\
\hline
\end{tabular}




\section{WHC-SD-WM-TRP-223 ReV 0}

MAY 05, 1994

3-WAY, $2 "$ CONVENTIONAI TEFLON BLOCK

ELEVATED TEMP. THERMAL CYCLE

STATIC IEAK TEST - NO EXTERNAL MOMENTS

CLAMPING TORQUE $=100.7$ FT-LBF

CHARGE PRESSURE $=250$ PSIG.

GRAPH NAME $=3$ WE250-2

TEMPERATURE $=400$ DEG. $F$

\begin{tabular}{|c|c|c|c|}
\hline $\begin{array}{l}\text { PRESSURE } \\
\text { PSIG }\end{array}$ & $\begin{array}{l}\text { ELAPSED } \\
\text { MINUTES }\end{array}$ & $\begin{array}{l}\text { ELAPSED } \\
\text { SECONDS }\end{array}$ & $\begin{array}{l}\text { ELAPSED } \\
\text { DECIMAI } \\
\text { MINUTES }\end{array}$ \\
\hline INPUT & INPUT & INPUT & COMPUTE \\
\hline $\begin{array}{l}250 \\
240 \\
240\end{array}$ & $\begin{array}{l}0 \\
2 \\
5\end{array}$ & $\begin{array}{r}0 \\
54 \\
55\end{array}$ & $\begin{array}{l}0.00 \\
2.90 \\
5.92\end{array}$ \\
\hline
\end{tabular}


MAY 06, 1994

3-WAY, 2" CONVENTIONAL TEFLON BLOCK

ELEVATED TEMP. THERMAL CYCLE

STATIC LEAK TEST - NO EXTERNAL MOMENTS

CLAMPING TORQUE $=100.7 \mathrm{FT}-\mathrm{LBF}$

CHARGE PRESSURE $=250$ PSIG.

GRAPH NAME $=3$ WE250-3

TEMPERATURE $=400 \mathrm{DEG} . \mathrm{F}$

\begin{tabular}{|c|c|c|c|}
\hline $\begin{array}{l}\text { PRESSURE } \\
\text { PSIG }\end{array}$ & $\begin{array}{l}\text { ELAPSED } \\
\text { MINUTES }\end{array}$ & $\begin{array}{l}\text { ELAPSED } \\
\text { SECONDS }\end{array}$ & $\begin{array}{l}\text { ELAPSED } \\
\text { DECIMAI } \\
\text { MINUTES }\end{array}$ \\
\hline INPUT & INPUT & INPUT & COMPUTE \\
\hline $\begin{array}{l}255 \\
260 \\
260\end{array}$ & & & $\begin{array}{l}0.00 \\
3.07 \\
6.05\end{array}$ \\
\hline
\end{tabular}


WHC-SD-WM-TRP-223 ReV 0

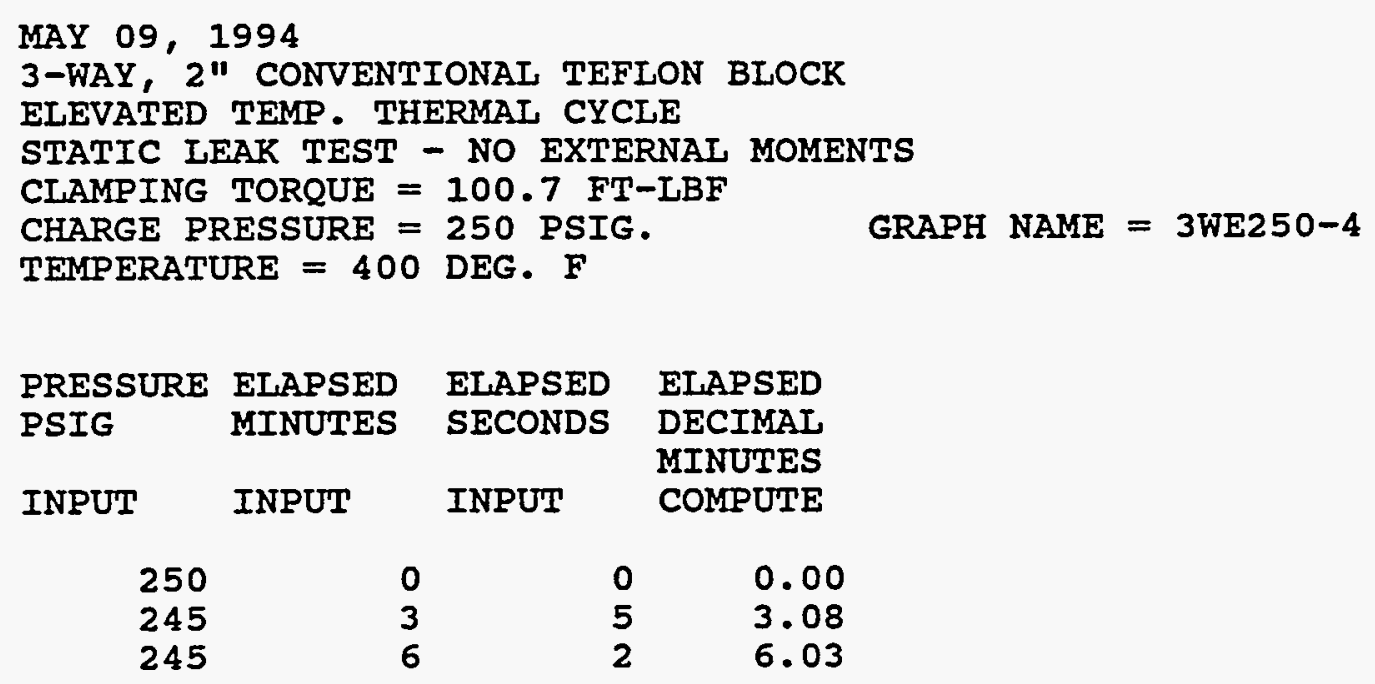

$\begin{array}{llll}245 & 6 & 2 & 6.03\end{array}$ 
MAY 10, 1994

3-WAY, 2" CONVENTIONAL TEFLON BLOCK

ELEVATED TEMP. THERMAL CYCLE

STATIC LEAK TEST - NO EXTERNAL MOMENTS

CLAMPING TORQUE $=100.7$ FT-LBF

CHARGE PRESSURE $=260$ PSIG.

GRAPH NAME $=\cdot 3$ WE260-5

TEMPERATURE $=400$ DEG. $\mathrm{F}$

\begin{tabular}{|c|c|c|c|}
\hline $\begin{array}{l}\text { PRESSURE } \\
\text { PSIG }\end{array}$ & $\begin{array}{l}\text { ELAPSED } \\
\text { MINUTES }\end{array}$ & $\begin{array}{l}\text { ELAPSED } \\
\text { SECONDS }\end{array}$ & $\begin{array}{l}\text { ELAPSED } \\
\text { DECIMAI } \\
\text { MINUTES }\end{array}$ \\
\hline INPUT & INPUT & INPUT & COMPUTE \\
\hline $\begin{array}{l}250 \\
250 \\
250\end{array}$ & & $\begin{array}{l}11 \\
21\end{array}$ & $\begin{array}{l}0.00 \\
2.18 \\
4.35\end{array}$ \\
\hline
\end{tabular}


WHC-SD-WM-TRP-223 ReV 0

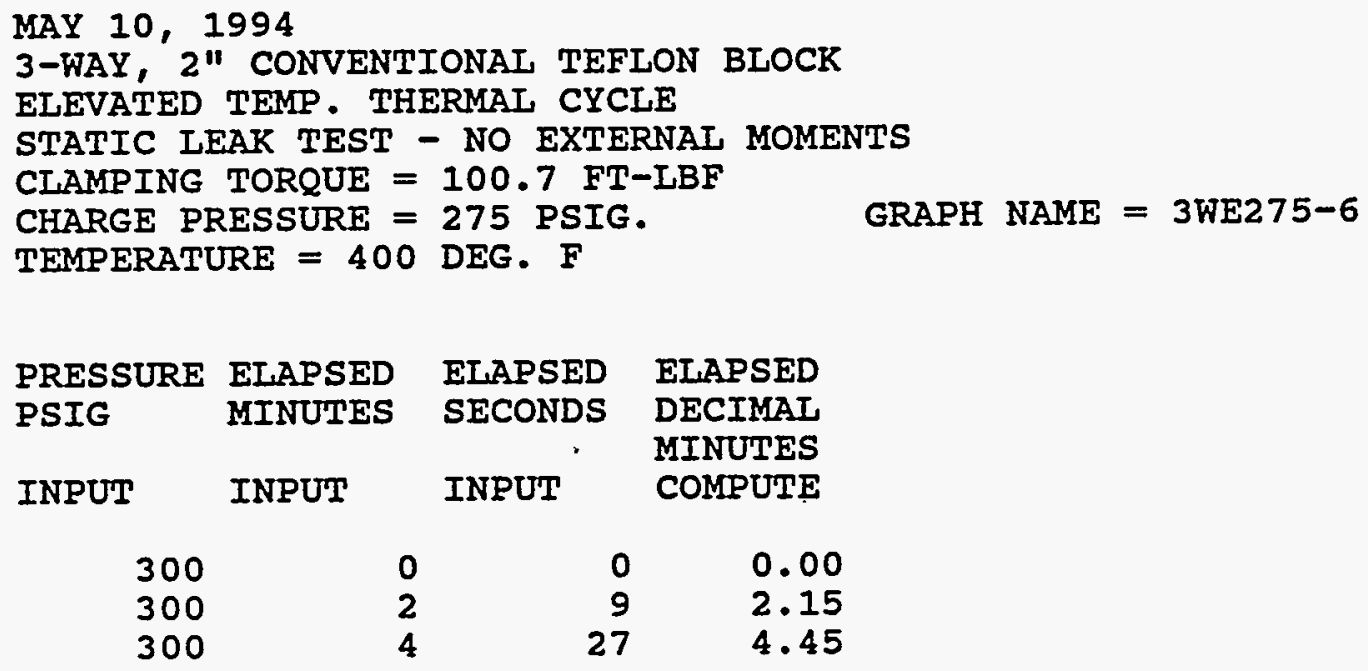

$\begin{array}{llll}300 & 4 & 27 & 4.45\end{array}$


WHC-SD-WM-TRP-223

Rev. 0

APPENDIX U: STATEMENT ATTESTING TO TEST PROCEDURES USED AND DATA CHECKS PERFORMED 
WHC-SD-WM-TRP-223

Rev. 0

\section{STATEMENT ATTESTING TO TEST PROCEDURES USED AND DATA CHECKS PERFORMED}

PERFORMANCE OF TESTS: Tests for this report were conducted during the period Apri1 27, 1994 to December 13, 1994. All of these tests were conducted in such a manner to meet the requirements of WHC-SD-TP-256 Rev. 0, "Jumper Connector Integral Seal Block Test PIan and Procedure, "Westinghouse Hanford Company, Richland, Washington, according to the procedure on pages 10-12 therein. Official issue date of the procedure was July 18, 1994. Tests conducted during the period April 27, 1994 to July 17, 1994 were performed under the auspices of the draft version of that procedure. To verify quality of data obtained during that period, it is hereby attested that there were no differences between the draft procedure and the final approved procedure that affected test methods or data collection. All tests were performed in the same laboratory, by the same personnel, using the same equipment, and the same method. All instruments used to collect data were in current calibration, traceable to the National Institute of Standards. Tests were performed by Test Technician Steven R. Jordan, assisted at various times by Senior Principal Engineer Edward S. Ruff.

QC WITNESS: WHC Quality Control Engineer Ernest N. Wegener witnessed testing performed on the 3-way ISB jumper connectors. He verified that instruments used to collect data were in current calibration, and that testing was performed in accordance with procedure. Testing performed on 2-in, 3-in, and 4-in single port ISB's was not witnessed by Mr. Hegener. For these tests, QC review of data was performed as described below.

DATA RECORD REVIEW: QC Engineer E.N. Hegener reviewed all laboratory notebook data entries for 2-in, 3-in, 4-in, and 3-way ISB's, and verified that it matched the data entered in spreadsheets used to graph results presented in the data appendices of this test report. Original data, as recorded during testing, is archived in the following documents:

Jordan, Steven R., 1994a, Laboratory Notebook \#HHC-N-817 (Hard Bound and Paginated), pp. 44-142, Westinghouse Hanford Company, Richland, Washington.

Jordan, Steven R., 1994b, Laboratory Notebook \#HHC-N-1088 (Hard Bound and Paginated), "3-Way Integral Seal Block Jumper Connector," Westinghouse Hanford Company, Richland, Hashington.

Jordan, Steven R., 1994c, PUREX Jumper Connector Integral' Seal Block Leak Test Data, WHC-SD-WM-DP-118, Rev. 0, Hestinghouse Hanford Company,

Richland, Washington.

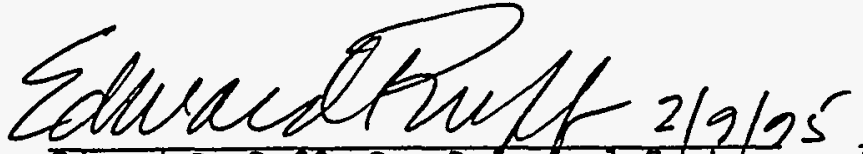

Edward S. Ruff, Sr. Princhpal Engineer Materials Engineering

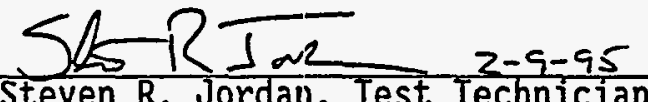

Steven R. Jordan, Test Technician Equipment Testing Laboratory 
WHC-SD-WM-TRP-223

Rev. 0

APPENDIX V: SOURCE REFERENCES FOR TEST CRITERIA

V-1 
From: $\quad$ Structural Margin Assessments

Phone: 372-2277

Date: December 6, 1993

Subject: QUALIFICATION OF MECHANICAL JUMPERS AND ELECTRICAL CONNECTIONS

To:

$\begin{array}{ll}\text { V. J. Cruz } & B 4-08 \\ \text { J. M. Light } & B 4-08 \\ \text { cc: T. J. Conrads } & H 5-55 \\ \text { S. K. Farnworth } & H 5-55 \\ \text { M. T. Husain } & \text { E6-37 } \\ \text { J. L. JuTyk } & H 5-57 \\ \text { D. S. Leach } & H 4-64 \\ \text { M. R. Lindquist } & H 5-57 \\ \text { J. R. Nicholson } & \text { E6-37 } \\ \text { E. C. Ocoma } & H 5-55 \\ \text { E. S. Ruff } & H 5-67 \\ \text { T. B. Salzano } & \text { E6-37 } \\ \text { File/LB } & \end{array}$

The qualification of the jumpers is in progress. So fiar, the effort has been directed in retrieving the historical work carried out by WHC, KEH, and PNL as well as other DOE sites on similar components. A summary of the findings with recommendations are presented herein.

The next step is to start the finite-element modeling and perform the analysis and evaluation. The experimental work on leakage test has also been initiated with the preparation of a test specification. Ed Ruff (from Materials and Welding Engineering group) is overseeing this phase of the project.

The findings of the review are itemized below with applicable recommendations, if any.

\section{General Review Comments}

The jumper qualifications carried out in the past, mainly concentrated on the jumper connector heads. No work has been found on qualifying the jumpers as a whole unit. Traditionally, AUTOPIPE has been used to calculate the reactions of the jumpers on the jumper connected head. Naturally, the seismic analysis would yield the resulting forces and moments from the jumper piping to the connector head. This approach assumes the connection of the jumper heads as rigid connectors for the piping. The qualification looks at the structural integrity of the connector head from these loads, applied torque to seal, pressure loads, and thermal loads.

The possibility of the seal failure, resulting in leakage, of the jumpers has not been addressed in the past. It needs to be determined 
if seal leakage during a seismic event is an issue. Action: D. Leach (date)

2. Proposed Work on MHTF Jumpers

The FDC Rev. 1 classifies the jumpers as safety class 2 . The design pressure and temperature (for the 2- and 3-inch waste transfer piping) is specified to be $400 \mathrm{psig}$ and $250^{\circ} \mathrm{F}$, respectively. It is assumed that the 4-inch and 3-way $1 / 2$ inch jumpers are under the same design conditions. The chemical compositions are listed in the FDC, Rev. 1, and will be used for seal selection. The seal material selection will be addressed under leakage test section.

Notes: The piping loads transferred to the connector heads are not specified yet. Worst case values used for PUREX and HWVP will be used for the analysis.

3. PUREX Jumpers

Structural analysis and evaluation was carried out for 2-inch jumpers for PUREX (HHC-SD-W028-DA-001). The analys is used ANSYS and was a rigorous analysis. The design pressure was $400 \mathrm{psig}$ and the design temperature was $240^{\circ} \mathrm{F}$. The seal used was Teflon. the evaluation used AISC and ASME allowables. The seal was not evaluated for integrity as only testing can qualify such a component.

4. HWVP Jumpers

Structural analysis and evaluation was carried out for 2-, 3-, and 4inch jumpers for HWVP. The report is not issued yet. It has been checked and independently reviewed. The analysis considered the critical components as identified in PUREX report. The operating pressure was $220 \mathrm{psig}$ and the design temperature was $390^{\circ} \mathrm{F}$. A series of leakage tests were also performed to find the minimum required torque to seal. The evaluation used AISC and ASME allowables. The seal material and its function were not addressed for seismic events. The connector head only was considered for seismic evaluation as one unit.

\section{Leakage Test for HWVP Jumpers}

A series of tests were run to bind the relationship between the applied torque, to the screw to seal the connector head, and the force on the block of the connector head. Furthermore, tests were run to find the torque valve to guarantee seal for 2-, 3-, and 4-inch connector heads under 250 psig pressure. The seal of choice was Teflon and original design of gasket retainers were used. 

at

WHC14

Subject: Project W320 Connector Needs

Message Contents

Ed,

This is in response to your last transmittal.

Safety Class: The current classification for these items of the Project is SC-3. If a higher level of "leak tightness" is certifyabie, we may want to upgrade in order to take the emphasis off of the containment value of the Tank Farm pits.

Nozzle Material: The project requires 300 series stainless, which is pretty standard.

Block Material: Same as above.

Cast or Machined Hooks: Either should be adequate. In researching the available components to build 4" connectors, I found that we' 11 be short 108 hooks for the Project needs. We may be in a bit of a jackpot with regard to casting houses anyway, so there is a strong chance we' 11 be going with machined hooks for the $4 "$ assemblies.

Temperature: The FDC requirements call for a 180 degree $F$ limit on temperature for purposes of structural design. The limit for routine operation is 120 degrees $F$. I wouldn't let this set the limit for overall testing.

Pressure: The pressure will be up about 350-400 psig. Everything is calculated right now and we're still kind of waiting to see what the pump vendors come back with based on our head and flow requirements.

Connector Sizes: He' 11 be using $1^{n}$ through $4^{\prime \prime}$ vertical and

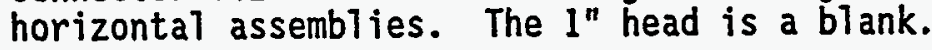

As far as filling out the data chart, I think we' 17 need to hold off on some of the items like "moment". KEH is doing an analysis to see what kind of thermally and mechanically induced loads will be on the nozzles and pit walls.

In talking with Tom, it seems that we need a meeting to really look at what testing is relevant, how it will be documented, and to what extremes it will be taken (e.g., ultimate destructive testing, etc.). If you folks set something up, give a call beforehand and I' 11 try to help you with whom to invite. Thanks. EGA 
[4] From: Thomas B Salzano at -KEH14 2/7/94 8:54AM (708 bytes: 11 in) To: M A Rezvani-Bafrouyeh at -WHC240, Edward S (Ed) Ruff at -HHC208

cc: Thomas J Conrads at -WHC74, Susan K Farnworth at -WHC208, Victor J Cruz at WHC231

Subject: JUMPER QUALIFICATION

$$
\text { Message Contents }
$$

The 4-in jumpers are for the Pit Exhaust System. The design temperature and pressure for this system are $120 \mathrm{~F}$ and $-10^{\prime \prime}$ of water, respectively.

The 2-in three way jumpers are for the Instrument Air System. The design temperature and pressure for this system are $125 \mathrm{~F}$ and $125 \mathrm{psig}$, respectively.

T. Salzano

372-2267 